\title{
gti.
}

\section{the Energy to Lead}

FINAL TECHNICAL REPORT

GTI PROJECT NUMBER 15347

\section{Low Cost Chemical Feedstocks Using an Improved and Energy Efficient Natural Gas Liquid (NGL) Removal Process}

Award Number:

Project Period:

Recipient Organization:

Partners:

Technical Contact:

Business Contact:

DOE Project Officer:

DOE Project Monitor:

DOE HQ Contact:

DOE Contract Specialist:

Distribution:
DE-FC36-03GO13150

$9 / 22 / 2003$ to $9 / 30 / 2009$

Gas Technology Institute (GTI)

1700 S. Mount Prospect Rd. Des Plaines, IL, 60018

Green Hi-Tek (GHT)

Illinois Institute of Technology (IIT)

Oak Ridge National Lab (ORNL)

Howard S. Meyer

1700 S. Mount Prospect Rd. Des Plaines, IL, 60018

Phone: 847-768-0955, Email:

howard.meyer@gastechnology.org

Nancy Bryla

1700 S. Mount Prospect Rd. Des Plaines, IL, 60018

Phone: 847/768-0911, Email: nancy.bryla@gastechnology.org

Bill Prymak, 303-275-4931, bill.prymak@go.doe.gov

Tim Ramsey,303-275-4933, tim.ramsey@go.doe.gov Bill Prymak, 303-275-4931, bill.prymak@go.doe.gov

Sara Wilson, 303-275-4922, sara.wilson@go.doe.gov

Unlimited 


\section{Legal Notice}

This information was prepared by Gas Technology Institute ("GTI") for United States Department of Energy (Sponsor).

Neither GTI, the members of GTI, the Sponsor, nor any person acting on behalf of any of them:

a. Makes any warranty or representation, express or implied with respect to the accuracy, completeness, or usefulness of the information contained in this report, or that the use of any information, apparatus, method, or process disclosed in this report may not infringe privately-owned rights. Conclusions and analysis of information gathered or generated for this report represent GTI's opinion based and with respect to which competent specialists may differ.

$b$. Assumes any liability with respect to the use of, or for any and all damages resulting from the use of, any information, apparatus, method, or process disclosed in this report; any other use of, or reliance on, this report by any third party is at the third party's sole risk. 


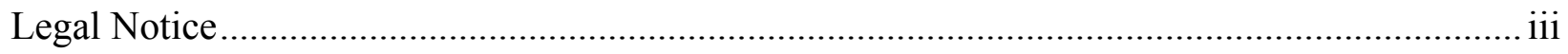

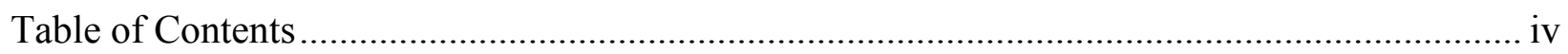

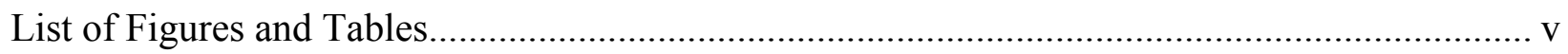

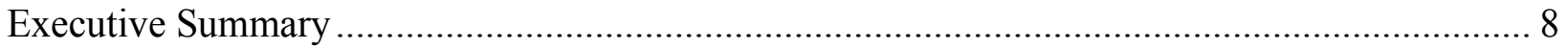

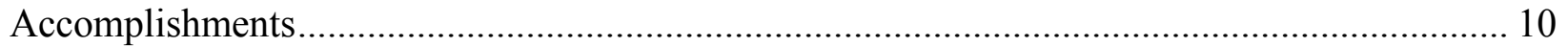

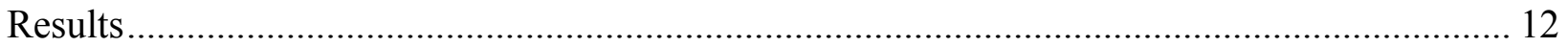

Task 1: Collect Relevant Physical Property and VLE Data for Preliminary Technical Verification and Design of Bench, Pilot and Commercial-Scale Units .................. 12

Task 2: Design and Construct Bench-Scale Unit for Laboratory Tests ................................ 15

Task 3: Conduct Bench-Scale Tests and Verify/Generate the Design Data for Pilot -Scale

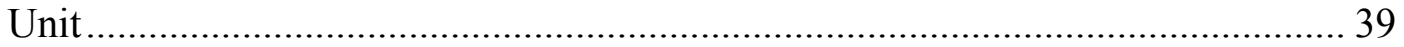

Task 5: Perform Theoretical and Experimental Study (R\&D Work) to Address Critical Process Development Issues ............................................................................... 60

Task 6: Design, Construct and Install a Pilot Unit and Conduct Field Tests ....................... 68

Task 7: Conduct Market Study and Prepare Commercialization Package........................... 80

Task 8: Attend Technical Meetings and Prepare Technical Reports ................................ 107

Attachment 1 - Request for Bid to Construct Pilot Plant Unit .............................................. 110

Attachment 2 - Proposal of FIROA Commercialization Strategy in the Environment of World Economic Crisis and Volatile Oil Pricing 143

Attachment 3 - Corrosion Performance of Aluminum 5182 Alloy in Calcium Chloride Solution

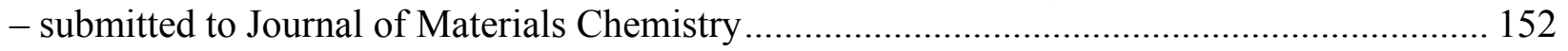

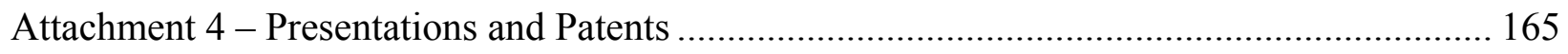




\section{List of Figures and Tables}

Page

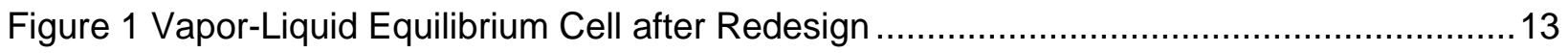

Figure 2 High-Pressure VLE Equipment....................................................................

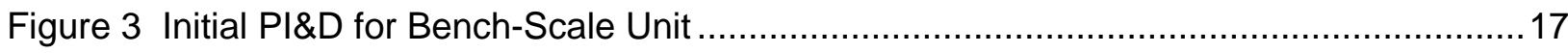

Figure 4 HYSYS Process Flow Diagram of Dehydration Section ........................................17

Figure 5 HYSYS Process Flow Section of Absorber Section .............................................2

Figure 6 Viscosity, Freezing Point and Weight Percent Curves of Aqueous Ethylene Glycol

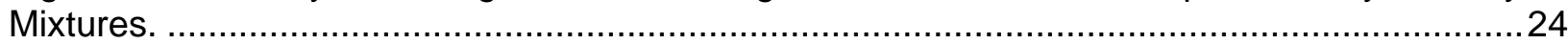

Figure 7 Design and Fabrication Schedule for Bench-Scale Unit........................................25

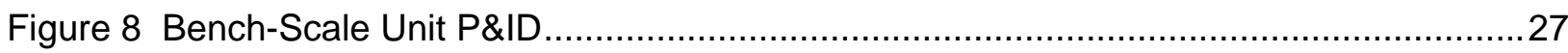

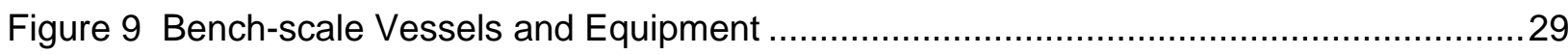

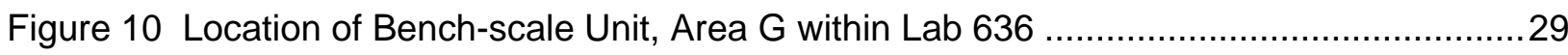

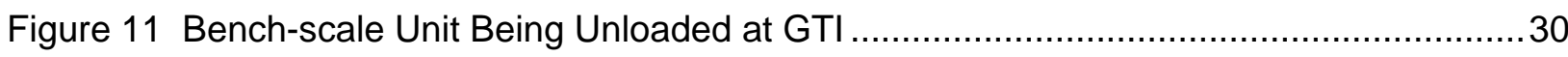

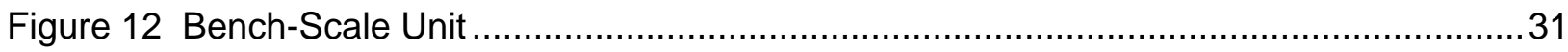

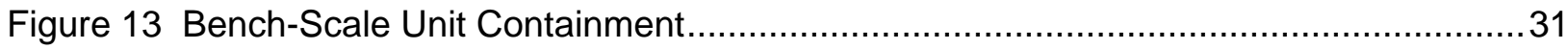

Figure 14 Absorber Column Prior to Heat Tracing and Insulation of Tubing/Piping ..................31

Figure 15 Bench-Scale Unit P\&ID.......................................................................... 44

Figure 16 Energy Cost for NGL Production as a Function of $C_{2}$ Recovery (gas richness - 2.2

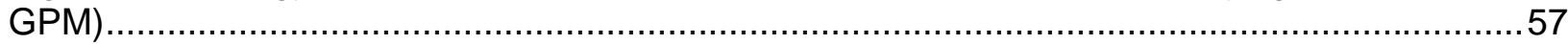

Figure 17 Energy Cost for NGL Production as a Function of $C_{2}$ Recovery (gas richness - 3.6

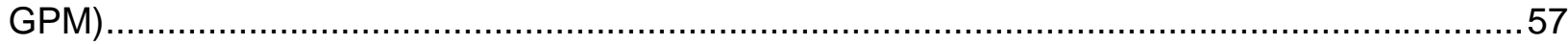

Figure 18 Percentage Comparison of the Overall Energy Cost of Various Processes ..............59

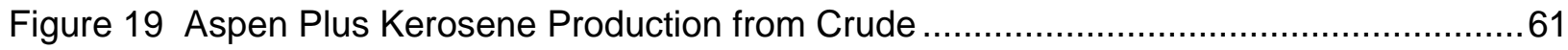

Figure 20 Methane Pressure Profile against Temperature in Syntroleum S-1.........................64

Figure 21 Methane Pressure Profile against Temperature in Sigma kerosene ........................64

Figure 22 Methane Solubility in Sigma Kerosene as a Function of a Dimensionless Time Unit (time per 2 seconds) at $-40^{\circ} \mathrm{F}$

Figure 23 Methane Solubility Testing In Syntroleum S-1 as a Function of a Dimensionless Time

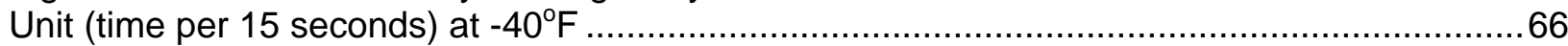

Figure $24 \mathrm{CO}_{2}$ Freezing Point for Various Levels of $\mathrm{CO}_{2}$ in the Feed Gas .............................67

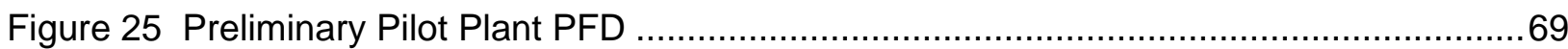

Figure 26 HYSYS Simulation for the Pilot Plant Unit .......................................................... 73

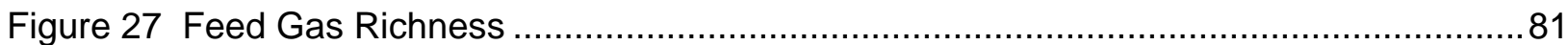

Figure 28 Energy Cost for NGL Production as a Function of $C_{2}$ Recovery (gas richness - 2.2 GPM)...... 
Figure 29 Energy Cost for NGL Production as a Function of $C_{2}$ Recovery (gas richness - 3.6 GPM).

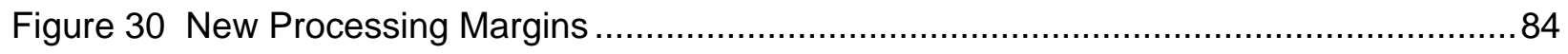

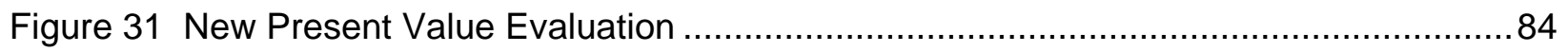

Figure 32 Simplified NGL Evaporation Scheme in ROS ............................................ 94

Figure 33 Energy Cost Savings for IROA Process for Rich Gas, 3.8 GPM NGL...................97

Figure 34 Cost Savings for IROA Process for Average Gas, 2.3 GPM NGL ........................98

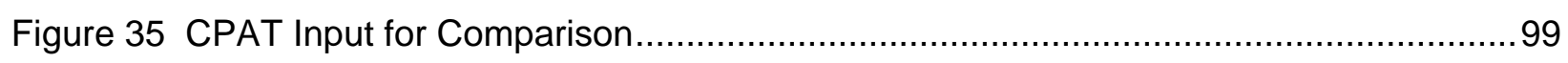

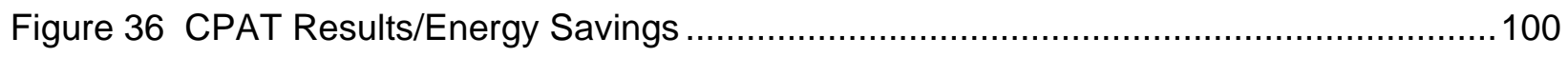

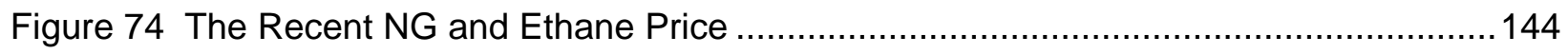

Figure 75 The Recent Propane and Butane Price ....................................................... 145

Figure 76 The Prices of Various NGL Components Compared with Crude Oil and Natural Gas 145

Figure 77 The Forecast of Chinese Gas Reserves and Domestic Production........................148

Figure 78 One of the Current Major Chinese Gas Project (Sichuan Gas Field and Pipeline).. 148

Figure 79 The Gas Fields and Major Gas Pipelines in China ........................................ 149

Figure 80 The New Kazakhstan-China Border- Guangdong Pipeline Under Construction......149

Table 1 Vendor Budgetary Quotes ....................................................................... 15

Table 2 Rich Oil Gas Feed Saturated with Water at $-40^{\circ} \mathrm{F}$ and 1000 psig............................ 18

Table 3 HYSYS Stream Summary for Dehydrator Section with EG Rate of $8 \mathrm{kgmol} / \mathrm{hr}$............18

Table 4 HYSYS Stream Summary for Dehydrator Section with EG Rate of $4 \ldots \ldots \ldots \ldots \ldots \ldots \ldots \ldots 19$

Table 5 HYSYS Stream Summary for Scrubber Section with Butane ..................................19

Table 6 HYSYS Stream Results of Methane Removal Section with Saturated Inlet Solvent

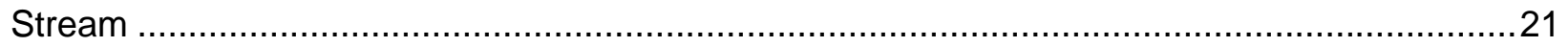

Table 7 HYSYS Stream Results of Absorber Section without Saturating Inlet Solvent Stream.. 22

Table 8 HYSYS Stream Summary for Dehydrator Section with EG Rate of $8 \mathrm{kgmol} / \mathrm{hr} . . . \ldots \ldots . . .25$

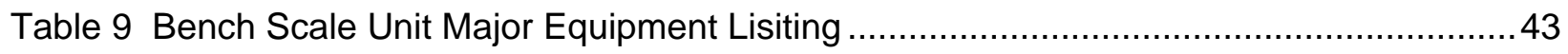

Table 10 Cases for Study and the Raw Gas Input Data .............................................46

Table 11 Glycol Solution Input Data ................................................................... 46

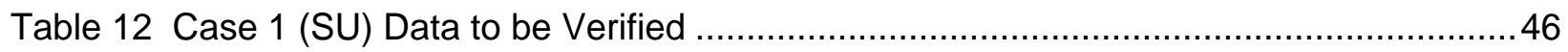

Table 13 Case 1 (SU) - Foam Data to be Verified (Evaluate the NGL condensate foaming) ${ }^{\star} . .47$

Table 14 Case 2 (R) Data to be Verified ........................................................................ 47

Table 15 Case 3 (L) Data to be Verified .................................................................. 47

Table 16 Cases to be Studied and the Raw Gas Input Data (IIT Report) ............................52 


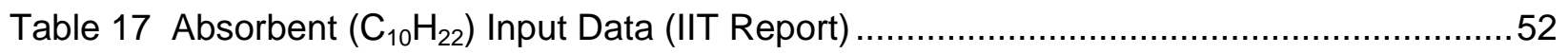

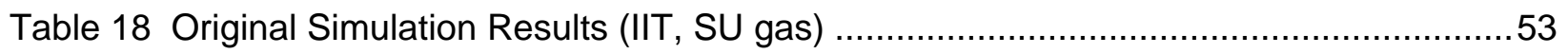

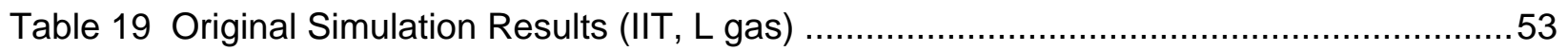

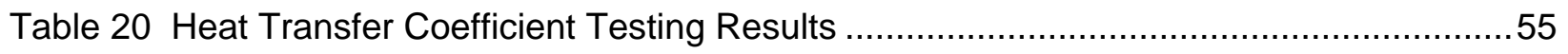

Table 21 Comparison of Calculated and US Patent \# 5,983,664 values .............................56

Table 22 Summary of the Sectional and Total Energy Cost of Various Processes ....................58

Table 23 Percentage Comparison of the Overall Energy Cost of Various Processes ...............58

Table 24 ASTM D-86 Comparison of Aspen Simulation of Kerosene Production from Mixed Crude Assay Blend specification and Synthetic Syntroleum Kerosene. ...............................6 62

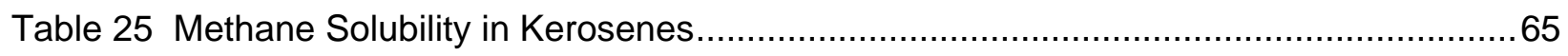

Table 26 Material and Energy Balance for Pilot Plant Unit .............................................. 74

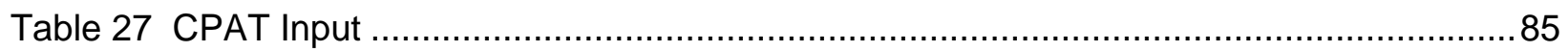

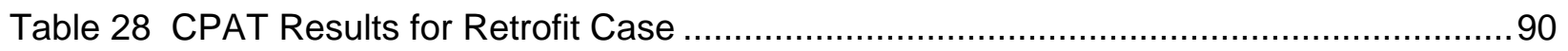

Table 29 Overall Energy Savings Compared to Previous Study ...........................................96

Table 47 Comparison of Total Energy Consumption: 1990-2030 (Unit: Million TOE) ...........146

Table 48 Chinese Energy Consumption Structure: 1990-2030 (Unit: Million TOE)..............146

Table 49 Comparison of Natural Gas Consumption: 1990--2030 (Unit: Million TOE).............147 


\section{Executive Summary}

The overall objective of this project is to develop a new low-cost and energy efficient Natural Gas Liquid (NGL) recovery process - through a combination of theoretical, bench-scale and pilot-scale testing - so that it could be offered to the natural gas industry for commercialization. The new process, known as the IROA process, is based on U.S. patent No. 6,553,784, which if commercialized, has the potential of achieving substantial energy savings compared to currently used cryogenic technology. When successfully developed, this technology will benefit the petrochemical industry, which uses NGL as feedstocks, and will also benefit other chemical industries that utilize gas-liquid separation and distillation under similar operating conditions. Specific goals and objectives of the overall program include: (i) collecting relevant physical property and Vapor Liquid Equilibrium (VLE) data for the design and evaluation of the new technology, (ii) solving critical R\&D issues including the identification of suitable dehydration and NGL absorbing solvents, inhibiting corrosion, and specifying proper packing structure and materials, (iii) designing, construction and operation of bench and pilot-scale units to verify design performance, (iv) computer simulation of the process using commercial software simulation platforms such as Aspen-Plus and HYSYS, and (v) preparation of a commercialization plan and identification of industrial partners that are interested in utilizing the new technology.

NGL is a collective term for $\mathrm{C}_{2}+$ hydrocarbons present in the natural gas. Historically, the commercial value of the separated NGL components has been greater than the thermal value of these liquids in the gas. The revenue derived from extracting NGLs is crucial to ensuring the overall profitability of the domestic natural gas production industry and therefore of ensuring a secure and reliable supply in the 48 contiguous states. However, rising natural gas prices have dramatically reduced the economic incentive to extract NGLs from domestically produced natural gas. Successful gas processors will be those who adopt technologies that are less energy intensive, have lower capital and operating costs and offer the flexibility to tailor the plant performance to maximize product revenue as market conditions change, while maintaining overall system efficiency.

Presently, cryogenic turbo-expander technology is the dominant NGL recovery process and it is used throughout the world. This process is known to be highly energy intensive, as substantial energy is required to recompress the processed gas back to pipeline pressure. The purpose of this project is to develop a new NGL separation process that is flexible in terms of ethane rejection and can reduce energy consumption by $20-30 \%$ from current levels, particularly for ethane recoveries of less than $70 \%$. The new process integrates the dehydration of the raw natural gas stream and the removal of NGLs in such a way that heat recovery is maximized and pressure losses are minimized so that high-value equipment such as the compressor, turbo-expander, and a separate dehydration unit are not required. GTI completed a techno-economic evaluation of the new process based on an Aspen-HYSYS simulation model. The evaluation incorporated purchased equipment cost estimates obtained from equipment suppliers and two different commercial software packages; namely, Aspen-Icarus and Preliminary Design and Quoting Service (PDQ\$). For a 100 MMscfd gas processing plant, the annualized capital cost for the new technology was found to be about $10 \%$ lower than that of conventional technology for $\mathrm{C}_{2}$ recovery above $70 \%$ and about $40 \%$ lower than that of conventional technology for $\mathrm{C}_{2}$ recovery below $50 \%$. It was also found that at around $40-50 \% \mathrm{C}_{2}$ recovery (which is economically 
justifiable at the current natural gas prices), the energy cost to recover NGL using the new technology is about $50 \%$ of that of conventional cryogenic technology. 


\section{Accomplishments}

This section includes a brief summary of significant accomplishments from the beginning of the project to the present. Items included are significant technical accomplishments, completed major go/no-go decision points and milestones, commercialization successes, patents applied for/received and any other important project highlights.

- A computer-simulation-based study was completed by GTI, IIT and GHT to compare the new IROA recovery process for natural-gas liquids (US patent US 6,553,784) with the latest design for Cryogenic Expansion Processes (CEP) (as described in US patent 5,983,664) for four different feed natural gas compositions [C2+: Super Rich (18\%), Rich (13\%), Lean $(8 \%)$, and Super Lean (3.7\%)] and four levels of C2 recovery $(2 \%, 35 \%, 70 \%$, and $95 \%)$.

0 The simulation results confirmed that the IROA process, unlike the traditional Refrigerated Oil Absorption process with low ethane recovery, could also recover up to 95\% of ethane similar to the Cryogenic Expansion Process.

o The IROA has the flexibility to reduce ethane recovery from $95 \%$ to $2 \%$ during operation with reduced energy consumption.

- Based on the annualized overall costs, the IROA was found to be more economical than the CEP in all cases except for higher $(95 \%)$ recoveries. Since the utility costs associated with IROA decrease with decreasing recovery of NGL, the IROA was found to be more economical than CEP at lower recovery levels.

- Further optimization studies identified two major areas of potential energy savings that make the IROA more economical for all levels of recovery:

o Maximizing the residue gas exit temperature which could reduce the refrigeration power requirement; and

o Separating the NGL condensate from the gas chiller to obtain a partial NGL liquid product which could reduce the power necessary for liquefying the NGL vapor from the Rich Oil Stripper (ROS).

- The Phase 1 project results were presented at the 2005 AIChE meeting in Atlanta, GA and to the DOE project review team.

- The original economic evaluation was performed using decane as the lean oil. Two alternative solvents have been identified to reduce the solvent costs and to ease modeling. Further testing was performed with kerosene and with a synthetic coal-derived lean oil to determine their applicability.

- Task 1 was completed during the second quarter of 2006 in that sufficient relevant physical property and VLE data were collected for preliminary technical verification and design of bench, pilot, and commercial-scale units.

- An engineering/fabricator was selected for the design and fabrication of the bench-scale unit. The unit was to be delivered to GTI at the end of January 2007.

- A request for quotes was submitted to nine $\mathrm{E} \& \mathrm{C}$ companies for the design and construction of the pilot plant. Three bids were received, but none was acceptable. GTI undertook responsibility for the design and construction of the pilot plant unit.

- The bench-scale unit was delivered to GTI's laboratory facility at the end of March 2007 with all completion to be performed at GTI by the fabrication personnel.

- A new pilot plant design package was issued as a request for bids to eight fabrication shops. Bids were received from two companies. 
- The status of the program was presented through the poster session, open AIChE meeting, and the closed DOE peer review meeting in April 2007.

- A meeting was held with EnCana Corporation on June 28, 2007 to discuss their hosting the pilot plant meeting. They appeared very interested.

- The bench-scale unit was remanded to GTI during December 2007 to complete installation and prepare for the HAZOP analysis.

- The bench-scale unit HAZOP analysis was completed during March 2008. While most of the issues can be resolved by operational control, several mechanical changes were required.

- A new patent application was approved for publication during January 2008. An analysis shows significant additional energy savings can be achieved for retrofit applications as well as for new plants. For example, it is possible to replace only the traditional dual-absorbent absorption column (with sponge oil stage) in existing plants with the IROA single-absorbent column to increase $\mathrm{C}_{2}$ recovery rate from $\sim 60 \%$ to $\sim 90 \%$, or to be adjustable between $35 \%$ $95 \%$. If an integrated demethanizer-deethanizer is used in retrofitting an existing plant, the residue gas re-compressor could be entirely eliminated or substantially reduced in size to lower the operating costs.

- Engineering modeling studies have shown that utilizing compact plate and fin heat exchanger technology could greatly reduce the cost and increase the energy efficiency of the IROA process.

- A design package was prepared for the plate frame heat transfer reactors that would be used to transfer the design philosophy to an engineering contactor.

- Construction of the Bench-Scale Unit was completed and commissioning was begun during November 2009.

- Contact was made with a commercial company that manufacturers compact plate and fin heat exchanger technology.

- Simulation modeling based on the newly applied-for patent application (FIROA) indicates about one third energy savings for the $95 \%$ ethane recovery from super rich natural gas case that was not economic under the previous configuration (IROA).

- Retrofit applications were examined as an early market penetration path. Incorporating the FIROA designs into straight refrigeration plants can significantly improve NGL recoveries, however, the savings for the FIROA progress are still greater than the straight refrigeration option. 


\section{Results}

This project is divided into eight tasks to be performed over six years.

\section{Task 1: Collect Relevant Physical Property and VLE Data for Preliminary Technical Verification and Design of Bench, Pilot and Commercial-Scale Units}

The accurate values of the distribution coefficients (commonly referred to as K-values) of hydrocarbons gases in various absorbing liquids (e.g., kerosene and N-decane) are important for accurate computer simulation of the new technology. Unfortunately, notable inconsistency existed among available K-value sets provided in various well-known handbooks, papers, and reports. A literature review was conducted to compile experimental $\mathrm{K}$ values for kerosene and $\mathrm{N}$ decane-natural gas systems for the temperature and pressure range relevant to this project. It was found that reliable experimental data was not available in the literature and experiments needed to be conducted at GTI to measure accurate VLE data using GTI's VLE unit. For the preliminary evaluation study, $\mathrm{K}$ values were calculated and compared with existing literature data using various equation-of-state based models such as Peng-Robinson (PR), Soave-Redlich-Kwong (SRK), ideal gas and other available literature correlations (e.g., Chao-Seader, McWilliams) and charts (e.g., DePriester). For the preliminary simulation work, PR model was selected because the predicted K-values using this model agree well with the existing experimental literature data for most systems.

Figure 1 is the schematic of the Vapor-Liquid Equilibrium Cell new configuration enabling both continuous and batch mode selection in the gas phase. Figure 2 is a photograph of the unit. A pressure transducer measures the partial pressure within the cell for both the batch and continuous mode operations. This gives the dynamic concentration of gas being absorbed into the liquid. The inlet and outlet gas composition was measured by a gas chromatograph as seen in Figure 1 when the cell is operating in the continuous mode. Gas samples were analyzed with a Varian 4900 Micro GC. The sample line has a Genie membrane filter to remove any liquid that might be carried with the gas before the sample was send to the Micro GC. This bypass flow and the vent from the Micro GC sample flow directed to a low-pressure vent scrubber before being discharged into the vent. Liquid samples were also taken at steady-state conditions for gas loading analysis in both the batch and continuous mode of operation. Liquid samples were analyzed with a Varian $3800 \mathrm{GC}$ custom modified to accept samples from the VLE system. The sampling valve was inside the thermally controlled chamber. The carrier gas from the GC was routed to the VLE chamber and to the sample valve. To ensure liquid homogeneity, mixing was accomplished with a variable speed stirrer. 


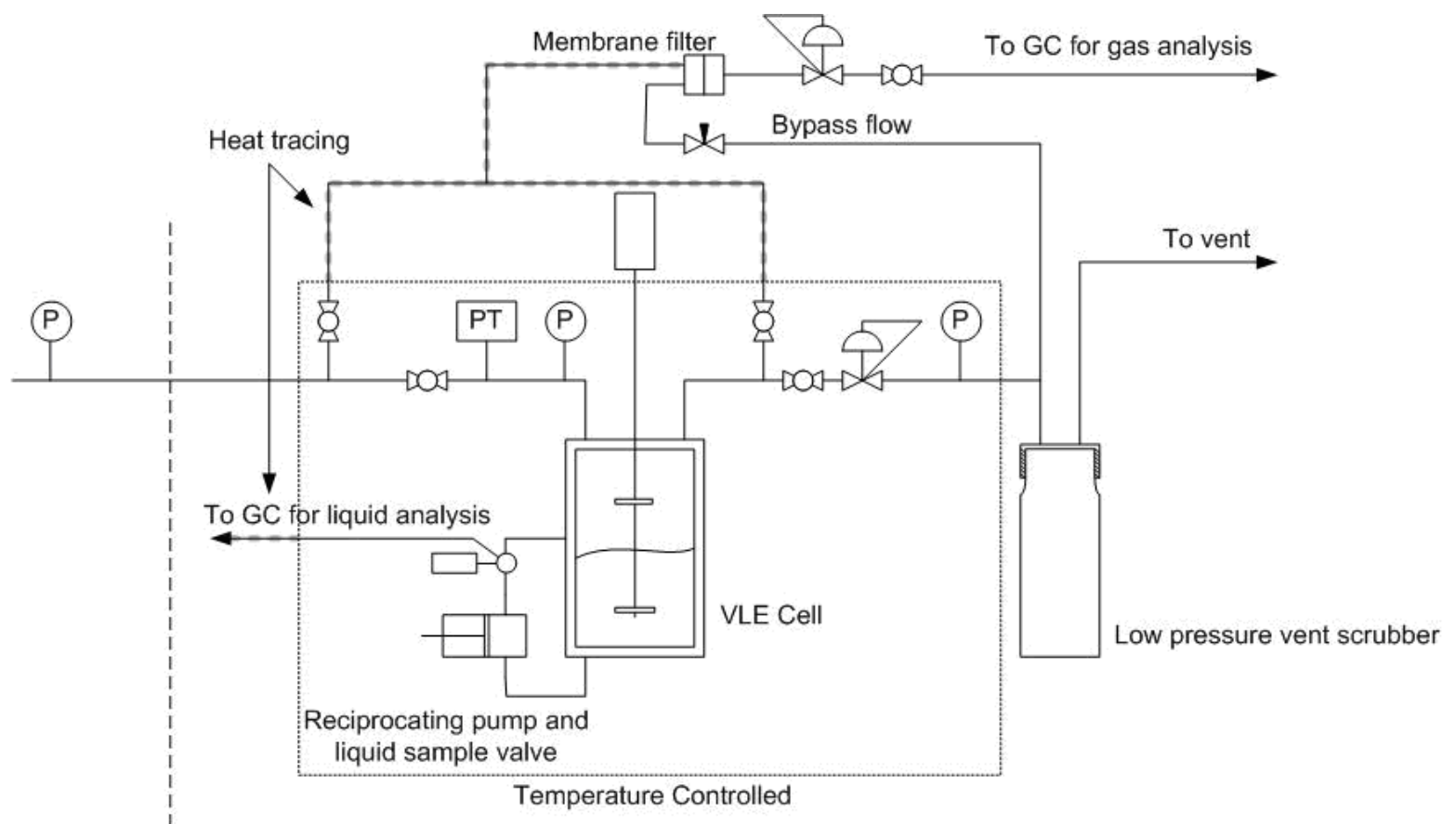

Figure 1 Vapor-Liquid Equilibrium Cell after Redesign

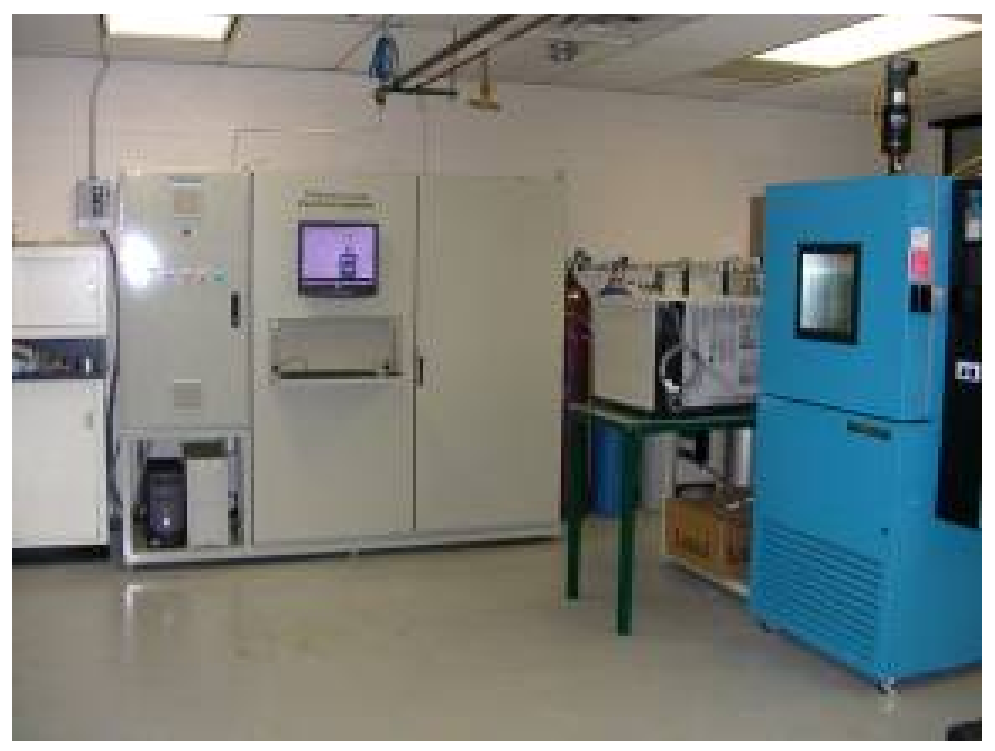

Figure 2 High-Pressure VLE Equipment

Obtaining solubilities and mass transfer properties in the solvent using this experimental technique required knowledge of either the gas or the liquid phase mass transfer coefficient. To interpret the absorption rate measurements and reduce those to kinetic constants will also require knowledge of the concentrations at the inlets and outlets as defined by the flux equations below. The mass transfer coefficients, $\mathrm{k}_{\mathrm{g}}$ and $\mathrm{k}_{\mathrm{l}}$, are characteristics of the mass transfer equipment. In order to obtain the gas mass transfer coefficient for the GTI reactor, one needs to also operate 
under continuous gas flow conditions. The newly redesigned VLE equipment will give us the details (temperature, pressure and composition) of the phases being contacted, the two equations in Equations 2 and 3 contain the fluxes $\mathrm{N}_{\mathrm{y}}$ and $\mathrm{N}_{\mathrm{x}}$ of each transferring component through the vapor and liquid phases, respectively and the concentration of the component in each phase at the interface, $\mathrm{y}^{\mathrm{i}}, \mathrm{x}^{\mathrm{i}}$ are still unknowns. If we combine these equations into a form using the over-all gas-based mass transfer coefficient, see Equation Set 1, we remove the extra two interface concentration unknowns.

$$
\begin{gathered}
N_{y}=k_{g}\left(y^{\text {bulk }}-y^{i}\right) \\
N_{x}=k_{l}\left(x^{i}-x^{b u l k}\right) \\
N_{y}=K_{g}\left(y^{\text {bulk }}-H x^{\text {bulk })}\right) \\
N_{x}=K_{l}\left(\frac{y^{\text {bulk }}}{H}-x^{\text {bulk })}\right)
\end{gathered}
$$

When we operate in batch in both gas and liquid in the reactor we integrate the following equation

to give

$$
\begin{gathered}
V_{g} \frac{d C_{g}^{b}}{d t}=-k_{l} a\left(m C_{g}^{b}-C_{l}^{b}\right) \\
\ln \left[\frac{C_{g}^{b}-C_{g}^{e q}}{C_{g}^{i}-C_{g}^{e q}}\right]=-\frac{\left(m V_{l}+V_{g}\right)\left(k_{l} a\right) t}{V_{g} V_{l}}
\end{gathered}
$$

for a dynamic solution to the value of the liquid mass transfer coefficient directly from the initial and time dependent concentration readings. Pressure readings at the initial and equilibrium points can then directly be used to obtain Henry's coefficient as follows

$$
H=\frac{P_{f}}{\left(P_{i}-P_{f}\right)} \frac{V_{l}}{V_{g}} R T
$$

which is the solubility multiplied by RT.

The feasibility simulations were based on ethylene glycol as the solvent for dehydration and decane as the absorbing solvent. Literature data as well as binary coefficients within the Aspen Plus and Aspen HYSYS simulation software packages were sufficiently defined for our current needs. Additional data will be obtained under Task 5 to address process development improvements such as alternative solvents. 


\section{Task 2: Design and Construct Bench-Scale Unit for Laboratory Tests}

A detailed P\&I diagram and equipment design data sheets was prepared as shown in Figure 3 A decision was made to place the bench-scale equipment in GTI's laboratory for testing rather than placing it in the field at this time. This will provide for both better control of feed conditions and reduce the costs of operating in the field away from GTI home offices.

A bid package was prepared and sent for bid. The process flow diagram (PFD) was completed, reviewed, refined, and distributed to several vendors for a budgetary bid. It was used primarily for communications and defining the unit, pressures, flow rates and temperatures. The flow diagram contains only the major components of the core system. The features of the bench-scale unit are given here. Four bids were received (See Table 1) and reviewed. The Vendor \#3 bid was selected for procurement. The initial estimated time for delivery of the bench-scale unit was April 2006.

\begin{tabular}{|c|c|}
\hline Vendor & Budgetary Quote \\
\hline Vendor \#1 & $\$ 177 \mathrm{k}$ \\
\hline Vendor \#2 & $\$ 350 \mathrm{k}$ \\
\hline Vendor \#3 & $\$ 100 \mathrm{k}$ \\
\hline Vendor \#4 & $\$ 180 \mathrm{k}$ \\
\hline
\end{tabular}

Table 1 Vendor Budgetary Quotes

As can be seen from in Figure 3, the main unit where dehydration and absorption will take place is a 4-inch-outer-diameter by 36-inch-high bench-scale packed bed reactor. It has both co- and counter-current capabilities. The dehydration can be run in counter-current mode, while the cocurrent mode will applied for the absorption section. The flow-rate of simulated feed gas through the packed bed was 20-100 scfh. The operating range for the bench-scale packed bed reactor is from -40 to $300^{\circ} \mathrm{F}$ and from atmospheric to $1000 \mathrm{psig}$.

Heat may also be generated when large amounts of solute are absorbed into the liquid phase, due to the heat of solution. The resulting change in temperature along the height of the absorber column may affect performance of this column. This problem can be avoided by adding cooling coils to the column or by using the excess chilled ethanol circulator used for the heat exchanger at $-40^{\circ} \mathrm{F}$. Using this ethanol in capacity to cool the jacketed small column may be sufficient to control temperature this way. A thermocouple, as shown in the diagram, will regulate the circulation, as necessary.

The cocurrent packed column for the absorber was chosen for several reasons. In cocurrent downflow, the gas is forced to move in a direction opposite to its natural direction/buoyancy, thereby enhancing its residence time and contact efficiency with the liquid. Of course, if the liquid velocity is not high enough, i.e. below the gas rise velocity, the gases will gradually accumulate at the column top. Cocurrent designs also have lower pressure drops and are not subject to the flooding limitations that are commonly experienced in countercurrent reactors. These features result in less expensive equipment costs. 
The counter-current packed column for the dehydrator was also chosen for several reasons. The main reason for counter-current flow is that a temperature gradient plays an important role in the separation. In order to minimize the methane exiting the bottom of the column, the bottom stage must be held above its boiling point, and thus the gas will help maintain this temperature. A heater and humidifier are placed prior to the inlet of the column as can be seen in Figure 3. A HYSYS simulation was constructed from the original IIT simulation and is shown in Figure 4. The size of the column used in HYSYS was in accordance to the packed column that will be constructed. This key difference between using the standard column templates and this three phase counterpart is that the default solver for three phase columns is the "Sparse Continuation" solver which is an advanced solver designed to handle three phase, non-ideal chemical systems, that other solvers cannot.

V-100 in Figure 3 will be the jacketed stainless steel hydrating vessel rated at 1000 psi with sparger and small ethanol circulator to allow for temperature dependent hydration of gases that saturates the dry rich gas before entering the dehydrator. The sensitivity of water removal was tested and a comparison can be seen by comparing Table 3 and Table 4 . The recovery of the hydrocarbons was not found to be sensitive to the amount of EG under these conditions as can be seen. The system is closed until a recovery of the rich is desired and n-butane was selected. It recovers all of the hydrocarbons, but methane well as illustrated in Table 5. This recovery is not sensitive to temperature, but is sensitive to the amount of butane used. Thus the more butane used, the more methane recovered in the liquid. The absorber section is illustrated in Figure 5 with the corresponding streams is summarized in Table 6. Table 7 illustrates that saturating the inlet solvent stream only improves performance by $6 \%$ under the same conditions. It will be left to experiment to determine the efficiency of saturation the accuracy of such prediction by HYSYS. The next section will cover some theory defining the results from experiment. 


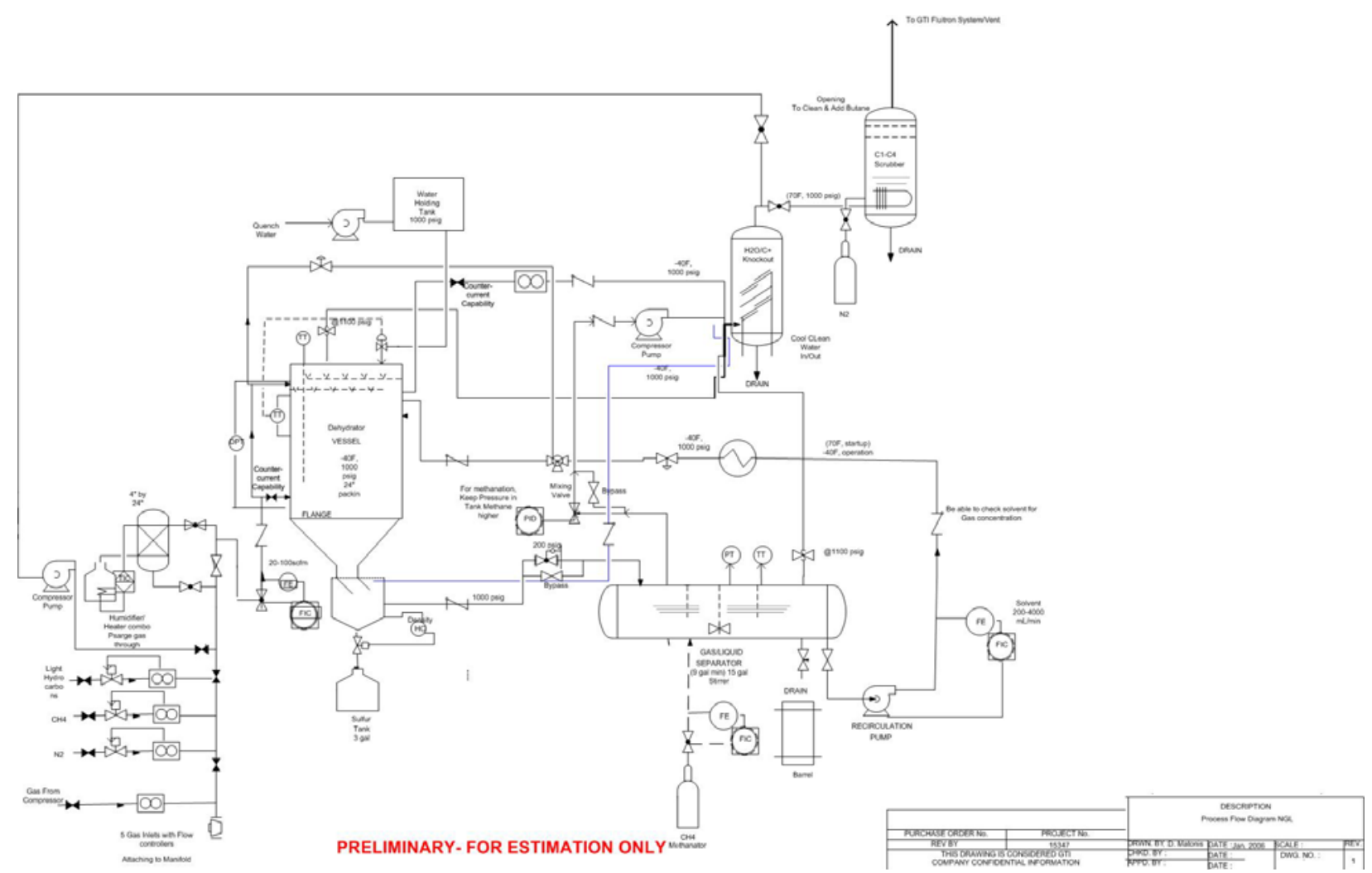

Figure 3 Initial PI\&D for Bench-Scale Unit

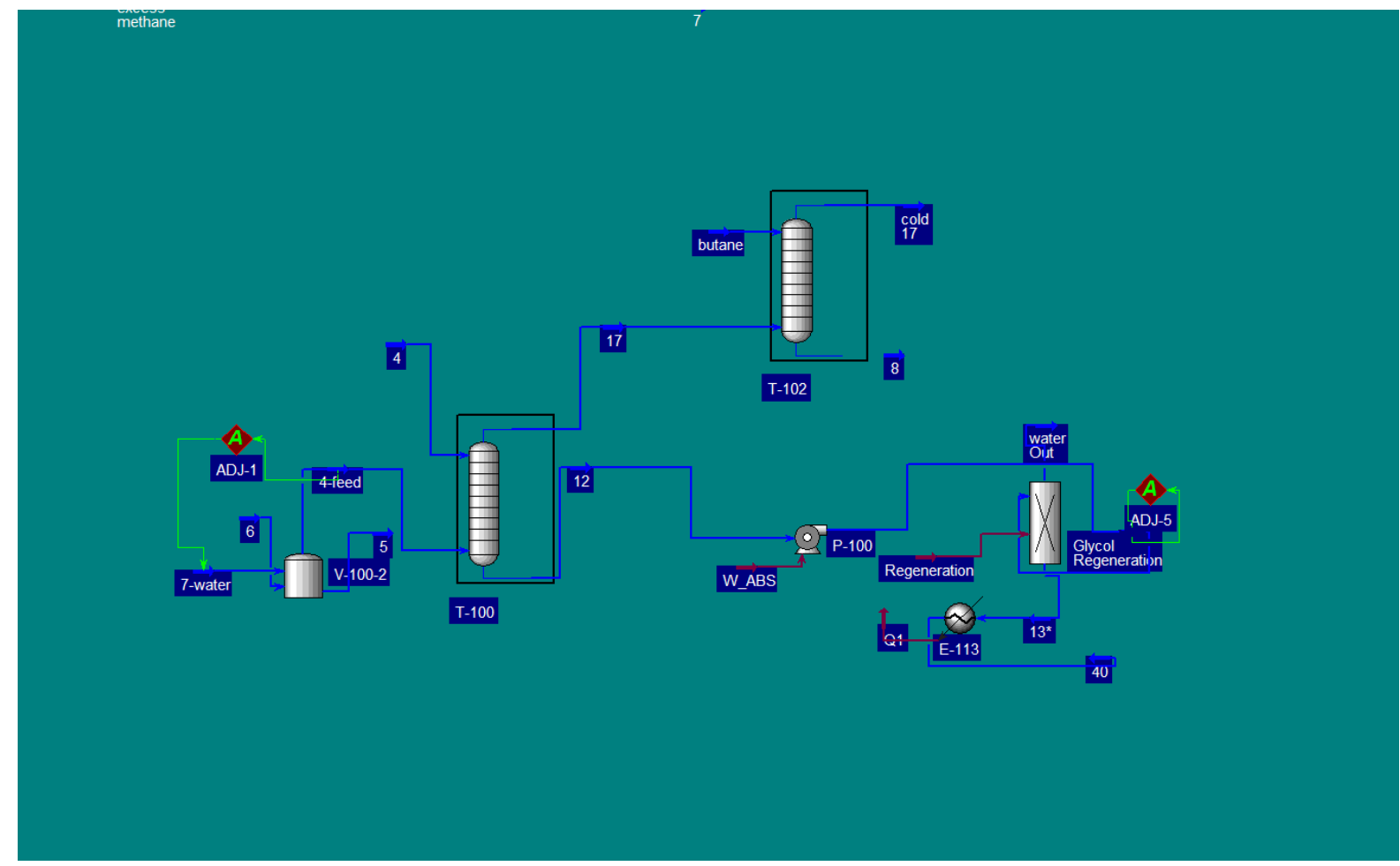

Figure 4 HYSYS Process Flow Diagram of Dehydration Section 


\begin{tabular}{|c|c|c|c|c|}
\hline Case & \multicolumn{4}{|c|}{ Rich } \\
\hline Liq. \% & \multicolumn{4}{|c|}{12.47 (IIT 12) } \\
\hline Composition & $\begin{array}{c}\text { Inlet } \\
\text { Gas }\end{array}$ & $\begin{array}{c}\text { Condensate } \\
\text { Fraction }\end{array}$ & $\begin{array}{c}\text { Condensate } \\
/ \text { Inlet Gas }\end{array}$ & $\begin{array}{c}\text { Condensate } \\
/ \text { Total } \\
\text { Recovery }\end{array}$ \\
\hline Unit & Tot=1.0 & Tot=1.0 & $\%$ & $\%$ \\
\hline $\mathrm{C}_{1}$ & 0.8695 & 0.0738 & 8.49 & $\mathrm{NA}$ \\
\hline $\mathrm{C}_{2}$ & 0.0792 & 0.0212 & 26.77 & 38.2 \\
\hline $\mathrm{C}_{3}$ & 0.0332 & 0.0165 & 49.70 & 50.7 \\
\hline $\mathrm{C}_{4}+$ & 0.0181 & 0.0132 & 72.93 & 73.7 \\
\hline Total & 1 & 0.1247 & $\mathrm{NA}$ & 48.1 \\
\hline
\end{tabular}

Table 2 Rich Oil Gas Feed Saturated with Water at $-40^{\circ} \mathrm{F}$ and 1000 psig

\begin{tabular}{|c|c|c|c|c|c|}
\hline & 4 & 4-feed & & 17 & 12 \\
\hline Flow Rate (kgmole/h) & 8 & 1016.347 & $\begin{array}{ll}\text { Flow } & \text { Rate } \\
(\text { kgmole/h) } & \end{array}$ & 1014.839 & 9.507633 \\
\hline Methane (kgmole/h) & 0 & 137.6933 & Methane (\%) & 99.9992 & $8.00 \mathrm{E}-04$ \\
\hline Ethane (kgmole/h) & 0 & 73.46217 & Ethane $(\%)$ & 99.99966 & $3.38 \mathrm{E}-04$ \\
\hline Propane (kgmole/h) & 0 & 25.03572 & Propane $(\%)$ & 99.9999 & $9.66 \mathrm{E}-05$ \\
\hline n-Butane (kgmole/h) & $\begin{array}{r}0 \\
8.00 \mathrm{E}-\end{array}$ & 9.496937 & n-Butane (\%) & 99.99998 & $2.01 \mathrm{E}-05$ \\
\hline $\mathrm{H}_{2} \mathrm{O}(\mathrm{kgmole} / \mathrm{h})$ & 02 & 1.73043 & $\mathrm{H}_{2} \mathrm{O}(\%)$ & 13.89306 & 86.10694 \\
\hline EGlycol (kgmole/h) & 7.92 & 0 & EGlycol (\%) & 0.16836 & 99.83164 \\
\hline n-Decane (kgmole/h) & 0 & 0 & n-Decane $(\%)$ & 0 & 0 \\
\hline DTRM-A (kgmole/h) & 0 & 0 & DTRM-A (\%) & 0 & 0 \\
\hline $\mathrm{n}-\mathrm{C}_{20}(\mathrm{kgmole} / \mathrm{h})$ & 0 & 0 & $n-C_{20}(\%)$ & 0 & 0 \\
\hline Nitrogen (kgmole/h) & 0 & 768.9286 & Nitrogen (\%) & 99.99471 & $5.29 \mathrm{E}-03$ \\
\hline n-Hexane (kgmole/h) & 0 & 0 & n-Hexane $(\%)$ & 0 & 0 \\
\hline
\end{tabular}

Table 3 HYSYS Stream Summary for Dehydrator Section with EG Rate of $8 \mathrm{kgmol} / \mathrm{hr}$ 


\begin{tabular}{|c|c|c|c|c|c|}
\hline \multicolumn{4}{|c|}{4 4-feed } & 17 & 12 \\
\hline Flow Rate (kgmole/h) & 4 & 1016.347 & $\begin{array}{l}\text { Flow } \\
\text { (kgmole/h) }\end{array}$ & 1015.022 & 5.325567 \\
\hline Methane (kgmole/h) & 0 & 137.6933 & Methane (\%) & 99.99965 & $3.49 \mathrm{E}-04$ \\
\hline Ethane (kgmole/h) & 0 & 73.46217 & Ethane $(\%)$ & 99.99987 & $1.34 \mathrm{E}-04$ \\
\hline Propane (kgmole/h) & 0 & 25.03572 & Propane $(\%)$ & 99.99997 & $3.41 \mathrm{E}-05$ \\
\hline n-Butane (kgmole/h) & $\begin{array}{r}0 \\
4.00 \mathrm{E}-\end{array}$ & 9.496937 & n-Butane $(\%)$ & 99.99999 & $6.29 \mathrm{E}-06$ \\
\hline $\mathrm{H}_{2} \mathrm{O}(\mathrm{kgmole} / \mathrm{h})$ & 02 & 1.73043 & $\mathrm{H}_{2} \mathrm{O}(\%)$ & 23.38903 & 76.61097 \\
\hline EGlycol (kgmole/h) & 3.96 & 0 & EGlycol (\%) & 0.318757 & 99.68124 \\
\hline n-Decane (kgmole/h) & 0 & 0 & n-Decane $(\%)$ & 0 & 0 \\
\hline DTRM-A (kgmole/h) & 0 & 0 & DTRM-A (\%) & 0 & 0 \\
\hline $\mathrm{n}-\mathrm{C}_{20}(\mathrm{kgmole} / \mathrm{h})$ & 0 & 0 & $\mathrm{n}-\mathrm{C}_{20}(\%)$ & 0 & 0 \\
\hline Nitrogen (kgmole/h) & 0 & 768.9286 & Nitrogen $(\%)$ & 99.99724 & $2.76 \mathrm{E}-03$ \\
\hline n-Hexane (kgmole/h) & 0 & 0 & n-Hexane $(\%)$ & 0 & 0 \\
\hline
\end{tabular}

Table 4 HYSYS Stream Summary for Dehydrator Section with EG Rate of 4 $\mathrm{kgmol} / \mathrm{hr}$

\begin{tabular}{|c|c|c|c|c|c|}
\hline & butane & 17 & & cold 17 & 8 \\
\hline $\begin{array}{l}\text { Flow } \\
\text { (kgmole/h) }\end{array}$ & 2000 & 1015.022 & $\begin{array}{ll}\text { Flow } & \text { Rate } \\
(\text { kgmole/h) } & \\
\text { Recovery } & \end{array}$ & 685.6335 & 2329.388 \\
\hline $\begin{array}{l}\text { Methane } \\
\text { (kgmole/h) }\end{array}$ & 0 & 137.6928 & Methane (\%) & 48.38361 & $5.16 \mathrm{E}+01$ \\
\hline $\begin{array}{l}\text { Ethane } \\
\text { (kgmole/h) }\end{array}$ & 0 & 73.46207 & Ethane $(\%)$ & 17.66972 & $8.23 \mathrm{E}+01$ \\
\hline $\begin{array}{l}\text { Propane } \\
\text { (kgmole/h) }\end{array}$ & 0 & 25.03571 & Propane $(\%)$ & 6.748349 & $9.33 \mathrm{E}+01$ \\
\hline $\begin{array}{l}\text { n-Butane } \\
\text { (kgmole/h) }\end{array}$ & 2000 & 9.496937 & n-Butane $(\%)$ & 2.417277 & $9.76 \mathrm{E}+01$ \\
\hline $\mathrm{H}_{2} \mathrm{O}(\mathrm{kgmole} / \mathrm{h})$ & $0.00 \mathrm{E}+00$ & 0.414086 & HYO (\%) & 36.4797 & 63.5203 \\
\hline $\begin{array}{l}\text { EGlycol } \\
\text { (kgmole/h) }\end{array}$ & 0 & $1.26 \mathrm{E}-02$ & EGlycol (\%) & 12.50012 & 87.49988 \\
\hline $\begin{array}{l}\text { n-Decane } \\
(\text { kgmole/h) }\end{array}$ & 0 & 0 & n-Decane $(\%)$ & 0 & 0 \\
\hline DTRM-A & & & & & 0 \\
\hline $\begin{array}{l}\text { (kgmole/h) } \\
\text { n-CY (kgmole/h) }\end{array}$ & $\begin{array}{l}0 \\
0\end{array}$ & $\begin{array}{l}0 \\
0\end{array}$ & $\begin{array}{l}\text { DTRM-A (\%) } \\
\text { n-CY (\%) }\end{array}$ & $\begin{array}{l}0 \\
0\end{array}$ & $\begin{array}{l}0 \\
0\end{array}$ \\
\hline $\begin{array}{l}\text { Nitrogen } \\
(\text { kgmole/h) } \\
\text { n-Hexane }\end{array}$ & 0 & 768.9073 & Nitrogen (\%) & 72.26032 & $2.77 \mathrm{E}+01$ \\
\hline (kgmole/h) & 0 & 0 & n-Hexane $(\%)$ & 0 & 0 \\
\hline
\end{tabular}

Table 5 HYSYS Stream Summary for Scrubber Section with Butane 


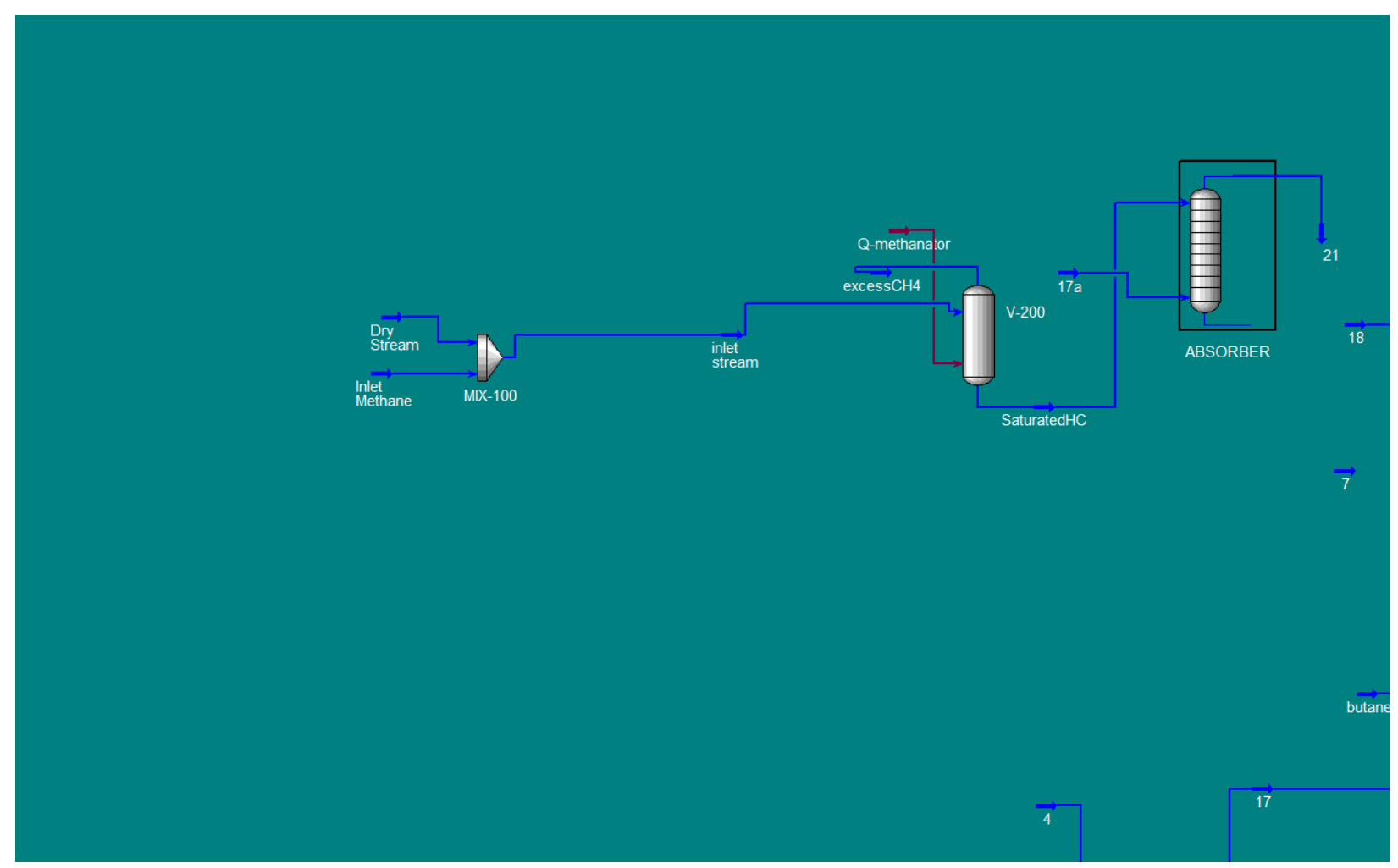

Figure 5 HYSYS Process Flow Section of Absorber Section 


\begin{tabular}{|c|c|c|c|c|}
\hline & $\begin{array}{l}\text { excess } \\
\text { methane }\end{array}$ & $17 *$ & 21 & 18 \\
\hline (kgmole/h) & 1260.116 & 2000 & 2191.946 & 1068.17 \\
\hline Methane & & & & \\
\hline $\begin{array}{l}\text { (kgmole/h) } \\
\text { Ethane }\end{array}$ & 498.1178 & 271.3101596 & 85.868 & $1.41 \mathrm{E}+01$ \\
\hline (kgmole/h) & 0.69706 & 144.7497685 & 48.53356 & $5.15 \mathrm{E}+01$ \\
\hline $\begin{array}{l}\text { Propane } \\
\text { (kgmole/h) }\end{array}$ & 0 & 49.33039731 & 19.03226 & $8.10 \mathrm{E}+01$ \\
\hline n-Butane & 2449942 & 18.71277889 & 5.571595 & $9.44 \mathrm{E}+01$ \\
\hline $\mathrm{H}_{2} \mathrm{O}$ (kgmole/h) & 0 & 0.815916314 & 77.57684 & 22.42316 \\
\hline EGlycol & & & & \\
\hline (kgmole/h) & $0.00 \mathrm{E}+00$ & $2.49 \mathrm{E}-02$ & 36.96526 & 63.03474 \\
\hline n-Decane & & & & \\
\hline $\begin{array}{l}\text { (kgmole/h) } \\
\text { DTRM-A }\end{array}$ & 758.8513 & 0 & $3.59 \mathrm{E}-03$ & 99.99641 \\
\hline (kgmole/h) & 0 & 0 & 0 & 0 \\
\hline $\mathrm{n}-\mathrm{C}_{20}(\mathrm{kgmole} / \mathrm{h})$ & 0 & 0 & 0 & 0 \\
\hline Nitrogen & & & & \\
\hline (kgmole/h) & 0 & 1515.056107 & 95.66815 & $4.33 \mathrm{E}+00$ \\
\hline $\begin{array}{l}\text { n-Hexane } \\
\text { (kgmole/h) }\end{array}$ & 0 & 0 & 0 & 0 \\
\hline
\end{tabular}

Table 6 HYSYS Stream Results of Methane Removal Section with Saturated Inlet Solvent Stream 


\begin{tabular}{|c|c|c|c|c|}
\hline $\begin{array}{ll}\text { Flow } & \text { Rate } \\
(\text { kgmole/h) } & \end{array}$ & $\begin{array}{l}\text { excess } \\
\text { methane } \\
\qquad 1001\end{array}$ & $17^{*}$ & 1694.885677 & 1306.114 \\
\hline $\begin{array}{l}\text { Methane } \\
\text { (komole/h) }\end{array}$ & 1 & 2713102 & 7956874636 & $204 \mathrm{E}+01$ \\
\hline $\begin{array}{l}\text { Ethane } \\
\text { (kgmole/h) }\end{array}$ & 1.134332 & 144.7498 & 37.20474668 & $628 \mathrm{E}+01$ \\
\hline $\begin{array}{l}\text { Propane } \\
(\text { kgmole } / \mathrm{h})\end{array}$ & 0 & 49.3304 & 12.63844931 & $8.74 \mathrm{E}+01$ \\
\hline $\begin{array}{l}\text { n-Butane } \\
(\text { kgmole/h) }\end{array}$ & 3.268414 & 18.71278 & 3.439308131 & $9.66 \mathrm{E}+01$ \\
\hline $\mathrm{H}_{2} \mathrm{O}(\mathrm{kgmole} / \mathrm{h})$ & 0 & 0.815916 & 71.96239544 & 28.0376 \\
\hline $\begin{array}{l}\text { EGlycol } \\
\text { (kgmole/h) }\end{array}$ & $0.00 \mathrm{E}+00$ & 2.49E-02 & 29.16840349 & 70.8316 \\
\hline $\begin{array}{l}\text { n-Decane } \\
\text { (kgmole/h) }\end{array}$ & 995.5973 & 0 & $1.92 \mathrm{E}-03$ & 99.99808 \\
\hline DTRM-A & & & & \\
\hline (kgmole/h) & 0 & 0 & 0 & 0 \\
\hline $\mathrm{n}-\mathrm{C}_{20}(\mathrm{kgmole} / \mathrm{h})$ & 0 & 0 & 0 & 0 \\
\hline $\begin{array}{l}\text { Nitrogen } \\
(\text { kgmole/h) }\end{array}$ & 0 & 1515.056 & 93.48379328 & $6.52 \mathrm{E}+00$ \\
\hline $\begin{array}{l}\text { n-Hexane } \\
\text { (kgmole/h) }\end{array}$ & 0 & 0 & 0 & 0 \\
\hline
\end{tabular}

Table 7 HYSYS Stream Results of Absorber Section without Saturating Inlet Solvent Stream

The number of stages required for a given separation is obtained from the application of equilibrium thermodynamics. The actual number of stages obtained from a packed tower is divided by the theoretical number stages to obtain the efficiency for the packed tower. When working with packings, efficiency is usually dealt with in terms of theoretical stages per unit depth of packing. The commonly used terms are HETP (height equivalent per theoretical plate) and $\mathrm{N}$ (number of theoretical stages per meter). As far as calculation of efficiency is concerned, most equations can be considered semi-empirical. The mass transfer capability of a packing is typically expressed as HETP, $\mathrm{HTU}, \mathrm{K}_{\mathrm{G}} \mathrm{a}$ or $\mathrm{K}_{\mathrm{L}} \mathrm{a}$, all of which are rate-controlled and can be converted from one another as follows ${ }^{1}$

$\mathrm{HTU}=\mathrm{G} / \mathrm{K}_{G} \mathrm{aP}=\mathrm{L} / \mathrm{K}_{\mathrm{L}} \mathrm{a} \rho_{\mathrm{L}}$

If $\mathrm{m} \mathrm{G} / \mathrm{L}=1$, then HETP and HTU are equal. If the equilibrium and operating lines are straight and parallel then

1 Leva, Max, "Tower Packings and Packed Tower Design", The United States Stoneware Company (1953) 


$$
\mathrm{HTU}=\operatorname{HETP}(1 / \mathrm{A}-1) / \ln (1 / \mathrm{A})
$$

$N T U=N=\frac{\log \left[\left(\frac{y_{1}-m x_{1}}{y_{2}-m x_{2}}\right)(1-1 / A)+1 / A\right]}{\log A} ; A=L / m G$

where $\mathrm{A}$ is the absorption factor.

Attempts have been made to generalize predictive methods for the mass transfer efficiency of packings using film, penetration and surface renewal models, but all have met with varying success. $^{2} \quad$ Therefore, bench-scale and pilot-scale plants are necessary to determine the hydrodynamics of the packing along the reactions specific to targeted application. This will lead to the number of theoretical plates needed for a given packing and reaction and then can be scaled up to according to the desired new conditions of gas and liquid flow rates by changes in the corresponding absorption factor, thus affecting the NTU.

The exact composition of the ethylene glycol solution for dehydration and the solvent to be tested for the absorption tower were finalized. The freezing point vs. composition curve for aqueous ethylene glycol solutions, Figure 6, indicates the occurrence of a eutectic, i.e., the point at which water and glycol crystallize together. On the dilute side of the eutectic, ice forms upon freezing, while on the concentrated side, solid glycol separates from solution when the freezing point is reached. Based on the computer simulation, $53 \%$ by weight ethylene glycol mixtures will be used in the bench and pilot scale units for testing as shown in Table 8 .

2 Bolles, W.L. and J.R. Fair I Chem Eng Symposium Series No. 56 p 3.3135 (1979) 


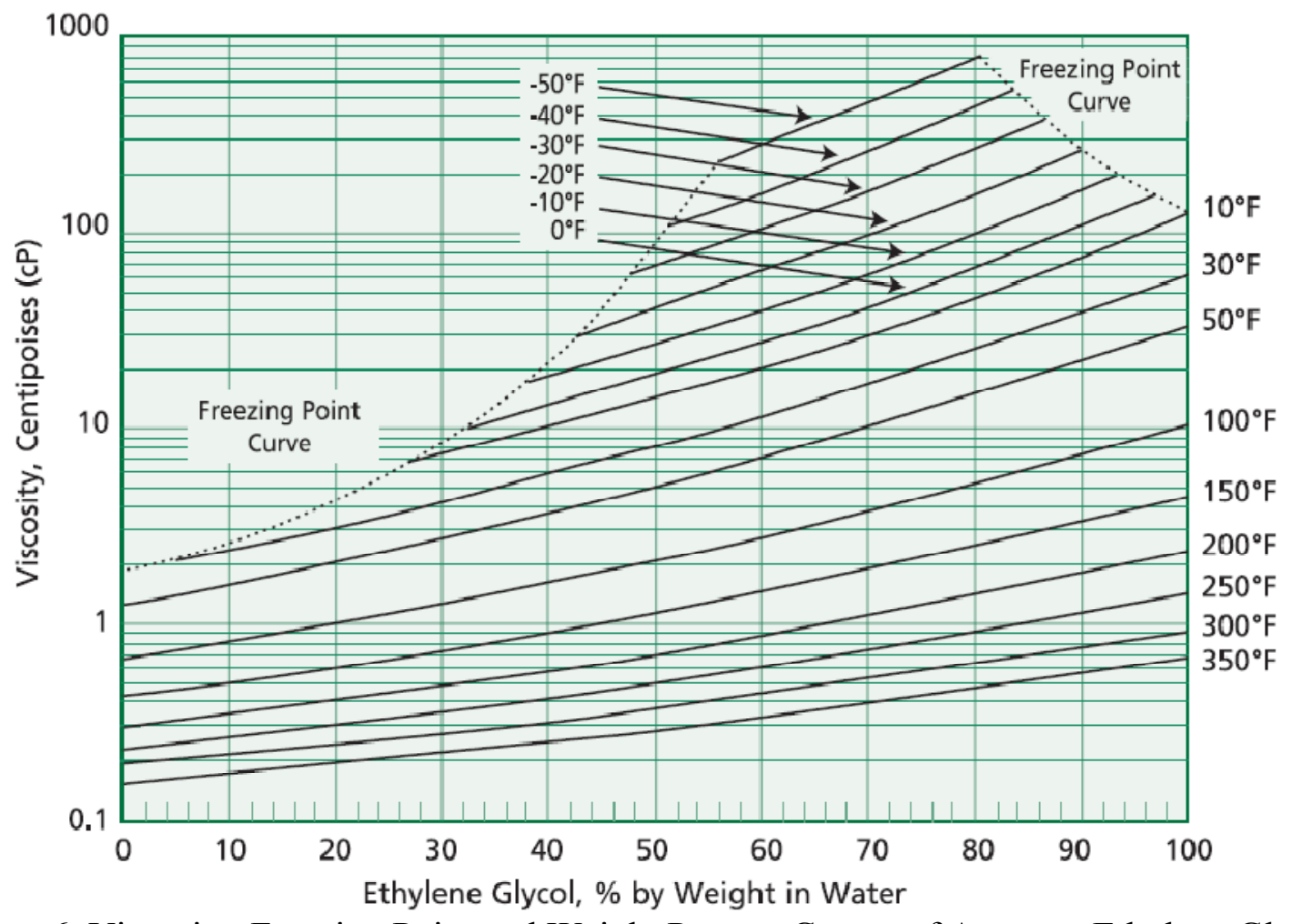

Figure 6 Viscosity, Freezing Point and Weight Percent Curves of Aqueous Ethylene Glycol Mixtures. 
Table 8 HYSYS Stream Summary for Dehydrator Section with EG Rate of $8 \mathrm{kgmol} / \mathrm{hr}$

\begin{tabular}{|c|c|c|c|c|}
\hline & 4 & sat-feed1 & 17 & 12 \\
\hline Flow Rate $(\mathrm{kg} / \mathrm{h})$ & 253.13 & 25.99 & 25.97 & 253.16 \\
\hline Vapor & 0 & 1 & 1 & 0 \\
\hline Temperature $[\mathrm{C}]$ & -42.78 & 35.00 & -34.98 & -34.98 \\
\hline $\begin{array}{l}\text { Pressure }[\mathrm{kPa}] \\
\text { mass fraction }\end{array}$ & 6996.08 & 6996.08 & 6996.08 & 6996.08 \\
\hline Methane & 0 & 0.7394 & 0.7401 & 0.0000 \\
\hline Ethane & 0 & 0.1262 & 0.1263 & 0.0000 \\
\hline Propane & 0 & 0.0775 & 0.0776 & 0.0000 \\
\hline n-Butane & 0 & 0.0559 & 0.0560 & 0.0000 \\
\hline $\mathrm{H}_{2} \mathrm{O}$ & 0.47 & 0.0010 & 0.0000 & 0.4701 \\
\hline EGlycol & 0.53 & 0.0000 & 0.0000 & 0.5299 \\
\hline
\end{tabular}

Detailed design work on the bench-scale unit is continuing by Vendor \#3 at a much slower rate than planned. We have observed that the equipment fabrication shops are operating at capacity due to economic conditions in allied industries and long delivery times on critical equipment components are being quoted. Progress will continue to be monitored and means to minimize delays will be investigated. The initial schedule for the bench-scale unit, given in Figure 7, estimated a delivery date to GTI by the end of October 2006.

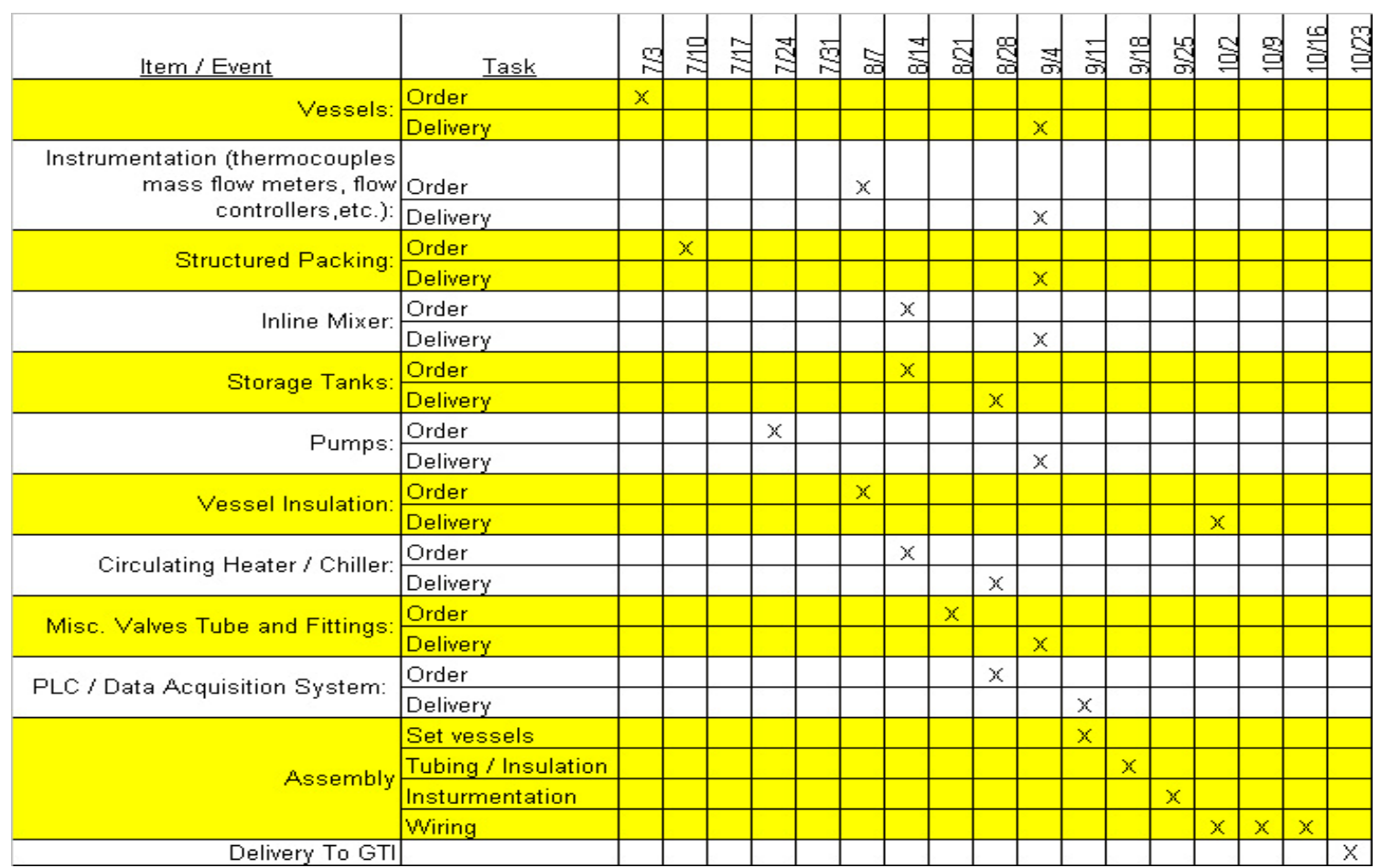

Figure 7 Design and Fabrication Schedule for Bench-Scale Unit

In the 1QFY2006, Vendor \#3 completed design and fabrication of the pressure vessels and procurement of other equipment according to the process and instrumentation diagram presented in Figure 8. The 
Bench Scale unit is a multi-purpose pilot plant specifically designed and built by Vendor \#3 to evaluate three processes. The three processes are outlined as follows:

\section{- Kerosene Absorption}

- $\quad$ Ethylene Glycol Dehydration

- $\quad \mathrm{H}_{2} \mathrm{~S} / \mathrm{SO}_{2}$ absorption/reaction and contaminant removal

Each of the three processes operates at high pressures, nominally 400 to 1000 psig with the plant designed to relieve at 1300 psig. The Kerosene Absorption and Ethylene Glycol Dehydration processes operate at cryogenic temperatures of $-40^{\circ} \mathrm{F}$ while the $\mathrm{H}_{2} \mathrm{~S} / \mathrm{SO}_{2}$ absorption/reaction (UCSRP-HP) process operates at $275^{\circ} \mathrm{F}$ and is designed to shutdown at $300^{\circ} \mathrm{F}$. Therefore all of the instruments, valves, pumps, pipes, tubes, vessels, and etc., need to be designed for these temperature, pressure and acid gas environments. These requirements are standard engineering practices followed by engineers throughout the industry.

Several of the vessels, pumps, coolant recirculator, and frame are seen in Figure 9. A trip was made to Vendor \#3 's workshop to inspect the fabricated vessels, meet with the assembly team, and emphasize our need to have the equipment delivered on time. A correction was made in the specification for the feed gas mass flow controllers. The original designs were set for the entire gas flow, including the nitrogen balance gas that can represent up to $95 \%$ of the gas flow. Progress continued to be monitored and means to minimize further delays were investigated. The schedule for the bench-scale unit estimated a delivery date to GTI before the end of February 2007. 


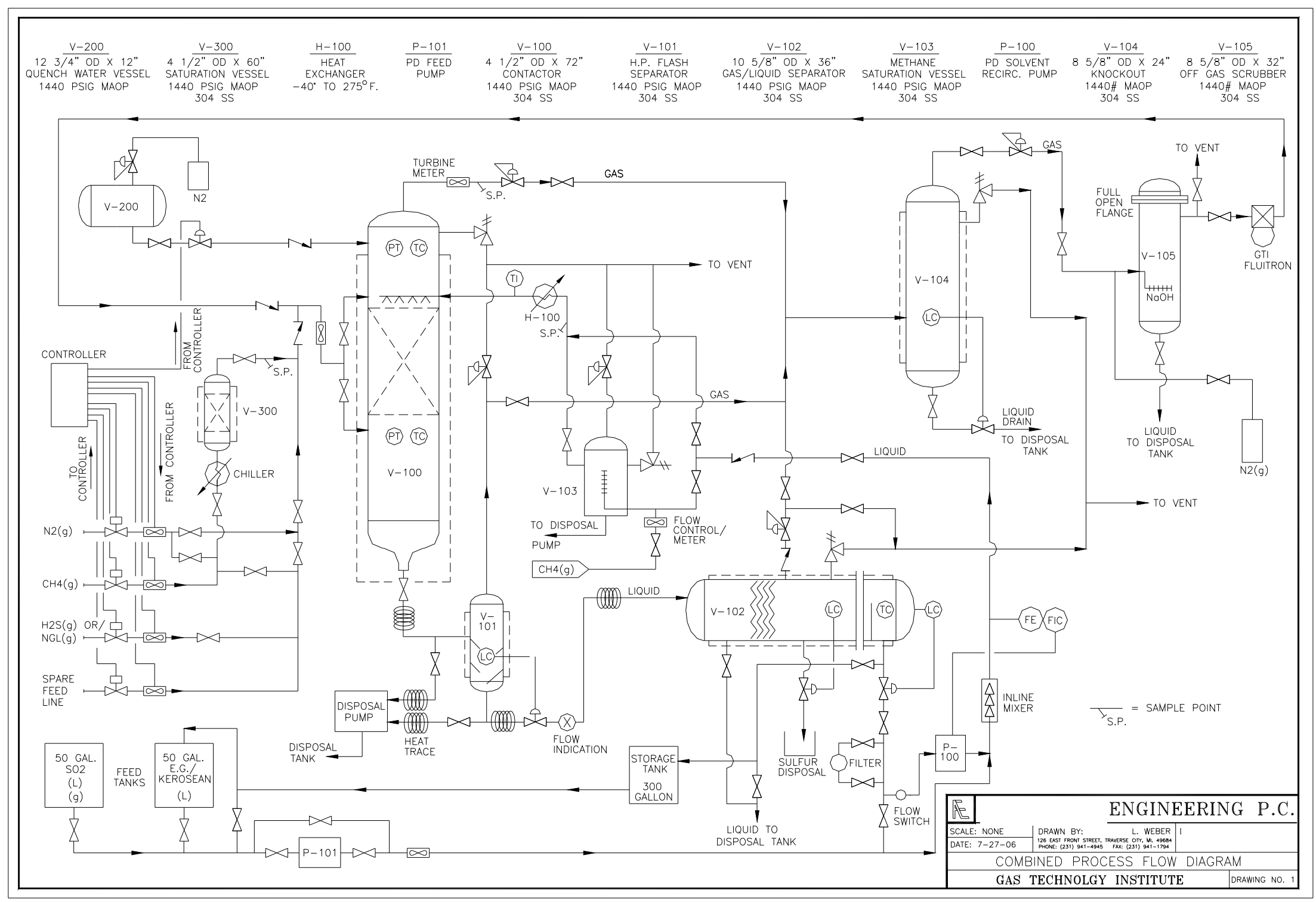

Figure 8 Bench-Scale Unit P\&ID

Page 27 
The location of the bench-scale unit was established within Laboratory 636 at GTI into Space $\mathrm{G}$ as shown on Figure 10. GTI removed laboratory benches and install the electrical connections and environmental protection required for safe operation of the bench unit, prior to delivery of the unit.

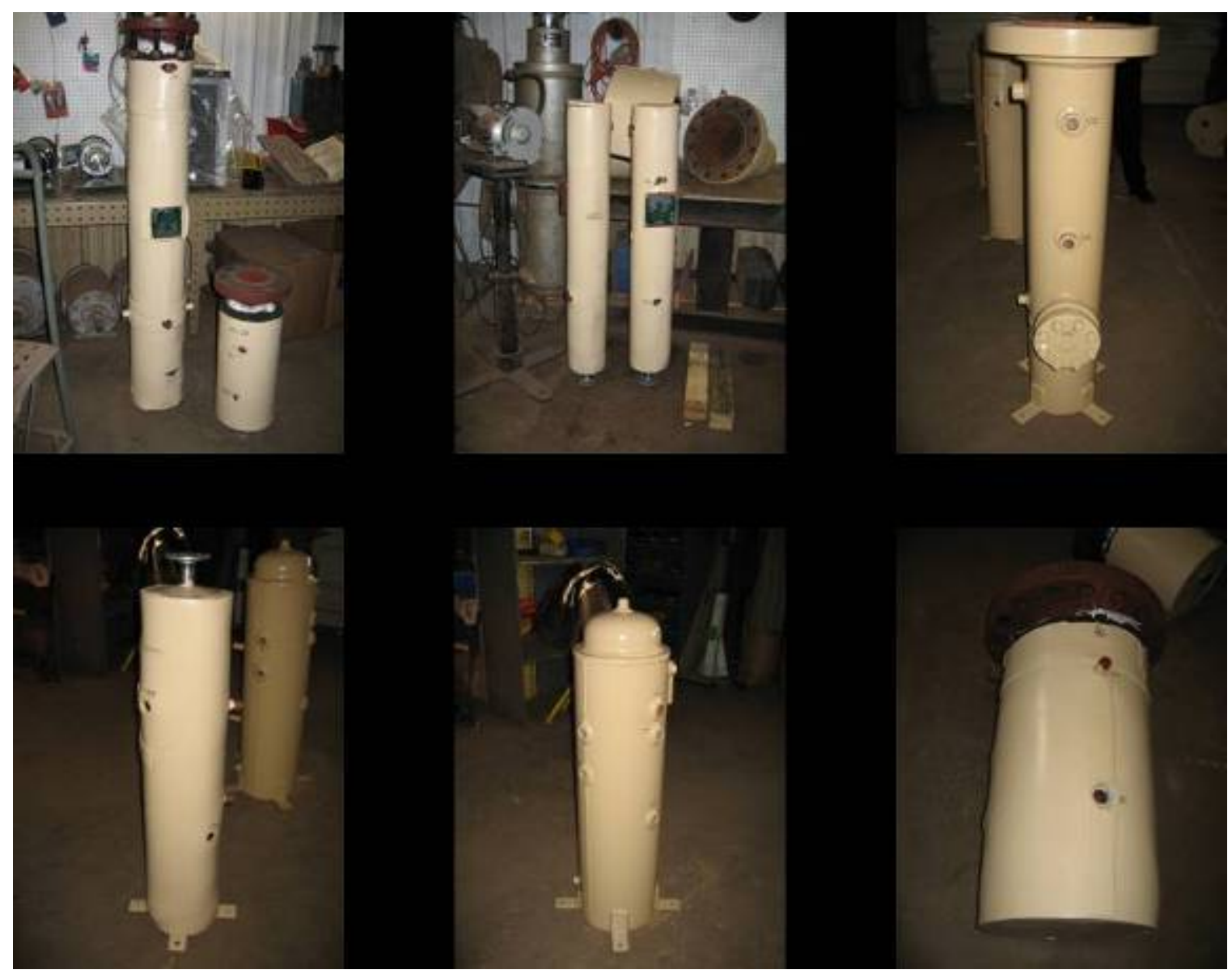




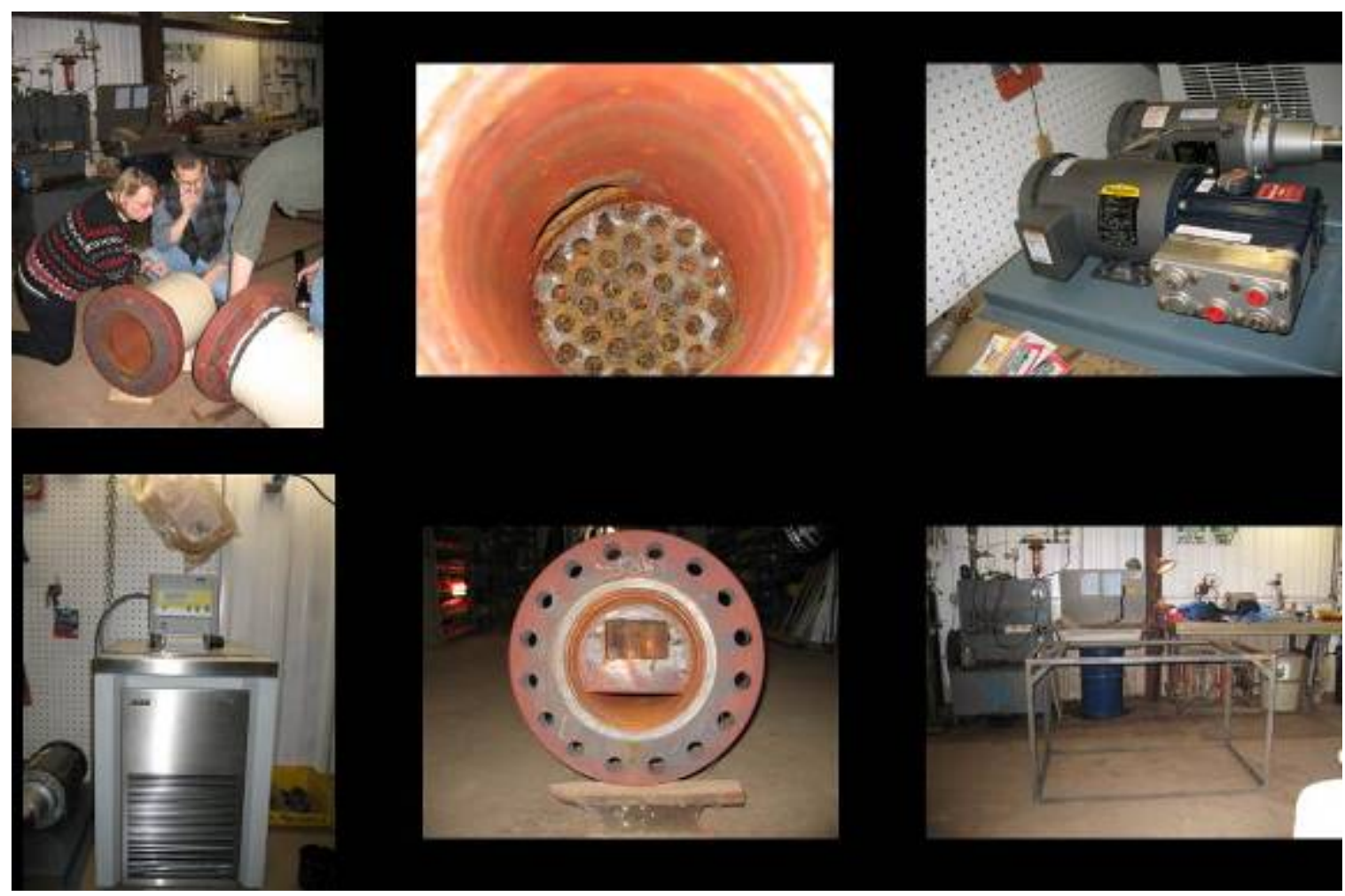

Figure 9 Bench-scale Vessels and Equipment

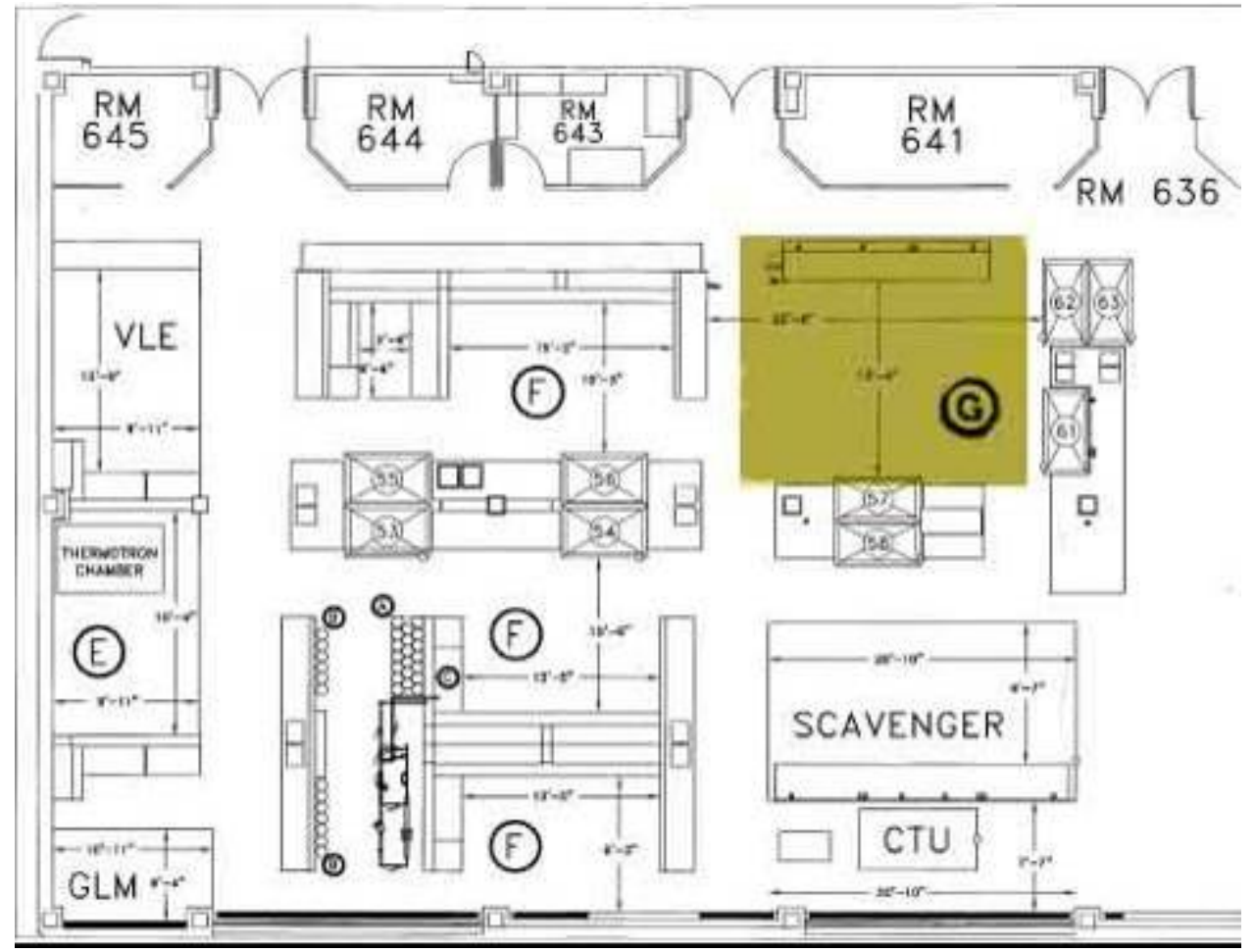

Figure 10 Location of Bench-scale Unit, Area G within Lab 636 
Vendor \#3 delivered the skid and major equipment to GTI Q2FY2007, see Figure 11. The installed equipment in GTI's gas processing laboratory can be seen in Figure 12 - Figure 14. For safe operations with hazardous gases, such as $\mathrm{CH}_{4}, \mathrm{H}_{2} \mathrm{~S}$ and $\mathrm{SO}_{2}$, the Bench Scale Unit has been contained in a Plexiglas enclosure, as seen in Figure 13. The ventilation system within the enclosure will ensure that any leaks are not released to the surrounding laboratory, but are properly handled. Vendor \#3 planned to complete the construction at GTI.

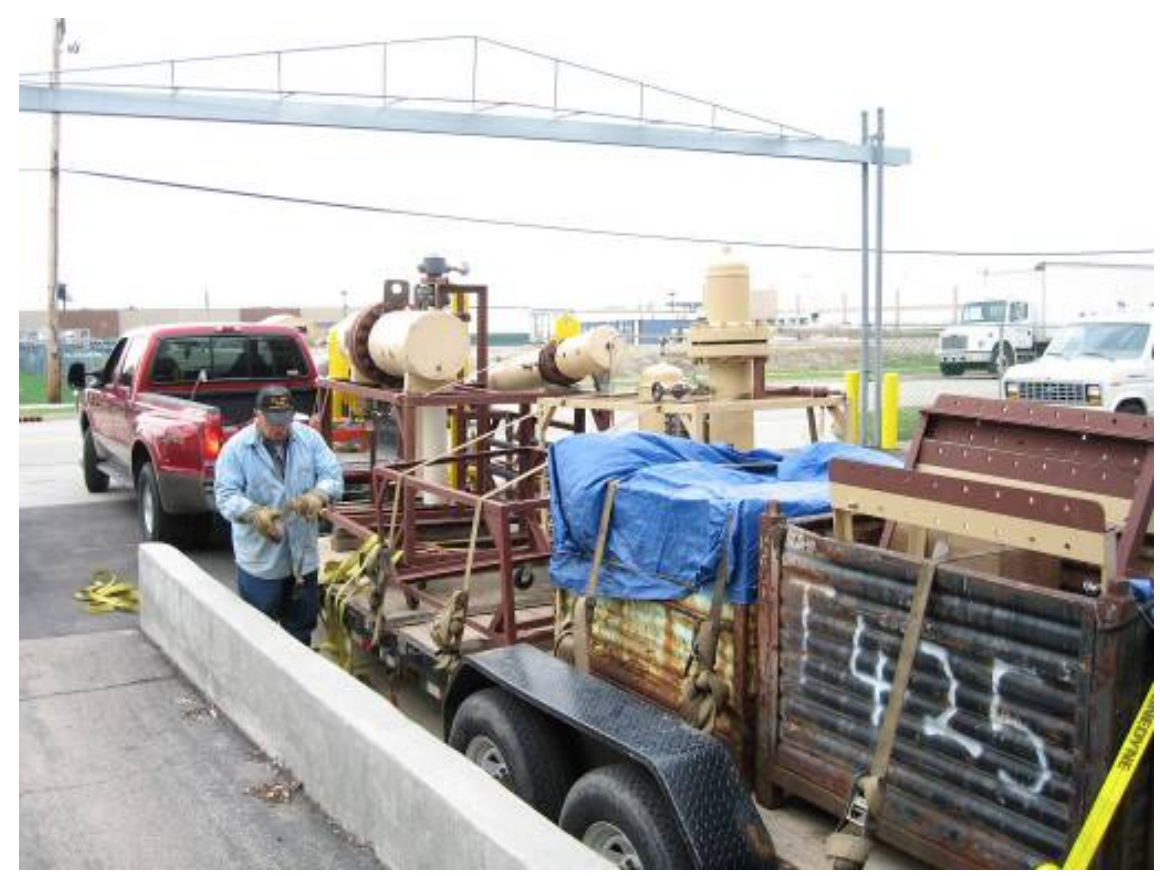

Figure 11 Bench-scale Unit Being Unloaded at GTI 


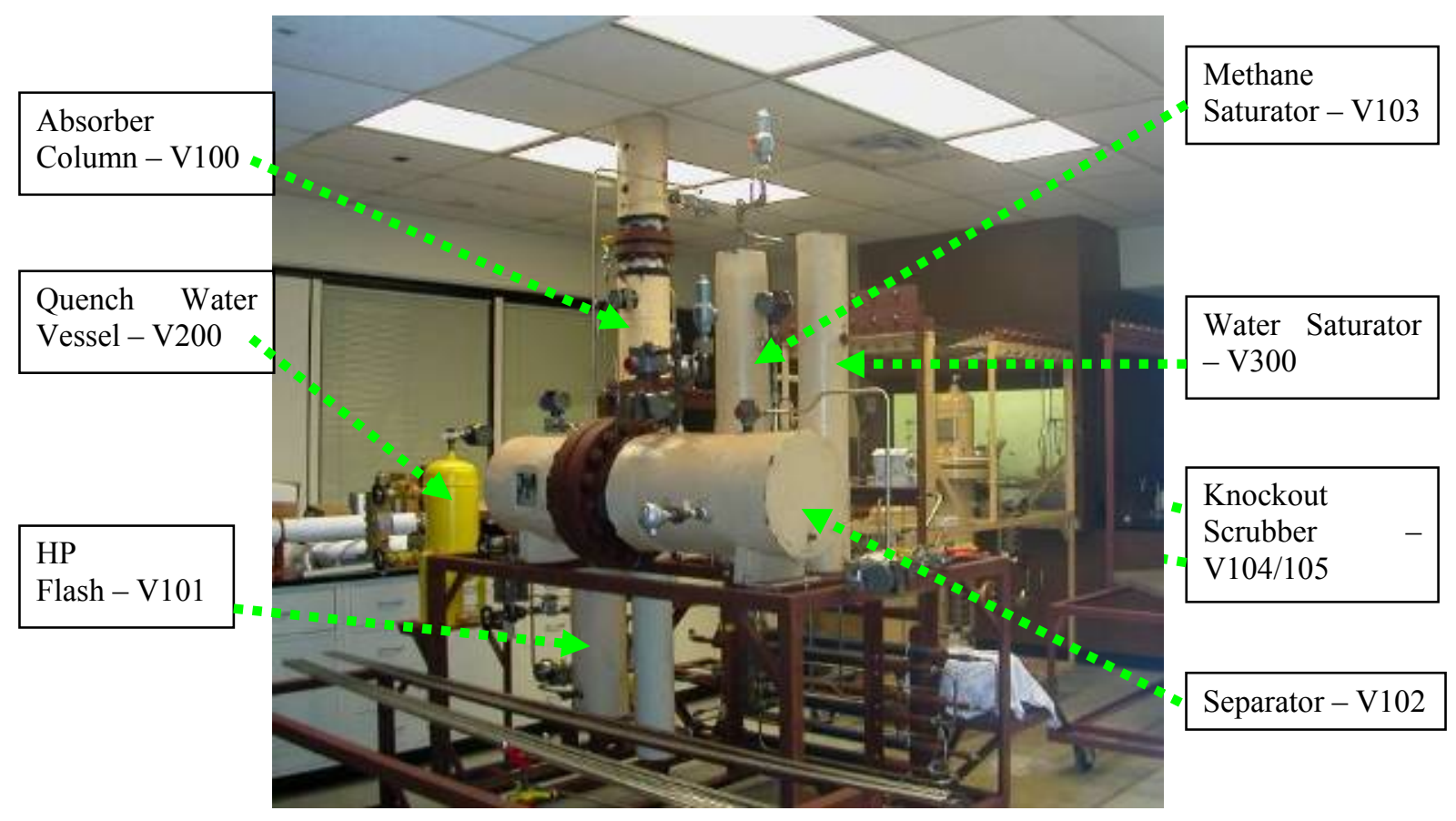

Figure 12 Bench-Scale Unit

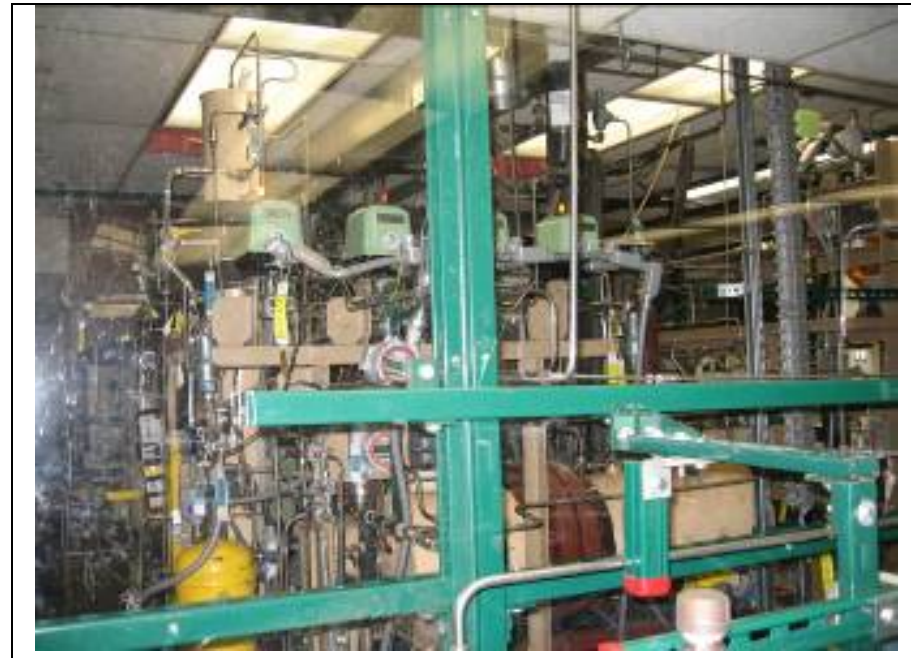

Figure 13 Bench-Scale Unit Containment

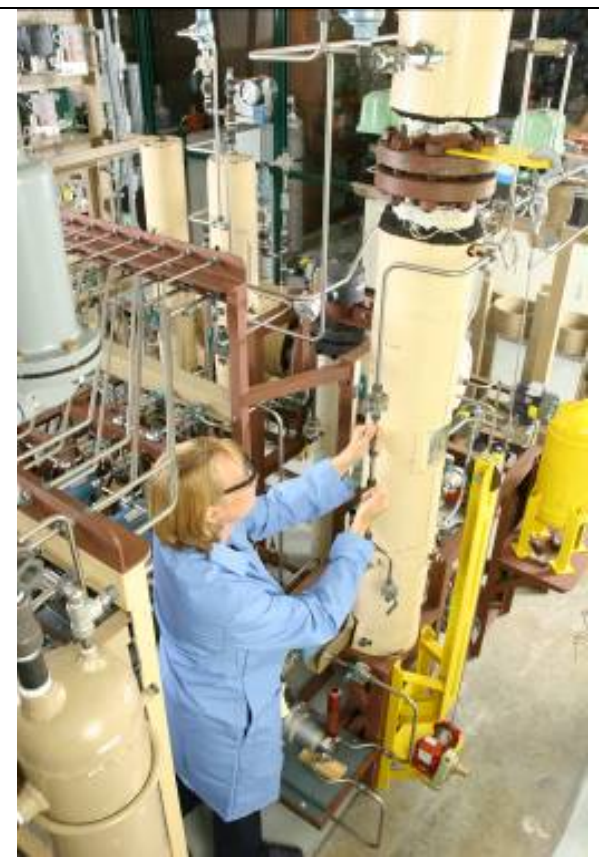

Figure 14 Absorber Column Prior to Heat Tracing and Insulation of Tubing/Piping

Q4FY2007, the bench scale unit was pressure tested for the gas phase. At this point we found that we needed an additional bypass line for better control of the gas flow rates coming from the main compressor unit. A proportional block valve, control and line were added. This addition 
was tested and demonstrated control of the gas flow rates along with completing the leak testing. All the leaks at the union were tightened and no other leaks were found in the unit.

During testing it was decided that it was not ideal to run with the compressor being manually controlled by the gas flow rate of the compressor. Controls were competing with the local flow rate controllers setting. A line and control were added to have the main compressor work off the system pressure. We verified that we were able to fully control the gas flow rates to unit's design specifications.

After this we began to test the plant for liquid flow rates and leaks. The debris from construction within the lines clogged up the proportional block valve leading back to the solvent tank. This caused a back up in the low pressure flash vessel, V-106, causing a pressure increase beyond the pressure relief valve setting of $120 \mathrm{psi}$. When the relief valve opened, the liquid was forced to go with the gas into the main vent. After cleaning system, we installed block valves on top of all the lines leading from the differential level flow transmitters to the top of the vessel. In this way, we can drain those lines quickly to be able to read the actual level in the vessel if the maximum fill line is reached. We also added automatic control that will open the proportional block valves to full-open in such cases for full drainage of the vessels as well as simultaneously shutting of the solvent feed to the unit thus preventing the liquid from filling up the vessels and going with the gas into the gas lines.

In preparation of the HAZOP analysis, Vendor \#3 was asked to provide P\&ID's and wiring schematics for the Bench Scale Unit. They issued a process flow diagram instead of a detailed P\&ID and had not prepared any schematics. GTI had to undertake the preparation of these drawings as required for a HAZOP.

The schematic drawings was contracted to Vector House. They manually traced and drew out a complete set of electrical drawings and to transferred the information to AutoCad and provided as many copies as GTI requires. In addition Vector House was contracted to correct a number of wiring issues such as replacing copper wire installed by Vendor \#3 instead of type $\mathrm{T}$ thermal couple wire for type $\mathrm{T}$ thermocouples and rewiring the Brooks mass flow meters with a feedback loop so that the operators will have PID loop control on the mass flow meters.

GTI's next task was to draw a set of P\&ID's as the plant was built. Small changes were ongoing and expected through the completion of the HAZOP in February 2008. All instruments, valves, pumps, vessels, and etc., were tabulated, labeled and documented. A prestart-up check-off list for valves and instruments was completed detailing the position (open or closed) that each valve and instrument needs to be in prior to executing the start-up procedure.

Once the documentation of all of the instruments, valves, pumps, pipes, tubes, vessels, etc. was completed, they were reviewed for size requirements, maximum and minimum operating temperature, maximum operating pressure and acid gas environments. The three day HAZOP review that commenced in February on the Bench-Scale Unit was finished on March 19, 2008. There were a total of 5 nodes studied. A node is a subset in the unit P\&IDs that can be broken into a smaller manageable piece that can be studied in greater detail. The HAZOP review examined a total of 63 process deviations in the 5 nodes. However, deviations can occur in more than one node such as high/low temperature, high/low flow, high/low pressure, etc. so some redundancies exist. The HAZOP review process generated a large list of recommendations that 
need to be addressed, covering 71 pages. These issues were addressed by the follow up Recommendation Commitment Meeting on March 24, 2008. All of the corrections and recommendations identified at the HAZOP review in both hardware and software were addressed.

Numerous engineering design flaws and equipment selection errors were discovered in the system Vendor \#3 fabricated. They are listed below followed by GTI's efforts to mitigate the issues in order to bring the plant back to standard engineering practices:

- On seven relief valves, the discharge ports, on liquid filled vessels, were plumbed to the vent header. In the event of a relief discharge, the vent header will fill up with liquid and dump contents on the roof.

o To mitigate this effort, a blow-down drum was installed. Then the relief valve discharge ports on liquid filled vessels were plumbed to the blow-down drum and the blow-down drum was vented to the vent header

- The relief valves protecting vessels from rupture were sized to discharge at 1440 psig while the maximum operating pressure of the vessels in the plant were designed for 1315 psig. The relief valve is required to be set at a lower pressure that the maximum allowable operating pressure of the device it is protecting.

0 To mitigate this, the relief valves were resized to discharge at $1300 \mathrm{psig}$.

- The two Chromalox heat exchangers were sized for 1000 psig at $300^{\circ} \mathrm{F}$. The plant is to operate at 1000 psig so the maximum operating pressure of the vessel needs to be greater than 1000 psig, specifically at least 1315 psig.

o To mitigate the effort two new Chromalox heat exchangers were specified with a maximum operating pressure of 1315 psig at $300^{\circ} \mathrm{F}$. The estimated cost was $\$ 43,404.70$.

- Two plastic 55 gallon feed drums and two plastic 165 gallon waste product drums were installed on the Bench Scale Unit. The Bench Scale Unit is on the second floor of the building at GTI with no secondary containment vessels.

o To mitigate any potential spill, secondary containment vessels were purchased and installed.

- The two plastic 55 gallon feed drums blocked the normal egress of a walkway resulting in a building code violation.

o To mitigate the issue, the two 55 gallon feed drums were moved and re-plumbed to provide adequate egress.

- Six Sharpe electric operated control valves do not meet the temperature and pressure requirements of the plant.

o The most cost effective way to mitigate this issue was to remove the valve seat and stem and replace them with ones that meet the temperature and pressure requirements of the plant.

- Twenty-five PGI bleeder valves did not meet the low temperature requirements of the plant and also they require valve stem extensions so they can be operated while insulated.

o Since the valve bodies did not meet the low temperature requirements of $-40^{\circ} \mathrm{F}$, new valves and valve stems are required to replace the old ones. A purchase order for the items has been submitted for procurement.

- Fifty Parker valve stems are not accessible to turn with insulation on them. 
o To mitigate the issue, fifty 2 " valve stem extensions were ordered and replaced with the shorter ones.

- Five Apollo valves did not meet the high temperature and high pressure requirements of the plant.

o Since the valve body did not meet the high temperature and high pressure requirements of the plant, they were replaced with Parker valves.

- Sixteen Parker check valves did not meet the acid gas requirements for the plant because they contained Viton seats. Viton is unsatisfactory for use in an acid gas environment.

o Since Parker does not provide Kalrez valve seats for its check valves, the valves were replaced with Swagelok check valves with Kalrez seats.

- A Nowata filter with brass internals did not meet the acid gas requirements for the plant. Brass is unsatisfactory for use in an acid gas environment.

o A new stainless steel Nowata filter was purchased with stainless steel internals and replaced the inferior model.

- The main feed pump P-101 was not relieved properly.

o A relief valve was installed so in the case of a high pressure event or a blockage downstream of the pump, the pump will recirculation the feed back into the feed tanks.

All AMSE Pressure Vessel code violations were mitigated by removing the block valve Vendor \#3 placed in between a pressure vessel and the relief valve it is protecting. Vendor \#3 stated that they wanted the ball valve for pressure checking the relief valve to make sure it was not leaking. It was their intent upon completion of the unit to leave these valves open and then remove the handle. However this procedure does not remove the AMSE Code violation.

Startup, shutdown, maintenance and operating procedures were written to comply with the recommendations from the noted deviations. In addition carbon bed maintenance procedures with a schedule to identify when the carbon bed is full of $\mathrm{H}_{2} \mathrm{~S}, \mathrm{SO}_{2}$ and etc were prepared. The carbon bed is the last defense in filling the ventilation system with large amounts of unwanted gases. Vector House was onsite to continue with the commissioning process of the Bench Scale Unit. There are a number of interlocks on pumps, temperature sensors, pressure sensors, vessel levels and etc. that need to discussed with Vector House to implement.

The HAZOP also identified that a differential pressure alarm needed to be installed in the vented hood area and interlocked to the unit. Signage and room monitors for the Fluitron down stairs were required. Also discussed was the need to ventilate the Fluitron room. The installation of a 30 minute UPS for the computer in the event of a power failure was also added.

Aker-Kvaerner, hired to perform piping fitting activities, completed piping of seven cylinder feeds and pressure regulators, completed the tubing connections on the two 165 gallon waste tanks, completed the tubing connections on the two 55 gallon feed tanks and completed piping to the moisture analyzer. Vector House has completed a full set of schematics for the Bench Scale Unit. The HAZOP review recommended that commissioning of the Bench Scale Unit can continue as Vendor \#3 built the unit. Any commissioning from changes made by the Recommendation Commitment Meeting were to be addresses as they arise. 
Aker-Kvaerner was hired on 3QFY2008 to perform the modifications to the unit as a result of the HAZOP recommendations, which included:

- Installing 990 psig relief valves on H-200 and H-300 Chromalox Heat exchangers

- Replacing 25 carbon steel valves with stainless steel valves

- Plumbing requirements as found deficient from the HAZOP

- Opening vessel V-105 was to be inspected with a boroscope to examine the sparger if one even exists. Vessels V-103 and V-300 will be removed and modified so a new sparger design can be inserted into each of the vessels. The liquid to gas contact in each vessel is only 2 inches, therefore the contact time is very short. In addition, there is no way a liquid level controller can control a 2 inch level in either of these tanks. In addition a level control device will be inserted in to vessels V-300 and V-200. Mott Corporation is currently in the process of designing the new sparger. Manufacturing the new spargers was the rate limiting step in bringing the plant online The spargers are required to disperse the acid gas into scavenger to neutralize the gas before it is discharged into the carbon bed

- Removing and modifying V-103 and V300 and weld in a 1 inch coupling for the new spargers

- Install level control devices V-200 and V-300

- Install P-103

- Install $\mathrm{pH}$ meter in V-105

- Install temperature thermal well for P-100 for an overheat temperature shutdown

- Remove and replace two back pressure regulators on V-106 with N.A.C.E. certified back pressure regulators

- Install filters before each Brooks mass flow meter and N.A.C.E. certified check valves downstream of the Brooks.

- Remove the block valve on the Bench Scale Vent System.

- A carbon bed was routed so it can be used while purging the unit when the sulfur and hydrocarbon processes are operational.

- A 120 psig relief valve was installed between the carbon bed and the inlet pressure reducing valve to protect the carbon bed from rupture. The vent system was rerouted because of an approximately 70 gallon ethylene glycol spill that occurred. The spill occurred as a result of a blocked vent system. While secondary containment captured about 40 gallons, approximately 30 gallons of glycol ended up on the floor,.

- A new emergency vent system that discharges directly to the outside of the building was completed. Previously, the emergency vent exhausted into the hood ventilation system. Potentially, 200 cubic feet of sour gas from the sulfur process could overwhelm this system in short order.

- Long-lead items related to the under-designed spargers for V-103, V-200 and V300 were received. The vessels were removed and modified. Level control devices for V-200 and V-300 were installed after the vessels were x-rayed and hydro-tested. Pump P-103 was installed.

- Vector House has completed controls programming for the dehydration, kerosene and sulfur modes of operation. Vector House has gone through the software 
programming that was written by Vendor \#3. It has been determined that the program written by Vendor \#3 suffers from a number of programming errors and will not run properly as Vendor \#3 had claimed when it was delivered. Vendor \#3 was not sure if the sulfur process would run but assured everyone the ethyleneglycol and kerosene processes would function properly. Vector House found that the sulfur process software was not completed. Work continues to debug and correct the Pre-HAZOP software. P\&ID's are continually being updated to reflect the communication between devices including interlocks.

- Installation of the 990 psig relief valves on H-200 and H-300 Chromalox heat exchangers was completed. Heat exchangers H-200 and H-300 can be isolated from the glycol and kerosene processes so they can be operated at 1000 psig. However the sulfur process will need to be operated at no more than 930 psig.

- Replacement of 25 carbon steel valves with stainless steel valves was completed.

- The Mott spargers were installed in vessels V-103 and V-300 and the vessels have been reinstalled and plumbed into the unit. Vessel V-200 and V-300 were installed with the new liquid level control devices. V-103 was re-engineered by raising the vessel higher than $\mathrm{V}-100$ so the process fluid will disengage from the process gas and flow downward into the process. In addition, a high pressure site glass was added to V-103 so the process fluid in the vessel could be visually monitored. The high pressure side of the differential pressure sensor on V-103 was moved to the top of V-100 so each of these vessels will operate at the same pressure. Each of these three vessels V-103, V-200 and V-300 has been x-rayed for cracks and hydro-tested.

- Installation of a thermal well at the output of P-100 was completed by the pipefitters.

- Installation of two back pressure regulators on the liquid and gas side of V-106 was completed.

- Installation of filters before each Brooks mass flow meter, N.A.C.E. certified check valves down-stream the Brooks mass flow meters, and plumbing requirements found deficient from the HAZOP was completed.

- Pressure testing was under way when a near miss safety incident occurred. Heat Exchanger H-300 was being pressure tested with 900 psig of nitrogen when a tube ripped loose from its ferrule. The large heat exchanger was isolated at the time but the nitrogen inside of the heat exchanger discharged into the hooded area with two contractors working in the area. A residual amount of ethylene-glycol was in the heat exchanger at the time due to previous Vendor \#3 pressure testing. One contractor had ethylene-glycol sprayed on his pants. The contractor removed his clothes, took a shower and changed into a clean set of clothes. All work was stopped and an accident investigation was initiated. It was determined that Vendor \#3 improperly tightened a ferrule on a stainless steel tube. A close look from the accident investigation showed the ferrule partially on the tube when it was tightened. This is not the first time we have observed this type of problem while pressure checking. We conducted a random check of 100 fittings to look for improperly tightened ferrule on tube connections. As a result of the random check, one ferrule was found on backwards, which could have resulted in a similar discharge, as discussed above. Therefore it was decided to check all of the 
tube fitting for properly tightened and seated ferrule tube fittings. Pressure testing was delayed until the ferrule check was completed. Twelve joints were found to be of substandard quality. In four joints, the ferrules were placed on backwards and, in the remaining joints, the ferrules were not fully on the tube. Therefore, the time and effort to examine each joint mitigated a potential high-pressure process fluid release into the laboratory area.

- A 220 volt 30 amp power line was routed from downstairs up to the unit and the power panel was bolted down as the heat trace insulation panel was installed.

- The software that Vendor \#3 wrote is currently running but needs restructure to a more efficient use of the programmable controls. Vector House was able to bump test the flow meters, pressure transmitters and thermometry. The Brooks mass flow meters were tested and signals verified in the I/O structure.

- One valve failed the pressure leak test due to a valve body leak that could not be repaired after numerous attempts. A new valve was ordered and installed.

- The electrician completed installing the heat trace lines. In addition, the electrician hard wired the liquid level controllers, pump P-103, pH probes, temperature probes and etc. as a result of HAZOP recommendations. All these devices will need to be tied into the computer software.

- The Neslab chiller RTD resistance thermistor was replaced after receipt of the unit sent free of charge from Neslab. However that did not resolve the problem with the chiller error message. A new electronic board was ordered and installed. We found a metal filing causing a short circuit in the Neslab readout.

- Both chiller loops were filled with Syntherm HF silicon oil and have been circulating the oil. A booster pump may be required for each of the chillers since the maximum flow rate of 3.5 gallons/minute and 7 gallons/minute was not achieved. This was due the head pressure on each of the system pumps. The Lauda heater/chiller was not cooling. The new booster pumps are positive displacement pumps so pump skids needed to be designed and built. The eight valves on the chiller side of the unit were exercised. The Brooks mass flow meters were tested and signals verified in the I/O structure.

- Vendor \#3 previously changed the controls of the Fluitron compressor from flow control to pressure control. In doing so, the functionality of other units in the area to operate with the Fluitron compressor was disabled. The Fluitron PLC requires a pressure signal from PT-6, a pressure transducer on the Bench Scale Unit in order to operate. Furthermore, the Bench Scale Unit will only operate in pressure control mode and not in flow control mode. The program for each process will look for the pressure signal from pressure control PLC. When that signal is unavailable, the program will stop. Although the Fluitron will be able to operate, Vendor \#3 's program operating the Bench Scale Unit was looking for a pressure control signal from the PLC installed on the compressor. Vector House has reprogrammed the control system to mitigate this problem and return the Fluitron compressor system controls back to its original state.

- The insulators installed the Armaflex HT insulation. Booster pumps will be required for each of the chillers since the maximum flow rates of 3.5 gallons/minute and 7 gallons/minute were not achieved, due the head pressure on each of the system pumps. 
- Water was discovered in the air lines which ruined the I to P controller. A new one was ordered and installed.

- Fifty gallons of a 50/50 volume \% mixture of water and ethylene glycol was loaded into the pump feed drum.

- The watchdog timer shut down alarm was installed to monitor the computer and the loss of ventilation alarm was installed.

- Booster pumps are required for each of the chillers since the maximum flow rate of 3.5 gallons/minute and 7 gallons/minute was not achieved, due to the head pressure on each of the system pumps. The booster pumps were delivered for the Neslab and Lauda Chillers. The pump skids were designed and assembled. The electrician completed the final electrical hook up on the pumps. Pressure testing of the pump skids was completed. The pumps were bumped to make sure that the flow was in the correct direction.

- The insulators completed insulating the booster pump skids. The software logic for booster pumps has been installed for the Neslab and Lauda Chillers. The pumps were started and circulated Syltherm heat transfer fluids at $-10^{\circ} \mathrm{F}$ through the heat exchangers and the jacketed vessels.

- The software controls for loss of ventilation was completed. An alarm annotation page was added to the main menu. In addition, a page was created in the main menu for all plant pressure transducers.

- Two emergency shutdown stop buttons were installed,. one near the exit door and one near the gas cylinder rack.

- LEL/H2S alarms were installed in the compressor room.

- Start up procedures were prepared to include proper isolation of about 12 valves for the Bench Scale Unit, the Scavenger Loop and the Gas/Liquid Membrane Unit while the Fluitron is running.

- Tags for the feed cylinders, feed drums and slop drums were installed.

- Chromalox heat exchangers were set to trip at $290{ }^{\circ} \mathrm{F}$ condition, no flow condition, and overpressure condition.

- The levels on V-102, V-104 and V-105 were set. The dehydration mode does not use these vessels.

The Bench-Scale Unit was commissioned 1QFY2009. 


\section{Task 3: Conduct Bench-Scale Tests and Verify/Generate the Design Data for Pilot -Scale Unit}

The initial commissioning activities include:

- A mixture of $40 \mathrm{vol} \%$ ethylene glycol and $60 \mathrm{vol} \%$ water was loaded into the pump feed drum. The mixture was changed from a $50 \mathrm{vol} \%$ / $50 \mathrm{vol} \%$ that was previously loaded into the feed drum.

- Ethylene glycol was circulated through V-100, V-101, V-106 and on to the 165gallon slop tanks. Good pump feed control was established; however a few new issues were identified. PRV-5 needed to be replaced with a new pressure relief valve because the cv was too low. SCV-101 was replaced because it was leaking. When the 165 gallon slop tanks were filling up, the drain plugs on the tanks leaked into the secondary containment vessel so those were fixed.

- Leak testing the unit with Snoop rendered the paper ID tags unreadable. The paper valve-, vessel-, and device-ID tags were removed and replaced with more durable plastic ones.

- A level was established in V-101 and V-106 and the scales for 0 to $100 \%$ operation were set up. Currently the gas side of the plant is being worked on by pushing nitrogen through vessels V-100, V-104, V-105 and through the Fluitron compressor and then through vessel V-300, the humidifier.

- A number of control parameters were changed in controlling the Fluitron since it was difficult to control pressure and flow in the gas loop. The controls can be returned to the way Vendor \#3 configured the unit to operate, if necessary.

- The Fluitron bypass control valve, BCV-1, was previously controlled by differential pressure transducer DPT-4. Now it uses flow transmitter FT-5 to control BCV-1. Therefore the Fluitron can pump gas at its designed flow rate and exactly the required amount of gas flow needed for the experiments can be siphoned off.

- The pressure on V-104 was being controlled by using flow transmitter FT-5. Now $\mathrm{V}-104$ is being controlled by the pressure transducer PT-8 that is located on V104.

- Control valve CCV-2 was previously controlled by V-100 bottom pressure transducer PT-3. Now it is a manual loading station where the operator can input a set point.

- Manual mode has been added to all control valves to give the operator more flexibility in operating the unit.

- The electrician replaced broken Liquid-Tite fittings, placed a Halar coated level probe in V-300 and installed a $\mathrm{pH}$ transmitter display.

- The water injection loop has been commissioned. The auto-fill on V-200 turns on and off pump P-102.

- The humidification loop has been commissioned.

- The auto-fill on V-300 turns on and off pump P-103. We initially had some trouble with P-103 pumping water but found a leaky pump head and repaired it.

- Manual loading stations were installed on P-104, P-105, CCV-2 and GCV-105A. These devices can now be placed in auto or manual. 
- It was determined that the differential pressure transducer on the tower V-100 was insufficient to measure any flooding issues that may arise.

- There was a 0-300 psid differential pressure transducer measuring the pressure between the upper tower and lower tower. At most the differential pressure will be in the range of about 2 psid. Therefore, the 0-300 psid dp cell was changed out to 0 to 100 inches water column and calibrated.

- Added the ability to change the "Control Action" from Direct (Cooling) to Reverse (Heating) for the four vessels cooled and heated by the Lauda HeaterChiller. Control action changes at $75^{\circ} \mathrm{F}$ for now.

- Eleven new tags were added to mark the unit filters.

- The heater thermocouples of the two unit process heaters H-200 and H-300 were paralleled to allow heater temperatures to be displayed on the computer screens and the Watlow temperature limit switches. A temperature control loop page was added for the two heaters.

- Calibrated the level transmitters for vessels V-102 and V-104 to 0-18 in $\mathrm{H}_{2} \mathrm{O}$ and $\mathrm{V}-105$ to $0-17$ in $\mathrm{H}_{2} \mathrm{O}$.

- Calibrated the old level transmitter for vessel V-103 to 0-100 in. $\mathrm{H}_{2} \mathrm{O}$. It has been reconfigured to measure the differential pressure between $\mathrm{V}-100$ top and $\mathrm{V}-103$ top to control the level in $\mathrm{V}-103$.

- Added a controlled emergency shutdown of the unit via three pushbutton switches: two mounted just inside of hallway doors on either side of the unit and a third switch located by the make up nitrogen cylinder. This will allow for thermal expansion of the process and cooling fluids if power is turned off to the unit. These switches parallel existing controlled shutdown logic. We will review this logic next month.

- Continued refining the unit control and display screens including the addition of process heaters $\mathrm{H}-200$ and $\mathrm{H}-300, \mathrm{~V}-104$ pressure control and level control, and $\mathrm{V}-102$ pressure and level control.

- Changed the Fluitron nitrogen flow control from V-104 outlet gas pressure to the unit inlet nitrogen flow meter, FT-5 via BCV-1 (the unit Fluitron by-pass valve).

- A stand alone fixed-point monitor with two remote sensing heads with audio and visual alarm was purchased and installed.

- The operating procedure to isolate or engage the units into the Fluitron compressor has been written.

- Trip set points have been installed.

- The alarm annunciate display page in RS View is work in progress. As the unit is operated this page will need to be monitored and updated. This page lets the operator know what alarm is shutting down the unit so action can be taken.

- Operating, Safety and Emergency shutdown procedures for determining flooding curves, mass and heat transfer have been written and testing started.

- Tests to determine flooding curves, operating liquid and gas flow rates started by evaluating pressure drop across the tower at different gas flow rates. No pressure drop data could be collected on V-100 due to unsteady gas flow rates.

- PID loop controls to all electrically controlled valves have been fine tuned to minimize oscillations in both gas and liquid flow rates 
- SCV-105B could not be fine-tuned due to a large pressure drop across the Spurger in the Off Gas Scrubber, V-105. Two valves were added to isolate/bypass V-105.

- With no detectable pressure drop across V-100, higher liquid flow rates were tried.

- It was determined that the liquid feed pump, P-101, could deliver only 35 gph even at its specified maximum power input. The suction line was changed from $1 / 2$ ' to 1 ' after consulting the pump manufacture. The pump can deliver with rates as high as $65 \mathrm{gph}$.

- Flooding curve determination stopped due to no recordable pressure difference across the tower. It was decided that heat and transfer coefficients would be determined as a function of liquid to gas $(\mathrm{L} / \mathrm{G})$ ratio

- Control loops for process Heaters H-200 and H-300 were activated as some heat transfer experiment required heating and/or cooling.

- Safe guards to protect many pieces of equipment from overheating or over pressurization have been put in place e.g. a minimum of 50 acfh of gas and $5 \mathrm{gph}$ of liquid solvent are required for continued operation of H-300 and H-200 to prevent overheating.

- Calibration of the moisture analyzer (Ametek) was completed in preparation for mass transfer tests.

- SCV-104A has been programmed to maintain a minimum pressure differential across the Fluitron for continued gas circulation in the sulfur process.

- GC calibration for ethane has been completed.

- The water/glycol mixture and kerosene could not be cooled to the desired temperature, $-40^{\circ} \mathrm{F}$, due to low chiller capacity, and short heat exchangers $\mathrm{H}-400$ and H-500. A $60 \mathrm{ft}$ shell and tube exchanger was designed and built to work in series with H-400 and H-500. Another chiller has been acquired to enhance liquid cooling.

- Level control switches in V-102 were installed. Programming for proper level control in V-102 has been completed together with the recycle pump P-100.

- An audible alarm station has been installed to alert operators of system malfunctions.

- All safe guards have been programmed into the PLC.

- Compressor room $\mathrm{H}_{2} \mathrm{~S} / \mathrm{LEL}$ monitor was installed and $\mathrm{H}_{2} \mathrm{~S}$ calibration done. The LEL sensor will be recalibrated with methane rather than pentane.

- The Bench Scale Unit is ready for the initial set experimental tests in the dehydration and absorption mode of operation. Mass and heat transfer experiments on the tower were started.

Experimental Plan of Glycol Refrigeration Dehydration and Flexible Kerosene Absorption

1. Introduction

The glycol refrigeration dehydration and flexible kerosene absorption are two of the most critical technologies in the Flexible Improved Refrigerated Oil Absorption (abbreviated as FIROA hereafter) process. A bench-scale experiment facility (abbreviated as BSE hereafter) has been designed and built for the experimental study of these technologies. A short packed column is set up for verification of the previous IIT simulation results and providing necessary experiment data for the design of a prototype unit for field tests. GTI assembled 
the Bench Scale Unit at GTI in accordance to the P\&ID illustrated in Figure 8 to collect these data. The major pieces of equipment in the Unit are listed in Table 9. As can be seen in Figure 12 and Figure 14, the main unit where absorption and reaction will take place is the absorber. It is a 4-inch-outer-diameter by 36-inch-high reactor column. The tower is packed with Sulzer Mellapak M250YTM Structured Packing. Two pumps are present in the system. The first pump is used to pump solvent into the top of the tower and the other pump is used to recycle or transfer solvent. Solvent hold-up in the column is regulated through a level transducer, and level controller assembly. Further, a pressure transducer is connected across the column to measure the pressure. The feed gases that will be used in the experiments will contain hydrogen sulfide, sulfur dioxide, and nitrogen. The flow rate of simulated feed gas through the packed bed was set to be between 20-100 scfm. The operating condition ranges for the bench-scale packed bed reactor is from -40 to $300^{\circ} \mathrm{F}$ and pressures from atmospheric to $1000 \mathrm{psig}$.

During general operations, nitrogen is used as the carrier gas. Nitrogen is supplied to the Unit from a circulating gas system that utilizes the Fluitron compressor to make up any pressure losses in the system. Seven mass flow controllers can meter gas components into the feed stream. The feed gas can be pre-saturated with water as needed using V-300. The gases are fed to the bottom of the column and flow countercurrent to the solvent flowing down the column. The treated gas stream exits the top of the column and goes through the knockout and scrubber prior to being reintroduced to the circulating gas system. The rich solvent goes through the flash and into a gas/liquid/liquid separator where the vapors, solvent and elemental sulfur are separated. The solvent can be recycled or collected for treating. The recycled and fresh solvent can be introduced at the top or bottom of the column to allow either co- or counter-current flow in the column. Gas and liquid samples can be taken throughout the Unit and instrumentation records the necessary temperature, pressure, and flow rates in order to establish energy and material balances. A data logger will collect the transmitted data and a bound laboratory record book will be used to maintain all manual measurements and notes from the operations. 
Table 9 Bench Scale Unit Major Equipment Lisiting

\begin{tabular}{|c|c|c|}
\hline Equip. Number & Equip. Name & Note \\
\hline & Fluitron Compressor & Located in basement \\
\hline & $\begin{array}{l}\text { Blowdown Drum } \\
\text { Feed Storage } \\
\text { Spent Solvent }\end{array}$ & \\
\hline $\mathrm{H}-100$ & Heat Exchanger & $1 / 2$ in. in $1 \frac{1}{2}$ in. shell $\times 3 \mathrm{ft}$ long \\
\hline $\mathrm{H}-100$ & Chiller & \\
\hline $\mathrm{H}-200$ & Heat Exchanger & \\
\hline $\mathrm{H}-300$ & Heat Exchanger & \\
\hline $\mathrm{H}-400(\mathrm{x} 2)$ & Heat Exchanger & $1 / 2$ in. in $1 \frac{1}{2}$ in. shell $\times 3 \mathrm{ft}$ long \\
\hline $\mathrm{H}-500(\mathrm{x} 3)$ & Heat Exchanger & $1 / 2$ in. in $1 \frac{1}{2}$ in. shell $\times 3 \mathrm{ft}$ long \\
\hline H-600 & Heat Exchanger & $1 / 2$ in. in $1 \frac{1}{2}$ in. shell $\times 3 \mathrm{ft}$ long \\
\hline $\mathrm{P}-100$ & PD Solvent Recirc. Pump & \\
\hline $\mathrm{P}-101$ & PD Feed Pump & \\
\hline $\mathrm{V}-100$ & Absorber Column & $4 \frac{1}{2}$ in. OD x 72 in. \\
\hline V-101 & HP Flash Separator & \\
\hline V-102 & Gas/Liquid/Liquid Separator & $105 / 8$ in. $O D \times 36$ in. \\
\hline V-103 & Methane Saturation Vessel & \\
\hline V-104 & Knockout & $85 / 8$ in. OD x 24 in. \\
\hline V-105 & Off Gas Scrubber & $85 / 8$ in. $O D \times 32$ in. \\
\hline V-106 & Knockout Pot & 7 in. OD x 30 in. \\
\hline V-200 & Quench Water Vessel & $123 / 4$ in. OD x 12 in. \\
\hline $\mathrm{V}-300$ & Saturation Vessel & $41 / 2$ in. OD x 60 in. \\
\hline
\end{tabular}




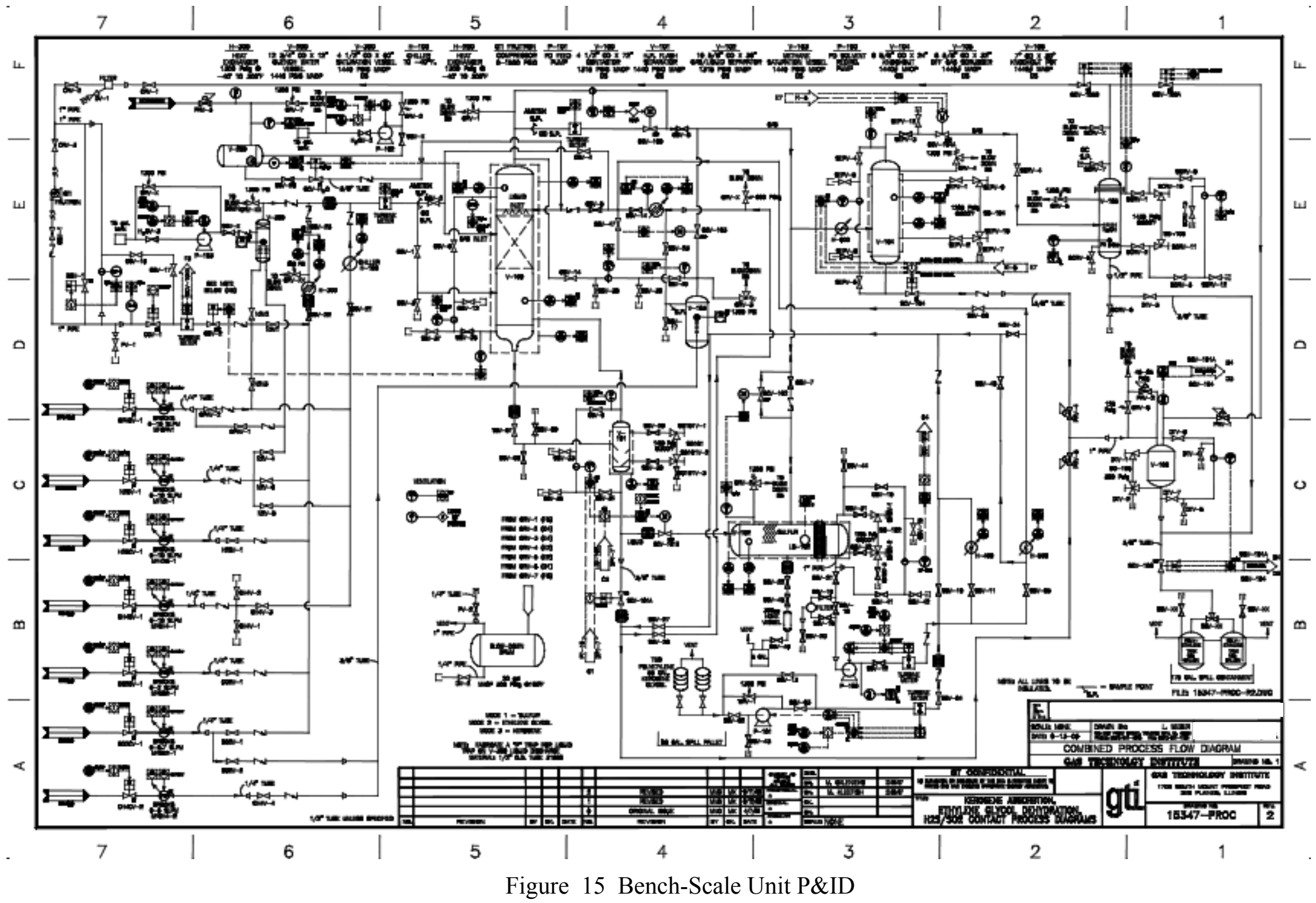


2. The Bases of the Proposed Experiments

(1) The BSE has a packed column of $4 \frac{1 / 2}{2}$ OD X 72" with 36 " high random packed packing materials of which the experiment with a raw gas stream between 20-100 scfm under $1000 / 315$ psig and $120^{\circ} \mathrm{F}$ to $-50^{\circ} \mathrm{F}$ could be conducted;

(2) The simulation data to be verified are based on IIT simulation report to GTI, Appendix B, with appropriate modifications according to potential FIROA commercialization requirements presented in various previous GHT-Conf reports;

(3) The raw gas input rates given in this proposal are assumed to be 0.1 MMscfd ( 70 $\mathrm{scfm}$ ), GTI may adjust the rates to meet the best experiment conditions according to actual BSE performance;

(4) Since the glycol refrigeration dehydration is basically a heat-transfer process which is not the design objective of packing performance, heat transfer capabilities of the packed column should be calibrated before dehydration.

3. Proposal on Glycol Refrigeration Dehydration Experiments

3.1 Introduction

The glycol refrigeration dehydration process differs radically from the traditional glycol absorption. In the traditional glycol dehydration process, a high purity glycol is used as a liquid desiccant. On the contrary, the glycol refrigeration dehydration uses very dilute $(\sim 16 \%$ mol) glycol-water solution as a coolant to raw gas to condense the moisture and dissolve the condensate in the solution so as to separate the water from the raw gas. The condensed water is then discharged with a small portion of the glycol solution coolant and evaporated. The present glycol refrigeration dehydration process, therefore, is a heat-transfer process, not an absorption process.

\subsection{Objectives}

(1) Demonstration of dehydration efficiency: The inlet raw gas, saturated with water vapor, should be cooled from $100^{\circ} \mathrm{F}$ down to an temperature above or slightly below the dew point of the NGL components of the raw gas, wherein more than $90 \%$ (depending on gas richness) of the moisture would be removed by the counterflowing glycol solution.

(2) Thermal calibration of packing material: Since the heat transfer characteristic data of the packing material are not generally available from the vendor, another objective of this BSE experiment is to calibrate the column V-100 to obtain the experimental heat-transfer efficient curves of the packing material.

(3) Demonstration of energy saving potential: In order to get maximum energy efficiency, the ideal average temperature gradient between the counter-flowing gas and liquid should always be kept about $5^{\circ} \mathrm{F}$.

(4) NGL Condensate Foaming Check: Determine the degree of foaming of the NGL condensate in the exit glycol solution. 
3.3 Cases to be studied

Table 10 Cases for Study and the Raw Gas Input Data

\begin{tabular}{|c|c|c|c|c|c|c|c|c|c|}
\hline Case & $\mathrm{T}$ & $\mathrm{P}$ & Flow Rate, $\mathrm{Q}_{0 \mathrm{G}}$ & $\mathrm{C}_{1}$ & $\mathrm{C}_{2}$ & $\mathrm{C}_{3}$ & $\mathrm{C}_{4+}$ & $\mathrm{H}_{2} \mathrm{O}$ & $\mathrm{Heat} \mathrm{Flow}$ \\
\hline Unit & ${ }^{\circ} \mathrm{F}$ & $\mathrm{psia}$ & $\mathrm{lbmol} / \mathrm{hr}$ & $1 \mathrm{bmol} / \mathrm{hr}$ & $1 \mathrm{bmol} / \mathrm{hr}$ & $\mathrm{lbmol} / \mathrm{hr}$ & $1 \mathrm{bmol} / \mathrm{hr}$ & $\mathrm{lbmol} / \mathrm{hr}$ & $\mathrm{MMBtu} / \mathrm{hr}$ \\
\hline $1(\mathrm{SU})$ & 100 & 900 & 11.004 & 9.012 & 1.066 & 0.494 & 0.418 & 0.014 & -0.3805 \\
\hline $2(\mathrm{R})$ & 100 & 900 & 11.004 & 9.556 & 0.870 & 0.364 & 0.200 & 0.014 & -0.3723 \\
\hline $3(\mathrm{~L})$ & 100 & 900 & 11.004 & 10.112 & 0.523 & 0.193 & 0.162 & 0.014 & -0.3672 \\
\hline
\end{tabular}

3.4 Other input data

Table 11 Glycol Solution Input Data

\begin{tabular}{|c|c|c|c|c|c|c|c|c|}
\hline Case & $\mathrm{T}$ & $\mathrm{P}$ & Flow Rate, $\mathrm{Q}_{0 \mathrm{~L}}$ & $\mathrm{H}_{2} \mathrm{O}$ & Glycol & Heat Flow & $\mathrm{H}_{\text {EXIT }}\left(95^{\circ} \mathrm{F}\right)$ & $\Delta \mathrm{H}$ \\
\hline Unit & ${ }^{\circ} \mathrm{F}$ & $\mathrm{psia}$ & $1 \mathrm{bmol} / \mathrm{hr}$ & $1 \mathrm{bmol} / \mathrm{hr}$ & $1 \mathrm{bmol} / \mathrm{hr}$ & $\mathrm{MMBtu} / \mathrm{hr}$ & $\mathrm{MMBtu} / \mathrm{hr}$ & $\mathrm{MMBtu} / \mathrm{hr}$ \\
\hline $1(\mathrm{SU})$ & 20 & 920 & $9.510( \pm 20 \%)$ & 8.005 & 1.505 & -1.282 & -1.269 & 0.013 \\
\hline $1(\mathrm{SU})$ - Foam & 0 & 920 & $9.510( \pm 20 \%)$ & 8.005 & 1.505 & -1.288 & -1.269 & 0.019 \\
\hline $2(\mathrm{R})$ & -6.5 & 920 & $7.027( \pm 20 \%)$ & 5.915 & 1.112 & -0.954 & -0.938 & 0.016 \\
\hline $3(\mathrm{~L})$ & -15 & 920 & $6.484( \pm 20 \%)$ & 5.458 & 1.026 & -0.882 & -0.865 & 0.017 \\
\hline
\end{tabular}

3.5 Simulation data to be verified

Table 12 Case 1 (SU) Data to be Verified

\begin{tabular}{|c|c|c|c|c|c|c|c|c|c|}
\hline Stream & $\mathrm{T}$ & $\mathrm{P}$ & Flow Rate, $\mathrm{Q}$ & $\mathrm{C}_{1}$ & $\mathrm{C}_{2}$ & $\mathrm{C}_{3}$ & $\mathrm{C}_{4+}$ & $\mathrm{H}_{2} \mathrm{O}$ & $\mathrm{Glycol}$ \\
\hline Unit & ${ }^{\circ} \mathrm{F}$ & $\mathrm{psia}$ & $1 \mathrm{bmol} / \mathrm{hr}$ & $\mathrm{lbmol} / \mathrm{hr}$ & $1 \mathrm{bmol} / \mathrm{hr}$ & $\mathrm{lbmol} / \mathrm{hr}$ & $1 \mathrm{bmol} / \mathrm{hr}$ & $\mathrm{lbmol} / \mathrm{hr}$ & $\mathrm{Mscfd}$ \\
\hline Exit Gas & 25 & 890 & $11.004-$ & $9.012-$ & $1.066-$ & $0.494-$ & $0.418-$ & $0.0004 *$ & 0 \\
\hline Exit Liquid & 95 & 900 & $9.523+$ & trace & trace & trace & trace & $8.018+$ & 1.505 \\
\hline
\end{tabular}

*Exclude entrained water/solution droplets. 
Table 13 Case 1 (SU) - Foam Data to be Verified (Evaluate the NGL condensate foaming)*

\begin{tabular}{|c|c|c|c|c|c|c|c|c|c|}
\hline Stream & $\mathrm{T}$ & $\mathrm{P}$ & Flow Rate, $\mathrm{Q}$ & $\mathrm{C}_{1}$ & $\mathrm{C}_{2}$ & $\mathrm{C}_{3}$ & $\mathrm{C}_{4+}$ & $\mathrm{H}_{2} \mathrm{O}$ & $\mathrm{Glycol}$ \\
\hline Unit & ${ }^{\circ} \mathrm{F}$ & $\mathrm{psia}$ & $\mathrm{lbmol} / \mathrm{hr}$ & $1 \mathrm{bmol} / \mathrm{hr}$ & $1 \mathrm{bmol} / \mathrm{hr}$ & $\mathrm{lbmol} / \mathrm{hr}$ & $1 \mathrm{bmol} / \mathrm{hr}$ & $1 \mathrm{bmol} / \mathrm{hr}$ & $\mathrm{Mscfd}$ \\
\hline Exit Gas & 5 & 890 & $<11.004^{*}$ & $\mathrm{TBD}$ & $\mathrm{TBD}$ & $\mathrm{TBD}$ & $\mathrm{TBD}$ & $0.0004^{*}$ & 0 \\
\hline Exit Liquid & $\mathrm{TBD}$ & 900 & $>9.523^{*}$ & $\mathrm{TBD}$ & $\mathrm{TBD}$ & $\mathrm{TBD}$ & $\mathrm{TBD}$ & $>8.018^{* *}$ & 1.505 \\
\hline
\end{tabular}

*Exclude entrained water/solution droplets.

**Not found in IIT simulation, only experimental results are expected.

Table 14 Case 2 (R) Data to be Verified

\begin{tabular}{|c|c|c|c|c|c|c|c|c|c|}
\hline Stream & $\mathrm{T}$ & $\mathrm{P}$ & Flow Rate, $\mathrm{Q}$ & $\mathrm{C}_{1}$ & $\mathrm{C}_{2}$ & $\mathrm{C}_{3}$ & $\mathrm{C}_{4+}$ & $\mathrm{H}_{2} \mathrm{O}$ & $\mathrm{Glycol}$ \\
\hline Unit & ${ }^{\circ} \mathrm{F}$ & $\mathrm{psia}$ & $\mathrm{lbmol} / \mathrm{hr}$ & $\mathrm{lbmol} / \mathrm{hr}$ & $\mathrm{lbmol} / \mathrm{hr}$ & $\mathrm{lbmol} / \mathrm{hr}$ & $\mathrm{lbmol} / \mathrm{hr}$ & $\mathrm{lbmol} / \mathrm{hr}$ & $\mathrm{lbmol} / \mathrm{hr}$ \\
\hline Exit Gas & -1.5 & 890 & 11.004 & $9.556-$ & $0.870-$ & $0.364-$ & $0.200-$ & $0.0003^{*}$ & 0 \\
\hline Exit Liquid & 95 & 900 & $7.040+$ & trace & trace & trace & trace & $5.928+$ & 1.112 \\
\hline
\end{tabular}

*Exclude entrained water/solution droplets.

Table 15 Case 3 (L) Data to be Verified

\begin{tabular}{|c|c|c|c|c|c|c|c|c|c|}
\hline Stream & $\mathrm{T}$ & $\mathrm{P}$ & Flow Rate, $\mathrm{Q}$ & $\mathrm{C}_{1}$ & $\mathrm{C}_{2}$ & $\mathrm{C}_{3}$ & $\mathrm{C}_{4+}$ & $\mathrm{H}_{2} \mathrm{O}$ & $\mathrm{Glycol}$ \\
\hline Unit & ${ }^{\circ} \mathrm{F}$ & $\mathrm{psia}$ & $\mathrm{lbmol} / \mathrm{hr}$ & $\mathrm{lbmol} / \mathrm{hr}$ & $\mathrm{lbmol} / \mathrm{hr}$ & $\mathrm{lbmol} / \mathrm{hr}$ & $\mathrm{lbmol} / \mathrm{hr}$ & $\mathrm{lbmol} / \mathrm{hr}$ & $\mathrm{lbmol} / \mathrm{hr}$ \\
\hline Exit Gas & -10 & 890 & $11.004-$ & $10.112-$ & $0.523-$ & $0.193-$ & $0.162-$ & $0.0002^{*}$ & 0 \\
\hline Exit Liquid & 95 & 900 & $6.497+$ & trace & trace & trace & trace & $5.471+$ & 1.026 \\
\hline
\end{tabular}

*Exclude entrained water/solution droplets. 


\subsection{Proposed Experimental Steps}

A. Thermal Calibration of the Packing Material (Use Case 3(L) inputs)

(1) Prepare input materials

a. For raw gas, prepare the estimated amount of Case 3(L) gas mixture sufficient for the entire run time, including the shake-down before reaching steady state for measurements;

b. For glycol solution, prepare $20 \%$ more than the estimated amount of Case $3(\mathrm{~L})$ to allow sufficient flow rate adjustment.

(2) Flow the raw gas and the glycol solution under the initial condition as described in Sec. 3.2 and 3.3 into the column until the flow is in stable condition.

(3) Measure the temperature of the exit gas stream (top) and the exit liquid (bottom).

(4) Adjust the glycol solution input rate until the temperature of the exit gas stream (top) reaches the simulation value $-10^{\circ} \mathrm{F}$ (given in Sec.3.4 Table 11), while keeping the temperature gradient at $5^{\circ} \mathrm{F}$ between inlet glycol solution and the exit gas, and leaving the exit liquid temperature unadjusted. This temperature is generally lower than simulated exit liquid temperature.

(5) Record all the measured data of the input and exit streams, including the components of the exit gas and liquid stream.

(6) Calculate and record the average heat transfer coefficient (h1) and the total heat transferred $(\Delta \mathrm{H} 1)$ of the packing section as well as the data taken in step (5). The data are the starting point of the packing thermal performance curve illustrating the relation of $h$ and $\Delta \mathrm{H}$ vs. flow rate of the input gas or liquid $\left(\mathrm{Q}_{0 \mathrm{G}(\mathrm{L})}\right.$ or $\left.\mathrm{Q}_{0 \mathrm{~L}(\mathrm{~L})}\right)$. (The subscript "(L)" indicates Lean Gas)

(7) Take the difference of the actual exit liquid temperature of step (4) and the simulated target temperature $\left(95^{\circ} \mathrm{F}\right)$. Divide the difference by 5 to obtain the temperature increments $(\Delta t)$ for next five runs.

(8) Repeat steps (4) with reducing both input gas and glycol solution flow at the same time, keeping the concentration of the solution and the temperature of the exit gas stream (top) constant, until the exit liquid temperature rises to $t+\Delta t$, and then repeat the steps (5) and (6) and get the 2 nd set data of the packing thermal performance curve.

(9) Repeat step (8) three more times to get the 3rd, 4th, 5th and 6th set data of the packing thermal performance curve. At the 6th run, the exit temperature of the bottom glycol solution should be $95^{\circ} \mathrm{F}$, while keeping the average temperature gradient between inlet raw gas and the exit glycol solution about $5^{\circ} \mathrm{F}$.

(10) Draw the packing thermal performance curve(s), to illustrate the relation of heat transfer coefficient, $h$, and total heat transferred, $(\Delta \mathrm{H})$, vs. the various set of input gas/liquid flow rates. These curves could be used for future engineering designs.

B. Carry Out Refrigeration Dehydration Experiments for Different Raw Gas Inputs (Cases $1(\mathrm{SU})$ and Case 2(R))

(1) Use the above calibration results for estimating the tentative estimated flow rates of the input raw gas $\mathrm{Q}_{0 \mathrm{G}}$ and glycol solution $\mathrm{Q}_{0 \mathrm{~L}}$ for direct comparison with IIT simulation results. Use the above calibration results for Lean gas, we could calculate the ratio of the 6th run input raw gas Q0G6(L) and the IIT simulation Q0G (L): $\mathrm{R}=\mathrm{Q}_{0 \mathrm{G} 6(\mathrm{~L})} / \mathrm{Q}_{0 \mathrm{G}(\mathrm{L})}=\mathrm{Q}_{0 \mathrm{G} 6(\mathrm{~L})} / 11.004$. Then the estimated flow rate for Case 
$1(\mathrm{SU}), \mathrm{Q}_{0 \mathrm{G}(\mathrm{SU})}=11.004 * \mathrm{R}$ and $\mathrm{Q}_{0 \mathrm{~L}(\mathrm{SU})}=9.51 * \mathrm{R}$; and for Case $2(\mathrm{R}), \mathrm{Q}_{0 \mathrm{G}(\mathrm{R})}=$ $11.004 * \mathrm{R}$ and $\mathrm{Q}_{\mathrm{OL}(\mathrm{R})}=7.027 * \mathrm{R}$.

(2) Calculate the input material quantity according to the above equations. The actual quantity prepared for both gas and glycol solution should increase $20 \%$ of the calculated value to allow adjustment needs. Flow the raw gas and the glycol solution into the column until the flow is in stable condition. Be aware the input glycol solution temperature for Cases $1(\mathrm{SU})$ is $20^{\circ} \mathrm{F}$. Measure the temperature of the exit gas stream (top) and the exit liquid (bottom). Adjust the glycol solution input rate until the temperature of the exit gas stream (top) reaches the relevant simulation value given in the relevant table above.

Check the exit liquid temperature, if not match the simulation value $\left(95^{\circ} \mathrm{F}\right)$, adjust both gas and liquid inputs in same proportion (keeping the ratio of gas to liquid input constant) while keeping the gas exit temperature constant, until the liquid exit temperature reaches $95^{\circ} \mathrm{F}$.

Record all the measured data of the input and exit streams, including the components of the exit gas and liquid stream.

Check the experimental data with simulation data to correct the discrepancies, and calculate the average heat transfer coefficient and total heat transferred for this case. Repeat all the above steps for the next case.

C. Carry Out Refrigeration Dehydration Experiments for Evaluation the NGL Condensate Foaming Problem (Cases 1(SU)-Foam)

The case 1(SU) - Foam experiment is designed to examine one of the pending problem of the possibility of NGL condensate foaming when condensed on a counter-flowing liquid film of glycol solution. No simulation data is available. The future commercial plant design could only depend on experimental data.

In FIROA process, this problem is circumvented with two-stage refrigeration dehydration: only the 1st stage use the glycol solution direct cooling to a temperature slightly above or below the NGL dew point to reduce the foaming to a minimum. (Ref. Case1(SU)) No significant amount of NGL condensate would appear in this sage, but over $90 \%$ of the water vapor in raw gas is removed. However, if the foaming problem is not so serious, a single stage approach could further simplify the overall system and save more capital investment. This argument leads to the proposed Case 1(SU) - Foam experiment.

In Case 1(SU) - Foam experiment, the separator V-101 is used as a 3-phase (Glycol solution, NGL condensate and entrained gas). The middle and the bottom liquid samples are taken separately from SSV-29 - SG101V-1 and SSV-30 - SG101V-2 and their components analyzed. Evaluation the degree of intermingling of NGL condensate and glycol solution in these samples may help to answer the foaming question.

The steps for this experiment are similar to the above except the last few additional foaming examination steps.

(1) Use the above calibration results for estimating the tentative estimated flow rates of the input raw gas $\mathrm{Q}_{0 \mathrm{G}}$ and glycol solution Q0L for comparison with IIT simulation results. In above calibration for Lean gas, find the ratio of the 6th run input raw gas $\mathrm{Q}_{0 \mathrm{G} 5(\mathrm{~L})}$ and the IIT simulation $\mathrm{Q}(\mathrm{L}): \mathrm{R}=\mathrm{Q}_{0 \mathrm{G} 6(\mathrm{~L})} / \mathrm{Q}_{(\mathrm{L})}=\mathrm{Q}_{0 \mathrm{G} 6(\mathrm{~L})} / 11.004$. Then for Case 1(SU)-Foam, $\mathrm{Q}_{\mathrm{OG}(\mathrm{SU})}=11.004 * \mathrm{R}$ and $\mathrm{Q}_{\mathrm{OL}(\mathrm{SU})}=9.51 * \mathrm{R}$.

(2) Prepare input materials according to the estimated quantities. The quantity of both 
gas and glycol solution should increase $30 \%$ to allow adjustment needs.

Flow the raw gas and the glycol solution into the column until the flow is in stable condition. Be aware the input gas temperature for Cases $1(\mathrm{SU})$-Foam is $0^{\circ} \mathrm{F}$.

(4) Measure the temperature of the exit gas stream (top) and the exit liquid (bottom);

(5) Adjust the glycol solution input rate until the temperature of the exit gas stream reaches the relevant simulation value given in the relevant table above.

(6) Check the exit liquid temperature, if not match the simulation value $\left(95^{\circ} \mathrm{F}\right)$, adjust both gas and liquid inputs simultaneously, keeping the ratio of gas to liquid input constant and the gas exit temperature constant, until the liquid exit temperature reaches $95^{\circ} \mathrm{F}$.

(7) Record all the measured data of the input and exit streams, including the components of the exit gas and liquid stream.

(8) Check the experimental data with simulation data to correct the discrepancies, and calculate the average heat transfer coefficient and total heat transferred for this case.

(9) Take samples from the separator V-101: the NGL condensate rich liquid sample from SSV-29 - SG101V-1 and the glycol solution liquid samples from SSV-30 SG101V-2.

(10) Analyze and record the respective components of the above two samples

(11) Evaluation and report the degree of intermingling of NGL condensate and glycol solution.

4. Flexible Kerosene Absorption Experiments

4.1 Introduction

The experiment of Flexible Kerosene Absorption in column V-100 seems straightforward. The vendor should have provided GTI the basic parameters such as the value of the equivalent theoretical plate (ETP) and the flow characteristics of the packing material. GTI has already selected the absorbent to be used (kerosene), and got the basic VLE data for BSE experiment. Using these data as the basis of the BSE, the simulation results could be verified in BSE experiments. However, because the packing height of the BSE column V-100 is very short, the value of ETP is rather small as compared with the actual 20 ETP used in full scale column of which the previous IIT simulation was based. The recovery rates of $\mathrm{C}_{2}$ obtained from $\mathrm{V}-100$ packing would be far below the required $95 \%$ in IIT simulation, and, hence, the BSE results could not be used for direct verification the IIT simulation performance of FIROA process.

To verify the change of IIT simulation values of the full-scale column from $95 \% \mathrm{C}_{2}$ recovery to $\sim 35 \%$, therefore, a few new simulations of the Absorber column (not the entire system) have to be carried out before the BSE Flexible Kerosene Absorption Experiments, the series of new simulations include (1) a full-scale column simulation of the full-scale Absorber like the IIT simulation, using kerosene as absorbent and keeping the working temperature at $40^{\circ} \mathrm{F}$ throughout the column; (2) a short-column simulation (V-100) using $\mathrm{C}_{10} \mathrm{H}_{22}$ as absorbent without the additional cooling of the column; and (3) a short-column simulation (V-100) using kerosene as absorbent. All simulations should be done for three different $\mathrm{C}_{2}$ recovery cases, i.e. $95 \%, 70 \%$, and $35 \%$ or the equivalent in short column case. Direct verification could only be carried out between the short-column simulation (3) and the BSE experiments results. The discrepancies of recovery efficiencies between short column simulations (3) and BSE experiment could be extrapolated to the full-size column simulation (1) using kerosene absorbent. Eventually, the comparison between full-size simulations using 
different types of absorbent $\left(\mathrm{C}_{10} \mathrm{H}_{22}\right.$ and kerosene $)$ could be obtained.

Considering the time and resource limitation, only two extreme cases need be verified, i.e. case 1(SU) and Case 3(L). Once the super-rich (SU) and lean (L) gas cases have been verified, the validity of the rich (R) gas simulation would be out of question.

\subsection{Objectives}

(1) Demonstration of the improved absorption efficiency and the flexibility of $\mathrm{C}_{2}$ recovery rates with changing the flow rate of the absorbent: The recovery rate of $\mathrm{C}_{2}$ could reach $95 \%$ with single absorbent, kerosene, and the recovery rate could be varied from $95 \%$ to $35 \%$ with varying the absorbent flow rate only.

(2) Verification of the experimental results with the simulation results: Since ETP of the 36 " packing of V-100 column is only a fraction of the actual column in previous IIT simulation ( $20 \mathrm{ETP})$, a modified simulation based on the vendor's data should be carried out for the selected kerosene absorbent. The BSE experiment data should be compared with this modified simulation results, and then extrapolated to predict the future performance of the actual full-size column. 
4.3 Cases to be studied

Table 16 Cases to be Studied and the Raw Gas Input Data (IIT Report)

\begin{tabular}{|c|c|c|c|c|c|c|c|c|}
\hline Case & $\mathrm{T}$ & $\mathrm{P}$ & Flow Rate, $\mathrm{Q}_{0 \mathrm{G}}$ & $\mathrm{C}_{1}$ & $\mathrm{C}_{2}$ & $\mathrm{C}_{3}$ & $\mathrm{C}_{4+}$ & $\mathrm{H}_{2} \mathrm{O}$ \\
\hline Unit & ${ }^{\circ} \mathrm{F}$ & Psia & $1 \mathrm{bmol} / \mathrm{hr}$ & $1 \mathrm{bmol} / \mathrm{hr}$ & $1 \mathrm{bmol} / \mathrm{hr}$ & $1 \mathrm{bmol} / \mathrm{hr}$ & $\mathrm{lbmol} / \mathrm{hr}$ & $\mathrm{lbmol} / \mathrm{hr}$ \\
\hline $1(\mathrm{SU})$ & -40 & 890 & 10.991 & 9.012 & 1.066 & 0.494 & 0.418 & 0.0004 \\
\hline $3(\mathrm{~L})$ & -40 & 890 & 11.991 & 10.112 & 0.523 & 0.193 & 0.162 & 0.0002 \\
\hline
\end{tabular}

4.4 Other Input Data

Table 17 Absorbent $\left(\mathrm{C}_{10} \mathrm{H}_{22}\right)$ Input Data (IIT Report)

\begin{tabular}{|c|c|c|c|c|c|c|c|c|}
\hline Case & $\mathrm{T}$ & $\mathrm{P}$ & Max. Flow Rate, $\mathrm{Q}_{0 \mathrm{~L}}$ & $\mathrm{C}_{10} \mathrm{H}_{22}$ & $\mathrm{C}_{1}$ & $\mathrm{C}_{2}$ & $\mathrm{C}_{3}$ & $\mathrm{C}_{4+}$ \\
\hline Unit & ${ }^{\circ} \mathrm{F}$ & $\mathrm{Psia}$ & $\mathrm{lbmol} / \mathrm{hr}$ & $1 \mathrm{bmol} / \mathrm{hr}$ & $\mathrm{lbmol} / \mathrm{hr}$ & $\mathrm{lbmol} / \mathrm{hr}$ & $\mathrm{lbmol} / \mathrm{hr}$ & $\mathrm{lbmol} / \mathrm{hr}$ \\
\hline 1 (SU) $95 \%$ & -38.5 & 900 & 2.285 & 1.735 & 0.543 & 0.003 & 0 \\
\hline $1(\mathrm{SU}) 70 \%$ & -38.5 & 900 & 1.100 & 0.829 & 0.261 & 0.006 & 0.004 & 0.003 \\
\hline 1 (SU) $35 \%$ & -38.5 & 900 & 0.420 & 0.208 & 0.096 & 0.091 & 0.020 & 0.005 \\
\hline $3(\mathrm{~L}) 95 \%$ & -38.5 & 900 & 3.077 & 2.339 & 0.0 .732 & 0.002 & 0 & 0.004 \\
\hline $1(\mathrm{~L}) 70 \%$ & -38.5 & 900 & 1.924 & 1.461 & 0.458 & 0.001 & 0 & 0.004 \\
\hline $1(\mathrm{~L}) 35 \%$ & -38.5 & 900 & 1.049 & 0.755 & 0.247 & 0.046 & 0 & 0.001 \\
\hline
\end{tabular}

4.5 Simulation results to be verified

IIT original simulation results, based on full-scale column, ETP 20 , and $\mathrm{C}_{10} \mathrm{H}_{22}$ absorbent (pre-saturated with $\mathrm{C}_{1}$ ) 
Table 18 Original Simulation Results (IIT, SU gas)

\begin{tabular}{|c|c|c|c|c|c|c|c|c|c|}
\hline \multicolumn{2}{|c|}{ Item } & $\mathrm{T}$ & $\mathrm{P}$ & Flow Rate, $Q_{0 \mathrm{G}}$ & $\mathrm{C}_{1}$ & $\mathrm{C}_{2}$ & $\mathrm{C}_{3}$ & $\mathrm{C}_{4+}$ & $\mathrm{C}_{10}$ \\
\hline Case & Stream & $\mathrm{F}$ & Psia & $\mathrm{lbmol} / \mathrm{hr}$ & $\mathrm{lbmol} / \mathrm{hr}$ & lbmol/hr & lbmol/hr & lbmol/hr & $\mathrm{lbmol} / \mathrm{hr}$ \\
\hline \multirow{3}{*}{$\begin{array}{c}1(\mathrm{SU}) \\
95 \% \mathrm{C}_{2}\end{array}$} & Gas Exit & -40 & 880 & 8.283 & 8.226 & 0.057 & 0 & 0 & 0 \\
\hline & RO Exit & -40 & 890 & 7.179 & 3.118 & 1.337 & 0.550 & 0.438 & 1.736 \\
\hline & Gas Return & -35 & 890 & 2.186 & 1.788 & 0.325 & 0.056 & 0.017 & 0 \\
\hline \multirow{3}{*}{$\begin{array}{c}1(\mathrm{SU}) \\
70 \% \mathrm{C}_{2}\end{array}$} & Gas Exit & -40 & 880 & 8.647 & 8.324 & 0.322 & 0 & 0.001 & 0 \\
\hline & RO Exit & -40 & 890 & 5.385 & 2.490 & 1.056 & 0.567 & 0.443 & 0.829 \\
\hline & Gas Return & -35 & 890 & 1.944 & 1.545 & 0.305 & 0.072 & 0.022 & 0 \\
\hline \multirow{3}{*}{$\begin{array}{c}1(\mathrm{SU}) \\
35 \% \mathrm{C}_{2}\end{array}$} & Gas Exit & -40 & 880 & 9.074 & 8.398 & 0.617 & 0.056 & 0.003 & 0 \\
\hline & RO Exit & -40 & 890 & 4.429 & 2.321 & 0.873 & 0,567 & 0.459 & 0.209 \\
\hline & Gas Return & -35 & 890 & 2.101 & 1.617 & 0.335 & 0.110 & 0.039 & 0 \\
\hline
\end{tabular}

Table 19 Original Simulation Results (IIT, L gas)

\begin{tabular}{|c|c|c|c|c|c|c|c|c|c|}
\hline \multicolumn{2}{|c|}{ Item } & $\mathrm{T}$ & $\mathrm{P}$ & Flow Rate, $Q_{0 G}$ & $\mathrm{C}_{1}$ & $\mathrm{C}_{2}$ & $\mathrm{C}_{3}$ & $\mathrm{C}_{4+}$ & $\mathrm{C}_{10}$ \\
\hline Case & Stream & ${ }^{\circ} \mathrm{F}$ & Psia & lbmol/hr & $\mathrm{lbmol} / \mathrm{hr}$ & $\mathrm{lbmol} / \mathrm{hr}$ & $\mathrm{lbmol} / \mathrm{hr}$ & $\mathrm{lbmol} / \mathrm{hr}$ & $\mathrm{lbmol} / \mathrm{hr}$ \\
\hline \multirow{3}{*}{$\begin{array}{c}3(\mathrm{~L}) \\
95 \% \mathrm{C}_{2}\end{array}$} & Gas Exit & -40 & 880 & 9.680 & 9.655 & 0.024 & 0 & 0.001 & 0 \\
\hline & RO Exit & -40 & 890 & 5.617 & 2.296 & 0.606 & 0.207 & 0.169 & 2.239 \\
\hline & Gas Return & -35 & 890 & 1.230 & 1.108 & 0.105 & 0.013 & 0.004 & 0 \\
\hline \multirow{3}{*}{$\begin{array}{c}3(\mathrm{~L}) \\
70 \% \mathrm{C}_{2}\end{array}$} & Gas Exit & -40 & 880 & 9.903 & 9.749 & 0.154 & 0 & 0 & 0 \\
\hline & RO Exit & -40 & 890 & 3.913 & 1.621 & 0.453 & 0.208 & 0.169 & 1.462 \\
\hline & Gas Return & -35 & 890 & 0.907 & 0.806 & 0.083 & 0.014 & 0.004 & 0 \\
\hline \multirow{3}{*}{$\begin{array}{c}3(\mathrm{~L}) \\
35 \% \mathrm{C}_{2}\end{array}$} & Gas Exit & -40 & 880 & 10.187 & 9.850 & 0.337 & 0 & 0 & 0 \\
\hline & RO Exit & -40 & 890 & 2.481 & 1.059 & 0.291 & 0.209 & 0.167 & 0.754 \\
\hline & Gas Return & -35 & 890 & 0.629 & 0.550 & 0.058 & 0.016 & 0.005 & 0 \\
\hline
\end{tabular}

A. GTI new simulation results, based on full-scale column, ETP 20, and kerosene absorbent (pre-saturated with $\mathrm{C}_{1}$ ) New Simulation Results (GTI, SU gas, with gas return)

B. GTI new simulation results, based on V-100 short column, ETP ??, and $\mathrm{C}_{10} \mathrm{H}_{22}$ absorbent (pure, not pre-saturated with $\mathrm{C}_{1}$ ) New BSE Simulation Results (GTI, SU gas, no gas return)

C. GTI new simulation results, based on V-100 short column, ETP ??, and kerosene absorbent (pure, not pre-saturated with $\mathrm{C}_{1}$ ) New BSE Simulation Results (GTI, SU gas, no gas return) 
4.6 Proposed experiment steps (For each type of raw gas)

(5) Prepare input materials

a. For raw gas, prepare the estimated amount of gas mixture sufficient for the entire run time, including the shake-down before reaching steady state for measurements;

b. For absorbent (kerosene), prepare $20 \%$ more than the estimated amount of $95 \%$ case to allow ample flow rate adjustment needs.

(6) Flow the raw gas and the absorbent under the initial condition as described in Sec. 4.3 and 4.4 into the column until the flow is in stable condition.

(7) Measure the temperature of the exit gas stream (top) and the exit rich oil (bottom).

(8) Take samples of the exit gas and the exit rich oil for analysis, and record the composition of these samples.

(9) Compare the experimental $\mathrm{C}_{2}$ recovery rate with the 4.5.D-1 simulation results. If the experiment $\mathrm{C}_{2}$ recovery rate does not reach the simulation highest $\mathrm{C}_{2}$ recovery rate, increase the absorbent flow and repeat step (2) through (5), until the desirable $\mathrm{C}_{2}$ recovery rate achieved in experiment.

(10) Record the set of data as the highest $\mathrm{C}_{2}$ recovery rate of the short column, equivalent to $95 \%$ of a full-size column.

(11) Decrease the absorbent flow and repeat the above experiment steps several times to obtain a series of $\mathrm{C}_{2}$ recovery rates and record all the data, until the kerosene flow rate reach its minimum simulation value.

(12) Draw a $\mathrm{C}_{2}$ recovery rate vs. absorbent flow rate curve, to identify the data corresponding to $95 \%, 70 \%$ and $35 \% \mathrm{C}_{2}$ recovery.

(13) Compare the experiment results with the simulation results and find the deviation range between them. Repeat the experiment to confirm the findings if necessary.

(14) Correct the short column simulation for the deviations to make a revised short column simulation.

(15) Extrapolate the revised BSE short column simulation to full-scale simulation with the kerosene absorbent.

(16) Compare the revised full-size simulation performance of kerosene absorber with IIT simulation with gas return $\mathrm{C}_{10} \mathrm{H}_{22}$ absorbent of the full-size column.

\section{Safety}

During the planned testing, there are a number of hazards that must be addressed. Prior to beginning the testing, the HAZOP team that performed the initial study in 2008 will meet to assure that all of the issues they identified have been properly resolved. A laboratory safety analysis will be performed on this experimental plan. The initial testing will be conducted with non-hazardous materials, including water and nitrogen, at near ambient temperatures to both train staff and assure that all the equipment is performing correctly. Next, tests at more severe temperature and pressure conditions will be conducted. Finally, hazardous gases, including $\mathrm{H}_{2} \mathrm{~S}$ and $\mathrm{SO}_{2}$, and flammable gases, including $\mathrm{NGL}$, will be performed.

Throughout the testing, the appropriate personnel protection equipment (PPE) will be required by all operating the Unit. Beyond the eye protection and clothing coveralls generally required by workers in the laboratory, those within the enclosure will require hard hats, steel toed shoes, and personnel gas detectors for $\mathrm{H}_{2} \mathrm{~S}$, combustibles, and oxygen levels. All operating personnel during the $\mathrm{H}_{2} \mathrm{~S}$ and $\mathrm{SO}_{2}$ testing will be required to have passed the $\mathrm{H}_{2} \mathrm{~S}$ Alive training or equivalent in addition to all required GTI and gas processing safety training. 
Tests were conducted to determination of the mass and heat transfer coefficients in the absorber column. The results of the heat transfer tests are shown in Table 20.

Table 20 Heat Transfer Coefficient Testing Results

\begin{tabular}{|c|c|c|c|c|}
\hline $\begin{array}{c}\text { liquid } \\
\text { flow } \\
(\mathrm{gpm})\end{array}$ & $\begin{array}{c}\text { gas flow } \\
(\mathrm{acfh})\end{array}$ & $\mathrm{L} / \mathrm{G}$ & $\begin{array}{c}\mathrm{H}_{\text {gas }} \\
\text { Btu/(lbas } \\
\text { packing })\end{array}$ & $\begin{array}{c}\mathrm{H}_{\text {liquid }} \\
\text { Btu/(lb } \\
\text { packing })\end{array}$ \\
\hline 10.1 & 273.7 & 0.1 & 0.2 & 0.85 \\
\hline 30.1 & 323.2 & 0.25 & 0.2 & 0.85 \\
\hline 50 & 273.7 & 0.46 & 0.2 & 0.84 \\
\hline 50 & 17.2 & 0.75 & 0.2 & 0.85 \\
\hline 49.9 & 125.1 & 1.04 & 0.2 & 0.84 \\
\hline
\end{tabular}

Additional data will be taken to quantify the mass transfer coefficient. 


\section{Task 4: Develop Computer Simulation for Preliminary Technical Verification and Bench-, Pilot-, and Commercial-Scale Design}

In order to test our capability and methodology to simulate an NGL recovery process, we first selected the currently used cryogenic process based on US patent 5,983,664 and performed simulation using HYSYS and ChemCad software packages. The data given in this patent are based on NGL recovery from 250 MMSCFD natural gas stream containing $85.76 \mathrm{~mol} \%$ methane, $7.81 \mathrm{~mol} \%$ ethane, $3.27 \mathrm{~mol} \%$ propane and $1.79 \mathrm{~mol} \%$ butane and higher hydrocarbons at $900 \mathrm{psia}$ and $120^{\circ} \mathrm{F}$. This process is based on expansion of the high-pressure natural gas to achieve the low temperature required for separation of methane from NGL. The simulation results from this study are summarized in Table 21.

Table 21 Comparison of Calculated and US Patent \# 5,983,664 values

\begin{tabular}{|c|c|c|c|c|}
\hline \multirow[t]{3}{*}{ Component } & \multirow[t]{3}{*}{$\begin{array}{l}\text { Inlet Natural Gas } \\
(\mathrm{Lb} \mathrm{Mol} / \mathrm{Hr})\end{array}$} & \multicolumn{3}{|c|}{$\begin{array}{l}\text { Natural gas Liquids Recovered } \\
\text { (Lb Mol/ Hr) }\end{array}$} \\
\hline & & \multirow{2}{*}{$\begin{array}{l}\text { Patent } \\
\text { Values }\end{array}$} & \multicolumn{2}{|c|}{ Simulation Results } \\
\hline & & & HYSYS & ChemCad \\
\hline Methane & 23542 & 58 & 69 & 161 \\
\hline Ethane & 2144 & 2068 & 2018 & 1949 \\
\hline Propane & 898 & 898 & 897 & 897 \\
\hline Butane & 491 & 491 & 490 & 491 \\
\hline $\begin{array}{l}\text { Residue } \\
\text { Compression (HP) }\end{array}$ & - & 16395 & 16486 & 16499 \\
\hline
\end{tabular}

The results presented in Table 21 confirm that our simulation technique was correct, as we were able to reproduce with sufficient accuracy the results given in the patent. Initially, the new process and the conventional cryogenic process for recovering NGL from natural gas were modeled using HYSYS and Aspen Plus. Since both these software give almost identical results further simulation work was restricted mainly to HYSYS. As expected, it took considerably long time (more than two months) to simulate the new process using HYSYS mostly because of its intricate heat integration/recovery loops and combined feed gas dehydration system. The data collected from various plant visits were extremely helpful in simulating this process. After successfully completing the base case simulation of the new process for 27 different cases were made representing three different ethane recoveries (from $2 \%$ to $95 \%$ ) and different gas richness (from 1 GPM to 5 GPM). Based on this analysis, some important modifications were incorporated in the initial process flowsheet to optimize heat recovery and make the process more flexible in terms of ethane rejection. The modified flowsheet was then used to generate simulation results for 48 different cases representing 4 different gas richness levels, 4 different ethane recoveries, and 3 different plant capacities. These results were later used to calculate the cost per gallon of NGL produced using the new process. These costs were compared with those of the conventional cryogenic process for different gas richness and different ethane recoveries. After completing this analysis, the finalized HYSYS model was used to generate equipment sizes and calculate equipment cost using Aspen-Icarus software. It was found that the cost of some equipment items generated from Aspen-Icarus is incorrect. The new model was then used for equipment sizing and costing using Aspen-Icarus and PDQ\$ costing packages. HYSYS based computer models for the new and conventional technologies were used to generate energy 
consumption data for different ethane recoveries and different gas richness. The results for two such cases (i.e., 2.2 and 3.6 GPM) are presented in Figure 16 and Figure 17. It can be seen from these figures that at around $40-50 \% \mathrm{C}_{2}$ recovery (which is economically justifiable at the current natural gas prices), the energy cost to recover NGL using the new technology is about $50 \%$ of that of conventional cryogenic technology.

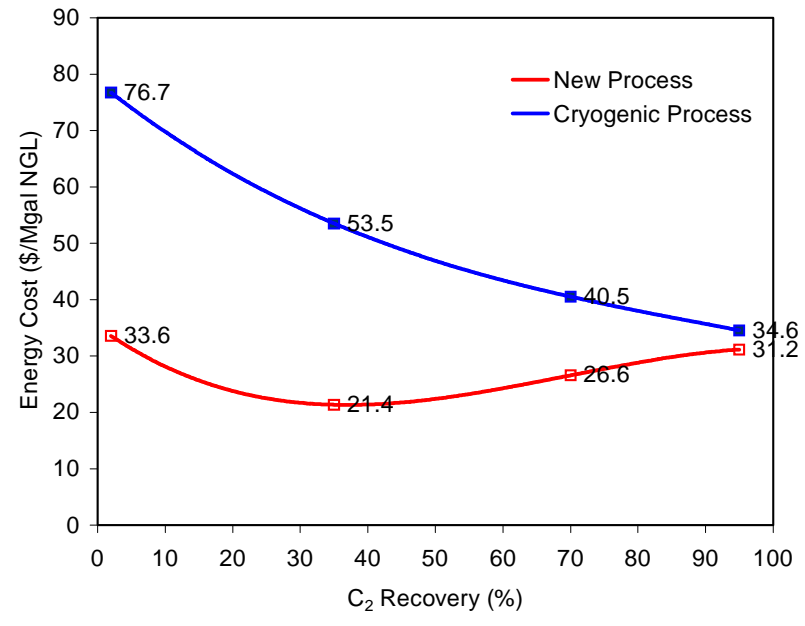

Figure 16 Energy Cost for NGL Production as a Function of $\mathrm{C}_{2}$ Recovery (gas richness 2.2 GPM)

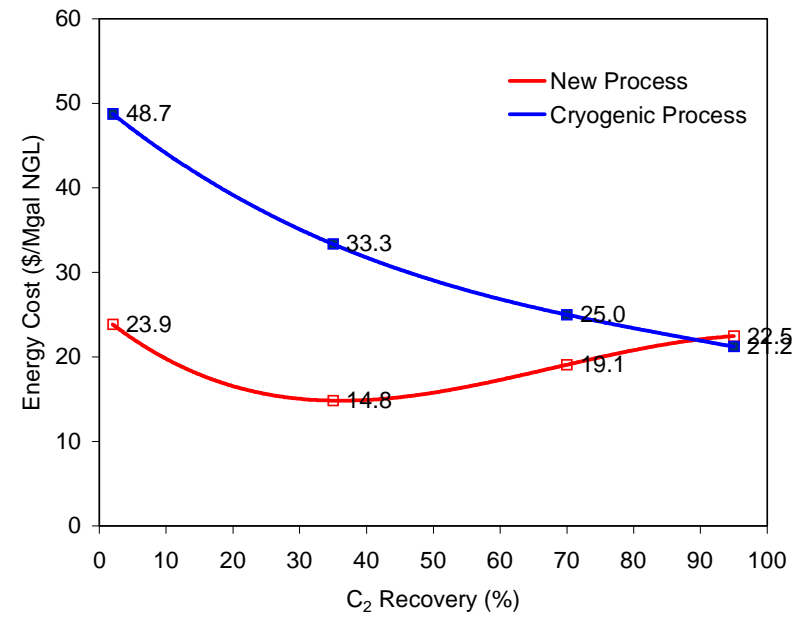

Figure 17 Energy Cost for NGL Production as a Function of $\mathrm{C}_{2}$ Recovery (gas richness - 3.6 GPM)

One target of the FIROA process is to get more energy savings to fully competitive in all cases with the CEP process. With the above-mentioned better energy-saving techniques, the present Advanced FIROA process gives more striking energy savings than the Basic FIROA process and original IROA process as shown in Table 22 and Table 23. For convenience, the original IROA data are taken as the bases for comparison. The red numbers indicate energy-saving and blue the opposite.

It is noted that while the total energy cost of the original IROA process for super-enrich gas at $95 \% \mathrm{C}_{2}$ recovery failed to compete with the CEP, the FIROA process gives obvious energy saving and unquestionable large margin of competitiveness over the CEP process in this case. It is, therefore, unnecessary to carry out the other cases where the IROA has already proved competitive over the CEP process with strikingly large margins. Figure 18 gives a picture of the percentage comparison of the total energy cost of various processes studied in this project. 
Table 22 Summary of the Sectional and Total Energy Cost of Various Processes

\begin{tabular}{|l|r|r|r|r|}
\hline \multicolumn{4}{|c|}{ HYSYS Simulation Results, in \$/Hr (Percentages of IROA are shown in parenthesis, energy-saving in red) } \\
\hline \multicolumn{1}{|c|}{ Utility Cost } & \multicolumn{1}{|c|}{ CEP $($ IIT) } & \multicolumn{1}{c|}{ IROA (IIT) } & Basic FIROA (GHT) & Advanced FIROA (GHT) \\
\hline Power $(\$ / H r)$ & $321.79(117.85)$ & $273.04(100)$ & $249.3(91.31)$ & $206(75.45)$ \\
\hline Heating Medium $(\$ / H r)$ & Nil $(0)$ & $77.59(100)$ & $44.3(57.09)$ & $35.2(45.37)$ \\
\hline Cooling Water $(\$ / H r)$ & $0.66(22.93)$ & $2.878(100)$ & $1.95(67.76)$ & $1.90(65.91)$ \\
\hline Total Utility Cost $(\$ / H r)$ & $322.45(91.21)$ & $353.51(100)$ & $296.5(83.87)$ & $243.1(68.77)$ \\
\hline
\end{tabular}

Table 23 Percentage Comparison of the Overall Energy Cost of Various Processes

\begin{tabular}{|l|r|r|r|r|}
\hline Total Utility Cost (\% of CEP) & 100 & 109.63 & 100 & 91.95 \\
\hline Total Utility Cost (\% of IROA) & 91.21 & 15.39 \\
\hline Total Utility Cost (\% of FIROA, Basic) & 108.75 & 119.23 & 63.87 & 100 \\
\hline Total Utility Cost (\% of FIROA, Adv.) & 132.64 & 145.42 & 81.99 \\
\hline
\end{tabular}




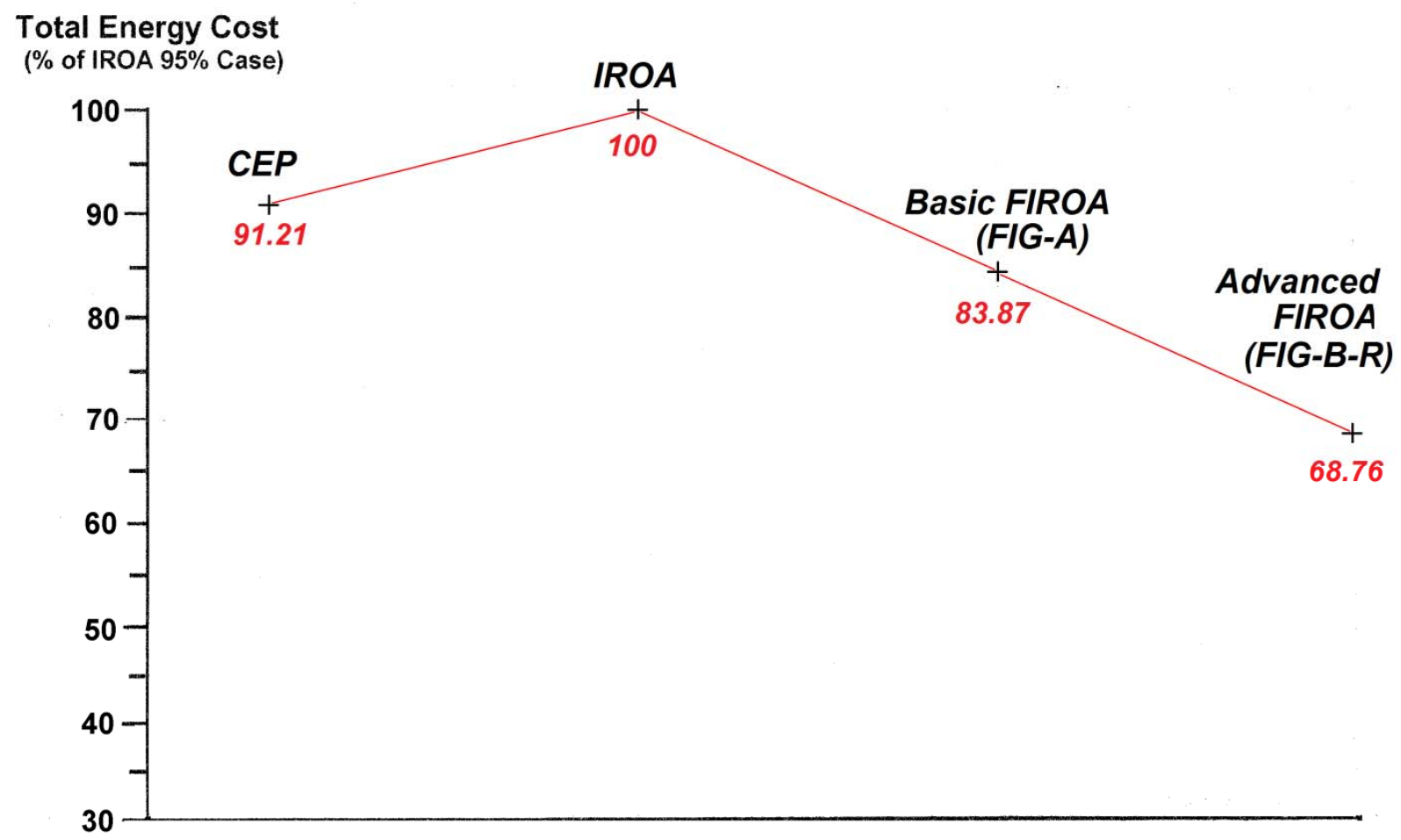

Figure 18 Percentage Comparison of the Overall Energy Cost of Various Processes 


\section{Task 5: Perform Theoretical and Experimental Study (R\&D Work) to Address Critical Process Development Issues}

Preliminary experimental work to evaluate the corrosion resistance of commercial aluminum alloys in a 30\% calcium chloride solution was completed at ORNL. Corrosion behavior of aluminum 5182 was evaluated in terms of weight change in $30 \%$ calcium chloride solution at the temperature ranging from 0 to $65^{\circ} \mathrm{C}$. The test results showed that $45^{\circ} \mathrm{C}$ is a threshold temperature for this material, which weight gain and loss occur above and below this temperature, respectively. This can be attributed to the different dominant reaction occurrence on the sample surface, corrosion and oxidation, which cause the weight change. Different surface morphologies, pitting and oxidation, were revealed by scanning electric microscopy (SEM). A paper was submitted to the Journal of Materials Chemistry on this work, entitled "Corrosion Performance of Aluminum 5182 Alloy in Calcium Chloride Solution". (See Attachment 3.)

The original solvent used in our simulation and economic evaluation was decane $\left(\mathrm{C}_{10}\right)$. With a boiling point of $345^{\circ} \mathrm{F}$ and molecular weight of 142.28 , it met the solvent claims within the process patent. Kerosene was selected as the first alternative solvent for the selective separation of methane from the hydrocarbons. Since kerosene is a blended material, supplies from different refiners may have slightly different constituents at varying concentrations of these constituents. Kerosene is defined as "A refined petroleum solvent (predominantly $\mathrm{C}_{9}-\mathrm{C}_{16}$ hydrocarbon, which is typically a mixture of $25 \%$ normal paraffins, $11 \%$ branched paraffins, $30 \%$ monocycloparaffins, $12 \%$ dicycloparaffins, $1 \%$ tricycloparaffins, $16 \%$ mononuclear aromatics and $5 \%$ dinuclear aromatics" 3 . The effect of these different components and concentrations on the absorption process is unclear.

An Aspen model was development to look at the properties of refined kerosene, see Figure 19. In addition to this, we can anticipate increasing refined fuel requirements into the future and their cost. Thus, we have also looked into kerosene-like products produced via non-conventional means.

One such pathway to producing a kerosene-like product today is from a synthesis process first developed in the 1920's known as Fischer-Tropsch. Fischer-Tropsch (FT) technology was originally developed in the 1920s using coal to create liquid fuels. Syntroleum Inc. uses their own Syntroleum Process ${ }^{\circledR}$ to convert stranded natural gas - using air instead of pure oxygen into ultra-clean liquid fuels. Syntroleum Inc. was contacted for their kerosene product that contains no aromatics or contaminants. Syntroleum supplied HP GC analysis for average molecular weight, iso/normal ratio, and average carbon ratio number in their kerosene mixture. The fuel is paraffinic and isoparaffinic, with no aromatics. This provided enough information for comparison to the refined kerosene in both simulation and testing. A comparison of ASTM D-86 boiling point range of the simulated refined kerosene and Syntroleum product is made in Table 24. Another spreadsheet will be developed later to define the Syntroleum product with no aromatics and a comparison of the predicted absorption properties of the solvents will be made.

3 NIOSH Pocket Guide, www.cdc.gov. 


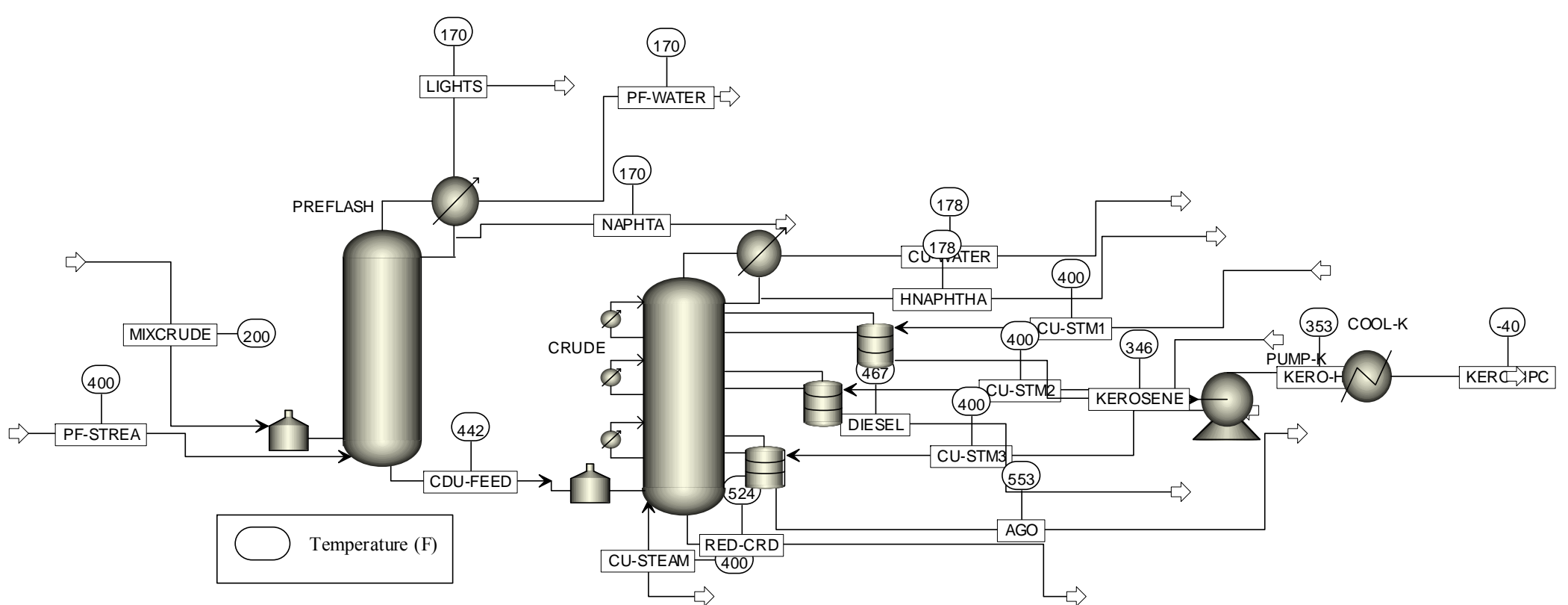

Figure 19 Aspen Plus Kerosene Production from Crude 


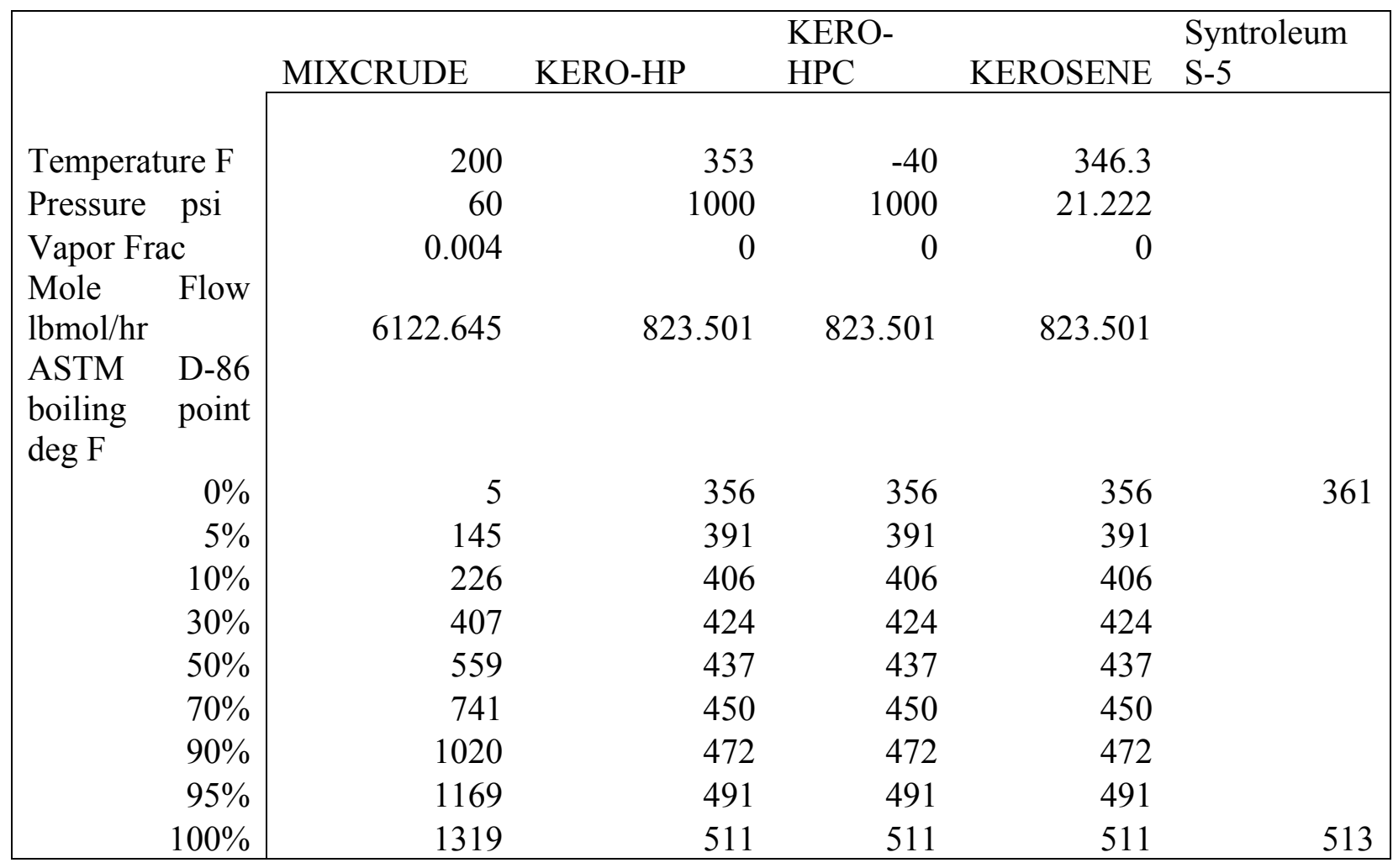

Table 24 ASTM D-86 Comparison of Aspen Simulation of Kerosene Production from Mixed Crude Assay Blend specification and Synthetic Syntroleum Kerosene.

A total of five experiments were conducted to study methane absorption and solubility in both Syntroleum S-1 and Sigma-Aldrich kerosenes using the equilibrium and regeneration pressures for the absorption process.

The vapor liquid equilibrium cell (VLE) was initially evacuated and was filled with exactly 260 $\mathrm{ml}$ of the test solvent at room temperature. Then, another vacuum was created, for approximately one minute, to displace air bubbles in the solvent before cooling the system to $-40^{\circ} \mathrm{F}$. When the VLE temperature was holding stable at $-40^{\circ} \mathrm{F}$, pure methane at room temperature was introduced into the cell, and the stirrer was turned on at a speed of $175 \mathrm{rpm}$. After the absorption had reached equilibrium, the VLE was depressurized at $-40^{\circ} \mathrm{F}$ and the solvent was released in to a $1000 \mathrm{ml}$ graduated beaker to record the volume and the mass. The $-40^{\circ} \mathrm{F}$ solvents were tested at both 44 and 400 psia.

Figure 20 through Figure 23 are representative of the solubilities of methane in both solvents, while Table 25 shows the calculated Henry's Law Constant derived from the data. Figure 20 clearly supports common physical solubility phenomena that are that solubility decreases with increasing temperature or decreasing pressure.

The Vapor Equilibrium Cell was taken apart for upgrading so that it could be operated at pressures up to 5,000 psia when it was discovered that the shaft was broken. The repaired shaft 
was reinstalled, but the system would not hold a pressure above 400 psi. Leak detection testing found several fittings added to allow easier addition and withdrawal of solvents, requiring plugging of the fittings.

The Vapor Equilibrium Cell was repaired and underwent pressure testing. The system was leak free at 500 psi. At 1500 psi, the leak rate was an acceptable 10 psi in 24 hours. Extreme temperature pressure transducers were purchased to take the pressure measurements within the environmental chamber. This will decrease the dead space line that was the result of having an external transducer.

The following is a summary of the modifications made to the VLE system for operation below 2500 psig:

1. The PPI autoclave "A" port plug was removed and a separate line from the autoclave to the 5000 psig pressure transducer for higher-pressure operation and leak-testing was performed.

2. The data acquisition and control software were modified for proper computer display of the new pressure transducer line, while maintaining the integrity of the old system.

3. The liquid sampling line at bottom of the autoclave was replaced with plugs until future need arises.

4. A 2500 psig mawp valve was installed to isolate lower-pressure transducer with more accuracy than the 5000 psig pressure transducer.

5. Connections were modified to not leak under leak check conditions; first with nitrogen(1000psig) and then with helium/nitrogen mixture(ca. $1770 \mathrm{psig}$ ).

6. The new total volume and associated error measurements were completed and verified.

7. The accuracy of the filling and emptying of the VLE was performed. 


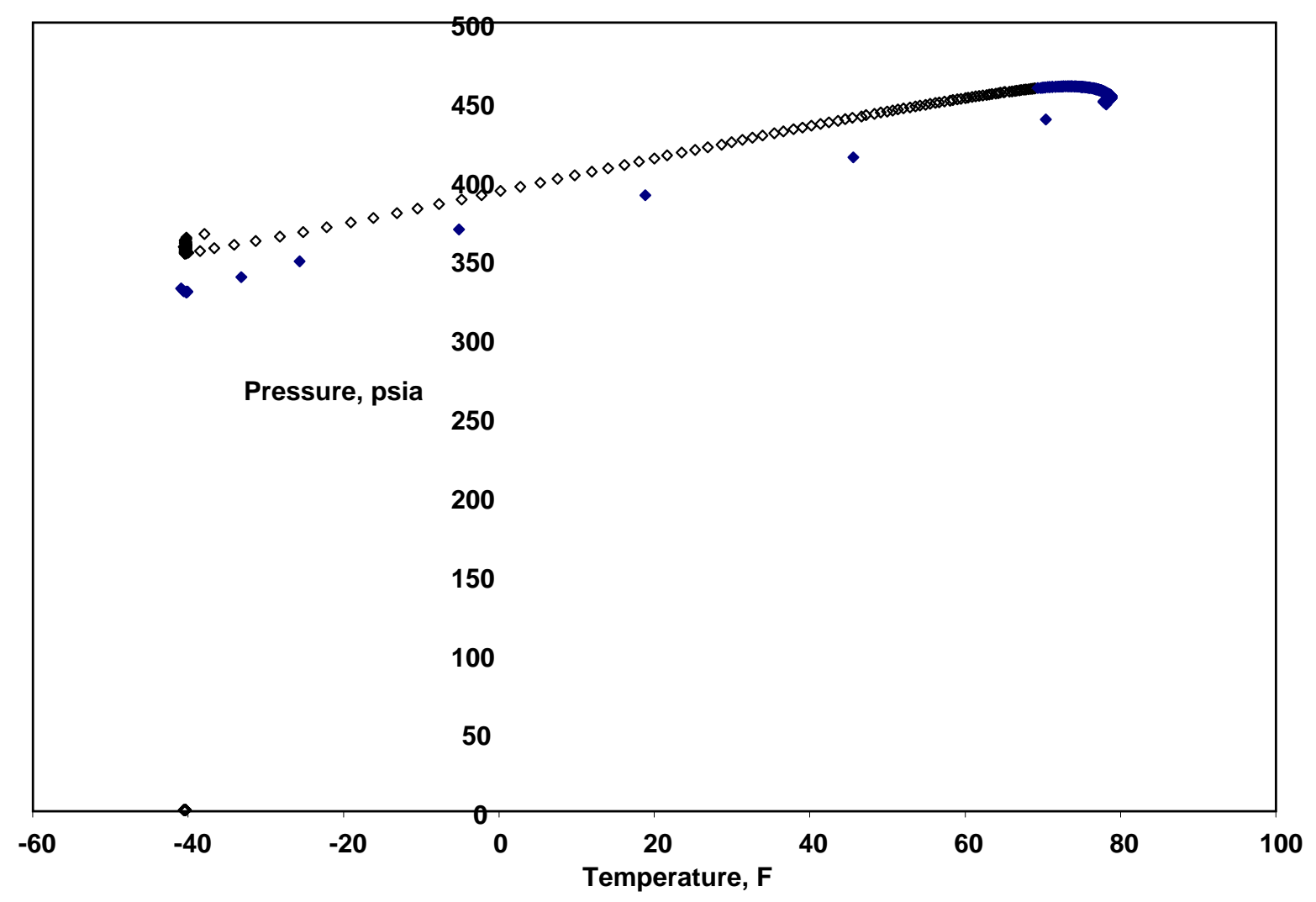

Figure 20 Methane Pressure Profile against Temperature in Syntroleum S-1

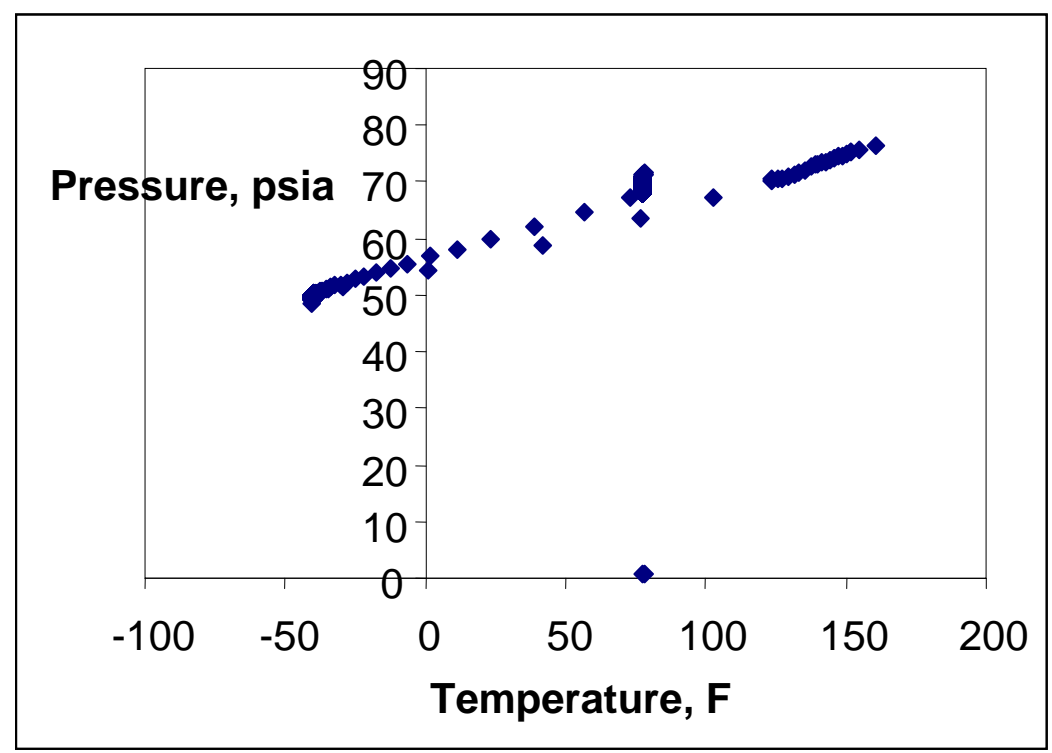

Figure 21 Methane Pressure Profile against Temperature in Sigma kerosene 
Table 25 Methane Solubility in Kerosenes

\begin{tabular}{|c|c|c|}
\hline & Syntroleum & Sigma Aldrich \\
\hline Temp, $K$ & 233 & 233 \\
\hline Stirrer Speed, rpm & 175 & 175 \\
\hline $\mathbf{P}_{\text {Regen, }}$ psia & 459.53 & 49.39 \\
\hline $\mathbf{P}_{\text {Equil, }}$ psia & 353.90 & 39.48 \\
\hline Henry constant, $\left(\mathbf{m}^{3} \mathrm{~Pa} / \mathrm{mol}\right)$ & 1753 & 2005 \\
& & \\
\hline
\end{tabular}

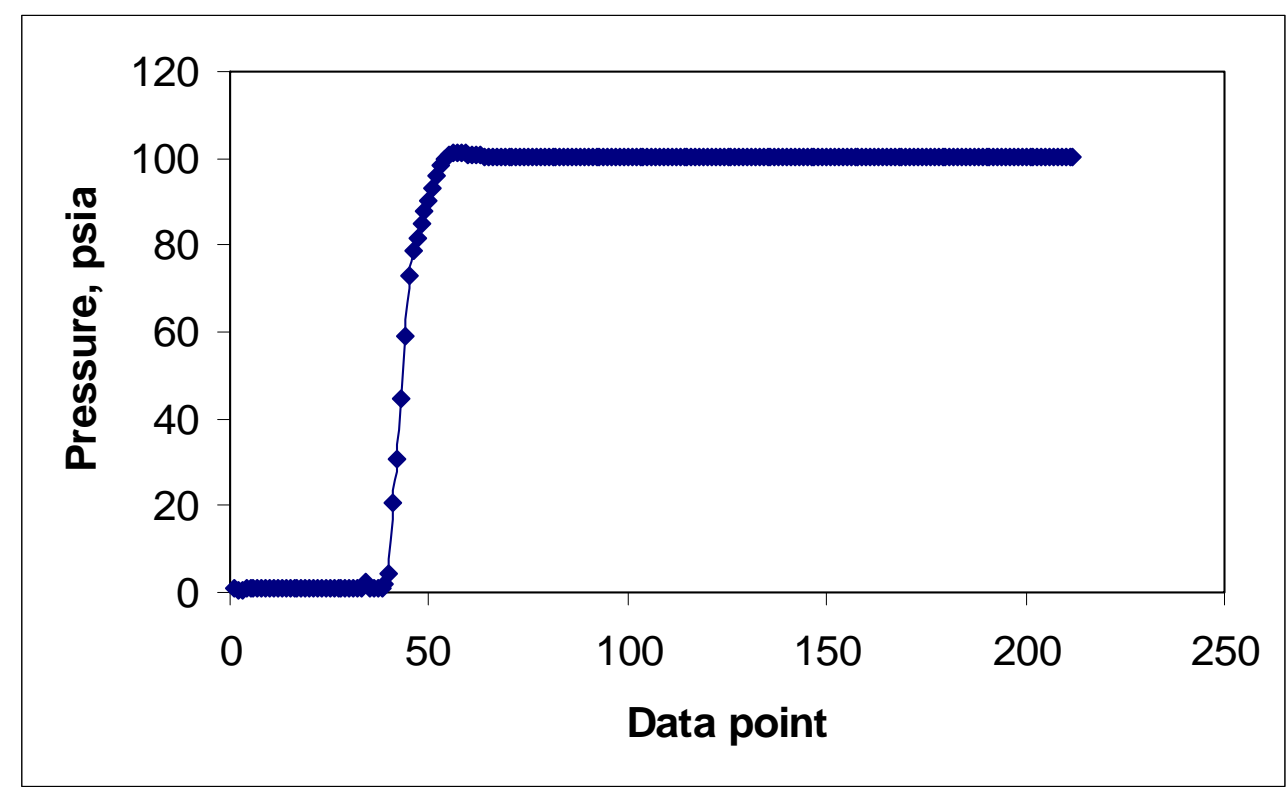

Figure 22 Methane Solubility in Sigma Kerosene as a Function of a Dimensionless Time Unit (time per 2 seconds) at $-40^{\circ} \mathrm{F}$ 


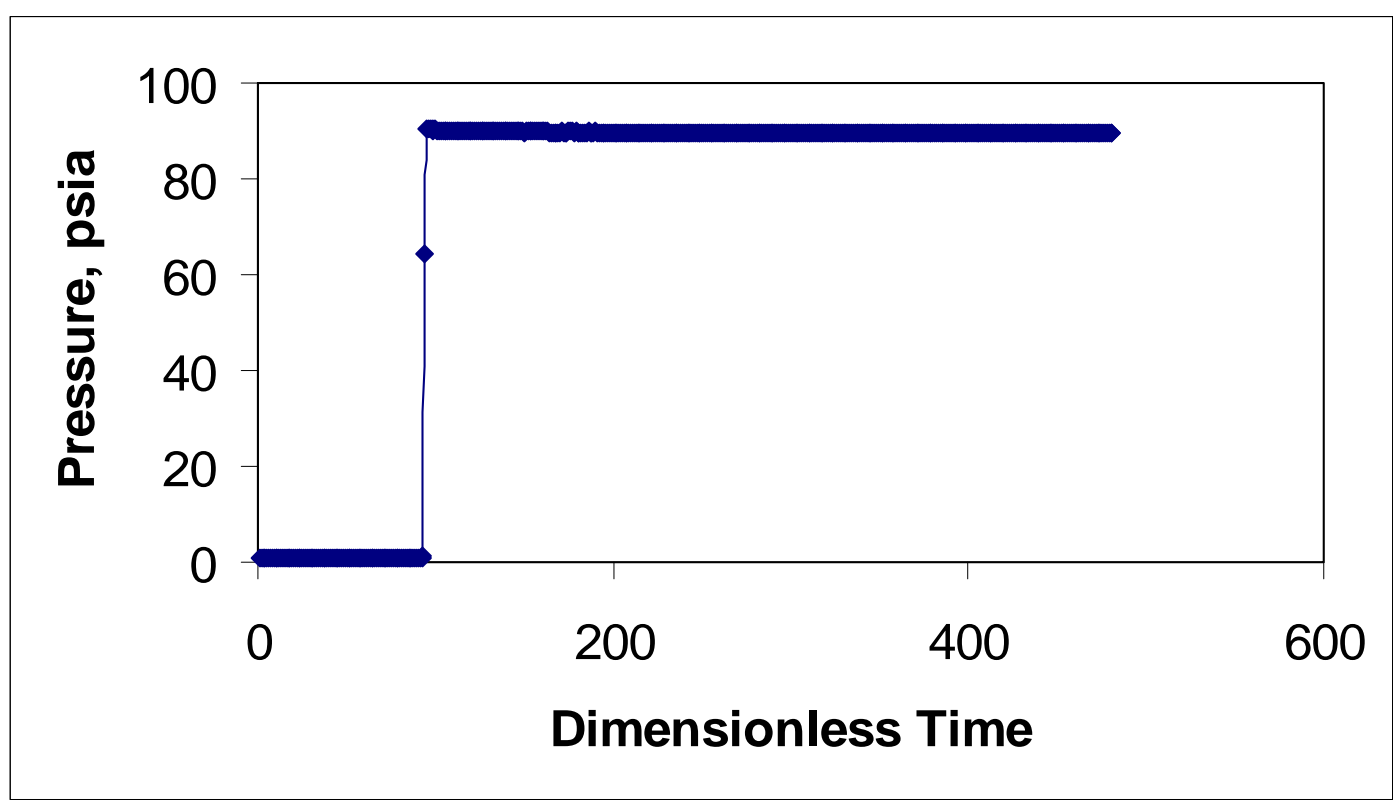

Figure 23 Methane Solubility Testing In Syntroleum S-1 as a Function of a Dimensionless Time Unit (time per 15 seconds) at $-40^{\circ} \mathrm{F}$

The big hurdle in the VLE unit has been the inability to test a gas in batch mode limiting the analysis to one gas component analysis in batch mode. A new GC with special external sample valving to permit only microliters of sample to be removed from the headspace which will not to disturb the equilibrium has been designed and special ordered from Shimadzu. In addition to this feature, the Shimadzu Gas Chromatograph (GC) is configured with three detectors.

The three detectors have the following characteristics for compound detection.

1. Thermal Conductivity Detector (TCD): A universal detector used primarily for the detection of fixed gases such as $\mathrm{CO}_{2}, \mathrm{CO}, \mathrm{N}_{2}, \mathrm{H}_{2}, \mathrm{CH}_{4}$ eluting from the column at concentrations ranging from $100 \mathrm{ppm}$ to 100 percent. The TCD is universal in nature but not very sensitive compared to other detectors, which is why the FID and FPD detectors (described below) were added, i.e. FID is sensitive to hydrocarbons while the FPD is sensitive to sulfur containing compounds.

2. Flame Ionization Detector (FID): A sensitive detector for hydrocarbons, such as heavier hydrocarbons, BTEX, glycols, etc. from a few ppb up to percent levels.

3. Flame Photometric detector (FPD): A sensitive detector for sulfur containing compounds such as $\mathrm{H}_{2} \mathrm{~S}, \mathrm{COS}, \mathrm{CH}_{3} \mathrm{SH}, \mathrm{C}_{2} \mathrm{H}_{5} \mathrm{SH}$, and $\mathrm{SO}_{2}$.

One issue that came up during the 2007 DOE peer review was the tolerance of the proposed process for carbon dioxide. For most NGL recovery processes, an acid gas removal process will be located upstream to remove $\mathrm{H}_{2} \mathrm{~S}$ and other sulfur compounds from the natural gas thereby minimizing the levels in the NGL product, as well. The AGR usually removes a significant fraction of the $\mathrm{CO}_{2}$. However, conventional turbo-expander systems must remove $\mathrm{CO}_{2}$ to the $100 \mathrm{~s}$ ppm level to prevent solid $\mathrm{CO}_{2}$ from forming at the cryogenic temperatures they achieve. This requires additional capital, operating, and fuel costs. 
The advance process that we are developing is not limited by the $\mathrm{CO}_{2}$ levels in the gas. Operating at $-40^{\circ} \mathrm{F}$, we are well above the solid/liquid $\mathrm{CO}_{2}$ phase line. Figure 24 shows the effect of pressure on the $\mathrm{CO}_{2}$ freezing point for a number of methane/carbon dioxide mixtures (shown as $\% \mathrm{CO}_{2}$ in the feed). This chart was generated using Aspen HYSYS simulation model for $\mathrm{CH}_{4}$ and $\mathrm{CO}_{2}$ mixtures varying between 5 and $100 \% \mathrm{CO}_{2}$, fed at $-40 \mathrm{~F}$ and pressures varying between 100 and 1000 psia. The chart shows that we will be operating 40 to $100^{\circ} \mathrm{F}$ above the $\mathrm{CO}_{2}$ freezing line and should therefore very tolerant to the any amount of $\mathrm{CO}_{2}$ in the feed.

$\mathrm{CO}_{2}$ Freezing Point for Various Levels of $\mathrm{CO}_{2}$ in the Feed Gas

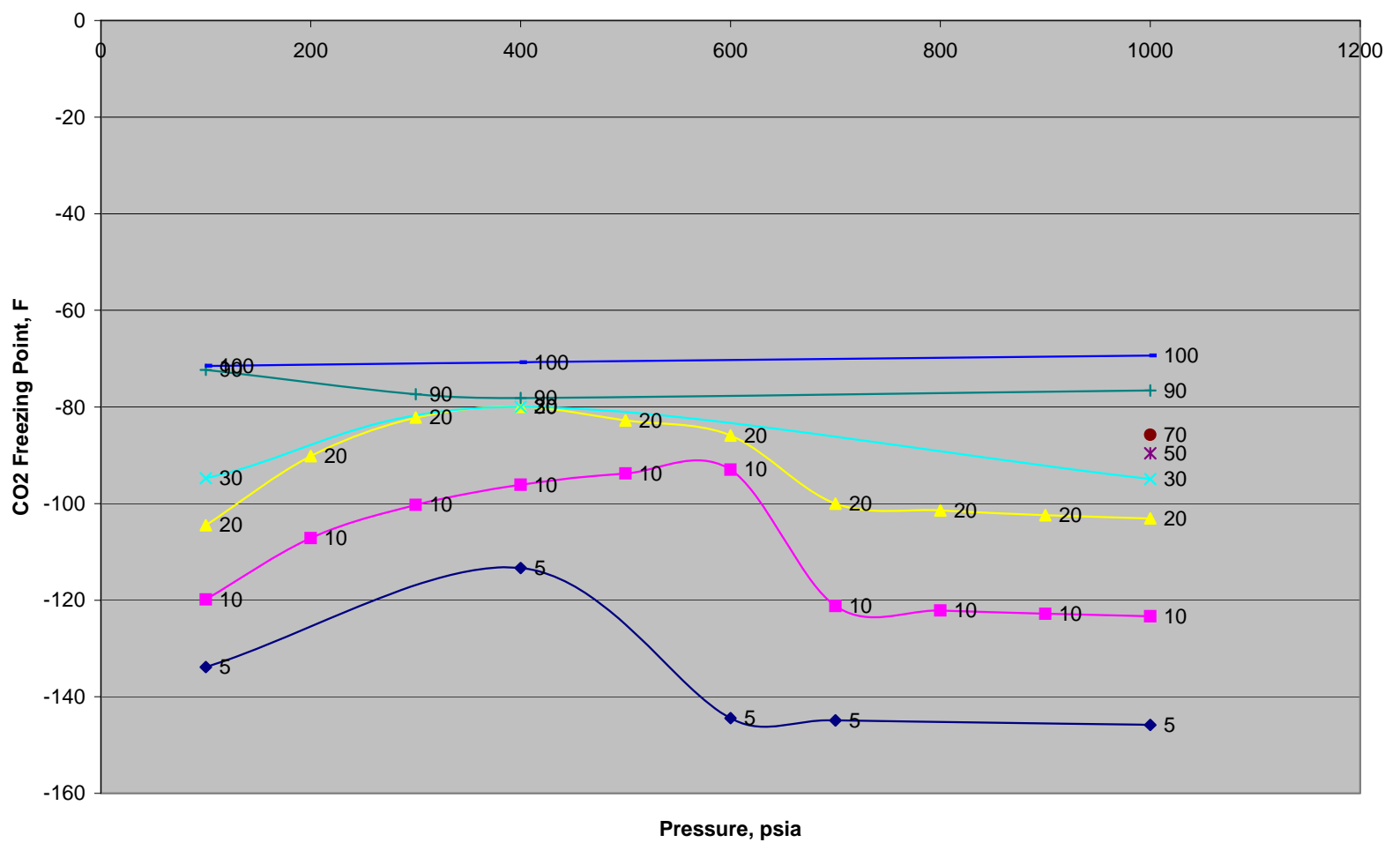

Figure $24 \mathrm{CO}_{2}$ Freezing Point for Various Levels of $\mathrm{CO}_{2}$ in the Feed Gas 


\section{Task 6: $\quad$ Design, Construct and Install a Pilot Unit and Conduct Field Tests}

Several meetings were held with natural gas producing and processing companies to identify a site for the pilot plant unit. Preliminary discussions were held with EnCana, BP, Chevron, and Kinder Morgan at the Laurance Reid Gas Conditioning Conference and at the Gas Processors Association Annual Meeting from the producing and processing side of the business as well as AkerKvaerner and the Washington Group from the engineering construction arena.

The design basis of the pilot plant unit is shown in Figure 25. The purpose of the pilot plant is to obtain design and operational data on the new pieces of equipment and process conditions that are not common practice in the industry and cannot be simulated with confidence. We will want to show that the simulation models can predict pilot performance. At approximately $1 \mathrm{MMscf} / \mathrm{d}$ feed rate, the pilot will not be a demonstration plant for the process. The pilot plant will not be fully heat integrated nor utilize the most energy efficient heat exchangers, for example, because they decrease the flexibility during testing. On the other hand, the pilot plant will have some additional unit operations to ensure the tests can be performed irrespective of efficiency or performance of individual aspects of the pilot plant operation as a whole. One example of this is to install a supplemental dehydration unit downstream of the test dehydrator. This will help ensure that we can deliver ice and hydratefree feed gas to the absorber system regardless of the performance of the test dehydrator. 


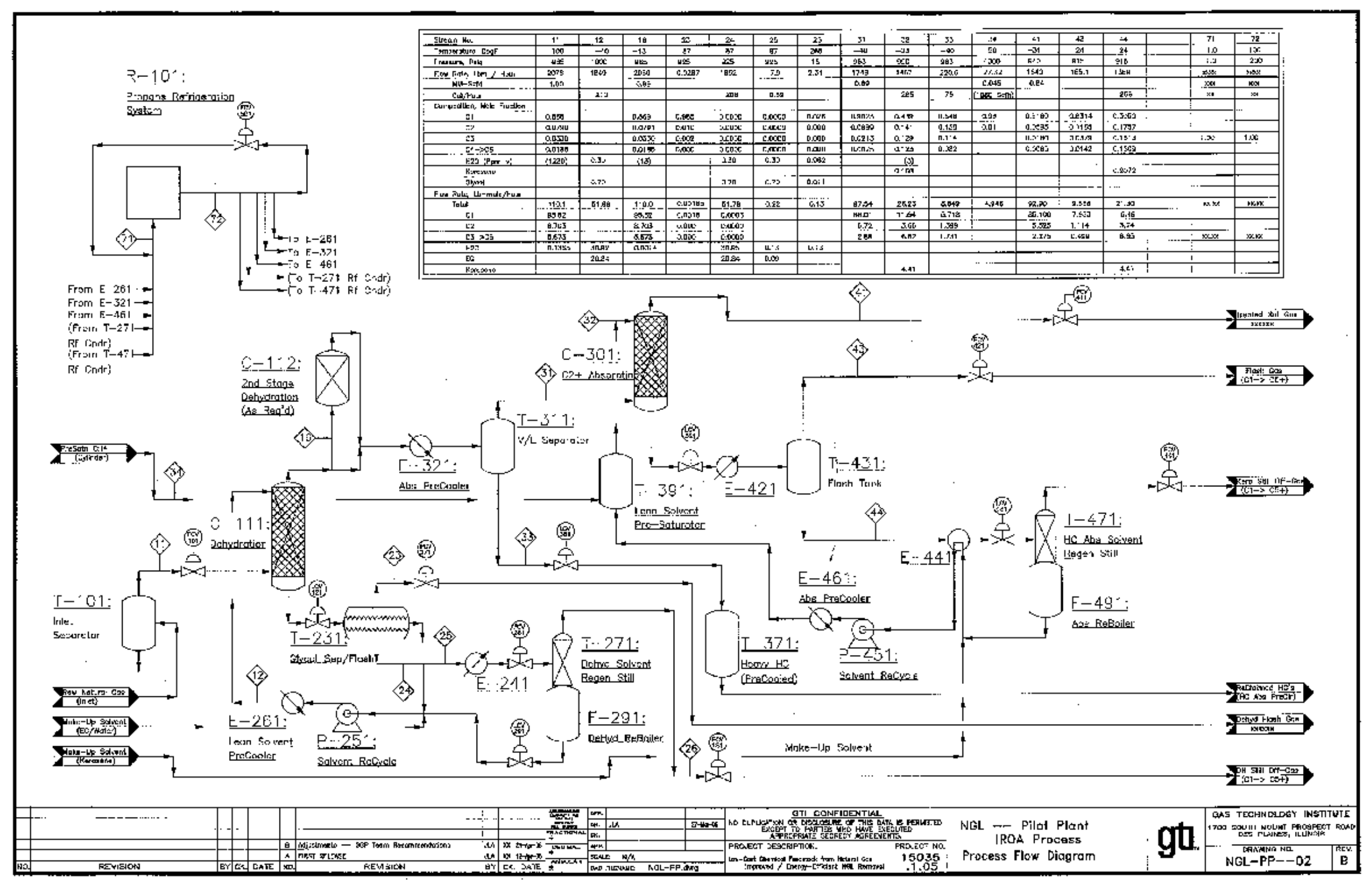

Figure 25 Preliminary Pilot Plant PFD 
A meeting was held with GTI and GHT to discuss the requirements for the pilot unit. It was agreed that the basic role for the pilot unit was on generating field data that are not readily available or not sufficiently calculated using a simulation program such as Aspen HYSYS or Aspen Plus.

The objectives of the pilot plant may be defined more concretely as: (1) The performance of a direct-contact type Dehydrator, operating with two different kinds of hydrate inhibitors Ethylene Glycol and Calcium Chloride; and (2) The flexibility of variable $\mathrm{C}_{2}$ recovery rates from $95 \%$ to $2 \%$ in the Absorber using a commercial absorbent operating at equilibrium composition:

(1) The performance of a direct-contact type Dehydrator, operating with two different kinds of hydrate inhibitors - Ethylene Glycol and Calcium Chloride.

Although ethyl glycol has been used extensively in gas processing plants, its high viscosity and foaming tendency, especially under low temperature (e.g., $\left.-40^{\circ} \mathrm{F}\right)$, may jeopardize its use as an heat transport medium under high flow rate in IROA dehydration column. An alternative hydrate inhibitor, i.e., calcium chloride $\mathrm{CaCl}_{2}$, therefore, has been proposed in the original patent and included in Task \#5 (R\&D) of this project. The field testing of this inhibitor solution would not require additional equipment or any change to the current system designed for ethylene glycol, because the properties of this inhibitor are in general better than ethylene glycol, and much better in viscosity (less than $1 / 7$ that of ethylene glycol solution at $-40^{\circ} \mathrm{F}$ ).

The $\mathrm{CaCl}_{2}$ solution has been used extensively in refrigeration industry as secondary refrigerant ("brine") for any years, its compatibility with ordinary structural materials under low temperature is well known. The corrosion of $\mathrm{CaCl}_{2}$ solution to aluminum alloy (material for plate-fin heat exchanger in future IROA plants) has been studied in ORNL for this project. The first round of experiments (without corrosion inhibitor) has been reported with encouraging results. A field testing, therefore, would be important to verify whether it is a better candidate to replace ethylene glycol in future commercial IROA plants. The most important physical properties of $\mathrm{CaCl}_{2}$ and glycol solutions are found in "ASHRAE Handbook of Fundamentals", 1974.

(2) The flexibility of variable $\mathrm{C}_{2}$ recovery rates from $95 \%$ to $2 \%$ in the Absorber.

This second objective should be included to verify the major advantage of the IROA process over both CEP and traditional ROA processes, i.e., the flexibility of $\mathrm{C}_{2}$ recovery during operations. In the process flow diagram and the P\&ID, all the Absorber and the lean oil regeneration equipment have already included, it is a logical inference that the system is designed to verify operational flexibility.

The Absorber section could be simplified (not to be totally eliminated) to control the budget within limit by (1) decrease the refrigeration load by pre-cooling with cold residue (product natural) gas; (2) replace the Pre-Saturator tower (C-311) with a Pre-Saturator Tank, just like many traditional ROA plant are currently using; and (3) eliminate the ROD because it would have little effect on the function of the Absorber. All the above measures together would be sufficient to control the cost within the budget limit. 
A request for quote (RFQ) document was prepared and is included as Attachment 1 to this report. The RFQ was submitted to nine engineering and construction $(\mathrm{E} \& \mathrm{C})$ companies asking for their quotes to be returned to GTI by August 17, 2006.

1. Process and mechanical engineering and drawings, project management of the required system;

2. Procurement, fabrication and assembly of all equipment and controls;

3. Installation of the pilot plant unit at an existing processing facility in Colorado; and

4. Assist in commissioning unit

Three bids were received in response to the RFQ. The cost estimates varied from $\$ 1,300,000$ to $\$ 2,300,000$. Two bids included $\$ 250,000$ for additional upfront engineering/design studies before they were comfortable fixing the cost of the pilot plant. GTI decided that the best route would be for the engineering study to be performed in-house with support from a small engineering company. Trimeric Engineering was hired to support the effort. Trimeric had been used previously under this contract to provide cost quotes for the economic evaluation. They provide similar engineering and design services to the gas-patch industry.

A meeting was held with Trimeric to review the requirements for the pilot plant unit. GTI specified that the dehydration unit should be designed for approximately $1 \mathrm{MMscf} / \mathrm{d}$ gas feed rate. The absorption process should first be sized to utilize as much of GTI's existing lean oil system test unit as practical to minimize costs and delivery times while meeting the requirements of the planned testing.

Part of the first step was to review previously cost estimates and estimate the expected costs for the pilot plant. Given the recent escalations in material and fabrication costs, as well as a general shortage of available labor for fabricating this type of equipment, it was likely that pilot plant costs will be higher than originally budgeted. GTI and Trimeric worked together to define any reductions in size or scope of the pilot plant that may be required in order to work within the sponsors' budget constraints for the overall pilot plant.

When the design basis was defined, including an understanding of which existing equipment, if any, can be re-used, the process design package (PDP) was prepared. The PDP is a package of requirements and specifications such that a typical fabrication shop could perform the detailed design of the vessels, piping, and modules that contain the process equipment and fabricate completed (or partially completed modules if GTI prefers to perform portions of the assembly inhouse) modules for shipment to GTI or GTI's test site.

The PDP included the following items:

- $\quad$ Finalized design basis

- $\quad$ Process flow diagrams (PFDs) \& scaled material balances

- $\quad$ Equipment size basis table

- $\quad$ Equipment site layout and module layout sketches

- $\quad$ Piping and Instrumentation Diagrams (P\&IDs)

- $\quad$ Equipment sketches, specifications and data sheets

- Control logic and instrumentation description

- $\quad$ Electrical requirements. 
$\bullet$

Following preparation of the PDP, GTI and Trimeric:

- $\quad$ Finalized the PDP

- $\quad$ Developed a list of candidate fabrication shops

- $\quad$ Issued the PDP and RFQ to fabrication shop(s)

- $\quad$ Coordinated with fabrication shop(s) during preparation of firm fixed price bid

The "super rich" design case from the earlier studies was used to form the basis for the pilot plant unit. The Aspen HYSYS simulation case was modified to allow sizing of equipment. The model case is seen in Figure 26 and the overall heat and material balances are given in Table 26 


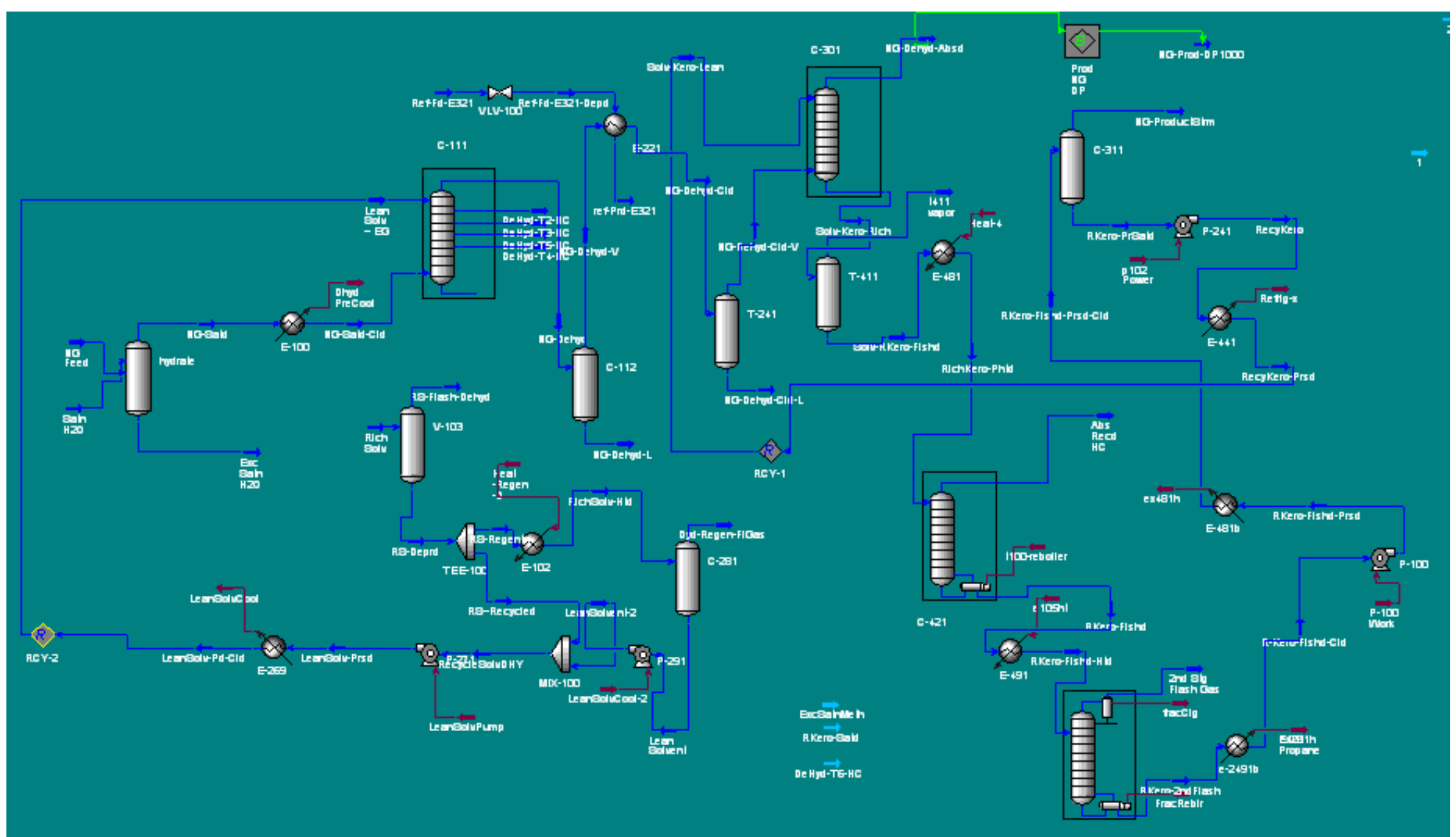

Figure 26 HYSYS Simulation for the Pilot Plant Unit 


\section{Name}

Vapour Fraction

Temperature [C]

Pressure [kPa]

Molar Flow [kgmole/h]

Mass Flow [kg/h]

Std Ideal Liq Vol Flow [m3/h]

Heat Flow $[\mathrm{kJ} / \mathrm{h}]$

$\begin{array}{lr}\text { Molar } & \text { Enthalpy } \\ {[\mathrm{kJ} / \mathrm{kgmole}]} & \\ \text { Actual Gas } & \text { Flow } \\ {\left[\mathrm{ACT} \mathrm{m}^{3} / \mathrm{h}\right]} & \end{array}$

Std Gas Flow [STD_m $\left.\mathrm{m}^{3} / \mathrm{h}\right]$

Name

Vapour Fraction
Temperature [C]
Pressure $[\mathrm{kPa}]$

Molar Flow [kgmole/h]

Mass Flow [kg/h]

Std Ideal Liq Vol Flow $[\mathrm{m} 3 / \mathrm{h}]$

Heat Flow $[\mathrm{kJ} / \mathrm{h}]$

$\begin{array}{lr}\text { Molar } & \text { Enthalpy } \\ {[\mathrm{kJ} / \mathrm{kgmole}]} & \\ \text { Actual Gas } & \text { Flow }\end{array}$

Table 26 Material and Energy Balance for Pilot Plant Unit NG Feed Satn $\mathrm{H}_{2} \mathrm{O}$ NG Satd Exc Satn NG Satd Lean Solv

$\begin{array}{rrrrrr} & & & \mathrm{H}_{2} \mathrm{O} & \mathrm{Cld} & --\mathrm{EG} \\ 1.00 & 0.00 & 1.00 & 0.00 & 1.00 & 0.00 \\ 38.61 & 40.56 & 37.96 & 37.96 & 37.22 & -40.00 \\ 6,894.76 & 6,963.71 & 6,894.76 & 6,894.76 & 6,860.29 & 7,101.60 \\ 49.90 & 4.54 & 49.96 & 4.47 & 49.96 & 15.53 \\ 939.80 & 81.72 & 940.91 & 80.61 & 940.91 & 532.96 \\ 2.89 & 0.08 & 2.89 & 0.08 & 2.89 & 0.50\end{array}$

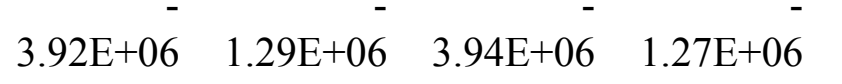
$7.87 \mathrm{E}+04$
15.73

$2.84 \mathrm{E}+05$

$7.89 \mathrm{E}+04 \quad 2.84 \mathrm{E}+05$

$15.69<$ empty>

$3.94 \mathrm{E}+06$

$5.49 \mathrm{E}+06$

$7.89 \mathrm{E}+04$

$<$ empty>

$1,179.76$

107.25

$1,181.21$

105.80

$<$ empty>

$3.53 \mathrm{E}+05$

$<$ empty $>$

$1,181.21$

367.21

NG Solv Kero

Dehyd

1.00

$-32.02$

$6,584.49$

29.74

481.27

1.60

Rich

$$
\text { NG }
$$

NG Prod

DP1000

0.00
-33.04
6.618 .97

-33.04
$6,618.97$

26.29

$2,691.76$

4.02

Cld

0.87

$-40.00$

$6,743.07$

49.90

939.83

2.89

1.00
-39.51
$6,894.76$

29.74

481.27

1.60

Rich Abs Recd

Kero Phtd

$$
\begin{array}{r}
0.16 \\
4.44 \\
6,515.55 \\
26.18 \\
2,689.85 \\
4.01
\end{array}
$$

$\mathrm{HC}$

1.00
2.07
$3,102.64$
10.74
195.80
0.62

1.00

2.07
2.64

95.80

0.62

$4.05 \mathrm{E}+06$

$8.11 \mathrm{E}+04$

11.60

$1,179.78$

Rkero

Flshd

$$
0.00
$$

281.92

$3,137.12$

15.44

$2,494.06$

3.39

$8.42 \mathrm{E}+05$

$2.35 \mathrm{E}+06$

$6.35 \mathrm{E}+06$

$4.19 \mathrm{E}+06$

$7.90 \mathrm{E}+04$

$2.41 \mathrm{E}+05$

$6.50<$ empty>
$8.39 \mathrm{E}+04$

8.39E +04

<empty>
$2.36 \mathrm{E}+06$

$7.95 \mathrm{E}+04$

$$
5.58
$$

$6.12 \mathrm{E}+06^{-}$

$2.34 \mathrm{E}+05$

$<$ empty>
$7.84 \mathrm{E}+04$

7.02
G- Rich Solv Solv Kero

1.00

$-7.10$

$0.00 \quad 0.00$
36.73

$36.73 \quad-40.00$

$6,825.81 \quad 8,515.03$

$\begin{array}{rr}15.59 & 12.66\end{array}$

$534.05 \quad 2,396.28$

$0.50 \quad 3.16$

$5.38 \mathrm{E}+06 \quad 5.14 \mathrm{E}+06$

$3.45 \mathrm{E}+05 \quad 4.06 \mathrm{E}+05$

<empty> <empty>

$368.63 \quad 299.39$

NG NG

Dehyd V Dehyd L

$$
\begin{array}{rr}
1.00 & 0.00 \\
-7.10 & -7.10 \\
6,756.86 & 6,756.86 \\
49.90 & 0.00 \\
939.83 & 0.00 \\
2.89 & 0.00
\end{array}
$$

$0.00 \mathrm{E}+00$

$4.05 \mathrm{E}+06$

$8.11 \mathrm{E}+04 \quad 3.50 \mathrm{E}+05$

11.60 <empty> 
[ACT m $3 / \mathrm{h}$ ]

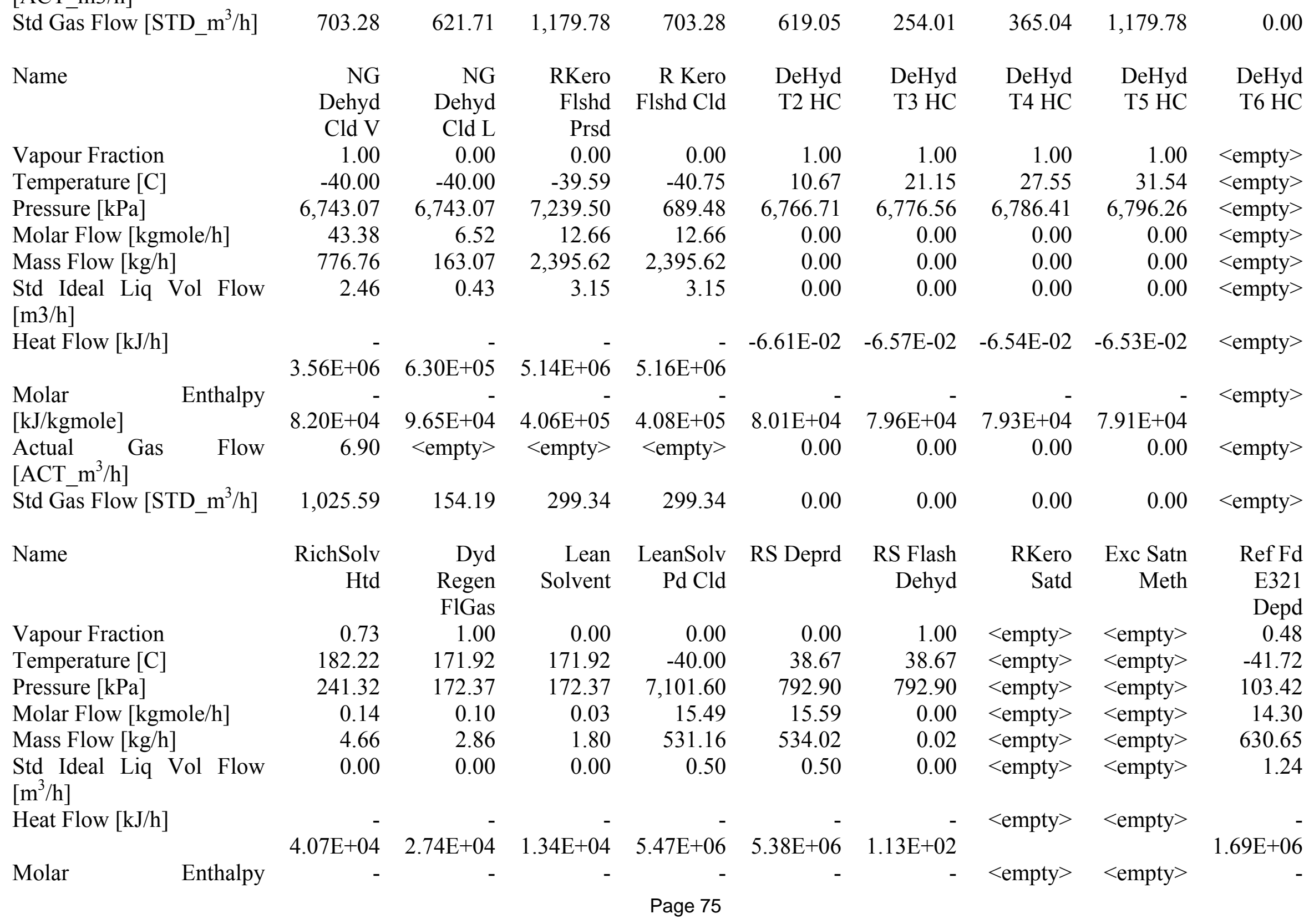


[kJ/kgmole]

Actual Gas

[ACT_m $\mathrm{m}^{3} / \mathrm{h}$ ]

Std Gas Flow [STD_m ${ }^{3} / \mathrm{h}$ ]

Name

Vapour Fraction

Temperature [C]

Pressure [kPa]

Molar Flow [kgmole/h]

Mass Flow [kg/h]

Std Ideal Liq Vol Flow $\left[\mathrm{m}^{3} / \mathrm{h}\right]$

Heat Flow [kJ/h]

$\begin{array}{lr}\text { Molar } & \text { Enthalpy } \\ {[\mathrm{kJ} / \mathrm{kgmole}]} & \\ \text { Actual Gas } & \text { Flow } \\ {[\text { ACT_m } / \mathrm{h}]} & \end{array}$

Std Gas Flow [STD_m ${ }^{3} / \mathrm{h}$ ]

Name

Vapour Fraction

Temperature [C]

Pressure [kPa]

Molar Flow [kgmole/h]

Mass Flow [kg/h]

Std Ideal Liq Vol Flow $\left[\mathrm{m}^{3} / \mathrm{h}\right]$

Heat Flow [kJ/h]
$2.99 \mathrm{E}+05$

$<$ empty>

3.22

ref Prd

E321

1.00

$-42.49$

99.97

14.30

630.65

1.24

$2.67 \mathrm{E}+05$

$3.97 \mathrm{E}+05$

$3.53 \mathrm{E}+05$

$3.45 \mathrm{E}+05$

2.16 <empty>

$<$ empty>

2.42

0.80

366.18

RS

E321

Recycled

RS NG

Regentn ProductSt

0.00

0.00

0.00

37.78
$1,482.37$

38.67

792.90

15.45

14.30

630.65

1.24

529.36

0.49

38.67

792.90

0.14

4.66

0.00

$\mathrm{rm}$
1.00
-40.00
$7,205.02$
0.00
0.00
0.00

$0.00 \mathrm{E}+00$

$1.55 \mathrm{E}+06 \quad 1.69 \mathrm{E}+06$

$5.33 \mathrm{E}+06$

$4.70 \mathrm{E}+04$

$1.09 \mathrm{E}+05$

265.28

$1.18 \mathrm{E}+05$

<empty>

$3.45 \mathrm{E}+05$

$<$ empty>

$3.45 \mathrm{E}+05$

<empty>

$4.06 \mathrm{E}+05$

0.00

$338.15 \quad 338.15$

2nd Stg RKero

Flash Gas

2ndFlash

1.00
69.43

0.00

313.64

723.95

689.48

2.78

98.43

0.23

$2,395.62$

3.15

365.38

3.22

0.00

RKero

Flshd

Prsd Cld

0.00

$-40.00$

7,205.02

12.66

2,395.62

3.15

t411

vapor

1.00

$-35.38$

$6,205.28$

0.11

1.91

0.01

Solv

RKero

Flshd

0.00

$-33.07$

$6,550.02$

26.18

$2,689.85$

4.01
$7.57 \mathrm{E}+04$

0.00

0.04

RKero

PrSatd

0.00
-40.00

$7,205.02$

12.66

$2,395.62$

3.15

$5.14 \mathrm{E}+06$

$4.06 \mathrm{E}+05$

$<$ empty>

299.34

Recycle

Solv

DHY

0.00

39.10

792.37

15.49

531.16

0.50

$5.13 \mathrm{E}+06$

$4.06 \mathrm{E}+05$

<empty>

299.34

Lean Solv

Prsd

0.00

39.62

$7,239.50$

15.49

531.16

0.50
$1.18 \mathrm{E}+05$

$<$ empty>

338.15

RKero

Prsd Flshd Htd

$0.00=0.00$

$-40.00 \quad 204.44$

$8,515.03 \quad 3,068.17$

$12.66 \quad 15.44$

$2,395.62 \quad 2,494.06$

$\begin{array}{ll}3.15 & 3.39\end{array}$

$5.14 \mathrm{E}+06 \quad 4.05 \mathrm{E}+06$

$4.06 \mathrm{E}+05 \quad 2.62 \mathrm{E}+05$

<empty> <empty>

$299.34 \quad 365.04$

LeanSolv Dhyd

ent 2 PreCool

$$
\begin{aligned}
0.00 & <\text { empty> } \\
172.03 & <\text { empty> } \\
792.37 & <\text { empty> } \\
0.03 & <\text { empty> } \\
1.80 & \text { <empty> } \\
0.00 & \text { <empty> }
\end{aligned}
$$




\begin{tabular}{|c|c|c|c|c|c|c|}
\hline & $2.54 \mathrm{E}+05$ & $3.00 \mathrm{E}+06$ & $5.14 \mathrm{E}+06$ & $9.00 \mathrm{E}+03$ & $6.34 \mathrm{E}+06$ & $5.35 \mathrm{E}+06$ \\
\hline Enthalpy & - & - & - & - & - & \\
\hline$[\mathrm{kJ} /$ kgmole $]$ & $9.14 \mathrm{E}+04$ & $2.37 \mathrm{E}+05$ & $4.06 \mathrm{E}+05$ & $8.00 \mathrm{E}+04$ & $2.42 \mathrm{E}+05$ & $3.45 \mathrm{E}+05$ \\
\hline $\begin{array}{l}\text { Actual Gas } \quad \text { Flow } \\
{\left[\text { ACT_m } \mathrm{m}^{3} / \mathrm{h}\right]}\end{array}$ & 10.89 & $<$ empty $>$ & $<$ empty $>$ & 0.02 & $<$ empty $>$ & <emnty > \\
\hline Std Gas Flow [STD_m $\left.\mathrm{m}^{3} / \mathrm{h}\right]$ & 65.70 & 299.34 & 299.34 & 2.66 & 619.05 & 366.1 \\
\hline Name & Heat 4 & $\begin{array}{l}\text { P } 100 \\
\text { Work }\end{array}$ & $\begin{array}{r}\text { E101 } \\
\text { Propane }\end{array}$ & $\begin{array}{r}\text { Heat } \\
\text { Regen D }\end{array}$ & $\begin{array}{r}\text { LeanSolv } \\
\text { Cool }\end{array}$ & Refrig \\
\hline Vapour Fraction & $<$ empty $>$ & $<$ empty $>$ & $<$ empty $>$ & $<$ empty $>$ & $<$ empty $>$ & $<$ empty $>$ \\
\hline Temperature $[\mathrm{C}]$ & $<$ empty $>$ & $<$ empty $>$ & $<$ empty $>$ & $<$ empty $>$ & $<$ empty $>$ & $<$ empty \\
\hline Pressure $[\mathrm{kPa}]$ & $<$ empty $>$ & $<$ empty $>$ & $<$ empty $>$ & $<$ empty $>$ & $<$ empty $>$ & $<$ empty \\
\hline Molar Flow [kgmole/h] & $<$ empty $>$ & $<$ empty $>$ & $<$ empty $>$ & $<$ empty $>$ & $<$ empty $>$ & $<$ empty \\
\hline Mass Flow $[\mathrm{kg} / \mathrm{h}]$ & $<$ empty $>$ & $<$ empty $>$ & $<$ empty $>$ & $<$ empty $>$ & $<$ empty $>$ & $<$ empty \\
\hline $\begin{array}{l}\text { Std Ideal Liq Vol Flow } \\
{\left[\mathrm{m}^{3} / \mathrm{h}\right]}\end{array}$ & $<$ empty $>$ & $<$ empty $>$ & $<$ empty $>$ & $<$ empty $>$ & $<$ empty $>$ & <empty \\
\hline Heat Flow $[\mathrm{kJ} / \mathrm{h}]$ & $2.16 \mathrm{E}+05$ & $2.61 \mathrm{E}+04$ & $2.16 \mathrm{E}+06$ & $6.23 \mathrm{E}+03$ & $1.26 \mathrm{E}+05$ & $1.20 \mathrm{E}+03$ \\
\hline $\begin{array}{l}\text { Molar } \\
{[\mathrm{kJ} / \text { kgmole }]}\end{array}$ & $<$ empty $>$ & $<$ empty $>$ & $<$ empty $>$ & $<$ empty $>$ & $<$ empty $>$ & cimpt \\
\hline $\begin{array}{l}\text { Actual Gas } \quad \text { Flow } \\
{\left[\text { ACT_m } \mathrm{m}^{3} / \mathrm{h}\right]}\end{array}$ & $<$ empty $>$ & $<$ empty $>$ & $<$ empty $>$ & $<$ empty $>$ & $<$ empty $>$ & <empty \\
\hline Std Gas Flow [STD_m $\mathrm{m}^{3} / \mathrm{h}$ ] & $<$ empty $>$ & $<$ empty $>$ & $<$ empty $>$ & $<$ empty $>$ & $<$ empty $>$ & $<$ empty \\
\hline Name & fracClg & $\begin{array}{r}\text { Frac } \\
\text { Reblr }\end{array}$ & $\begin{array}{r}\text { LeanSolv } \\
\text { Pump }\end{array}$ & $\begin{array}{r}\text { Lean Solv } \\
\text { Cool } 2\end{array}$ & ex481h & ex29 \\
\hline Vapour Fraction & $<$ empty $>$ & $<$ empty $>$ & $<$ empty $>$ & $<$ empty> & $<$ empty $>$ & $<$ empty \\
\hline Temperature $[\mathrm{C}]$ & $<$ empty $>$ & $<$ empty $>$ & $<$ empty $>$ & $<$ empty $>$ & $<$ empty $>$ & $<$ empty \\
\hline Pressure $[\mathrm{kPa}]$ & $<$ empty $>$ & $<$ empty $>$ & $<$ empty $>$ & $<$ empty $>$ & $<$ empty $>$ & $<$ empty \\
\hline Molar Flow [kgmole/h] & $<$ empty $>$ & $<$ empty $>$ & $<$ empty $>$ & $<$ empty $>$ & $<$ empty $>$ & <empty \\
\hline Mass Flow $[\mathrm{kg} / \mathrm{h}]$ & $<$ empty $>$ & $<$ empty $>$ & $<$ empty $>$ & $<$ empty $>$ & $<$ empty $>$ & $<$ empty \\
\hline $\begin{array}{l}\text { Std Ideal Liq Vol Flow } \\
{\left[\mathrm{m}^{3} / \mathrm{h}\right]}\end{array}$ & $<$ empty $>$ & $<$ empty $>$ & $<$ empty $>$ & $<$ empty $>$ & $<$ empty $>$ & $<$ empty \\
\hline \multirow[t]{2}{*}{ Heat Flow $[\mathrm{kJ} / \mathrm{h}]$} & $3.91 \mathrm{E}+04$ & $8.27 \mathrm{E}+05$ & $4.25 \mathrm{E}+03$ & $1.52 \mathrm{E}+00$ & $1.85 \mathrm{E}+03$ & $2.16 \mathrm{E}+06$ \\
\hline & & & & Page 77 & & \\
\hline
\end{tabular}

\begin{tabular}{|c|c|c|}
\hline $5.34 \mathrm{E}+06$ & $1.34 \mathrm{E}+04$ & \\
\hline & - & $<$ empty $>$ \\
\hline $3.45 \mathrm{E}+05$ & $3.97 \mathrm{E}+05$ & \\
\hline$<$ empty $>$ & $<$ empty $>$ & $<$ empty $>$ \\
\hline 366.18 & 0.80 & $<$ empty $>$ \\
\hline p102 & e105ht & $\mathrm{t} 100$ \\
\hline Power & & reboiler \\
\hline$<$ empty $>$ & $<$ empty $>$ & $<$ empty $>$ \\
\hline$<$ empty $>$ & $<$ empty $>$ & $<$ empty $>$ \\
\hline$<$ empty $>$ & $<$ empty $>$ & $<$ empty $>$ \\
\hline$<$ empty $>$ & $<$ empty $>$ & $<$ empty $>$ \\
\hline$<$ empty $>$ & $<$ empty $>$ & $<$ empty $>$ \\
\hline$<$ empty $>$ & $<$ empty $>$ & $<$ empty $>$ \\
\hline $5,440.26$ & - & $1.82 \mathrm{E}+06$ \\
\hline & $5.88 \mathrm{E}+05$ & \\
\hline$<$ empty $>$ & $<$ empty $>$ & $<$ empty $>$ \\
\hline$<$ empty $>$ & $<$ empty $>$ & $<$ empty $>$ \\
\hline$<$ empty $>$ & $<$ empty $>$ & $<$ empty $>$ \\
\hline
\end{tabular}




\begin{tabular}{|c|c|c|c|c|c|c|c|}
\hline $\begin{array}{l}\text { Molar } \\
{[\mathrm{kJ} / \mathrm{kgmole}]}\end{array}$ & Enthalpy & $<$ empty $>$ & $<$ empty $>$ & $<$ empty $>$ & $<$ empty $>$ & $<$ empty $>$ & $<$ empty $>$ \\
\hline $\begin{array}{l}\text { Actual Gas } \\
{\left[\mathrm{ACT} \_\mathrm{m}^{3} / \mathrm{h}\right]}\end{array}$ & Flow & $<$ empty $>$ & $<$ empty $>$ & $<$ empty $>$ & $<$ empty $>$ & $<$ empty $>$ & $<$ empty $>$ \\
\hline Std Gas Flow [S & $\left.\mathrm{m}^{3} / \mathrm{h}\right]$ & $<$ empty $>$ & $<$ empty $>$ & $<$ empty $>$ & $<$ empty $>$ & $<$ empty $>$ & $<$ empty $>$ \\
\hline
\end{tabular}


Two bids were received. They both were for approximately $\$ 850,000$. This would require the building of the pilot plant over two fiscal years to meet funding limitations. We are examining the possibility of splitting the pilot plant to two construction/operational campaigns, one with just the dehydration step with its greater technical risk and the other performed later for the NGL recovery.

Discussions were initiated with EnCana Corporation, Colorado, as a potential host of the pilot plant unit. A meeting was held on June 28, 2007 in EnCana's Denver office. The facility personnel were very interested in participating in the program. They sent typical gas compositional data for three of their processing plants in the Denver area. The plant that looked the most interesting has a feed gas that could be delivered at 1126 psi to our test unit. The gas contains about 6 gallons of NGL/M scf of natural gas. The saturated gas would have a higher heating value of $1222 \mathrm{Btu} / \mathrm{scf}$.

Further work on the pilot plant was deferred until sufficient BSE data were available to verify the benefits of the technology. 


\section{Task 7: $\quad$ Conduct Market Study and Prepare Commercialization Package}

In order to understand the existing NGL recovery technologies and discuss the recent market trends in this field, GTI organized a plant visit from Nov. 10 to 13, 2003 in which three key personnel involved in this project (one from GHT and two from GTI) visited three different commercial plants for NGL recovery and held technical discussion with a group of senior process/design engineers from a major engineering company with expertise in design and construction of these plants. The three plants visited during this trip were:

1. refrigerated lean oil absorption plant at King Ranch, South Texas, owned by ExxonMobil Corp., gas processing capacity $=925 \mathrm{MMscfd}$;

2. cryogenic plant at Spindle, CO, owned by Duke Energy Field Services (DEFS), gas processing capacity $=40 \mathrm{MMscfd}$;

3. J-T plant at Platteville, CO, owned by Duke Energy Field Services (DEFS), gas processing capacity $=50$ MMscfd.

The engineering company visited during this trip was Washington Group International Inc. (WGI), Denver, CO. During the meeting, various technical and marketing aspects of the proposed technology were discussed with WGI's experts in NGL recovery. WGI has performed an engineering feasibility study for NGL recovery using the refrigerated lean oil absorption process. The data collected from the plant visit was used in the preliminary evaluation of the new technology.

GTI project manager attended a 3 day course on NGL recovery and NGL marketing organized by Oil Price Information Service (OPIS) held on February 17-19, 2004 at Houston, TX and visited Dynegy's NGL fractionation plant located at Mont Belview near Houston, TX, as a part of the course. During this course and plant visit some useful plant data and information related to natural gas processing technology, NGL trading contracts, fluctuations in NGL market prices and its effect on $\mathrm{C}_{2}$ recovery were collected. Important plant data and market information on the existing NGL recovery technologies were also collected during the GPA meeting when one of GTI engineer associated with this project visited three different NGL recovery plants operated by Enterprise ${ }^{\circledR}$ located in St. Mary Parish, LA. The names of the plants visited are:

1. Neptune 1: Capacity $=350$ MMscfd, Technology = Cryogenic Turbo-expander (IPSI process), Built in 2001.

2. Neptune 2: Capacity $=350$ MMscfd, Technology = Cryogenic Turbo-expander (IPSI process), Built in 2004.

3. Calumet Gas Processing Plant: Capacity $=1300$ MMscfd, Technology $=$ Refrigerated Lean Oil Process, Built in 1970s.

A comprehensive survey of various state, federal and private sources was done to compile a list of all NGL recovery plants in North America (including the U.S., Mexico and Canada). These plants were categorized in terms of technology used; gas throughput; energy consumption; and NGL recovery (particularly $\mathrm{C}_{2}$ and $\mathrm{C}_{3}$ recovery). The data for capital and operating costs and plant staffing were also collected for various existing NGL recovery processes. In order to compare the new technology with the cryogenic technology on a similar cost basis, an economic analysis of the process based on annual capital return was performed. 
Based on the survey data, four gas compositions were selected to represent various "richness" of natural gas in NGL content, as shown in Figure 27. The graph also shows how the compositional fraction is represented in the overall natural gas resource base.

Figure 27 Feed Gas Richness

IIT completed a comprehensive computer simulation of the IROA process. This study included comparison of the IROA and TurboExpander $(\mathrm{T} / \mathrm{E})$ processes at four different feed natural gas compositions $\left[\mathrm{C}_{2}^{+}:\right.$Super Rich(18\%), Rich(13\%), Lean( $\left.8 \%\right)$, and Super Lean(3.7\%)] and four levels of $\mathrm{C}_{2}$ recovery $(2 \%, 35 \%, 70 \%$, and $95 \%)$.

The both processes were simulated at all operating conditions specified by GTI using a commercially available process simulation software (HYSYS 3.1). The operating condition for the IROA process included all four natural gas compositions at $95 \% \mathrm{C}_{2}$ recovery, while those for the $\mathrm{T} / \mathrm{E}$ process all gas compositions and recovery levels. These process simulations provided all the process variables and various utility requirements. The total utility costs were determined based on unit costs of various utilities provided by GTI which included $0.042 \$ / \mathrm{kWh}$ for electricity, $0.00005 \$ /$ gal for cooling water, @ $80^{\circ} \mathrm{F}$ and $6 \$ / \mathrm{MMBTU}$ for heating above $500^{\circ} \mathrm{F}$, and waste heating below 500 $\mathrm{F} @ 1 \mathrm{HP} / 1000 \mathrm{BTU} / \mathrm{hr}$.

Equipment sizing and costing was also performed for a selected number of conditions specified by GTI using ASPEN Icarus 12.0. These cases included three gas compositions (Super Rich, Rich, and Lean) at $95 \%$ recovery for IROA and $95 \%$ as well as $35 \%$ recoveries for $\mathrm{T} / \mathrm{P}$. In cases where the estimated equipment costs provided by ASPEN Icarus 12.0 were believed to be inaccurate (based on literature data), correction were made using the estimates provided by matche.com. 
The annualized overall costs of the processes were determined using different interest rate ranging from $5 \%$ to $20 \%$.

Based on the annualized overall costs, IIT concluded that the IROA appears to be more economical than T/E for higher NGL recoveries (e.g. @95\%). However, since the utility costs associated with $\mathrm{T} / \mathrm{E}$ decreases with decreasing recovery of NGL, assuming utility cost of CEP to be independent of the recovery level (as indicated by GTI) the IROA can be more economical than T/E at lower recovery levels. Purchased equipment cost estimates were obtained using a new commercially available software called Preliminary Design and Quoting Service (PDQ\$). This software estimates costs for fabricated equipment and catalog items that are based on vendor information. Code designed vessels, reactors, towers, and exchangers are provided with fabricator cost breakdown and detailed engineering specifications for ordering. The software is based on reliable non-indexed, non-factored cost data directly from vendors and can be used for preliminary project evaluation or final engineering cost estimates of chemical plants and petroleum refineries.

In this work, the cost estimates obtained using PDQ\$ were compared with those from AspenIcarus and equipment vendors. In general, the estimates from PDQ \$ were found to be in very good agreement with those from vendors. The overall estimate compared within $9 \%$ of the vendor quoted costs. As for the estimates from Aspen-Icarus, good agreement was found in estimated cost values and vendor quotes for standard items such as pressure vessels and towers. However, this agreement was not very good for major items such as compressors and heat exchangers. For this reason, the cost estimates from PDQ\$ were selected as the basis for economic evaluation of the new and conventional processes.

The cost estimates were used to calculate the annualized capital cost for 100 MMscfd natural gas processing plant based on new and conventional NGL recovery technologies. It was found that the annualized capital cost for the new technology is about $10 \%$ lower than that of conventional technology for $\mathrm{C}_{2}$ recoveries above $70 \%$ and about $40 \%$ lower than that of conventional technology for $\mathrm{C}_{2}$ recoveries below $50 \%$. These results when combined with the operating cost reductions due to lower energy consumption for the new process (see Figure 28 and Figure 29) may reduce the cost of NGL extraction from natural gas using new technology by about $40-50 \%$ at $\mathrm{C}_{2}$ recoveries lower than $50 \%$. 


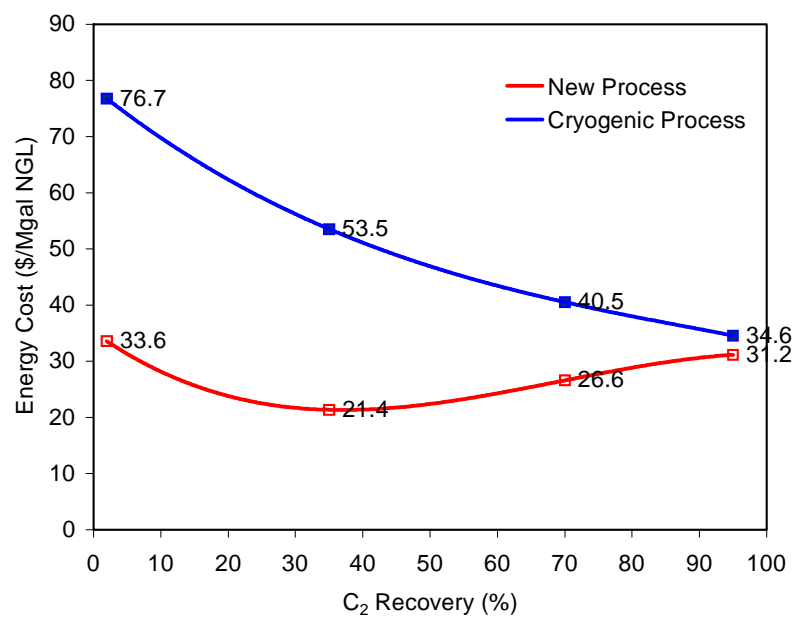

Figure 28 Energy Cost for NGL Production as a Function of $\mathrm{C}_{2}$ Recovery (gas richness -2.2

GPM)

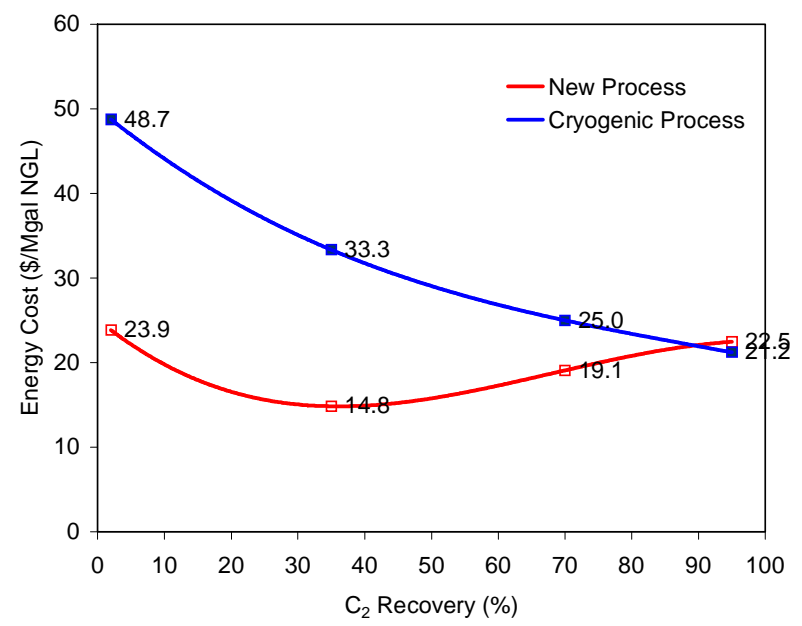

Figure 29 Energy Cost for NGL Production as a Function of $\mathrm{C}_{2}$ Recovery (gas richness 3.6 GPM)

The net processing margins were calculated to compare the New Process with Cryogenic Expansion Process. For each technology, 35\% and 95\% recovery of the $\mathrm{C}_{2}$ components were determined, as shown on Figure 30. Net Processing Margins are the differences between the revenue streams from the NGL and product gas and the processing capital, operating, and shrinkage costs. The higher the margins, the more the processor makes on the processing of the stream. As illustrated, the New Process has higher margins for the range of natural gas prices. 


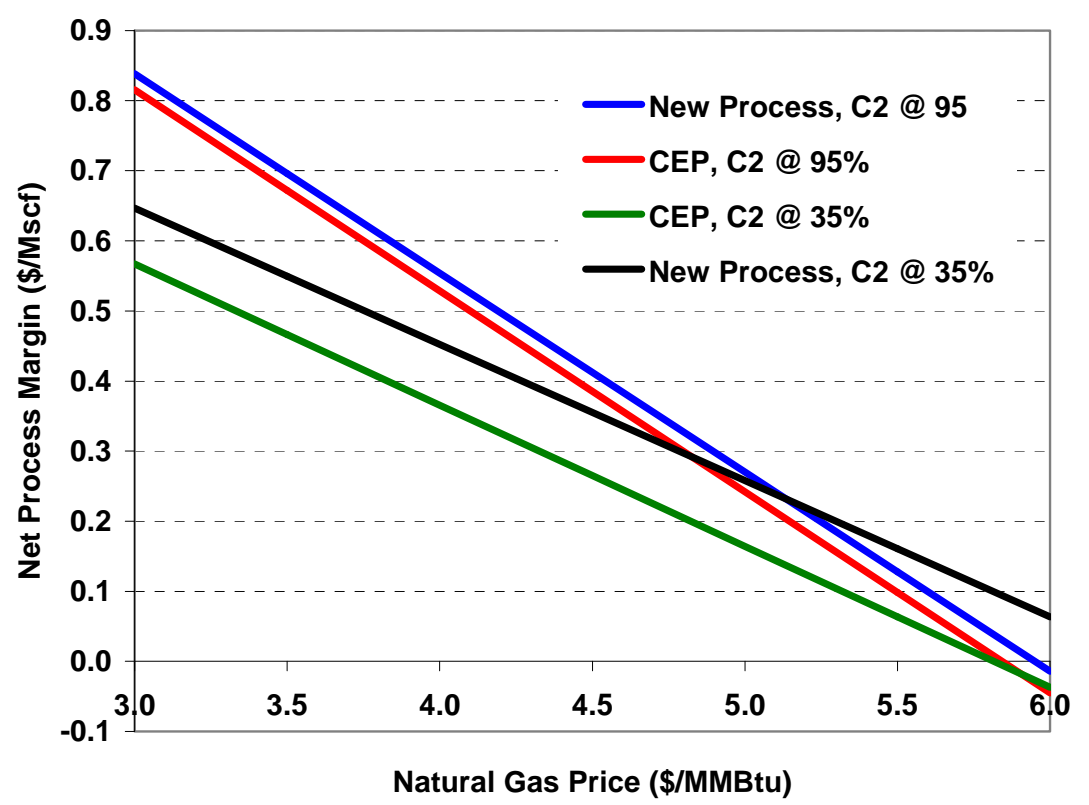

Figure 30 New Processing Margins

A net present value evaluation was also performed to compare the processes. A project life of 15 years, a discount rate of $10 \%$ and 2004 NGL prices were assumed as shown in Figure 31. Gas prices of $\$ 4.50$ and $\$ 5.00 / \mathrm{MM}$ Btu were used to compare 35,70 and $95 \% \mathrm{C}_{2}$ recovery cases. In each case, the New Process had a greater than 10\% NPV advantage over the conventional technology. For the low $\mathrm{C}_{2}$ recovery cases, the New Process NPV was over three times that of the conventional technology at the higher gas prices and 1.7 times greater at the lower gas price.

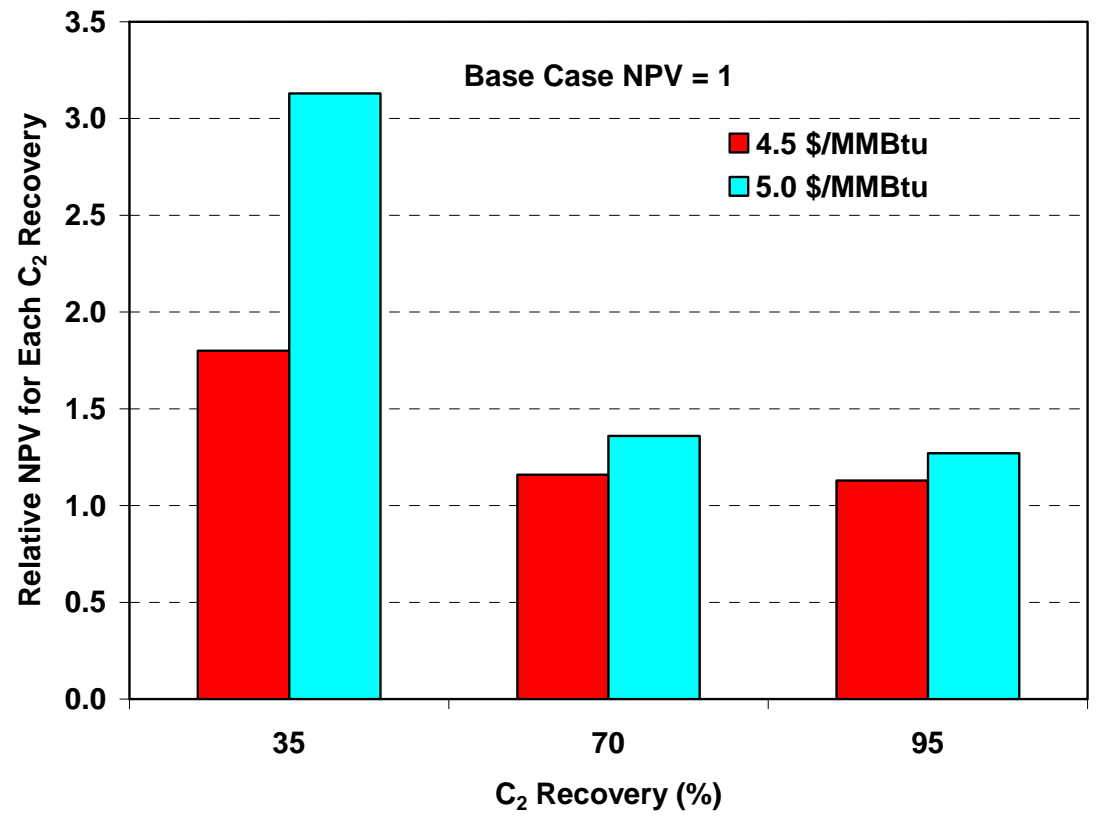

Figure 31 New Present Value Evaluation 
At the request of the reviewers at the DOE review meeting held in April 2005 in association with the Annual AIChE meeting, GTI was requested to submit a retrofit case for CPAT analysis. (see Table 27 and Table 28) The new technology case had an IRR of 500\% and NPV of \$35.8MM compared to the "do nothing" case with an IRR of $100 \%$ and NPV of $\$ 29.4 \mathrm{MM}$. The process would be saving about $\$ 1 \mathrm{MM} / \mathrm{y}$ on an investment of about $\$ 1.8 \mathrm{MM}$. The model calculates this at 2.3 years payout. With even slightly greater energy savings per accelerated units deployed than in our base case for the technology, the model only deploying one plant of a total potential 125 plants. These results are counter-intuitive; significant penetration should be achieved with these favorable economics. BCS, International was asked for guidance on the CPAT case, but has not resolved the issue.

Table 27 CPAT Input

\section{Project: Retrofit Market for IROA}

- Default

- Edited

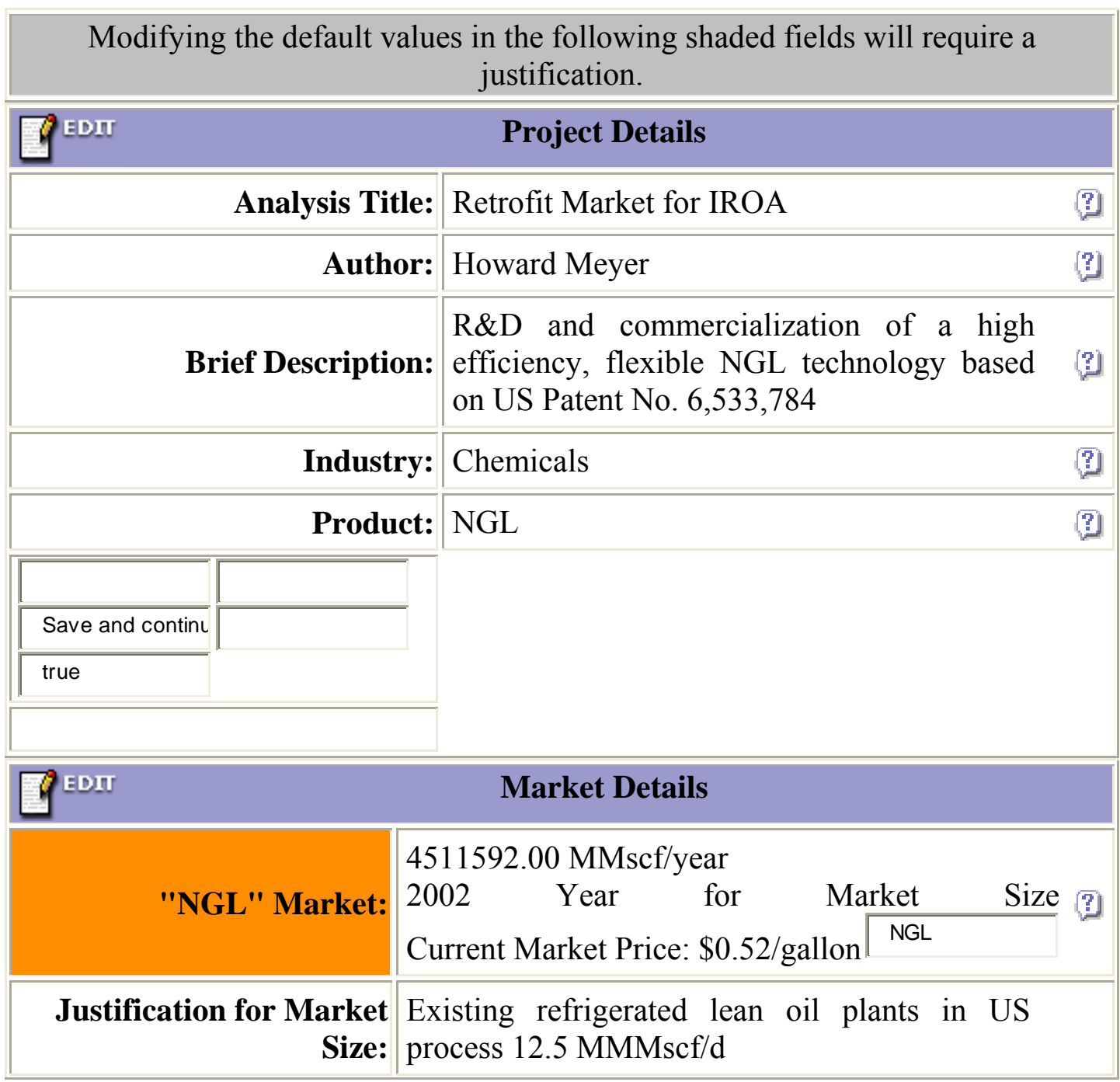




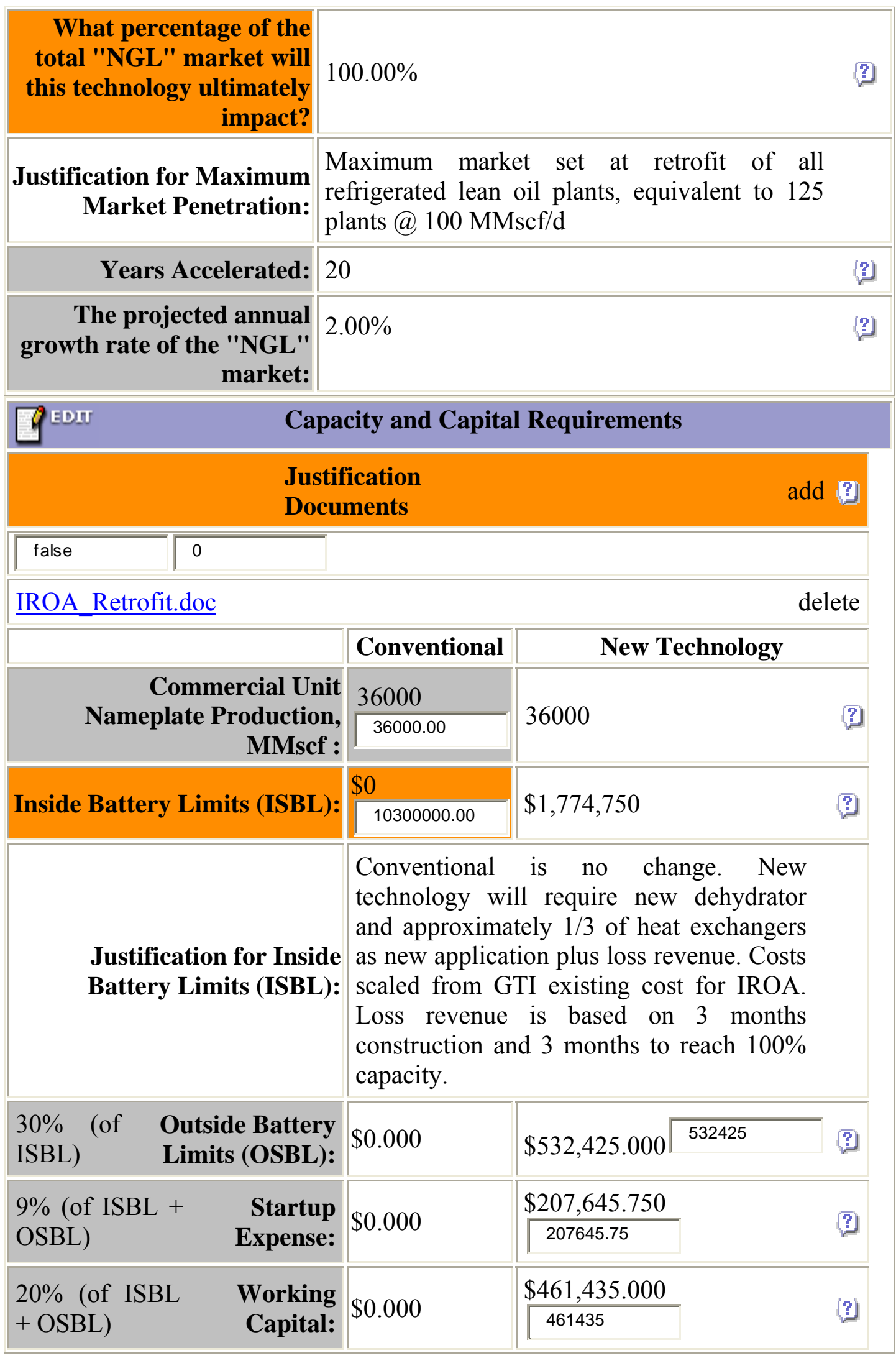




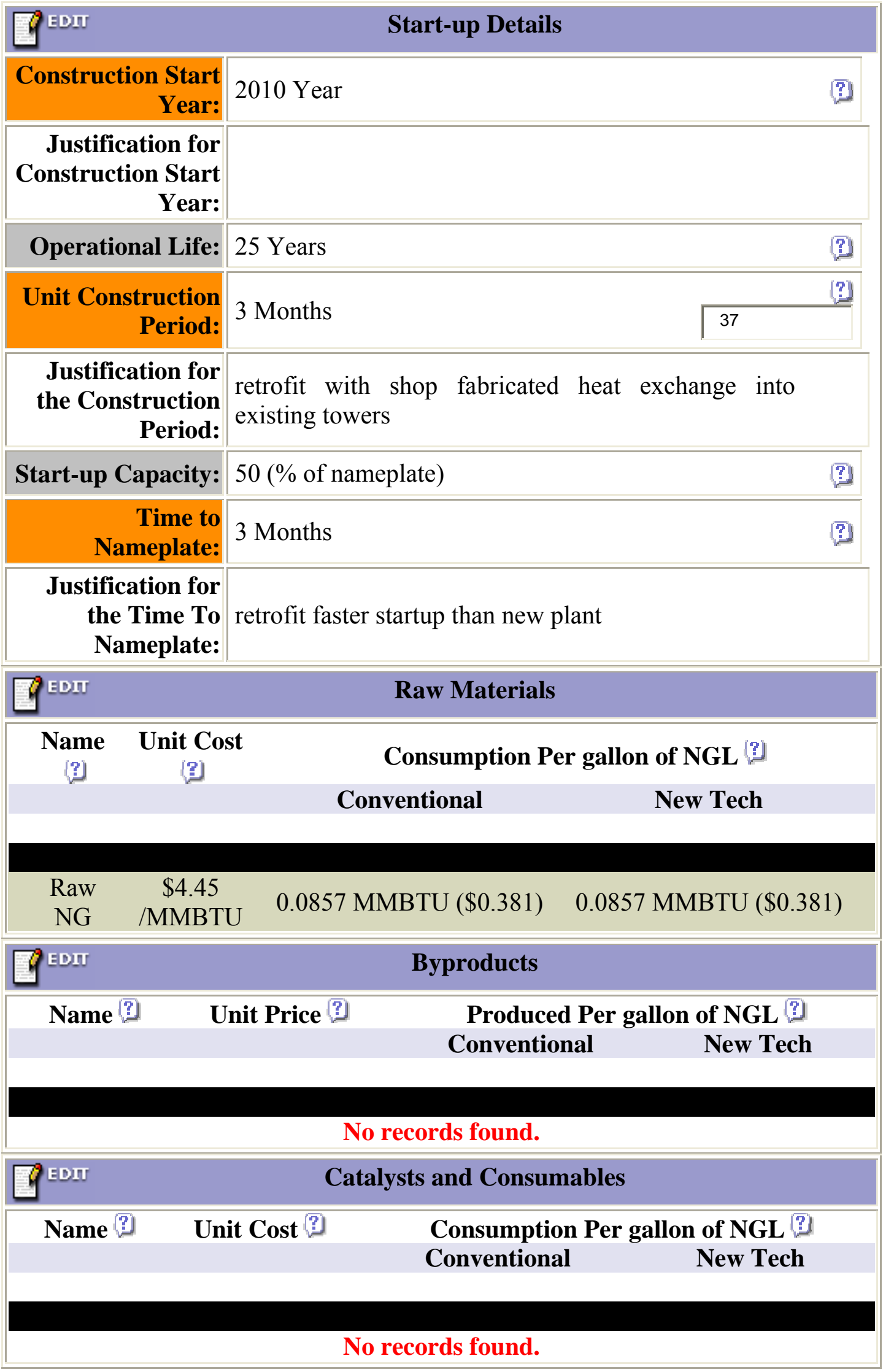




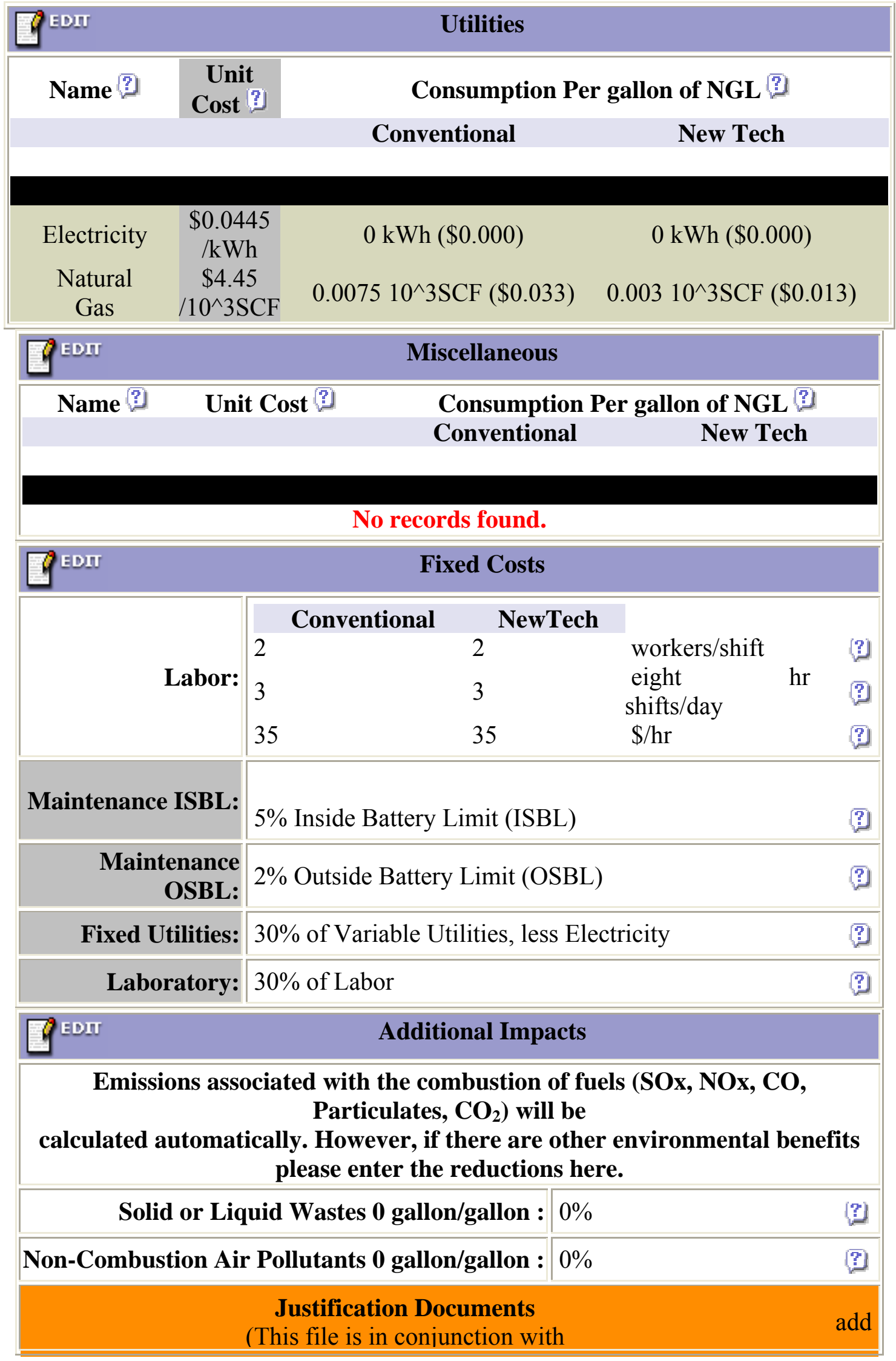


Final Progress Report

DE-FC36-03G013150

the Capacity and Capital

Requirements Justification

Document)

Page 89 
Table 28 CPAT Results for Retrofit Case

\begin{tabular}{|c|c|c|c|c|c|}
\hline \multicolumn{6}{|c|}{ Project Summary Results for New Technology, Part I } \\
\hline \multicolumn{6}{|c|}{ Energy Impacts for NGL } \\
\hline & 2010 & 2015 & 2020 & 2025 & 2030 \\
\hline \begin{tabular}{|l} 
MARKET \\
(MMscf) \\
\end{tabular} & $5,286,049$ & $5,836,225$ & $6,443,664$ & $7,114,326$ & $7,854,791$ \\
\hline \begin{tabular}{|l} 
MARKET \\
PENETRATION \\
\end{tabular} & $0.70 \%$ & $0.60 \%$ & $0.60 \%$ & $0.50 \%$ & $0.00 \%$ \\
\hline \begin{tabular}{|l} 
ACCELERATED \\
UNITS \\
DEPLOYED \\
\end{tabular} & 1 & 1 & 1 & 1 & 0 \\
\hline $\begin{array}{l}\text { ENERGY } \\
\text { SAVINGS }\end{array}$ & & & & & \\
\hline $\begin{array}{l}\frac{\text { Heat/Steam }}{\text { Energy Savings }} \\
\text { (trillion Btu) }\end{array}$ & 0.3 & 0.3 & 0.3 & 0.3 & 0 \\
\hline $\begin{array}{l}\text { Electricity } \\
\text { Savings (trillion } \\
\text { Btu) } \\
\end{array}$ & 0 & 0 & 0 & 0 & 0 \\
\hline $\begin{array}{l}\text { Feedstock Energy } \\
\text { Savings } \\
\text { (trillion Btu) }\end{array}$ & 0 & 0 & 0 & 0 & 0 \\
\hline
\end{tabular}

\begin{tabular}{|c|c|c|}
\hline \multicolumn{2}{|c|}{ Energy Cost as a \% of Product Price } \\
\hline $\begin{array}{c}\text { Conventional } \\
\text { Technology }\end{array}$ & $\begin{array}{c}\text { New } \\
\text { Technology }\end{array}$ \\
\hline 6.4 & 2.6 \\
\hline
\end{tabular}




\begin{tabular}{|c|c|c|c|c|c|c|c|}
\hline \multicolumn{8}{|c|}{ New Technology Estimated Annual Operating Cash Flows } \\
\hline $\begin{array}{l}\text { Project } \\
\text { Capital: } \\
\$ 1,774,75 \\
0\end{array}$ & $\perp$ & $\begin{array}{l}\text { Ancillar } \\
\text { y } \\
\text { Capital: } \\
\$ 532,425 \\
\end{array}$ & & $\begin{array}{l}\text { IRR: } \\
500.0 \\
\% \\
\end{array}$ & & \begin{tabular}{l}
\multicolumn{1}{c}{ Total } \\
NPV $=\$$ \\
$35,839,70$ \\
$\underline{2}$
\end{tabular} & \\
\hline $\begin{array}{l}100 \% \\
\text { Capacity } \\
\text { Annual } \\
\text { Costs for: }\end{array}$ & $\begin{array}{l}\text { Raw } \\
\text { Materials: } \\
\$ 22,254,93 \\
6\end{array}$ & & $\begin{array}{l}\text { Utilities: } \\
\$ 779,05 \\
3 \\
\end{array}$ & & $\begin{array}{l}\text { Consumable } \\
\text { s: } \$ 0\end{array}$ & & $\begin{array}{l}\text { Labor: } \\
\$ 306,60 \\
0\end{array}$ \\
\hline
\end{tabular}

Conventional Technology Estimated Annual Operating Cash Flows

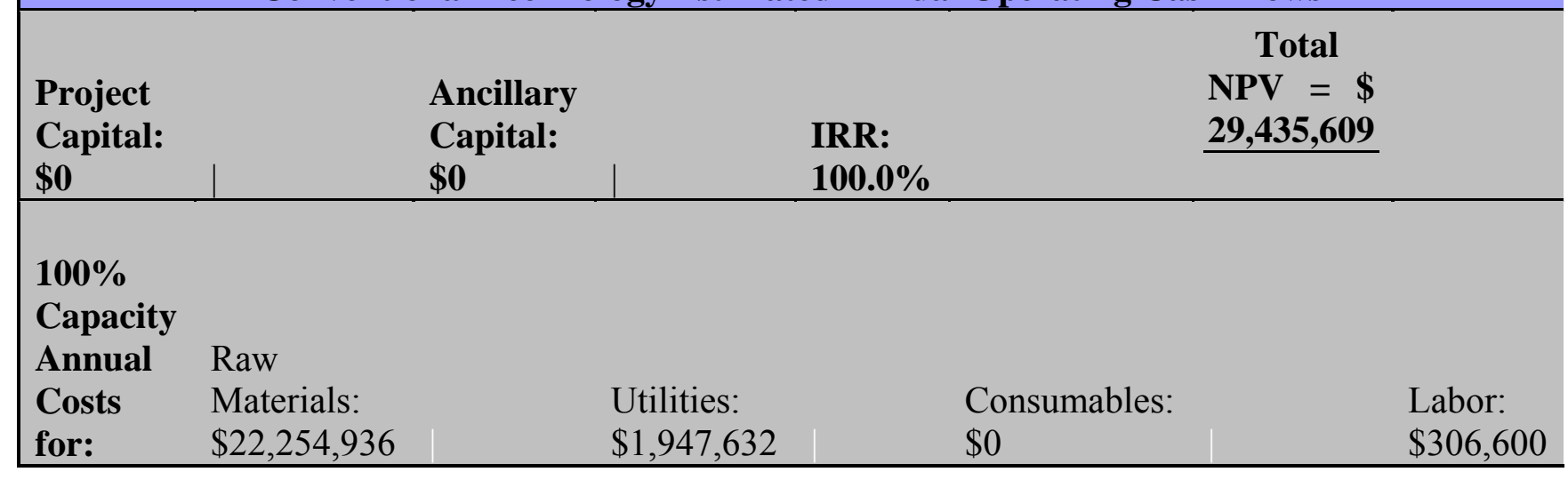




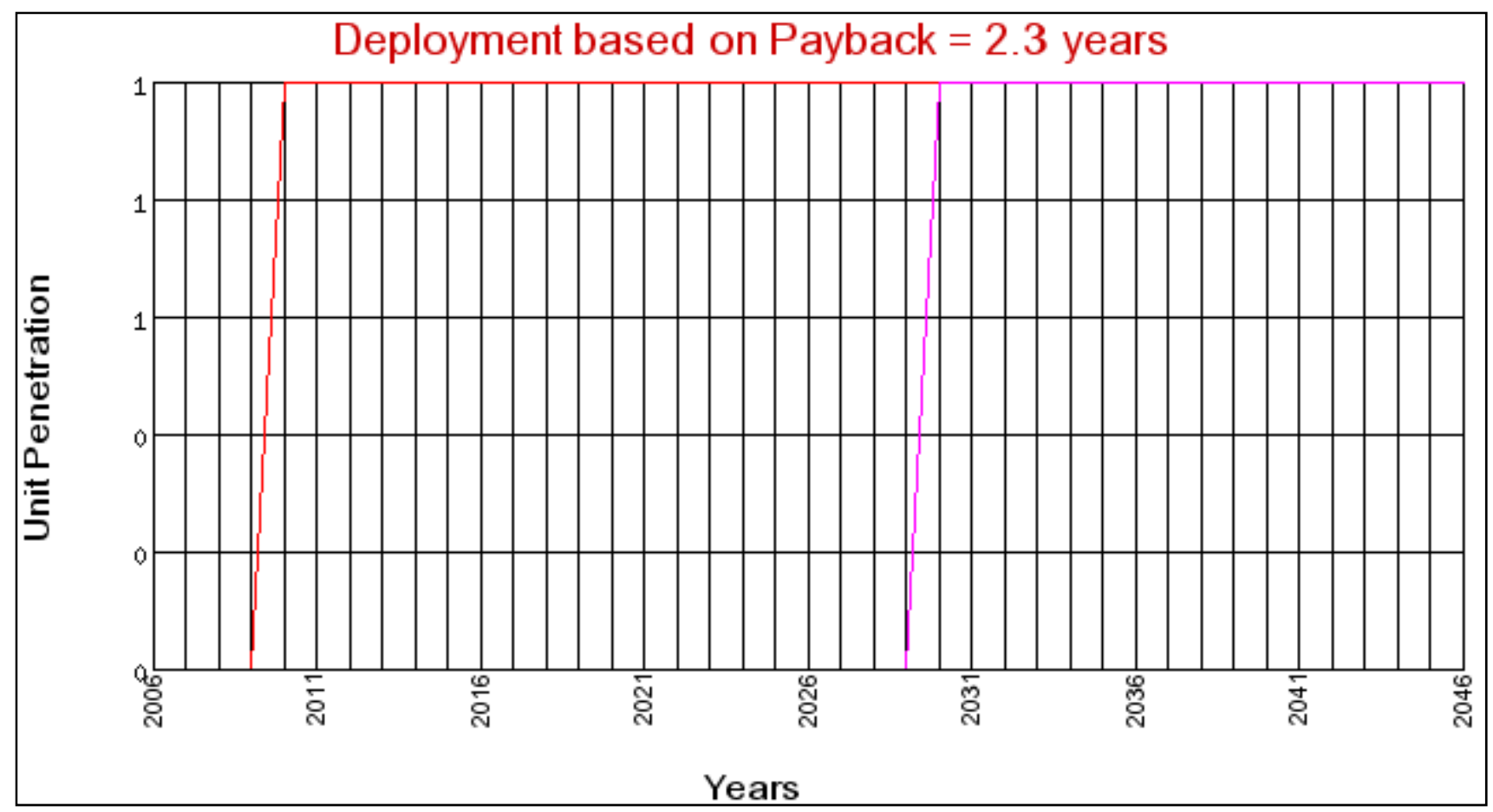


In the first phase of this project, IIT completed a comprehensive computer simulation of the IROA process covering 12 cases, comprising three different NGL contents and each with four operational modes. The final results were reported in September 2004. The simulation results provided a sound basis for the GTI evaluation of the IROA process. In April 2005, GTI presented the evaluation results at the AIChE 2005 Spring Meeting and to DOE reviewers. With DOE favorable appraisal and formal approval, the second phase of the IROA project was initiated.

Although the comprehensive simulation was appropriate for general IROA process evaluation, the flowsheet has not been fully optimized. In particular, the opportunity to better exploit energy use of the system was not studied in depth. There is room for improvements. In the second phase of this project, the simulation were updated for energy use in the IROA process.

The following two major areas of potential energy savings have been identified:

1) Maximizing the residue gas exit temperature could reduce the refrigeration power requirement; and

2) Separating the NGL condensate from gas chiller to get partial NGL liquid product could reduce the power necessary for liquefying the NGL vapor from ROS.

GHT has used a number of different approaches to estimate the impact on the energy usage of flowsheet changes. The updated energy savings are summarized here.

For the optimization of the refrigeration power required in cooling inlet gas, the following formula has been used:

Where $\mathrm{S}=$ Energy savings, MMBtu/hr

$$
\mathrm{S}=\left(85-\mathrm{T}_{2}\right) *\left(\mathrm{H}_{2}-\mathrm{H}_{21}\right) /\left(\mathrm{T}_{2}-\mathrm{T}_{21}\right)
$$

$85=$ Optimum residue gas exit temperature, ${ }^{\circ} \mathrm{F}$

$\mathrm{T}_{2}, \mathrm{~T}_{21}=$ Actual residue gas exit temperature of IIT stream $\# 2$ and $\# 21$, respectively, ${ }^{\circ} \mathrm{F}$

$\mathrm{H}_{2}, \mathrm{H}_{21}=$ Enthalpy for stream \#2 and \#21, respectively, MMBtu/hr.

For the optimization of NGL recovery energy, the NGL condensates in gas cooling process is separated from the inhibitor medium and processed in a separate condensate demethanizer (CD). The IROA system diagram has been revised accordingly.

Three areas of energy savings have been calculated separately:

1) The reduction of the NGL vapor compressor (P-6) power;

2) The pumping power change due to the change of absorbent flow rate; and

3) The reduction of the heat duty for NGL vapor evaporation in ROS and subsequent cooling water for NGL vapor liquefaction.

The reduction of the NGL vapor compressor (P-6) power is determined by two factors:

The first factor is straightforward; the compressor power will be proportional to the amount of NGL vapor throughput.

The second factor is a novel feature of the new system design. As mentioned above, the injection of the low temperature partial NGL condensate stream \#8 into the compressed and cooled NGL vapor stream \#34b. The mixing of these two streams lower the mixture temperature and liquefies 
the vapor NGL at lower pressure. This would further reduce the energy requirement of the NGL compressor P-6. Detailed analysis has been conducted to determine the effect of this factor.

The pumping power of absorbent depends on three parameters. For a given absorber tower working under given temperature and pressure conditions, the absorbent flow rate is determined mainly with the gas flow rate, as well as the required $\mathrm{C}_{2}$ recovery rate and the concentration of $\mathrm{C}_{2}$ relative to $\mathrm{C}_{1}$. While all three of the parameters in the new system design decreased, their effects on energy savings are different. The first two have positive savings when decreased and the last one having negative savings. Without running the HYSYS (or Aspen) software, the calculation of the approximate gas absorption efficiency was interpolate from the existing IIT simulation results of gases with different $\mathrm{C}_{2}$ composition.

The evaporation is carried out in multiple steps, i.e., first, through the expansion valve (Vp-2, as shown in Figure 32), and then in the downward flow path through many heated plates inside the ROS tower. To estimate the potential energy savings of NGL evaporation in rich oil due to the removal of NGL condensates before the Absorber, a simplified scheme of the mid-section of the ROS, where the net up-flowing NGL vapor and the net down-flowing absorbent (eventually exit as Lean Oil at the bottom), is used as shown in Figure 32.

Taking the overall energy balance equation:

Evaporation Heat $=\mathrm{H}_{\mathrm{NGL}} * \mathrm{~F}_{\mathrm{NGL}}+\mathrm{H}_{\mathrm{LO}} * \mathrm{~F}_{\mathrm{LO}}-\mathrm{H}_{\mathrm{RO}} * \mathrm{~F}_{\mathrm{RO}}$

Where $\mathrm{H}_{\mathrm{RO}}=$ Enthalpy of rich oil before expansion,

$\mathrm{H}_{\mathrm{NGL}}=$ Enthalpy of NGL at the post expansion temperature of rich oil,

$\mathrm{H}_{\mathrm{LO}}=$ Enthalpy of lean oil at the post expansion temperature of rich oil, and

$\mathrm{F}_{\mathrm{NGL}}, \mathrm{F}_{\mathrm{LO}}, \mathrm{F}_{\mathrm{RO}}=$ the respective flow rate of NGL vapor, lean oil and rich oil.

The energy savings of evaporation heat is the difference of the evaporation heats calculated from the simulation conditions and the updated cases in which the NGL contents in rich oil is reduced due to the removal of NGL condensates before Absorber.

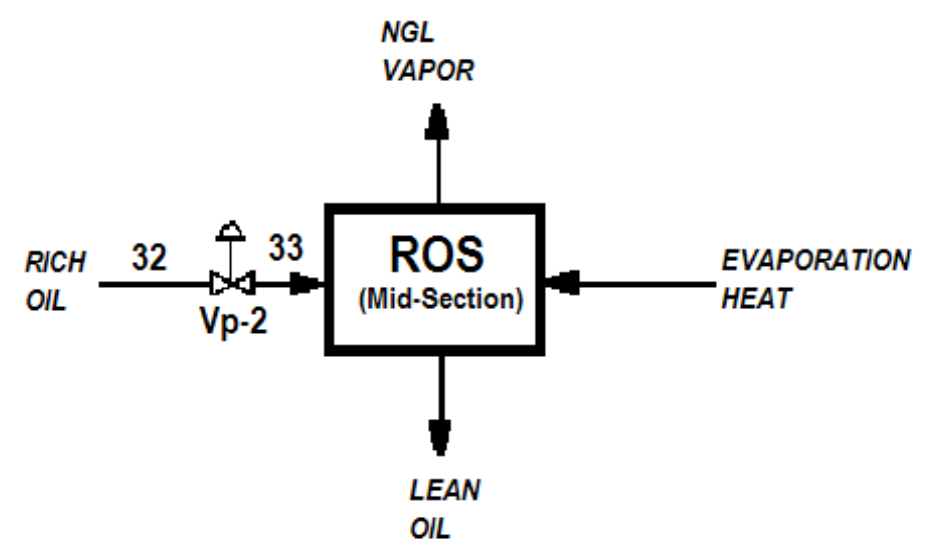

Figure 32 Simplified NGL Evaporation Scheme in ROS 
The overall energy savings and the contribution of each area are summarized in the following Table 29.

In Figure 33 and Figure 34, the significant energy savings are shown. The updated energy costs are the lowest as compared with the previous simulation energy costs (middle curve) as well as those of the cryogenic process (highest curve). Previously, the IROA energy cost at $95 \% \mathrm{C}_{2}$ was greater than that of the cryogenic process. The updated IROA energy cost at $95 \% \mathrm{C}_{2}$ recovery is now lower. Please note that the percentage savings given in Table 29 are calculated based on the total IROA energy cost, compared to the previous IROA definition. When calculated on the cost savings between IIT costs and cryogenic process, the percentage of additional savings in Table 29 to the previous savings over cryogenic process are much larger. For example in Figure 34, the previous IIT IROA energy cost savings to cryogenic process at $70 \% \mathrm{C}_{2}$ recovery is $\$ 13.95 / \mathrm{Mgal}$. The updated IROA energy cost savings to cryogenic process becomes $\$ 20.02 / \mathrm{Mgal}$, or a $56.5 \%$ increase over the previous IROA energy savings. In contrast, the savings in Table 29 is just $21.67 \%$. The previous IIT energy savings data taken in this example is close to what GTI used in evaluation table reported to DOE. It is believed, therefore, the updated IROA simulation results would give much better economical performance than previously evaluated. 
Table 29 Overall Energy Savings Compared to Previous Study

\begin{tabular}{|c|c|c|c|c|c|c|c|c|c|c|c|c|c|c|}
\hline Area & Item & Unit & SR95 & SR70 & SR35 & SR02 & R95 & R70 & $\mathrm{R} 35$ & R02 & A95 & A70 & A35 & A02 \\
\hline IIT Base & Total E-Cost & \$/hr & 353.91 & 286.82 & 184.98 & 215.09 & 327.76 & 236.46 & 135.93 & 148.36 & 278.71 & 190.1 & 123.21 & 131.91 \\
\hline \multirow{3}{*}{$\begin{array}{c}\text { Residue Gas } \\
\text { Energy } \\
\text { Recovery }\end{array}$} & Ref. Savings & hp & $\mathbf{0}$ & 668.14 & 782.201 & 260.95 & 178.02 & 468.80 & 328.27 & 328.27 & 134.03 & -63.45 & 489.36 & 832.40 \\
\hline & E-Cost Sav. & \$/hr & $\mathbf{0}$ & 28.06 & 32.85 & 10.96 & 7.48 & 19.69 & 13.79 & 13.79 & 5.63 & -2.66 & 20.55 & 34.96 \\
\hline & $\%$ of Total & $\%$ & 0 & 9.8 & 17.8 & 5.1 & 2.3 & 8.3 & 10.1 & 9.2 & 2.0 & -1.4 & 16.7 & 26.5 \\
\hline \multirow{11}{*}{$\begin{array}{c}\text { Separation of } \\
\text { NGL } \\
\text { Condensates } \\
\text { Before } \\
\text { Absorber }\end{array}$} & Comp. P-Sav. & hp & 1220.49 & 1036.75 & 597.06 & 34.1547 & 590.57 & 541.72 & 482.92 & 62.69 & 218.76 & 238.65 & 151.32 & 133.59 \\
\hline & E-Cost Sav. & \$/hr & 51.26 & 43.54 & 25.08 & 1.43 & 24.80 & 22.75 & 20.28 & 2.63 & 9.19 & 10.02 & 6.36 & 5.61 \\
\hline & $\%$ of Total & $\%$ & 14.5 & 15.2 & 13.6 & 0.7 & 7.6 & 9.6 & 14.9 & 1.8 & 3.3 & 5.3 & 5.2 & 4.3 \\
\hline & $\begin{array}{c}\text { Abs.Power } \\
\text { av. }\end{array}$ & hp & -76.94 & -26.04 & $\mathbf{0}$ & $\mathbf{0}$ & -16.13 & 6.41 & $\mathbf{0}$ & $\mathbf{0}$ & $\mathbf{0}$ & $\mathbf{0}$ & $\mathbf{0}$ & $\mathbf{0}$ \\
\hline & E.-Cost Sav. & $\$ / \mathbf{h r}$ & -3.23 & -1.09 & $\mathbf{0}$ & $\mathbf{0}$ & -0.68 & 0.27 & $\mathbf{0}$ & $\mathbf{0}$ & $\mathbf{0}$ & $\mathbf{0}$ & $\mathbf{0}$ & $\mathbf{0}$ \\
\hline & $\%$ of Total & $\%$ & -0.9 & -0.4 & 0 & 0 & -0.2 & 0.1 & 0 & 0 & 0 & 0 & 0 & 0 \\
\hline & Evap. E. Sav. & $\underset{\text { hr }}{\text { MMBtu/ }}$ & 1.07 & 1.71 & 2.58 & 0.14 & -0.17 & 0.41 & 0.78 & -0.17 & -1.92 & -0.73 & -0.03 & -0.47 \\
\hline & E. Cost Sav. & $\$ / \mathbf{h r}$ & 6.37 & 10.26 & 15.49 & 0.84 & -1.03 & 2.48 & 4.66 & -1.03 & -11.50 & -4.37 & -0.21 & -2.80 \\
\hline & $\%$ of Total & $\%$ & 1.8 & 3.6 & 8.4 & 0.4 & -0.3 & 1.1 & 3.4 & -0.7 & -4.1 & -2.3 & -0.2 & -2.1 \\
\hline & $\begin{array}{l}\text { Sub-Total } \\
\text { Sav. }\end{array}$ & \$/hr & 54.39 & 52.71 & 40.56 & 2.27 & 23.10 & 25.50 & 24.94 & 1.60 & -2.31 & 5.65 & 6.15 & 2.81 \\
\hline & $\%$ of Total & $\%$ & 15.4 & 18.4 & 21.9 & 1.1 & 7.1 & 10.8 & 18.4 & 1.1 & -0.8 & 3.0 & 5.0 & 2.1 \\
\hline \multirow{2}{*}{$\begin{array}{l}\text { Total Energy } \\
\text { Savings }\end{array}$} & Total E. sav. & $\$ / \mathbf{h r}$ & 80.77 & 73.42 & 13.23 & 30.56 & 45.19 & 38.73 & 15.39 & 3.31 & 2.99 & 26.70 & 37.77 & 80.77 \\
\hline & $\%$ of Total & $\%$ & 28.2 & 39.7 & 6.2 & 9.3 & 19.1 & 28.5 & 10.4 & 1.2 & 1.6 & 21.7 & 28.6 & 28.2 \\
\hline
\end{tabular}




\section{Energy Cost}

\$ / Mgal NGL

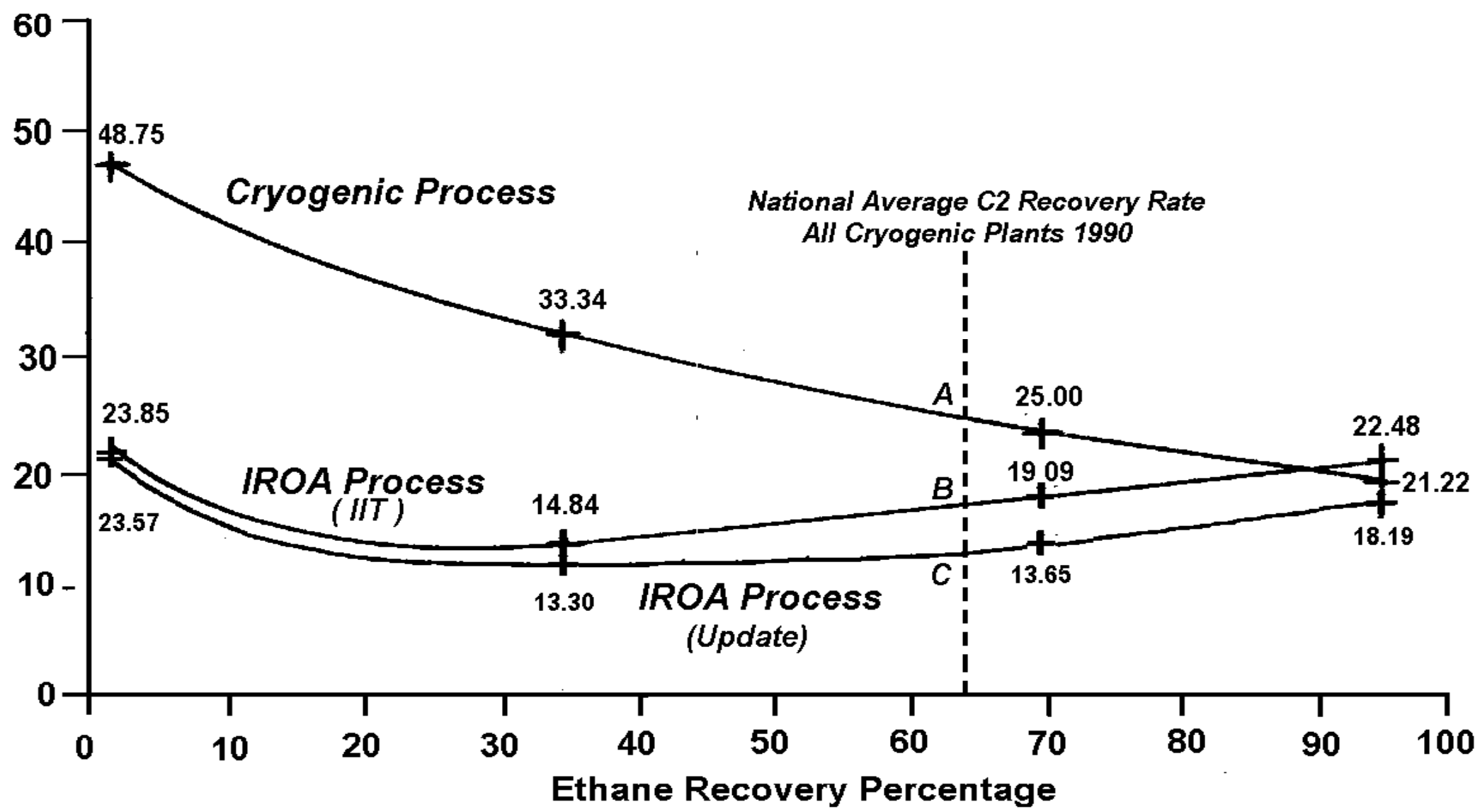

Figure 33 Energy Cost Savings for IROA Process for Rich Gas, 3.8 GPM NGL 


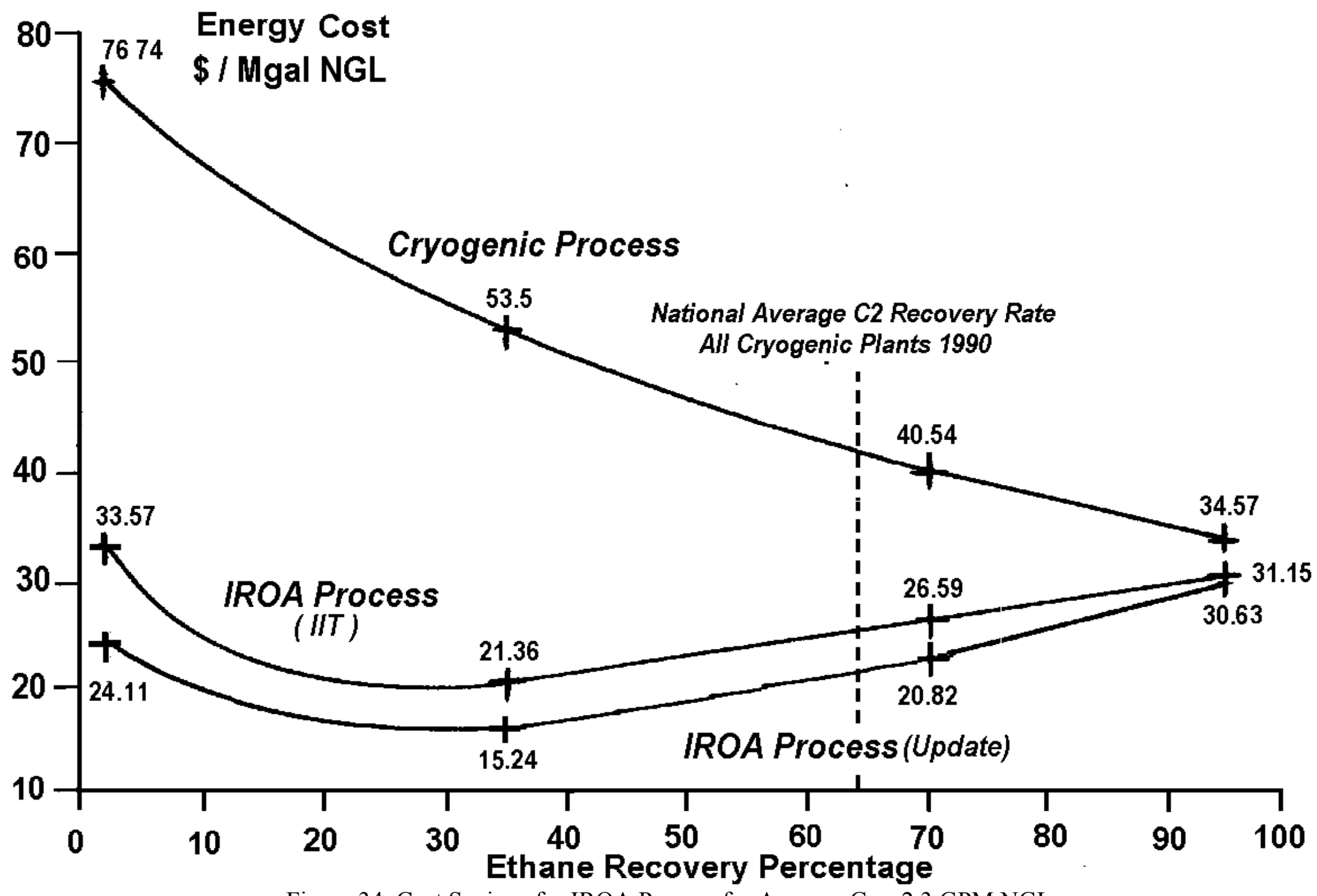

Figure 34 Cost Savings for IROA Process for Average Gas, 2.3 GPM NGL 
During 3QFY2006, the commercial feasibility of the technology was reviewed, based on the DOE CPAT web-based software program. Two cases were run based on a comparison with cryogenic systems and a retrofit comparison into existing lean oil facilities.

\begin{tabular}{|llrlr|}
\hline & IROA & & \multicolumn{2}{c|}{ Turbo-Expander } \\
\hline Project Capital (MM) & $\$$ & 10.30 & $\$$ & 10.30 \\
Ancillary Capital (MM) & $\$$ & 3.09 & $\$$ & 3.09 \\
IRR & $75.6 \%$ & & $64.6 \%$ & \\
Annual Operating Costs & & & & \\
Raw Materials (MM) & $\$$ & 265.15 & $\$$ & 265.15 \\
Utilities (MM) & $\$$ & 1.11 & $\$$ & 3.34 \\
Consumables (MM) & $\$$ & - & $\$$ & - \\
Labor (MM) & $\$$ & 0.31 & $\$$ & 0.31 \\
\hline
\end{tabular}

Figure 35 CPAT Input for Comparison 


\begin{tabular}{|c|c|c|c|c|c|}
\hline \multicolumn{6}{|c|}{ Project Summary Results for New Technology, Part I } \\
\hline \multicolumn{6}{|c|}{ Energy Impacts for Natural Gas Liquids } \\
\hline & 2010 & 2015 & 2020 & 2025 & 2030 \\
\hline 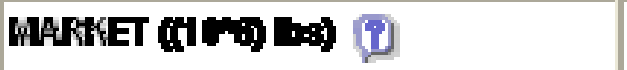 & $111+8.2$ & 115,0103 & 119,0903 & $123,317,3$ & $127,894.2$ \\
\hline MARAET PBETRATOW (D) & Drs & abs & $19.2 \%$ & 2005 & $290 \times 5$ \\
\hline ACCEERATE WTS DELOYEV (D) & 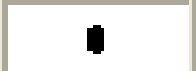 & 48 & 107 & 19 & 173 \\
\hline \multicolumn{6}{|l|}{ BERGY SAWASS (D) } \\
\hline 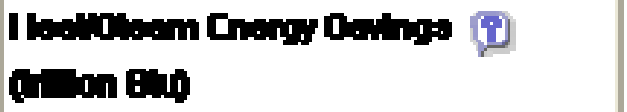 & ato & 1299 & 30.23 & $\mathbf{4 3 2}$ & 1907 \\
\hline 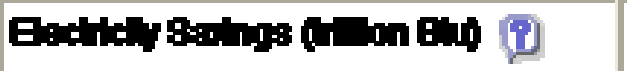 & Do & Don & Don & no & Do \\
\hline \multicolumn{6}{|l|}{ TACI BEROY SAVWBS (D) } \\
\hline 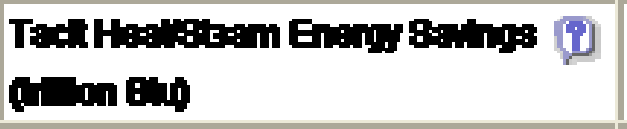 & Do & 1299 & 30.23 & $\mathbf{4 2 2}$ & 1907 \\
\hline 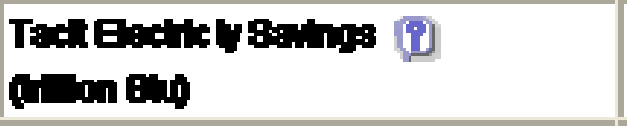 & 010 & $\mathbf{0 0}$ & $\mathbf{0 0}$ & Do & 000 \\
\hline 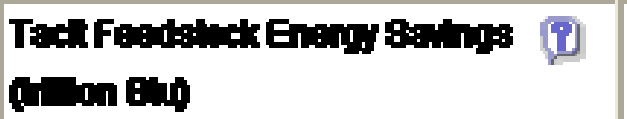 & مضD & Do & 00 & Don & 10 \\
\hline
\end{tabular}

Figure 36 CPAT Results/Energy Savings 


\begin{tabular}{|c|c|c|c|c|c|}
\hline \multicolumn{6}{|c|}{ Project Summary Results for New Technology, Part I } \\
\hline \multicolumn{6}{|c|}{ Energy Impacts for Natural Gas Liquids } \\
\hline & 2010 & 2016 & 2020 & 2026 & 2030 \\
\hline WARTE (10M) (D) & 28,75000 & 2,75000 & 26,7500 & 26,7500 & 28,7500 \\
\hline MARAGE PQETRATOW (D) & acs & $120 \times 6$ & $320 \%$ & $620 \%$ & $723 \%$ \\
\hline ACCER:ATE UTS DELOYEV (D) & - & 15 & 40 & ex & g] \\
\hline \multicolumn{6}{|l|}{ BERGYSAYABS (P) } \\
\hline 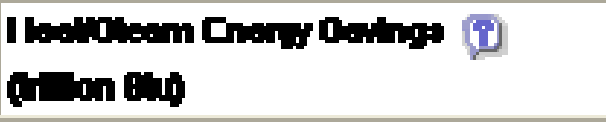 & on & 4.24 & 11.30 & $183 x$ & 2542 \\
\hline 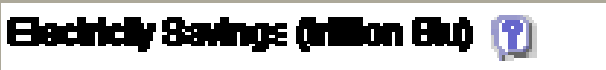 & an & 000 & 000 & 000 & avo \\
\hline \multicolumn{6}{|l|}{ TACI B BRGY SAVASS (D) } \\
\hline 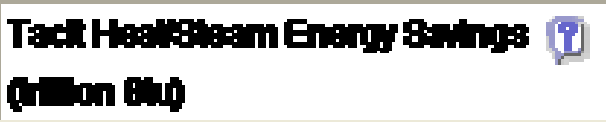 & 0.0 & 4.24 & 11.30 & $183 x$ & $\mathbf{2 5} 4 \mathbf{2}$ \\
\hline 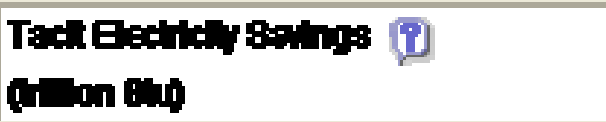 & ow & 000 & 000 & 000 & 000 \\
\hline 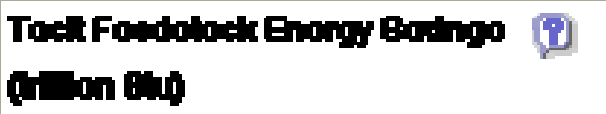 & a) & $\infty$ & 000 & 000 & on \\
\hline
\end{tabular}


Dr. Lu, GHT, prepared a series of market studies and commercial packages. The conclusions from several of the recent studies are included here.

\section{A. The Flexible IROA System Diagrams for Simulation in the Commercialization Package}

The Application of the Generic FIROA Systems for Simulation

Since the two generic systems selected in current simulation work represent all the meritorious features of the FIOA process, particularly the flexibility and energy savings, they could be applied to a wide variety of potential customer demands.

For new plant designs, these systems offer the bases of plants processing different raw gases over a wide range of NGL richness and gas quality, particularly for low quality gases. In current simulation, a rich gas will be used as example, but the same model is well applicable to other kind of raw gas as already shown in previous IIT simulation.

For retrofitting of existing plants, it is particularly interesting to note that it is possible to apply any individual section or a couple of selected sections to enhance the productivity, improve the efficiency or reduce energy consumption of said plants. For example, it is possible to replace only the traditional dual-absorbent absorption column (with sponge oil stage) in existing plants with the FIROA single-absorbent column to increase $\mathrm{C}_{2}$ recovery rate from $\sim 60 \%$ to $\sim 90 \%$, or adjustable between 35\%-95\%. Should an integrated demethanizer-deethanizer be used in retrofitting the existing plant, the residue gas re-compressor could be entirely eliminated or substantially reduced in size to lower the operation costs.

The flexibility of application of IROA process could greatly enhance its commercialization capabilities in a fluctuating gas market.

\section{B. Preliminary Study on the Performance of the Plate-Fin Exchanger-Reactor for ROD of IROA Process IROA Com-Ref-001}

Concluding Remarks and Further Work

Based on the above preliminary estimation of PF evaporators and the comparison with traditional tower re-boiler combinations, we can state that:

1) The PF evaporator is compact and efficient;

2) The PF evaporator is much smaller and lighter than the traditional tower-re-boiler combination, and is quite suitable for skid-mounting and transportation;

3) The application of the PF technology to IROA plants (including RODs, ROS, and other exchangers) would make the future IROA plants, both new and retrofits, extreme competitive and attractive.

4) The cost of a PF evaporator is expected to be much lower than that estimated for a conventional ROD-1 column-re-boiler combination. The IIT simulation estimated that the combination equipment would comprise about $20 \%$ of the total equipment cost of IROA plant.

\section{Preliminary Study on the Performance of the Plate-Fin Type Cascade ROS and COD of IROA Process}


Conclusion Remarks and Further Work

Based on the above preliminary performance evaluation of PF CROS, we observe that:

1) The PF CROS could greatly reduce the NGL vapor compression power requirement, and thus result in significant capital and operational cost savings.

2) The PF CROS is compact and efficient;

3) The PF CROS is much smaller and lighter than the traditional tower-re-boiler combination, and is quite suitable for skid-mounting and transportation;

4) The application of the PF technology to IROA plants (including RODs, ROS, and other exchangers) would make future commercial IROA plants extremely competitive, including both building new plants and the retrofitting of existing plants.

5) By using hybrid dual-sectional units, the PF CROS cost is likely to be lower than the estimated cost of traditional equipment as given in the IIT simulation.

\section{Major FIROA Plate-Fin Heat-Exchanger Reactor and Input/Output Data}

\section{Conclusion Comments}

The three major PFHER to be used in future FIROA plants are proposed according to a few minor modifications current industrial practice. No novel technology has been involved. The advantages of the applications of PFHER have been already discussed in previous studies. Additional information would be provided by GHT on requests.

\section{E. Commercialization Package that Expands the Advantages of the FIROA Process.}

\section{Further Improvements to be Verified in Next Simulation}

The merits of the FIROA Basic System has been successfully illustrated with the most critical SU $95 \% \mathrm{C}_{2}$ recovery case. Any other type of gas could be simulated with the same simulation package when needs arise. No further optimization computation would be performed with the Basic system because most of the inefficient thermodynamic processes identified in the Basic System have already been improved in the Advanced System. To carry out the simulation of the Advanced System would lead to eliminate these inefficiencies and obtain the best optimum results of the FIROA System.

The key to the more advanced system is the replacement of the ROD, ROS and COD with relevant "Cascade-columns" to further increase the mass- and heat-transfer efficiency of the PFHERs. The computer simulation results of these individual cascade columns, based on inputs data from previous IIT data, have been reported in the FIROA patent application document. These data have been updated with recent GHT simulation date and more encouraging results obtained. The most striking advantages are: (1) a higher fraction of NGL condensate would be recovered in COD as liquid product; (2) a much lower stripping lean oil required in ROD-UP that would save the subsequent RO processing heat consumption; (3) much less low pressure $(\sim$ 450 psia) residue gas from ROD that would save residue gas re-compression power; and (4) much less low pressure ( 100 psia) NGL gas that would further reduce the NGL compressor power consumption. 
The revised Advanced System is in fact more concise as compared with the Basic System because the cascade equipment combined the inefficient piece with the more efficient piece into a single most efficient integrated piece.

It is noted that the cascade COD in Advance System is the integration of the three items \#35, \#35a and \#30 in Basic System, cascade ROD in Advance System is the integration of the three items \#45, \#55a and \#55b in Basic System, and cascade ROS in Advance System is the integration of the three items \#66a, \#66b and \#67 in Basic System.

It is noted that that the advanced system looks simpler than the basic system as a whole, but the interior multi-stream flow channel configuration of each piece of major equipment is complicated. Fortunately the state-of-the-art PFHER manufacturers have already made such technology available to many industries, including the gas industry, e.g. the "Cold Box" in many CEP plants.

\section{F. Proposal on the Strategy of the Final Phase of Current FIROA Project For Accelerating Market Entry under Volatile Economic Environments}

\section{The Core Technology of FIROA Process and the Proposal on Early Involvement of a PFHER Supplier in FIROA Commercialization Efforts}

The core technology of FIROA Process is the extensive and innovative use of various types of highly efficient PF exchanger technologies, i.e., the plate-fin heat exchangers (PFHX), plate-fin exchanger-reactors (PFHR), and the more complicated PFHR for "cascade-columns". Without these PF exchanger technologies, the FIROA process could not be able to gain all its attractive features.

Although the PF exchanger technology has been widely adopted in various modern industrial sectors, including, the petroleum-gas industry, aero-space industry, marine industry, energy industry, food industry, etc., it always has to be customer-designed and could not have a universal, standardized data bank providing required PF exchanger design bases covering all kinds of PF applications. As a result, should FIROA process be commercialized, even a complete HYSYS simulation could not be work out without the participation of a PF exchanger supplier providing these customer-design data inputs to said simulation software.

As a result, GHT has made a proposal on the early involvement of a competent PFHER supplier in FIROA commercialization efforts for GTI reference. The reasons follow.

1. From technical view point, to include one small test PFHER (preferably the ROD or COD) unit in our pilot unit will demonstrate the feasibility and economy of the PFHER and shorten the market entrance time.

2. From economic view point, to invite one interested supplier to join the project could provide in-kind resources and services to accelerate our pilot unit task at lower cost because the supplier will supply the PFHER at its own cost.

3. Under current global economy depression environment, the necessity of the early involvement of a PFHER supplier could not only reduce the pressure of the limitation of remaining budget and time for our task on pilot unit construction and tests, but also help the follow-up commercialization progress. 
4. To identify a competent partner, please check Chart E\&C experience and credit with ECCAN and KER and compare it with other similar suppliers familiar to GTI to select the most appropriate partner. If no other better competitor identified, the Chart E\&C could be one qualified candidate.

\section{G. Comparison of the HYSYS Simulation of the ISRP and the Traditional SRP}

\section{Conclusion Comments}

The preliminary comparison of the HYSYS simulation of ISRP and traditional SRP illustrates the striking energy savings of the ISRP over the traditional one over a broad range of refrigeration temperatures before the condensate separator. The major contribution of such energy savings comes from the much less residue gas recompressing power requirements as expected. However, at the lowest end of the refrigeration temperatures (e.g. $-83{ }^{\circ} \mathrm{F}$ ), the advantage sharply decrease. The ISRP, therefore, could not be used as an alternative highly efficient recovery process to FIROA process for $\mathrm{C}_{3}+$ NGL products.

\section{H. HYSYS Simulation of the Advanced FIROA Process (GHT-Conf-116).}

Further Work for the Complete HYSYS Simulation Package of FIROA Process

1. Beside the Data of the various components of the system model, a complete FIROA HYSYS simulation also requires the Equipment Design Data, the Equipment Cost Data, and the Annualized Cost Data for further engineering design and the comparison with the CEP and IROA (IIT) simulation results. Since the key equipment of current FIROA process comprise of customized PFHER that require equipment data provided solely by qualified supplier, it is impossible to complete the simulation as usual without their inputs. It is, therefore, to immediate identify adequate PFHER supplier to accelerate the completion of the FIROA simulation work and facilitate the preparation of commercialization documents. One of these potential qualified suppliers, the Chart Energy \& Chemical, has been contacted recently and they showed interests and to try their own specific PFHER software to verify the FIROA simulation results and help GHT proceed the subsequent simulation task elements, in their modified HYSYS system. They have been requested to contact GTI project leader directly to talking for such cooperation opportunity.

2. To illustrate the advantages of the advanced FIROA process, the most unfavorable case, i.e. the Super-Rich Oil at $95 \% \mathrm{C}_{2}$ recovery, was selected for this trial run to compare with $\mathrm{CEP}$ and the original IROA process. For inlet gas of other richness and $\mathrm{C}_{2}$ recovery rates, similar simulation model could easily be carried for the future commercialization package. For commercialization purposes, at least one practically case of an existing gas field should be simulated to provide an example of the applicable of the advanced FIROA process. The best way is to ask ENCAN select one of its existing gas plants and provide a set of real operation data (including input gas and output data, operation parameters and energy, etc.) to GHT for the next HYSYS simulation.

3. GHT would also start the simulation study on the potential benefits of retrofitting existing Straight Refrigeration (SRP) and CEP with the cascade PFHER technique used in the 
advanced FIROA process. The study would explore the cascade PFHER market beyond the traditional ROA plants.

\section{HYSYS Simulation of Retrofitting the Straight Refrigeration Process for NGL Separation}

\section{Scope of HYSYS Simulation for ISRP}

Since the study on retrofitting the traditional SRP is an effort to explore a new area of FIROA and PFHER applications, a relatively broader scope of search has been planned. Altogether 12 cases, including 3 different richness of raw gases (Super-rich, Rich and Lean) and 4 different refrigeration temperatures $\left(-55,-65,-75\right.$, and $\left.-85^{\circ} \mathrm{F}\right)$ were planned to be simulated. For the rich and super-rich gas, some of the low temperature range are not applicable, because of the liquefaction of the entire raw gas which would not be acceptable. To provide such low temperature refrigeration, a two-stage refrigerator with $\mathrm{C}_{2} / \mathrm{C}_{3}$ mixture refrigerant will be used. The HYSYS simulation of the refrigeration process with mixed refrigerant will be carried out separately to simplify the calculation.

The preliminary results of the ISRP simulation showed that the recovery rates of NGL components are remarkable at each temperature. Should $\mathrm{C}_{2}$ product is not desirable, as the market price dropping, the bottom temperature of the COD2 could be adjusted to obtain the most favorable results.

As mentioned above, the current simulation could not be fully completed. A complete ISRP HYSYS simulation requires the Equipment Design Data, the Equipment Cost Data, and the Annualized Cost Data for further engineering design and the comparison with the CEP and IROA (IIT) simulation results. Since the key equipment of the ISRP comprises of customized PFHER that require equipment data provided solely by qualified supplier, it is impossible to complete the simulation as usual without their inputs. It is, therefore, necessary to immediate identify adequate PFHER supplier to accelerate the completion of the on-going simulation work and facilitate the preparation of commercialization documents. One of these potential qualified suppliers, the Chart Energy \& Chemical, has been contacted recently and they showed interests to try their own specific PFHER software to verify the future ISRP simulation results to facilitate GTI commercialization efforts. 


\section{Task 8: $\quad$ Attend Technical Meetings and Prepare Technical Reports}

The project kick-off meeting was held at GTI's Des Plaines office on Wednesday, January 28, 2004 from 9.00 AM to 5.00 PM. The meeting was attended by representatives from DOE Golden Office, GTI, ORNL, GHT and IIT. During the meeting project tasks and milestones were discussed and the results of the initial work on the literature review and process simulation performed by GTI, IIT and GHT were reviewed. Delegates attending the meeting were also given a tour of GTI's lab facility that will be used to perform experimental work related to this project. Electronic copies of the technical presentations were submitted to DOE project manager.

GTI project manger attended a 3 day course on NGL recovery and NGL marketing organized by Oil Price Information Service (OPIS) held on February 17-19, 2004 at Houston, TX and visited an NGL fractionation plant as a part of the course. He also attended the annual meeting of the Gas Processors Association (GPA) held on March 14-17, 2004 at New Orleans, LA and also visited three gas processing plants in the area and collected data which are being used for computer simulation under task 4.

A comprehensive PI questionnaire and CPAT energy saving results were submitted to DOE Golden office for FY05 ITP Chemicals and Subprogram Portfolio Review. The GTI project manager attended the Annual Gas Processors Association (GPA) convention (March 13-17, 2005, San Antonio, Texas) and collected latest information about various technical issues related to NGL recovery and the status of NGL industry in the U.S. At the meeting, he visited two NGL recovery plants located near San Antonio.

Please also see Publications/Presentations below.

\section{- Patents:}

Based on the latest computer simulation results for the new process, a new patent application was filed by GHT on Dec. 20, 2004. On January 19, 2005, the GHT re-issue application of US Patent $\# 6,553,784$ was filed at USPTO to add 6 process-related claims in the original patent. The reissue was believed to be vital for the commercialization of said patent, and, hence, important for current project.

The Reissued Patent RE39,826 E was published by United States Patent Office (USPTO) on September 11, 2007. The Reissued Patent is an amended version of US Patent No. 6,553,784, with additional process claims. An electronic copy of the reissued patent is attached to this report.

In view of the participation of ENCAN in present project and the vast potential of IROA application in the Canadian gas processing market, GHT recently submitted an application of the US patent pending "A Flexible Hydrocarbon Gas Separation Process and Apparatus" (US Appl. No. 11/713,757) to the Canadian Intellectual Property Office (CIPO) on September 16, 2007. The contents of this patent application package are identical to that submitted previously to the USPTO. 
The US Patent Office sent a notice on the publication of a new patent application for IROA technology dated January 24, 2008. The Pub. No. is US 2008/0016909 A1, entitled "Flexible Hydrocarbon Gas Separation Process and Apparatus" and is attached.

\section{- Publications/Presentations:}

A poster presentation was made on the DOE's FY05 ITP Chemicals and Subprogram Portfolio Review Meeting. Phase-1 results and a technical paper were presented at AIChE Spring National Meeting (April 10-14, 2005, Atlanta, GA). The paper was entitled, "Techno-Economic Evaluation of an Improved and Energy Efficient Natural Gas Liquid (NGL) Removal Process." and the presentation is attached.

Presentations were made at the Spring 2007 American Institute of Chemical Engineering meeting and DOE peer review meeting held on April 25, 2007. The extended abstract and presentation are attached.

A phone review meeting was held on September 21, 2007 between DOE-EERE/ITP and GTI personnel. The scope of the meeting was to cover ten on-going programs, including this project. This will be an on-going communications tool. Status reports, including commercialization plans, were updated periodically throughout the program with the support of Pacific Northwest Laboratory personnel.

H. Meyer and D. Leppin attended the DOE-ITP Peer Review Meeting Presentation on October 29, 2008 in Washington DC on how the activities ITP supports contribute to their overall mission to reduce U.S. industrial energy intensity and enhance competitiveness. The presentation is attached.

Bill Prymak, DOE-Golden Project Manager, visited GTI on December 4, 2008 to see the benchscale unit and be updated on the project.

\section{- $\quad$ Extending Applications:}

A request for a commercial design package was received during the 1QFT2005 from an engineering contractor for a major North American gas processor. The application was for recovery of NGL from a refinery gas. They became aware of the new technology based on the paper presented at the 2005 AIChE meeting in Atlanta and requested the package to be developed with a focus on propylene recovery from a refinery off-gas stream. Approximately $30 \%$ of world propylene production is currently contained in refinery by-product streams. Four cases were developed with propylene recoveries from 70 to $99.5 \%$. The application introduces the gas stream into the process at a relatively low pressure, approximately $100 \mathrm{psig}$. Solvent pumps were the major energy consumers in the design. The most commonly technologies used for this application are based on compressing the feed to increase the recovery, including turboexpander and proposed membranes. No compression of the feed or product gas was required in our design, with a potential energy savings. Also, since the degree of dehydration and its accompanying regeneration is not as extreme as required for a cryogenic process, additional energy savings should be achievable. We were unable to obtain the general findings of the 
comparison study to determine if this is a significant energy saving benefit that should be captured in CPAT.

A proposal was submitted to NineSigma, Inc., entitled; " $\mathrm{C}_{4}-\mathrm{C}_{7}$ Hydrocarbon Separation from Atmospheric Gases Using IROA". They were seeking a refinery gas cleanup technology for an unnamed Fortune 500 company. IROA process has the capability to dehydrate low pressure, water saturated gases that tend to be too wet for other technologies. We received notice that the proposal was rejected.

\section{- Commercial Contact:}

GTI participated in a phone conversation with John Balsam, NHT, about a commercialization readiness assessment they are performing on this technology for DOE's Industrial Technologies Program. After the phone interview, we received and responded to questions and comments on the Commercialization Readiness Assessment Framework survey NHT prepared.

GTI received an inquiry from Nova Chemicals concerning the applicability and status of this program. As a large chemical company, they are investigating non-traditional NGL recovery processes to help them plan for their feedstock supply costs. Information was shared and a contact established. 
Pilot Plant for IROA Recovery of NGL - GTI Project 15035.1.05/DOE Project 15347.1.01 Process Design of Facilities for Field Testing

Jim Aderhold

23-May-2006

Rev. 1 10-July-2006

\section{Summary}

Natural gas liquids (NGL) are removed at natural-gas processing plants for two purposes: to prevent condensation in downstream transportation and to enhance the value of these $\mathrm{C}_{2}$ to $\mathrm{C}_{5}{ }^{+}$ hydrocarbons as refining or chemical feedstocks.

The Gas Technology Institute (GTI) is the primary contractor on a project to develop an improved process for NGL recovery, which is referred to as the Improved Refrigerated Oil Absorption (IROA) process. One phase of this project is to design and construct a pilot plant for the IROA process. The objectives of this pilot plant are (1) to verify the IROA process with a slip stream of actual (sweetened) natural gas, at conditions in the vicinity of optimal process conditions (as determined from the bench-scale IROA studies), through attended operations of up to several shifts in duration, and (2) to provide a platform for testing of IROA equipment concepts.

A Design Basis is presented for this nominal 1 MMscfd pilot plant. The primary research questions for development of the IROA process are in the Dehydration Section. More specifically, the equipment which require verification are the non-isothermal / direct-cooling approach in the dehydration column and the second-stage dehydration / cooler, with ethylene glycol (and/or calcium chloride solution) spray, to prevent hydrate formation. Therefore, detailed design should initially focus on these areas. The extent of the Lean Oil Absorption Section will depend on the funds remaining in the project budget. NGL will be recovered at several points in the pilot plant; flow rates and composition will be determined for mathematical calculation of recoveries. Utilities will be limited to those typically available in commercial gas plants, which are usually found in remote locations.

A Process Flow Diagram (PFD), Material Balance, Piping and Instrumentation Diagrams (P\&ID's), and Process Description are provided for the IROA pilot-plant system. This pilot plant will be located at a commercial gas processing facility, and its objectives will be (1) to meter a slip-stream of sweetened natural gas at high pressure and at a controlled flow rate, (2) to dehydrate the natural gas to less than 2 ppmv of water content, and (3) to absorb $\mathrm{C}_{2}+$ hydrocarbon in a distillate boiling-range petroleum-derived solvent to yield a hydrocarbon dewpoint in the range of $-75^{\circ} \mathrm{F}$. 


\section{Introduction / Background Information}

"Natural Gas Liquids" (NGL) is a collective term for the mixtures of ethane $\left(\mathrm{C}_{2}\right)$, propane $\left(\mathrm{C}_{3}\right)$, butane $\left(\mathrm{C}_{4}\right)$, and natural gasoline $\left(\mathrm{C}_{5}{ }^{+}\right)$which is extracted from natural gas (Abbasian et al, 2004) [1]. The separation or extraction of NGL from a natural-gas stream is done primarily for two reasons: (1) to prevent condensation of higher hydrocarbons during transmission of natural gas through pipelines and / or (2) to enhance the ultimate value of the raw natural gas stream containing mainly methane. If the heavier hydrocarbon liquids are left in the gas, they are valued at the gas sales price along with the lighter gaseous components.

Separated NGL is primarily used as chemical feed stocks, in oil refining and petrochemical industries, with some NGL converted to blending components for gasoline. The economic values of separated NGL components, when sold as chemical feedstocks, are typically much higher than the heating value when left in a natural gas although the differential changes with the various markets. The major industrial uses of NGL as chemical feedstock include:

- Ethane, propane, normal butane and natural gasoline, used as feedstock for production of ethylene

- Iso-butane, used as feedstock for the production of propylene oxide and methyl tertiary butyl ether (MTBE)

- N-butane, used as feedstock for the production of acetic acid and maleic anhydride

- Refinery-produced propylene and butylenes, consumed by chemical companies.

The average worldwide NGL production in 2002 was 6.8 million barrels per day. The United States is the largest NGL producer, with a capacity of 1.9 million barrels per day from about six hundred gas processing plants. The domestic natural gas production is projected to increase from 19.5 to 25.1 trillion cubic feet between 2001 and 2020 -- an average rate of increase of $1.3 \%$ per year. This will require about twenty new gas-processing plants of 500 million standard cubic feet per day to be built in the US by year 2020 .

As summarized by (Lee et al, 1999) [2], in the 1950's and 1960's, a simple oil-absorption process was commonly utilized to recover propane in the twenty-five to fifty percent ranges. The process used light oil to absorb NGL components from the feed-gas stream, in an absorption column, typically operated at the ambient temperature and at a pressure close to the sales-gas pipeline pressure. In the late-1980's, a proprietary, selective solvent process was introduced, based on the refrigerated lean-oil-absorption process. A lighter oil, which is used as the physical solvent, is selectively tailored for recovery of desirable hydrocarbons from a gas stream (Mehra process).

Beginning around 1960, as the demand for ethane increased, new processes were sought to recover ethane most efficiently. For ethane recover up to fifty percent and propane recovery up to ninety percent, a simple propane refrigeration system provides refrigeration at temperatures to $-40^{\circ} \mathrm{F}$. (For higher recovery of ethane, a cascade refrigeration cycle would be required - for example, for eighty percent ethane recovery, refrigeration to approximately $-90^{\circ} \mathrm{F}$ would be required.) 
The use of turbo-expanders in cryogenic expansion gas-processing plants began in the early 1960 's, as a result of technological improvements in the manufacture of practical mechanical designs, which were suitable continuous operations in a variety of operating conditions. By 1970, most new gas processing plants were being designed to incorporate the particular advantages of an expander, which produced useful work, normally used partially to re-compress residue or export gas. Coincident with the development of development of reliable turbo-expanders was the development of compact, efficient, and relatively-inexpensive plate-fin or brazed aluminum heat exchangers.

In recent years, the oil and gas prices kept rising and the profit margin of ethane recovery dropped substantially. The cryogenic expansion plants have to decrease their ethane production with more energy-intensive and expensive ethane-rejection operations. [4] The interests revived on the improvements over the traditional refrigerated oil absorption process that could operate with flexible ethane recovery rate to better meet the fluctuating NGL market. [5]

An improved NGL recovery process has been developed by Yingzhong Lu, as described in US $6,553,784$ B2 (2003) that provides an answer to such a challenge. [3] This improved process will be referred to as the Improved Refrigerated Oil Absorption (IROA) process, or as the Comprehensive Natural Gas Processor (NGP). A project has been initiated under DOE sponsorship (DE-FC36-03GO13150), with the Gas Technology Institute (GTI) as the primary contractor, to develop the information required for quantification of the energy savings with the improved process and for commercialization of this technology.

In the first phases of this project, a computer-simulation-based study was completed by Abbasian et al (2004) at Illinois Institute of Technology (IIT) to compare the new NGP / IROA recovery process for natural-gas liquids with the latest design for Cryogenic Expansion Processes (CEP) (as described in US patent 5,983,664). This HYSYS study estimated both capital equipment costs and utility costs, at various operating conditions for four different feed natural gas compositions $\left[\mathrm{C}_{2}^{+}\right.$: Super Rich (18\%), Rich (13\%), Lean (8\%), and Super Lean (3.7\%)] and four levels of $\mathrm{C}_{2}$ recovery $(2 \%, 35 \%, 70 \%$, and $95 \%)$. The IIT simulation results confirmed that the IROA process, unlike the traditional Refrigerated Oil Absorption process with low ethane recovery, could also recover up to $95 \%$ of ethane similar to the Cryogenic Expansion Process. In addition, the IROA has the flexibility to reduce ethane recovery from $95 \%$ to $2 \%$ during operation with reduced energy consumption. Due to the limitation of time and resources, the process conditions and the flow diagram used in NGP/IROA simulation were not optimized by IIT.

Table 1. Natural Gas Composition for HYSYS Studies

\begin{tabular}{|l|l|l|l|l||}
\hline \multirow{2}{*}{$\begin{array}{l}\text { Natural Gas } \\
\text { Component }\end{array}$} & \multicolumn{4}{|l||}{ Natural Gas Composition ( Mol \%) } \\
\cline { 2 - 5 } & Super Rich & Rich & Lean & Super Lean \\
\hline Methane & 81.9 & 87.0 & 92.0 & 96.3 \\
\hline Ethane & 9.7 & 7.9 & 4.8 & 2.2 \\
\hline Propane & 4.5 & 3.3 & 1.8 & 0.8 \\
\hline Butane+ & 3.8 & 1.8 & 1.5 & 0.7 \\
\hline Total NGL & $\mathbf{1 8 . 0}$ & $\mathbf{1 3 . 0}$ & $\mathbf{8 . 0}$ & 3.7 \\
\hline
\end{tabular}


Equipment sizing and costing was also performed for a selected number of conditions specified by GTI using ASPEN Icarus 12.0. These cases included three gas compositions (Super Rich, Rich, and Lean) at 95\% recovery for CEP and $95 \%$ as well as 35\% recoveries for NGP. Based on IIT simulation results, the annualized overall costs of the processes were determined using different interest rate ranging from $5 \%$ to $20 \%$. Based on the annualized overall costs, CEP appears to be more economical than NGP for higher NGL recoveries (e.g. @95\%). However, since the utility costs associated with NGP decreases with decreasing recovery of NGL, the NGP can be more economical than CEP at lower recovery levels.

At the beginning of the second phase of this project, studies of the IIT simulation results indicated that the updated IROA simulation results would give much better economic performance than previously evaluated. [6] Under improved conditions, it was shown that even for high NGL recoveries up to $95 \%$, IROA process would be more economical as compared with the optimized Cryogenic Expander Process. 


\section{Pilot Plant Design Basis -- General Comments}

The objectives of the IROA Pilot-Plant are to verify:

(1) The performance of a direct-contact type Dehydrator, operating with two different kind of hydrate inhibitors - ethylene glycol and calcium chloride; and

(2) The flexibility of variable $\mathrm{C}_{2}$ recovery rates from $95 \%$ to $2 \%$ in the Absorber using a commercial absorbent operating at equilibrium composition,

- with a slip stream of actual (sweetened) natural gas

- in the vicinity of optimal process conditions (as determined from the bench-scale IROA studies) for different $\mathrm{C}_{2}$ recovery rates

- through attended operations, up to several shifts in duration

- to provide a platform for testing of IROA equipment concepts

In the initial planning for this project, it was projected that the capacity of this pilot plant would be between one and five million standard cubic feet per day (1-5 MMscfd). However, the process design efforts have indicated that, in order to keep columns at reasonable diameters and costs within budget, it will be necessary to maintain pilot-plant capacity at the low end of the range. Therefore, the design basis has the inlet gas rate to the dehydration section at 1 MMscfd.

The studies with the IROA pilot plant will focus on the unique equipment / features of this process: determining concept viability and quantifying the product yields and qualities at conditions close to those projected for commercial operations. For development of the IROA process, the primary research questions are in the Dehydration Section, specifically, the (1) nonisothermal / direct-cooling approach in the dehydration column and (2) and the second-stage dehydration / cooler, with ethylene glycol (and/or calcium chloride solution) spray, to prevent hydrate formation. Therefore, if necessary to maintain costs within the project budget, the flow rate to the Lean-Oil Absorption section of the pilot-plant might be reduced by bypassing some of the dehydrated natural gas.

For several reasons, heat integration will not be implemented into the IROA pilot plant design. First, the bench-scale studies have not been accomplished yet, so the precise process conditions for each piece of equipment have not been determined. Second, as discussed below, all the equipment on a full IROA process will not be included in the pilot plant and the flow rate through the Lean Oil section of the pilot plant may be decreased during the detailed design of the pilot plant. Third, tight heat integration will make the pilot plant difficult to operate.

The IROA pilot plant will not include all the columns needed for fractionation of the NGL liquids recovered into saleable product streams, such as ethane, propane / LPG (liquefied petroleum gas), butanes, and natural gasoline. Rather, the flow rates and the compositions for the several NGL streams on the pilot plant will be measured, and these streams will be blended mathematically to develop on overall NGL recovery stream and the measures of individual component recoveries. There may be some feed / product heat exchangers for the solvent regeneration systems, in order to minimize the overall refrigeration requirements.

Since the IROA pilot plant is intended for operation at a commercial gas plant, all the pilot-plant equipment and instrumentation must be rated for a classified, hazardous environment (Class I, 
Division 2). Furthermore, the host gas plant is most likely to be in a remote location, so limited utilities will likely be available. Therefore, the utilities for the IROA pilot plant design should be chosen as follows:

Utilities likely available

- $\quad$ Electricity:

480 VAC (3-phase)

120 VAC (1-phase) by IROA step-down transformer

- $\quad$ Flare, for relief systems

- Refrigeration (self-contained, as section of IROA unit)

Utilities likely not available (not used on IROA design)

- Steam

- Cooling water

The product streams from the IROA pilot plant will be measured for flow rate, composition, temperature and pressure, and bomb samples will be obtained for detailed characterization subsequently. The product streams from the IROA pilot plant will then be returned back to the host plant: the processed natural gas on a continuous basis and the NGL streams dumped back on a regular basis, according to levels in the several accumulators. The return streams routing will need to be determined in design. 


\section{Process Description : IROA}

There are three primary sections to the proposed IROA pilot-plant system: (1) dehydration, (2) absorption, and (3) refrigeration. Therefore, there are three Piping and Instrumentation Diagrams (P\&ID's) for the IROA NGL pilot plant, as given in the figures at the end of each section below.

\section{Dehydration Section:}

The P\&ID for the Dehydration Section is given in the first figure below, which is labeled as Drawing No. NGL-PP-01-A (Revision D), dated 16-May-2006.

The initial solvent for the IROA Dehydration Section will be a blend of ethylene glycol (EG) in water. In order to flow at the lowest temperatures planned for the IROA pilot-plant experiments (that is, between -40 and $-55^{\circ} \mathrm{F}$ ), the concentration of ethylene glycol in the dehydration solvent will have to be between sixty and eighty weight percent, within the non-crystallization range. Therefore, the nominal concentration of the dehydration solvent is assumed to be seventy percent (weight) or forty-one percent (molar) ethylene glycol. Later tests will be conducted with calcium chloride as the desiccant. The corresponding nominal concentration of calcium chloride solution is thirty percent (weight) or 4.9 percent (molar) $\mathrm{CaCl}_{2}$.

Sweetened natural gas is first directed into the T-101, Inlet-Gas Separator, to remove any entrained liquids that may accompany the natural gas from upstream processing. For steady-state operation of the IROA NGL pilot plant, the level of liquid entrainment in the feed gas is expected to be relatively low or negligible. This feed-gas separator is provided mainly for startup and to protect for operating upsets. Therefore, it will be provided with only a sight glass and level alarm, and it will be drained manually -- probably into the gas plant's liquid slop system. Feed gas flow rate will be measured by a flow element, and controlled though a flow-control valve and automated flow-control loop. The natural gas feed to the IROA NGL pilot plant will need to be pre-processed to less than 4 ppmv hydrogen sulfide content, and this natural gas is expected to be fully saturated with moisture, at a pressure between 900 and 1100 psig -- that is, between 1000 and 1500 ppmv of water, or 50 to $75 \mathrm{lbs}-\mathrm{H}_{2} \mathrm{O}$ per MMscf.

The feed gas, at ambient temperatures of up to $100^{\circ} \mathrm{F}$, will then be routed directly to the first stage of dehydration -- it is fed into the bottom of the C-111 column, which will be loaded with either random or structured packing. Recycled, cooled dehydration solvent will be fed into the top of the $\mathrm{C}-111$ at about $-40^{\circ} \mathrm{F}$ and will flow counter-current to the wet natural gas flow. Therefore, the first-stage of dehydration will be highly non-isothermal. The up-flowing natural gas will be cooled to between 0 and $-20^{\circ} \mathrm{F}$, and the down-flowing solvent will be heated to between 70 and $95^{\circ} \mathrm{F}$. It will be necessary to maintain the gas outlet temperature high enough, so that condensation of $\mathrm{C}_{3}{ }^{+}$hydrocarbons does not occur in the first-stage of dehydration. For example, for a mid-range of $\mathrm{C}_{2}{ }^{+}$concentration in the feed natural gas, the gas outlet temperature will between -10 and $-15^{\circ} \mathrm{F}$. The gas-outlet temperature can be adjusted by varying the rate and / or the temperature of the solvent fed to the C-111 column.

The rich solvent from the bottom of the $\mathrm{C}-111$ column will be routed though a level-control valve to the T-271 separator, which will be designed (1) to skim off any hydrocarbon liquids 
from process upsets and (2) to provide some hold-up volume for the dehydration solvent in the pilot plant. The T-271 separator should be sized large enough to hold the entire inventory of dehydration solvent, when an experimental run is completed on the IROA pilot plant.

Since the pick-up of water in the dehydration solvent is relatively low, compared to the solvent circulation rate, most of the recovered solvent will be cooled and re-circulated to the C-111 column. Only a slip stream will be withdrawn from the circulating dehydration solvent for regeneration (water removal), as will be covered in more detail below.

The cooling of the rich solvent is carried out in two steps. The solvent is cooled first in E-26X with the cold Product Natural Gas left the top of Lean Oil Pre-saturator C-311, and then in E-261 with liquid propane refrigerant to $-40^{\circ} \mathrm{F}$. The exit temperature of Product Natural Gas left E-26X should be as high as possible (up to $\sim 85^{\circ} \mathrm{F}$ ) to help minimize the required refrigeration power. [6]

For the product gas out of the top of the C-111, first-stage dehydration column, the dew-point / hydrate-formation temperature will be only five to ten degrees below the gas outlet temperature. For example, for the moderately-rich feed gas, the first-stage dehydration will have a product gas temperature of about $-10^{\circ} \mathrm{F}$ and a hydrate-formation temperature of about -15 to $-20^{\circ} \mathrm{F}$. Therefore, further dehydration of the natural gas is required before the gas can be fed to the hydrocarbon absorption column, which operates close to isothermally at $-40^{\circ} \mathrm{F}$. Two options are provided for the second-stage of dehydration on the IROA NGL pilot plant: (A) cooling / dehydration with EG- or $\mathrm{CaCl}_{2}$-sprayed cooling surfaces or (B) adsorption of moisture. The former option is integral to the development of the IROA NGL process, while the later is a backup, standard-technology route, for cases where development work is focused on the Absorption section of the IROA technology.

Option A for second-stage dehydration involves cooling the product gas from first-stage dehydration in a heat exchanger to about $-40^{\circ} \mathrm{F}$ (with low-temperature refrigerant), with solvent sprayed on the cooled surfaces to prevent ice formation. The amount of solvent ( $\mathrm{EG}$ or $\mathrm{CaCl}_{2}$ solution) that is required to absorb the remaining moisture in the gas feed to the second-stage dehydration is relatively low, but it would be very difficult to assure full coverage of all heatexchange areas with a low solvent flow rate. Therefore, Option A for second-stage dehydration will consist of a high solvent circulation rate, with relatively low solvent make-up and solvent withdrawal rates. The partially-dehydrated natural gas will pass through a heat exchanger, E-221, which is shown only as a "box" on the dehydration P\&ID at this point. (The choice between shell and tube, plate-fin, or some other type will be made with the engineering / construction contractor.)

The key feature in Option A will be solvent spray (or some other mechanism to generate a solvent film) on the natural-gas side of heat exchange area to capture moisture and prevent ice formation. The recovered solvent will drain into a sump on the heat exchanger (or in a separate vessel) and be circulated back to the heat exchange surfaces. Cooled solvent will be added to the circulating solvent system, at a very low flow rate, through the FIC-322 flow control loop. An interface detector, LI-221, will periodically signal for the removal of some of the circulation solvent for regeneration. 
The condensed $\mathrm{C}_{3}{ }^{+}$hydrocarbons will be separated from the cooled, dehydrated natural gas in the T-241 Separator, and the gas stream will be routed to the IROA pilot-plant Absorption section. The recovered $\mathrm{C}_{3}{ }^{+}$hydrocarbon liquids will overflow into the T-261 NGL Receiver, which is equipped with level indicator LI-272, to allow quantification of the rate of NGL condensation in the second stage of dehydration / pre-cooling before the IROA Absorption section. A "peanut" drum and manual drain will also be provided on the T-241 separator to return any carryover solvent back to the dehydration solvent regeneration system.

Option B for second-stage dehydration involves a much-simpler (less operator-intensive) dehydration system, which will remove the few remaining ppm of moisture in the first-stage dehydration natural gas product for several hours to a few days of pilot-plant operation. Specifically, the product gas from the first-stage dehydration column, C-111, is routed to the C112 column, which is packed with molecular sieve, or some other type of adsorbent, which picks up the moisture selectively over the hydrocarbon components of the natural gas stream. When calcium chloride solution is used as inhibitor solvent in C-111, the solid desiccant in C-112 may use anhydrate $\mathrm{CaCl}_{2}$ pellets which will become liquid when absorbing moisture and then be discharged. The natural gas can then be cooled in the E-221 heat exchanger, without the ice formation concerns that are present in the Option A approach. It is anticipated that the Option B approach with C-112, might be employed when the IROA NGL pilot plant is being used to study the operation of the Absorption Section. There is a bypass line around the $\mathrm{C}-112$, for the product gas to proceed directly to the E-221 heat exchanger for Option A second-stage dehydration studies.

As alluded to above, regeneration of the dehydration solvent is accomplished by routing a slip stream of the circulating dehydration solvent towards the C-281 Dehydration Solvent Regeneration Column. The pressure is reduced, and the solvent is heated to drive the water off from the EG-water solution. By driving off essentially all the water from the solvent sent to the regenerator, the solvent flow rate to regeneration can be minimized, which reduces the cooling load for the regenerated solvent. The circulating solvent will have to be checked periodically for EG concentration, and the flow to the regeneration through FIC-281 will be increased or decreased, depending on whether the EG concentration in the circulating solvent is below or above the target EG concentration (approximately seventy percent). 
P\&ID -- Dehydration Section -- Version D -- 16-May-2006 (Amended by GHT, 10-June-2006)

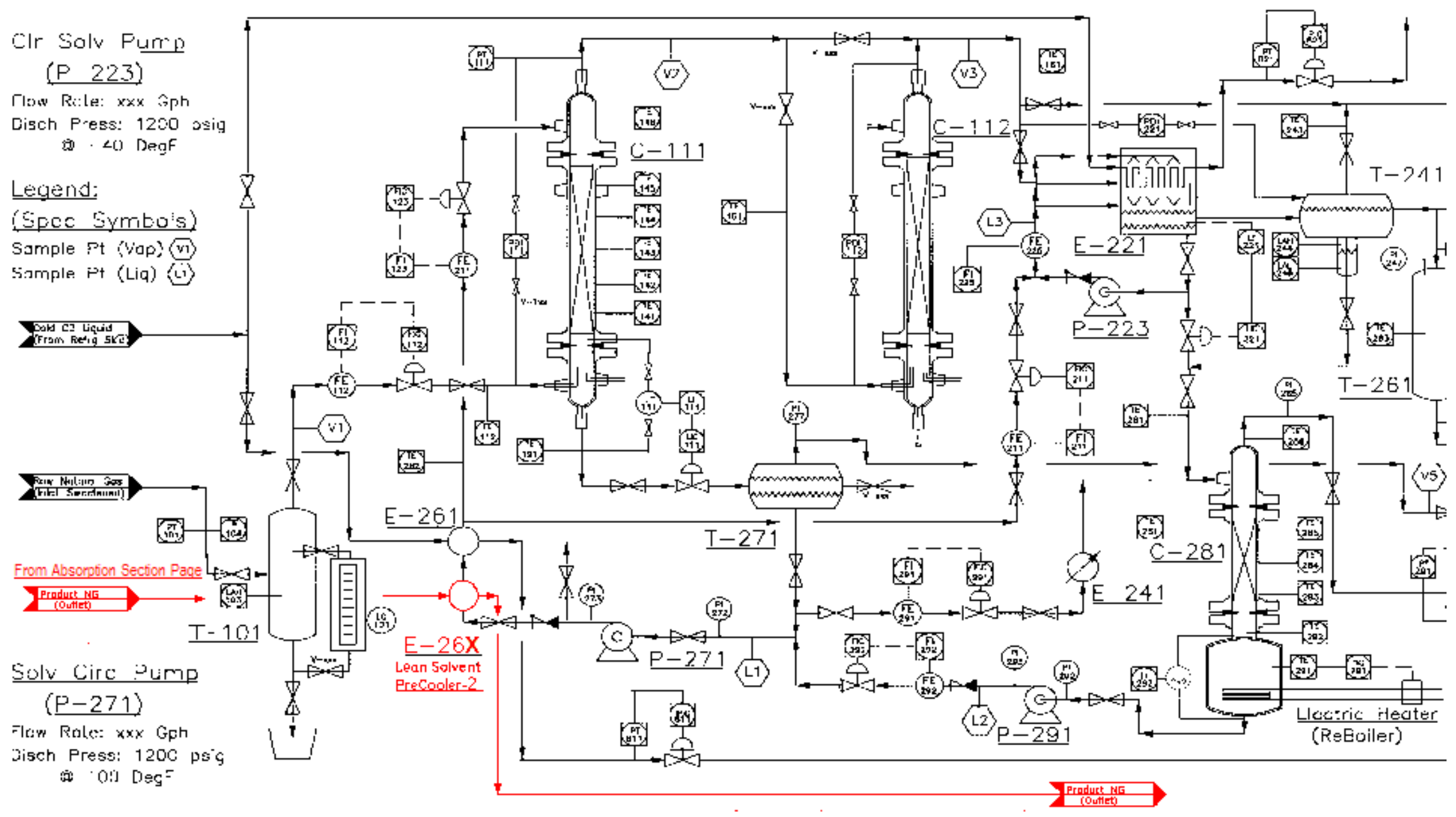




\section{$\underline{\text { Lean Oil Absorption Section: }}$}

The P\&ID for the Absorption section is given in the figure below this section, which is labeled as Drawing No. NGL-PP-01-B (Revision D), dated 16-May-2006.

For the current design effort, it is assumed that the solvent for the Lean Oil Absorption section will be a standard kerosene / jet fuel product from one of the major petroleumrefining companies. (One of the objectives of the bench-scale studies is to determine the best lean-oil solvent, but the bench-scale program will not be completed in time to affect the design of the IROA pilot plant.) In most cases, the major refiners produce and market a single light distillate product, which will meet the specifications for No. 1 Diesel Fuel, Kerosene, and Jet-A. Some typical specifications for these products are the following:

Table 2. Typical Properties for Lean Oil (Kerosene / Jet A)

\begin{tabular}{|c|c|c|}
\hline Density & API Gravity & 41 \\
\hline & SO & 0.82 \\
\hline Viscosity & $\operatorname{cs}$ at $-30^{\circ} \mathrm{F}$ & 11 \\
\hline Freeze Pt & $\mathrm{F}$ & -55 \\
\hline \multicolumn{3}{|l|}{ Distillation } \\
\hline & $10 \mathrm{Pct}_{1}^{\circ} \mathrm{F}$ & 360 to 390 \\
\hline & $50 \mathrm{Pct}_{1}^{\circ} \mathrm{F}$ & 410 to 430 \\
\hline & $90 \mathrm{Pct}^{\circ}{ }^{\circ} \mathrm{F}$ & 460 to 480 \\
\hline & $\mathrm{EP},{ }^{\circ} \mathrm{F}$ & 510 to 540 \\
\hline Flash Pt & $\mathrm{F}$ & 120 to $130+$ \\
\hline \multicolumn{3}{|c|}{ NFPA Hazard Classification } \\
\hline & Health & 1 to 2 \\
\hline & Flammability & 2 \\
\hline & Reactivity & 0 \\
\hline
\end{tabular}

In order to control the flow rate to the Lean-Oil Absorption Section independently from the front-end natural-gas feed rate to the Dehydration Section, the FIC-301 flow-control loop allows some of the dehydrated natural gas to be bypassed around the Lean-Oil Absorption Section. Dehydrated, cooled $\left(-40^{\circ} \mathrm{F}\right)$ natural gas is first directed through FE301 into the bottom of the C-301 Lean Oil Absorption Column. Recycled lean oil (also at $-40^{\circ} \mathrm{F}$ ) is fed into the top of the C-301 column, and, therefore, the solvent and the gas travel counter-currently. This column will be operated essentially isothermally, and the heavier hydrocarbons $\left(\mathrm{C}_{2}^{+}\right.$- that is, ethane, propane, butane, and higher molecular weight hydrocarbons, or NGL's) will be absorbed into the lean oil. The design of C-301 should based on very high recovery rate $(95-100 \%)$ of $\mathrm{C}_{3}{ }^{+}$, and variable recovery rate of $\mathrm{C}_{2}$ from a maxima of $95 \%$ to around $30 \%$ at reduced absorbent flow while always keeping $\mathrm{C}_{3}$ recovery rate higher than $95 \%$.

The dried and low-dew-point gas product from the top of the C-301 column is routed to the bottom of the C-311 Pre-Saturator Column. The regenerated lean oil, with most of the $\mathrm{C}_{2}$ to $\mathrm{C}_{7}$ hydrocarbons removed, is fed to the top of the $\mathrm{C}-311$ column. The purpose of this operation is to pre-saturate the lean oil with methane, which releases a significant amount of heat in the presaturator rather than in the column. If the saturation occurred in 
the C-301 column, the heat rise would have a negative impact on the extent of NGL removal in the Lean Oil Absorber. The methane-saturated lean oil is then pumped, by P421, through the E-441 heat exchanger to the top of the C-301 column. The flow rate of the product gas exiting C-311 is then measured by FE-381, and the pressure of the product gas is reduced through the PIC-381 system. It will likely be returned to the suction side of the compressor(s) in the host natural gas plant for recovery and eventual sales gas.

The rich solvent from the bottom of C-301 (saturated with methane and NGL) is reduced in pressure through the LIC-301 system, and the rich solvent is then routed to the T-411 Absorber Solvent Flash Drum. This drum will most likely operate between 500 and 800 psig, and will be the first stage of releasing and recovering the $\left(\mathrm{C}_{2}^{+}\right) \mathrm{NGL}$ 's from the rich solvent. The flow rate will be measured for the gas flashed off in the T-411 drum through FE-411, and the composition of this stream will be sampled to quantify the amount of $\mathrm{C}_{2}^{+}$ heavy hydrocarbons for the NGL recovery calculations.

On the T-411 Flash Drum, a small "peanut" drum is provided on the bottom, in case small amounts of the dehydration solvent are entrained with the dehydrated gas fed to the Absorption section. An interface detector / alarm, LAH-437, will be provided in the bottom of the T-411 drum to detect a buildup of the dehydration solvent. On alarm, the recovered dehydration solvent would be manually drained back to the Dehydration Section, until the low level for the interface is detected on LAL-439.

The solvent liquid from the T-411 flash drum will be reduced in pressure, through the LIC-431 system, to about 500 psig. This rich solvent is then routed to the top of the C421 Rich Oil Demethanizer (ROD), which is intended to remove the remainder of the methane from the rich solvent. For the pilot-plant, the quality of the over-heads from the ROD tower is not critical, but rather measurement of the flow rate and the composition for the NGL material balance. Therefore, the column is rather simple with no overhead condenser / no reflux and with a simple integral reboiler to provide heat in the bottom of the column. The overheads will be returned continuously to the host gas processing plant. The temperature of the re-boiler and the pressure of the ROD will be controllable for ethane rejection operation test when the required ethane recovery rate will be reduced from $30 \%$ to $2 \%$.

To maximize recovery of the NGL in the Lean Oil Absorption column, the $\mathrm{C}_{2}$ through $\mathrm{C}_{5}$ content of the rich solvent has to be reduced as much as practical. Therefore, the bottom liquids from the ROD tower are reduced in pressure through the LIC-432 system and heated through the E-481, and then fed to the middle of the C-451 Lean Oil Fractionator. This column will be operated at approximately 100 psig, with an overhead condenser / reflux, and with a reboiler.

The overhead product from the C-431 Fractionator, which will be mostly NGL, will be analyzed for specific components of the NGL's, and the flow rate will be measured by FE-431. This will allow material balance on the NGL components, and calculation of removal efficiencies for individual components. 
The lean solvent from the bottom of the C-431 Fractionator will be discharged on level control into the T-481 Lean Solvent Hold Drum. This drum should be sized large enough so the solvent inventory of the entire Lean Oil Absorption Section can be dumped into the T-481 vessel at the end of an experimental test run. Therefore, as the Lean Oil Absorption Section is brought to steady-state, the level in T-481 will decrease.

The lean solvent from the T-481 holding drum / accumulator pressurized through the P471 pump to greater than $950 \mathrm{psig}$, and the lean solvent is then cooled. For the IROA pilot plant, it will be necessary to control the equipment temperatures independently from one another, in order to study several processing variables independently. Therefore, very little heat integration between equipment has been incorporated in the process design. However, the cooling of the regenerated, lean solvent will be the largest user of the refrigerant system, and two "feed/effluent" types of heat exchangers (E-491 and E-481) have been specified in the process design, in case it is necessary to control the refrigeration duty to "reasonable" (economically viable) levels for the refrigeration system. Finally, the regenerated solvent is then cooled to about $-40^{\circ} \mathrm{F}$ through the E-461 heat exchanger.

The cooled regenerated solvent is then routed to the C-311 Pre-Saturator, as described above. The liquid effluent from the bottom of the C-311 column is repressured to greater than 1000 psig through the P-421 pump and then re-cooled to $-40^{\circ} \mathrm{F}$, through the E-441 refrigerated heat exchanger. 
P\&ID-Absorption Section-Version D-16-05-06 (Amended \#2 by GHT, 10-10-06) (ROD eliminated and T-412 replaced C-311)

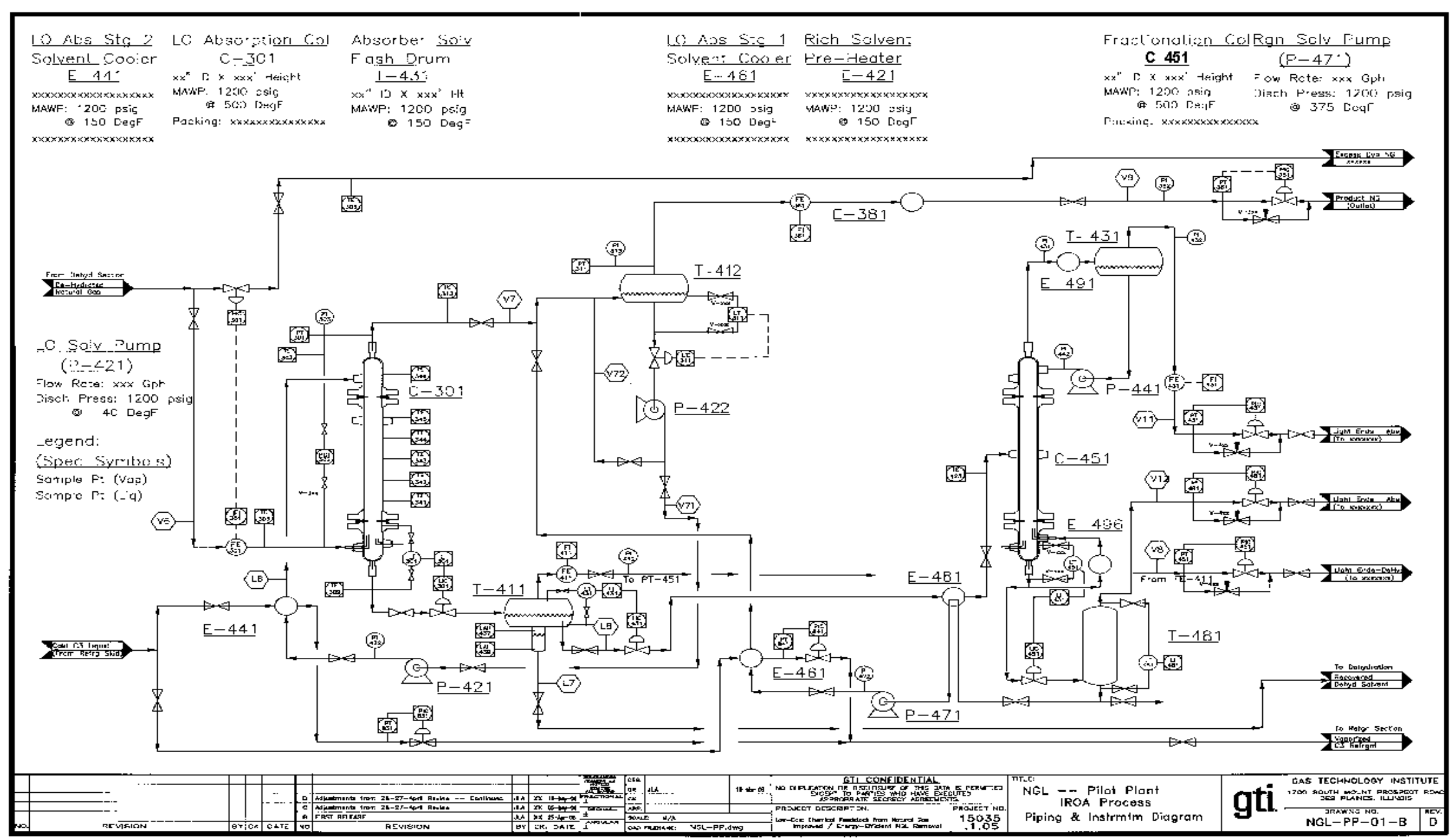




\section{Refrigeration Section:}

For the IROA Pilot Plant, the refrigeration will be likely be supplied as a separate system, which should be able to be started up and lined out initially, with no load / no circulation through the dehydration nor absorption sections of the pilot plant. In other words, the refrigeration package will have to contain, at a minimum, the following equipment:

-- Refrigerant compressor (inlet pressure 5 to 15 psig;

likely outlet pressure 150 to $250 \mathrm{psig}$ )

-- Compressor driver (most likely electric motor: $460 \mathrm{VAC} / 3$-phase / $60 \mathrm{~Hz}$; natural-gas engine acceptable, at reasonable cost)

-- Instrumentation and Control panel (suitable for Class I, Division 2 area; capable of sending data / alarm signals to main IROA pilot-plant control / data-acquisition system)

-- Propane condenser (air-cooled)

-- Propane receiver

-- Suction Knock-Out Drum

-- Piping and valves on refrigeration skid (per ANSI B31.3) 


\section{One-Stage Refrigeration System}

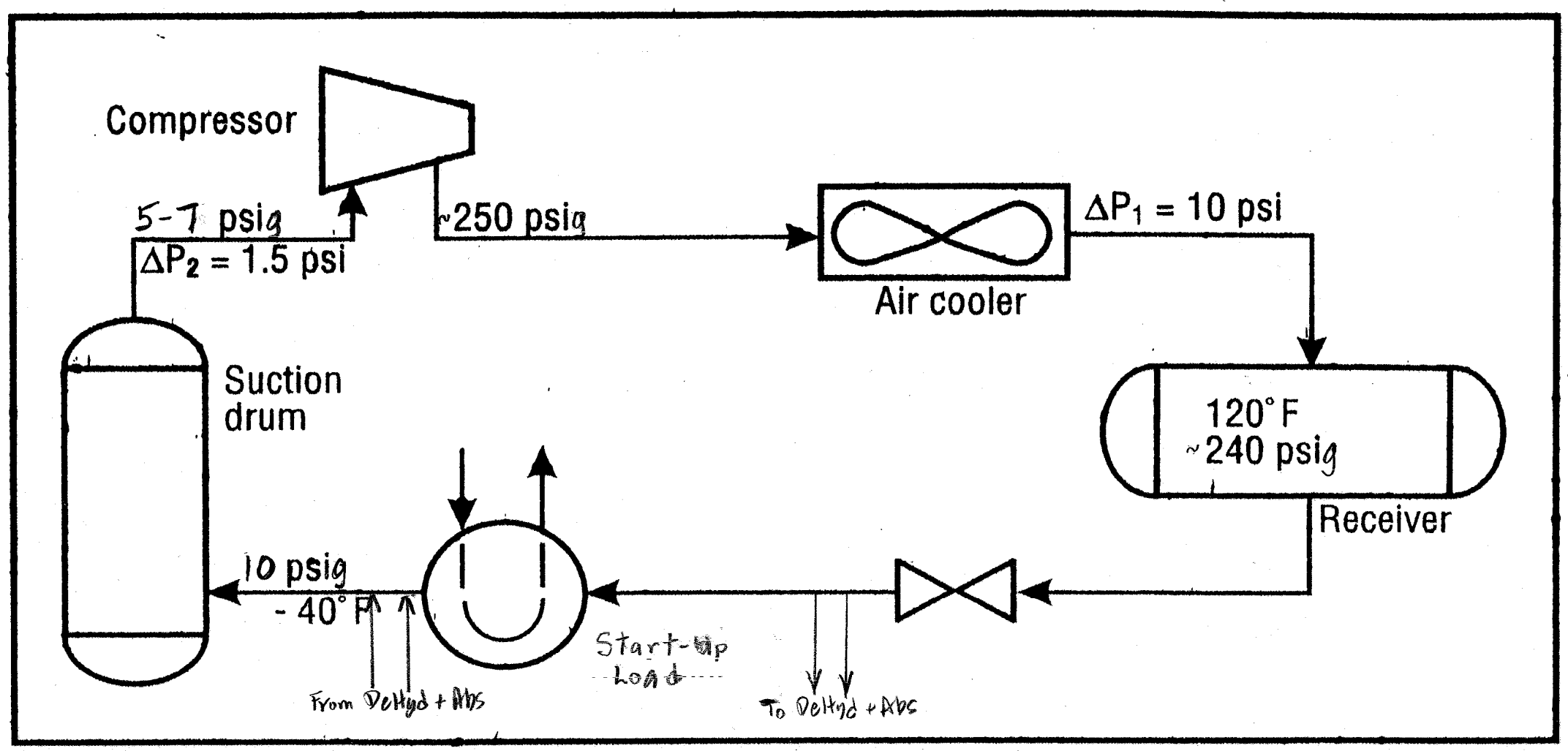


Final Progress Report

DE-FC36-03GO13150

$\underline{\text { Process Flow Diagram (PFD) / Material Balance }}$

Page 127 
PFD --Process Flow Diagram -- Version E -- 23-May-2006 (Amended \#2 by GHT, 10-June-2006)

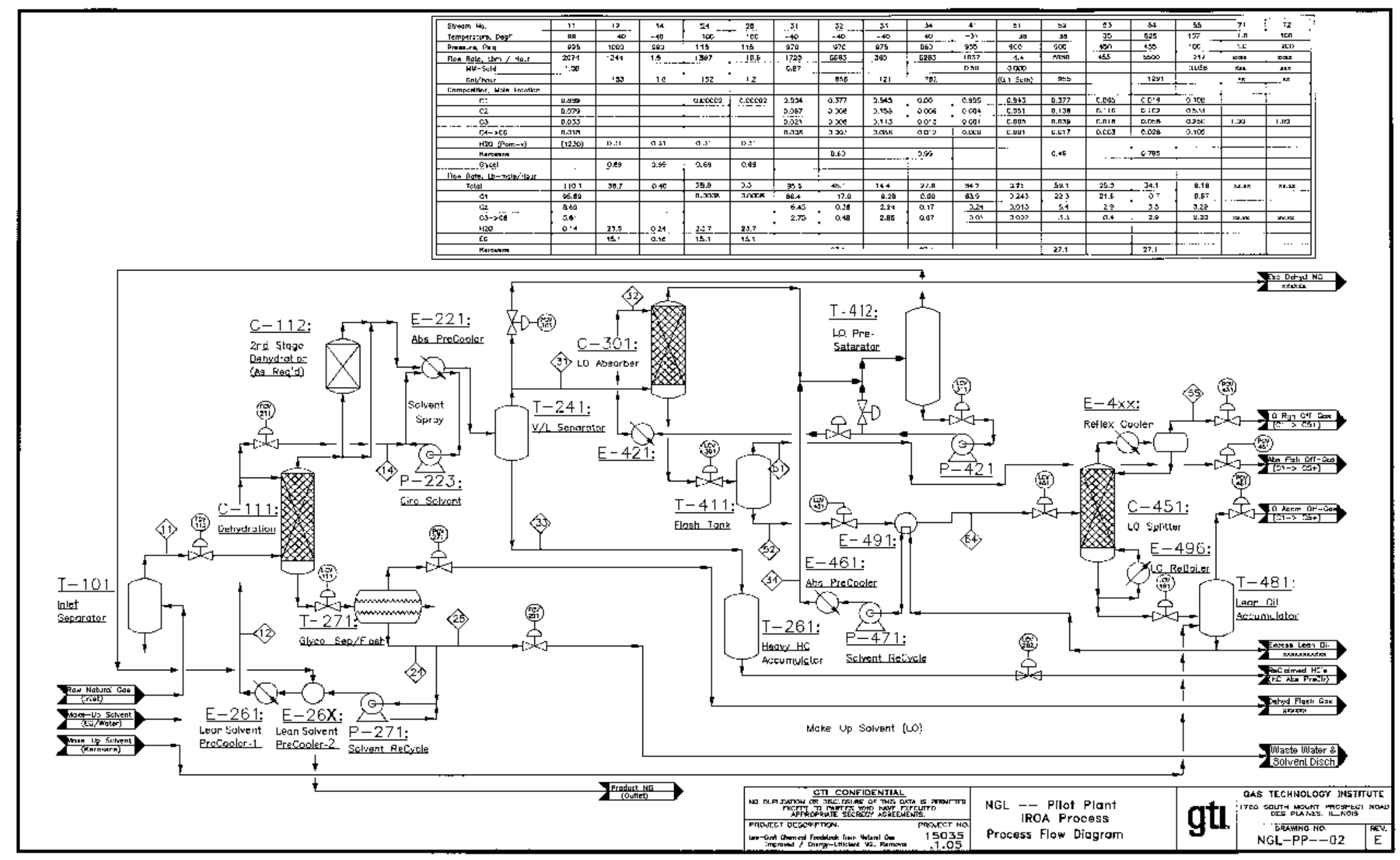


PFD -- Material Balance on Major Streams -- Version E -- 23-May-2006

\begin{tabular}{|c|c|c|c|c|c|c|c|c|c|c|c|c|c|c|c|c|c|}
\hline Stream No. & 11 & 12 & 14 & 24 & 25 & 31 & 32 & 33 & 34 & 41 & 51 & 52 & 53 & 54 & 55 & 71 & 72 \\
\hline Temperature, DegF & 99 & -40 & -40 & 100 & 100 & -40 & -40 & -40 & -40 & -31 & -38 & -38 & 35 & 525 & 157 & 1.0 & 100 \\
\hline $\begin{array}{l}\text { Pressure, Psig } \\
\end{array}$ & 995 & 1000 & 990 & 115 & 115 & 970 & 970 & 975 & 960 & 955 & 900 & 900 & 450 & 455 & 100 & 1.0 & 200 \\
\hline Flow Rote, Lbm / Hour & 2074 & 1344 & 1.5 & 1367 & 10.6 & 1720 & 5563 & 360 & 5283 & 1037 & 4.4 & 5950 & 455 & 5500 & 217 & $\mathrm{xx \textrm {xx }}$ & $x \times x \times$ \\
\hline MM-Scfd & 1.00 & & & & & 0.87 & & & & 0.58 & 0.000 & & & & 0.056 & $x \times x$ & $x \times x$ \\
\hline Gal/Hour & & 153 & 1.6 & 152 & 1.2 & & 858 & 121 & 782 & & $(0.1 \mathrm{Scth})$ & 955 & & 1291 & & $x x$ & $\mathrm{xx}$ \\
\hline Composition, Mole Fraction & & & & & & & & & & & & & & & & & \\
\hline $\mathrm{c1}$ & 0.869 & & & 0.00002 & 0.00002 & 0.904 & 0.377 & 0.645 & 0.00 & 0.995 & 0.943 & 0.377 & 0.865 & 0.019 & 0.108 & & \\
\hline $\mathrm{C} 2$ & 0.079 & & & & & 0.067 & 0.008 & 0.156 & 0.006 & 0.004 & 0.051 & 0.108 & 0.116 & 0.102 & 0.533 & & \\
\hline $\mathrm{C3}$ & 0.033 & & & & & 0.021 & 0.008 & 0.113 & 0.013 & 0.001 & 0.005 & 0.039 & 0.016 & 0.056 & 0.250 & 1.00 & 1.00 \\
\hline $\mathrm{C} 4->\mathrm{C} 6$ & 0.018 & & & & & 0.008 & 0.007 & 0.086 & 0.012 & 0.000 & 0.001 & 0.017 & 0.003 & 0.028 & 0.105 & & \\
\hline $\mathrm{H} 2 \mathrm{O}(\mathrm{Ppm}-\mathrm{v})$ & (1230) & 0.31 & 0.31 & 0.31 & 0.31 & & & & & & & & & & & & \\
\hline Kerosene & & & & & & & 0.60 & & 0.99 & & & 0.46 & & 0.795 & & & \\
\hline Glycol & & 0.69 & 0.69 & 0.69 & 0.69 & & & & & & & & & & & & \\
\hline Flow Rote, Lb-mole/Hour & & & & & & & & & & & & & & & & & \\
\hline Total & 110.1 & 38.7 & 0.40 & 38.8 & 0.3 & 95.6 & 45.1 & 14.4 & 27.9 & 64.2 & 0.26 & 59.1 & 25.0 & 34.1 & 6.16 & $x x \cdot x x$ & $x \times . x x$ \\
\hline c1 & 95.69 & & & 0.0008 & 0.0008 & 86.4 & 17.0 & 9.28 & 0.00 & 63.9 & 0.243 & 22.3 & 21.6 & 0.7 & 0.67 & & \\
\hline $\mathrm{C2}$ & 8.69 & & & & & 6.45 & 0.36 & 2.24 & 0.17 & 0.24 & 0.013 & 6.4 & 2.9 & 3.5 & 3.29 & & \\
\hline $\mathrm{C3}->\mathrm{C} 6$ & 5.61 & & & & & 2.75 & 0.48 & 2.85 & 0.67 & 0.01 & 0.002 & 3.3 & 0.4 & 2.9 & 2.20 & $x \mathrm{x} . \mathrm{xx}$ & $x x . x x$ \\
\hline $\mathrm{H} 2 \mathrm{O}$ & 0.14 & 23.5 & 0.24 & 23.7 & 23.7 & & & & & & & & & & & & \\
\hline$E G$ & & 15.1 & 0.16 & 15.1 & 15.1 & & & & & & & & & & & & \\
\hline Kerosene & & & & & & & 27.1 & & 27.1 & & & 27.1 & & 27.1 & & & \\
\hline
\end{tabular}


Control / Data Acquisition System: The Control System should be designed to keep the pilot plant under control with minimal operator interaction during steady-state periods but allow operator control of the pilot plant during testing periods. A localized control panel should be provided with emergency shutdown provisions to the host plant control system. The Data Acquisition System must record all flow, temperature, and pressure elements for later data analysis.

Other Considerations: Time is of great importance for the design and construction of the pilot plant. A bid that provides means to shorten the time to have the pilot plant system ready for operation will be favorably considered. Any improvements to the underlying design of the pilot plant that will make it easier to operate, able to obtain more commercially-viable data, or improve on the general process economics will be considered. 


\section{References:}

1. Abbasian, Javad, Manish Bhargava, and Rahul Mago, "Support Studies for Evaluation of an Improved Natural Gas Liquids (NGL) Removal Process," Prepared by Illinois Institute of Technology for Gas Technology Institute (US-DOE Contract: DE-FC36-03GO13150), September, 2004.

2. Lee, R. J., J. Yao, and D. G. Elliot, "Flexibility, efficiency to characterize gas-processing technology," Oil and Gas Journal, Special Supplement, Petroleum of the 21st Century, December 13, 1999

3. Lu, Yingzhong, US Patent 6,553,784 - B2, "Comprehensive Natural Gas Processor", April 29, 2003.

4. John Wilkinson, et al., Next Generation Processes for NGL/LPG Recovery", Hydrocarbon Engineering, May, 2002.

5. Techno-Economic Evaluation of An Improved and Energy-Efficient Natural Gas Liquid (GNGL) Removal Process, GTI, Presented at 2005 AIChE Spring National Meeting, Atlanta, GA, April 11-14th, 2005.

6. GTI Quarterly Report, Jan.1 - Mar.31, 2006, 


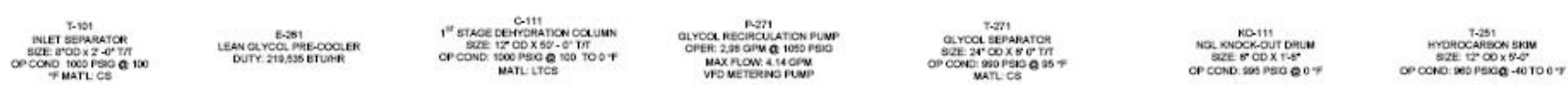

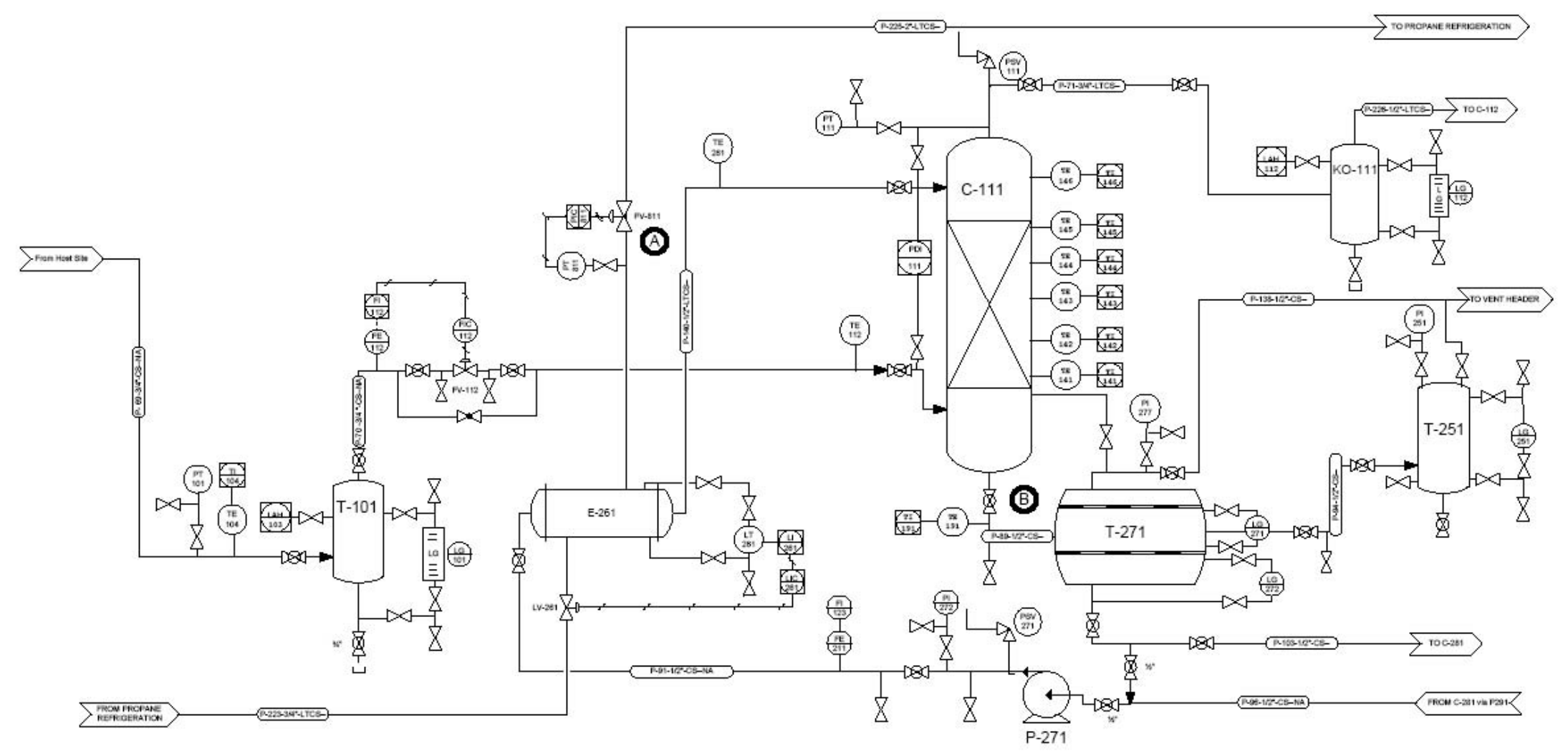

\begin{tabular}{|c|c|c|c|c|c|c|c|c|c|c|c|}
\hline 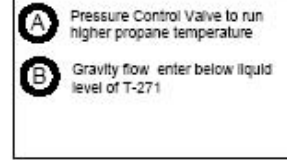 & & $m$ & 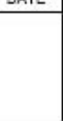 & DESCRPPTION & BY & CHECKEO & APPROVED & \multicolumn{2}{|c|}{$\begin{array}{c}\text { TRMARRC } \\
\text { CORPORATION } \\
\text { P.O. Box } 826 \\
\text { Buda. Texas } 78610 \\
\end{array}$} & 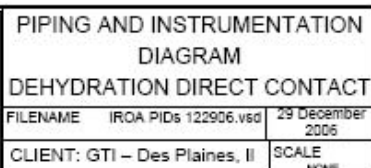 \\
\hline
\end{tabular}




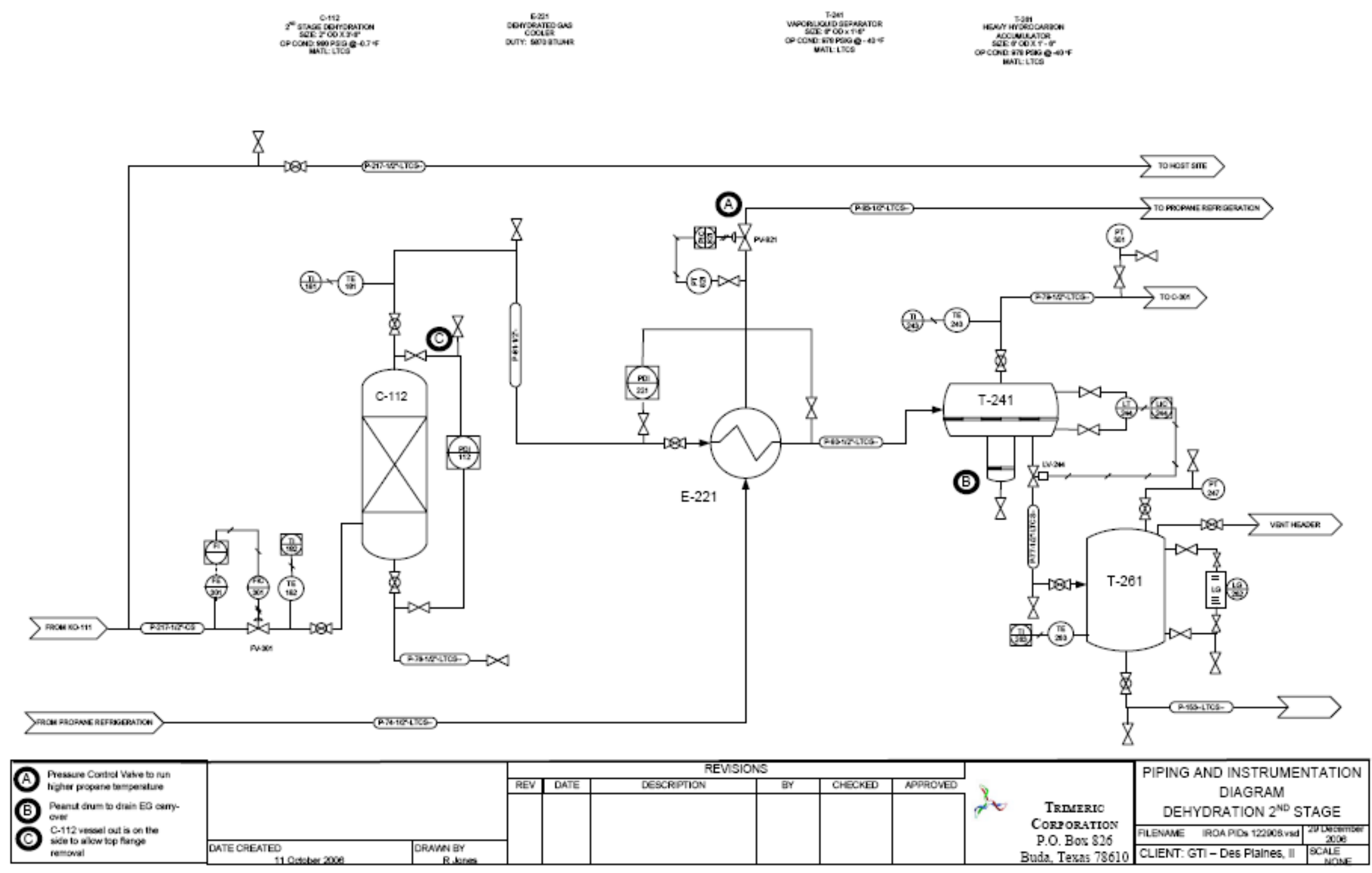




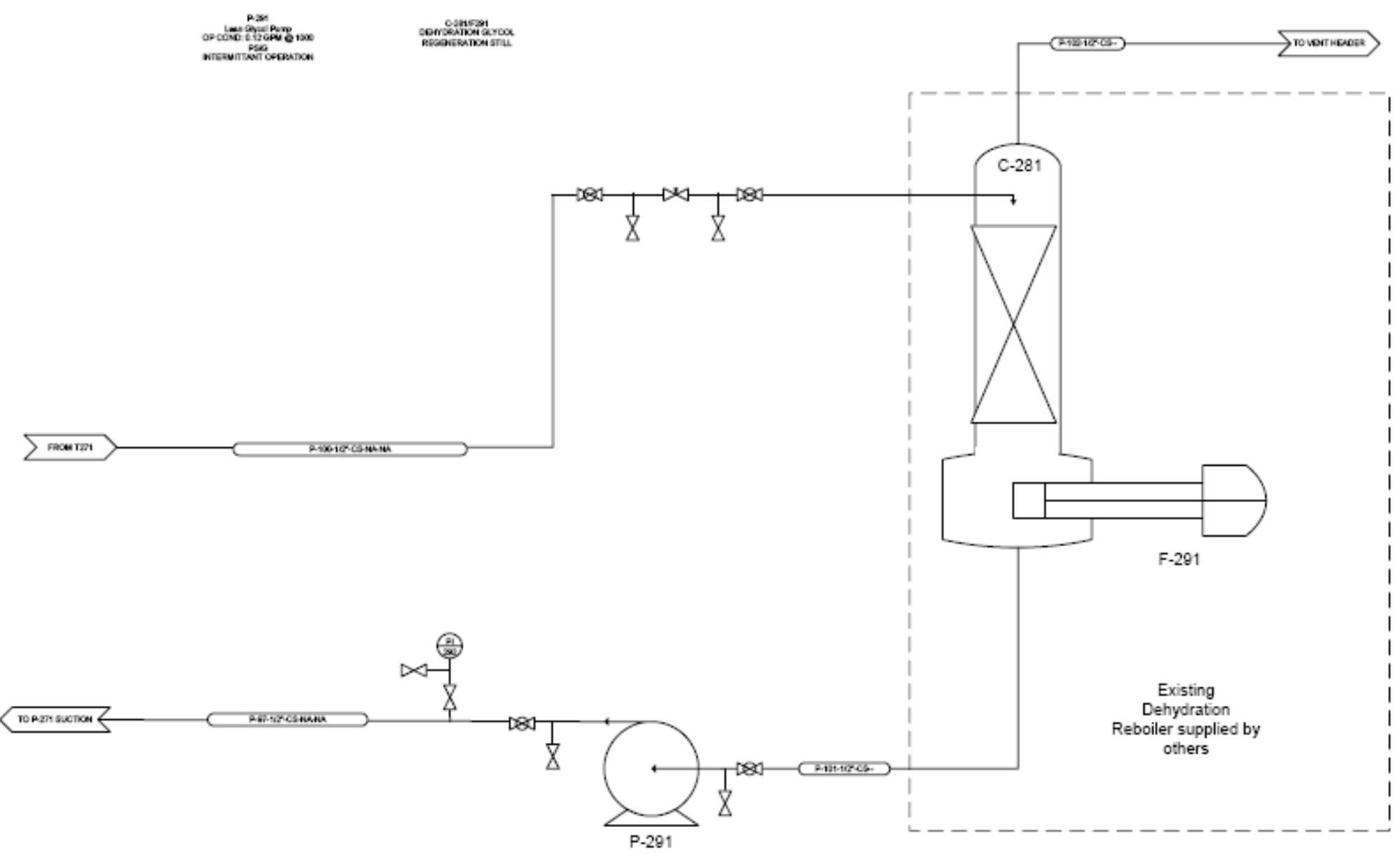

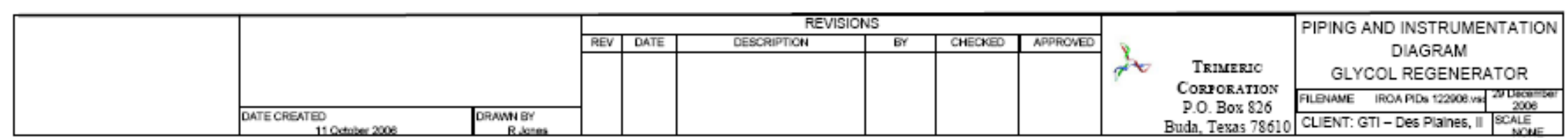



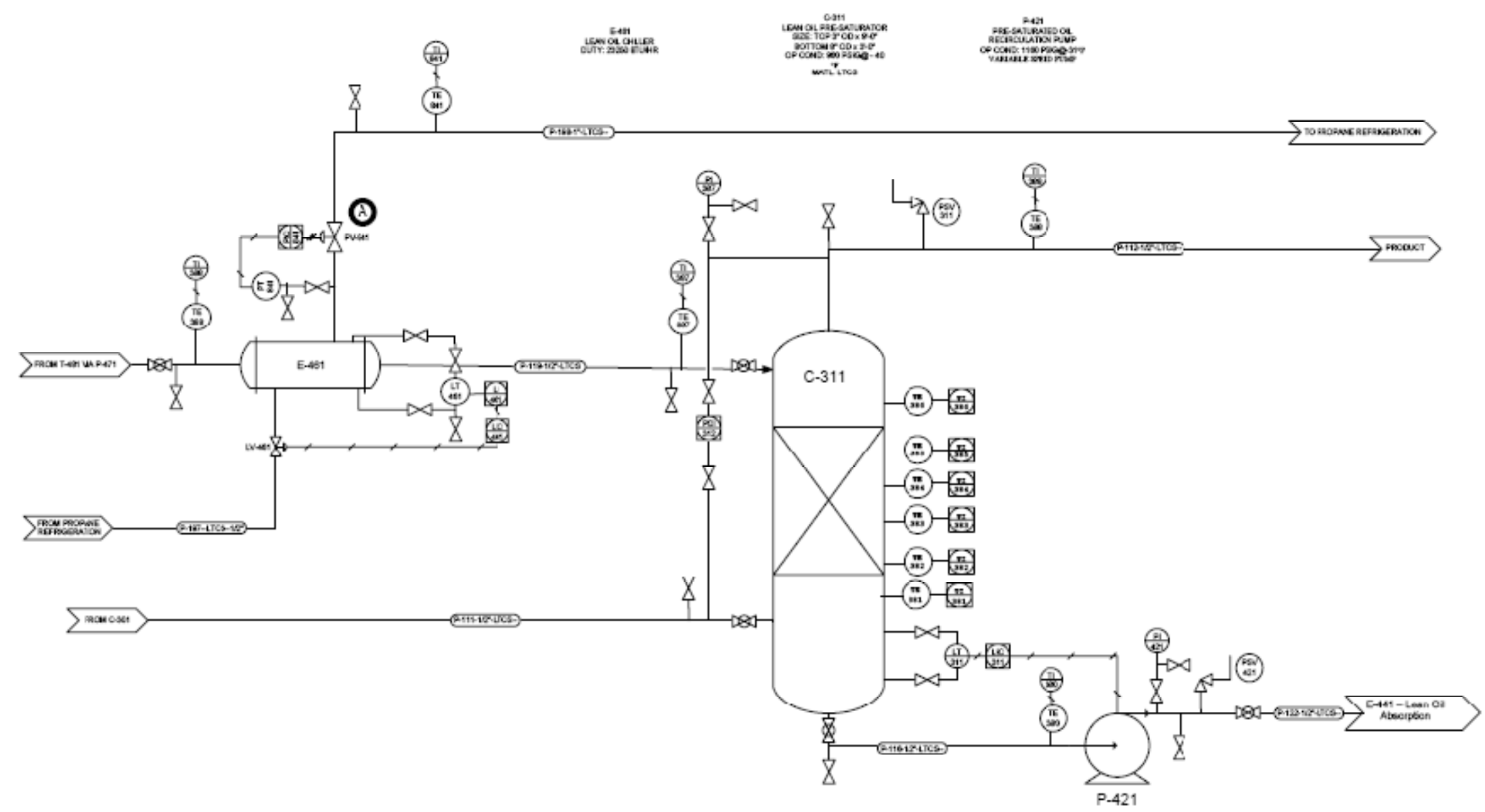

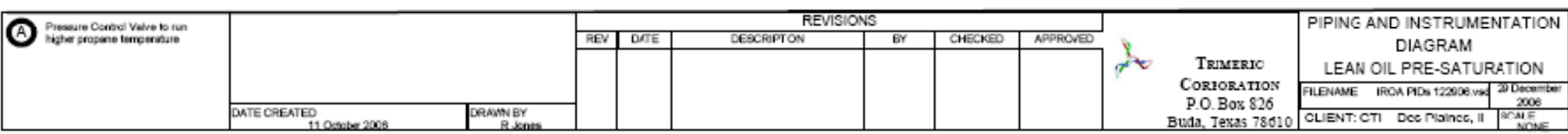




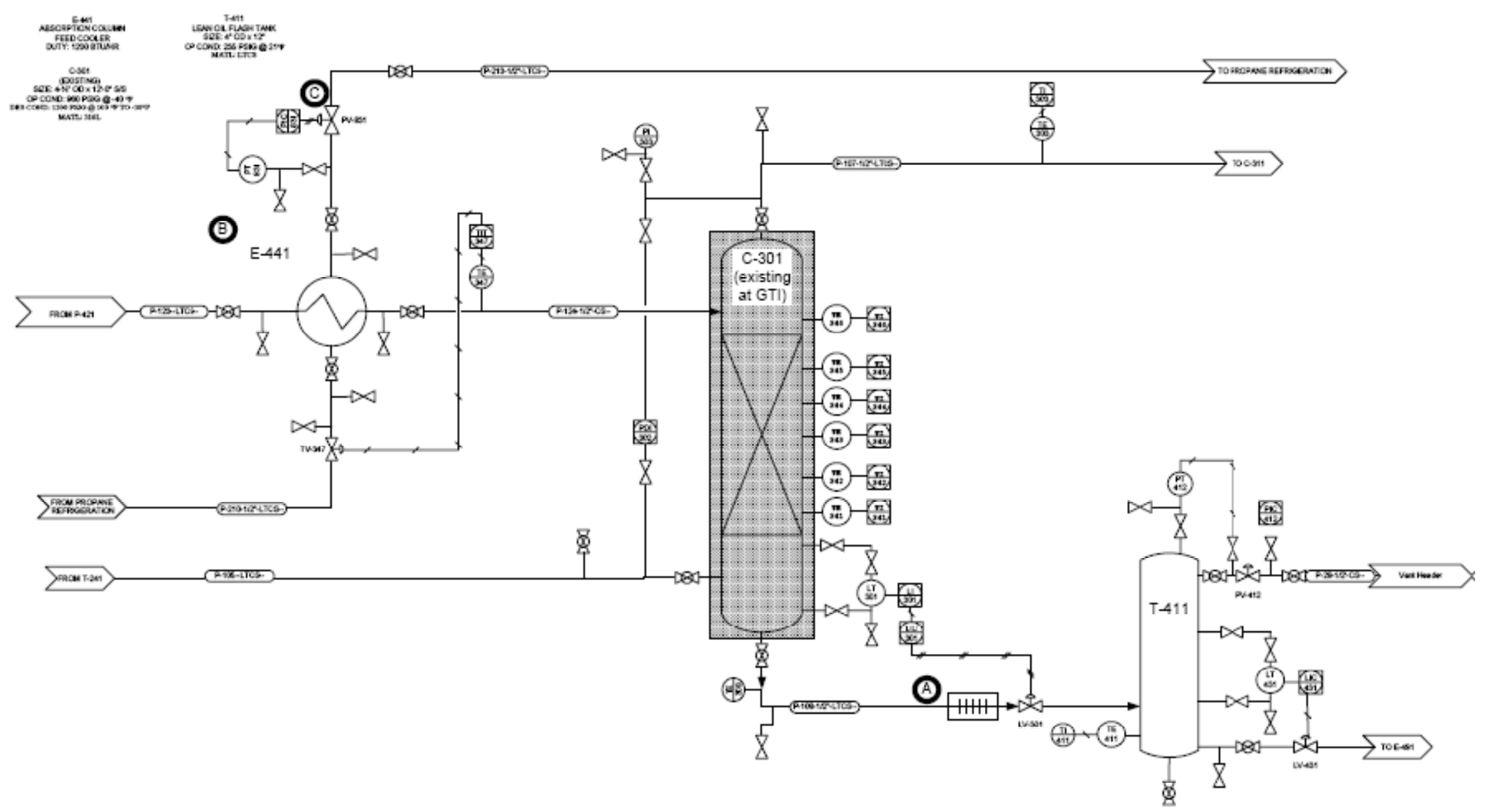

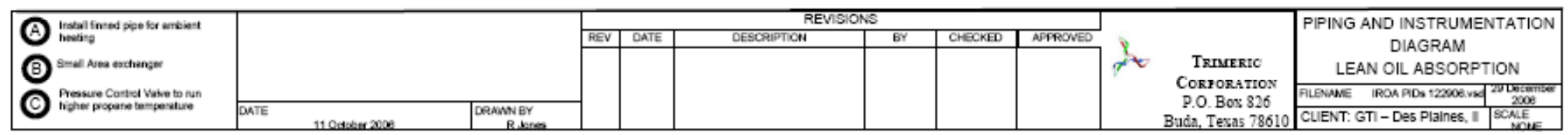




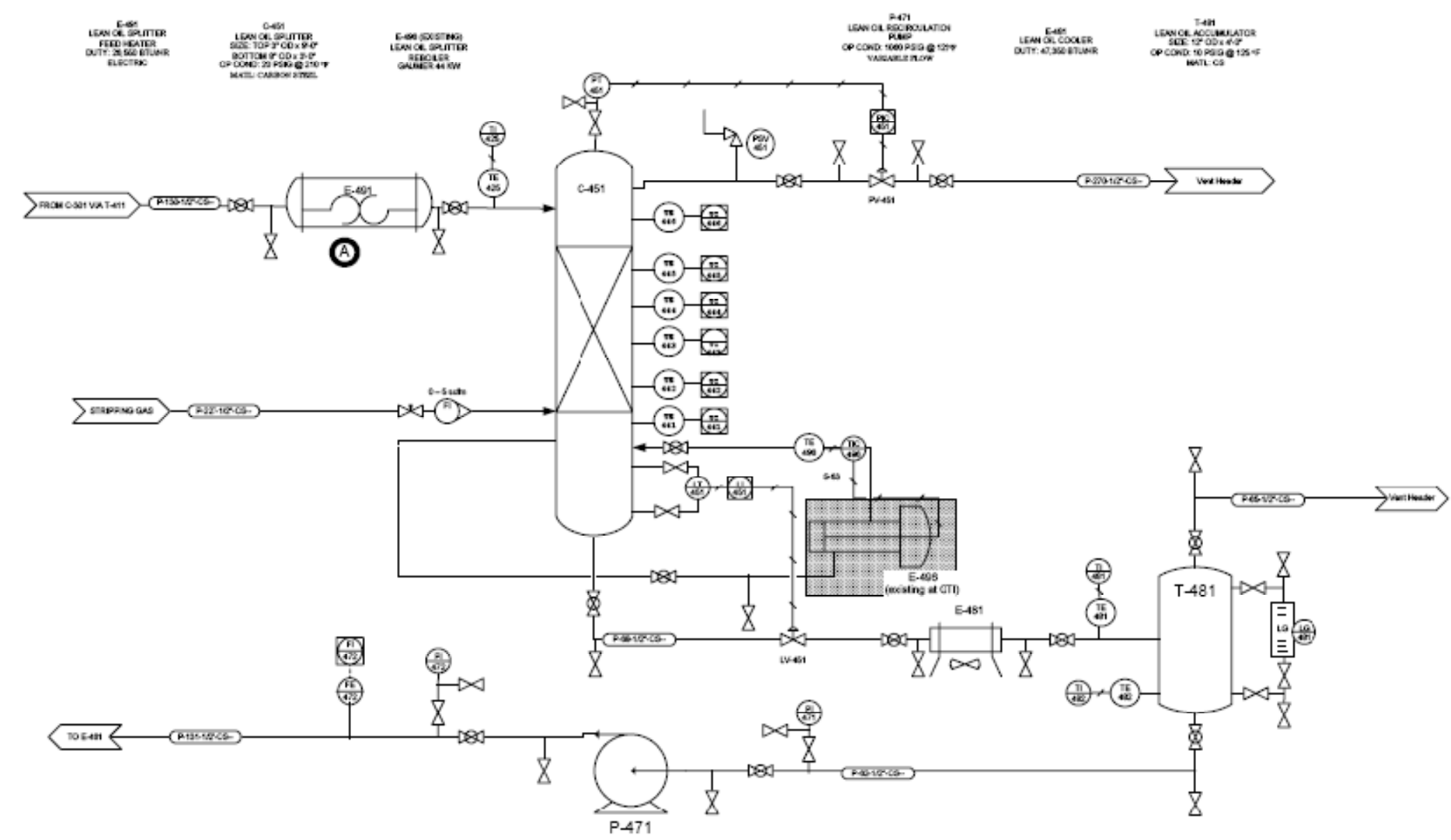

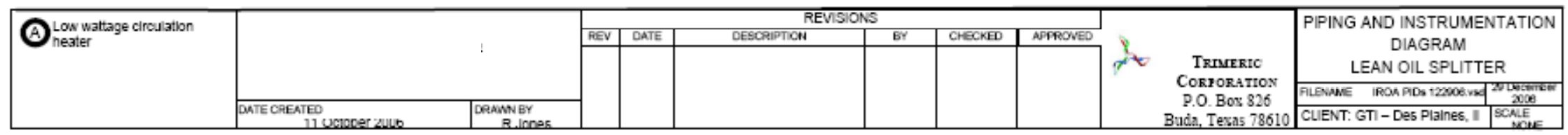




\begin{tabular}{|c|c|c|c|c|c|c|c|c|}
\hline \multicolumn{2}{|c|}{ stream numbel } & 1 & 2 & $2 \mathrm{~b}$ & 3 & 4 & 5 & 7 \\
\hline Nane & Units & NG-estd & NG-Denyd & NG-Detyd-Cid-V-Oplt & RG-Den,d-Cld & NG-Detyd-Clat $\mathrm{V}$ & NG-D:hyd-CIs-L & Rich solv \\
\hline Vapour Fraction & & 1 & 1 & 1 & 0.8593 & 1 & 0 & 0 \\
\hline Tenperatur: & $F$ & 100.3 & 0.6793 & 0.6793 & -40 & 40 & -40 & $\$ 4.71$ \\
\hline \begin{tabular}{|l|} 
Pressure \\
\end{tabular} & 080 & 1002 & 980 & 980 & 971 & 978 & 978 & 990 \\
\hline Mass Fiow & boar & 2074 & 2072 & 85.77 & 85.77 & 70.89 & 14.88 & 1512 \\
\hline Hest Flow & Stu/hr & $-3.74 E+06$ & $-3.87 E+05$ & $-1.60 E+05$ & $-1,64 E+05$ & $-1,41 E+05$ & $-2.47 E+04$ & $-5.81 E+06$ \\
\hline Molar Enthzipy & Bthntemole & $-3.39 \mathrm{E}+04$ & $-3.52 \mathrm{E}+04$ & $-3.52 \mathrm{E}+0.6$ & $-3.61 E+04$ & $-3.53 \mathrm{E}+04$ & $-4.15 E+04$ & $-1.51 E+05$ \\
\hline Acts/ Oss Flow & ACFM & 9.234 & 6.038 & 0.25 & & 0.1681 & & \\
\hline Srd Gas Filiw & UNSCFD & $1.00 \mathrm{E}+00$ & $100 E+01$ & $4.15 \mathrm{E}-02$ & $4.15 E-02$ & $3.61 \mathrm{E}-02$ & $5.42 \mathrm{E}-03$ & 4.11E-01 \\
\hline \multicolumn{9}{|l|}{ PROPERTIEO } \\
\hline Molsecular W elght & & 18.93 & 18.84 & 18.84 & 18.54 & 17.91 & 25.01 & 35.75 \\
\hline Masa Denaty & bita & 3.744 & 5.712 & 5.719 & 7.974 & 7.028 & 22.18 & 67.74 \\
\hline Mass Enthspy & Ethnt & -1801 & -1858 & -1868 & -1915 & -1969 & .1658 & $\leftarrow 222$ \\
\hline Mass Entrooy & Eth/b-F & 1.907 & 1.767 & 1.767 & 1.659 & 1.781 & 1.079 & -0.1644 \\
\hline Hest Capadty & Ethilumolef & 12.21 & 16.43 & 16.43 & 22.12 & 22.17 & 21.76 & 25.83 \\
\hline Mass Hest Capaciti & Ethab-F & 0.643 & 2.8721 & 2.8721 & 1.174 & 1238 & 0.87 & 0.7225 \\
\hline Opesific Hest & BthitmoleF & 12.21 & 16.43 & 16.43 & 22.12 & 22.17 & 21.76 & 25.83 \\
\hline Act LIa. FIOW & |บรดPM & 0 & & & $8.36 E=02$ & & $8.36 \mathrm{E} \cdot 02$ & 2.967 \\
\hline 2 Factor & & & 2.6553 & 2.6553 & & & & $8.79 E-02$ \\
\hline$C_{p N / 2 \mathrm{r}}$ & & 1.544 & 2.222 & 2.222 & 2.659 & 3.151 & 1.1 & 1.102 \\
\hline Hest of $V a p$ & Ethibmols & 3281 & 1959 & 1959 & 1969 & & 7146 & 1.22E-04 \\
\hline 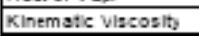 & ca: & 0.2272 & 2.1431 & 2.1431 & & 0.1182 & C.1561 & 3.455 \\
\hline Masa Heat of Vas. & Eth/b & 1742 & 104 & 104 & 1045 & & 165.8 & \\
\hline \begin{tabular}{|l|} 
Ourace Telsion \\
\end{tabular} & dyneicm & & & & 4.643 & & 4.643 & 53.38 \\
\hline Thermal Conductlvity & Bth/hr-At:F & $2.32 \mathrm{E} \cdot 02$ & 2.13E-5: & $2.13 E-02$ & & $2.2 \% \mathrm{E} \cdot 02$ & $5.02 \mathrm{E}-02$ & 0.2668 \\
\hline \begin{tabular}{|l|l|l} 
Vlacosity \\
\end{tabular} & $\angle P$ & $1.36 \mathrm{E} \cdot 02$ & $1.31 E-02$ & $1,31 E-02$ & & $1.31 \mathrm{E}-02$ & $5.55 \mathrm{E}-02$ & 3.749 \\
\hline Mss: CV & Btulib-F & 0.4198 & 0.3924 & 1.3924 & 0.4357 & 0.1929 & 0.7906 & 0.5557 \\
\hline COMPONENTS & \multirow{2}{*}{\multicolumn{4}{|c|}{ Overall Phese Mass flow }} & & & & \\
\hline \begin{tabular}{|l|} 
Hergane \\
\end{tabular} & & & & 63.5567 & 63.5697 & 573936 & 6.1621 & 0.097 \\
\hline Emane & bohr & 261.2996 & 251.2966 & 10.8177 & 10.8177 & 8.0348 & 2.7829 & 0.003 \\
\hline \begin{tabular}{|l|} 
Prozane \\
\end{tabular} & bonr & 162.0673 & $1 \equiv 0.0671$ & 5.6268 & $6.62 \mathrm{ig}$ & 3.65 & 2.9668 & 0.2002 \\
\hline -Butene & woar & 57.6274 & 57.6274 & 2.3858 & 2.3858 & 0.5701 & 1.4157 & 0 \\
\hline n-Bitane & bar & 57.6274 & 57.6274 & 23958 & 2.3658 & 0.8333 & 1.5525 & 0 \\
\hline TEQyycol & owar & 0 & 0 & 0 & 0 & 0 & 0 & 0 \\
\hline EQycol & $10 \mathrm{Mr}$ & 0 & 2.0004 & 0 & 0 & 0 & 0 & 1125.5502 \\
\hline H2O & towar & 2.441 & 2.0411 & 2.0017 & 0 & 0 & 0 & 485.2072 \\
\hline $\mathrm{H} 2 \mathrm{~s}$ & tomer & 0.0112 & 2.0087 & 2.0004 & 0.0024 & 0.8003 & 0.0001 & 0.0025 \\
\hline$n-\mathrm{C} 11$ & Bowr & 0 & 0 & 0 & 0 & 0 & 0 & 0 \\
\hline$n-\mathrm{C} 12$ & bowr & 0 & 0 & 0 & 0 & 0 & 0 & 0 \\
\hline$n-\mathrm{C} 13$ & tonar & 0 & 0 & 0 & 0 & 0 & 0 & 0 \\
\hline$n-C 14$ & bour & 0 & 음 & 0 & 0 & o & 0 & 0 \\
\hline$n-\mathrm{C} 15$ & bour & 0 & 0 & 0 & 0 & 0 & 0 & 0 \\
\hline$n-C 10$ & toonr & 0 & 0 & 0 & 0 & 0 & 0 & 0 \\
\hline \begin{tabular}{|l|l} 
Total \\
\end{tabular} & tonar & 2074.3332 & 2571.831 & 85.7738 & 85.7721 & 70.892 & 14.88 & 1611.8601 \\
\hline
\end{tabular}




\begin{tabular}{|c|c|c|c|c|c|c|c|c|c|}
\hline \multicolumn{2}{|c|}{ Stream rumber } & 8 & 12 & 13 & 15 & 16 & 17 & 18 & 19 \\
\hline Name & Units & LeanSolk-Prsd & LearSolv-Pc-Cld & RS-Flash-Denyd & RS-Risgentn & RS-Recycled & LeanSolvert-2 & RecycleSolwoHY & Lean Solvent: \\
\hline Vapour Fration & & 0 & 0 & 1 & 0 & 0 & 0 & 0 & 0 \\
\hline Tenperatur: & $F$ & 99.95 & -40 & 98.31 & 98.31 & 98.31 & 341.7 & 99.02 & 341.5 \\
\hline Pressure & p5la & 1053 & 1030 & 115 & 115 & 115 & 114.9 & 114.9 & 25 \\
\hline Lass Flow & bonr & 1605 & 1606 & 0.08434 & 1073 & 1601 & 4.909 & 1605 & 4.909 \\
\hline Heg FIOD & Btumr & $-6.77 E+06$ & $-6.93 E+06$ & $-1.68 \mathrm{E}+02$ & $-4.53 E+04$ & $-6.76 \mathrm{E}+06$ & $-1.57 E+04$ & $-6.78 E+06$ & $-1.5 \pi+[4$ \\
\hline Molar Eninapy & Btulibmole & $-1.51 E+05$ & $-1.54 E+05$ & $-3.25 E+04$ & $-1.51 E+05$ & $-1.51 \mathrm{E}+05$ & $-1.71 E+C 6$ & $-1.51 E+05$ & $-1.71 \mathrm{E}+05$ \\
\hline Actlal Gas FiOW & ACFM & & & 0004409 & & & & & \\
\hline Sto Gas Fiow & MUISCFD & 4.09E-01 & $\angle 09 E-0^{\circ}$ & 470E-05 & $2.73 E-03$ & $4.08 E-01$ & $8.36 \mathrm{E}-0 \mathrm{C}$ & 4 D9E-01 & $8.36 E-04$ \\
\hline PROPERTES & & & & & & & & & \\
\hline Molecular Weight & & 35.79 & 35.79 & 16.34 & 3575 & 35.75 & 53.5 & 35.79 & 53.5 \\
\hline Mass Densky & bit:3 & 67.62 & 71.36 & 0.3188 & 67.42 & 57.42 & 61.49 & 57.41 & 61,44 \\
\hline Mass Enenalpy & Btulb & -4215 & -4313 & -1990 & -4222 & -4222 & -3192 & .4219 & -3.92 \\
\hline Mass Entrogy & Btulb-F & -0.1539 & .0 .3587 & 2.467 & -0.1589 & -0.1589 & .0 .2552 & -0.1589 & -0.2552 \\
\hline Hea. Capascty & BtulibmoleF & 25.93 & 24.01 & 8.961 & 25.95 & 25.95 & 42.65 & 25.98 & 4267 \\
\hline Mass Heat Capacty & Btulb-F & 0.7247 & 0.6708 & 0.5485 & 0.726 & 0.726 & 0.7972 & 0.726 & 0.7976 \\
\hline Specinic Heat & BtulbmoleF & 25.93 & 24.01 & 8.961 & 25.95 & 25.95 & 42.65 & 25.98 & 42.67 \\
\hline Act Liq. Flow & USGPM & 2.961 & 2.806 & 0 & $1.98 \mathrm{E}-02$ & 2.961 & $9.95 E-03$ & 2.97 & $9.96 E-03$ \\
\hline Z Factor & & $9.25 \mathrm{E} \cdot 02$ & & & & & $1.16 \mathrm{E}-02$ & $1,02 E-02$ & \\
\hline $\mathrm{Cp} / \mathrm{Cr}$ & & 1.102 & 1.067 & 1.314 & 1.104 & $\because .104$ & 1.078 & 1.104 & 1.049 \\
\hline Hex of Vap. & Btulibmole & $1.16 E+04$ & $1.18 \mathrm{E}+\mathrm{QS}$ & 5742 & $1.97 E+04$ & $1.97 \mathrm{E}+04$ & $206 \mathrm{E}+04$ & $1.97 E+04$ & $2.32 \Xi+04$ \\
\hline KInematic Viscosily & CSI & 3.207 & & 2.3 & 3.287 & 3.287 & 0.5126 & 3.259 & 0.5136 \\
\hline Mass Heat of Vap. & Btulb & 323.6 & 329.4 & & & & 384.1 & & 433.5 \\
\hline Surface Tension & dyre:cm & 53.05 & 61.63 & & 53.17 & 53.17 & 35.44 & 53.11 & 3545 \\
\hline Themal Conductinty & Btunr-tr:F & $0.26 \div 1$ & 0.243 & $206 E-02$ & 0.2571 & 0.2671 & 0.1421 & 0.267 & 0.1422 \\
\hline Viscosily & $\infty$ & 3.474 & 49.64 & 117E-02 & 3.55 & 3.55 & 0.5049 & 3.52 & 0.5254 \\
\hline Mass CV & Btulb-F & 0.6574 & 0.6287 & 0.4174 & 0.6577 & 0.6577 & 0.7395 & 0.6577 & 0.7505 \\
\hline CONPONENTS & Overal Phase & lass now & & & & & & & \\
\hline lifarnane & Berr & 0.0161 & 0.0161 & 0.0808 & 0.0001 & 0.0161 & 0 & 0.0161 & 0 \\
\hline Ethene & 10\%rr & 0.0001 & 0.0001 & 0.0029 & 6 & 0.0001 & 0 & 0.0001 & 0 \\
\hline Propane & Boir & 0 & 0 & 0.0002 & 8 & 0 & 2 & 0 & 0 \\
\hline F-euane & bowr & 0 & 0 & 0 & 0 & 0 & 0 & 0 & 0 \\
\hline$n \cdot$ Gutane & oirs & 0 & 0 & 0 & 0 & 0 & 0 & 0 & 0 \\
\hline TEGIycol & Bomr & 0 & 0 & 0 & 0 & 0 & 0 & 0 & 0 \\
\hline EGyed & bons & 1123.6418 & 1123.6413 & 0 & 7.4963 & $11 \cdot 9.0539$ & 4.588 & 1123.6418 & 4.588 \\
\hline $\mathrm{H}_{2} \mathrm{O}$ & Bomr & 482.2995 & 482.2995 & 0.0005 & 3.2287 & 4861.9781 & 0.3214 & 432.2995 & 0.3214 \\
\hline $\mathrm{H} 2 \mathrm{~S}$ & bows & 0.0025 & 0.0025 & 0 & 6 & 0.0025 & 0 & 0.0025 & 0 \\
\hline$n-C^{-11}$ & bovr & 0 & 0 & 0 & 0 & 0 & 0 & 0 & 0 \\
\hline$n-C^{4} 2$ & 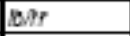 & 0 & 0 & 0 & 0 & 0 & 0 & 0 & 0 \\
\hline$n-C^{13}$ & borr & 0 & 0 & 0 & 0 & 0 & 0 & 0 & 0 \\
\hline$n-C^{-14}$ & $6 \%$ & 0 & 0 & 0 & 0 & 0 & 0 & 0 & 0 \\
\hline$n-C^{4} 5$ & owr & 0 & 0 & 0 & 0 & 0 & 0 & 0 & 0 \\
\hline$n-C^{+}-8$ & $6 \%$ r & 0 & 0 & 0 & 0 & 0 & 0 & 0 & 0 \\
\hline Total & Domr & 1605.9601 & 1605.9601 & 0.0843 & 10.7251 & 1601.0507 & 4.9094 & $1605.960^{\circ}$ & 4.9794 \\
\hline
\end{tabular}




\begin{tabular}{|c|c|c|c|c|c|c|c|c|}
\hline \multicolumn{2}{|c|}{ stream number } & 23 & 27 & $3: 1$ & 32 & 33 & 34 & 35 \\
\hline Name: & units & $\mathrm{NG}-$ veryo-V & uya-regen-r16as & NGG-LUenyo-L & NG, venya-ciLL-VMrant & NGi-Lenyo-AD50 & NGj-rrocuctstrm & sov-kero-kicn \\
\hline Vapour Fraction & & 1 & 1 & 0 & 1 & 1 & 1 & D \\
\hline Temperature & 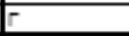 & -0.0793 & 341.0 & -0.6793 & -0.0793 & $-3 C .71$ & -20.00 & -34.05 \\
\hline Pressure & psta & 980 & 25 & 990 & 980 & 960 & 959.9 & 960 \\
\hline D.ass Flow & bohr & 95.77 & 5.816 & 0.001721 & 1096 & 55.56 & 11.16 & 298.4 \\
\hline Heat Flon & Btuinr & $-1.60 E+05$ & $-2,39 E+04$ & $-1.18 E+01$ & $-3.71 E+06$ & $-1.17 \mathrm{E}+05$ & $-8.68 E+04$ & $-3.00 \mathrm{E}+05$ \\
\hline Mnarar Fninalpy & Fituhnmole & $-3.50 F+\Pi 4$ & $-115 F+n=5$ & $-124 F+n 5$ & $-3.50 F+n$ & $-3 \Delta 1 F+n d$ & $-3 \Delta n F+n \Delta$ & $-111 \mathrm{nF}+155$ \\
\hline Actual Gas Flow & ACFM & 0.25 & 1.175 & & 5.798 & 0.1831 & 0.1462 & \\
\hline Sto Gas Flow & MMSCFD & $4.15 \mathrm{E}-02$ & $1.90 \mathrm{E}-03$ & $8.64 E-07$ & $9.60 \mathrm{E}-01$ & $3.11 E-02$ & $2.33 E-02$ & $2.49 \mathrm{E}-02$ \\
\hline \multicolumn{9}{|l|}{ PROPERTIES } \\
\hline Wolecular Welight & & 18.84 & 27.93 & 18.14 & 18.84 & 16.27 & 16.09 & 109 \\
\hline Mass Denslty & bitl3 & 5.719 & $8.25 \mathrm{E}-02$ & 65.06 & 5.719 & 5.058 & 4.694 & 47.37 \\
\hline Mass En:halpy & Btunb & -1868 & -4114 & -6851 & -1868 & -2098 & -2109 & -1006 \\
\hline Mass Entrogy & Btulb-F & 1.767 & 1.269 & 0.5391 & 1.767 & 1.98 & 2.011 & 0.41 \\
\hline Heat Capacty & Btulbmole F & 16.43 & 13.58 & 18.73 & 16.43 & 14.42 & 13.36 & 48.91 \\
\hline Mas6 Heat Capachy & Btulb-F & 0.8721 & 0.4362 & 1.032 & 0.8721 & 0.8861 & 0.8259 & 0.4435 \\
\hline Specille Heat & BtulbmolefF & 16.43 & 13.58 & 18.73 & 16.43 & 14.42 & 13.36 & $48: 91$ \\
\hline Act Llq. Flow & USGPM & & & $3.30 E-06$ & & & & 0.7854 \\
\hline$Z$ Facter & & 0.6553 & & $5.55 \mathrm{E}-02$ & 0.6553 & 0.6804 & 0.7119 & 0.4847 \\
\hline $\mathrm{C}_{\mu} \mathrm{C}_{\mathrm{V}}$ & & 2.222 & 1.103 & 1.112 & 2.222 & 2.207 & 2.057 & 1.042 \\
\hline Heat of Vap. & Btulbmole & 1959 & 30950 & $1.22 \mathbf{E}+04$ & 1959 & & & $5.87 \mathrm{E}+04$ \\
\hline Filnemstic Viscosily & cost & 0.1410 & 0.290 & $2.4 C 5$ & 0.1431 & 0.147 & 0.1575 & 2.05 \\
\hline Mass Heat $\alpha$ Vap. & Btulb & 104 & 1109 & 672.8 & & & & \\
\hline Surfase Tonsion & dynaicm & & & 70.18 & & & & 16.13 \\
\hline Therma Conductivity & Btuingr-t.t.F & $2.13 E-02$ & $1.68 \mathrm{E}-02$ & 0.3103 & $2.13 E-02$ & $2.06 E-02$ & $2.05 \mathrm{E}-02$ & $6.45 E-02$ \\
\hline Mlsnnality & $\infty$ & 1.3กF- $\cap 2$ & $11 \mathrm{nF}-\mathrm{nD}$ & $2.5,59$ & $1.31 \mathrm{~F}-\mathrm{n} 2$ & $110 F-n ?$ & $118 \mathrm{R}-\Omega 2$ & 21163 \\
\hline Mass CV & Btulb-F & 0.3924 & 0.4111 & 0.928 & 0.3924 & 0.4014 & 0.4035 & 0.4303 \\
\hline COMPONENTS & \multicolumn{8}{|c|}{ Overall Phase Ma35 fow } \\
\hline Bethane & Bomr & 63.5557 & 0.0001 & 0 & 1471.6065 & 53.9254 & 40.9099 & 16.5783 \\
\hline $\begin{array}{l}\text { Ethane } \\
\text { Prooane }\end{array}$ & $\begin{array}{l}\text { bohr } \\
\text { toms }\end{array}$ & $\begin{array}{c}10.8177 \\
6.6268\end{array}$ & $\begin{array}{l}0 \\
0\end{array}$ & $\begin{array}{l}0 \\
0\end{array}$ & $\begin{array}{l}250.4789 \\
153.4403\end{array}$ & $\begin{array}{l}1.5431 \\
0.0539\end{array}$ & $\begin{array}{l}0.1791 \\
0.0415\end{array}$ & $\begin{array}{l}7.8838 \\
3.8515\end{array}$ \\
\hline Fuutane & bir & 2.3958 & 0 & 0 & 55.2416 & 0.018 & 0.0151 & 1.1727 \\
\hline n-Eutane & binr & 2.3958 & 0 & 0 & 55.2416 & 0.015 & 0.0126 & 1.0835 \\
\hline TEGiycol & bohr & 0 & 0 & 0 & 0 & 0 & 0 & 0 \\
\hline EGiycol & bohr & 0 & 2.9083 & 0 & 0.0004 & $\overline{0}$ & 0 & $\bar{D}$ \\
\hline $\mathrm{H}_{2} \mathrm{O}$ & bohr & 0 & 2.9072 & 0.0017 & 0.0394 & 0 & 0 & 0 \\
\hline $\mathrm{H} 2 \mathrm{~S}$ & bohr & 0.0004 & 0 & 0 & 0.00184 & 0 & 0 & 0.0003 \\
\hline$n-c 11$ & Bomr & 0 & 0 & 0 & 0 & 0 & 0 & D \\
\hline$n-\mathrm{C} / 2$ & $\cos \theta$ & 0 & 0 & 0 & 0 & 0 & 0 & 0.0100 \\
\hline$n-C 13$ & bohr & 0 & 0 & 0 & 0 & 0.0001 & 0.0001 & 45.0501 \\
\hline$n 014$ & benr & 0 & 0 & 0 & 0 & 0 & 0 & 81.4763 \\
\hline$n-C 15$ & omr & 0 & 0 & 0 & 0 & 0 & 0 & 92.8942 \\
\hline$n-c 10$ & bohr & 0 & 0 & D & 0 & 0 & 0 & 12.4229 \\
\hline Total & bohr & 85.7721 & 5.8156 & 0.0017 & 1986.0572 & 55.5557 & 41.1584 & 298.4307 \\
\hline
\end{tabular}




\begin{tabular}{|c|c|c|c|c|c|c|c|c|}
\hline \multicolumn{2}{|c|}{ stream number } & 36 & 38 & 39 & 45 & 47 & 48 & 49 \\
\hline Name & Unts & RKero-Flshd-Htd & RKero-2ndFlash & $\mathrm{C}-1$ & RIKero-Flishd-Prsd-Cld & RKerofishd-Prsd & Rivero-Prsatd & Racykero \\
\hline Vapour Fraztion & & 0.0905 & 0 & 1 & 0 & 0 & 0 & 0 \\
\hline Teniperature & $F$ & 212 & 399.2 & 208.5 & $-\angle 0$ & 125 & -30.85 & .30 .34 \\
\hline Pressure & pola & 2248 & 29.01 & 29.01 & 1045 & 1050 & 959.9 & 1160 \\
\hline Mass Flow & bosr & 279.1 & 268.5 & 11.32 & 263.5 & 268.5 & 2829 & 262.9 \\
\hline Heat Flow & Btwhr & $-2.27 \mathrm{E}+05$ & $-1.83 E+c 5$ & $-1.42 \mathrm{E} \div \mathrm{04}$ & $-2.46 E+05$ & $-225 E+0 E$ & $-2.76 E+C 5$ & $-276 E+05$ \\
\hline Moly Enthapy & Btulomole & $-1.35 E+05$ & $-1.37 E+C 5$ & $-3.58 \mathrm{E}+\mathrm{C4}$ & $-1.85 E+05$ & $-1.59 \mathrm{E}+0 \mathrm{E}$ & $-26 E+C 5$ & $-126 E+05$ \\
\hline Actsal Gas Fiow & ACFIA & & & 1.623 & & & & \\
\hline Sid Gas Fin & DAMSCFD & $1.54 \mathrm{E}-02$ & $1.22 \mathrm{E}-02$ & $3.62 \mathrm{E}-03$ & $1.22 E-02$ & $1.22 \mathrm{E}-02$ & $200 \mathrm{E}-02$ & 2 COEE-02 \\
\hline PROPERTES & & & & & & & & \\
\hline Molacular Welght & & 1655 & 201.3 & 28.52 & 201.3 & 201.3 & 129.1 & 129.1 \\
\hline Mass Densty & brt:3 & 25.28 & 38.94 & $\therefore 16 \mathrm{E}-01$ & 5084 & 46.77 & 43.47 & 48.52 \\
\hline Mass Enthajpy & Bturb & -814 & -579.7 & -1254 & -916.9 & -939.4 & -975.4 & .974 .4 \\
\hline Mass Entrooy & Bturb-F & 0.5576 & 0.678 & 1.666 & 0.2343 & $C .4491$ & 0.3805 & 0.3306 \\
\hline Heat Capacity & Btulamole $\mathrm{F}$ & 94.65 & 135.5 & 14.58 & 8485 & 104.2 & 57.26 & 57.25 \\
\hline Mass Heat Capaclty & Btu/b-F & 0.5719 & 0.6732 & 0.511 & 0.4215 & c.5174 & 0.4434 & 0.4433 \\
\hline Spectilc Heat & Btulamole $\mathrm{F}$ & 94.65 & 135.5 & 14.58 & 8485 & 104.2 & 57.26 & 57.25 \\
\hline Act. LIq. Filon & USGPM & 0.7902 & 0.8597 & & 0.6565 & 6.7159 & 0.7277 & 0.727 \\
\hline ZFrator & & & & 0.9928 & 0.9169 & 6.7205 & 0.5557 & 0.6701 \\
\hline Cpick & & 1.005 & 1.839 & 1.164 & 1.114 & 1.151 & 1.036 & 1.288 \\
\hline Heat of Vạ. & Bturbande & 6. $87 \mathrm{E}+04$ & $629 E+04$ & 11120 & & & $678 E+C 4$ & \\
\hline KInematic Nscosity & cS: & & & 6.75 & 8.611 & 1.817 & 4.017 & 4.07 \\
\hline Mas5s Heat x Vap. & Bturlo & & & 369.9 & & & & \\
\hline Surtace Tenslan & dynelcm & 17.02 & 12.13 & & 3041 & 23.16 & 18.42 & 18.41 \\
\hline Themal Conouctivy & Btunnr-4t-F & & $5.74 E-02$ & $.97 E-02$ & $8.66 E-02$ & $7.55 \mathrm{E}-02$ & $6.64 \mathrm{E}-\mathrm{D2}$ & $664 \mathrm{E}-02$ \\
\hline Vscosity & $\mathrm{CP}$ & & 0.2908 & $.26 E-02$ & 7.613 & 1.361 & 3.119 & 3.163 \\
\hline Mass Cv & Btulb-F & 0.569 & 0.3661 & 0.4392 & 0.3785 & .4497 & 0.428 & 0.3441 \\
\hline COMPONENTS & Overal Prase & Mass fow & & & & & & \\
\hline Metiane & bars & 2.1651 & 0.073 & 2.8004 & 0.073 & 0.073 & 13.0886 & 13.0886 \\
\hline Ethane & basr & 3.9116 & 0.0433 & 3.8684 & 0.0433 & 0.0433 & 1.4073 & 1.4073 \\
\hline Propane & bosr & 3.0952 & 0.2333 & 2.8624 & 0.2333 & C.2338 & 0.2463 & 0.2463 \\
\hline F-Buane & b/sr & 1.0813 & 0.2179 & 0.8634 & 0.2179 & C. 2179 & 0.2208 & 0.2206 \\
\hline$n$-Butane & borr & 1.024 & 0.2632 & 0.7608 & 0.2532 & C. 2632 & 0.2655 & 0.2655 \\
\hline TEGycol & basr & 0 & 0 & 0 & 0 & 0 & 0 & 0 \\
\hline EGycal & bust & 0 & 0 & 0 & 0 & 0 & 0 & 0 \\
\hline $\mathrm{H}_{2} \mathrm{O}$ & Barr & 0 & 0 & 0 & 0 & 0 & 0 & 0 \\
\hline $\mathrm{H} 2 \mathrm{~S}$ & bors & 0.0002 & 0 & 0.0002 & 0 & 0 & 0 & 0 \\
\hline$n-\mathrm{Cl1}$ & b/rr & 0 & 0 & 0 & 0 & 0 & 0 & 0 \\
\hline$n-C 12$ & Basr & 6.0178 & 5.991 & 0.0198 & 5.991 & 5.991 & 5.991 & 5.991 \\
\hline$n-\mathrm{C} 13$ & barr & 45.05 & 44.9863 & 0.0637 & 44.9863 & 44.9863 & 44.9863 & 44.9863 \\
\hline$n-C 14$ & bisr & 81.4763 & 81.4291 & 0.0473 & 81.4291 & 81.4291 & 81.4291 & 81.4291 \\
\hline$n-\mathrm{C} 15$ & Bonr & 92.8942 & 92.8649 & 0.0292 & 92.8649 & 92.8549 & 92.8649 & $\$ 2.8649$ \\
\hline$n-C 10$ & bisr & 42.4294 & 42.423 & 0.0054 & 42.423 & 42.423 & 42.423 & 42.423 \\
\hline Total & borr & 279.1101 & 266.5255 & 11.3219 & 268.5255 & 268.5255 & 282.9223 & 232.9226 \\
\hline
\end{tabular}

Page 141 


\begin{tabular}{|c|c|c|c|c|c|c|}
\hline \multicolumn{2}{|c|}{ Stream number } & 50 & 53 & 57 & 61 & 63 \\
\hline Name & Units & Recykero-Prsd & t411 vapor & R-Kero-Fisind-Cld & Solv-RKero-Flshd & Stip Gas \\
\hline Vapour Fraction & & 0 & 1 & 0 & 0 & 1 \\
\hline Temperature & $F$ & -40 & 21.46 & 122 & 21.46 & 77 \\
\hline Fressure & psia & 1150 & 234.8 & 24.01 & 234.8 & 29.01 \\
\hline Mass Flow & binr & 2329 & 19.29 & 268.5 & 279.1 & 0.7074 \\
\hline Heat Flow & Btu'hr & $-2.77 E+05$ & $-3.54 E+04$ & $-2.27 \mathrm{E}+05$ & $-2.55 E+05$ & $-1.42 \mathrm{E}+03$ \\
\hline \begin{tabular}{|l|l|} 
Molar Enthajyy \\
\end{tabular} & Btulamole & $-1.26 E+05$ & $-3.37 E+04$ & $-1.70 \mathrm{E}+05$ & $-1.51 E+05$ & $-3.22 \mathrm{E}+04$ \\
\hline Actual Gas Fiow & ACFIA & & 0.3592 & & & 0.1453 \\
\hline Stod Gas Fow & DAMSCFD & $2.00 E-02$ & $9.57 E-03$ & $1.22 \mathrm{E}-02$ & $1.54 E-02$ & $4.02 \mathrm{E}-04$ \\
\hline \multicolumn{7}{|l|}{ PROPERTIES } \\
\hline 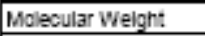 & & 129.1 & 18.37 & 201.3 & 165.5 & 16.04 \\
\hline \begin{tabular}{|l|} 
Mas5 Densily \\
\end{tabular} & Don:3 & 43.83 & 0.8952 & 46.38 & 43.19 & $8.12 \mathrm{E}-02$ \\
\hline Mas5 Entha/py & Bturlo & -978.7 & -1834 & -344.9 & -914.3 & -2008 \\
\hline Mas5 Entropy & Btulo-F & 0.3708 & 2.083 & 0.4477 & 0.3828 & 2.647 \\
\hline Heat Capachy & Btulbmole $F$ & 56.52 & 9.617 & 104.2 & 76.78 & 8.637 \\
\hline Ma55 Heat Capaclty & Btulo-f & 0.4377 & 0.5235 & 0.5176 & 0.4639 & 0.5384 \\
\hline Specilic Heat & Btuliomole $F$ & 56.52 & 9.617 & 104.2 & 76.78 & 8.637 \\
\hline Act. LIQ. Flow & USGPM & 0.7216 & & 0.7218 & 0.7222 & \\
\hline ZFactor & & 0.6745 & & $1.67 E-02$ & & 0.9956 \\
\hline Cpicr & & 1.25 & 1.37 & 1.151 & 1.027 & 1.307 \\
\hline Heat of Vap. & Btulamole & & 4126 & $5.04 E+04$ & $8.84 E+04$ & 3417 \\
\hline KInematlc Viscosity & cSt & 4.588 & 0.7237 & 1.863 & 3.395 & 8.685 \\
\hline \begin{tabular}{|l} 
Mas5 Heat of Vap. \\
\end{tabular} & Bturb & & & & & \\
\hline Surtace Tenslon & dyneicm & 18.69 & & 23.28 & 22.84 & \\
\hline Themal Conouctuly & Btunnr-at-F & $6.67 \mathrm{E}-02$ & $1.66 E-02$ & $7.67 \mathrm{E}-02$ & $7.53 E-02$ & $1.96 \mathrm{E}-02$ \\
\hline Viscosity & CP & 3.593 & $1.04 E-02$ & 1.384 & 2.621 & $1.13 \mathrm{E}-02$ \\
\hline Mas5 Cv & Bturb-F & 0.3502 & 0.382 & 0.4499 & 0.4519 & 0.4121 \\
\hline COMPONENTS & \multicolumn{6}{|c|}{ Overal Phase Mass flow } \\
\hline Methane & Bing & 13.0886 & 14.4123 & 0.073 & 2.1661 & 0.7074 \\
\hline Ethane & Bhr & 1.4073 & 3.9721 & 0.0433 & 3.9116 & 0 \\
\hline Propane & Bhir & 0.2463 & 0.7552 & 0.2338 & 3.0962 & 0 \\
\hline Futane & binr & 0.2208 & 0.0914 & 0.2179 & 1.0813 & 0 \\
\hline n-Eutane & bing & 0.2655 & 0.0595 & 0.2632 & 1.024 & 0 \\
\hline TEGycol & Bhin & 0 & 0 & 0 & 0 & 0 \\
\hline EGyycal & Bhin & 0 & 0 & 0 & 0 & 0 \\
\hline $\mathrm{H}_{2} \mathrm{O}$ & Bhir & 0 & 0 & 0 & 0 & 0 \\
\hline H2S & binr & 0 & 0.0001 & 0 & 0.0002 & 0 \\
\hline$n-C 11$ & binr & 0 & 0 & 0 & 0 & 0 \\
\hline$n \cdot C 12$ & Bhin & 5.991 & 0 & 5.991 & 6.0108 & 0 \\
\hline$n-C 13$ & binr & 44.9863 & 0 & 44.9863 & 45.05 & 0 \\
\hline$n-C 14$ & binr & 81.4291 & 0 & 81.4291 & 81.4763 & 0 \\
\hline$n-C 15$ & binr & 92.8649 & 0 & 92.8649 & 92.8942 & 0 \\
\hline$n-c 10$ & binr & 42.423 & 0 & 42.423 & 42.4294 & 0 \\
\hline Total & binr & 282.9228 & 19.2906 & 268.5255 & 279.1401 & 0.7074 \\
\hline
\end{tabular}


Attachment 2 - Proposal of FIROA Commercialization Strategy in the Environment of World Economic Crisis and Volatile Oil Pricing 
Proposal of FIROA Commercialization Strategy in the Environment of World Economic Crisis and Volatile Oil Pricing

Dr. Yingzhou Lu, GHT

January 25, 2008 -- GHT Conf114

\section{Introduction}

For energy sector and energy consuming commodities, a historical revolution is now underway. Unprecedented national energy independence programs are being proposed and/or carried out. Beside the bold renewable energy programs just declared by the new Administration, all kinds of energy-saving programs, such as hybrid and plug-in automobiles, also attracted the attention of both the government and the public. The present report would analyze and propose how to seize the opportunity for the commercialization of our new FIROA process.

\section{The Recent NGL Price Fluctuation Emphasizes the Advantage of FIROA Process in} North American Market

The Canada National Energy Board recently disclosed a report on the price of natural gas and NGL components. [1] However, the price of NG and ethane are given in C\$/GJ as gaseous fuel in Figure 37, but those of propane and butane are given in $\mathrm{C} \$ / \mathrm{m}^{3}$ or $\mathrm{C} \$ / \mathrm{bbl}$ as liquid feedstock in Figure 38 to compare with crude oil. To make better comparison between ethane and other NGL components, the price of ethane is converted into $\mathrm{C} \$ / \mathrm{m}^{3}$ as liquid at $-88{ }^{\circ} \mathrm{C}$ to compare with other NGL component price values as shown in Figure 39, wherein the price of NG retained at the same volumetric price ratio to ethane as Figure 37.

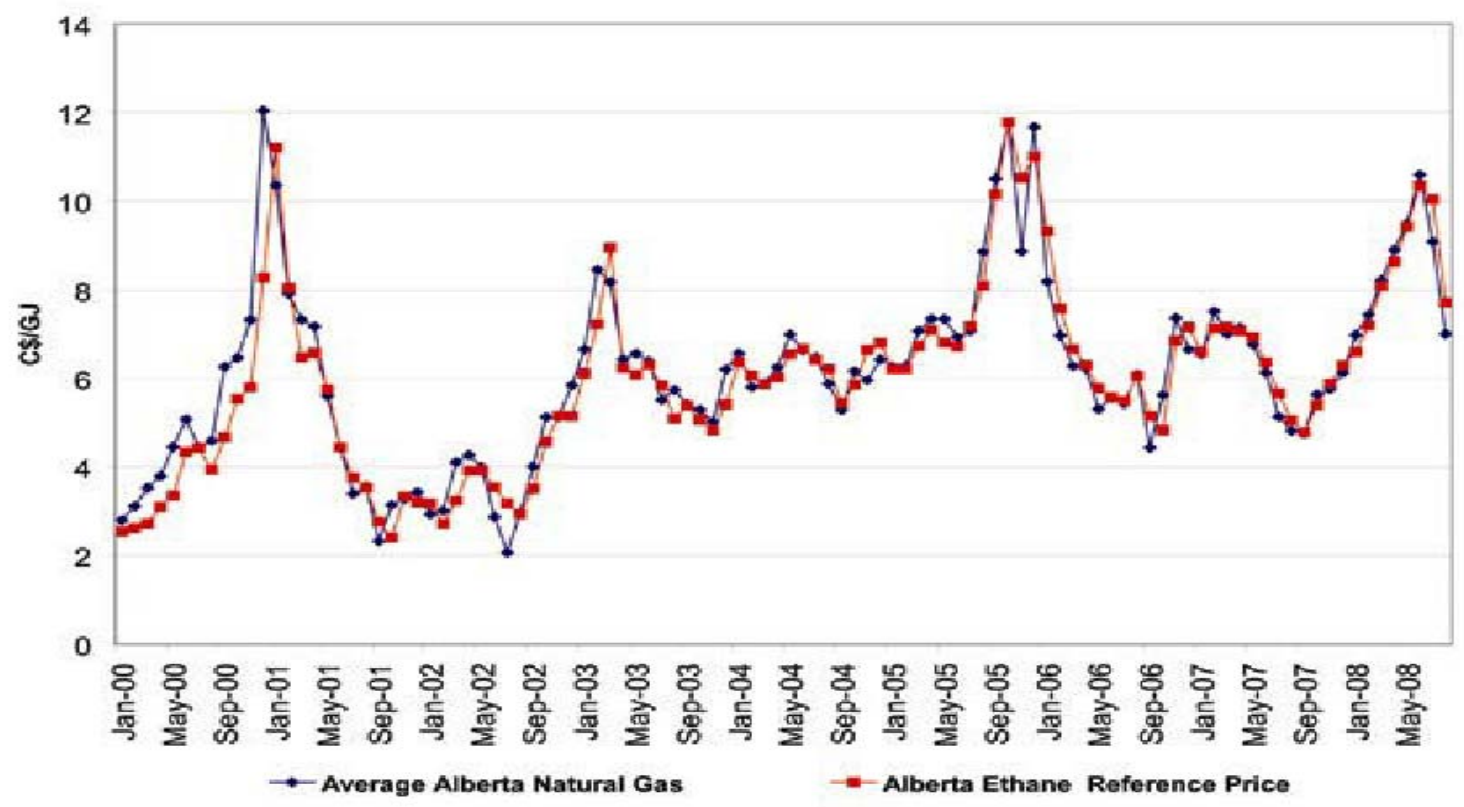

Figure 37 The Recent NG and Ethane Price 


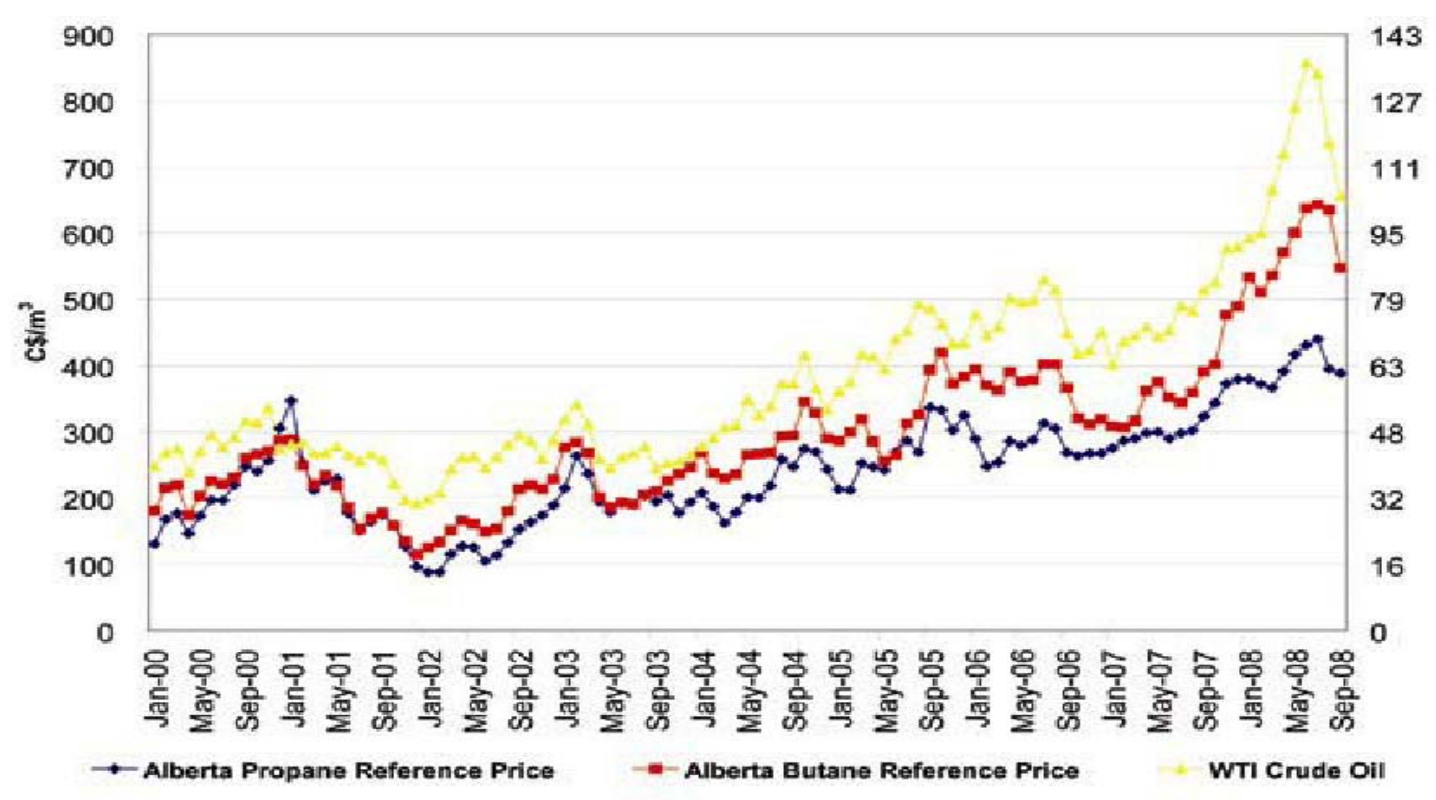

Figure 38 The Recent Propane and Butane Price

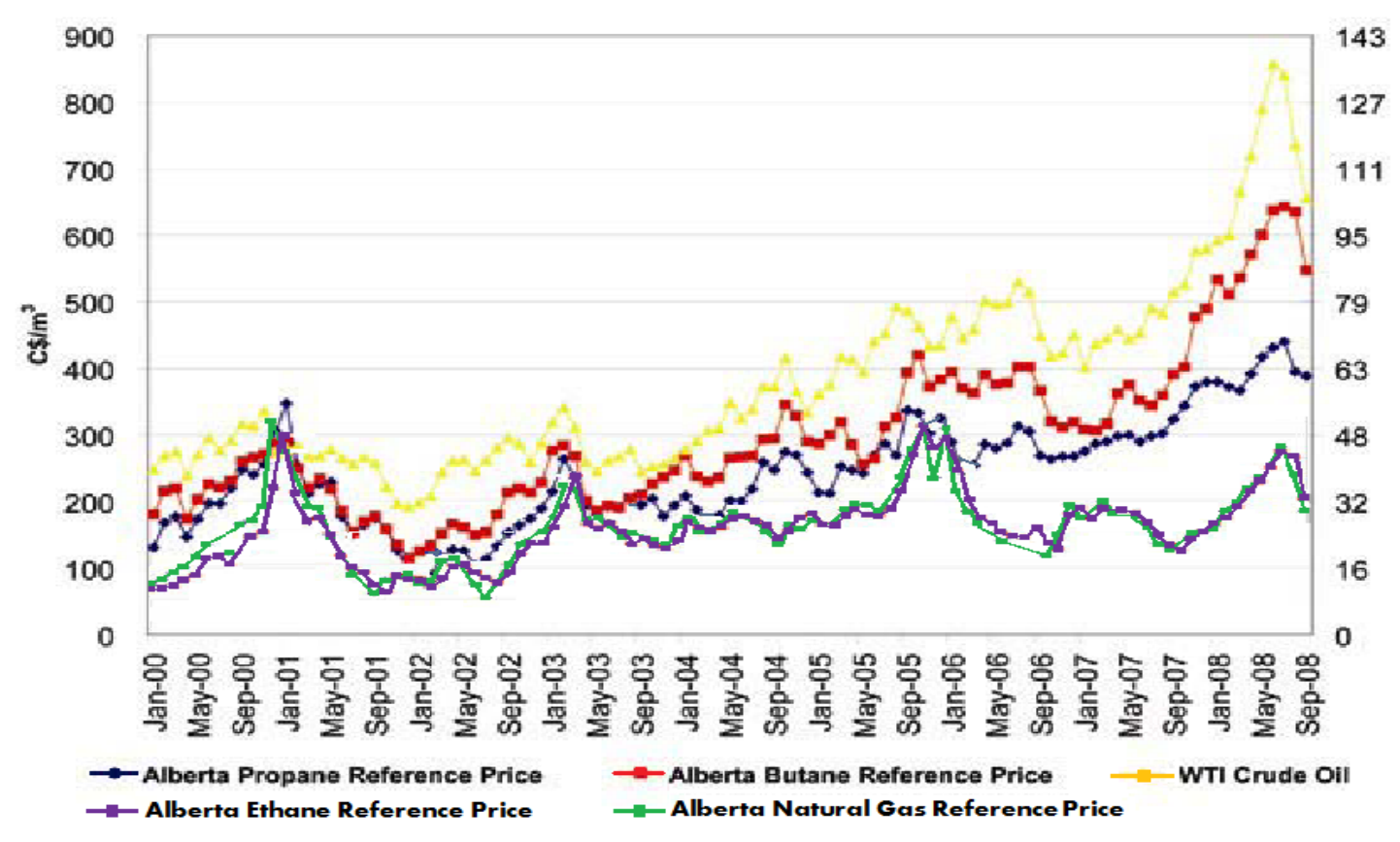

Figure 39 The Prices of Various NGL Components Compared with Crude Oil and Natural Gas

Since the price of propane and butane varied closer to the price of crude and the price of ethane closer to NG, the later varying according to its own market demands, it is obvious that when crude oil price peaked and dipped after 2005, the price gap between ethane (also NG) and other NGL components widened abruptly. Because the crude oil price will subject to many factors other than pure demand-supply relationship, e.g. the OPEC 
policy, the geo-politic conflicts, etc., the future crude price will continue to fluctuate beyond reasonable forecasts. The similar pattern may repeat again and again for next decades. Under such condition, the energy-savings advantage of FIROA over Cryogenic process will be more striking than before in near future.

For commercialization of FIROA, GHT plans the following:

1. Collect and evaluate more recent NGL price data, particular the US market data;

2. Update and evaluate more recent cryogenic energy cost data in US;

3. Update the FIROA energy-saving curves as compared with recent cryogenic energy cost data;

4. Update the long term energy-savings data by 2030 previously submitted to DOE;

5. Use the data obtained in items 3 and 4 above in GTI commercialization package.

\section{The Emerging NG Market in Mainland China and Its Implications to FIROA} Commercialization

According to the IEA "World Energy Outlook - 2008" [2], the natural gas consumption history and outlook of USA and China (Mainland) data are compared in Table 1 through 3 below.

Table 30 Comparison of Total Energy Consumption: 1990-2030 (Unit: Million TOE)

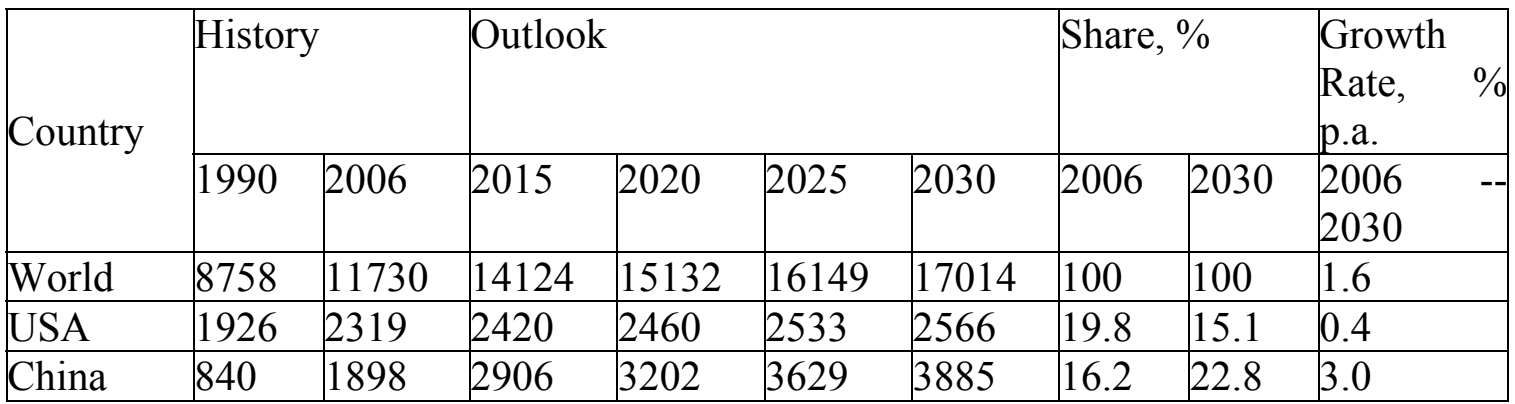

It is noticed that in Table 30 the Chinese total energy consumption will increase about $3 \%$ yearly through next two decades to fueling its economy expanding rapidly at about $8 \%$ per year. At the same time, the US energy consumption increases only about $0.4 \%$ per year. Before 2015, the Chinese energy consumption will exceed the US and become the largest energy consumer in the world.

In Table 2 the energy structure in China is presented. Coal consumption accounts for $64 \%$ of the total in 2006 and the share remains almost unchanged to $63 \%$ by 2030 . On the contrary, oil share increases from $19 \%$ to $21 \%$, and gas from $3 \%$ to $5 \%$. The most rapid increasing is "other renewable", but its share by 2030 is very small $(\sim 1 \%)$. Nuclear power is the second rapid growing sector $(7.3 \%$ p.a.), but only provides $2 \%$ energy of the total. It is also noticed that the fast increase of the share of gas will exceed biomass soon after the year 2030. It is a landmark in such a huge developing country that its energy structure has been modernized.

Table 31 Chinese Energy Consumption Structure: 1990 - 2030 (Unit: Million TOE)

\begin{tabular}{|c|c|c|c|c|c|c|c|c|c|}
\hline \multirow[t]{2}{*}{ Fuel Type } & \multicolumn{2}{|c|}{ History } & \multicolumn{4}{|c|}{ Outlook } & \multicolumn{2}{|c|}{ Share, \% } & $\begin{array}{l}\text { Growth Rate, } \\
\% \text { p.a. }\end{array}$ \\
\hline & 1990 & 2006 & 2015 & 2020 & 2025 & 2030 & 2006 & 2030 & $2006--2030$ \\
\hline Coal & 534 & 1244 & 1898 & 2139 & 2341 & 2441 & 64 & 63 & 3.0 \\
\hline
\end{tabular}




\begin{tabular}{|l|l|l|l|l|l|l|l|l|l|}
\hline Oil & 116 & 352 & 553 & 639 & 725 & 814 & 19 & 21 & 3.6 \\
\hline Gas & 13 & 49 & 103 & 132 & 161 & 188 & 3 & 5 & 3.6 \\
\hline Nuclear & 0 & 14 & 46 & 55 & 66 & 78 & 1 & 2 & 7.3 \\
\hline Hydro & 11 & 37 & 61 & 71 & 80 & 88 & 2 & 2 & 3.6 \\
\hline Biomass & 200 & 277 & 230 & 224 & 227 & 237 & 12 & 6 & 0.2 \\
\hline $\begin{array}{l}\text { Other } \\
\text { Renewable }\end{array}$ & 0 & 4 & 15 & 22 & 30 & 40 & 0 & 1 & 10.4 \\
\hline
\end{tabular}

The comparison of gas consumption between US and China is shown in Table 32. It is noticed that while the net growth rate of NG consumption in US is negligible $(0.1 \%$ p.a.) between 2006 and 2030, the Chinese gas consumption will rapidly increase 3.7 fold at an average yearly rate of $5.8 \%$ within the next two decades. The net increase of natural gas consumption per year is about $25 \%$ of the current annual gas consumption in the US. Should China decide to build new gas processing plants to feed its rapid growing domestic market, about one quarter of total capacity of the current US plants could be constructed in China within next two decades. Because there is no commercial scale NGL extraction plant inside China at the time being and the Chinese companies are eager to absorb advanced western technology, the potential market for FIROA process in China is quite attractive. An alternative forecast of the Chinese domestic gas production is given by one Chinese source in Figure 40. [3,4] The numbers roughly match those given in Table 32 where LNG imports are included.

Table 32 Comparison of Natural Gas Consumption: 1990--2030 (Unit: Million TOE)

\begin{tabular}{|l|l|l|l|l|l|l|l|l|l|}
\hline \multirow{2}{*}{ Country } & History & \multicolumn{3}{l}{ Outlook } & \multicolumn{2}{l}{ Share, \% } & $\begin{array}{l}\text { Growth Rate, } \\
\% \text { p.a. }\end{array}$ \\
\cline { 2 - 13 } & 1990 & 2006 & 2015 & 2020 & 2025 & 2030 & 2006 & 2030 & $2006--2030$ \\
\hline USA & 438 & 501 & 536 & 517 & 515 & 518 & 22 & 20 & 0.1 \\
\hline China & 13 & 49 & 103 & 132 & 161 & 188 & 3 & 5 & 5.8 \\
\hline Ratio, $\%$ & 3 & 10 & 20 & 26 & 31 & 37 & 13.6 & 25 & 580 \\
\hline
\end{tabular}

Steady Increase of China's Domestic Natural Gas Proven Reserves (trillion cubic meters)

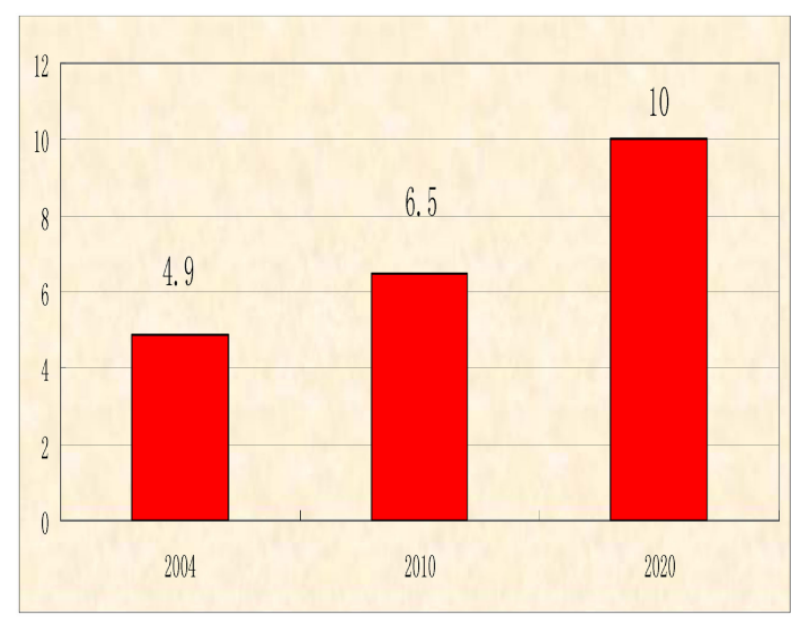

Steady Increase of China's Domestic Natural

Gas Production (100 million cubic meters)

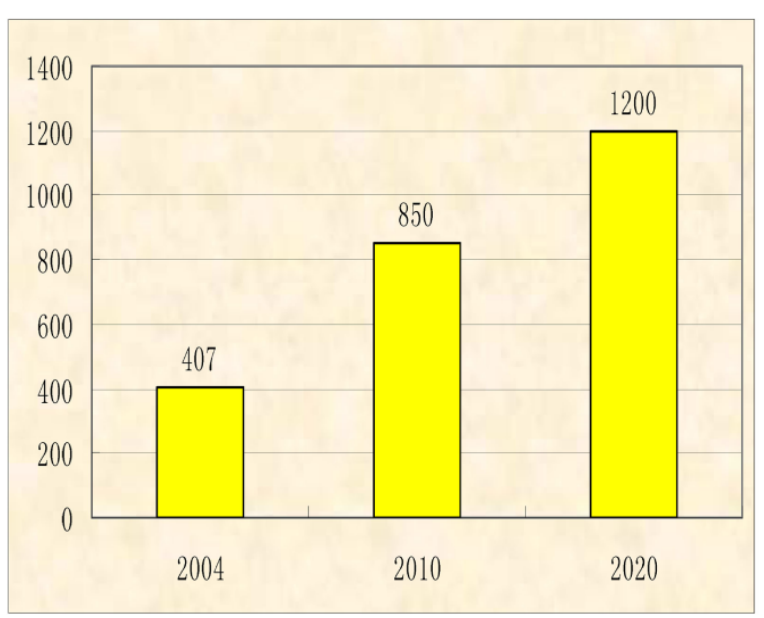


Figure 40 The Forecast of Chinese Gas Reserves and Domestic Production

The Chinese government recently declared an ambitious 4 trillion Yuan ( $\$ 570$ billion) economic stimulation plan, among which the construction of a national natural gas network is one of the priority projects. Most recently a huge Puguang gas field with proven reserve $>0.6$ trillion cubic meters has been discovered in Sichuan province, west China, and a major pipeline from Sichuan to Shanghai (over $1600 \mathrm{~km}$ ) is under construction. The total investment amounts to US\$2.6 billion. Since the sulfur content is high, six gas sweetening plants have been planned. Yet no NGL separation plan has been reported. The location of the gas field and the planned pipeline are shown in Figure 41. [5]

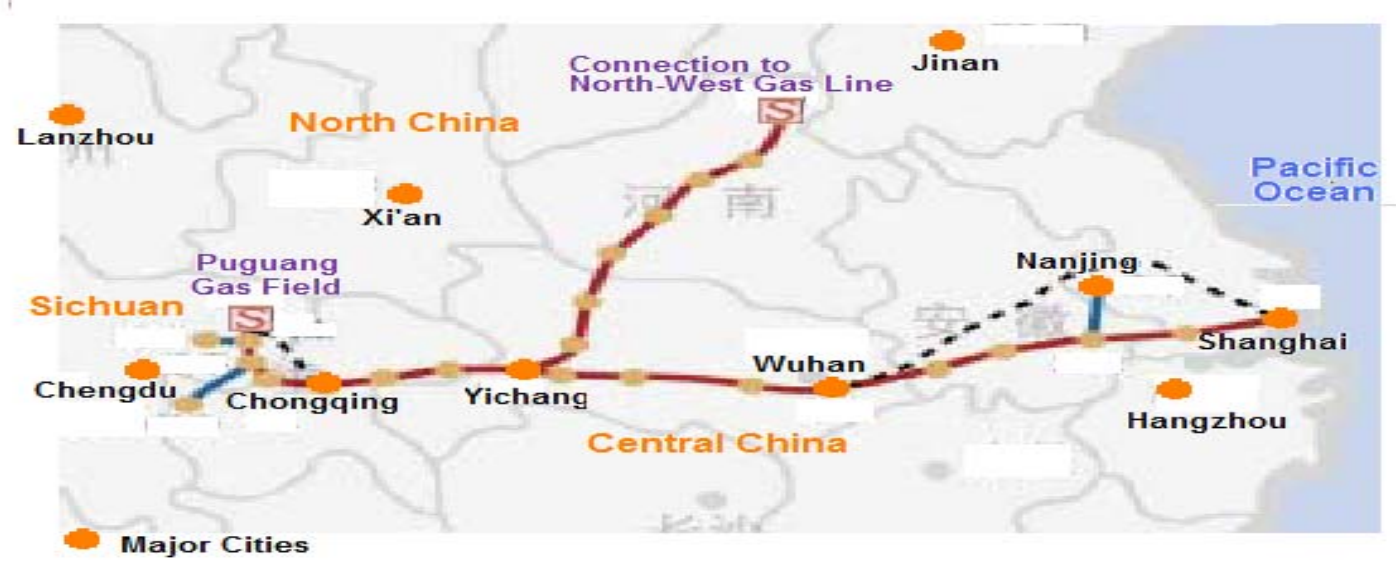

Figure 41 One of the Current Major Chinese Gas Project (Sichuan Gas Field and Pipeline)

In addition to the newly explored Sichuan gas field, there are three North China gas fields and long-distance pipelines already in operation as shown in Figure 42[3] The longest pipeline extends $4000 \mathrm{~km}$ from Urumchi (Xinjiang Province) to Shanghai, with a capacity of 17 Billion cubic meters per year. Two others extend 935 and 847 $\mathrm{km}$ from Shan'xi Province to Beijing, with capacity of 12 and 3.6 billion cubic meters per year, respectively. Another long-distance pipeline, as shown in Figure 43, extends $8678 \mathrm{~km}$ from North-west China-Kazakhstan border to Guangzhou (South China), with capacity over 20 billion cubic meters per year is under construction. [6] Beside other domestic gas field and pipelines, many international gas pipelines are under active planning stage, including Turkmen-Uzbek-Kazakhstan-China pipeline in Central Asia (total 7000 km) [7]; Burma-China pipeline in South Asia; Russia-West China and Russia-East China pipelines. The details of the entire plan are not available at present. 


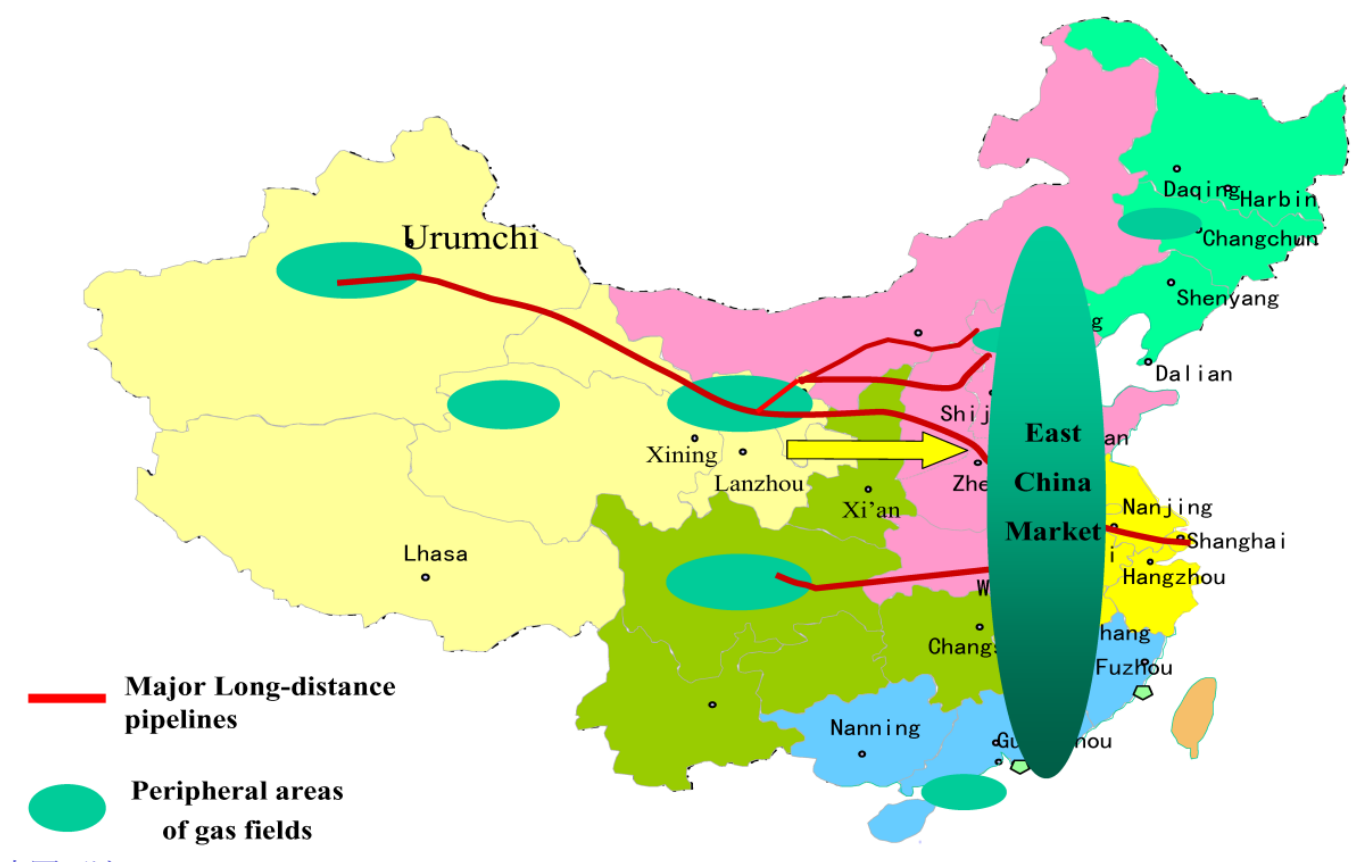

Figure 42 The Gas Fields and Major Gas Pipelines in China

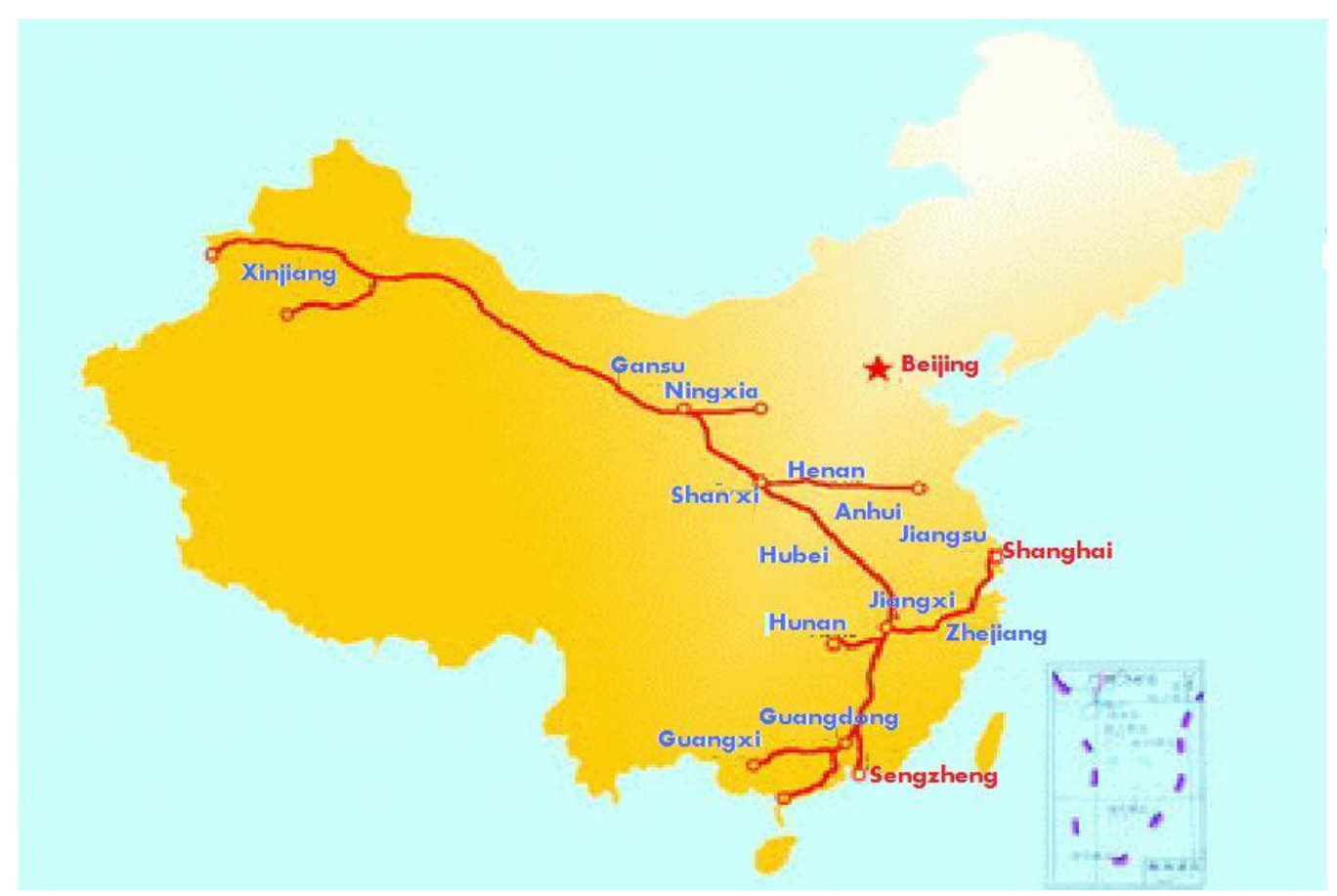

Figure 43 The New Kazakhstan-China Border- Guangdong Pipeline Under Construction

The Chinese oil and natural gas exploration and transmission are managed by a few big national companies controlled by government. The most important four companies are the China National Petroleum Corporation (CNPC), China Petroleum Chemical Corporation (SNPC_- "S" stands for "Sino"), China Ocean Oil Corporation (COOC), and 
China Petroleum Engineering Construction Corporation (CPECC). The last one is in charge of all oil and gas related engineering construction works, domestic and abroad. All major projects, however, have to be reviewed and endorsed by the China's National Development and Reform Commission (CNDRC). Certain connections with the government agencies or officers are important in marketing efforts, particularly with other competitive new technologies.

As a rapid growing developing country, China is particularly enthusiastic to introducing all kinds of new technologies. However, the protection of intellectual properties is always an issue during cooperation. For this reason, all GHT inventions patented or applied for patent in the US have already been patented or applied for patent in China to ease the commercialization of patents, including the FIROA process.

Even though the FIROA process has not been demonstrated in the US yet, the marketing efforts in China should begin as early as possible for two reasons:

1) To gather more information of the gas market, the existing Chinese relevant plans and regulations, and Chinese contacts for preparation of the FIROA commercialization efforts in vast Chinese and Asia market;

2) To explore the possibility of cooperative partner and/or support for the demonstration stage of FIROA process beyond the current DOE project immediately after the completion of current project. Since China has a hybrid economic system different from the developed countries, its behavior in present economic turmoil may be different. Such opportunity should also be examined.

\section{Proposal of FIROA Commercialization Strategy in World Economic Crisis}

Based on the above information and analyses, the following steps of preparation of FIROA Commercialization are proposed;

1. Complete the Pilot Unit field tests and gather the actual data of the FIROA, including a small teat unit of the Plate-fin type Heat Exchanger Reactor (to be provided by Chart Energy \& Chemicals)

2. Gather more necessary NGL components price data in USA and update the FIROA energy-savings curves and previous DOE long-term energy-saving forecast form to provide the basis of FIROA merits claim.

3. Prepare the first commercialization package, based on above information.

4. Identify an existing ENCAN facility that could be partially retrofitted with FIROA technology to demonstrate the merits of the new process;

5. Approach potential investors, both domestic or abroad, for construction of the demonstration unit;

6. Compete the demonstration operation and gather more actual data on FIROA merits;

7. Prepare the final commercialization package, based on above information.

8. Begin the commercialization in both domestic and abroad markets.

\section{References}

1. National Energy Board, How Canadian Market Works, www.neb-ne.gc.ca

2. IEA, World Energy Outlook - 2008, IEA Publication, 12 November, 2008

3. Su Shulin, The Development of China's Natural Gas Market, China National Petroleum Company (CNPC), June 2005 
4. Liu Chenglin, et al. Potential Recoverable Natural Gas Resources in China, Petroleum Science, May 2008, pp.83-86

5. Report on Sichuan Gas Field, www.ChinaNews.com, 08-31-2007

6. 2nd West-East Gas Pipeline in China Begins Construction, www.wenweipo.com, 11-13-2008.

7. Central Asia - China Gas Pipeline, Wikipedia, 1-10-2009. 
Attachment 3 - Corrosion Performance of Aluminum 5182 Alloy in Calcium Chloride Solution - submitted to Journal of Materials Chemistry 


\title{
Corrosion Performance of Aluminum 5182 Alloy in Calcium Chloride
}

\author{
Solution \\ Y. Cui ${ }^{1 *}$, Q.Y. $\operatorname{Han}^{2}$, M. Du ${ }^{2}$ \\ ${ }^{1}$ Department of Materials Science and Engineering, The University of Tennessee, \\ Knoxville, TN 37996, USA \\ ${ }^{2}$ Metals \& Ceramics Division, Oak Ridge National Laboratory, Oak Ridge, TN 37831, USA
}

\begin{abstract}
Corrosion behavior of aluminum 5182 was evaluated in terms of weight change in $30 \%$ calcium chloride solution at the temperature ranging from 0 to $65^{\circ} \mathrm{C}$. The test results show that $45^{\circ} \mathrm{C}$ is a threshold temperature for this material, which weight gain and loss occur above and below this temperature, respectively. This can be attributed to the different dominant reaction occurrence on the sample surface, corrosion and oxidation, which cause the weight change. Different surface morphologies, pitting and oxidation, were revealed by scanning electric microscopy (SEM).
\end{abstract}

\section{Introduction}

Aluminum alloys are widely used in the industries for their excellent mechanical and physical properties such as wide range of strength values, high strength-to-weight ratio, and excellent conductor of heat and electricity et al. [1-3]. When considering the operational performance of aluminum alloys in the engineering projects, one of the most important points to be considered is corrosion resistance [4-5]. Corrosion resistance of aluminum in aqueous media can be attributed to a rapidly formed surface oxide film. This corrosive film protects aluminum from most chemicals in various corrosive conditions. Corrosion associated with aluminum alloys is very sensitive to some aggressive anions such as chloride; the role of the aggressive anions is in the breakdown of passive oxide films on metals leading to localized corrosion. The localized corrosion mechanism is still not quite understood and still has lot of unanswered questions [6-8]. Even though the corrosion problem related to the aluminum and its alloys is one of the main concerns and the literature available on this topic is quite substantial, however, fewer reports related to the calcium chloride 
solution used in the corrosion test can be founded. Often, chloride ion-containing solution for the corrosion testing is usually used 3.5\% sodium chloride solution, including the ASTM standard requirement [9-11]. With the increase of utilizations in the industries, more and more aluminum alloys are used in the heat-exchangers which contain sodium chloride solutions.

The objective of this study is to evaluate the corrosion behavior of aluminum 5182 in terms of weight change in $30 \%$ calcium chloride solution at the temperature ranging from 0 to $65^{\circ} \mathrm{C}$. Optical light microscopy (OLM), scanning electron microscopy (SEM), and energy dispersive spectroscopy (EDS) were used for characterization of the microstructures.

\section{Experimental Procedures}

The material used in this experiment was commercial aluminum 5182, containing $4.65 \% \mathrm{Mg}, 0.37 \% \mathrm{Mn}, 0.25 \% \mathrm{Fe}, 0.25 \% \mathrm{Si}$, and less than $0.1 \% \mathrm{Cu}$. Typical microstructure of this material is that there are a lot of $\mathrm{Mg}_{2} \mathrm{Si}$ disperse second-phase in the matrix to improve mechanical and physical properties [12].

The corrosion test specimens $\left(25 \mathrm{~mm}^{2}\right)$ extracted from the test materials (thickness $=4 \mathrm{~mm}$ ) were ground and polished to a 600-grit surface finish and sharp edges were rounded. Before corrosion testing, the exposed surface and weight of the specimen were measured, and weighed to the nearest $0.00001 \mathrm{~g}$ using a high quality analysis balance (ASTM standard requires $0.001 \mathrm{~g}$ ). Corrosion immersion testing was carried out in a 30\% calcium chloride solution at the temperature from 0 to $65^{\circ} \mathrm{C}$ for 48 hours. A water bath with a temperature control accuracy of $\pm 0.1 \mathrm{C}^{\circ}$ was used for the test. The flask containing testing solution was transferred to the water bath and hold until the system came to the equilibrium at the tested temperature. Then, the specimen was placed in a 
glass holder and immersed in the test solution. No more than one specimen was placed in a test container. At the end of the test, the specimen was removed, rinsed and cleaned together with a soft nylon brush under running water, then placed in methanol with ultrasonic agitation to remove corrosion products and dry. Then, the sample weight was measured for the calculation of corrosion rate based on the following formula:

$$
\mathrm{R}=\left(\mathrm{W}_{\mathrm{i}}-\mathrm{W}_{\mathrm{f}}\right) / \mathrm{A} \times 100 \%
$$

Where:

$$
\begin{aligned}
& \mathrm{R}=\text { Corrosion Rate } \\
& \mathrm{W}_{\mathrm{i}}=\text { Initial mass of sample; } \\
& \mathrm{W}_{\mathrm{f}}=\text { Final mass of sample after corrosion testing; } \\
& \mathrm{A}=\text { area, } \mathrm{mm}^{2}
\end{aligned}
$$

\section{Results and Discussion}

Figure 1 shows the testing results obtained from immersion corrosion testing in $30 \%$ calcium chloride solutions after 48 hours. The results are summarized in the form of weight change $\left(\mathrm{mg} / \mathrm{cm}^{2}\right)$ as a function of temperature $\left({ }^{\circ} \mathrm{C}\right)$. It is to be noted that with the increase of temperature, weight loss increases gradually, and reaches the maximum value at $45^{\circ} \mathrm{C}$. Two duplicated tests were performed at this temperature and further confirmed the test results. When the temperature is above $45^{\circ} \mathrm{C}$, the sample shows no more weight loss and changes to weight gain. $45^{\circ} \mathrm{C}$ is a corner point of this plot for weight loss and weight gain, which reflects the different dominant factors controlling the weight change on the sample surface during corrosion testing. To further test the relationship between weight change and testing time, continuous corrosion testing was performed in 48,96 , 
192 and 384 hours at $25^{\circ} \mathrm{C}$ and $55^{\circ} \mathrm{C}$ respectively in $30 \%$ calcium chloride solutions. Testing procedure and examination are the same. Figure 2 shows the weight loss and weight gain as a function of exposed time at $25^{\circ} \mathrm{C}$ and $55^{\circ} \mathrm{C}$, respectively. With the increase of exposed time, the differences of weight loss and weight gain are increased as compared to the less exposed time, which means that the pitting corrosion and the compound formation increases significantly on the sample surface.

Figure 3 shows the typical morphology of the weight loss sample surface at $25^{\circ} \mathrm{C}$ in $30 \%$ calcium chloride solution after 384 hours. It is obvious that the corrosion attack occurred along the grain boundaries and covalence to form large pits with the time increase. Aluminum does not develop much pitting at lower temperature (less than $15^{\circ} \mathrm{C}$ ) because its pitting potential at the lower temperature in this solution in considerably more noble. In the presence of aggressive anions $\left(\mathrm{Cl}^{-}\right)$pitting potential is sensitive to temperature. With the increase the temperature, the weight loss increases gradually, because pitting initiation and propagation developed significantly, and the weight change (weight loss) is mainly controlled by the pitting corrosion.

As the temperature increases above $45^{\circ} \mathrm{C}$, the weight changes (weight gain) on the surface can be attributed to the formation of chemical compounds on the sample surface. A metallographic sample extracted from the transverse section after continuous corrosion testing revealed that $10 \mu \mathrm{m}$ oxide film existed next to the aluminum plate, as shown in the Figure 4. It is composed of two layers [13]; inner layer and outer layer. The inner layer next to the metal is a compact amorphous barrier layer of a thickness. Covering the barrier layer is a thicker more permeable outer layer of hydrated oxide. With the increase of testing time, the increased weight gain can be attributed to the increase of the oxide 
thickness, which will result in the loss in tensile strength and loss of effective cross section. Typical SEM morphology of 5182 sample surface after corrosion testing was revealed in Figure 5. It is to be noted that the oxide films are not uniformly distributed on the sample surface. EDS spectrum in Figure 6 (a) and (b) shows the characterization of compositions in locations A and B in Figure 5. Location A is rich of elements Ca, Al, $\mathrm{O}$ and $\mathrm{Cl}$, which reflects the composition of compound formed on the surface. Location $\mathrm{B}$ is probably the pore location with less oxide film and its composition is that of the alloy composition based on the EDS spectrum. Thus, weight gain above $45^{\circ} \mathrm{C}$ is mainly controlled by the oxidation formation.

\section{Conclusions}

Corrosion testing was performed in terms of weight change to evaluate the corrosion behavior of 5182 aluminum in $30 \%$ calcium chloride solutions at the temperature ranging from 0 to $65^{\circ} \mathrm{C}$. The corrosion test results reveal that weight loss and weight gain as a function of temperature and exposure time occurred below and above $45 \mathrm{C}$, respectively. This can be attributed as results of pitting corrosion and compound formation on the sample surface. Metallurgical evaluation shows that there is an oxide films rich of $\mathrm{O}, \mathrm{Ca}, \mathrm{Cl}$, and $\mathrm{Al}$ elements on the surface of weight gain test samples, which can be regarded as the compounds of corrosion products.

\section{References}

1.

2.

3. 
4. J.R. Davis. Corrosion of Aluminum and Aluminum Alloys, ASM International, First Edition, 1999.

5.

6. E. E. Stansbury and R.A.Buchanan: 'Fundamentals of Electrochemical Corrosion', 2000, ASM International, 277-279.

7. A. John Sedriks: 'Corrosion of Stainless Steels', 2nd Edn, 16-17; 1996, A WileyInterscience Publication, John Wiley \& Sons, Inc.

3. H. E. Canavan, S. Y. Yu, P. M. Natishan, D. E. Ramaker, and William E. O'Grady, "Surface analysis of the oxide film on aluminum single crystals exposed to chloride solution,” U. S. Naval Research Laboratory, Technical Report.

8. D.A. Jones. Principles and prevention of Corrosion, Macmillan Publishing Company, 1992, pp. 209.

9. G44, Practice for evaluating stress corrosion cracking resistance of metals and alloys by alternate immersion in $3.5 \%$ sodium chloride solution.

10. Test method for determining susceptibility to stress corrosion cracking of highstrength aluminum alloy products.

11.

12. J.R. Davis, Davis \& Associates, ASM Specialty Handbook, Aluminum and Aluminum Alloys, ASM International, Third Edition, May 1994, pp. 494.

13. M.S. Hunter and P. Fowle, J. Electrochem. Soc., Vol. 103, 1956, p.482. 


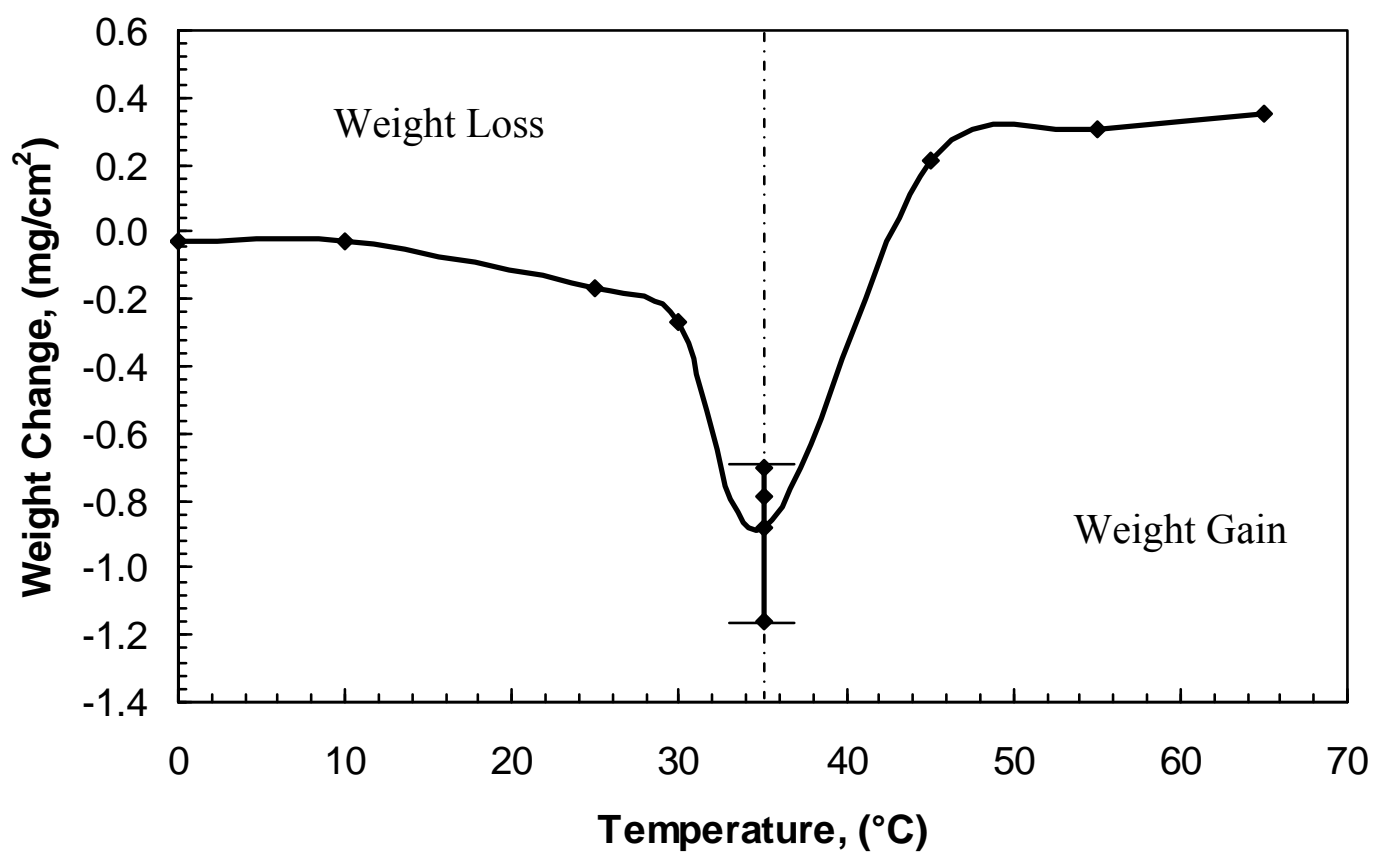

Figure 1. Weight change of aluminum 5182 is as a function of temperature at $30 \%$ calcium chloride for 48 hours. 

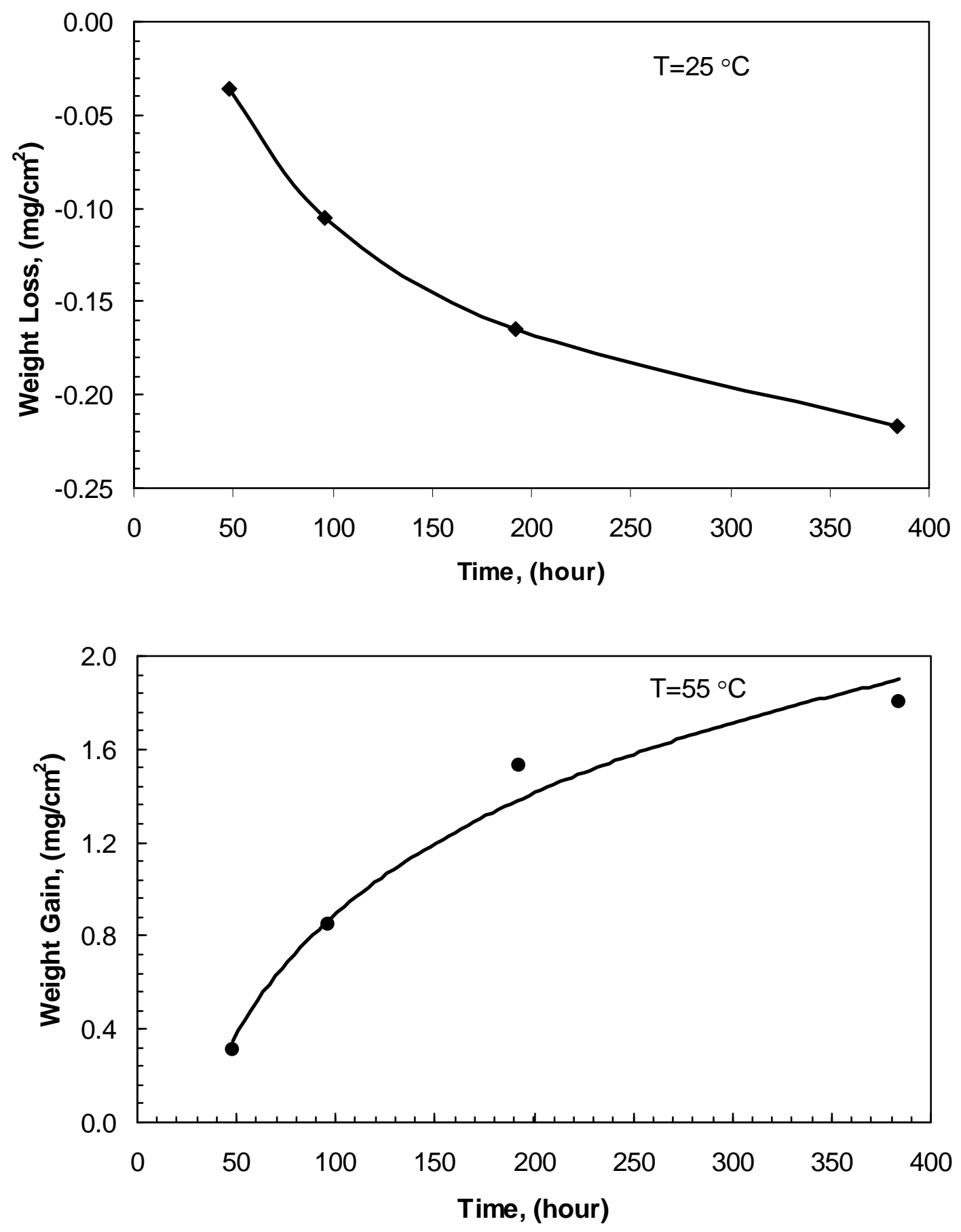

Figure 2. Weight loss at $25^{\circ} \mathrm{C}$ and weight gain at $55^{\circ} \mathrm{C}$ is as a function of exposed time at $30 \%$ calcium chloride. 
Final Progress Report

DE-FC36-03G013150

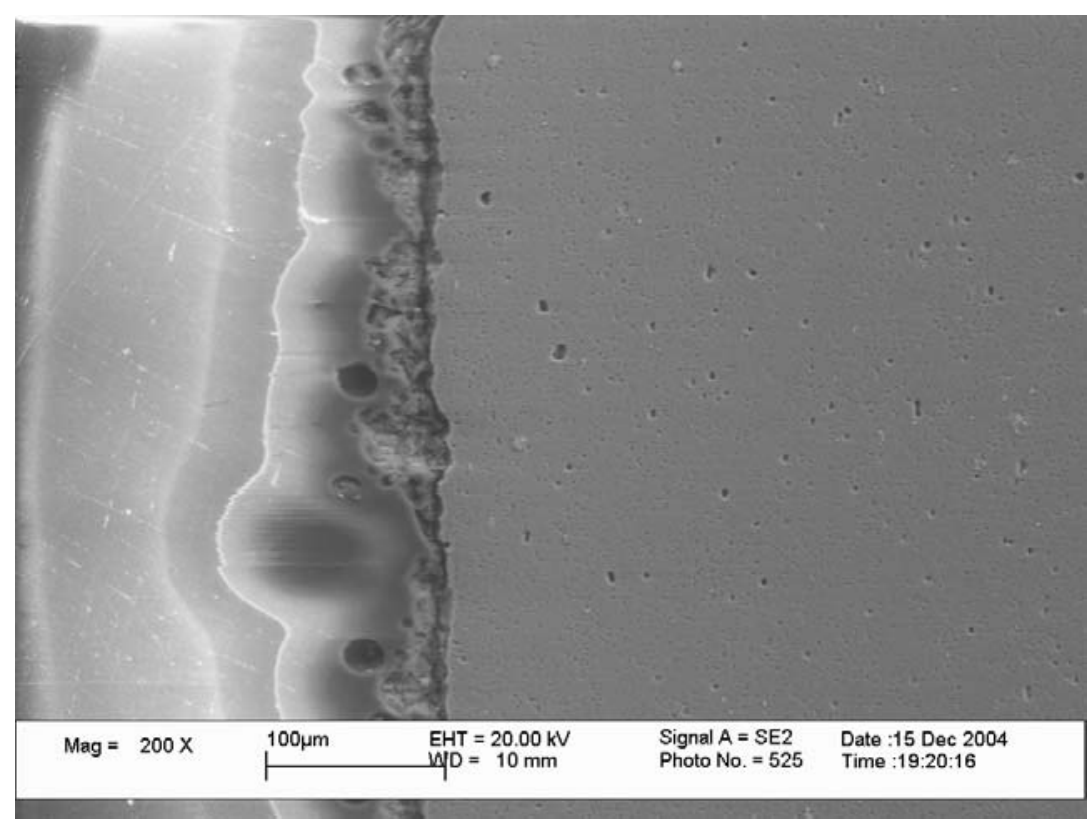

Figure 4. SEM depiction of sample transverse section. 


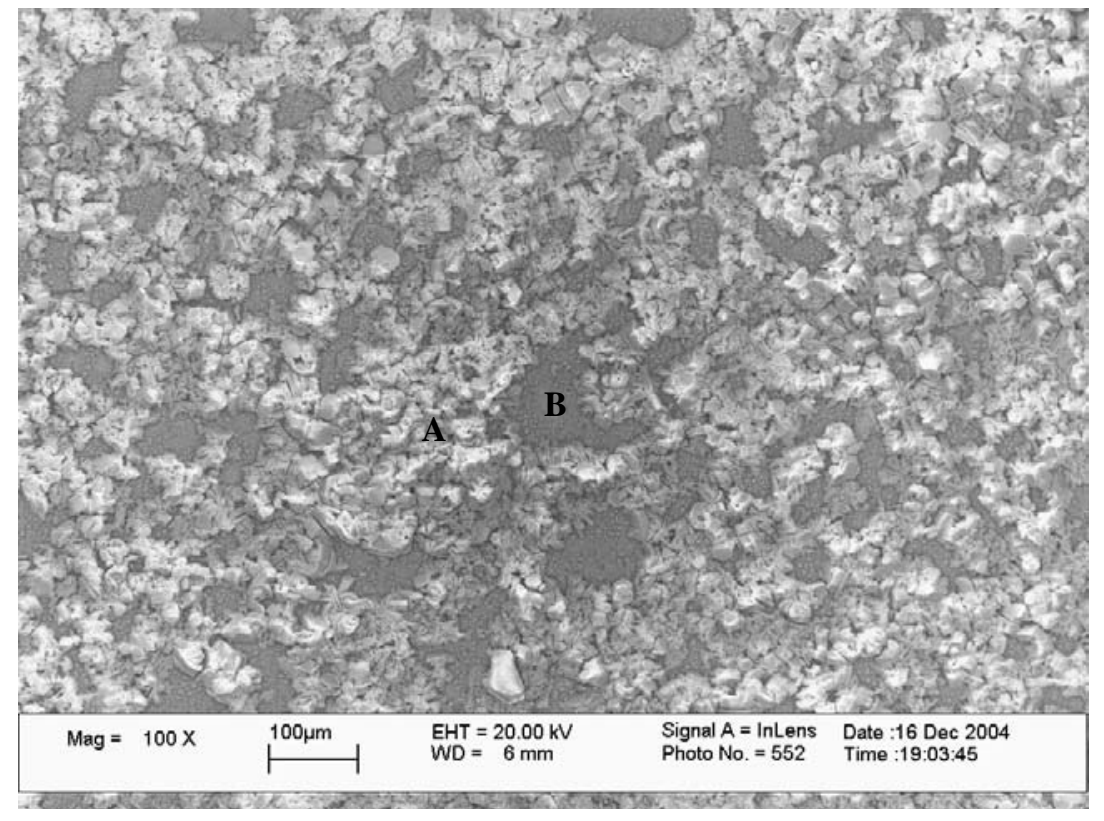

Figure 5. SEM surface morphology of 5182 sample after 384 hours in 30\% calcium chloride solution. 


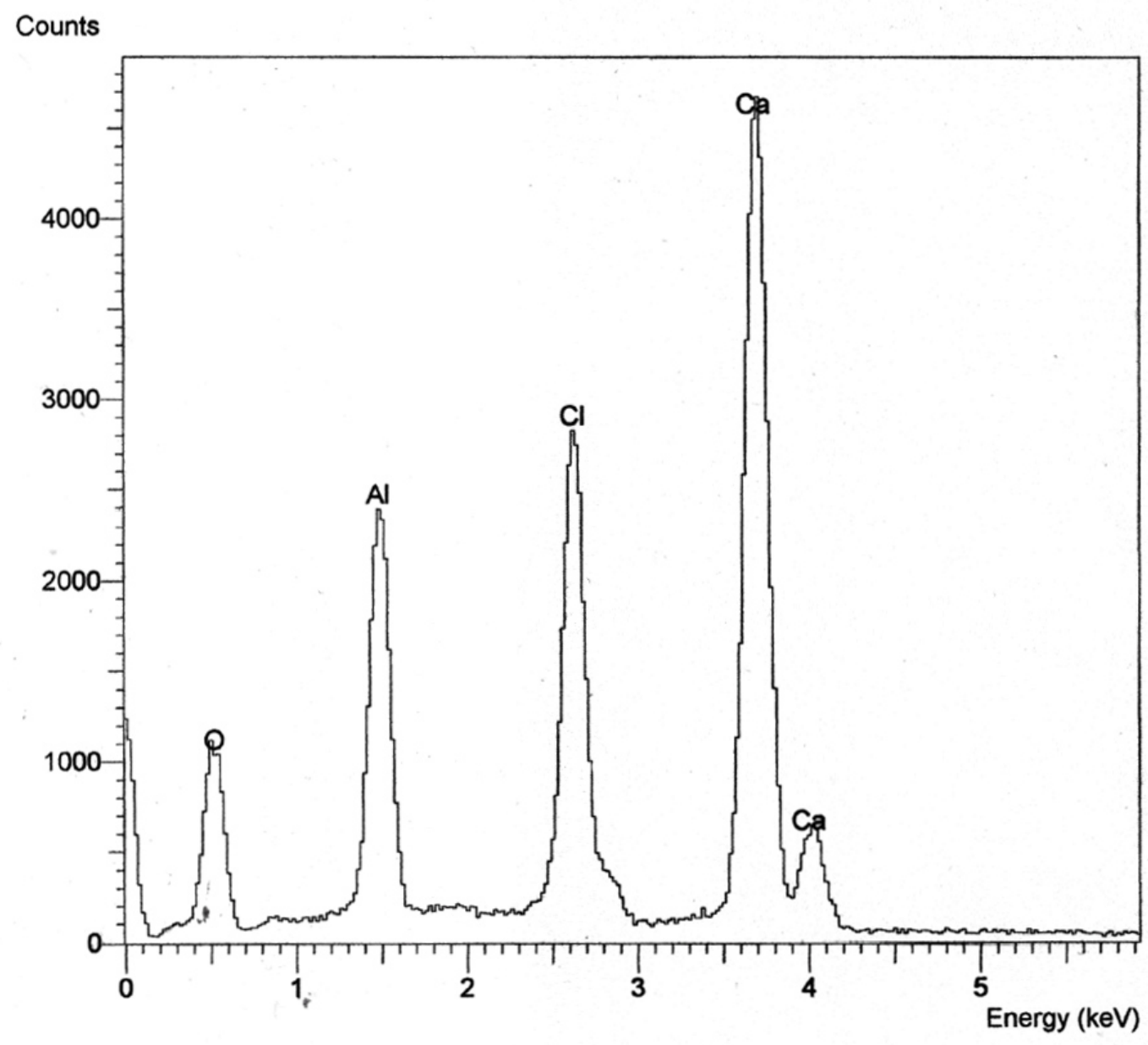

Figure 6(a). EDS spectrum for location A in Figure 5. 


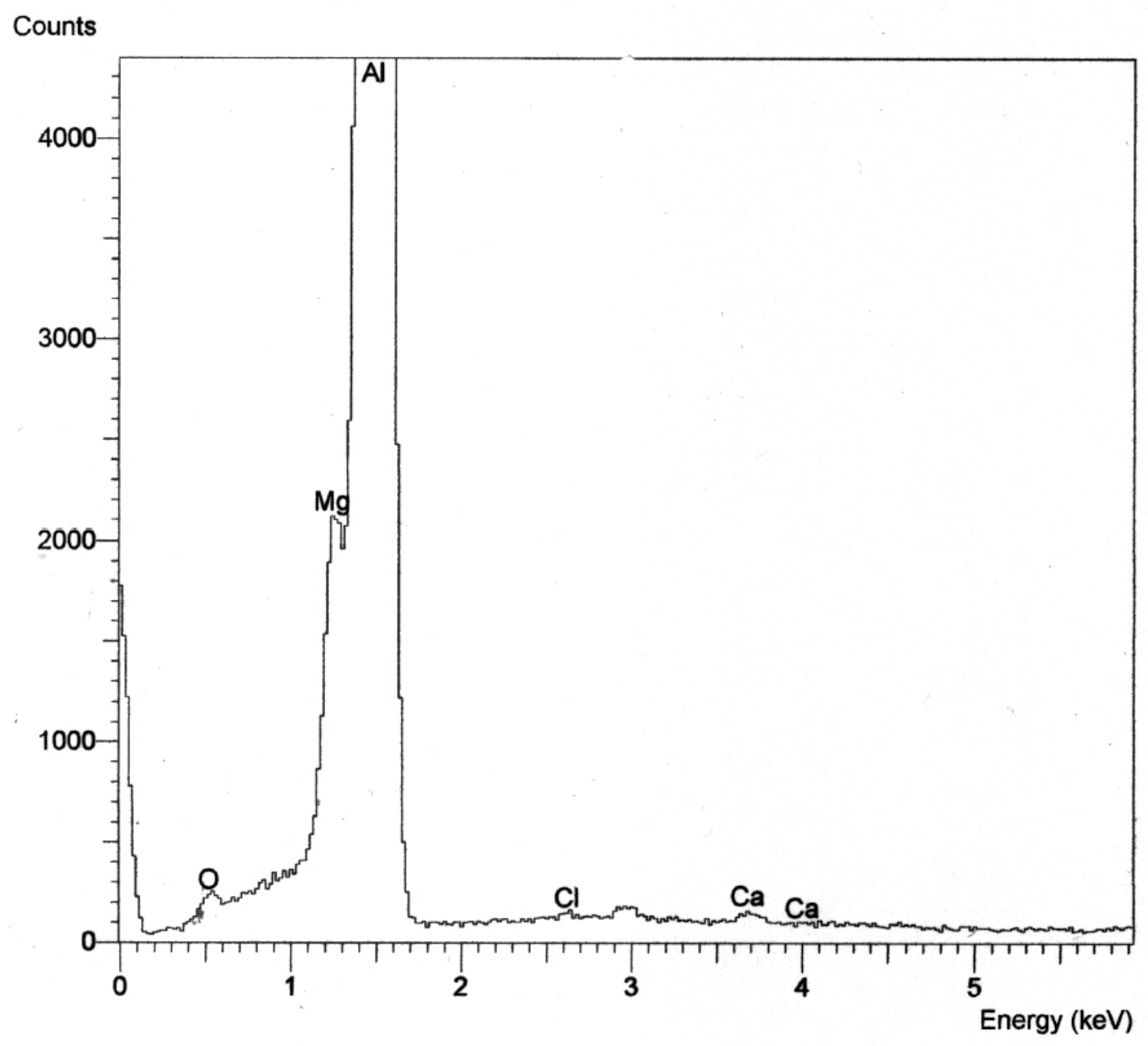

Figure 6(b). EDS spectrum for location B in Figure 5. 
Techno-Economic Evaluation of an Improved and Energy Efficient Natural Gas Liquid (NGL) Removal Process

Aqil Jamal, Howard Meyer, and Dennis Leppin Gas Technology Institute Yingzhong Lu, Green Hi-Tek Javad Abbasian, Illinois Inst. Of Technology

April 11-14th, 2005 2005 AIChE Spring National Meeting, Atlanta, GA 


\section{Outline}

> Project Objectives, Scope and Motivation

$>$ Background

> Technical Approach

$>$ Accomplishments

$>$ Conclusions

$>$ Future Work 


\section{Project Objectives}

$>$ Develop a new low-cost and energy efficient NGL recovery process (as per U.S. patent $6,553,784$ ) through combination of theoretical, bench-scale and pilot-scale testing which will ensure a supply of low cost chemical feedstocks for the U.S. petrochemical industry 


\section{Project Scope}

$>$ Phase -1 ( 1 yr)

$>$ develop a preliminary computer simulation model to verify the process performance and compare it with conventional cryogenic technology

$>$ Conduct techno-economic evaluation of the process

$>$ Phase -2 (4 yrs)

$>$ design, construct and operate bench and pilot-scale units to demonstrate the technology and develop scale-up data

$>$ conduct critical R\&D related to the development of the new process

$>$ update computer simulation model based on bench and pilot-scale test data

$>$ perform market study, develop process economics and commercialization path 


\section{Project Motivation}

$>$ NGL extraction industry provide the majority of the petrochemical feedstocks

$>$ In times of high natural gas prices in relation to hydrocarbon liquids prices, these plants will either shutdown or leave NGLs in the natural gas leading to supply shortage and high prices for petrochemical feedstocks

$>$ New technology is needed to enable stable and economical supply of chemical feedstocks 


\section{Outline}

> Project Objectives, Scope and Motivation

$>$ Background

$>$ Technical Approach

> Accomplishments

$>$ Conclusions

$>$ Future Work 
$>$ Mixture of $\mathrm{C}_{2}+$ hydrocarbons extracted from natural gas

$>$ Primarily used as chemical feedstocks in oil refining and petrochemical industries with some use as home heating fuel

$>$ Chemical feedstock usage

$>$ Ethane, propane, normal butane and natural gasoline for ethylene production

$>$ Isobutane for propylene oxide and MTBE production

$>$ Normal butane for acetic acid and maleic anhydride production

$>$ Refinery-produced propylene and butylenes consumed by chemical companies. 


\section{NGL Market}

$>$ NGL production in 2004

> Worldwide - 6.3 MMbpd

> U.S. $-1.9 \mathrm{MMbpd}$ (the largest producer in the world)

$>$ To be economical the value of NGL extracted > fuel shrinkage + processing cost

$>$ For a new plant process margins must also be enough to justify the capital expenditure

$>$ Rising NG prices make high $\mathrm{C}_{2}$ recoveries uneconomical 


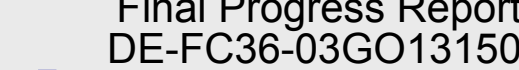 A Fluctuating NGL Market}
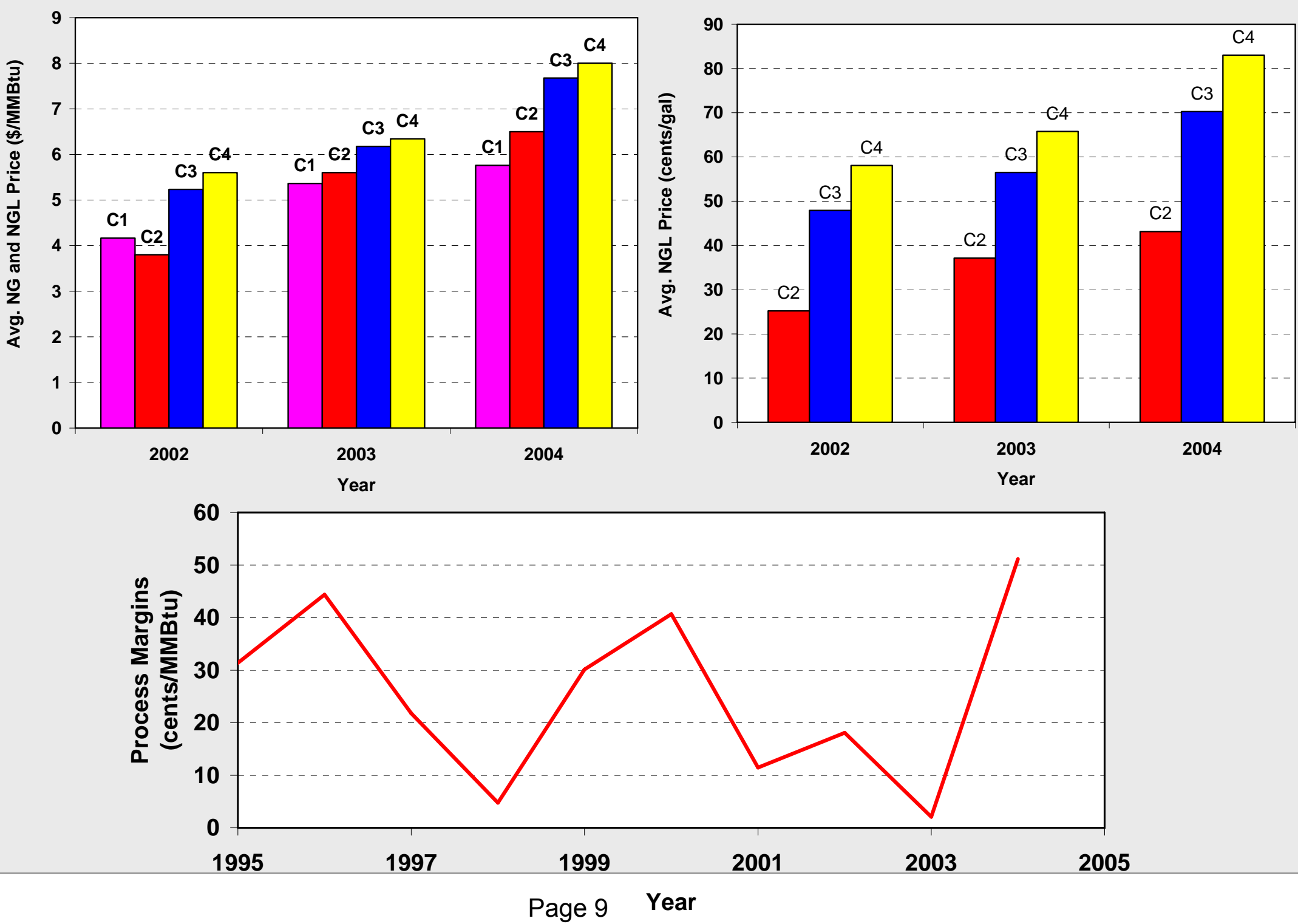


\section{Rising NG Prices Make High $\mathrm{C}_{2}$ Recoveries Uneconomical}

2003 Mont Belvieu Avg. NGL Prices

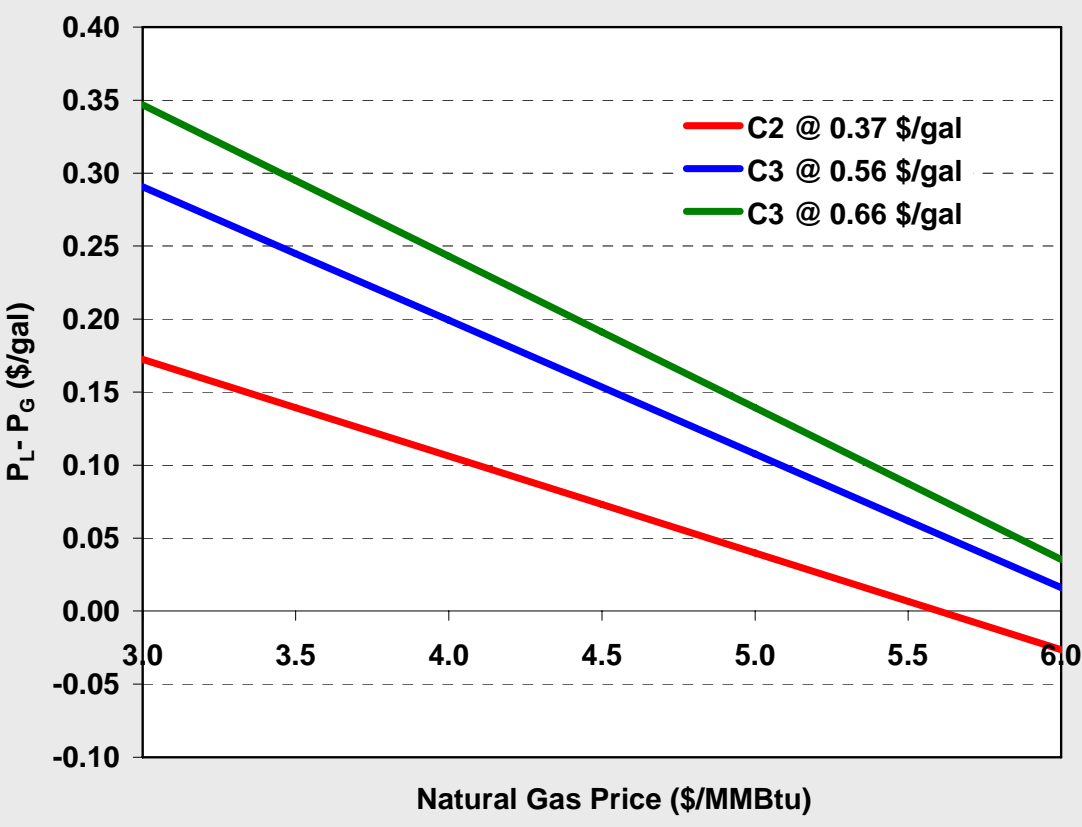

2004 Mont Belvieu Avg. NGL Prices

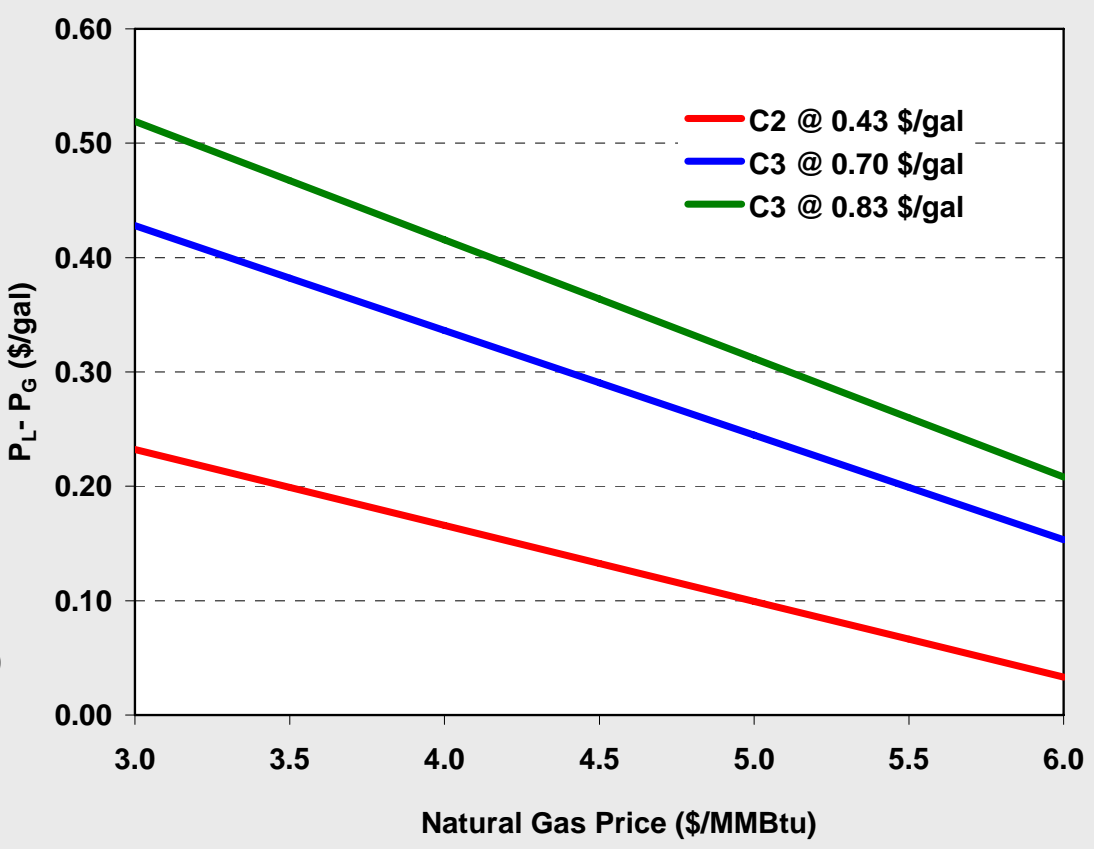




\section{NGL Process Selection Criteria}

$>$ Process selection depends on the:

$>$ Gas richness

$>\mathrm{C}_{2} \& \mathrm{C}_{3}$ recoveries

$>$ Pipe line pressure and quality specifications

$>$ Means for moving NGLs to market

$>$ Capital and operating cost depends on the process selected 


\section{NGL Recovery Technologies}

$>$ Straight Refrigeration Process

$>$ Used for hydrocarbon dewpointing

$>$ Recoveries: $\mathrm{C}_{3}=70-90 \%, \mathrm{C}_{4}+=90-95 \%$

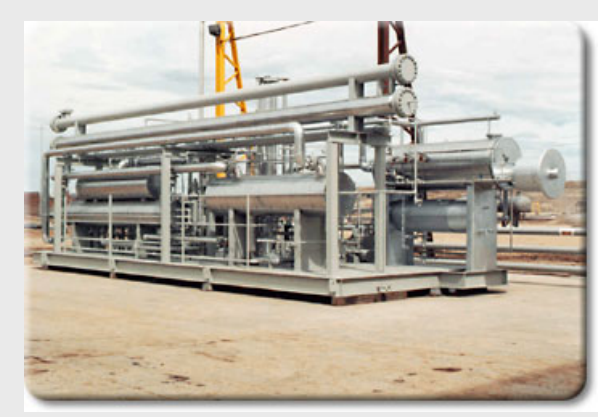

$>$ Refrigerated Lean Oil Absorption Process

$>$ Recoveries: $\mathrm{C}_{2}=40-50 \%, \mathrm{C}_{3}=80-90 \%, \mathrm{C}_{4}+=100 \%$

$>$ Most plants were built in 1960s

$>$ Cryogenic Expansion Process (CEP)

$>$ Recoveries: $C_{2}=80-95 \%, C_{3}=90-100 \%, C_{4}+=100 \%$

$>$ Commonly used worldwide

$>$ Energy intensive

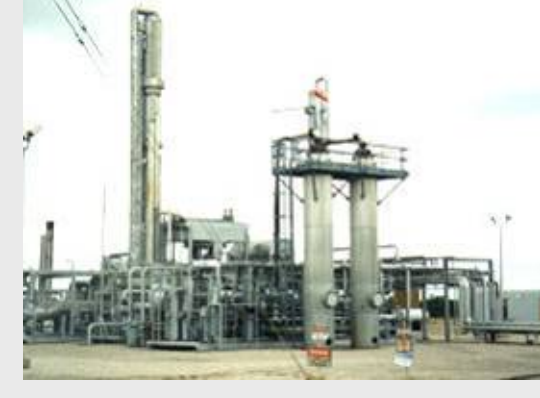




\section{Outline}

$>$ Project Objectives, Scope and Motivation

$>$ Background

> Technical Approach

$>$ Accomplishments

$>$ Conclusions

$>$ Future Work 


\section{Technical Approach}

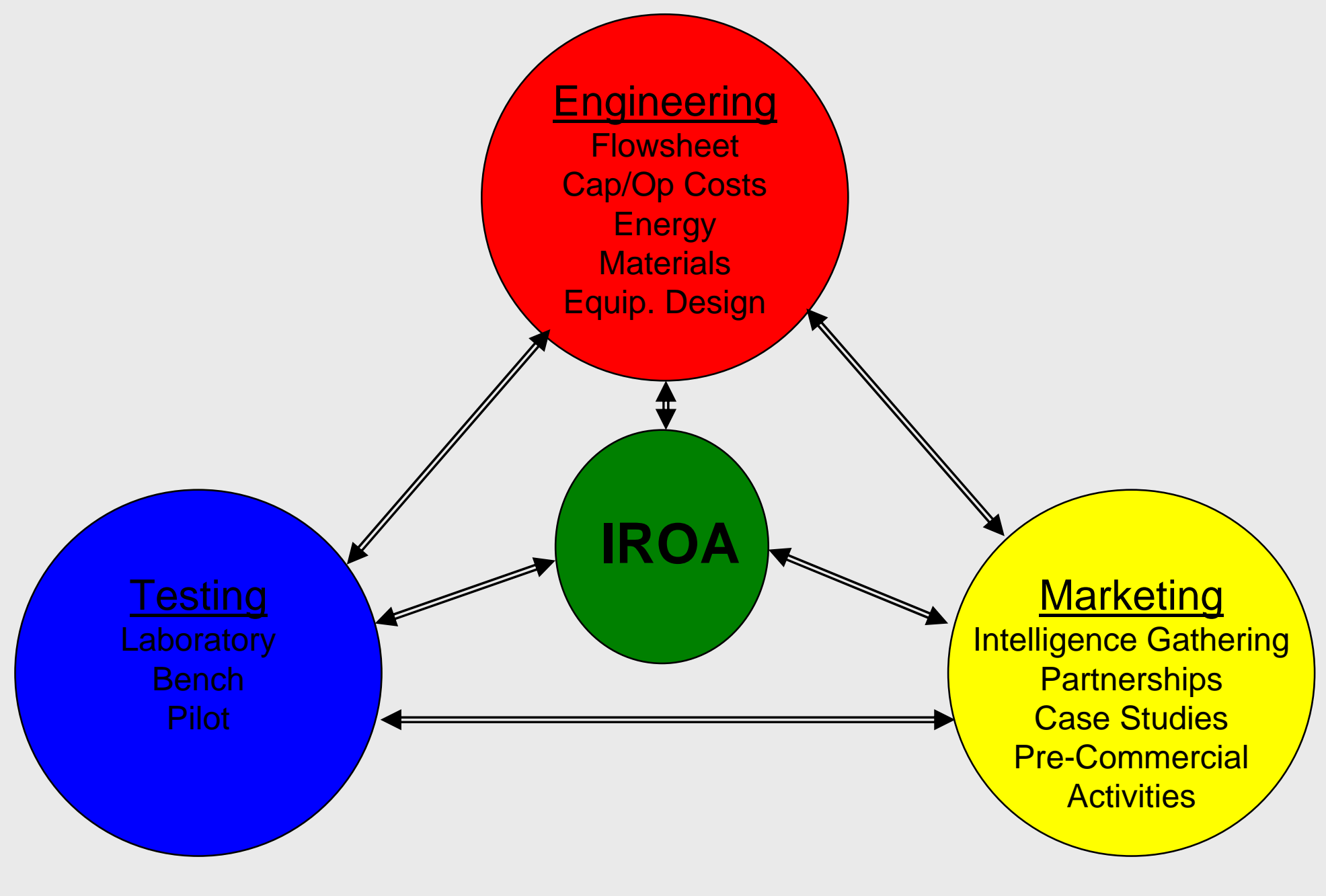

Page 14 


\section{Outline}

> Project Objectives, Scope and Motivation

$>$ Background

> Technical Approach

$>$ Accomplishments

$>$ Conclusions

$>$ Future Work 


\section{IROA Technology}

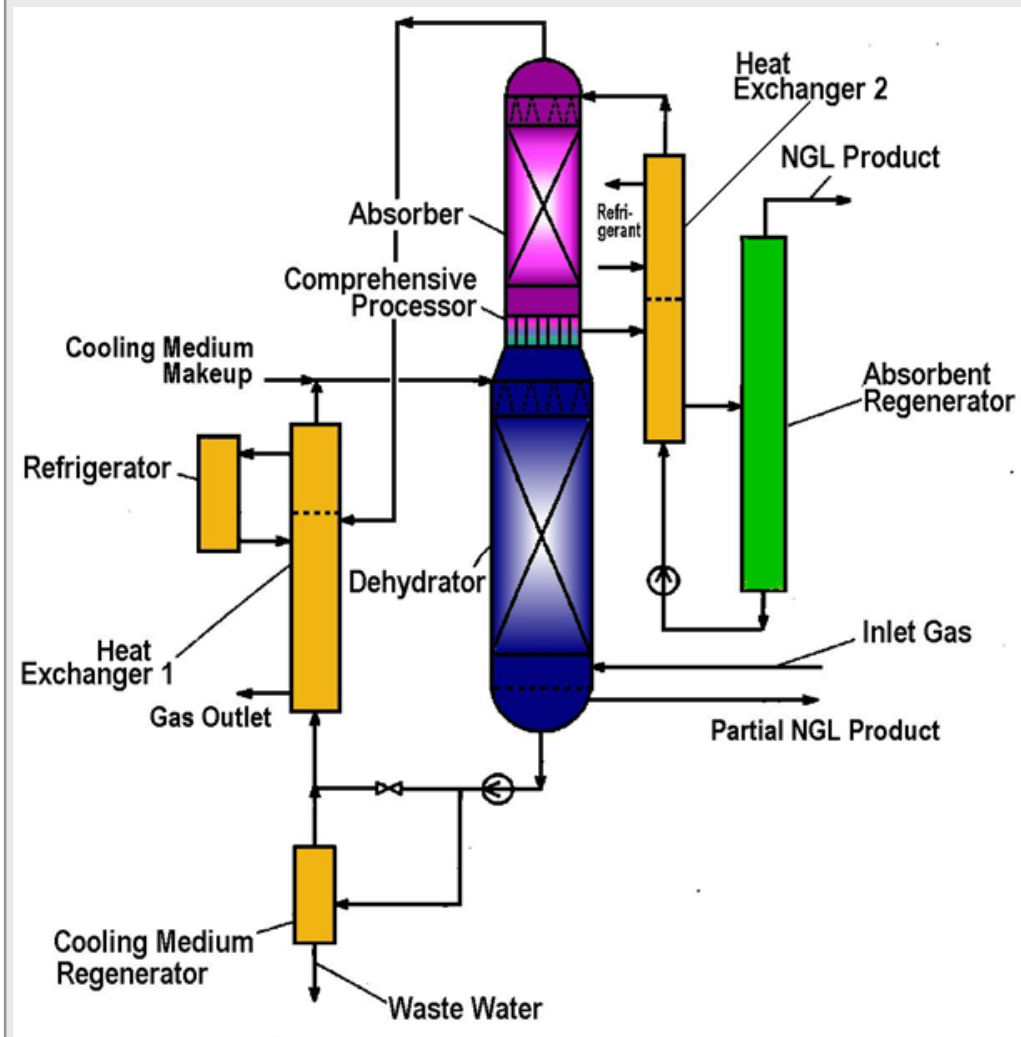

US Patent \# 6,553,784
> Main Features

$>$ Integrates dehydration of feed gas with NGL recovery

> Uses a single absorbent

$>$ Absorber runs isothermally

$>$ Better heat integration by recycling lean oil within internal heat recovery loops

$>$ More compact plant because IROA uses high efficiency packed columns and plate-fin type compact heat exchangers 


\section{IROA Technology}

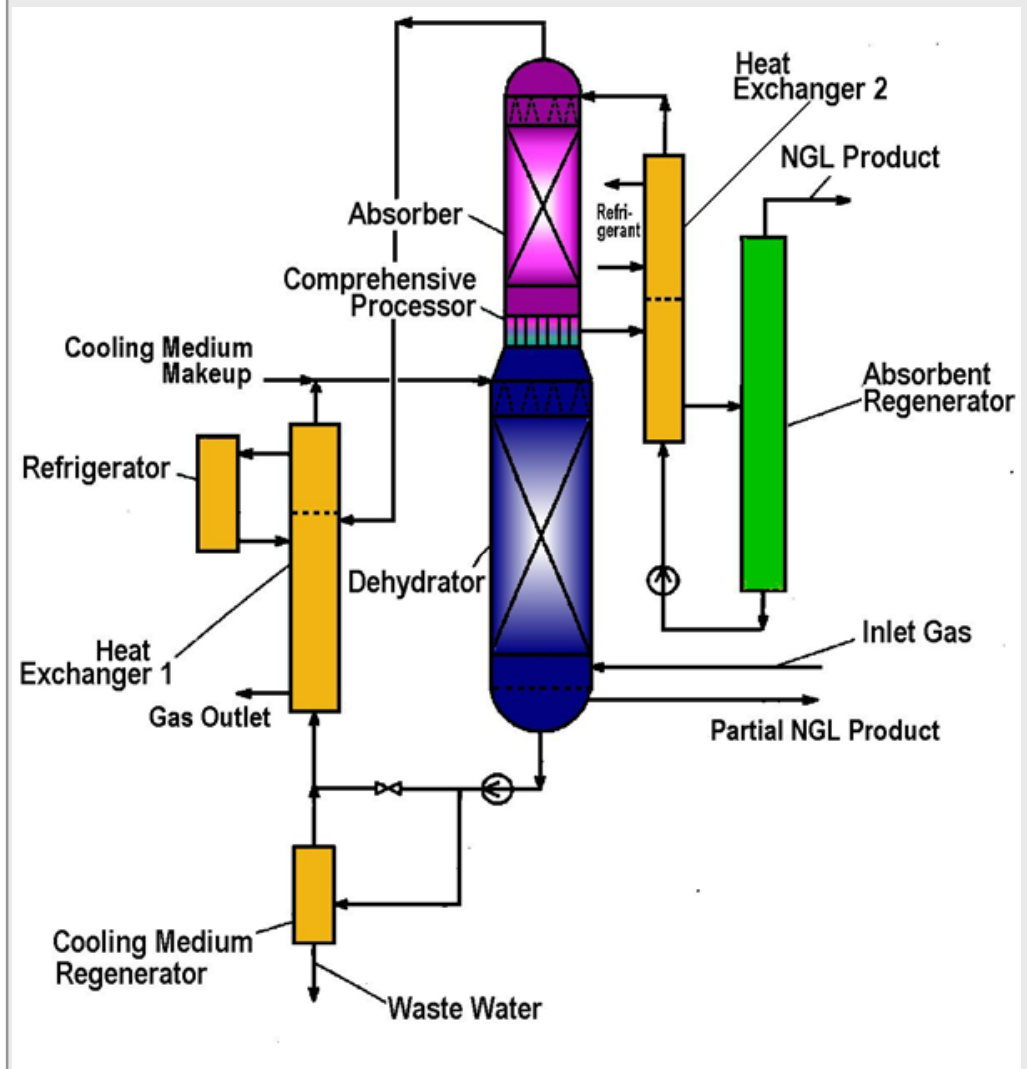

US Patent \# 6,553,784
$>$ Main Advantages

$>$ NGLs are recovered at moderate refrigeration temperatures ( - $\left.40^{\circ} \mathrm{F}\right)$

$>$ Negligible pressure losses

$>\mathrm{C}_{2}$ rejection is achieved by simply reducing absorbent flowrate

$>$ No separate dehydration unit required

$>$ More $\mathrm{CO}_{2}$ tolerant 


\section{Base Case Cryo Process}

Final Progress Report

DE-FC36-03G013150

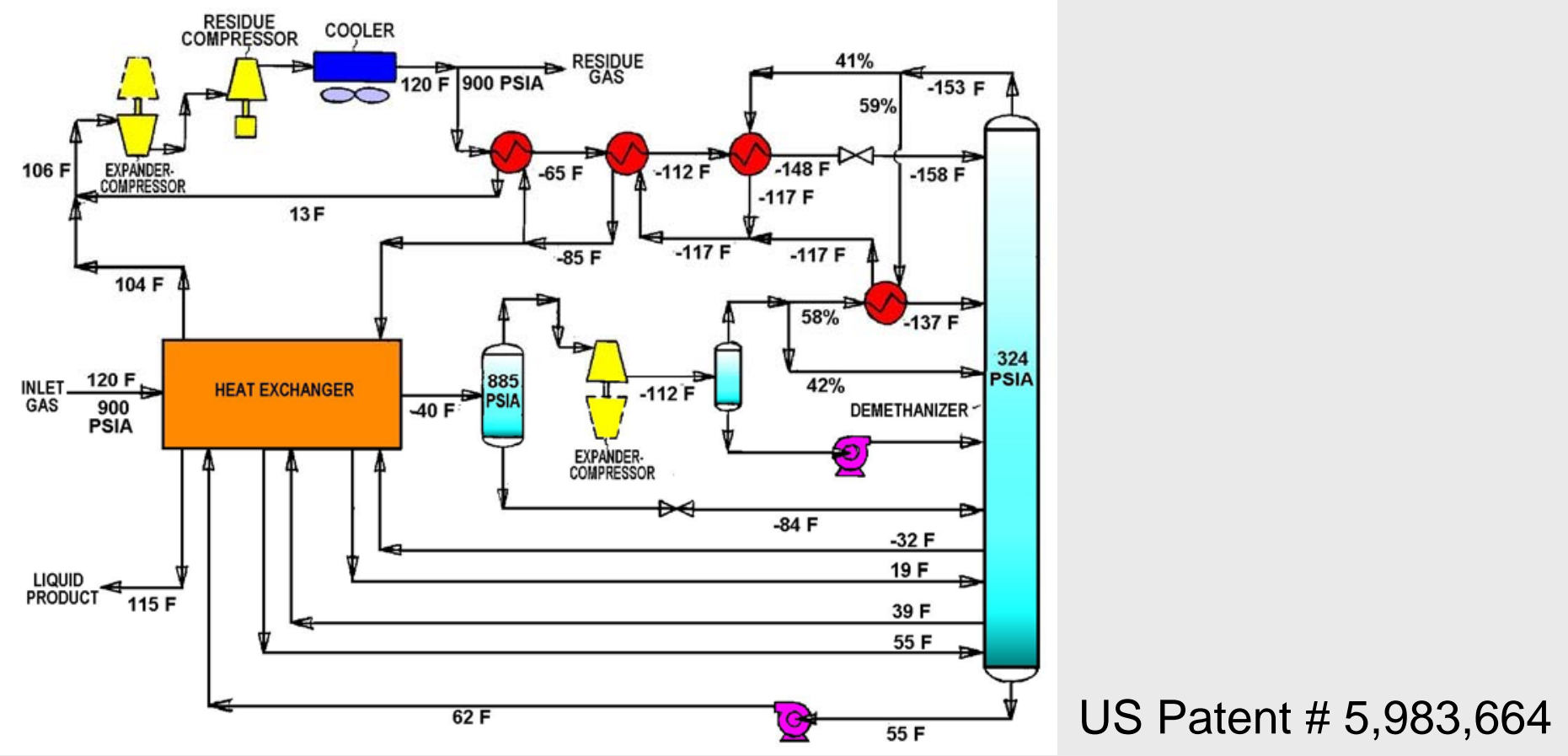

$>$ NG feed stream pressure is reduced by about 60 $65 \%$ to cool the inlet gas to cryogenic temperatures even for low $\mathrm{C}_{2}$ recoveries

$>$ High energy penalties to recompress the residue gas 


\section{Basis: Energy Consumption Cost}

$>$ Energy consumption includes

$>$ Refrigeration

$>$ Reboiler heat

$>$ Cooling water @80 F

$>$ Both power and heat consumptions are represented in terms of BTU of fuel gas consumed

$>$ Gas price $=\$ 5 / \mathrm{MMBTU}$

$>$ Heat rate of gas engine driven compressors $=7,000$ BTU/bhp-hr

$>$ Waste heat recovery $=1,000$ BTU/bhp-hr 


\section{Final Progress Report DE-FC36-03G013150 \\ Basis: Gas Feed Conditions}

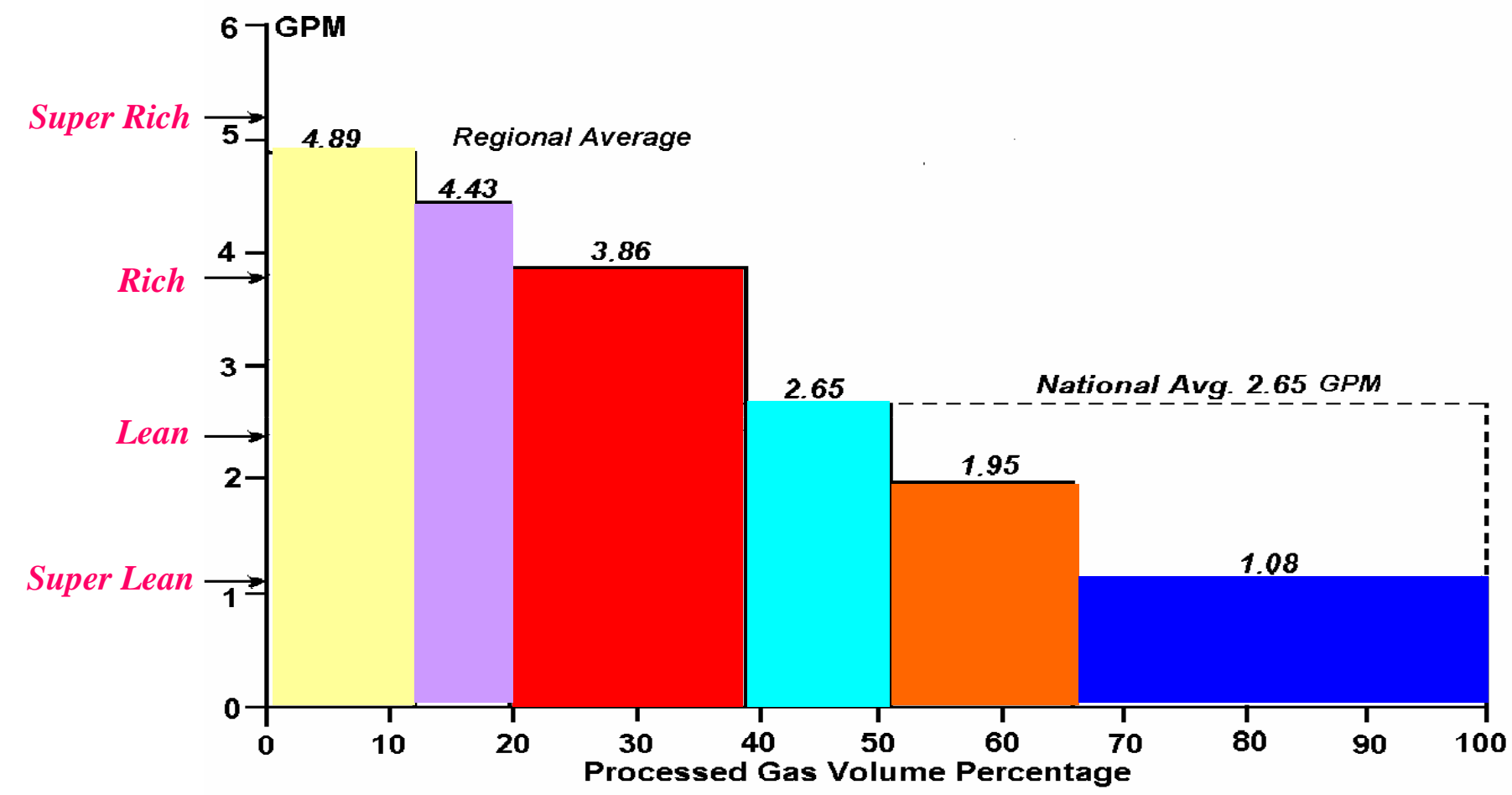




\section{Simulation Cases}

Plant Size $=100$ MMscfd

\begin{tabular}{|c|c|c|c|c|}
\hline $\begin{array}{l}\text { NGL Content } \\
\text { (GPM) }\end{array}$ & \multicolumn{4}{|c|}{$\begin{array}{c}C_{2} \text { Recovery } \\
(\%)\end{array}$} \\
\hline $\begin{array}{c}5.1 \\
\text { (Super Rich) }\end{array}$ & $\begin{array}{c}2 \\
\text { (IROA) }\end{array}$ & $\begin{array}{c}35 \\
\text { (IROA) }\end{array}$ & $\begin{array}{c}70 \\
\text { (IROA) }\end{array}$ & $\begin{array}{c}95 \\
\text { (CEP, IROA) }\end{array}$ \\
\hline $\begin{array}{c}3.6 \\
\text { (Rich) }\end{array}$ & $\begin{array}{c}2 \\
\text { (IROA) }\end{array}$ & $\begin{array}{c}35 \\
\text { (IROA) }\end{array}$ & $\begin{array}{c}70 \\
\text { (IROA) }\end{array}$ & $\begin{array}{c}95 \\
\text { (CEP, IROA) }\end{array}$ \\
\hline $\begin{array}{c}2.2 \\
\text { (Lean) }\end{array}$ & $\begin{array}{c}2 \\
\text { (IROA) }\end{array}$ & $\begin{array}{c}35 \\
\text { (IROA) }\end{array}$ & $\begin{array}{c}70 \\
\text { (IROA) }\end{array}$ & $\begin{array}{c}95 \\
\text { (CEP, IROA) }\end{array}$ \\
\hline $\begin{array}{c}1.0 \\
\text { (Super Lean) }\end{array}$ & $\begin{array}{c}2 \\
\text { (IROA) }\end{array}$ & $\begin{array}{c}35 \\
\text { (IROA) }\end{array}$ & - & $\begin{array}{c}95 \\
\text { (CEP) }\end{array}$ \\
\hline Costing & No & Yes & No & Yes \\
\hline
\end{tabular}




\section{Energy Savings}

Gas Richness $=2.2$ GPM

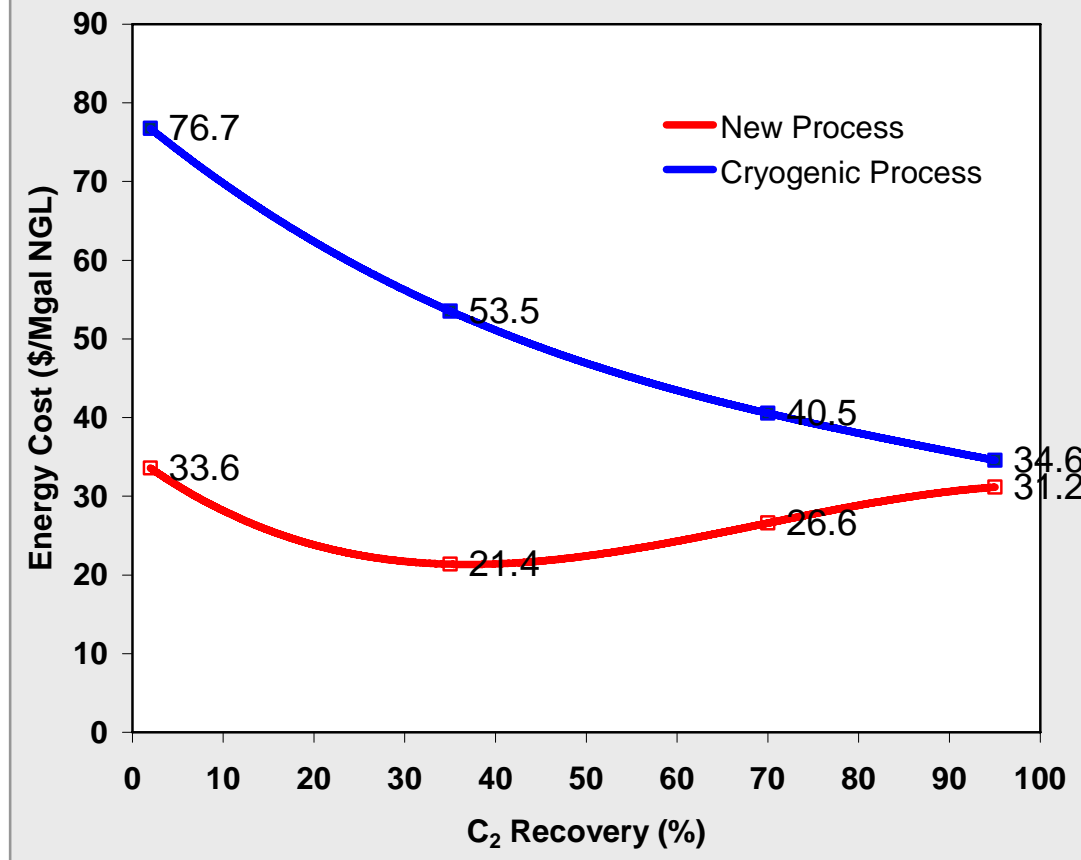

Gas Richness = 3.6 GPM

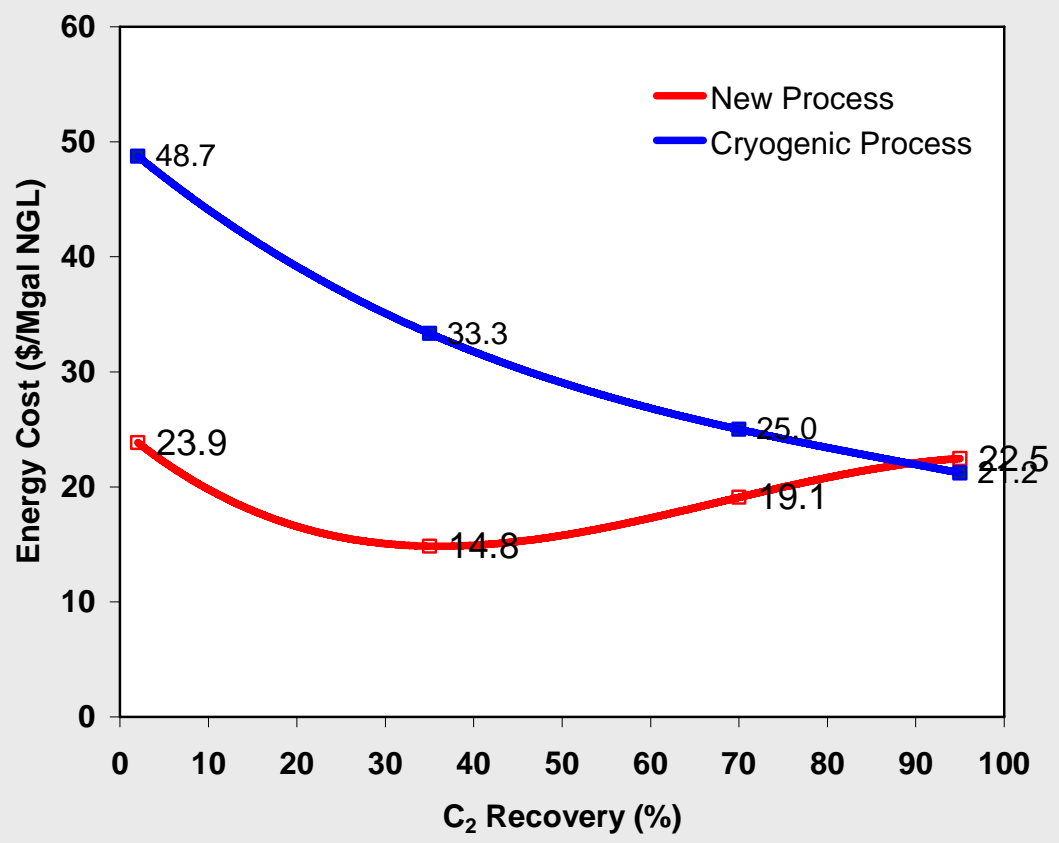




\section{Equipment Cost Comparison}

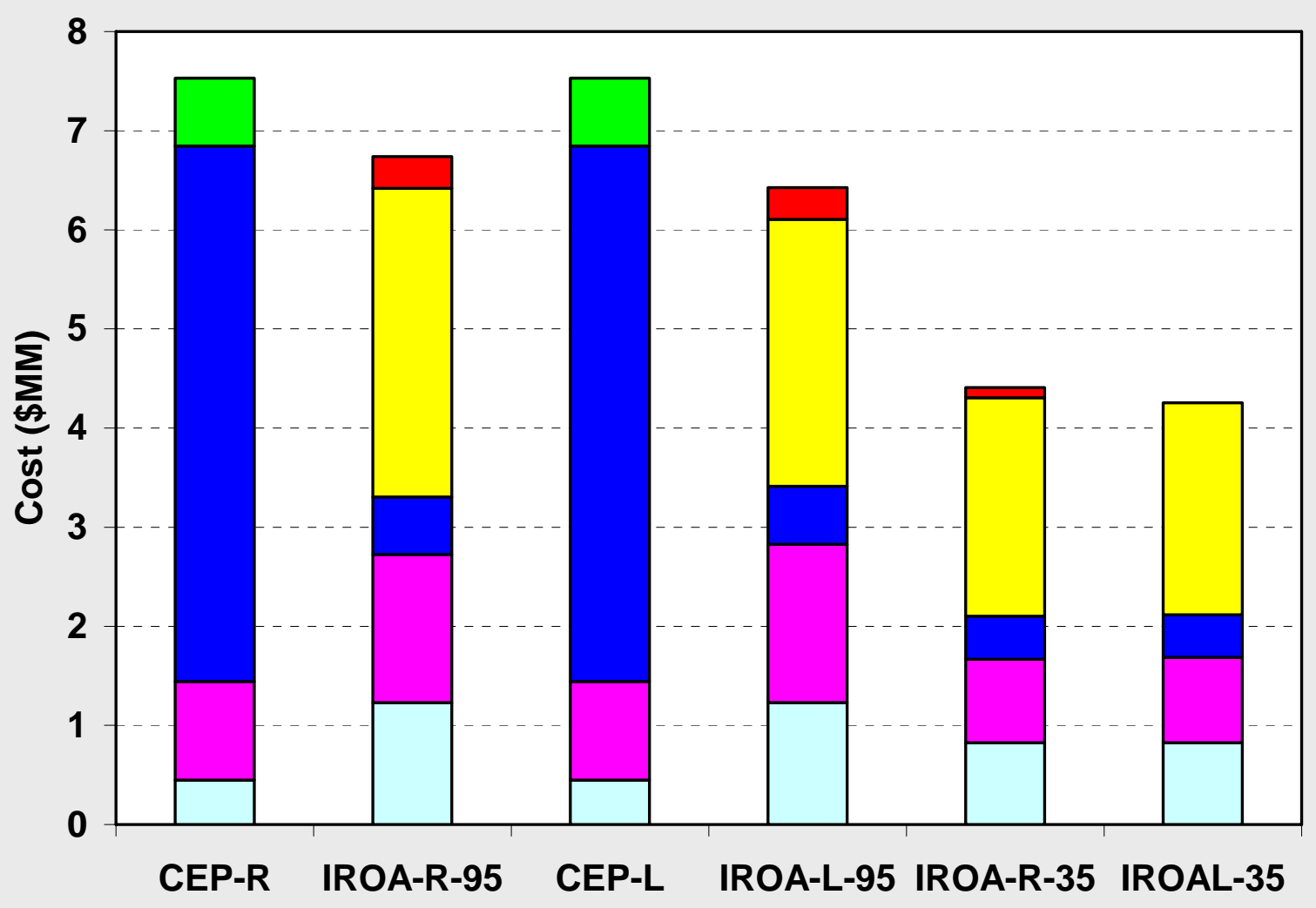

\section{$\square$ Vessels}

$\square$ Refrigration $\square$ Heat Exchangers

$\square$ Mole Sieve Dehydration
口Compressors \& Pumps

$\square$ Hot Oil Heater 


\section{Net Process Margin}

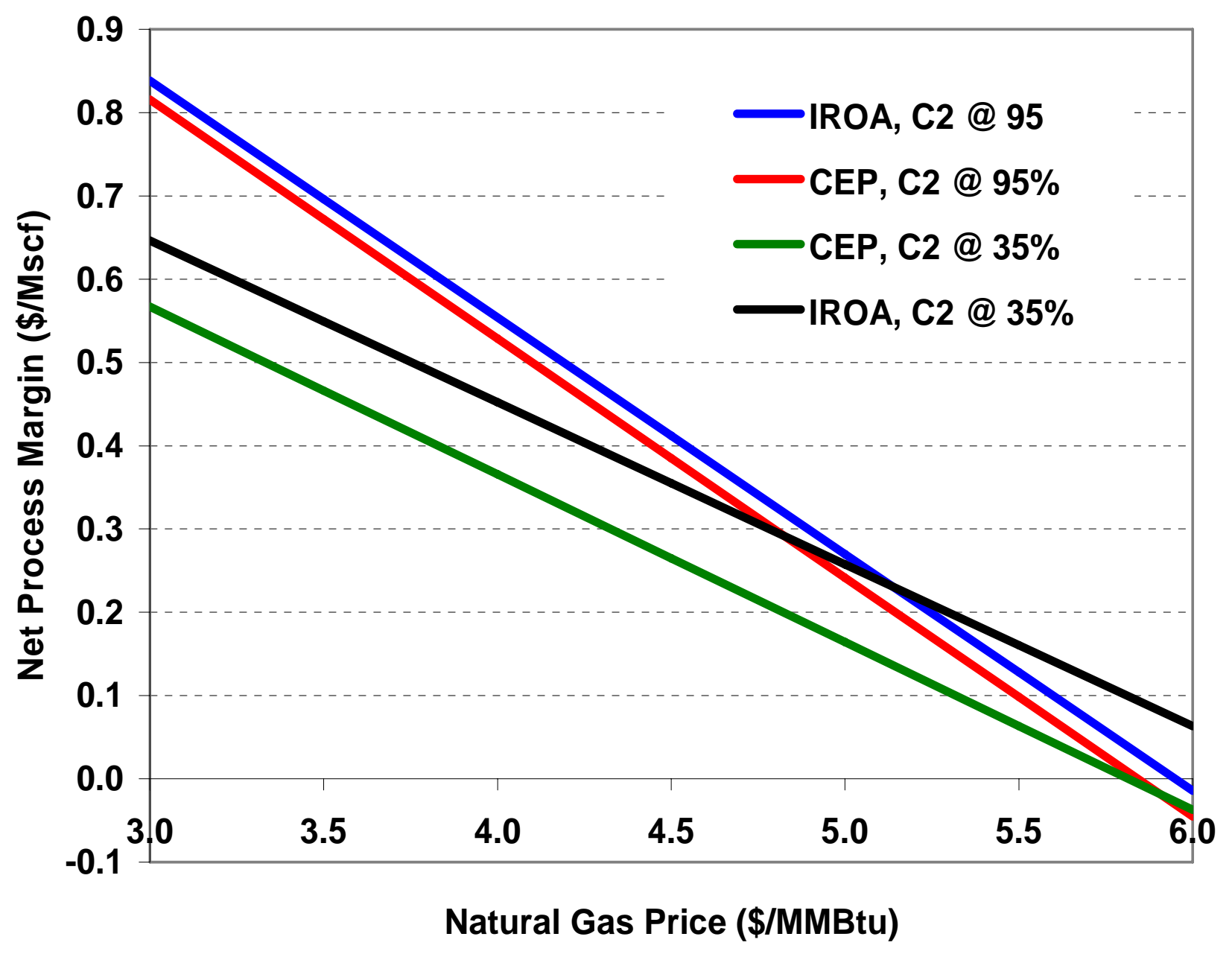

Page 24 


\section{Greater than $10 \%$ NPV Advantage}
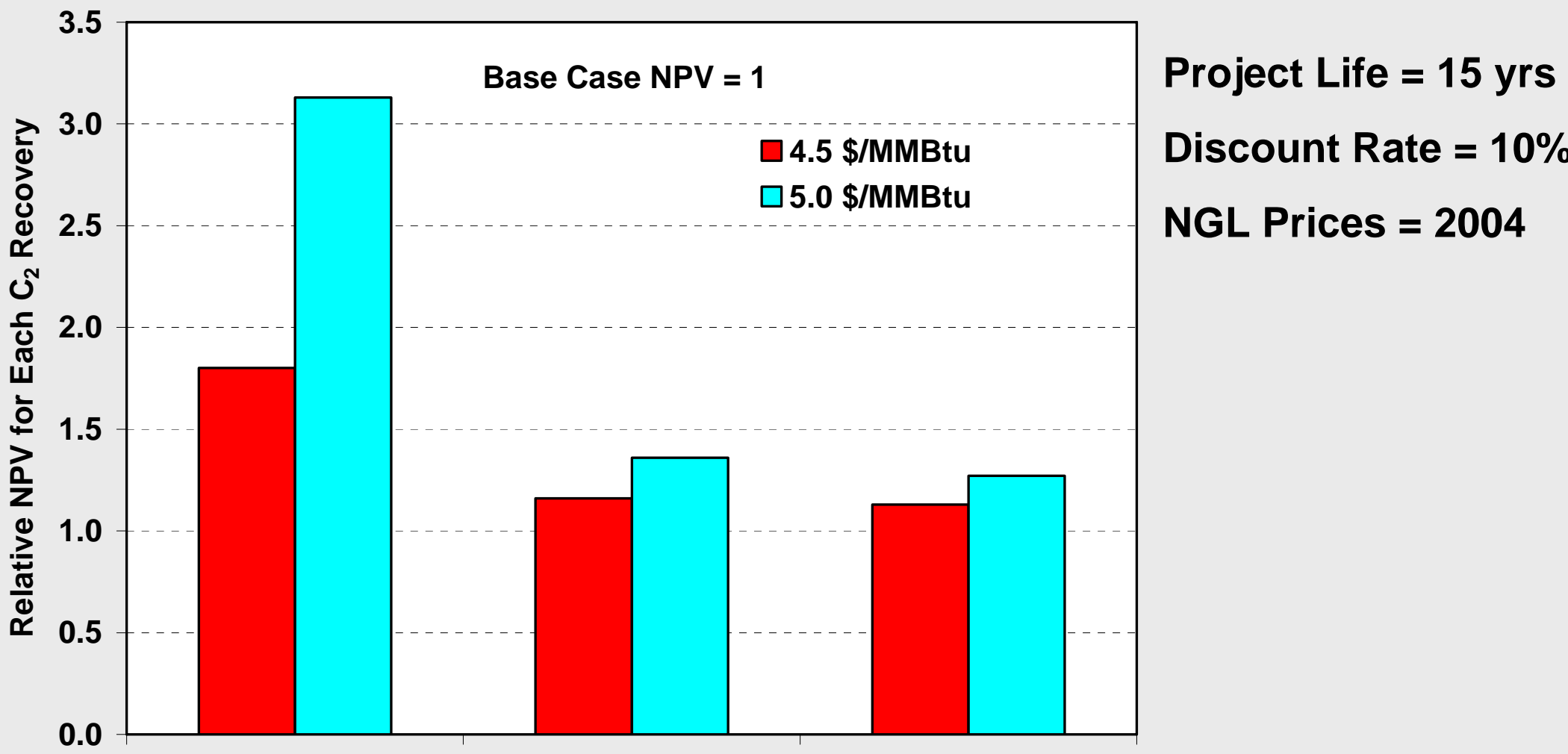

Discount Rate $=\mathbf{1 0} \%$

NGL Prices $=2004$

35

70

95

Base Case NPV Value

$\mathrm{C}_{2}$ Recovery (\%)

\begin{tabular}{|c|c|c|}
\hline$C_{2}$ Recov. & $\begin{array}{c}\text { NPV @ } \\
4.5 \text { \$/MMBtu } \\
(\$ M M)\end{array}$ & $\begin{array}{c}\text { NPV @ } @ \\
5.0 \text { \$/MMBtu } \\
(\$ M M)\end{array}$ \\
\hline 35 & 39.9 & 15.6 \\
70 & 57.38 & 26.8 \\
95 & 69.06 & 34.34 \\
\hline
\end{tabular}




\section{IROA Energy Saving Potential}

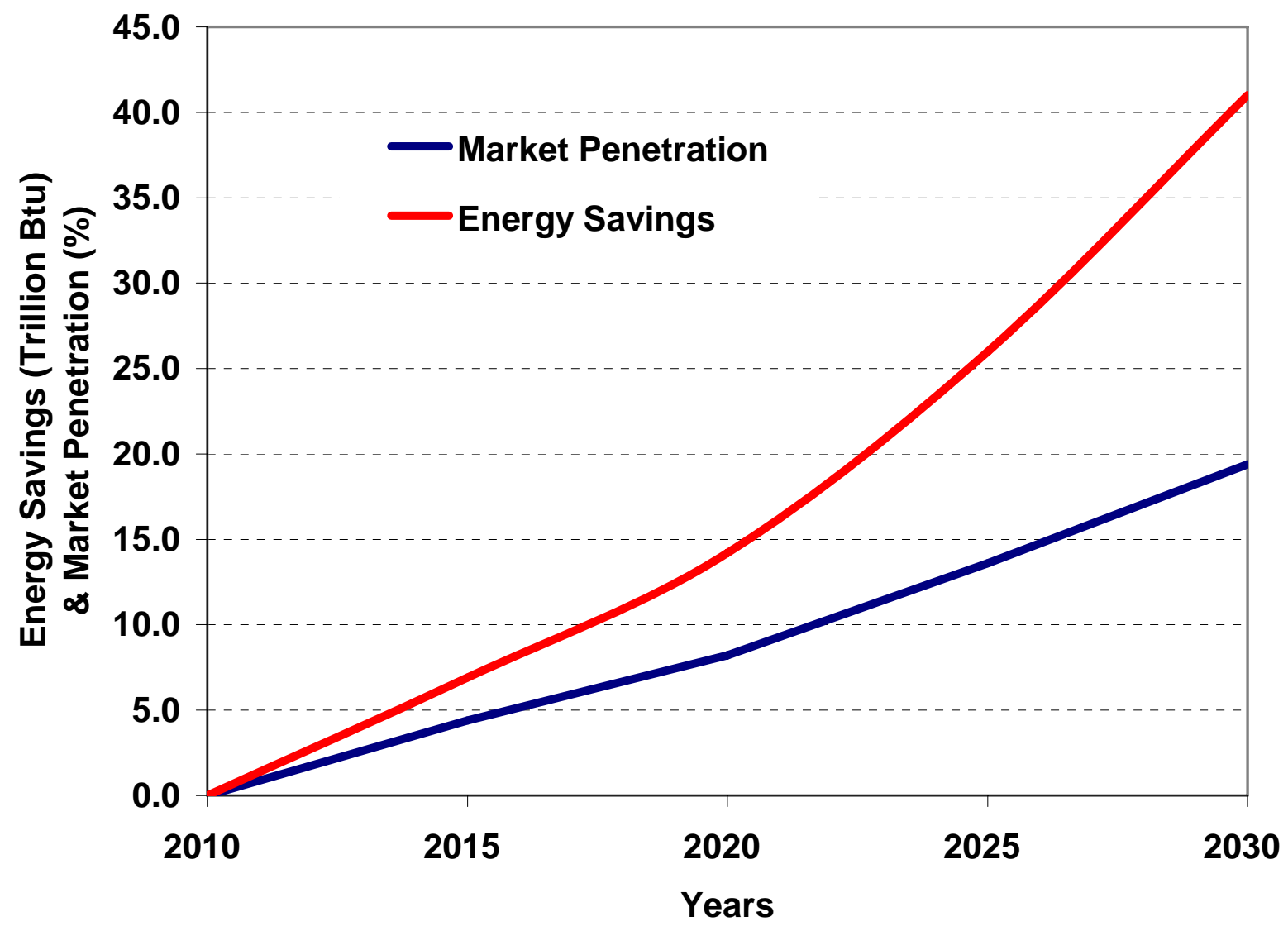




\section{U.S. NGL Demand Favors Low er C $\mathrm{C}_{2}$ Recovery}

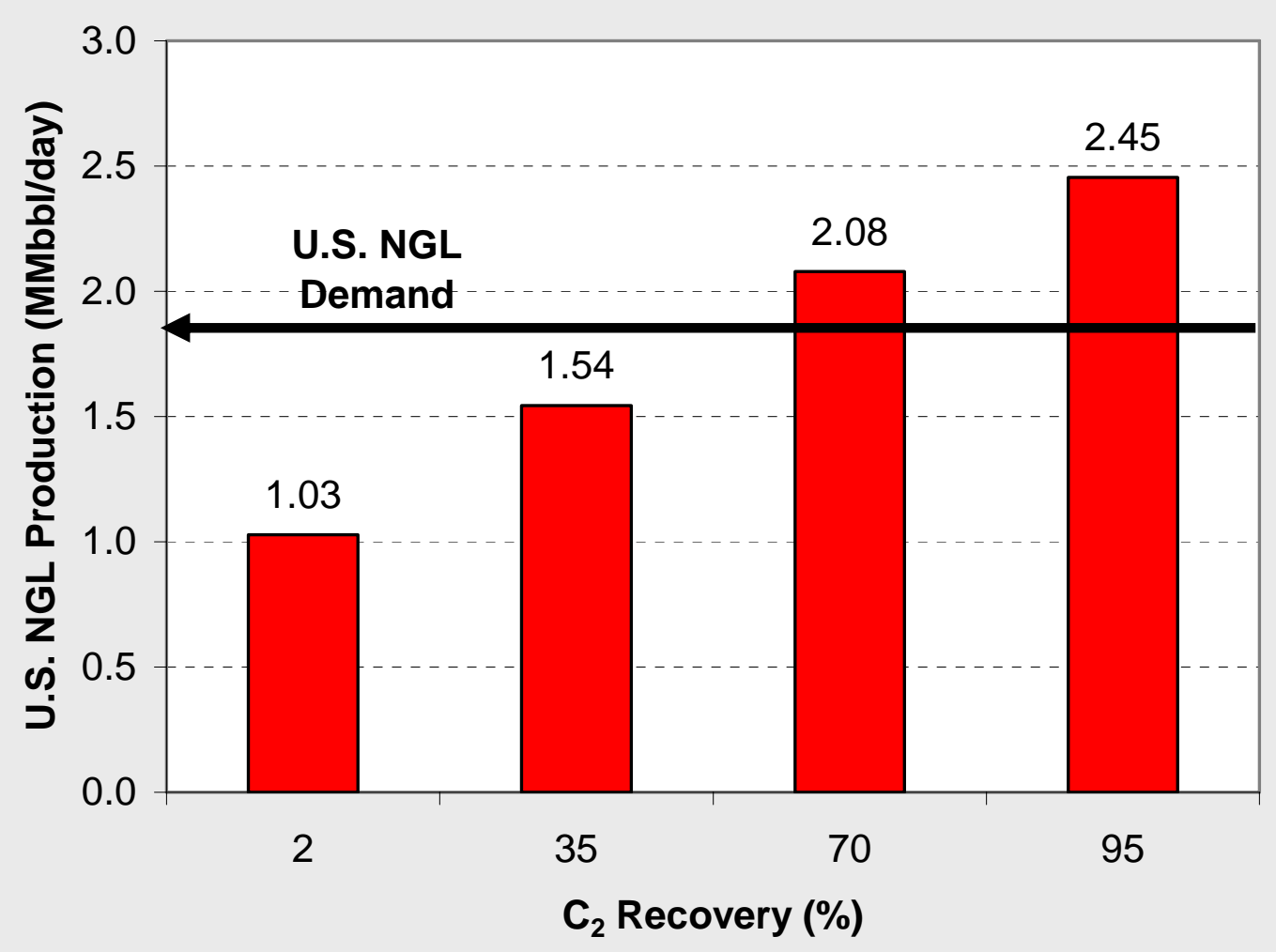

Based on gas richness of 2.2 GPM (National Avg.) 


\section{Conclusions}

$>$ Phase-1 of the project has been successfully completed

$>$ IROA technology is expected to -

$>$ Provide lower cost, more flexible gas processing plants of any sizes for varying inlet gas conditions

$>$ Substantial energy savings for $\mathrm{C}_{2}$ rejection operations

$>$ Application of new, efficient equipment in gas processing industry: such as plate-fin heat exchangers, high-efficiency structured packing for absorption/distillation columns

$>$ Acceptance of IROA by gas processors will lead to secure supplies of NGLs with varying gas prices 


\section{Future Work}

$>$ Conduct VLE experiments to generate K-value data for Kerosene-hydrocarbon mixtures

$>$ Conduct bench-scale tests using a natural gas slip stream at various operating conditions and obtain scale-up data

$>$ Update the simulation model and optimize the design for maximizing energy efficiency

$>$ Design and operate the pilot-scale unit

$>$ Continue to engage with gas processing industry to commercialize the IROA technology 


\section{Acknowledgements}

$>$ The authors gratefully acknowledge the support and funding from:

$>$ U.S. DOE (Energy Efficiency and Renewable Energy) Contract DE-FC36-03G013150

$>$ Gas Research Institute Contract 8372

$>$ The authors also gratefully acknowledge the advise from:

> Mr. Johnny Johnson, The Washington Group

$>$ Ms. Susan King, Duke Energy Field services

$>$ Dr. Pervaiz Nasir, Enterprise Product Partners

$>$ Mr. Ajay Chandra, Purvin \& Gertz 
Low Cost Chemical Feedstocks Using an Improved and Energy Efficient Natural Gas Liquid (NGL) Removal Process (DOE Award \# DE-FC36-03G013150)

Aqil Jamal, Howard Meyer, and Dennis Leppin Gas Technology Institute Yingzhong Lu, Green Hi-Tek Javad Abbasian, Illinois Inst. Of Technology

April 11-14, 2005 Chemicals Subprogram Portfolio Review 2005 AIChE Spring National Meeting, Atlanta, GA, 


\section{Project Objectives}

$>$ Develop a new low-cost and energy efficient NGL recovery process (as per U.S. patent $6,553,784$ ) through combination of theoretical, bench-scale and pilot-scale testing which will ensure a supply of low cost chemical feedstocks for the U.S. petrochemical industry 


\section{Project Scope}

$>$ Phase -1 ( 1 yr)

$>$ develop a preliminary computer simulation model to verify the process performance and compare it with conventional cryogenic technology

$>$ Conduct techno-economic evaluation of the process

$>$ Phase -2 (4 yrs)

$>$ design, construct and operate bench and pilot-scale units to demonstrate the technology and develop scale-up data

$>$ conduct critical R\&D related to the development of the new process

$>$ update computer simulation model based on bench and pilot-scale test data

$>$ perform market study, develop process economics and commercialization path 


\section{Project Motivation}

$>$ NGL extraction industry provide the majority of the petrochemical feedstocks

$>$ In times of high natural gas prices in relation to hydrocarbon liquids prices, these plants will either shutdown or leave NGLs in the natural gas leading to supply shortage and high prices for petrochemical feedstocks

$>$ New technology is needed to enable stable and economical supply of chemical feedstocks 


\section{Project Budget and Spending}

\begin{tabular}{|c|c|c|c|c|c|c|}
\hline \multicolumn{7}{|c|}{ (in \$1000) } \\
\hline $\begin{array}{c}\text { Total } \\
\text { Project } \\
\text { Cost }\end{array}$ & $\begin{array}{c}\text { Total } \\
\text { Federal } \\
\text { Funding }\end{array}$ & $\begin{array}{c}\text { Total GTI } \\
\text { Co-funding } \\
\text { (in-cash) }\end{array}$ & $\begin{array}{c}\text { Total Federal } \\
\text { Share of } \\
\text { Outlays as of } \\
12 / 31 / 04\end{array}$ & $\begin{array}{c}\text { Total GTI } \\
\text { Share of } \\
\text { Outlay as } \\
\text { of 12/31/04 }\end{array}$ & $\begin{array}{c}\text { Original } \\
\text { Federal } \\
\text { Spending } \\
\text { Estimate }\end{array}$ & $\begin{array}{c}\text { Original } \\
\text { GTI } \\
\text { Spending } \\
\text { Estimate }\end{array}$ \\
\hline $\mathbf{3 , 5 6 5 . 0}$ & $\mathbf{2 , 4 9 5 . 0}$ & $\mathbf{1 , 0 6 9 . 0}$ & $\mathbf{3 1 4 . 5}$ & $\mathbf{4 9 . 0}$ & $\mathbf{3 4 5 . 4}$ & $\mathbf{3 0 0 . 0}$ \\
\hline
\end{tabular}

$>$ FYO5 budget estimates

$>\mathrm{DOE}=\$ 640 \mathrm{~K}($ Available $=\$ 254 \mathrm{~K}$, Required $=\$ 386 \mathrm{~K})$

$>\mathrm{GTI}=\$ 560 \mathrm{~K}$

$>$ FYO6 budget estimates

$>\mathrm{DOE}=\$ 590 \mathrm{~K}$

$>\mathrm{GTI}=\$ 460 \mathrm{~K}$ 


\section{Project Tasks \& ScheduPe}

\begin{tabular}{|c|c|c|c|c|c|c|c|c|c|c|c|c|c|c|c|c|c|c|c|c|c|}
\hline \multirow{2}{*}{$\begin{array}{c}\text { Task } \\
\#\end{array}$} & \multirow[t]{2}{*}{ Description } & \multicolumn{4}{|c|}{2004} & \multicolumn{4}{|c|}{2005} & \multicolumn{4}{|c|}{2006} & \multicolumn{4}{|c|}{2007} & \multicolumn{4}{|c|}{2008} \\
\hline & & 1 & 2 & 3 & 4 & 1 & 2 & 3 & 4 & 1 & 2 & 3 & 4 & 1 & 2 & 3 & 4 & 1 & 2 & 3 & 4 \\
\hline 1 & $\begin{array}{l}\text { Collect relevant physical property and } \\
\text { VLE data for preliminary technical } \\
\text { verification and design of bench, pilot } \\
\text { and commercial-scale units }\end{array}$ & - & & - & 1 & - & & & - & - & - & & & & & & & & & & \\
\hline 2 & $\begin{array}{l}\text { Design and construct bench-scale unit } \\
\text { for laboratory tests }\end{array}$ & & & & - & & 2 & & & & & & & & & & & & & & \\
\hline 3 & $\begin{array}{l}\text { Conduct bench-scale tests and } \\
\text { verify/generate the design data for } \\
\text { pilot-scale unit }\end{array}$ & & & & & & & & 3 & - & - & & & & & & & & & & \\
\hline 4 & $\begin{array}{l}\text { Develop computer simulation for } \\
\text { preliminary technical verification and } \\
\text { bench, pilot and commercial-scale } \\
\text { design }\end{array}$ & - & & - & - & - & & & - & - & - & - & & - & & & & 6 & & - & \\
\hline 5 & $\begin{array}{l}\text { Perform theoretical and experimental } \\
\text { study (R\&D work) to address critical } \\
\text { process development issues }\end{array}$ & & & - & - & & & & - & - & & - & & - & . & & - & & & & \\
\hline 6 & $\begin{array}{l}\text { Design, construct and install a pilot } \\
\text { unit and conduct field tests }\end{array}$ & & & & & & & & - & - & 4 & - & & & 5 & & & & & & \\
\hline 7 & $\begin{array}{l}\text { Conduct market study and prepare } \\
\text { commercialization package }\end{array}$ & & & & & & & & & & & & & & & & & & & 7 & \\
\hline 8 & $\begin{array}{l}\text { Attend technical meetings and prepare } \\
\text { technical reports }\end{array}$ & & & - & - & & & & - & - & & - & & - & & & - & & & & 8 \\
\hline
\end{tabular}




\section{Project Performance}

\begin{tabular}{|c|l|c|}
\hline$\#$ & \multicolumn{1}{|c|}{ Milestone } & Status \\
\hline 1 & $\begin{array}{l}\text { Preliminary computer simulation model developed and } \\
\text { initial technical verification of the process completed }\end{array}$ & Completed \\
\hline 2 & Design and construction of bench-scale unit completed & To be completed by $06 / 05$ \\
\hline 3 & $\begin{array}{l}\text { Bench-scale tests completed and computer simulation } \\
\text { model updated }\end{array}$ & $\begin{array}{c}\text { VLE tests started, bench- } \\
\text { scale testing } \mathbf{0 6} / 05\end{array}$ \\
\hline 4 & Construction of pilot scale unit completed & On time \\
\hline 5 & $\begin{array}{l}\text { Field-tests completed, process design verified and } \\
\text { computer simulation model updated }\end{array}$ & On time \\
\hline 6 & Final computer simulation model completed & On time \\
\hline 7 & Engineering and commercialization package completed & On time \\
\hline 8 & Final report prepared and submitted to DOE & On time (9/30/2008) \\
\hline
\end{tabular}

$>$ Exercised due diligence and delayed bench-scale unit construction until agreed upon process design was achieved

$>$ Expected project completion date - 9/30/2008 


\section{Team Participation}

\begin{tabular}{|l|l|}
\hline Team Member & \multicolumn{1}{c|}{ Tasks/Contributions } \\
\hline GTI & - Over all project management and technical oversight of subcontractors work. \\
(Tasks 1-8) & $\begin{array}{l}\text { - Gathering of commercial data, cost and marketing information } \\
\text { - Development of research tasks and schedule } \\
\text { - Technical assistance in the development of computer model and continued model update } \\
\text { - Techno-economic evaluation and market study } \\
\text { - Design, construction, commissioning and operation of bench and pilot-scale units }\end{array}$ \\
\hline - Data analysis and preparation of reports and publications
\end{tabular}




\section{Commercialization Plan}

$>$ GTI has signed an exclusive agreement with the current owner of the technology (i.e., GHT) and has completed preliminary techno-economic evaluation

$>$ GTI plans to publish some of these results in the gas processing-related conferences (e.g., GPA, LRGCC) where most of the NGL producers and natural gas processors are represented

$>$ After successful completion of bench-scale testing, GTI will approach potential industrial partners for assistance in commercialization of this technology

$>$ Eventually, GTI plans to license the technology to an E\&C or process technology licensors (e.g. , UOP) to enable end users to acquire it 


\section{Project status}

$>$ Phase-1 is successfully completed

$>$ Continued development is justified

$>$ Co-funding for Phase-2 has been secured

$>$ Bench-scale unit has been designed and sized and ready for fabrication 


\section{gti}

\section{AN IMPROVED AND ENERGY EFFICIENT NATURAL GAS LIQUIDS (NGL) REMOVAL PROCESS}

Howard S. Meyer, Diana Matonis, and Dennis Leppin Gas Technology Institute, Des Plaines, IL

Yinghong Lu, Green Hi-Tek, Oak Ridge, TN AIChE Spring Meeting, Houston, TX April 25, 2007 


\section{Outline}

$>$ Project Objectives, Scope and Motivation

$>$ Background

$>$ Technical Approach

$>$ Accomplishments

$>$ Conclusions

$>$ Future Work 


\section{Project Objectives}

$>$ Develop a new low-cost and energy efficient NGL recovery process (as per U.S. patent $6,553,784$ ) through combination of theoretical, bench-scale and pilot-scale testing which will ensure a supply of low cost chemical feedstocks for the U.S. petrochemical industry 


\section{Project Scope}

$>$ Phase -1 (1 yr)

- develop a preliminary computer simulation model to verify the process performance and compare it with conventional cryogenic technology

- Conduct techno-economic evaluation of the process

$>$ Phase -2 (4 yrs)

- design, construct and operate bench and pilot-scale units to demonstrate the technology and develop scale-up data

- conduct critical R\&D related to the development of the new process

- update computer simulation model based on bench and pilot-scale test data

- perform market study, develop process economics and commercialization path 


\section{Project Motivation}

$>$ NGL extraction industry provide the majority of the petrochemical feedstocks

$>$ In times of high natural gas prices in relation to hydrocarbon liquids prices, these plants will either shutdown or leave NGLs in the natural gas leading to supply shortage and high prices for petrochemical feedstocks

$>$ New technology is needed to enable stable and economical supply of chemical feedstocks 


\section{Outline}

$>$ Project Objectives, Scope and Motivation

$>$ Background

$>$ Technical Approach

$>$ Accomplishments

$>$ Conclusions

$>$ Future Work 


\section{What is NGL?}

$>$ Mixture of $\mathrm{C}_{2}+$ hydrocarbons extracted from natural gas

$>$ Primarily used as chemical feedstocks in oil refining and petrochemical industries with some use as home heating fuel

$>$ Chemical feedstock usage

- Ethane, propane, normal butane and natural gasoline for ethylene production

- Isobutane for propylene oxide and MTBE production

- Normal butane for acetic acid and maleic anhydride production

- Refinery-produced propylene and butylenes consumed by chemical companies. 


\section{NGL Market}

$>$ NGL production in 2005

- Worldwide - 6.3 MMbpd

- U.S. - 1.8 MMbpd (the largest producer in the world)

$>$ To be economical the value of NGL extracted $>$ fuel shrinkage + processing cost

$>$ For a new plant process margins must also be enough to justify the capital expenditure

$>$ Rising NG prices make high $\mathrm{C}_{2}$ recoveries uneconomical 


\section{Well Head Natural Gas Prices}

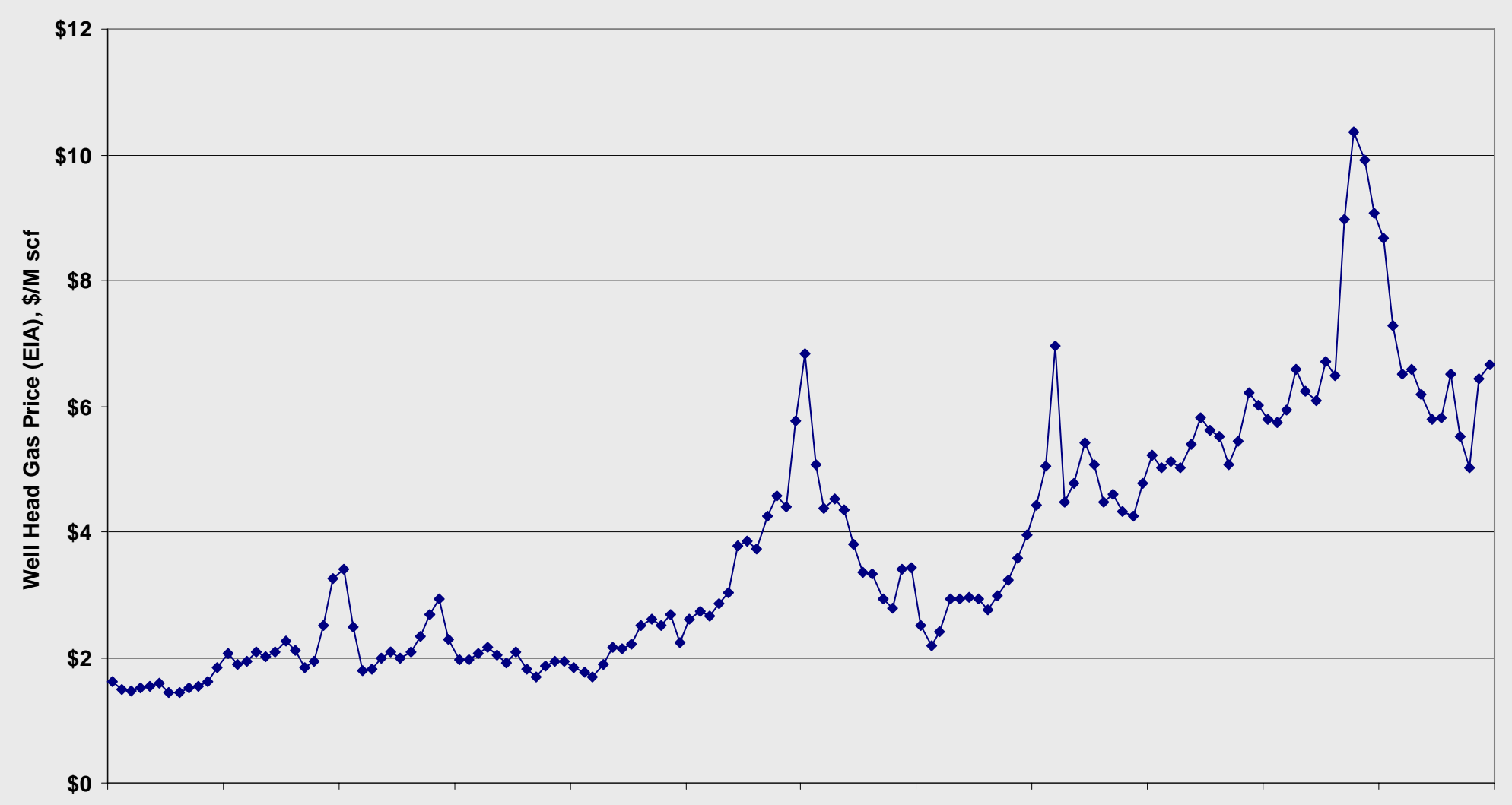

Jan-1995 Jan-1996 Dec-1996 Dec-1997 Jan-1999 Jan-2000 Dec-2000 Dec-2001 Jan-2003 Jan-2004 Dec-2004 Dec-2005 Jan-2007 


\section{Fuel Value of NGL}

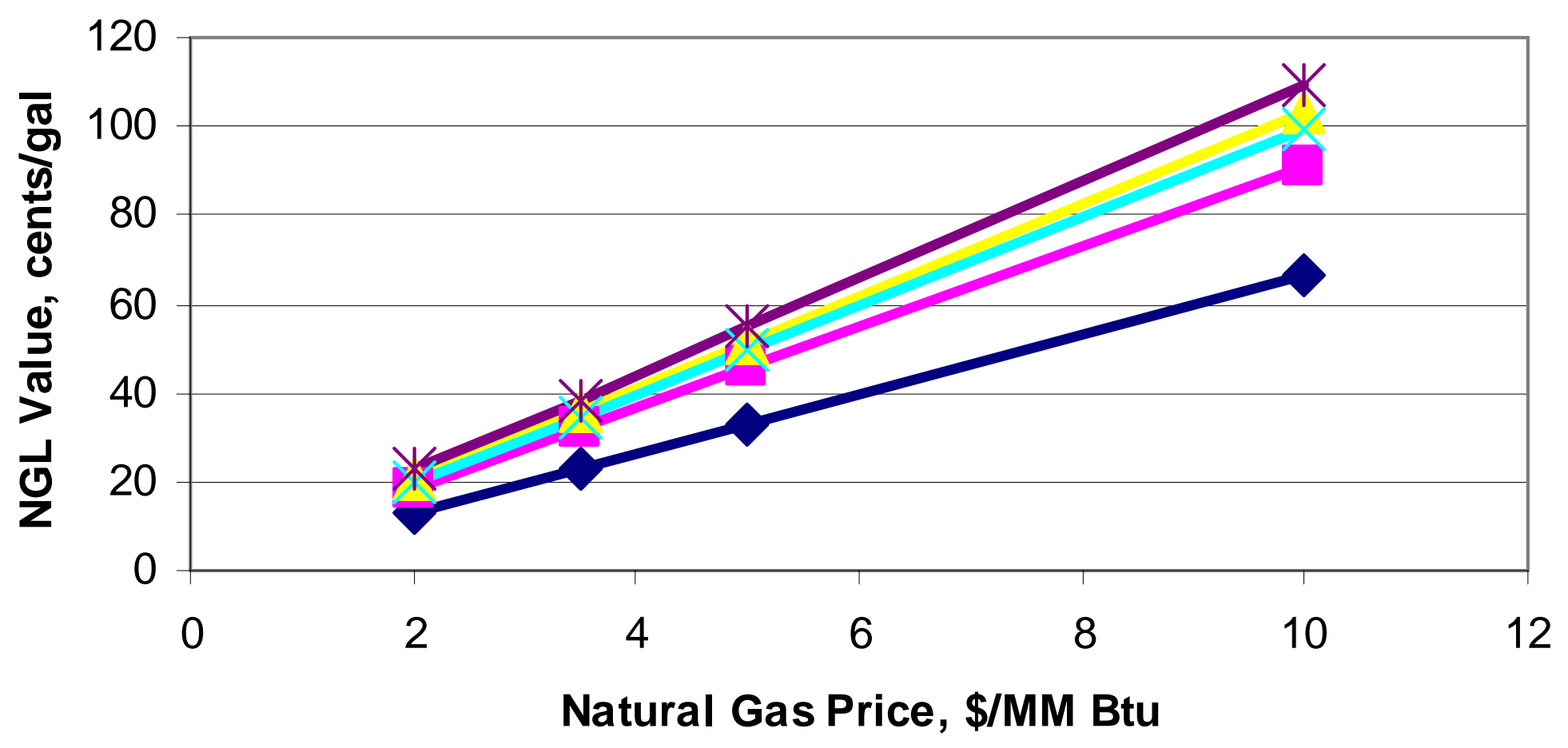

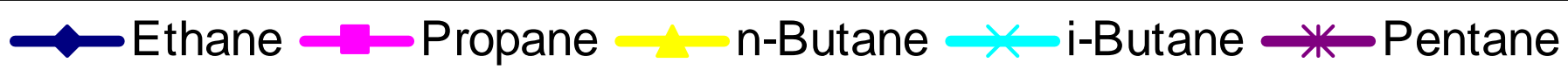




\section{Barnes \& Clicks - Gas \\ Processing Margins - Ethane}

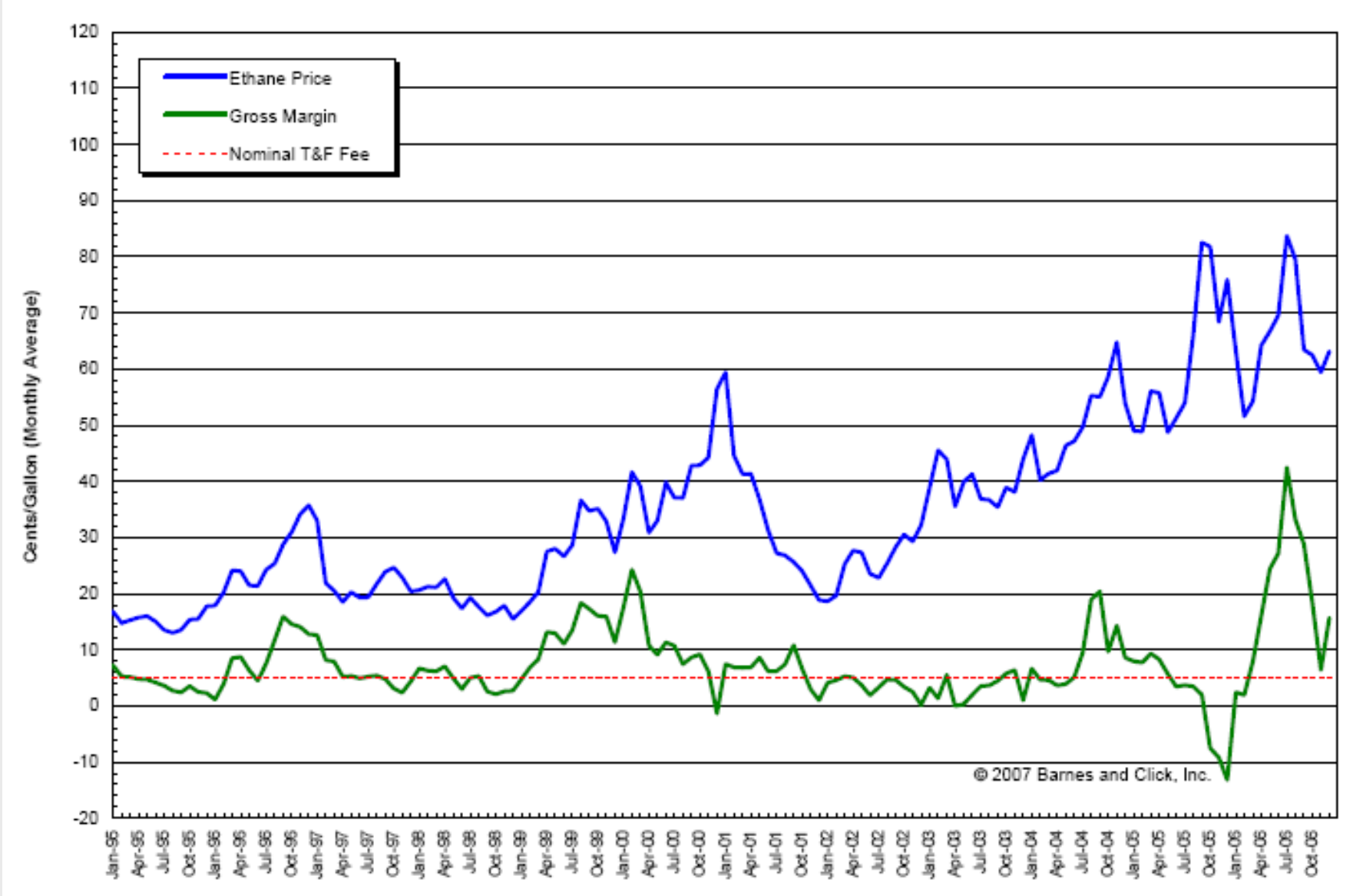




\section{NGL Process Selection Criteria}

$>$ Process selection depends on the:

- Gas richness

$-\mathrm{C}_{2} \& \mathrm{C}_{3}$ recoveries

- Pipe line pressure and quality specifications

- Means for moving NGLs to market

$>$ Capital and operating cost depends on the process selected 


\section{NGL Recovery Technologies}

$>$ Straight Refrigeration Process

- Used for hydrocarbon dewpointing

- Recoveries: $\mathrm{C}_{3}=70-90 \%, \mathrm{C}_{4}+=90-95 \%$

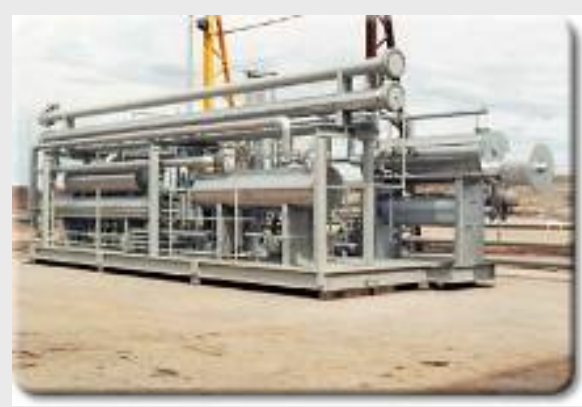

$>$ Refrigerated Lean Oil Absorption Process

- Recoveries: $\mathrm{C}_{2}=40-50 \%, \mathrm{C}_{3}=80-90 \%, \mathrm{C}_{4}+=100 \%$

- Most plants were built in 1960s

$>$ Cryogenic Expansion Process (CEP)

- Recoveries: $C_{2}=80-95 \%, C_{3}=90-100 \%, C_{4}+=100 \%$

- Commonly used worldwide

- Energy intensive 


\section{Outline}

$>$ Project Objectives, Scope and Motivation

$>$ Background

$>$ Technical Approach

$>$ Accomplishments

$>$ Conclusions

$>$ Future Work 


\section{Technical Approach}

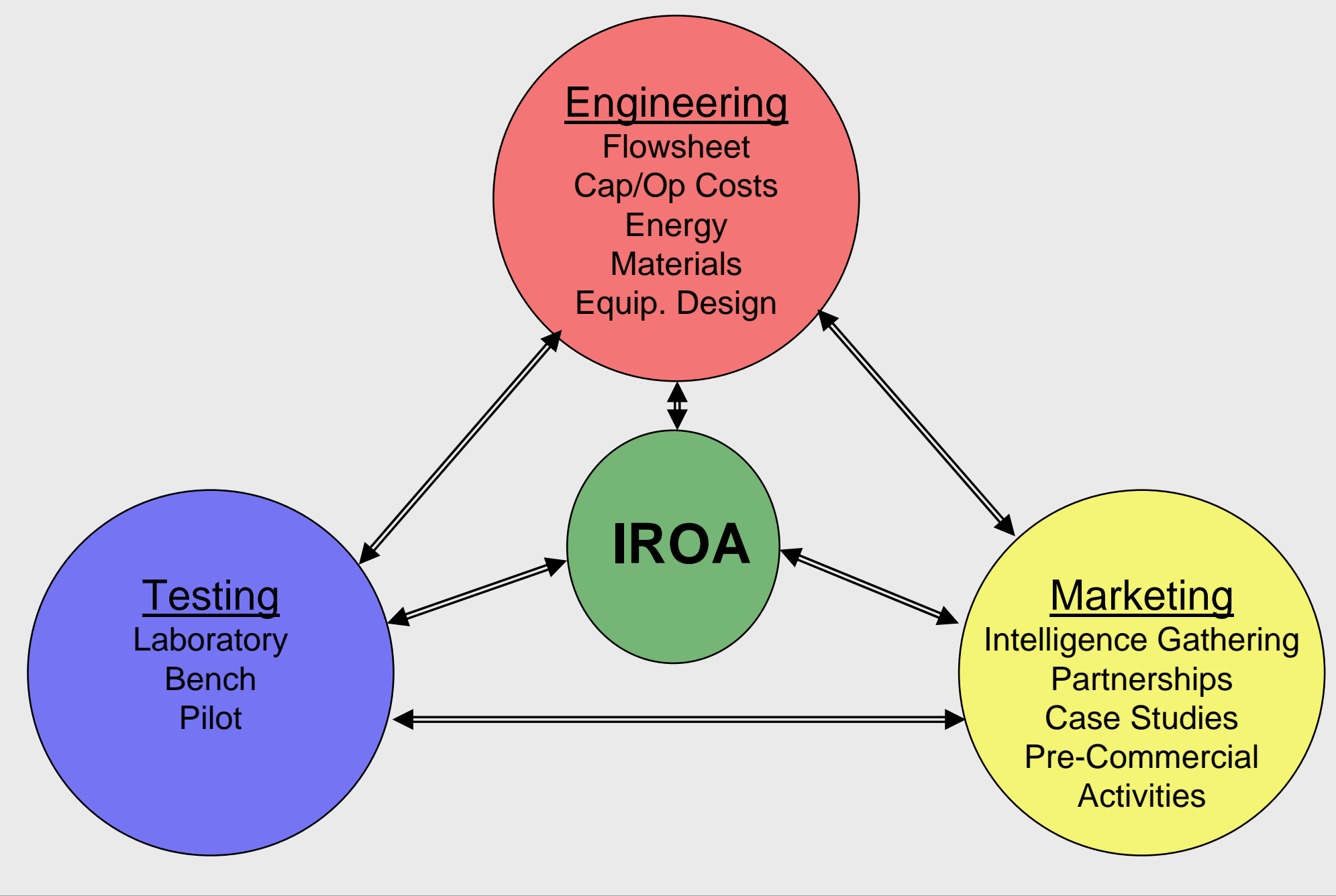




\section{Outline}

$>$ Project Objectives, Scope and Motivation

$>$ Background

$>$ Technical Approach

$>$ Accomplishments

$>$ Conclusions

$>$ Future Work 


\section{IROA Technology}

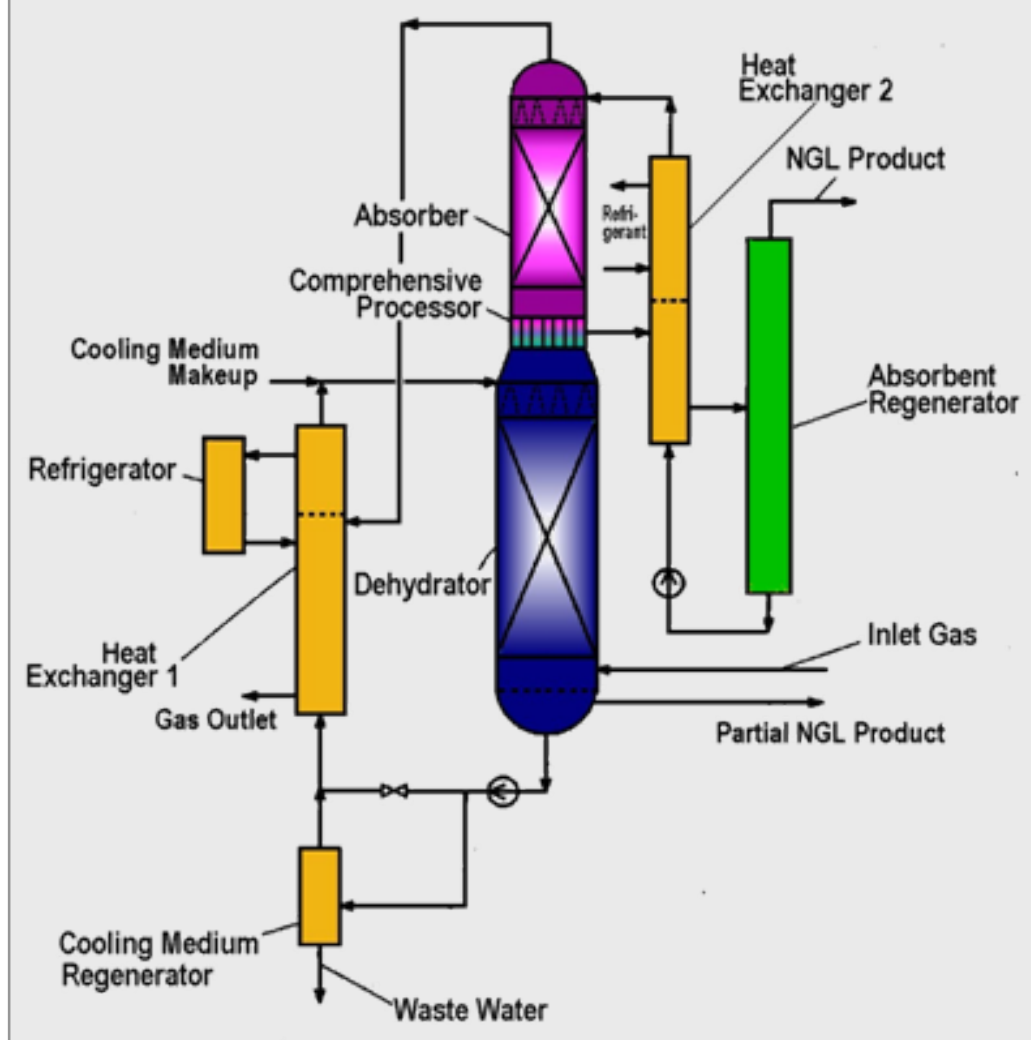

US Patent \# 6,553,784
$>$ Main Features

- Integrates dehydration of feed gas with NGL recovery

- Uses a single absorbent

- Absorber runs isothermally

- Better heat integration by recycling lean oil within internal heat recovery loops

- More compact plant because IROA uses high efficiency packed columns and plate-fin type compact heat exchangers 


\section{IROA Technology}

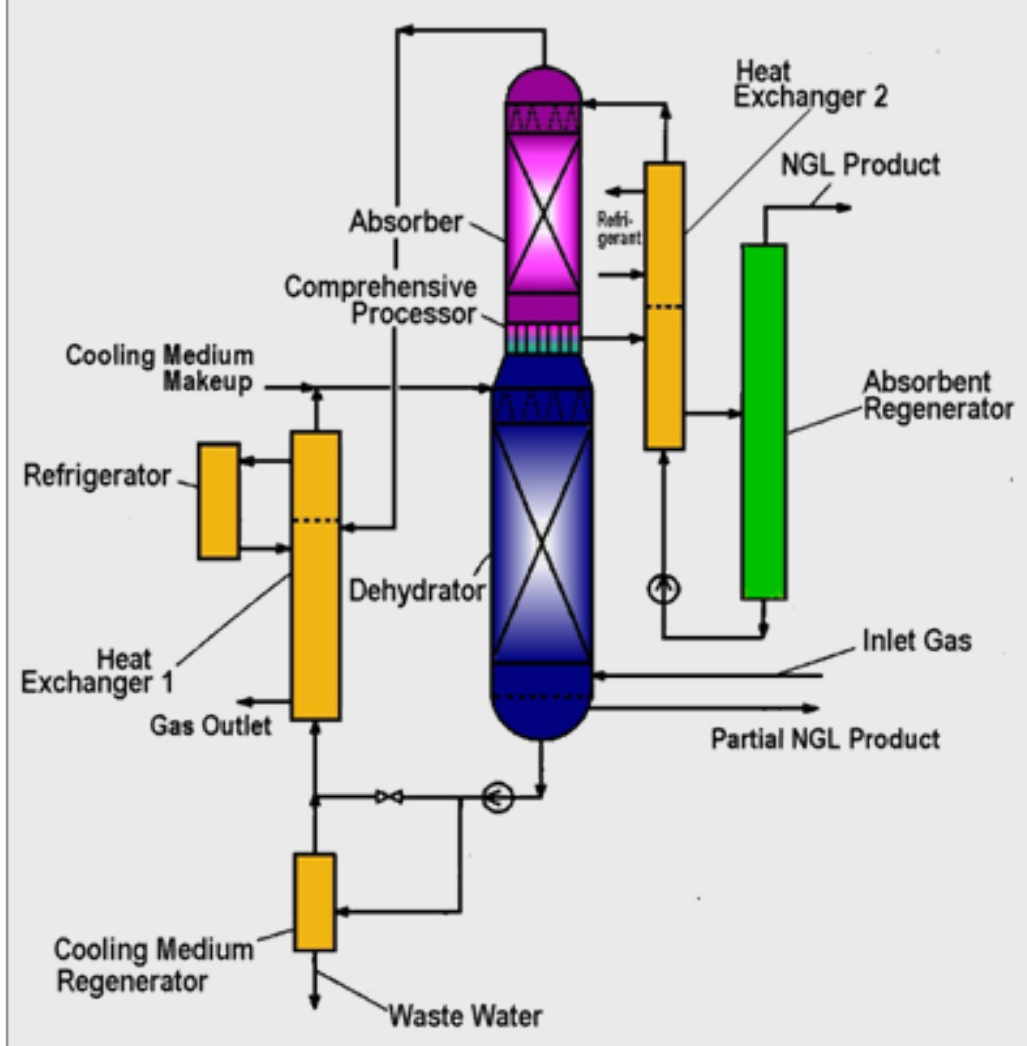

US Patent \# 6,553,784
$>$ Main Advantages

- NGLs are recovered at moderate refrigeration temperatures ( - $\left.40^{\circ} \mathrm{F}\right)$

- Negligible pressure losses

$-\mathrm{C}_{2}$ rejection is achieved by simply reducing absorbent flowrate

- No separate dehydration unit required

- More $\mathrm{CO}_{2}$ tolerant 


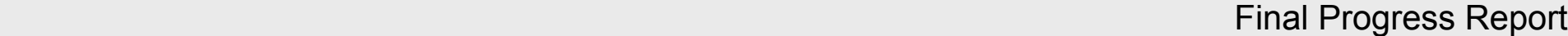

Base Case Cryo Proces's

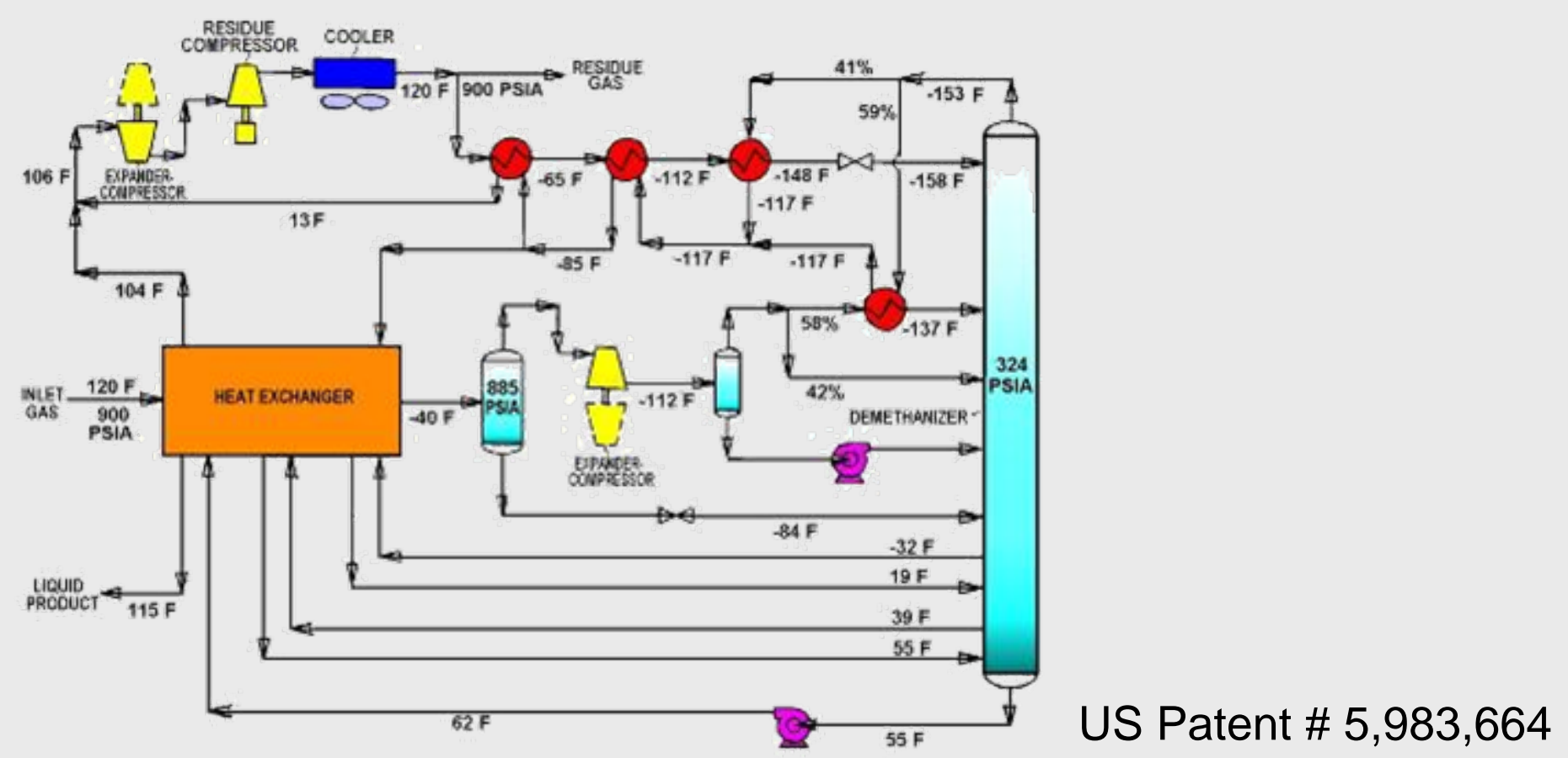

$>$ NG feed stream pressure is reduced by about 60 $65 \%$ to cool the inlet gas to cryogenic temperatures even for low $\mathrm{C}_{2}$ recoveries

$>$ High energy penalties to recompress the residue gas 


\section{Basis: Energy Consumption Cost}

$>$ Energy consumption includes

- Refrigeration

- Reboiler heat

- Cooling water @80 of

$>$ Both power and heat consumptions are represented in terms of BTU of fuel gas consumed

$>$ Gas price $=\$ 5 / \mathrm{MMBTU}$

$>$ Heat rate of gas engine driven compressors $=$ 7,000 BTU/bhp-hr

$>$ Waste heat recovery $=1,000 \mathrm{BTU} / \mathrm{bhp}-\mathrm{hr}$ 


\section{Final Progress Report DE-FC36-03GO13150 \\ Basis: Gas Feed Conditions}

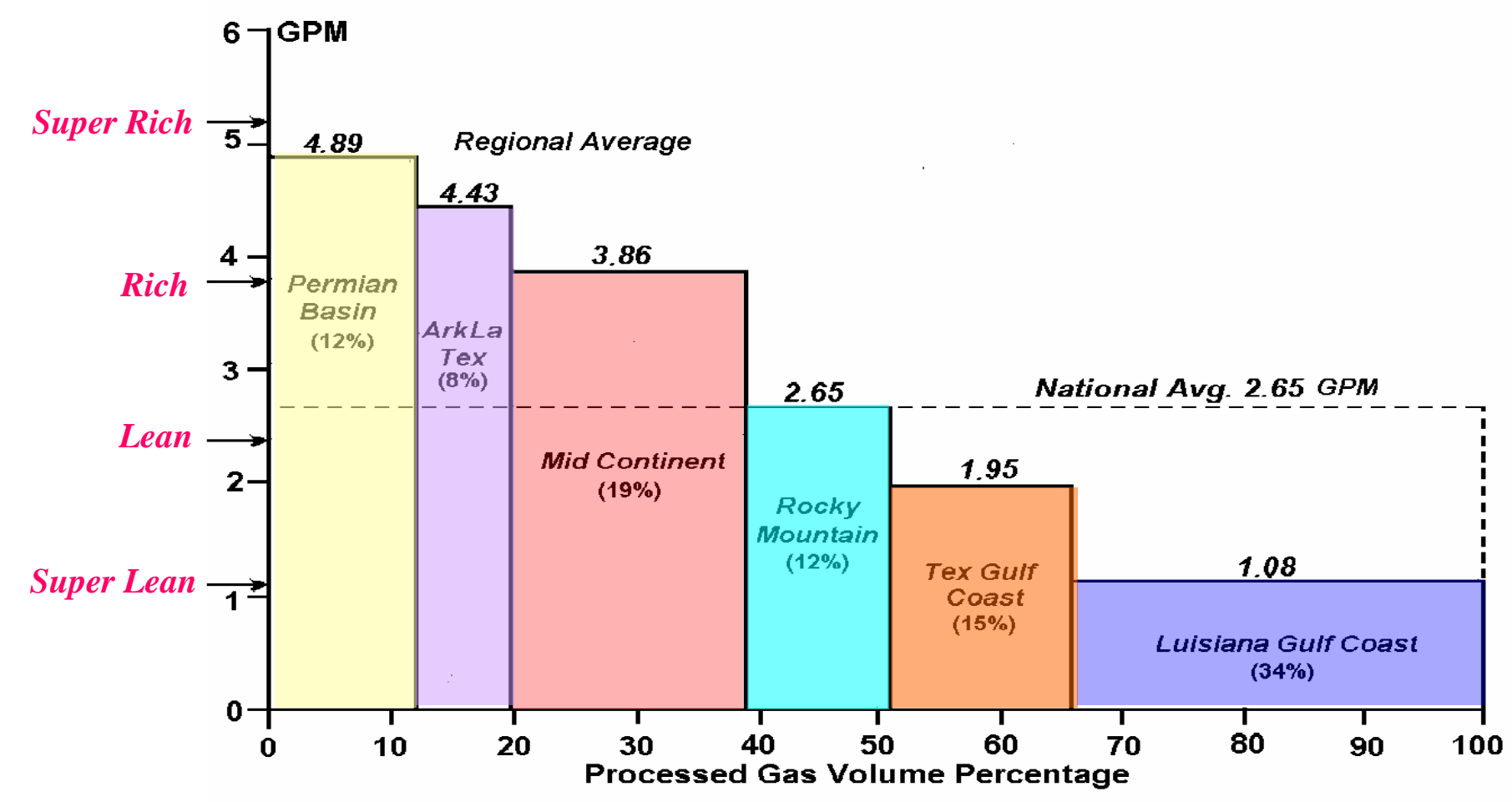




\section{Simulation Cases}

Plant Size $=100$ MMscfd

\begin{tabular}{|c|c|c|c|c|}
\hline $\begin{array}{c}\text { NGL Content } \\
\text { (GPM) }\end{array}$ & \multicolumn{4}{|c|}{$\begin{array}{c}\mathrm{C}_{2} \text { Recovery } \\
(\%)\end{array}$} \\
\hline $\begin{array}{c}5.1 \\
\text { (Super Rich) }\end{array}$ & $\begin{array}{c}2 \\
\text { (IROA) }\end{array}$ & $\begin{array}{c}35 \\
\text { (IROA) }\end{array}$ & $\begin{array}{c}70 \\
\text { (IROA) }\end{array}$ & $\begin{array}{c}95 \\
\text { (CEP, IROA) }\end{array}$ \\
\hline $\begin{array}{c}3.6 \\
\text { (Rich) }\end{array}$ & $\begin{array}{c}2 \\
\text { (IROA) }\end{array}$ & $\begin{array}{c}35 \\
\text { (IROA) }\end{array}$ & $\begin{array}{c}70 \\
\text { (IROA) }\end{array}$ & $\begin{array}{c}95 \\
\text { (CEP, IROA) }\end{array}$ \\
\hline $\begin{array}{c}2.2 \\
\text { (Lean) }\end{array}$ & $\begin{array}{c}2 \\
\text { (IROA) }\end{array}$ & $\begin{array}{c}35 \\
\text { (IROA) }\end{array}$ & $\begin{array}{c}70 \\
\text { (IROA) }\end{array}$ & $\begin{array}{c}95 \\
\text { (CEP, IROA) }\end{array}$ \\
\hline $\begin{array}{c}1.0 \\
\text { (Super Lean) }\end{array}$ & $\begin{array}{c}2 \\
\text { (IROA) }\end{array}$ & $\begin{array}{c}35 \\
\text { (IROA) }\end{array}$ & - & $\begin{array}{c}95 \\
\text { (CEP) }\end{array}$ \\
\hline Costing & No & Yes & No & Yes \\
\hline
\end{tabular}




\section{Recovery Costs for 2.2 GPM Gas}

\subsection{G P M}

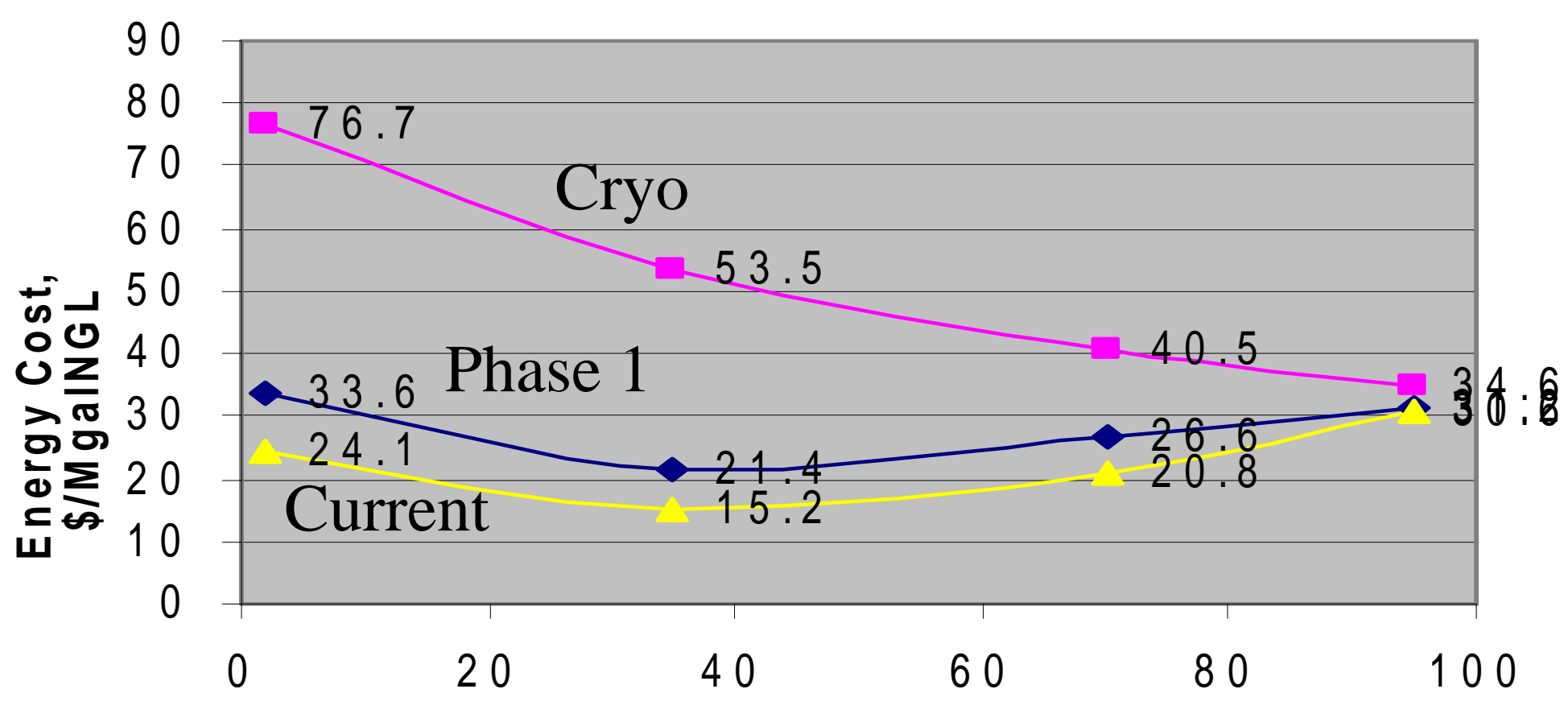

Ethane Recovery, \% 


\section{Recovery Costs for $3.6 \mathrm{GPM}$ Gas}

$$
3.6 \text { G P M }
$$

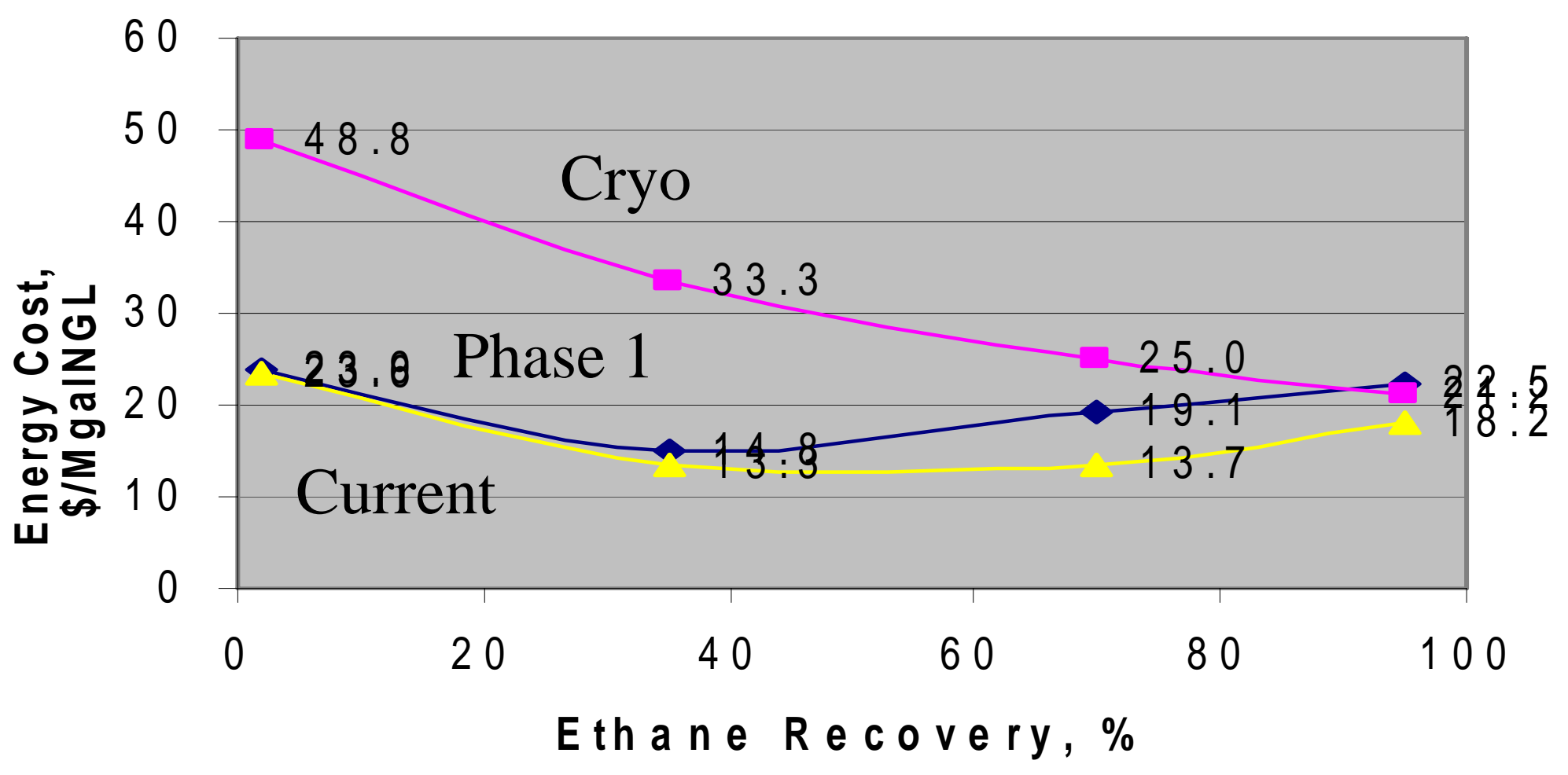




\section{U.S. NGL Demand Favors Low Low $\mathrm{C}_{2}$ Recovery}

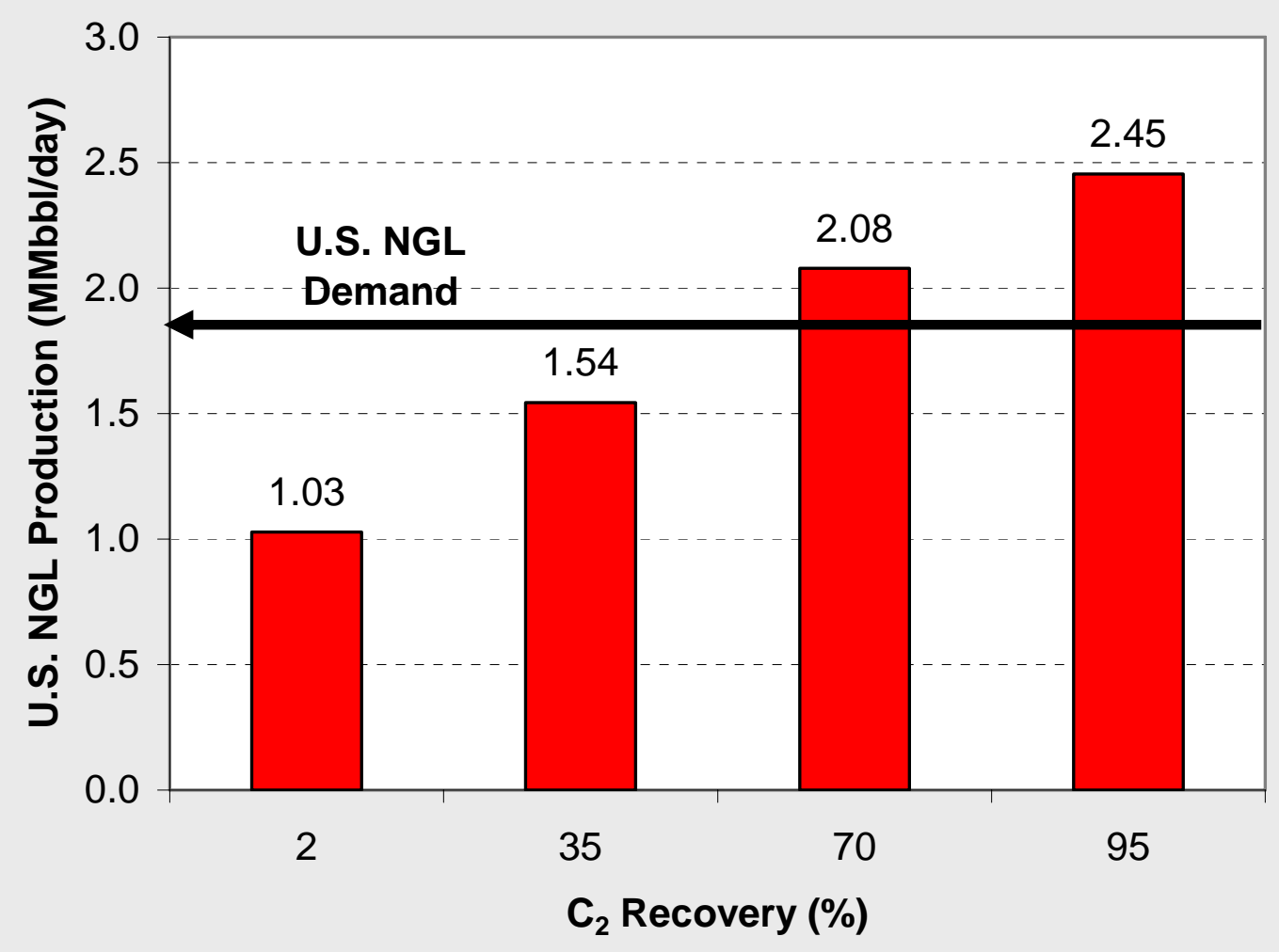

Based on gas richness of 2.2 GPM (National Avg.) 
Final Progress Report

DE-FC36-03G013150

VLE Equipment
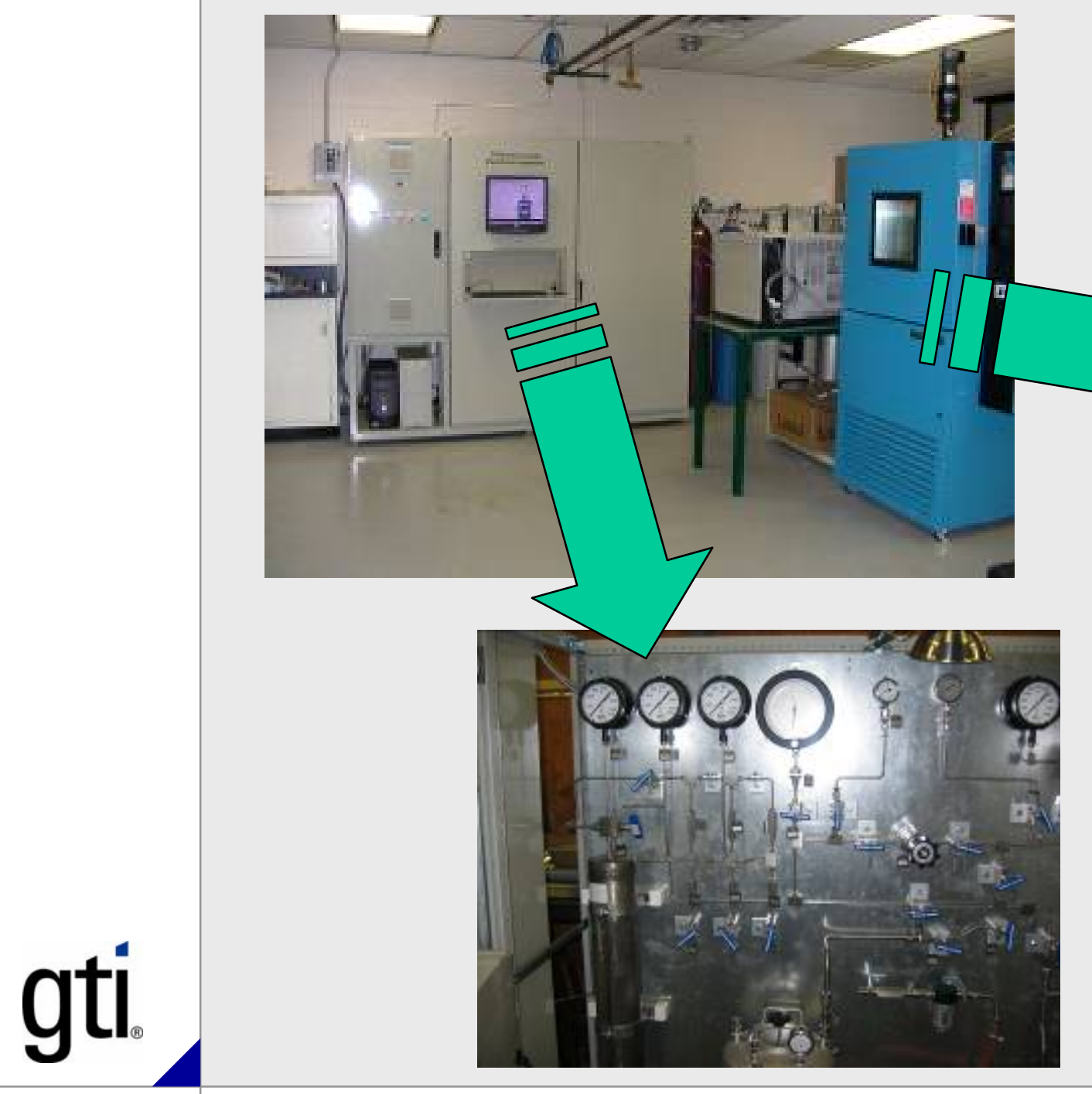


\section{Preliminary Methane Solubility in Kerosenes}
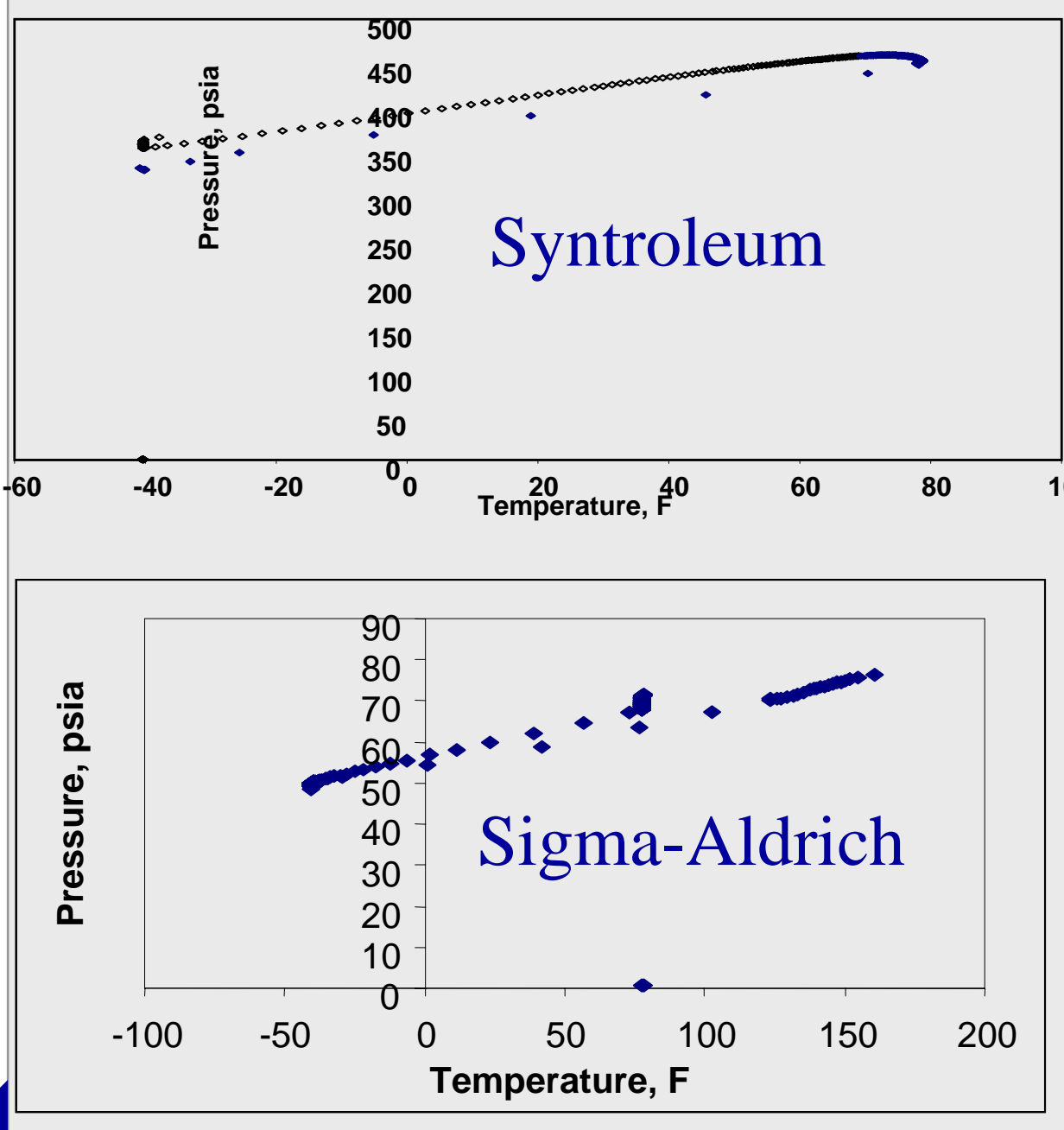

\begin{tabular}{|l|c|c|}
\hline & Syntroleum & $\begin{array}{c}\text { Sigma } \\
\text { Aldrich }\end{array}$ \\
\hline Temp, K & 233 & 233 \\
\hline $\begin{array}{l}\text { Stirrer } \\
\text { Speed, } \\
\text { rpm }\end{array}$ & 175 & 175 \\
\hline$P_{\text {Regen, psia }}$ & 459.53 & 49.39 \\
\hline$P_{\text {Equil }}$, psia & 353.90 & 39.48 \\
\hline $\begin{array}{l}\text { Henry } \\
\text { constant, } \\
\text { (m }{ }^{3} \text { Pa/mol) }\end{array}$ & 1753 & 2005 \\
\hline
\end{tabular}




\section{Bench-scale Unit Design}

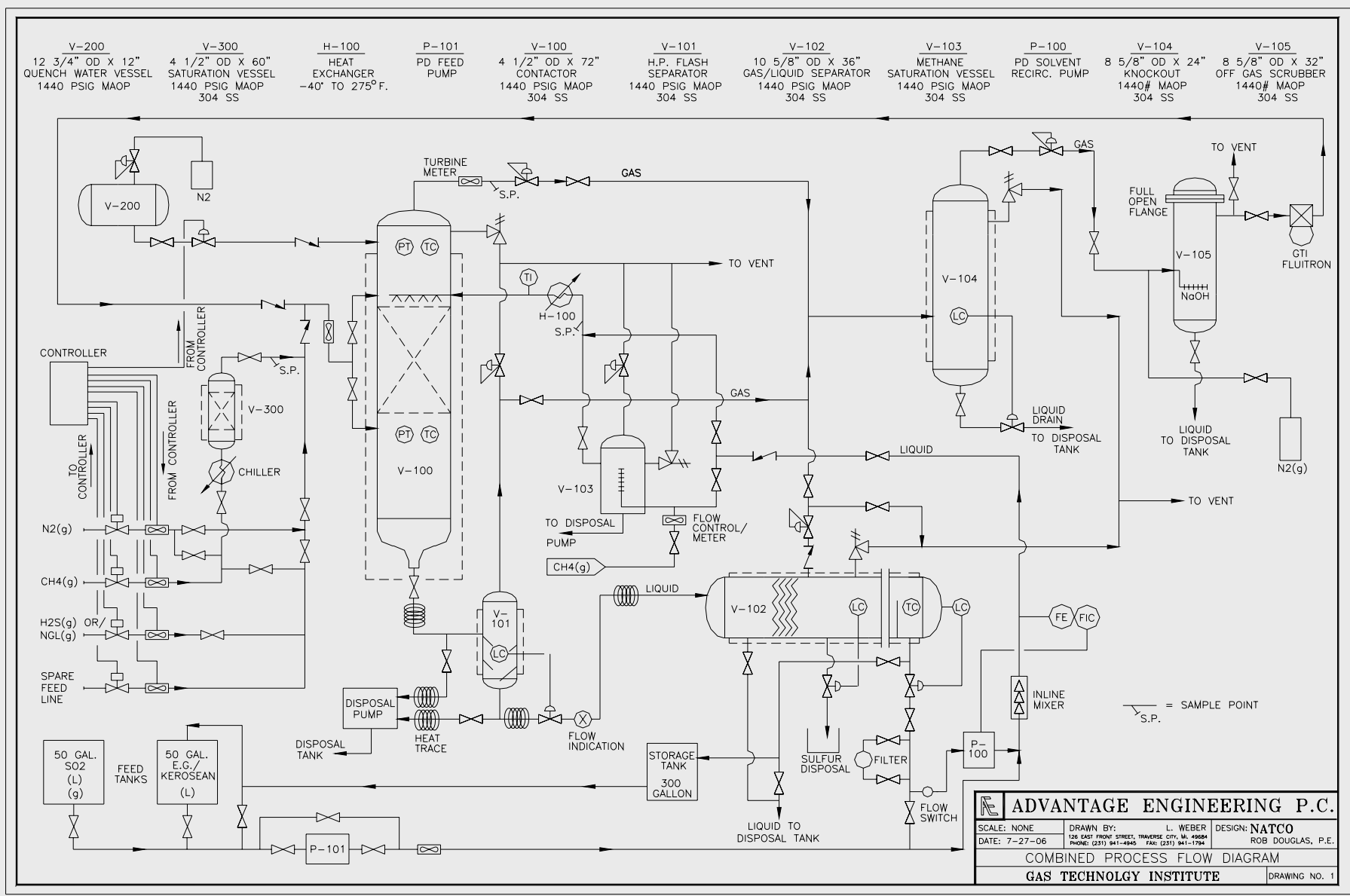




\section{Bench-scale Unit at GTI}

\section{Absorber Column - V100}

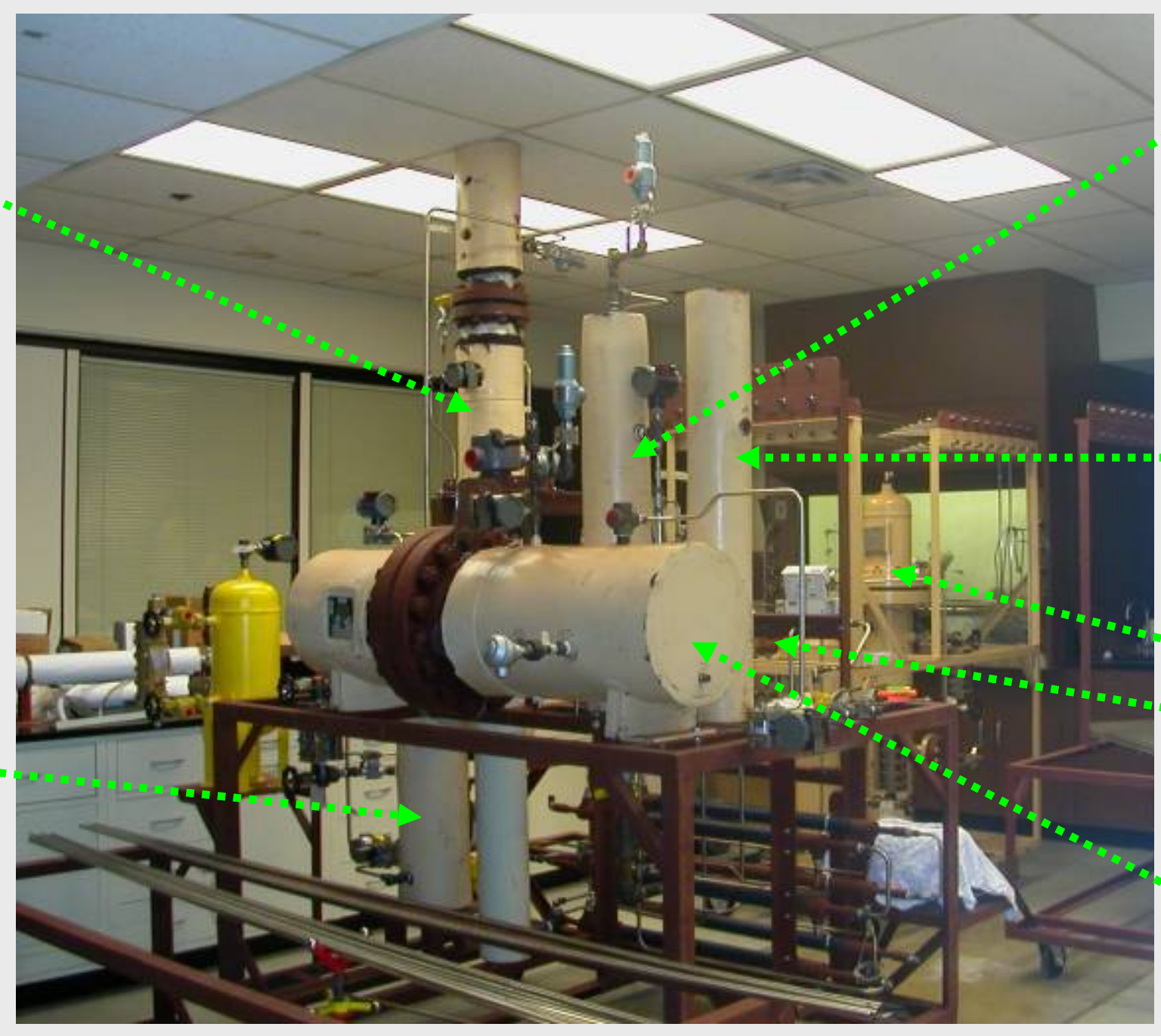

Page 69
HP

Flash V101
Methane Saturator V103

Water

Saturator V300

Knockout Scrubber V104/105 Separator V102 


\section{Pilot Plant - Dehydration Section}

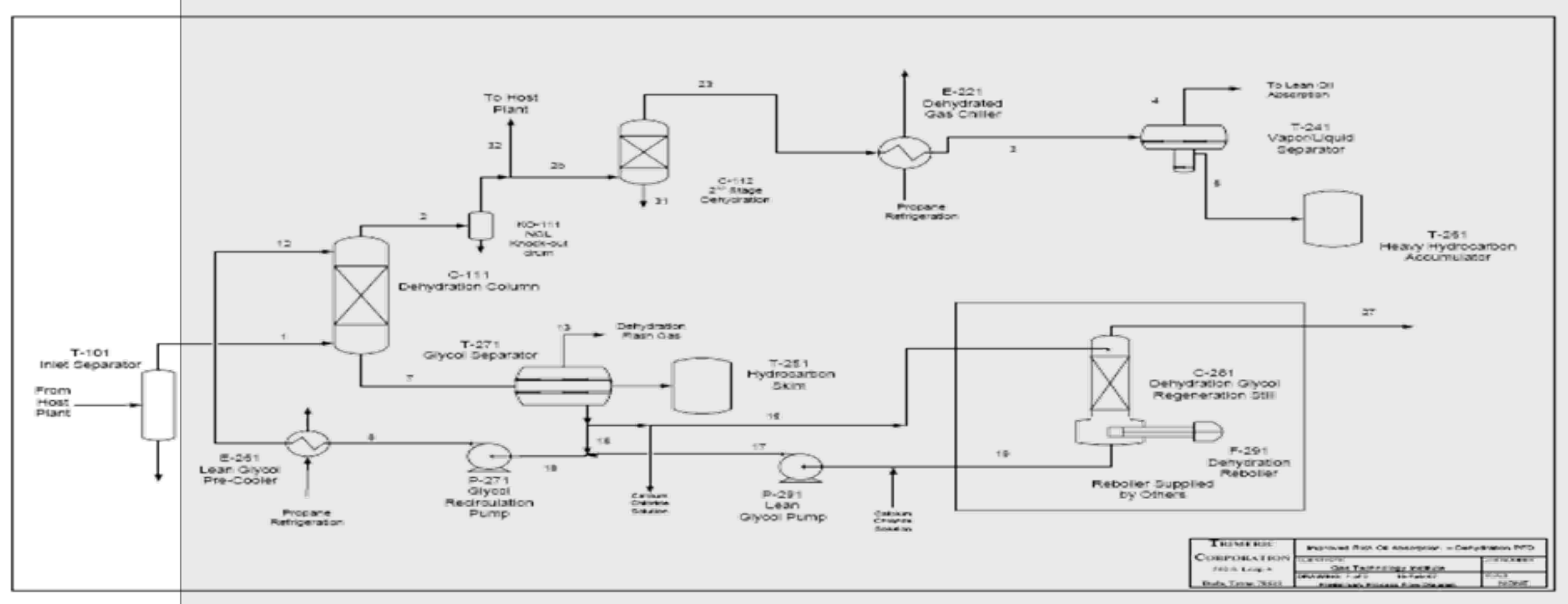

gti 


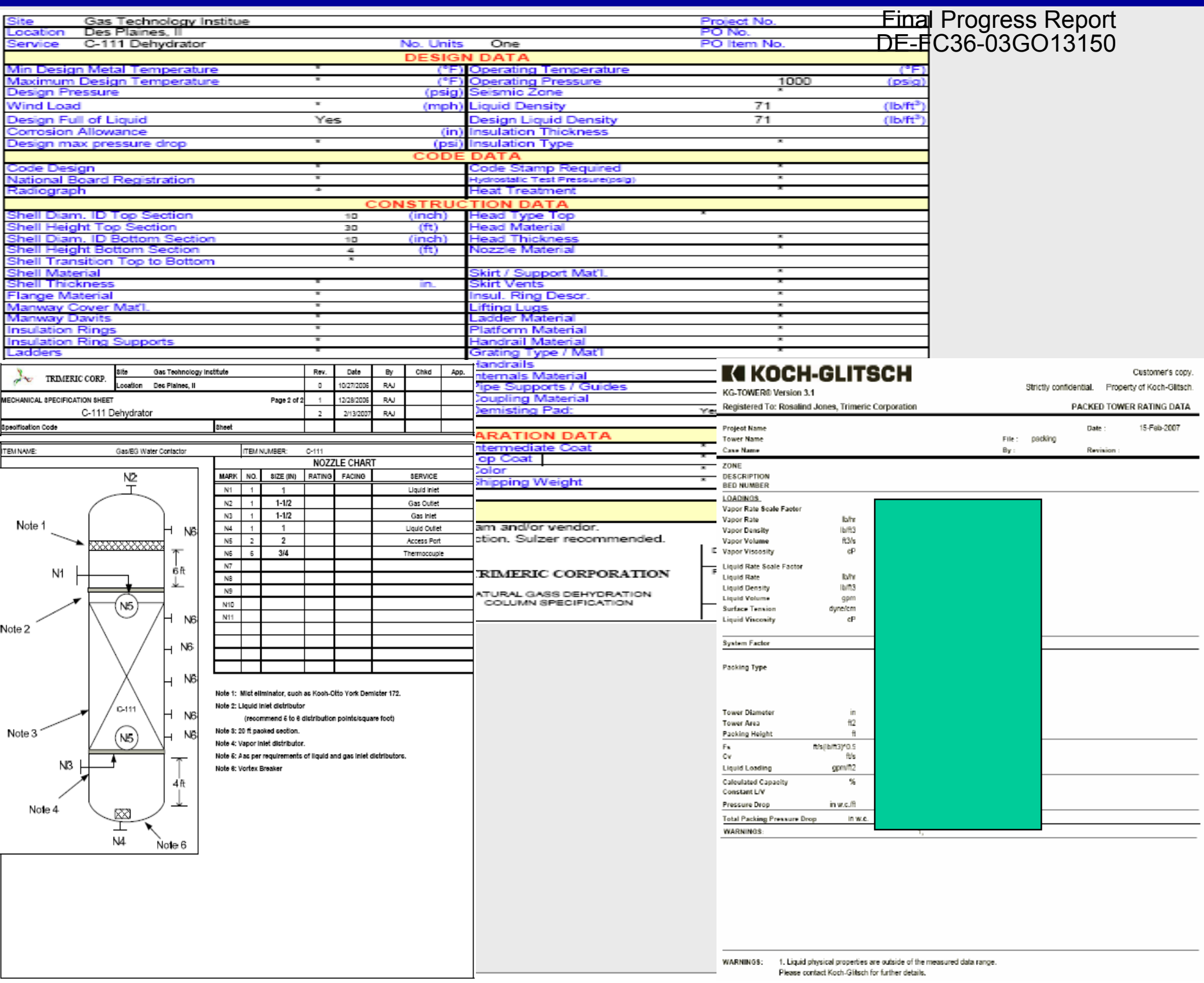

Page 71 


\section{Conclusions}

$>$ Phase-1 of the project has been successfully completed

$>$ IROA technology is expected to -

- Provide lower cost, more flexible gas processing plants of any sizes for varying inlet gas conditions

- Substantial energy savings for $C_{2}$ rejection operations

- Application of new, efficient equipment in gas processing industry: such as plate-fin heat exchangers, high-efficiency structured packing for absorption/distillation columns

$>$ Acceptance of IROA by gas processors will lead to secure supplies of NGLs with varying gas prices 


\section{Future Work}

$>$ Complete VLE experiments to generate Kvalue data for kerosene-hydrocarbon mixtures

$>$ Conduct bench-scale tests using a natural gas slip stream at various operating conditions and obtain scale-up data

$>$ Update the simulation model and optimize the design for maximizing energy efficiency

$>$ Design and operate the pilot-scale unit

$>$ Continue to engage with gas processing industry to commercialize the IROA technology 


\section{Acknowledgements}

$>$ The authors gratefully acknowledge the support and funding from:

- U.S. DOE (Energy Efficiency and Renewable Energy) Contract DE-FC36-03G013150

- Gas Research Institute Contract 8372

$>$ The authors also gratefully acknowledge the advise from:

- Mr. Johnny Johnson, The Washington Group

- Ms. Susan King, Pearl Development Corp

- Dr. Pervaiz Nasir, Enterprise Product Partners

- Mr. Ajay Chandra, Purvin \& Gertz 
Low Cost Chemical Feedstocks Using an Improved and Energy Efficient Natural Gas Liquid (NGL) Removal Process (DOE Award \# DE-FC36-03G013150)

Howard Meyer, Diana Matonis, and Dennis Leppin

\author{
Gas Technology Institute \\ Yingzhong Lu, Green Hi-Tek
}

April 25, 2007

Chemicals Subprogram Portfolio Review 2007 AIChE Spring National Meeting, Houston, TX 


\section{Project Objectives}

$>$ Develop a new low-cost and energy efficient NGL recovery process (as per U.S. patent $6,553,784$ ) through combination of theoretical, bench-scale and pilot-scale testing which will ensure a supply of low cost chemical feedstocks for the U.S. petrochemical industry 


\section{Project Scope}

$>$ Phase -1 (1 yr)

$>$ develop a preliminary computer simulation model to verify the process performance and compare it with conventional cryogenic technology

$>$ Conduct techno-economic evaluation of the process

$>$ Phase -2 (4 yrs)

$>$ design, construct and operate bench and pilot-scale units to demonstrate the technology and develop scale-up data

$>$ conduct critical R\&D related to the development of the new process

$>$ update computer simulation model based on bench and pilot-scale test data

$>$ perform market study, develop process economics and commercialization path 


\section{Project Motivation}

$>$ NGL extraction industry provide the majority of the petrochemical feedstocks

$>$ In times of high natural gas prices in relation to hydrocarbon liquids prices, these plants will either shutdown or leave NGLs in the natural gas leading to supply shortage and high prices for petrochemical feedstocks

$>$ New technology is needed to enable stable and economical supply of chemical feedstocks 


\section{Project Schedule Issues}

$>$ Construction of bench-scale unit has caused major delay in project schedule and budgets

$>$ Competitive bid on design and construction from 4 vendors in 1QFY06

$>$ NATCO selected as low bid, NGL experienced, 04/06 delivery date with $\mathrm{PO}$ issued 12/05

> Unit delivered to GTI 03/07, Estimated cost $\$ 550 \mathrm{~K}$

$>$ Design of pilot-plant unit performed prior to bench-scale unit testing

$>$ Bids received from $3 \mathrm{E} / \mathrm{C}$ companies ranging from $\$ 1.3$ to $2.3 \mathrm{MM}$ in $1 \mathrm{QFY} 2007$

$>$ Decision to perform more engineering, concentrate on dehydration step, rebid, coming in at $\$ 850 \mathrm{~K}$ 


\section{Project Budget and Spending}

\begin{tabular}{|c|c|c|c|c|c|c|c|}
\hline \multicolumn{3}{|c|}{ Budget Period } & \multirow[b]{2}{*}{$\begin{array}{c}\text { Proposed } \\
\text { DOE } \\
\text { Spending }\end{array}$} & \multicolumn{3}{|c|}{ Actual Spending to $3 / \mathbf{3 1 / 2 0 0 7}$} & \multirow[b]{2}{*}{$\begin{array}{l}\text { DOE Funding } \\
\text { Limitation }\end{array}$} \\
\hline Year & Start Date & End Date & & $\begin{array}{l}\text { DOE } \\
\text { Amount }\end{array}$ & $\begin{array}{l}\text { GTI Cost } \\
\text { Share }\end{array}$ & TOTAL & \\
\hline 0 & & 9/30/2003 & $\$ 0$ & $\$ 0$ & $\$ 0$ & $\$ 0$ & $\$ 345,000$ \\
\hline 1 & $10 / 1 / 2003$ & 9/30/2004 & $\$ 525,500$ & $\$ 249,952$ & $\$ 35,617$ & $\$ 285,569$ & $\$ 599,000$ \\
\hline 2 & 10/1/2004 & 9/30/2005 & $\$ 491,000$ & $\$ 223,252$ & $\$ 154,908$ & $\$ 378,160$ & $\$ 836,278$ \\
\hline 3 & 10/1/2005 & 9/30/2006 & $\$ 800,300$ & $\$ 284,371$ & $\$ 173,638$ & $\$ 458,009$ & $\$ 936,278$ \\
\hline 4 & $10 / 1 / 2006$ & $9 / 30 / 2007$ & $\$ 513,900$ & $\$ 96,799$ & $\$ 290,237$ & $\$ 387,036$ & $\$ 1,508,774$ \\
\hline 5 & $10 / 1 / 2007$ & 9/30/2008 & $\$ 248,060$ & & & & \\
\hline & & & $\$ 2,578,760$ & $\$ 854,374$ & $\$ 654,400$ & $\$ 1,508,774$ & \\
\hline
\end{tabular}




\section{Cumulative Budget and Spending}

\begin{tabular}{|rrr|rrrr|}
\hline \multicolumn{3}{|c|}{ Budget Period } & \multicolumn{4}{c|}{ Cumulative as of 3/31/2007 } \\
\hline Year & $\begin{array}{r}\text { Start } \\
\text { Date }\end{array}$ & End Date & $\begin{array}{r}\text { Proposed Cum } \\
\text { Spending }\end{array}$ & $\begin{array}{r}\text { DOE Cum } \\
\text { Amount }\end{array}$ & Cum TOTAL & $\begin{array}{r}\text { GTI Cum } \\
\text { Cost } \\
\text { Share }\end{array}$ \\
\hline 0 & & $9 / 30 / 2003$ & $\$ 0$ & $\$ 0$ & $\$ 0$ & $\$ 0$ \\
1 & $10 / 1 / 2003$ & $9 / 30 / 2004$ & $\$ 525,500$ & $\$ 249,952$ & $\$ 285,569$ & $\$ 35,617$ \\
\hline $\mathbf{2}$ & $\mathbf{1 0 / 1 / 2 0 0 4}$ & $\mathbf{9 / 3 0 / 2 0 0 5}$ & $\mathbf{\$ 1 , 0 1 6 , 5 0 0}$ & $\mathbf{\$ 4 7 3 , 2 0 4}$ & $\mathbf{\$ 6 6 3 , 7 2 9}$ & $\mathbf{\$ 1 9 0 , 5 2 5}$ \\
\hline 3 & $\mathbf{1 0 / 1 / 2 0 0 5}$ & $\mathbf{9 / 3 0 / 2 0 0 6}$ & $\mathbf{\$ 1 , 8 1 6 , 8 0 0}$ & $\mathbf{\$ 7 5 7 , 5 7 5}$ & $\mathbf{\$ 1 , 1 2 1 , 7 3 8}$ & $\mathbf{\$ 3 6 4 , 1 6 3}$ \\
\hline 4 & $10 / 1 / 2006$ & $9 / 30 / 2007$ & $\$ 2,330,700$ & $\$ 854,374$ & $\$ 1,508,774$ & $\$ 654,400$ \\
\hline 5 & $10 / 1 / 2007$ & $9 / 30 / 2008$ & $\$ 2,578,760$ & & & \\
\hline
\end{tabular}




\section{FY 2007 Budget and Impact}

$>$ \$685K Requested for $4 / 1 / 2007$ to $9 / 30 / 2007$

$>$ \$385K Requested for $10 / 1 / 2007$ to $12 / 31 / 2007$

$>$ \$300K DOE Funds secured to $9 / 30 / 2007$

$>$ \$300K GTI Cash cofunding

$>$ Impact: Delay of pilot plant to next fiscal year 


\section{Original Project Tasks \& Schedule}

\begin{tabular}{|c|c|c|c|c|c|c|c|c|c|c|c|c|c|c|c|c|c|c|c|c|c|}
\hline \multirow{2}{*}{$\begin{array}{c}\text { Task } \\
\text { \# }\end{array}$} & \multirow[t]{2}{*}{ Description } & \multicolumn{4}{|c|}{2004} & \multicolumn{4}{|c|}{2005} & \multicolumn{4}{|c|}{2006} & \multicolumn{4}{|c|}{2007} & \multicolumn{4}{|c|}{2008} \\
\hline & & 1 & 2 & 3 & 4 & 1 & 2 & 3 & 4 & 1 & 2 & 3 & 4 & 1 & 2 & 3 & 4 & 1 & 2 & 3 & 4 \\
\hline 1 & $\begin{array}{l}\text { Collect relevant physical property and } \\
\text { VLE data for preliminary technical } \\
\text { verification and design of bench, pilot } \\
\text { and commercial-scale units }\end{array}$ & & & & 1 & & & & & & & & & & & & & & & & \\
\hline 2 & $\begin{array}{l}\text { Design and construct bench-scale unit } \\
\text { for laboratory tests }\end{array}$ & & & & & & 2 & & & & & & & & & & & & & & \\
\hline 3 & $\begin{array}{l}\text { Conduct bench-scale tests and } \\
\text { verify/generate the design data for } \\
\text { pilot-scale unit }\end{array}$ & & & & & & & & 3 & & & & & & & & & & & & \\
\hline 4 & $\begin{array}{l}\text { Develop computer simulation for } \\
\text { preliminary technical verification and } \\
\text { bench, pilot and commercial-scale } \\
\text { design }\end{array}$ & & & & & & & & & & & & & & & & & 6 & & & \\
\hline 5 & $\begin{array}{l}\text { Perform theoretical and experimental } \\
\text { study (R\&D work) to address critical } \\
\text { process development issues }\end{array}$ & & & & & & & & & & & & & & & & & & & & \\
\hline 6 & $\begin{array}{l}\text { Design, construct and install a pilot } \\
\text { unit and conduct field tests }\end{array}$ & & & & & & & & & & 4 & & & & 5 & & & & & & \\
\hline 7 & $\begin{array}{l}\text { Conduct market study and prepare } \\
\text { commercialization package }\end{array}$ & & & & & & & & & & & & & & & & & & & 7 & \\
\hline 8 & $\begin{array}{l}\text { Attend technical meetings and prepare } \\
\text { technical reports }\end{array}$ & & & & & & & & & & & & & & & & & & & & 8 \\
\hline
\end{tabular}




\section{Project Performance}

\begin{tabular}{|c|c|c|c|}
\hline$\#$ & Milestone & $\begin{array}{l}\text { Schedule at } \$ 300 \mathrm{~K} \text { for } \\
\text { FY07 and FY08 }\end{array}$ & $\begin{array}{l}\text { Schedule at } \$ 850 K \text { for } \\
\text { both FY07 and FY08 }\end{array}$ \\
\hline 1 & $\begin{array}{l}\text { Preliminary computer simulation } \\
\text { model developed and initial technical } \\
\text { verification of the process completed }\end{array}$ & Completed & Completed \\
\hline 2 & $\begin{array}{l}\text { Design and construction of bench-scale } \\
\text { unit completed }\end{array}$ & $\begin{array}{l}\text { Delivered to GTI } 03 / 07 \\
\text { Commissioning by } 05 / 07\end{array}$ & $\begin{array}{l}\text { Delivered to GTI } 03 / 07 \\
\text { Commissioning by } 05 / 07\end{array}$ \\
\hline 3 & $\begin{array}{l}\text { Bench-scale tests completed and } \\
\text { computer simulation model updated }\end{array}$ & $\begin{array}{l}\text { Scheduled to complete } \\
\qquad 06 / 08\end{array}$ & $\begin{array}{l}\text { Scheduled to complete } \\
\qquad 10 / 07\end{array}$ \\
\hline 4 & $\begin{array}{l}\text { Construction of pilot scale unit } \\
\text { completed }\end{array}$ & $\begin{array}{l}\text { Scheduled to complete by } \\
\qquad 12 / 09\end{array}$ & $\begin{array}{l}\text { Scheduled to complete by } \\
\qquad 11 / 07\end{array}$ \\
\hline 5 & $\begin{array}{l}\text { Field-tests completed, process design } \\
\text { verified and computer simulation } \\
\text { model updated }\end{array}$ & $\begin{array}{c}\text { Schedule to initiate testing } \\
\text { by } 04 / 10 \text {, testing by } 04 / 11 \text {, } \\
\text { modeling by } 08 / 11\end{array}$ & $\begin{array}{c}\text { Schedule to initiate } \\
\text { testing by } 02 / 08, \text { testing } \\
\text { by } 06 / 08, \text { modeling by } \\
07 / 08\end{array}$ \\
\hline 6 & $\begin{array}{l}\text { Final computer simulation model } \\
\text { completed }\end{array}$ & $\begin{array}{l}\text { Scheduled to complete by } \\
\qquad 06 / 12\end{array}$ & $\begin{array}{l}\text { Schedule to complete by } \\
\qquad 08 / 08\end{array}$ \\
\hline 7 & $\begin{array}{l}\text { Engineering and commercialization } \\
\text { package completed }\end{array}$ & $\begin{array}{l}\text { Scheduled to complete by } \\
\qquad 12 / 12\end{array}$ & $\begin{array}{l}\text { Schedule to complete by } \\
\qquad 09 / 08\end{array}$ \\
\hline 8 & $\begin{array}{l}\text { Final report prepared and submitted } \\
\text { to DOE }\end{array}$ & $\begin{array}{l}\text { Scheduled to complete by } \\
\qquad 03 / 13\end{array}$ & On time $(9 / 30 / 2008)$ \\
\hline
\end{tabular}




\section{CPAT Economics}

\begin{tabular}{lcccc} 
& & IROA & \multicolumn{2}{c}{ Turbo-Expander } \\
Project Capital (MM) & $\$$ & 10.30 & $\$$ & 10.30 \\
Ancillary Capital (MM) & $\$$ & 3.09 & $\$$ & 3.09 \\
IRR & & $75.6 \%$ & & $64.6 \%$
\end{tabular}

Annual Operating

Costs

$\begin{array}{lllll}\text { Raw Materials (MM) } & \$ & 265.15 & \$ & 265.15\end{array}$

Utilities (MM)

$\begin{array}{llll}\$ & 1.11 & \$ & 3.34\end{array}$

Consumables (MM)

$\$$

$-\quad \$$

$\$$

$0.31 \$$

0.31 


\section{CPAT Energy Savings}

\begin{tabular}{|c|c|c|c|c|c|}
\hline \multicolumn{6}{|c|}{ Project Summary Results for New Technology, Part I } \\
\hline \multicolumn{6}{|c|}{ Energy Impacts for Natural Gas Liquids } \\
\hline & 2010 & 2015 & 2020 & 2025 & 2030 \\
\hline MARKET $\left(\left(10^{n} 6\right)\right.$ lbs) $[7]$ & $111,066.2$ & $115,008.3$ & $119,090.3$ & $123,317.3$ & $127,694.2$ \\
\hline MARKET PENETRATION $[\overline{7}]$ & $0.0 \%$ & $8.6 \%$ & $19.2 \%$ & $26.6 \%$ & $29.0 \%$ \\
\hline ACCELERATED UNITS DEFLOVED & 0 & 46 & 107 & 153 & 173 \\
\hline \multicolumn{6}{|l|}{ ENERGY SAVINGS ?] } \\
\hline $\begin{array}{l}\text { Heat'Steam Energy Savings } 7] \\
\text { (trillion Btu) }\end{array}$ & 0.00 & 12.99 & 30.23 & 43.22 & 48.87 \\
\hline Electricity Savirgs (trillion Btu) 7 & 0.00 & 0.00 & 0.00 & 0.00 & 0.00 \\
\hline \multicolumn{6}{|l|}{ TACIT ENERGYSAVINGS } \\
\hline $\begin{array}{l}\text { Tacit Heat'Steam Energy Savings }[7] \\
\text { (trillIIon Btu) }\end{array}$ & 0.00 & 12.99 & 30.23 & 43.22 & 48.87 \\
\hline $\begin{array}{l}\text { Tacit Electricity Savings } \\
\text { (trillion Btu) }\end{array}$ & 0.00 & 0.00 & 0.00 & 0.00 & 0.00 \\
\hline $\begin{array}{l}\text { Tacit Feedstock Energy Savings } \\
\text { (trillion Btu) }\end{array}$ & 0.00 & 0.00 & 0.00 & 0.00 & 0.00 \\
\hline
\end{tabular}




\section{CPAT Energy Costs}

\begin{tabular}{|c|c|}
\hline \multicolumn{2}{|c|}{ Energy Cost as a \% of Product Price } \\
\hline Comentional Technology $?$ & New Technology $?$ \\
\hline 1.2 & 0.4 \\
\hline
\end{tabular}




\section{CPAT Pollutant Reductions}

\begin{tabular}{|c|c|c|c|c|c|}
\hline \multicolumn{6}{|c|}{ Project Summary Results for New Technology, Part II } \\
\hline \multicolumn{6}{|c|}{ Energy Impacts for Natural Gas Liquids } \\
\hline & 2010 & 2015 & 2020 & 2025 & 2030 \\
\hline \multicolumn{6}{|l|}{$\begin{array}{l}\text { POLLUTANT REDUCTIONS } 7] \\
\text { (Whetric Tons) }\end{array}$} \\
\hline Carbon (tCE/yr*1 $0^{\text {ng }}$ ) & 0.00 & 0.21 & 0.50 & 0.71 & 0.80 \\
\hline Nitrogen Oxides (thy) & 0.00 & 2.00 & 4.65 & 6.66 & 7.53 \\
\hline Sulfur Oxides (tyr) & 0.00 & 5.60 & 13.03 & 18.63 & 21.06 \\
\hline Carbon Monoxide (thy) & 0.00 & 0.13 & 0.30 & 0.42 & 0.48 \\
\hline Volatile Organic Compounds (tyr) & 0.00 & 0.03 & 0.06 & 0.09 & 0.10 \\
\hline Particulates(thy) & 0.00 & 0.09 & 0.22 & 0.32 & 0.36 \\
\hline
\end{tabular}

gti 


\section{Retrofit CPAT Case}

> Assumptions:

$>$ Retrofit into 125 existing refrigerated lean oil plants

- Penetration set to $100 \%$ to see maximum case

- All plants converted within 25 years

$>$ Retrofit case

- Replacing the dehydration tower and $\sim 1 / 3$ of the heat exchangers

- 3 months of off-line

- 3 months from $50 \%$ start up to $100 \%$ capacity

$>$ Conventional case

- As is/do nothing

- \$1 capital (program requires some value) 


\section{CPAT Retrofit Economics}

$\begin{array}{lrrrr} & & \text { IROA } & \text { Turbo-Expander } \\ \text { Project Capital (MM) } & \$ & 1.8 & \$ 0.000001 \\ \text { Ancillary Capital (MM) } & \$ & .53 & \$ & 0 \\ \text { IRR } & & 500 \% & 100 \%\end{array}$

\section{Annual Operating}

Costs

Raw Materials (MM)

$\$ \quad 265.15$

\$ 265.15

Utilities (MM)

$\$$

1.11

$\$ \quad 3.34$

Consumables (MM)

$\$$

Labor (MM)

$\$$

0.31

$\$$

$\$ \quad 0.31$ 


\section{CPAT Retrofit Energy Savings}

\begin{tabular}{|c|c|c|c|c|c|}
\hline \multicolumn{6}{|c|}{ Project Summary Results for New Technology, Part I } \\
\hline \multicolumn{6}{|c|}{ Energy Impacis for Natural Gas Liquids 7} \\
\hline & 2010 & 2015 & 2020 & 2025 & 2030 \\
\hline MAFIKET $\left(\left(10^{\wedge} 6\right)\right.$ lbs $)$ & $26,750.0$ & $26,750]$. & $26,7 \in 0.0$ & $26,750.0$ & $26,750.0$ \\
\hline MAFIET PENETRATION & $0.0 \%$ & $12.0 \%$ & $32 .[\%$ & $52.0 \%$ & $72.0 \%$ \\
\hline ACCELERATED UNITS DEPLOYED $]$ & 0 & 15 & 4[ & 65 & 90 \\
\hline \multicolumn{6}{|l|}{ ENERGY SAVIGS } \\
\hline $\begin{array}{l}\text { Heat'Steam Energy Savings } 7 \\
\text { (trillion Htu) }\end{array}$ & 0.00 & 4.24 & 11.30 & 18.36 & 25.42 \\
\hline Electricity Saw ngs (trillion Btu) $\$$ & 0.00 & 0.00 & 0.00 & 0.00 & 0.00 \\
\hline \multicolumn{6}{|l|}{ TACIT ENERGY SAVINGS } \\
\hline $\begin{array}{l}\text { T:abil HeabSlearr Erıryy Savirugs } \\
\text { (trillion Btu) }\end{array}$ & 0.00 & 4.24 & 11.30 & 18.36 & 25.42 \\
\hline $\begin{array}{l}\text { Tacit Electricity Savings } 9] \\
\text { (trillion Btu) }\end{array}$ & 0.00 & 0.00 & 0.00 & 0.00 & 0.00 \\
\hline $\begin{array}{l}\text { Tacit Feedstosk Energy Savings } ? \\
\text { (trilıon Htu) }\end{array}$ & 0.00 & 0.00 & 0.00 & 0.00 & 0.00 \\
\hline
\end{tabular}




\section{CPAT Retrofit Energy Costs}

\section{Energy Cost as a \% of Product Price}

Corventional Technology ?

1.2
New Technology?

0.4 


\section{CPAT Retrofit Pollutant Reductions}

\begin{tabular}{|c|c|c|c|c|c|}
\hline \multicolumn{6}{|c|}{ Project Summary Results for New Technology, Part II } \\
\hline \multicolumn{6}{|c|}{ Energy Impacts for Natural Gas Liquids } \\
\hline & 2010 & 2015 & 2020 & 2025 & 2030 \\
\hline \multicolumn{6}{|l|}{$\begin{array}{l}\text { POLLUTANT REDUCTIONS } ?] \\
\text { (Wetric Tons) }\end{array}$} \\
\hline Carbon (CES/r $\left.10^{\mathrm{ng}} \mathrm{g}\right)$ & 0.00 & 0.07 & 0.19 & 0.30 & 0.42 \\
\hline Nirogen Oxides (thr) & 0.00 & 0.65 & 1.74 & 2.83 & 3.92 \\
\hline Btlful Oxides ịtyly & 0.00 & 1.83 & 4.87 & 7.91 & 10.96 \\
\hline Carbon Monoxide (thy) & 0.00 & 0.04 & 0.11 & 0.18 & 0.25 \\
\hline Volatile Organ c Compourds (tyr) & 0.00 & 0.01 & 0.02 & 0.04 & 0.05 \\
\hline Particulates(thr) & 0.00 & 0.03 & 0.08 & 0.13 & 0.19 \\
\hline
\end{tabular}

gti 


\section{Commercialization Plan}

$>$ GTI has signed an exclusive agreement with the current owner of the technology (i.e., GHT) and has completed preliminary techno-economic evaluation

$>$ GTI plans to publish some of these results in the gas processing-related conferences (e.g., GPA, LRGCC) where most of the NGL producers and natural gas processors are represented

$>$ After successful completion of bench-scale testing, GTI will approach potential industrial partners for assistance in commercialization of this technology

$>$ Eventually, GTI plans to license the technology to an E\&C or process technology licensors (e.g. , UOP) to enable end users to acquire it 


\section{Team Participation}

\begin{tabular}{|c|c|}
\hline Team Member & Tasks/Contributions \\
\hline $\begin{array}{l}\text { GTI } \\
\text { (Tasks 1-8) }\end{array}$ & $\begin{array}{l}\text { - Over all project management and technical oversight of subcontractors work. } \\
\text { - Gathering of commercial data, cost and marketing information } \\
\text { - Development of research tasks and schedule } \\
\text { - Technical assistance in the development of computer model and continued model update } \\
\text { - Techno-economic evaluation and market study } \\
\text { - Design, construction, commissioning and operation of bench and pilot-scale units } \\
\text { - Data analysis and preparation of reports and publications }\end{array}$ \\
\hline $\begin{array}{l}\text { GHT } \\
\text { (Tasks 1-8) }\end{array}$ & $\begin{array}{l}\text { - Providing confidential technical data to GTI about IROA process } \\
\text { - Technical assistance in model development and review of the results } \\
\text { - Assistance in process designing pf bench and pilot-scale units } \\
\text { - Assistance in data analysis and development of the test plan }\end{array}$ \\
\hline $\begin{array}{l}\text { IIT } \\
(\text { Tasks } 1,4 \& 5)\end{array}$ & $\begin{array}{l}\text { - Development of process simulation model using HYSYS and Aspen } \\
\text { - Literature search and assistance in measuring VLE data and bench-scale testing. }\end{array}$ \\
\hline ORNL (Task 5) & - Assistance in material selection and corrosion tests \\
\hline EnCana & - To provide site for pilot testing \\
\hline KBR & - To review bench and pilot-scale test results \\
\hline
\end{tabular}




\section{EnCana (change from Duke Energy as field host)}

$>$ Net proved reserves of 12.4 Tcf natural gas and 1.1 MMM Bbl crude oil, bitumen and NGLS

$>$ EnCana owns and operates various gas gathering and processing facilities.

$>$ Colorado - 3 plants processing $~ 600 \mathrm{Mcf} / \mathrm{d}$

> Utah - 1 plant processing $60 \mathrm{MMcf} / \mathrm{d}$

> Wyoming - 1 plant processing $70 \mathrm{MMcf} / \mathrm{d}$

$>$ Texas - gathering facilities

> Canada - 50 plants processing 4,000 MMcf/d 


\section{Project status}

$>$ Phase 1 was successfully completed

$>$ Continued development is justified

$>$ Lab testing alternative solvents

$>$ Process modeling improving

$>$ Bench-scale unit delivered to GTI

> Pilot-scale unit purchase order on hold for budget

$>$ Economic models identify advantages at high $\mathrm{C}_{2}$ recoveries 


\section{(12) United States Patent} Lu
(10) Patent No.: $\quad$ US 6,553,784 B2

(45) Date of Patent: Apr. 29, 2003
(54) COMPREHENSIVE NATURAL GAS PROCESSOR

(76) Inventor: Yingzhong Lu, 104 Harland Ct., Oak Ridge, TN (US) 37830

(*) Notice: Subject to any disclaimer, the term of this patent is extended or adjusted under 35 U.S.C. 154(b) by 0 days.

(21) Appl. No.: 09/683,946

(22) Filed: Mar. 6, 2002

\section{Prior Publication Data}

US 2003/0037567 A1 Feb. 27, 2003

(30) Foreign Application Priority Data

Aug. 23, 2001

(CH)

$01124271 \mathrm{~A}$

(51) Int. Cl. ${ }^{7}$

F25J 3/00

(52) U.S. Cl. 62/632;62/633

(58) Field of Search $62 / 620,622,625$, $62 / 632-633,635,618$

\section{References Cited}

\section{U.S. PATENT DOCUMENTS}

$3,837,143$ A $* 9 / 1974$ Sutherland et al. $95 / 162$

4,421,535 A * 12/1983 Mehra

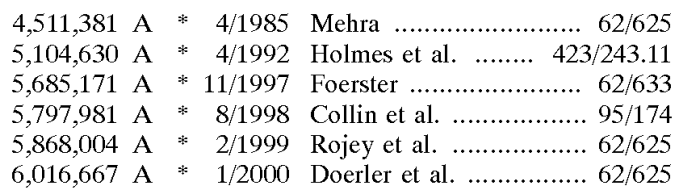

* cited by examiner

Primary Examiner-William C. Doerrler

\section{ABSTRACT}

The present invention related to an apparatus for efficient and cost-effective comprehensive processing of natural gas, including the removal of moisture and the recovery of the higher hydrocarbons components $\left(\mathrm{C}_{2}{ }^{+}\right)$. The said apparatus comprises the following major components: an integrated natural gas processor with a dehydration section and a higher hydrocarbons absorption section; a heat transport medium cooler; an absorbent cooler; a fractional distiller for separating the light oil from the heavy oil absorbent; an inhibitor regenerator; and a refrigeration unit. The present invention provides a low-cost natural gas comprehensive processor that is universally applicable to both terrestrial and off-shore natural gas exploitation. The said apparatus also provides an efficient and cost-effective natural gas dehydrator when the dehydration section is used independently without incorporating the absorption section.

7 Claims, 3 Drawing Sheets

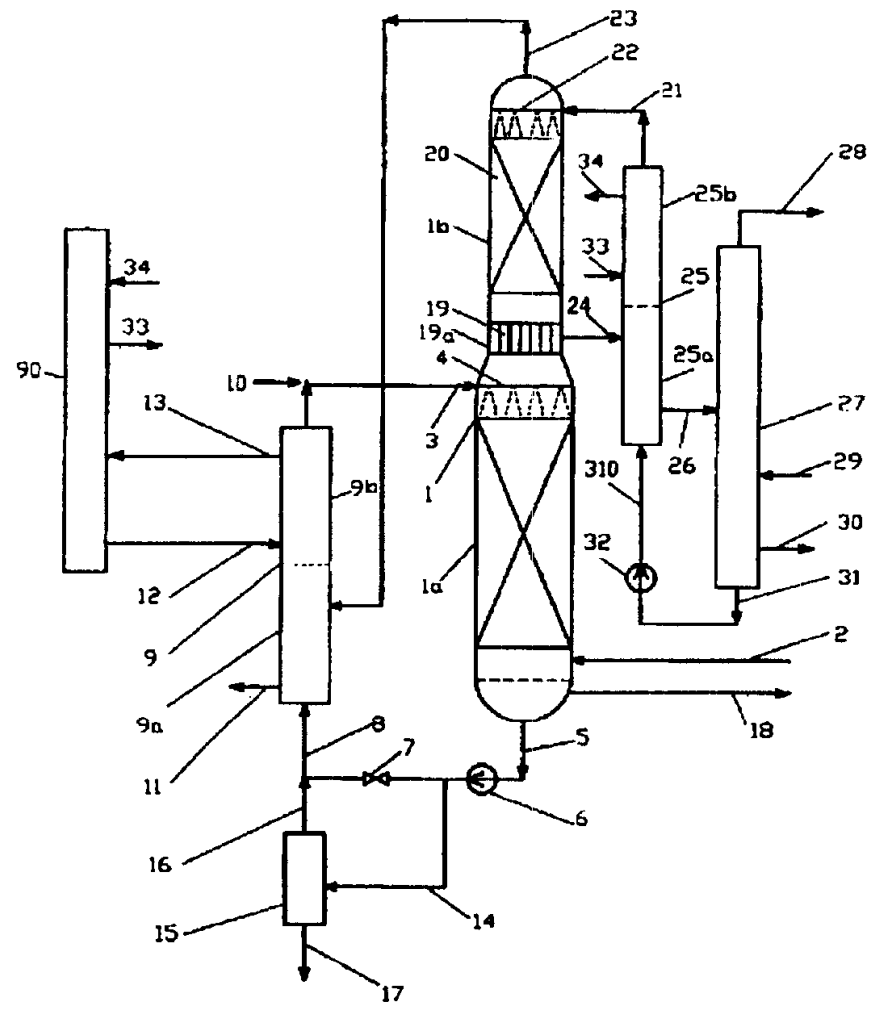


Final Progress Report

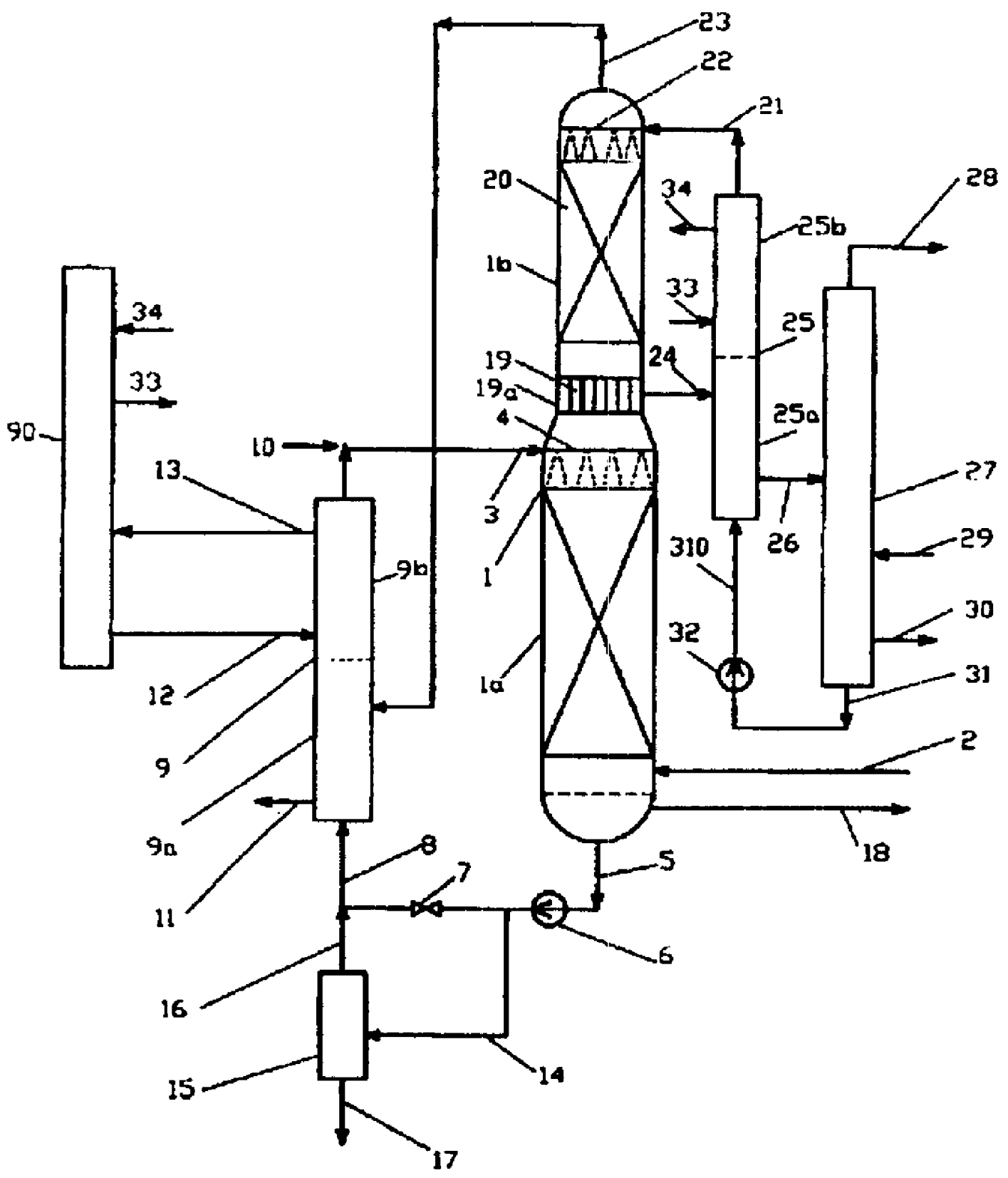

FIG 1 
Final Progress Report

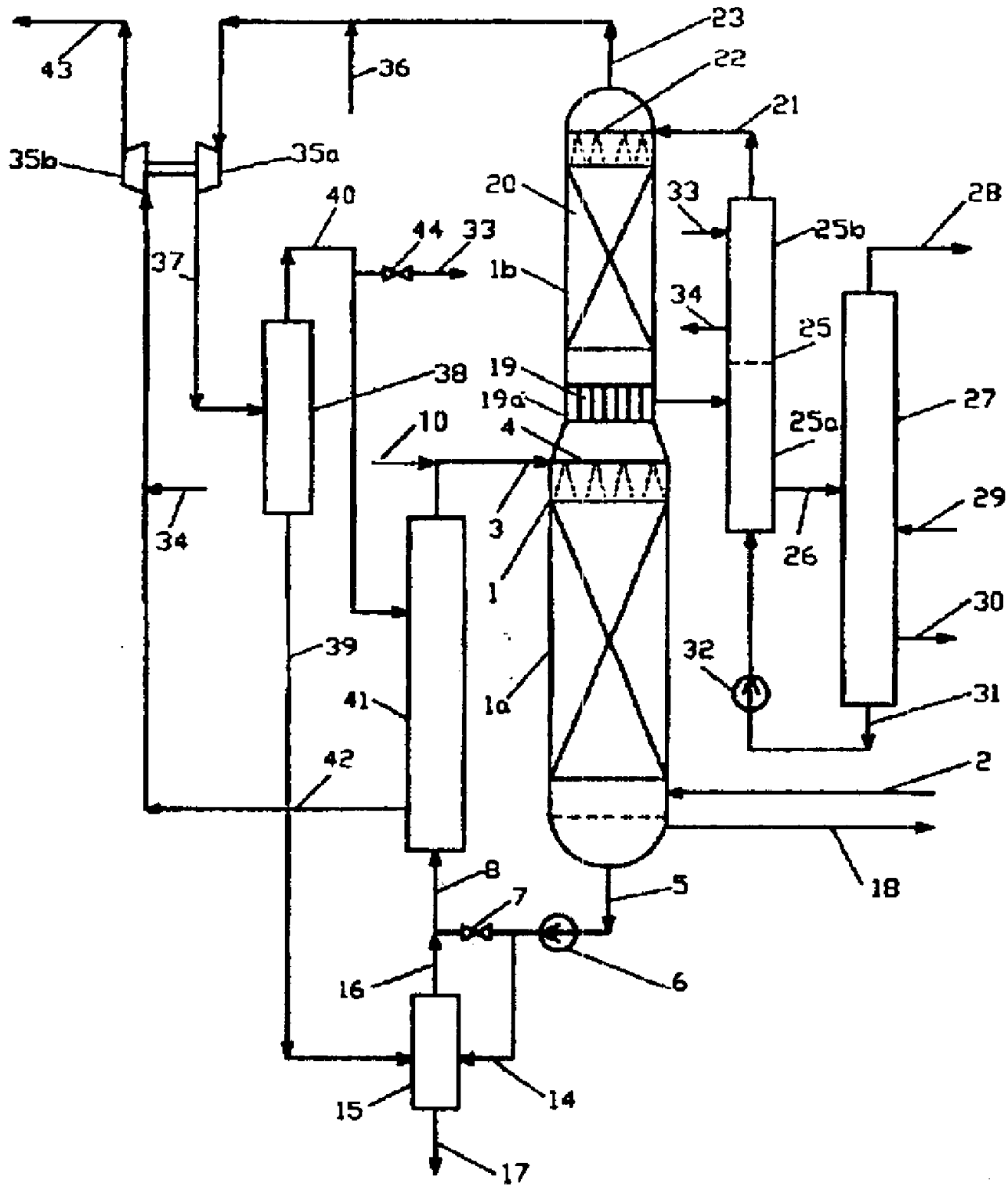

FIG 2 
Final Progress Report

DE-FC36-03G013150

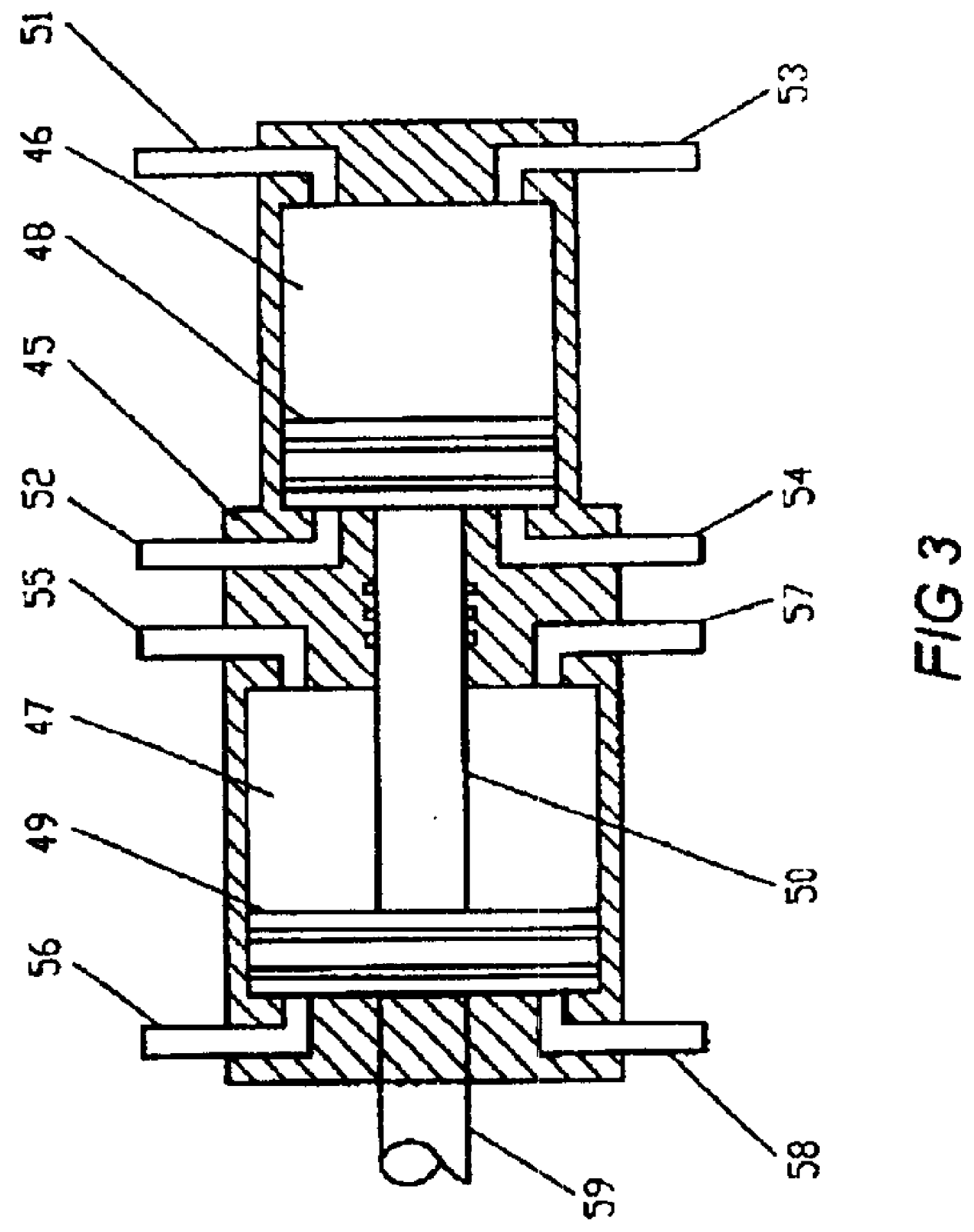




\section{1 COMPREHENSIVE NATURAL GAS PROCESSOR}

\section{BACKGROUND OF INVENTION}

The reduction of $\mathrm{CO}_{2}$ emission is one of the greatest concerns in combating the catastrophic "global warming" trend. As a result, the world puts much emphasis on the exploitation of "clean energy" with less or non-emission for both industrial and domestic uses. Natural gas (hereafter abbreviated as "NG"), as compared with coal and petroleum, is considered the most economic "clean" fuel that is used on a large, industrial scale at present and in the near future. In addition, the discovery of huge amount of ocean-bed gashydrates increases the recoverable resources of NG substantially. It is expected that, in the long run, the global NG consumption may eventually exceeds all other fossil fuels.

NG is a mixture of hydrocarbon gases, consisting of mainly methane $\left(\mathrm{C}_{1}\right)$ and a smaller fraction of heavier gaseous hydrocarbons (i.e., ethane, $\mathrm{C}_{2}$; propane, $\mathrm{C}_{3}$; butane, $\mathrm{C}_{4}$; pentane and higher, $\mathrm{C}_{5}{ }^{+}$; sometimes $\mathrm{C}_{3}+$ is called "light oil" as a whole. However, the economic values of these higher hydrocarbon components, when separated and sold as chemical feedstock, are usually much higher than burnt as a fuel. A number of NG processing plants, therefore, have been constructed to extract these valuable materials.

The state-of-the-art NG processing plants generally work on a cryogenic process for efficiently separating the higher hydrocarbon gases In this process, a huge volume of NG is cooled down by expansion to a very low cryogenic temperature around $-150^{\circ} \mathrm{F}$. Such a process is extremely energy-consuming, and the facility usually comprises many pieces of expensive equipment, notably the molecular-sieve dehydrator, the multiple-flow finned-plate heat exchanger, and the turbo expander-compressor. High capital and operational costs are thus resulted. As a consequence, only a limited fraction of the NG could be processed before consumed as a fuel. Most of the valuable higher hydrocarbon contents was improperly used.

In the past two decades, a number of US patents have been granted in this field, for example, the 13 US patents entitled "hydrocarbon Processing" presented by late Roy E. Campbell, et al., i.e., U.S. Pat. Nos. 4,140,504; 4,157,904; $4,171,964 ; 4,278,457 ; 4,854,955 ; 4,869,740 ; 4,889,545$; $5,555,784 ; 5,568,737 ; 5,771,712 ; 5,881,569 ; 5,983,664$; and $6,182,469$. However, most of these patents only proposed some specific improvements to the same cryogenic process. No substantial break-through in NG processing technology has ever been proposed. A more efficient and cost-effective technology for NG procession, therefore, is desirable.

The recent developments in NG refrigeration dehydration technology, e.g., those presented in U.S. Pat. No. 5,664,426, "Regenerative Gas Dehydrator;" 1997, and U.S. Pat. No. 6,158,242, "Gas Dehydration Method and Apparatus," 2000, provided the basis of a break-through in the NG processing technology. These patents make possible to perform refrigeration dehydration and refrigeration absorption in a single unit.

Accordingly, it is an objective of the present invention to provide a comprehensive NG processor, based on the refrig- 60 eration dehydration and absorption technologies, for efficient and cost-effective comprehensive processing of NG. The said processor could simultaneously perform the removal of moisture and the recovery of the higher hydrocarbons $\left(\mathrm{C}_{2}^{+}\right)$in a single piece of equipment, thus substan- 6 tially reducing the capital and operational costs of the $\mathrm{NG}$ processing plant.

\section{2}

Another objective of the present invention is to provide an energy-saving comprehensive NG processor that, when processing high pressure NG, does not need external energy for refrigeration.

5 A further objective of the present invention is to provide a high-efficiency free-piston expander-compressor to provide the required refrigeration.

\section{SUMMARY OF INVENTION}

10 With regard to the above and other objectives, the present invention provides a comprehensive $\mathrm{NG}$ processor to simultaneously perform refrigeration dehydration and refrigeration absorption of higher hydrocarbon gases with maximum recovery rate at minimum energy consumption. The final 5 product is a gaseous mixture enriched in higher hydrocarbons with minimum residual methane.

The said apparatus comprises the following major components: an integrated NG processor (hereafter abbreviated as "processor) with a refrigeration dehydration section 20 (hereafter abbreviated as "dehydrator") and a refrigeration absorption section (hereafter abbreviated as "absorber"); a heat-transport medium (hereafter abbreviated as "medium") cooler; an absorbent cooler; a fractional distiller; a gashydrate inhibitor (hereafter abbreviated as "inhibitor") regenerator; and a refrigeration unit.

The principle of the operations of the comprehensive NG processor follows. The inlet moisture-laden NG, flowing upward from the bottom of the dehydrator, is cooled down to the desired dewpoint temperature by directly contacting a down-flowing, adequately dispersed low-temperature medium stream. The medium is an aqueous solution containing an inhibitor. The moisture in the inlet NG is condensed on the surface of the medium droplets. The medium, diluted with the condensates, is re-concentrated in an inhibitor regenerator and recycled. The dehydrated NG continues to flow upward into the absorber wherein the higher hydrocarbon gases are absorbed with a down-flowing, adequately dispersed low-temperature absorbent (e.g., heavy oil) stream. The light oil-laden absorbent (hereafter abbreviated as "rich oil") then enters the fractional distiller wherein the absorbed higher hydrocarbons is separated as the final product. The recovered absorbent is cooled in the absorbent cooler and recycled to the absorber of the processor. The processed NG, basically free from higher hydrocarbons (hereafter abbreviated as "lean NG"), is re-heated and eventually delivered to the NG transportation pipeline. The refrigeration unit provides the required refrigeration for both medium cooler and absorbent cooler.

50 When the pressure of the inlet NG is sufficiently high, the required refrigeration could be provided with expanding the dehydrated high pressure NG. In such a "self-refrigeration" case, no external energy is required.

In case of the pressure difference between the inlet NG 55 and the NG transportation pipeline is small, a high-efficiency free-piston NG expander-compressor is proposed in the present invention to provide the required self-refrigeration.

\section{BRIEF DESCRIPTION OF DRAWINGS}

The above and other features and advantages of the present invention will now be further described in the following detailed description section in conjunction with the attached drawings in which:

FIG. 1 illustrates one preferred embodiment of the com5 prehensive NG processor of the present invention wherein a separate industrial refrigeration unit is used to provide the required refrigeration. 
3

FIG. 2 illustrates another preferred embodiment of the comprehensive $\mathrm{NG}$ processor of the present invention wherein an integrated NG expander-compressor is used to provide the required self-refrigeration.

FIG. 3 illustrates the high-efficiency free-piston NG expander-compressor for providing the required selfrefrigeration.

\section{DETAILED DESCRIPTION}

FIG. 1 illustrates one preferred embodiment of the comprehensive $N G$ processor of the present invention wherein a separate industrial refrigeration unit is used to provide the required refrigeration.

The said apparatus comprises the following major components: a processor 1 comprising a dehydrator $1 a$ and an absorber $\mathbf{1} b$; a medium cooler 9 comprising a pre-cooler $9 a$ and a deep-cooler $\mathbf{9} b$; an absorbent cooler $\mathbf{2 5}$ comprising a pre-cooler $25 a$ and a deep-cooler $25 b$; a fractional distiller 27; an inhibitor regenerator 15, and a refrigeration unit $\mathbf{9 0 .}$

The inlet NG, laden with moisture and all the higher hydrocarbon components, i.e., $\mathrm{C}_{2}, \mathrm{C}_{3}, \mathrm{C}_{4}$, and $\mathrm{C}_{5}{ }^{+}$, enters the dehydrator $1 a$ from the bottom via the raw $\mathrm{NG}$ inlet pipeline $\mathbf{2}$ and flows upward.

A low-temperature medium, containing an inhibitor, 25 enters from the top of the dehydrator via the medium inlet pipeline 3. The medium is distributed or dispersed with the medium distributor 4 over the whole cross-section of the dehydrator and flows downward.

The medium is an aqueous solution of an inhibitor, such as an ionic salt or an organic compound. The concentration of the said inhibitor should be sufficient high to prevent the formation of gas-hydrates/ice over the entire temperature range of the dehydrator operations.

The medium is either sprayed as finely divided droplets or is dispersed with a packed column to provide extensive contacting surfaces for cooling the up-flowing NG. The moisture in the NG condenses on the dispersed medium surfaces and dissolves into the inhibitor solution. The slightly diluted medium is eventually discharged from the bottom of the dehydrator via the medium discharge pipeline 5.

The discharged medium is re-pressurized with the pump 6. A major portion of the re-pressurized medium passes through the regulation valve 7 and is sent to the primary side of the pre-cooling section $9 a$ of the medium cooler 9 via the medium transfer pipeline $\mathbf{8}$.

A small fraction of the re-pressured medium is diverted via the effluent transfer pipelines $\mathbf{4}$ into the inhibitor regenerator 15 wherein the diluted inhibitor solution is re-concentrated. The highly concentrate inhibitor solution is sent via the inhibitor recycle pipeline $\mathbf{1 6}$ and mixes with the medium flowing in the medium transfer pipeline 8 . The wastewater separated in the regenerator is discharged via the wastewater discharge pipeline $\mathbf{1 7}$.

In the medium cooler, the medium is first pre-cooled with the cold lean NG reflux coming from the integrated NG processor via the lean NG outlet pipeline 23. The re-heated lean NG is delivered via the lean NG delivery pipeline $\mathbf{1 1}$ to the NG transportation pipeline (not shown).

The pre-cooled medium continues to flow upward into the primary side of the deep-cooler $9 b$ wherein it is deep-cooled to the required low-temperature with the refrigerant (or brine) provided with the industrial refrigerator 90. The refrigerant enters the secondary side of the deep-cooler via the refrigerant inlet pipeline $\mathbf{1 2}$ and leaves via the refrigerant

\section{4}

outlet pipeline 13. The deep-cooled medium is recycled into the dehydrator via the medium inlet pipeline 3 . The makeup medium is introduced via the medium makeup pipeline $\mathbf{1 0}$.

In case the concentration of the higher hydrocarbons in NG is so high that the light oil gas partially condenses into liquid in the dehydrator $1 a$. The mixed condensates of water in the medium and light oil is collected at the bottom of the dehydrator. The light oil layer flowing over the liquid medium is discharged via the light oil outlet $\mathbf{1 8}$ as a part of the final product.

Now return to the absorber $1 b$ of the integrated NG processor. The dewpoint of the dehydrated NG when leaving from the top of the dehydrator is close to the entrance temperature of the deep-cooled medium. The cold dehydrated NG enters the absorber from the bottom, and flows upward through a series of bypass pipes 19 in the enriched oil collector 19a. The up-flowing dehydrated NG comes into contact with the down-flowing cold absorbent running through a packed column 20. A steam of the deep-cooled absorbent enters from the top of the absorber via the absorbent inlet pipeline 21. The absorbent is distributed by the absorbent distributor 22. The temperature of the absorbent at the top of the absorber is kept slightly about the dewpoint of the dehydrated NG to avoid gas-hydrate formation.

With such a counter-extraction process in the absorber, the recovery rates of the light oil gases $\left(\mathrm{C}_{3}+\right)$ are very high. A reasonable fraction of ethane $\left(\mathrm{C}_{2}\right)$ is also recovered. At the same time, the absorption rate of methane is relatively low. As mentioned above, the lean NG leaves the top of the absorber via the lean NG outlet pipeline $\mathbf{2 3}$, and enters the secondary side of the pre-cooler $\mathbf{9} a$ of the medium cooler $\mathbf{9}$.

The rich oil flows out from the absorber $1 b$ via the rich oil outlet pipeline $\mathbf{2 4}$ and enters the secondary side of the pre-cooler 25 $a$. The rich oil absorbs heat from the recycling absorbent flowing in the primary side of the pre-cooler. The rich oil leaves the pre-cooler via the rich oil transfer pipeline 26 and enter the fractional distiller 27 wherein the final product, a gaseous mixture enriched in higher hydrocarbons, is separated from the absorbent. The separated higher hydrocarbons gas mixture is delivered via the product outlet pipeline $\mathbf{2 8}$ to a refiner (not shown).

The energy required for the fractional distillation process is provided with a heating medium entering the distiller via ${ }_{45}$ the heat medium inlet pipeline 29 and leaving by the heat medium outlet pipeline $\mathbf{3 0}$.

The recovered absorbent, leaving the fractional distiller via the absorbent outlet pipeline $\mathbf{3 1}$, is re-pressurized with a pump 32. The absorbent enters the primary side of the absorbent cooler 25 via the absorbent recycle pipeline $\mathbf{3 1 0}$.

The recycled absorbent flows upward through the primary side of the absorbent cooler $\mathbf{2 5}$. It is first pre-cooled with the cold rich oil flowing in the secondary side of the pre-cooler $\mathbf{2 5} a$, and then deep-cooled with the refrigerant flowing in the 55 secondary side of the deep-cooler $25 b$. The refrigerant enters the secondary side of the absorbent deep-cooling section via the refrigerant inlet pipeline $\mathbf{3 3}$ and leaves via the outlet pipeline 34 . The refrigerant is provided with the industrial refrigerator 30 .

60 FIG. 2 illustrates another preferred embodiment of the comprehensive NG processor of the present invention, in which an integrated NG expander-compressor is used to provide the required "self-refrigeration". The said embodiment is applicable when the pressure of the lean NG is 5 sufficiently higher than the NG pressure required in the NG transport pipeline. The lean NG may be expanded in three different kinds of gas expansion devices. 


\section{5}

According to the magnitudes of the pressure difference between inlet NG and the dehydrated NG transportation pipeline, there are three options for the NG expansion devices. (1) When the said pressure difference is quite large, a simple expansion valve could be used to expand the inlet NG to a pressure above or equal to the transportation pipeline pressure and obtain the desired low temperature for refrigeration. In this case, the de-pressurized NG needs no re-compression. (2) When the said pressure difference is moderately high, the inlet NG has to be expanded below the transportation pipeline pressure to obtain the desired low temperature for refrigeration. A portion of the expansion energy needs to be recovered for re-compression the de-pressurized NG. In this case, a turbo expandercompressor is preferred. (3) When the said pressure difference is rather small, but still relevant, the expansion energy must be recovered to the maximum extent for $\mathrm{NG}$ re-compression. In this case, the high efficiency free-piston expander-compressor, as described in the following FIG. 3, is recommended.

It should be noted, for both cases (2) and (3), an external powered NG compressor may also be incorporated, as appropriate, for re-compressing the de-pressurized NG to the required pressure of the $N G$ transport pipeline.

Return to FIG. 2 wherein a turbo NG expandercompressor as mentioned in the case (2) is illustrated as an example.

Because most components of the comprehensive NG processor in FIG. 2 are identical to those in FIG. 1, they are labeled with the same numbers in FIG. 2. Only the dissimilar components of the self-refrigeration unit are labeled with different numbers and will be described in details below. These dissimilar components include the turbo expander $\mathbf{3 5} a$ and compressor $35 b$, the medium cooler $\mathbf{4 1}$, and the filter 38 .

The lean NG, left the absorber $1 b$ via the lean NG outlet pipeline $\mathbf{2 3}$ and mixed with the inhibitor introduced via the inhibitor injection pipeline $\mathbf{3 6}$, enters the turbo expander $\mathbf{3 5} a$ and is expanded. Gas expansion causes the NG temperature sharply dropped to the required low temperature. A small amount of the residual moisture is condensed into tinny liquid droplets entrained in the chilly lean NG. The chilly lean NG enters the filter $\mathbf{3 8}$ via the de-pressurized NG transfer pipeline 37. The liquid droplets are separated as an effluent, and the latter is discharged into the inhibitor regenerator 15 via the effluent pipeline 39. The dried chilly lean NG enters the secondary side of the medium cooler 41 via the chilly lean NG inlet pipeline 40. The chilly lean NG absorbs the heat from the recycled medium and flows into the compressor $35 b$ via the de-pressurized NG return pipeline 42 . A portion of the chilly NG is diverted via the bypass valve 44 and bypass pipeline 33 to the absorbent cooler 25 , and returns via the bypass return pipeline 34 . The lean NG is then re-compressed to the required pressure and delivered via the lean NG delivery pipeline $\mathbf{4 3}$ to the NG transportation pipeline (not shown).

As described above, the system in FIG. 2 does not require any external energy to provide the self-refrigeration.

Having described the features and the advantages of the various embodiments of the present invention as a comprehensive NG processing apparatus, it should be pointed out 60 that the dehydration section with its accessories could also be operated independently as a pure NG dehydrator, without incorporating the absorption section and its accessories.

FIG. 3 illustrates the high-efficiency free-piston NG expander-compressor for self-refrigeration.

The light alloy body $\mathbf{4 5}$ of the said free piston expandercompressor comprises two cylinders with different diam-

\section{6}

eters. The smaller cylinder $\mathbf{4 6}$ is the expander, and the larger cylinder 47 the compressor. Two free pistons, 48 and 49 , are rigidly connected with a short hollow shaft $\mathbf{5 0}$ to form a single integrated moving part. Since the latter is a compact, light-weighted component, very high frequency operation and high mechanical efficiency are feasible. For a highpressure NG, the size of such a free piston machine is relatively small. For example, for an apparatus processing $500,000 \mathrm{~m}^{3}$ STP per day, under an initial pressure of $10 \mathrm{MPA}$ 10 and an exit pressure of 5 MPA, the maximum diameter of the free piston expander-compressor will be in the order of 12 $\mathrm{cm}$ when working at 4,000 strokes per minute.

In FIG. 3, the NG inlet pipelines $\mathbf{5 1}$ and $\mathbf{5 2}$ and the outlet pipelines 53 and 54 of the expander, as well as the inlet 5 pipelines 55 and 56 and the outlet pipelines 57 and 58 of the compressor are connected to the relevant cylinders as illustrated. The associated valves controlling these inlet pipelines and outlet pipelines are similar to those used in modern high-speed internal combustion engine. These valves are not shown in FIG. 3.

In case that the pressure difference between the inlet NG and the outlet NG to the pipeline is too small so that additional external compressing energy is required, a viable option is to connect the said free piston with extending the shaft $\mathbf{5 9}$, as shown by the dotted line, to a conventional reciprocating piston-type gas engine, not shown in FIG. 3.

In summary, the present invention is related to an apparatus for efficient and cost-effective comprehensive processing of NG, including the removal of moisture and the recovery of the higher hydrocarbons $\left(\mathrm{C}_{2}^{+}\right)$, in a single integrated processing unit. The present invention provides a low-cost comprehensive NG processor that is universally applicable to both terrestrial and off-shore NG exploitation.

Having describes the present invention and preferable embodiments thereof, it will be recognized that numerous variations, substitutions and additions may be made to the present invention by those ordinary skills without departing from the spirit and scope of the appended claims.

What is claimed is:

1. A comprehensive gas processor for removing the moisture and recovering the higher hydrocarbons (i.e., $\mathrm{C}_{2}{ }^{+}$) therein either on-situ in a gas field or in a plant comprising:

(a) an integrated gas processor comprsing two sections working on a hybrid process, i.e., an integration of two different processes within a single casing:

i) a refrigeration-dehydration section working on refrigeration process wherein the inlet gas contacts with a counter-flowing stream of dispersed cold heat-transport medium containing a non- or lowvolatile hydrate inhibitor with boiling point higher than $180^{\circ} \mathrm{C}$. and the moisture of said gas is condensed and removed with the cold heat-transport medium; and

ii) an absorption section working on low-temperature absorption process wherein the dehydrated gas contacts with a counter-flowing stream of dispersed liquid absorbent with a hydrocarbon gas solubility higher than $20 \mathrm{scf} / \mathrm{gal}$ wherein the higher hydrocarbons (i.e., $\mathrm{C}_{2}^{+}$) are absorbed;

(b) a heat-transport medium cooler comprising a precooling stage and a deep-cooling stage wherein in said pre-cooling stage said heat-transport medium is precooled with the cold outlet gas left said integrated gas processor and in said deep-cooling stage the medium is deep-cooled with the refrigerant provided with a refrigerator; 


\section{US $6,553,784 \mathrm{~B} 2$}

\section{7}

(c) an absorbent cooler comprising a pre-cooling stage and a deep-cooling stage wherein in said pre-cooling stage said recycling absorbent is pre-cooled with the cold outlet absorbent left said integrated gas processor and in said deep-cooling stage the absorbent is deepcooled with the refrigerant provided with a refrigerator;

(d) a fractional distiller for separating the absorbed higher hydrocarbons as a product from the outlet absorbent left said integrated gas processor and then the separated absorbent is recycled back to said integrated gas processor;

(e) an inhibitor regenerator for concentrating the lowvolatile hydrate inhibitor to be recycled and discharging the wastewater;

(f) a refrigerator for providing the refrigerant to said deep-cooling stages of said heat-transport medium cooler and said absorbent cooler;

(g) a pipeline for delivering the recovered higher hydrocarbons; and

(h) a gas inlet pipeline and a pipeline for delivering the processed gas.

2. A comprehensive gas processor of claim 1 wherein the dehydration section of said integrated processor and its accessories (comprising said heat-transport medium cooler, said inhibitor regenerator, said refrigerator, and said gas inlet-pipeline and a pipeline for delivering the processed 25 gas) are operated independently as a gas dehydrator without incorporating the absorption section.

3. A comprehensive gas processor of claim 1 wherein said heat-transport medium is an aqueous solution of calcium chloride or other ionizing salts and the regeneration rate of 30 said solution is less than 5 liter per $\mathrm{kg}$ of wastewater to be discharged.

\section{8}

4. A comprehensive gas processor of claim 1 wherein said heat-transport medium is an aqueous solution of ethylene glycol or other organic compounds with boiling points higher than $180^{\circ} \mathrm{C}$. and the regeneration rate of said solution is less than 5 liter per $\mathrm{kg}$ of wastewater discharged.

5. A comprehensive gas processor of claim 1 wherein said absorbent is heavy oil (i.e., hydrocarbon mixture with molecular weight higher than 100) or other organic com10 pounds with hydrocarbon gas solubility higher than 20 scf/gal liquid.

6. A comprehensive gas processor of claim 1 when working on inlet gas pressure greater than $5.0 \mathrm{MPa}$ wherein said refrigerant to said deep-cooling stages of said heattransport medium cooler and said absorbent cooler is provided with a gas expansion device when the inlet gas pressure is greater than $5.0 \mathrm{MPa}$.

7. A gas expansion device of claim 6 wherein said 20 expansion device is a triple-sectional free-piston gas expander-compressor-booster comprising:

(a) a gas expansion cylinder and a gas compression cylinder;

(b) a co-shaft gas expansion piston and gas compression piston; and

(c) a co-shaft gas-fueled booster piston-engine providing supplemental power for compressing said expanded gas to the required delivery pipeline pressure. 


\section{(19) United States \\ (12) Reissued Patent} Lu
(10) Patent Number:

(45) Date of Reissued Patent:
US RE39,826 E

Sep. 11, 2007

\section{(54) COMPREHENSIVE NATURAL GAS PROCESSING}

(76) Inventor: Yingzhong Lu, 104 Harland Ct., Oak Ridge, TN (US) 37830

(21) Appl. No.: 11/038,003

(22) Filed: Jan. 19, 2005

Related U.S. Patent Documents

Reissue of:

(64) Patent No:

$6,553,784$

Issued:

Appl. No.:

Apr. 29, 2003

$09 / 683,946$

Filed:

Mar. 6, 2002

(30) Foreign Application Priority Data

Aug. 23, $2001 \quad$ (CN)

01124271

(51) Int. Cl.

F25J $3 / 00$

$(2006.01)$

(52) U.S. Cl. 62/632; $62 / 633$

(58) Field of Classification Search $62 / 632$ $62 / 635,636,620,622,625,633$ See application file for complete search history.

\section{References Cited}

\section{U.S. PATENT DOCUMENTS}

$3,837,143 \mathrm{~A} * 9 / 1974$ Sutherland et al. $95 / 162$

$4,421,535 \mathrm{~A} * 12 / 1983$ Mehra $62 / 625$

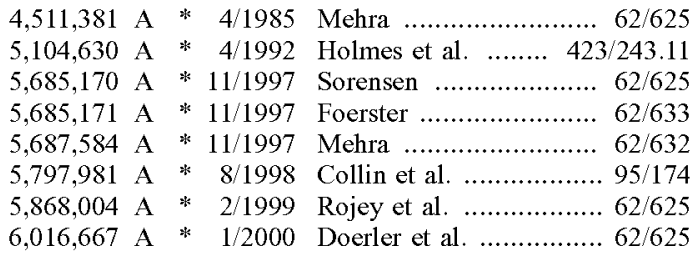

* cited by examiner

Primary Examiner-William C. Doerrler

\section{ABSTRACT}

The present invention related to a process and an apparatus for efficient and cost-effective comprehensive processing of natural gas, including the removal of moisture and the recovery of the higher hydrocarbons components $\left(\mathrm{C}_{2}{ }^{+}\right)$. The said apparatus comprises the following major components: an integrated natural gas processor with a dehydration section and a higher hydrocarbons absorption section; a heat transport medium cooler; an absorbent cooler; a fractional distiller for separating the light oil from the heavy oil absorbent; an inhibitor regenerator; and a refrigeration unit. The present invention provides a low-cost natural gas comprehensive [processor] processing that is universally applicable to both terrestrial and off-shore natural gas exploitation. The said apparatus also provides an efficient and cost-effective natural gas dehydrator when the dehydration section is used independently without incorporating the absorption section.

12 Claims, 3 Drawing Sheets

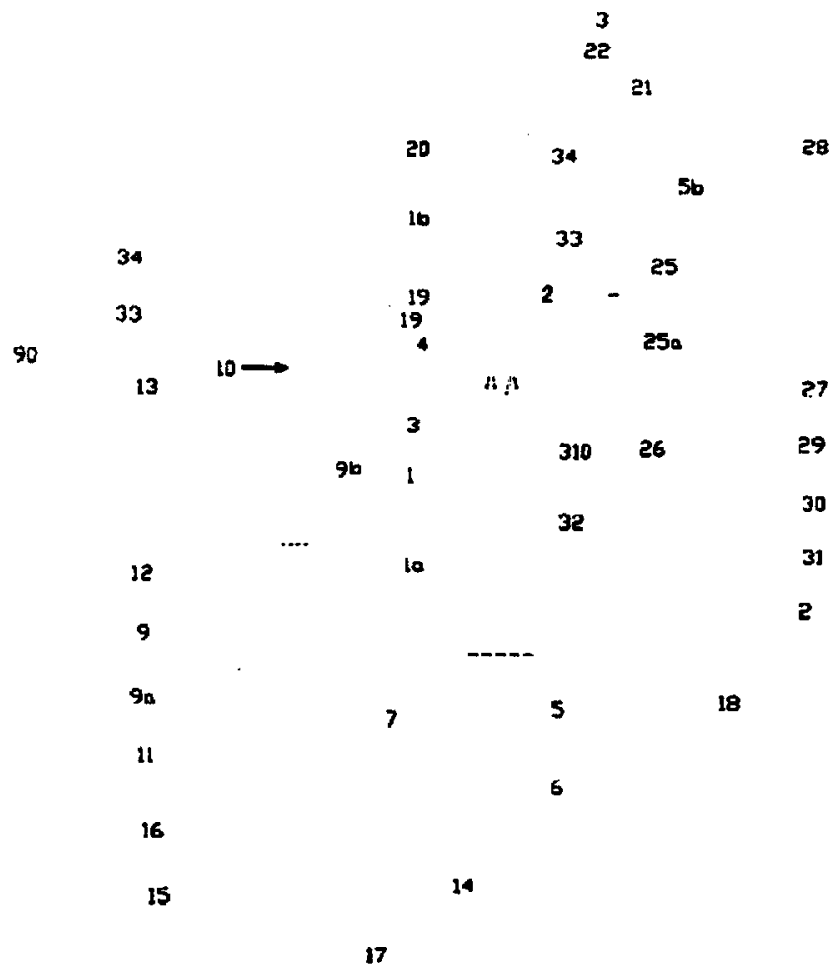


Final Progress Report DE-FC36-03G013150

U.S. Patent Sep. 11, 2007 Sheet 1 of 3

US RE39,826 E

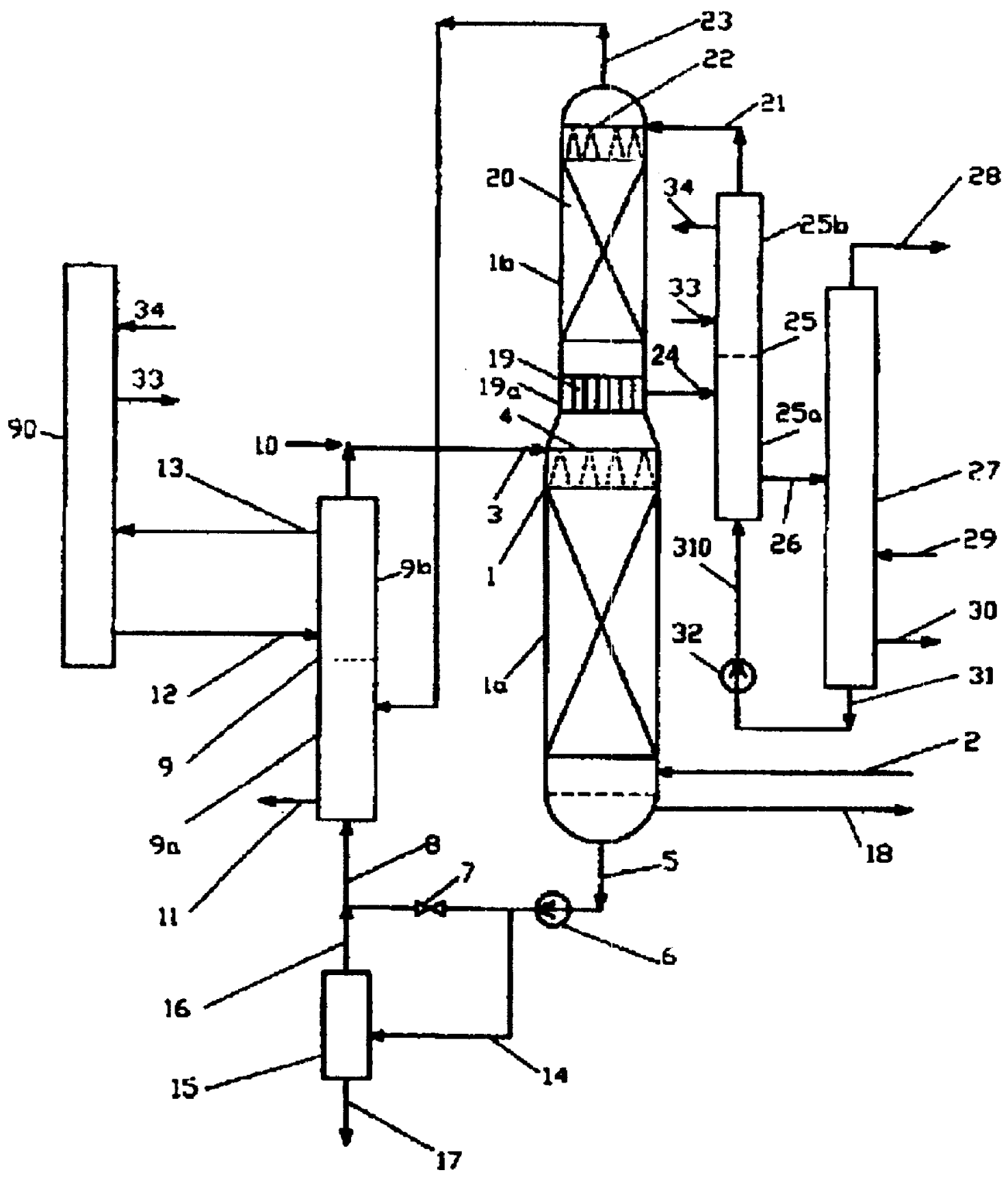

FIG 1 
Final Progress Report DE-FC36-03G013150

U.S. Patent Sep. 11, 2007 Sheet 2 of 3

US RE39,826 E

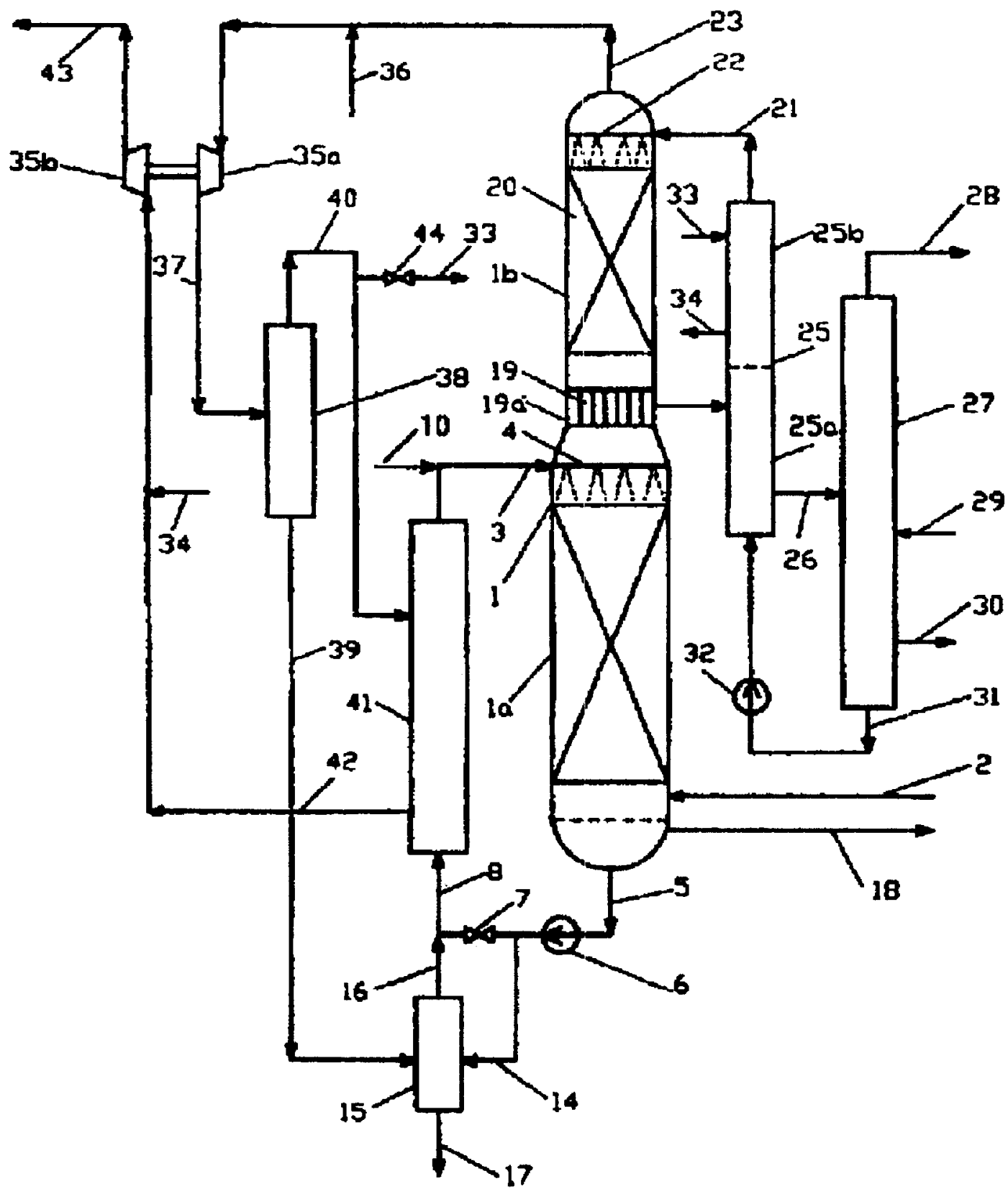

FIG 2 
Final Progress Report

DE-FC36-03G013150

U.S. Patent Sep. 11, 2007 Sheet 3 of 3 US RE39,826 E

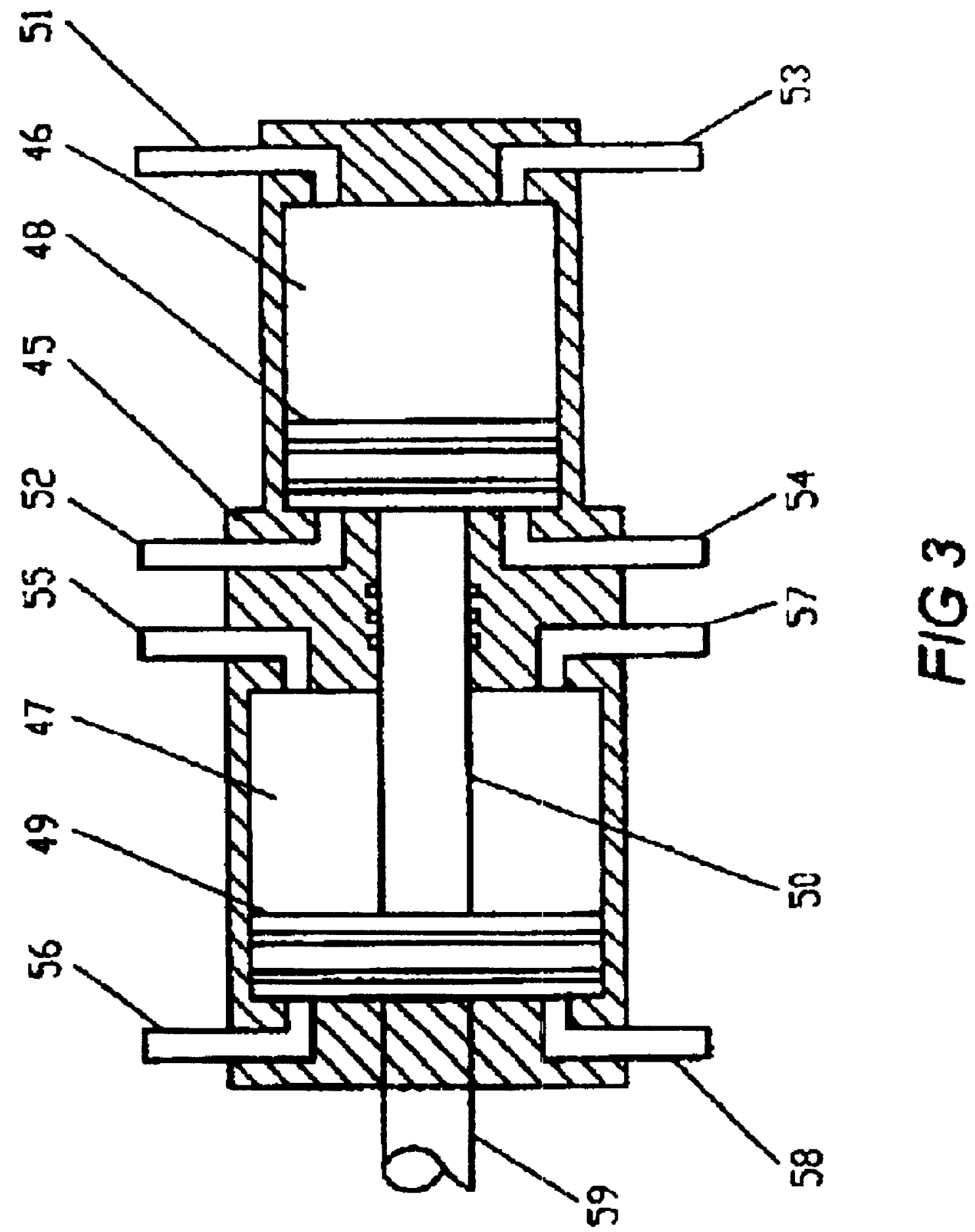




\section{1 \\ COMPREHENSIVE NATURAL GAS PROCESSING}

\begin{abstract}
Matter enclosed in heavy brackets [ ] appears in the original patent but forms no part of this reissue specification; matter printed in italics indicates the additions made by reissue.
\end{abstract}

\section{BACKGROUND OF INVENTION}

The reduction of $\mathrm{CO}_{2}$ emission is one of the greatest concerns in combating the catastrophic "global warming" trend. As a result, the world puts much emphasis on the exploitation of "clean energy" with less or non-emission for both industrial and domestic uses. Natural gas (hereafter abbreviated as "NG"), as compared with coal and petroleum, is considered the most economic "clean" fuel that is used on a large, industrial scale at present and in the near future. In addition, the discovery of huge amount of ocean-bed gashydrates increases the recoverable resources of NG substantially. It is expected that, in the long run, the global NG consumption may eventually exceeds all other fossil fuels.

$\mathrm{NG}$ is a mixture of hydrocarbon gases, consisting of mainly methane $\left(\mathrm{C}_{1}\right)$ and a smaller fraction of heavier gaseous hydrocarbons (i.e., ethane, $\mathrm{C}_{2}$; propane, $\mathrm{C}_{3}$; butane, $\mathrm{C}_{4}$; pentane and higher, $\mathrm{C}_{5}{ }^{+}$; sometimes $\mathrm{C}_{3}+$ is called "light oil" as a whole. However, the economic values of these higher hydrocarbon components, when separated and sold as chemical feedstock, are usually much higher than burnt as a fuel. A number of NG processing plants, therefore, have been constructed to extract these valuable materials.

The state-of-the-art NG processing plants generally work on a cryogenic process for efficiently separating the higher hydrocarbon gases In this process, a huge volume of NG is cooled down by expansion to a very low cryogenic temperature around $-150^{\circ} \mathrm{F}$. Such a process is extremely energy-consuming, and the facility usually comprises many pieces of expensive equipment, notably the molecular-sieve dehydrator, the multiple-flow finned-plate heat exchanger, and the turbo expander-compressor. High capital and operational costs are thus resulted. As a consequence, only a limited fraction of the NG could be processed before consumed as a fuel. Most of the valuable higher hydrocarbon contents was improperly used.

In the past two decades, a number of US patents have been granted in this field, for example, the 13 US patents entitled "hydrocarbon Processing" presented by late Roy E. Campbell, et al., i.e., U.S. Pat. Nos. 4,140,504; 4,157,904; $4,171,964 ; 4,278,457 ; 4,854,955 ; 4,869,740 ; 4,889,545$; $5,555,784 ; 5,568,737 ; 5,771,712 ; 5,881,569 ; 5,983,664$; and $6,182,469$. However, most of these patents only proposed some specific improvements to the same cryogenic process. No substantial break-through in NG processing technology has ever been proposed. A more efficient and cost-effective technology for NG procession, therefore, is desirable.

The recent developments in NG refrigeration dehydration technology, e.g., those presented in U.S. Pat. No. 5,664,426, "Regenerative Gas Dehydrator," 1997, and U.S. Pat. No. 6,158,242, "Gas Dehydration Method and Apparatus," 2000, provided the basis of a break-through in the NG processing 60 technology. These patents make possible to perform refrigeration dehydration and refrigeration absorption in a single unit.

Accordingly, it is an objective of the present invention to provide a comprehensive NG process and a processor, based 65 on the refrigeration dehydration and absorption technologies, for efficient and cost-effective comprehensive

\section{2}

processing of NG. The said processor could simultaneously perform the removal of moisture and the recovery of the higher hydrocarbons $\left(\mathrm{C}_{2}^{+}\right)$in a single piece of equipment, thus substantially reducing the capital and operational costs of the NG processing plant.

Another objective of the present invention is to provide an energy-saving comprehensive NG process and a processor that, when processing high pressure $\mathrm{NG}$, does not need external energy for refrigeration.

10 A further objective of the present invention is to provide a high-efficiency free-piston expander-compressor to provide the required refrigeration.

\section{SUMMARY OF INVENTION}

With regard to the above and other objectives, the present invention provides a comprehensive NG process and a processor to simultaneously perform refrigeration dehydration and refrigeration absorption of higher hydrocarbon gases with maximum recovery rate at minimum energy consumption. The final product is a gaseous mixture enriched in higher hydrocarbons with minimum residual methane.

The said apparatus comprises the following major components: an integrated NG processor (hereafter abbreviated as "processor) with a refrigeration dehydration section (hereafter abbreviated as "dehydrator") and a refrigeration absorption section (hereafter abbreviated as "absorber"); a heat-transport medium (hereafter abbreviated as "medium") cooler; an absorbent cooler; a fractional distiller; a gashydrate inhibitor (hereafter abbreviated as "inhibitor") regenerator; and a refrigeration unit.

The principle of the operations of the comprehensive NG processor follows. The inlet moisture-laden NG, flowing upward from the bottom of the dehydrator, is cooled down to the desired dewpoint temperature by directly contacting a down-flowing, adequately dispersed low-temperature medium stream. The medium is an aqueous solution containing an inhibitor. The moisture in the inlet NG is condensed on the surface of the medium droplets. The medium, diluted with the condensates, is re-concentrated in an inhibitor regenerator and recycled. The dehydrated NG continues to flow upward into the absorber wherein the higher hydrocarbon gases are absorbed with a down-flowing, adequately dispersed low-temperature absorbent (e.g., heavy oil) stream. The light oil-laden absorbent (hereafter abbreviated as "rich oil") then enters the fractional distiller wherein the absorbed higher hydrocarbons is separated as the final product. The recovered absorbent is cooled in the absorbent cooler and recycled to the absorber of the processor. The processed NG, basically free from higher hydrocarbons (hereafter abbreviated as "lean NG"), is re-heated and eventually delivered to the NG transportation pipeline. The refrigeration unit provides the required refrigeration for both 5 medium cooler and absorbed cooler.

When the pressure of the inlet NG is sufficiently high, the required refrigeration could be provided with expanding the dehydrated high pressure NG. In such a "self-refrigeration" case, no external energy is required.

In case of the pressure difference between the inlet $\mathrm{NG}$ and the NG transportation pipeline is small, a high-efficiency free-piston NG expander-compressor is proposed in the present invention to provide the required self-refrigeration.

\section{BRIEF DESCRIPTION OF DRAWINGS}

The above and other features and advantages of the present invention will now be further described in the 
3

following detailed description section in conjunction with the attached drawings in which:

FIG. 1 illustrates one preferred embodiment of the comprehensive NG [processor] processing of the present invention wherein a separate industrial refrigeration unit is used to provide the required refrigeration.

FIG. 2 illustrates another preferred embodiment of the comprehensive NG [processor] processing of the present invention wherein an integrated NG expander-compressor is used to provide the required self-refrigeration.

FIG. 3 illustrates the high-efficiency free-piston NG expander-compressor for providing the required selfrefrigeration.

\section{DETAILED DESCRIPTION}

FIG. 1 illustrates one preferred embodiment of the comprehensive NG [processor] processing of the present invention wherein a separate industrial refrigeration unit is used to provide the required refrigeration.

The said apparatus comprises the following major components: a processor 1 comprising a dehydrator $1 \mathrm{a}$ and an absorber $1 \mathrm{~b}$; a medium cooler 9 comprising a pre-cooler $9 \mathrm{a}$ and a deep-cooler $9 \mathrm{~b}$; an absorbent cooler 25 comprising a pre-cooler 25a and a deep-cooler 25b; a fractional distiller 27; an inhibitor regenerator $\mathbf{1 5}$, and a refrigeration unit $\mathbf{9 0 .}$

The inlet $\mathrm{NG}$, laden with moisture and all the higher hydrocarbon components, i.e., $\mathrm{C}_{2}, \mathrm{C}_{3}, \mathrm{C}_{4}$, and $\mathrm{C}_{5}{ }^{+}$, enters the dehydrator 1a from the bottom via the raw $\mathrm{NG}$ inlet pipeline $\mathbf{2}$ and flows upward.

A low-temperature medium, containing an inhibitor, enters from the top of the dehydrator via the medium inlet pipeline 3. The medium is distributed or dispersed with the medium distributor 4 over the whole cross-section of the dehydrator and flows downward.

The medium is an aqueous solution of an inhibitor, such as an ionic salt or an organic compound. The concentration of the said inhibitor should be sufficient high to prevent the formation of gas-hydrates/ice over the entire temperature range of the dehydrator operations.

The medium is either sprayed as finely divided droplets or is dispersed with a packed column to provide extensive contacting surfaces for cooling the up-flowing NG. The moisture in the NG condenses on the dispersed medium surfaces and dissolves into the inhibitor solution. The slightly diluted medium is eventually discharged from the bottom of the dehydrator via the medium discharge pipeline 5.

The discharged medium is re-pressurized with the pump 6. A major portion of the re-pressurized medium passes through the regulation valve 7 and is sent to the primary side of the pre-cooling section $9 \mathrm{a}$ of the medium cooler 9 via the medium transfer pipeline $\mathbf{8}$.

A small fraction of the re-pressured medium is diverted via the effluent transfer pipelines 4 into the inhibitor regenerator $\mathbf{1 5}$ wherein the diluted inhibitor solution is re-concentrated. The highly concentrate inhibitor solution is sent via the inhibitor recycle pipeline $\mathbf{1 6}$ and mixes with the medium flowing in the medium transfer pipeline 8 . The wastewater separated in the regenerator is discharged via the wastewater discharge pipeline 17.

In the medium cooler, the medium is first pre-cooled with the cold lean NG reflux coming from the integrated NG processor via the lean NG outlet pipeline 23 . The re-heated lean NG is delivered via the lean NG delivery pipeline $\mathbf{1 1}$ to the NG transportation pipeline (not shown).

\section{4}

The pre-cooled medium continues to flow upward into the primary side of the deep-cooler $9 \mathrm{~b}$ wherein it is deep-cooled to the required low-temperature with the refrigerant (or brine) provided with the industrial refrigerator 90 . The refrigerant enters the secondary side of the deep-cooler via the refrigerant inlet pipeline 12 and leaves via the refrigerant outlet pipeline 13. The deep-cooled medium is recycled into the dehydrator via the medium inlet pipeline 3 . The makeup medium is introduced via the medium makeup pipeline $\mathbf{1 0}$.

In case the concentration of the higher hydrocarbons in NG is so high that the light oil gas partially condenses into liquid in the dehydrator 1a. The mixed condensates of water in the medium and light oil is collected at the bottom of the dehydrator. The light oil layer flowing over the liquid medium is discharged via the light oil outlet 18 as a part of 15 the final product.

Now return to the absorber $\mathbf{1 b}$ of the integrated $\mathrm{NG}$ processor. The dewpoint of the dehydrated NG when leaving from the top of the dehydrator is close to the entrance temperature of the deep-cooled medium. The cold dehy-

20 drated NG enters the absorber from the bottom, and flows upward through a series of bypass pipes 19 in the enriched oil collector 19a. The up-flowing dehydrated NG comes into contact with the down-flowing cold absorbent running through a packed column 20. A steam of the deep-cooled absorbent enters from the top of the absorber via the absorbent inlet pipeline 21. The absorbent is distributed by the absorbent distributor 22 . The temperature of the absorbent at the top of the absorber is kept slightly about the dewpoint of the dehydrated NG to avoid gas-hydrate for30 mation.

With such a counter-extraction process in the absorber, the recovery rates of the light oil gases $\left(\mathrm{C}_{3}+\right)$ are very high. A reasonable fraction of ethane $\left(\mathrm{C}_{2}\right)$ is also recovered. At the same time, the absorption rate of methane is relatively low. 35 As mentioned above, the lean NG leaves the top of the absorber via the lean NG outlet pipeline 23, and enters the secondary side of the pre-cooler 9 a of the medium cooler 9 .

The rich oil flows out from the absorber $1 \mathrm{~b}$ via the rich oil 40 outlet pipeline $\mathbf{2 4}$ and enters the secondary side of the pre-cooler 25a. The rich oil absorbs heat from the recycling absorbent flowing in the primary side of the pre-cooler. The rich oil leaves the pre-cooler via the rich oil transfer pipeline 26 and enter the fractional distiller 27 wherein the final product, a gaseous mixture enriched in higher hydrocarbons, is separated from the absorbent. The separated higher hydrocarbons gas mixture is delivered via the product outlet pipeline $\mathbf{2 8}$ to a refiner (not shown).

The energy required for the fractional distillation process is provided with a heating medium entering the distiller via the heat medium inlet pipeline 29 and leaving by the heat medium outlet pipeline $\mathbf{3 0}$.

The recovered absorbent, leaving the fractional distiller via the absorbent outlet pipeline 31, is re-pressurized with a pump 32. The absorbent enters the primary side of the absorbent cooler 25 via the absorbent recycle pipeline $\mathbf{3 1 0}$.

The recycled absorbent flows upward through the primary side of the absorbent cooler 25. It is first pre-cooled with the cold rich oil flowing in the secondary side of the pre-cooler $6025 \mathrm{a}$, and then deep-cooled with the refrigerant flowing in the secondary side of the deep-cooler $\mathbf{2 5} \mathrm{b}$. The refrigerant enters the secondary side of the absorbent deep-cooling section via the refrigerant inlet pipeline $\mathbf{3 3}$ and leaves via the outlet pipeline 34. The refrigerant is provided with the industrial 65 refrigerator $\mathbf{3 0}$.

FIG. 2 illustrates another preferred embodiment of the comprehensive NG [processor] processing of the present 
5

invention, in which an integrated NG expander-compressor is used to provide the required "self-refrigeration". The said embodiment is applicable when the pressure of the lean NG is sufficiently higher than the NG pressure required in the NG transport pipeline. The lean NG may be expanded in three different kinds of gas expansion devices.

According to the magnitudes of the pressure difference between inlet NG and the dehydrated NG transportation pipeline, there are three options for the NG expansion devices. (1) When the said pressure difference is quite large, a simple expansion valve could be used to expand the inlet $\mathrm{NG}$ to a pressure above or equal to the transportation pipeline pressure and obtain the desired low temperature for refrigeration. In this case, the de-pressurized NG needs no re-compression. (2) When the said pressure difference is moderately high, the inlet NG has to be expanded below the transportation pipeline pressure to obtain the desired low temperature for refrigeration. A portion of the expansion energy needs to be recovered for re-compression the de-pressurized NG. In this case, a turbo expander- 20 compressor is preferred. (3) When the said pressure difference is rather small, but still relevant, the expansion energy must be recovered to the maximum extent for $\mathrm{NG}$ re-compression. In this case, the high efficiency free-piston expander-compressor, as described in the following FIG. 3 , is recommended.

It should be noted, for both cases (2) and (3), an external powered NG compressor may also be incorporated, as appropriate, for re-compressing the de-pressurized NG to the required pressure of the NG transport pipeline.

Return to FIG. 2 wherein a turbo NG expandercompressor as mentioned in the case (2) is illustrated as an example.

Because most components of the comprehensive NG processor in FIG. 2 are identical to those in FIG. 1, they are labeled with the same numbers in FIG. 2. Only the dissimilar components of the self-refrigeration unit are labeled with different numbers and will be described in details below. These dissimilar components include the turbo expander $35 \mathrm{a}$ and compressor $35 \mathrm{~b}$, the medium cooler $\mathbf{4 1}$, and the filter 38 .

The lean NG, left the absorber $1 \mathrm{~b}$ via the lean NG outlet pipeline $\mathbf{2 3}$ and mixed with the inhibitor introduced via the inhibitor injection pipeline 36 , enters the turbo expander $35 \mathrm{a}$ and is expanded. Gas expansion causes the NG temperature sharply dropped to the required low temperature. A small amount of the residual moisture is condensed into tinny liquid droplets entrained in the chilly lean NG. The chilly lean NG enters the filter $\mathbf{3 8}$ via the de-pressurized NG transfer pipeline 37. The liquid droplets are separated as an 50 effluent, and the latter is discharged into the inhibitor regenerator 15 via the effluent pipeline 39 . The dried chilly lean NG enters the secondary side of the medium cooler 41 via the chilly lean NG inlet pipeline $\mathbf{4 0}$. The chilly lean NG absorbs the heat from the recycled medium and flows into 55 the compressor $35 \mathrm{~b}$ via the de-pressurized NG return pipeline 42. A portion of the chilly NG is diverted via the bypass valve 44 and bypass pipeline 33 to the absorbent cooler 25 , and returns via the bypass return pipeline $\mathbf{3 4}$. The lean NG is then re-compressed to the required pressure and delivered 60 via the lean NG delivery pipeline $\mathbf{4 3}$ to the NG transportation pipeline (not shown).

As described above, the system in FIG. 2 does not require any external energy to provide the self-refrigeration.

Having described the features and the advantages of the 65 various embodiments of the present invention as a comprehensive NG processing apparatus, it should be pointed out

\section{6}

that the dehydration section with its accessories could also be operated independently as a pure NG dehydrator, without incorporating the absorption section and its accessories.

FIG. 3 illustrates the high-efficiency free-piston NG expander-compressor for self-refrigeration.

The light alloy body $\mathbf{4 5}$ of the said free piston expandercompressor comprises two cylinders with different diameters. The smaller cylinder $\mathbf{4 6}$ is the expander, and the larger cylinder $\mathbf{4 7}$ the compressor. Two free pistons, 48 and 49, are 10 rigidly connected with a short hollow shaft $\mathbf{5 0}$ to form a single integrated moving part. Since the latter is a compact, light-weighted component, very high frequency operation and high mechanical efficiency are feasible. For a highpressure NG, the size of such a free piston machine is relatively small. For example, for an apparatus processing $500,000 \mathrm{~m}^{3}$ STP per day, under an initial pressure of $10 \mathrm{MPA}$ and an exit pressure of $5 \mathrm{MPA}$, the maximum diameter of the free piston expander-compressor will be in the order of 12 $\mathrm{cm}$ when working at 4,000 strokes per minute.

In FIG. 3, the NG inlet pipelines $\mathbf{5 1}$ and $\mathbf{5 2}$ and the outlet pipelines $\mathbf{5 3}$ and $\mathbf{5 4}$ of the expander, as well as the inlet pipelines 55 and $\mathbf{5 6}$ and the outlet pipelines $\mathbf{5 7}$ and $\mathbf{5 8}$ of the compressor are connected to the relevant cylinders as illustrated. The associated valves controlling these inlet pipelines and outlet pipelines are similar to those used in modern high-speed internal combustion engine. These valves are not shown in FIG. 3.

In case that the pressure difference between the inlet NG and the outlet NG to the pipeline is too small so that 30 additional external compressing energy is required, a viable option is to connect the said free piston with extending the shaft $\mathbf{5 9}$, as shown by the dotted line, to a conventional reciprocating piston-type gas engine, not shown in FIG. 3.

In summary, the present invention is related to an appa35 ratus for efficient and cost-effective comprehensive processing of $\mathrm{NG}$, including the removal of moisture and the recovery of the higher hydrocarbons $\left(\mathrm{C}_{2}^{+}\right)$, in a single integrated processing unit. The present invention provides a low-cost comprehensive NG processor that is universally 40 applicable to both terrestrial and off-shore NG exploitation.

Having describes the present invention and preferable embodiments thereof, it will be recognized that numerous variations, substitutions and additions may be made to the present invention by those ordinary skills without departing 5 from the spirit and scope of the appended claims.

What is claimed is:

1. A comprehensive gas processor for removing the moisture and recovering the higher hydrocarbons (i.e., $\mathrm{C}_{2}^{+}$) therein either on-situ in a gas field or in a plant comprising:

(a) an integrated gas processor comprising two sections working on a hybrid process, i.e., an integration of two different processes within a single casing;

i) a refrigeration-dehydration section working on refrigeration process wherein the inlet gas contacts with a counter-flowing stream of dispersed cold heat-transport medium containing a non- or lowvolatile hydrate inhibitor with boiling point higher than $180^{\circ} \mathrm{C}$. and the moisture of said gas is condensed and removed with the cold heat-transport medium; and

ii) an absorption section working on low-temperature absorption process wherein the dehydrated gas contacts with a counter-flowing stream of dispersed liquid absorbent with a higher hydrocarbon gas solubility [higher] greater than $20 \mathrm{scf} / \mathrm{gal}$ wherein the higher hydrocarbons (i.e., $\mathrm{C}_{2}{ }^{+}$) are absorbed[.] under said absorption conditions; 
7

(b) a heat-transport medium cooler comprising a precooling stage and a deep-cooling stage wherein in said pre-cooling stage said heat-transport medium is precooled with the cold outlet gas left said integrated gas processor and in said deep-cooling stage the medium is deep-cooled with the refrigerant provided with a refrigerator;

(c) an absorbent cooler comprising a pre-cooling stage and a deep-cooling stage wherein in said pre-cooling stage said recycling absorbent is pre-cooled with the cold outlet absorbent left said integrated gas processor and in said deep-cooling stage the absorbent is deepcooled with the refrigerant provided with a refrigerator;

(d) a fractional distiller for separating the absorbed higher hydrocarbons as a product from the outlet absorbent 15 left said integrated gas processor and then the separated absorbent is recycled back to said integrated gas processor;

(e) an inhibitor regenerator for concentrating the lowvolatile hydrate inhibitor to be recycled and discharg- 20 ing the wastewater;

(f) a refrigerator for providing the refrigerant to said deep-cooling stages of said heat-transport medium cooler and said absorbent cooler;

(g) a pipeline for delivering the recovered higher hydro- 25 carbons; and

(h) a gas inlet pipeline and a pipeline for delivering the processing gas.

2. A comprehensive gas processor of claim 1 wherein the dehydration section of said integrated processor and its 30 accessories (comprising said heat-transport medium cooler, said inhibitor regenerator, said refrigerator, and said gas inlet-pipeline and a pipeline for delivering the processed gas) are operated independently as a gas dehydrator without incorporating the absorption section.

3. A comprehensive gas processor of claim 1 wherein said heat-transport medium is an aqueous solution of calcium chloride or other ionizing salts [and the regeneration rate of said solution is less than 5 liter per $\mathrm{kg}$ of wastewater to be discharged].

4. A comprehensive gas processor of claim 1 wherein said heat-transport medium is an aqueous solution of ethylene glycol or other organic compounds with boiling point[s] higher than $180^{\circ} \mathrm{C}$. [and the regeneration rate of said solution is less than 5 liter per $\mathrm{kg}$ of wastewater discharged.]

5. A comprehensive gas processor of claim 1 wherein said absorbent is heavy oil (i.e., hydrocarbon mixture with molecular weight higher than 100) or other organic compounds with hydrocarbon gas solubility higher than 20 scf/gal liquid.

6. A comprehensive gas processor of claim 1 when 50 working on inlet gas pressure greater than $5.0 \mathrm{MPa}$ wherein said refrigerant to said deep-cooling stages of said heattransport medium cooler and said absorbent cooler is provided with a gas expansion device with the inlet gas pressure is greater than $5.0 \mathrm{MPa}$.

7. A gas expansion device of claim 6 wherein said expansion device is a triple-sectional free-piston gas expander-compressor-booster comprising:

(a) a gas expansion cylinder and a gas compression cylinder;

(b) a co-shaft gas expansion piston and gas compression piston; and

(c) a co-shaft gas-fueled booster piston-engine providing supplemental power for compressing said expanded gas to the required delivery pipeline pressure.

8. A continuous process for separating moisture and hydrocarbons higher than methane from a natural gas

\section{8}

stream at pipeline or wellhead pressure, comprising removing said moisture and said hydrocarbons heavier than methane as a $\mathrm{C}_{2}{ }^{+}$mixture, including the following steps:

(a) Cooling said natural gas stream by directly contacting the natural gas stream with a low temperature counterflowing heat-transport medium containing an aqueous solution of a gas-hydrate inhibitor at pipeline or wellhead pressure and at a rate sufficient to cool said gas to the low temperature required by the absorption Step (c), and the majority of water moisture is condensed and dissolved into the heat-transport medium and, at the same time, a portion of higher hydrocarbons is also condensed as a liquid that is insoluble to the heat transport medium

(b) Separating the insoluble condensed higher hydrocarbons liquid from the condensed water (already dissolved into the heat-transport medium) as a portion of the product stream;

(c) Extracting said dehydrated, depleted cold natural gas stream leaving Step (a) by flowing counter-flow to the cold natural gas stream a cold absorbent with a higher hydrocarbon (i.e., $\mathrm{C}_{2}^{+}$) solubility greater than $20 \mathrm{scf} /$ gal under said absorption conditions and at a rate sufficient to produce a rich absorbent stream containing $\mathrm{C}_{2}^{+}$mixture and a small portion of methane, and a cold residue natural gas stream of gas transport pipeline quality;

(d) Regenerating said rich absorbent by fractionating the rich absorbent leaving Step (c) at reduced pressure and separating from said absorbent the absorbed $\mathrm{C}_{2}{ }^{+} \mathrm{mix}$ ture as a product stream;

(e) Cooling the heat-transport medium leaving Step (a) to said sufficiently low temperature in two steps: first with the cold stream of the residue gas leaving Step $(c)$, then with the refrigerant provided by an external refrigerator;

(f) Separating the condensed water moisture from said heat transport medium by evaporating a small portion of the heat-transport medium leaving Step (a) under reduced pressure, and recycling the concentrated inhibitor solution to the heat-transport medium stream;

(g) Recycling the regenerated absorbent leaving Step (d) by compressing it to pipeline or well-head pressure, and cooling it to the required low temperature in two steps: first with the rich absorbent leaving Step (c), then with the refrigerant provided by an external refrigerator; and

(h) Delivering the residue gas leaving Step (e) into the gas transport pipeline.

9. The process of claim 8 , wherein the cold stream of the residue gas leaving Step (c) is expanded to a lower pressure and much lower temperature to provide sufficient internal refrigeration.

10. The process of claim 9, wherein in Step (e) the 5 heat-transport medium leaving Step (a) is cooled to said sufficiently low temperature with the cold stream of the said expanded residue gas.

11. The process of claim 8 , wherein in step (a) the said heat transport medium is an aqueous solution of calcium chloride or other ionizing salts with a potential regeneration 60 rate less than 5 liter per $\mathrm{kg}$ of wastewater to be discharged.

12. The process of claim 8, wherein in step (a) the said heat transport medium is an aqueous solution of ethylene glycol or other organic compounds with a potential regeneration rate less than 5 liter per $\mathrm{kg}$ of wastewater to be 65 discharged. 


\section{(19) United States \\ (12) Patent Application Publication Lu}

(10) Pub. No.: US 2008/0016909 A1

(43) Pub. Date:

Jan. 24, 2008

(54) FLEXIBLE HYDROCARBON GAS SEPARATION PROCESS AND APPARATUS

(76) Inventor: Yingzhong Lu, Oak Ridge, TN (US)

Correspondence Address:

LU YINGZHONG

104 HARLAND COURT

OAK RIDGE, TN 37830

(21) Appl. No.:

$11 / 713,757$

(22) Filed:

Mar. 5, 2007

(30)

Foreign Application Priority Data

Jul. 19, 2006

$(\mathrm{CN})$ 200610099387.6

Publication Classification

(51) Int. Cl.

F25J 3/08

(2006.01)
(52) U.S. CI.

$62 / 623$

(57)

ABSTRACT

The present invention related to a flexible hydrocarbon gas separation process that could dehydrate the water-saturated hydrocarbon gas mixture and recover thereof the required higher hydrocarbons (NGL) therein with a controllable ethane recovery rate (ranging from $>95 \%$ to $<2 \%$ ) while keeping high recovery rate of all other heavier components. The flexible process comprises the following steps: deepcooling and dehydrating the raw gas and get the NGL condensate; flowing the deep-dehydrated gas into the flexible absorber to get the rich oil with desirable ethane content; completely demethanizing and partially deethanizing as desired the rich oil and the NGL condensate to get purified rich oil and purified NGL condensate, respectively; separating the NGL vapor from the purified rich oil; cooling and compressing the NGL vapor; mixing the NGL vapor with the purified NGL condensate; and liquefying the mixture to get the final NGL product. The present invention also provides a flexible apparatus with highly efficient components for the flexible process.

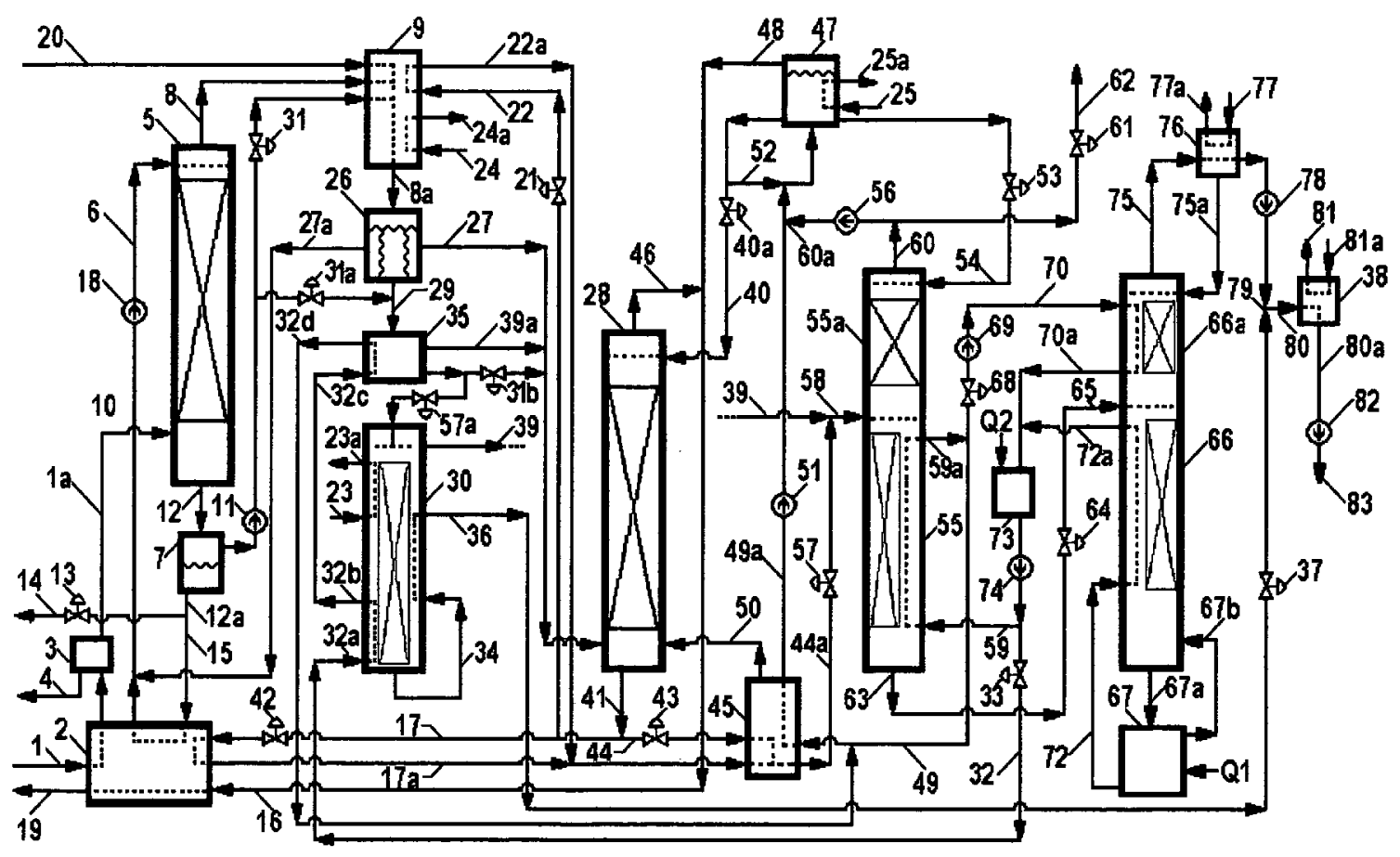




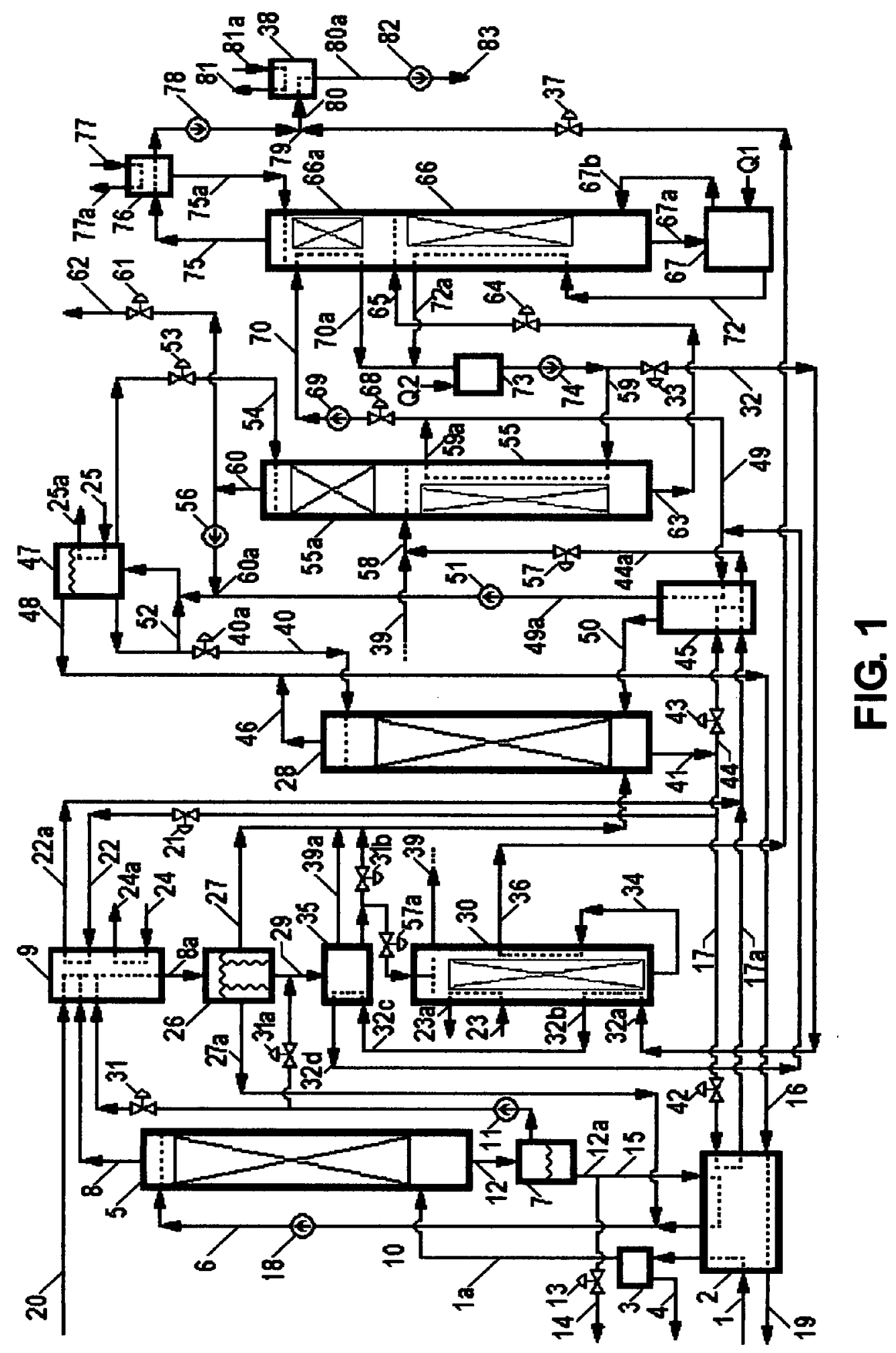




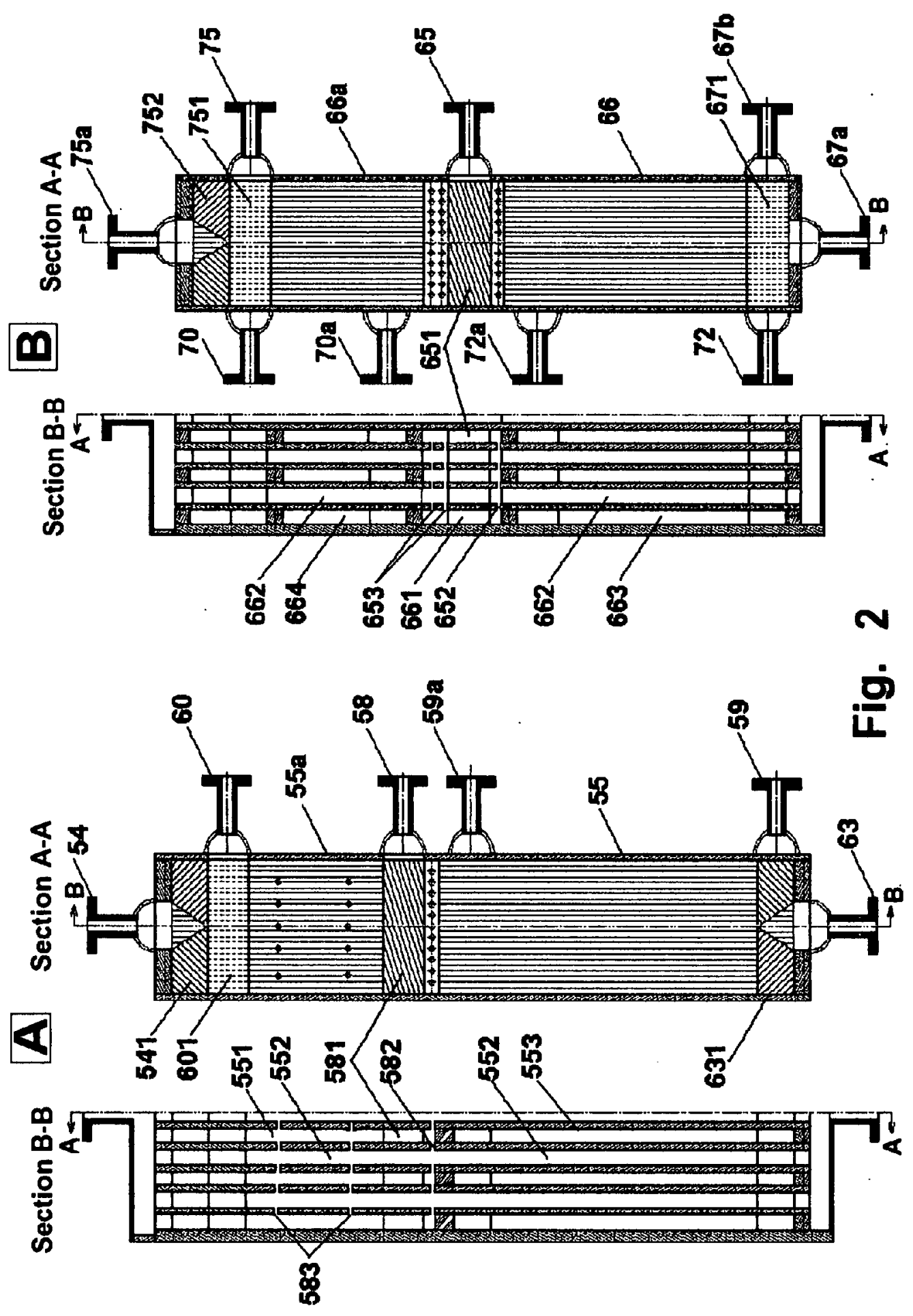




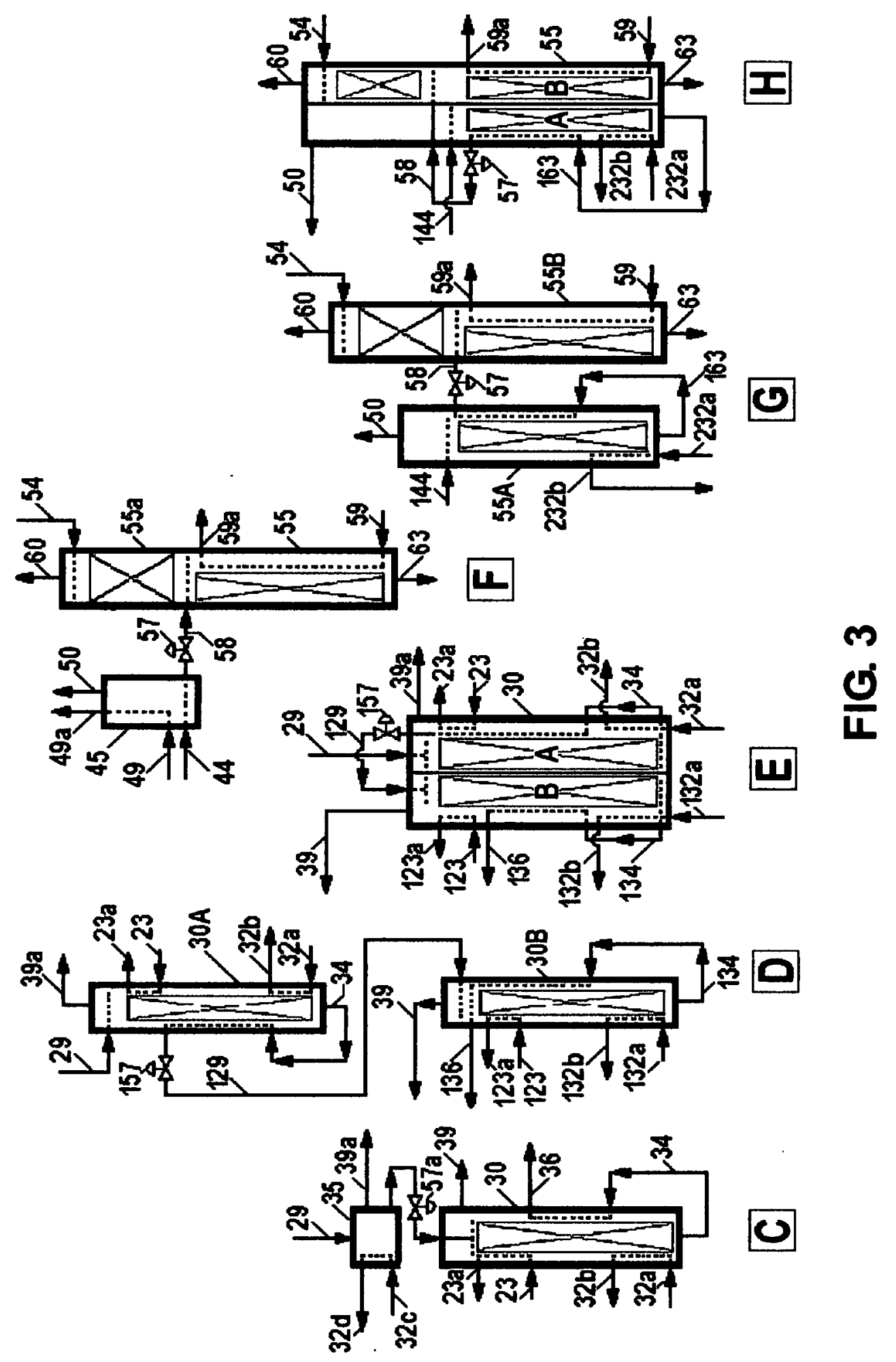


Patent Application Publication Jan. 24, 2008 Sheet 4 of 8 US 2008/0016909 A1

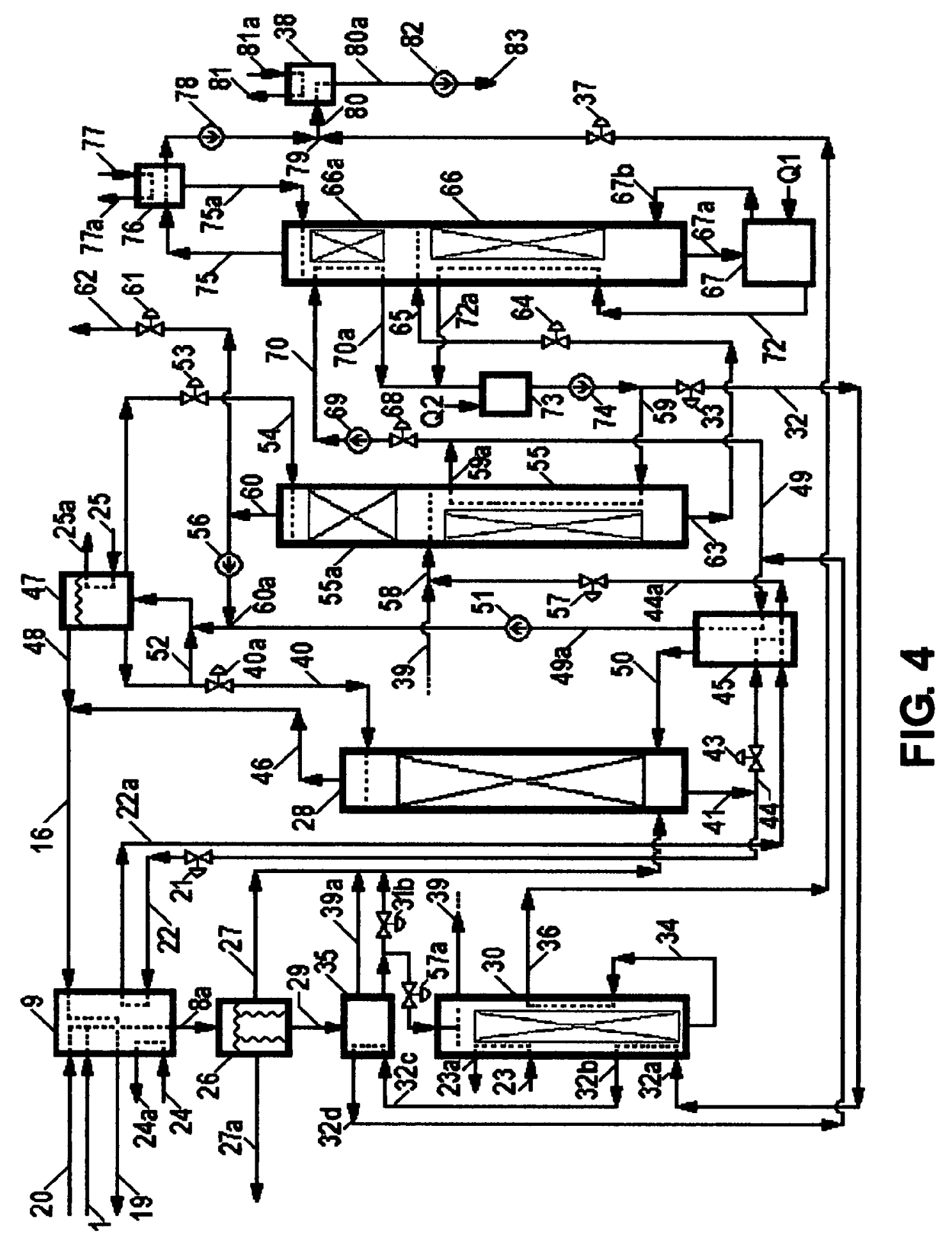




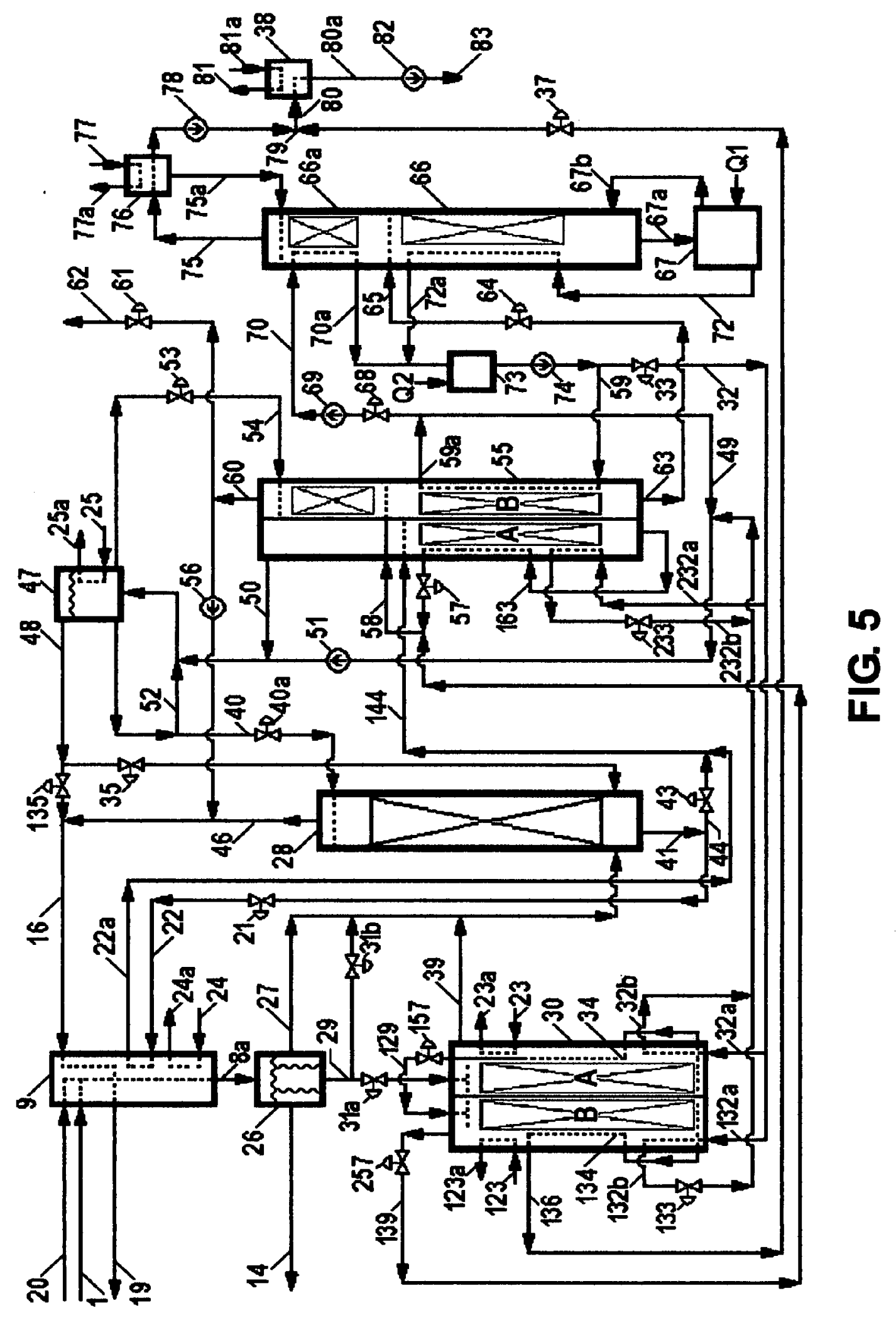




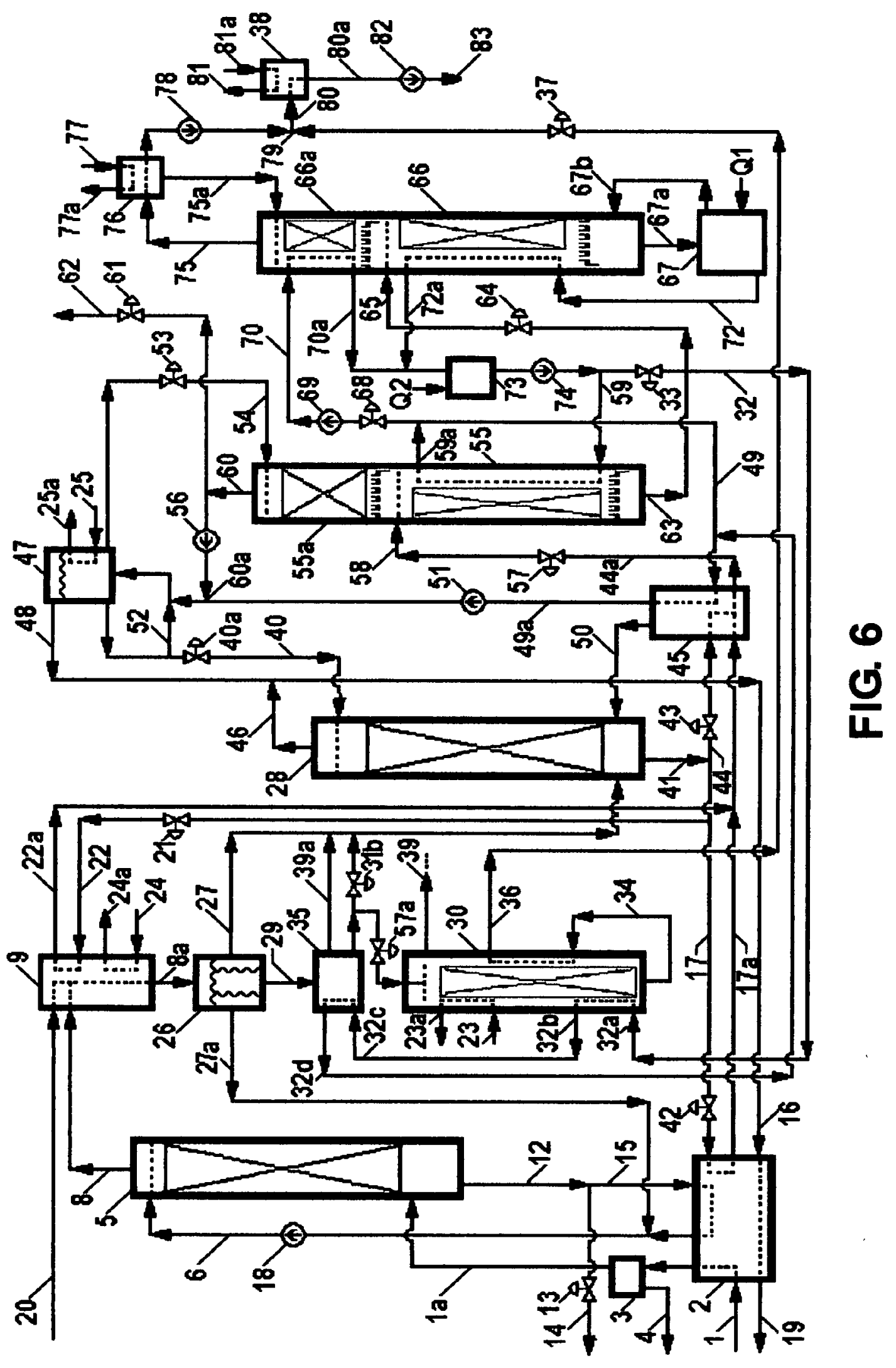




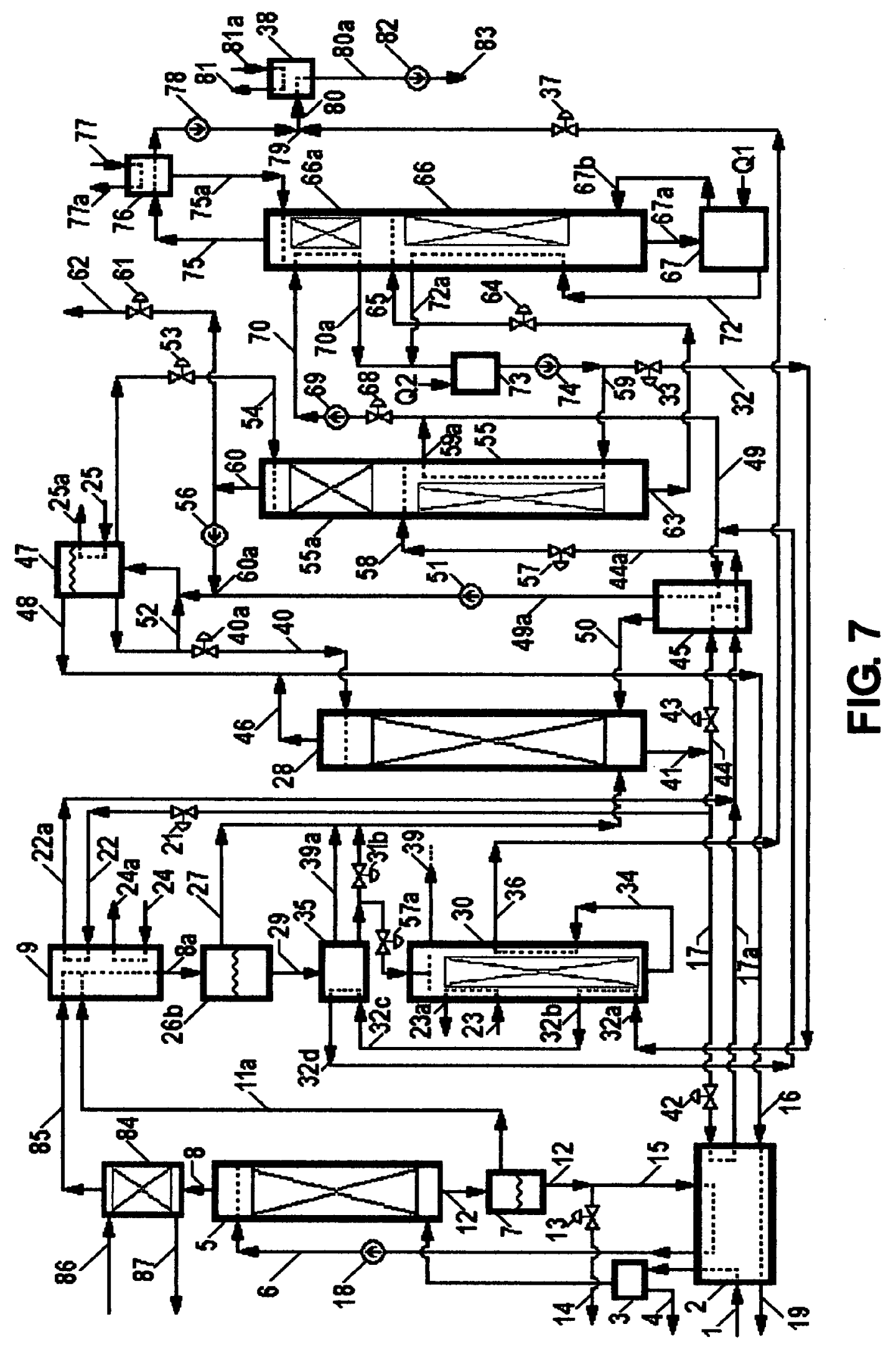




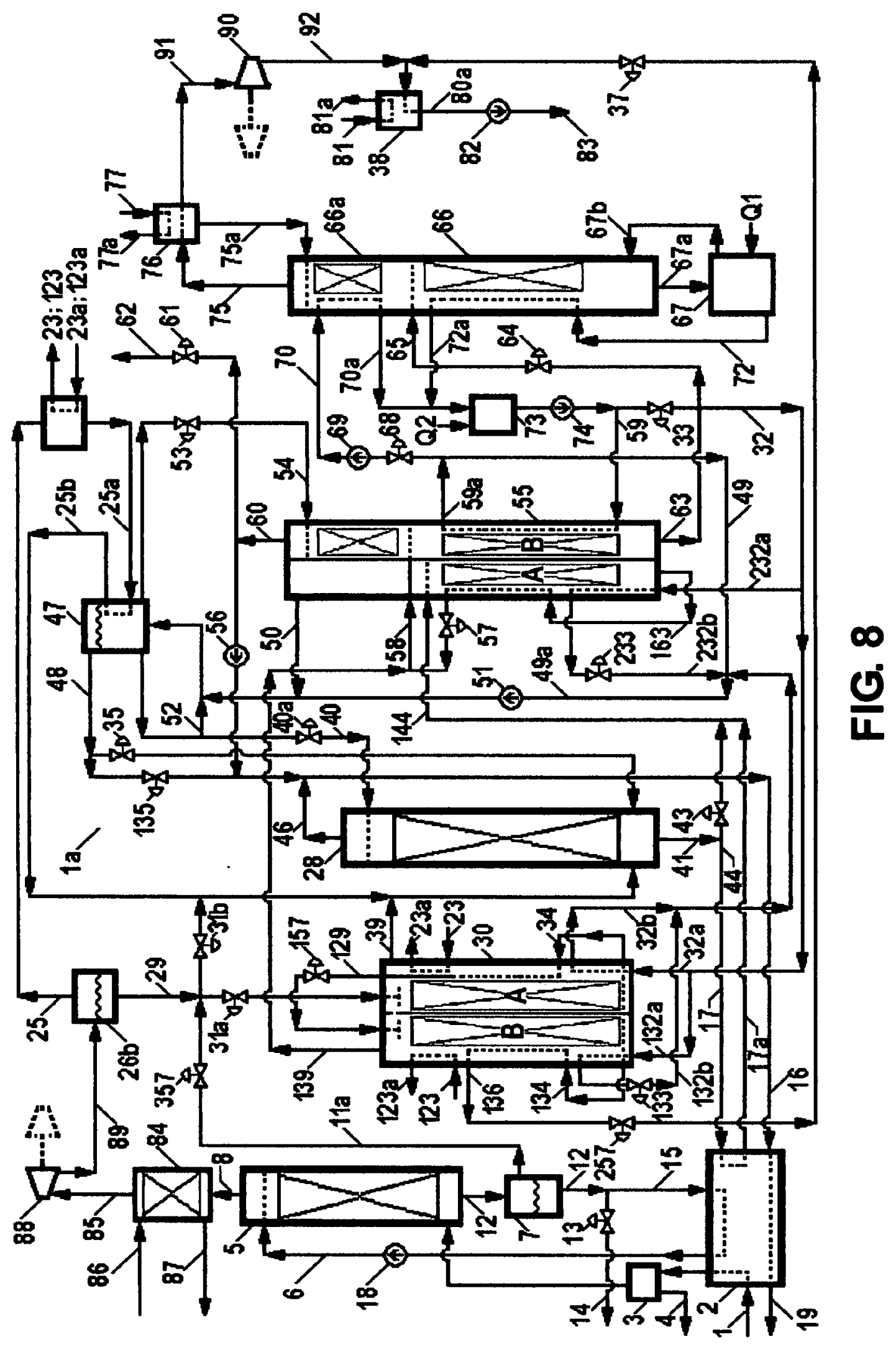




\section{FLEXIBLE HYDROCARBON GAS SEPARATION PROCESS AND APPARATUS}

\section{BACKGROUND OF THE INVENTION}

[0001] The rapid globalization of world economy, expansion of world population and rise of living standards lead to tremendous energy demand. Since the present oil and gas supply could hardly always match the rapidly rising market demand, their prices are escalating and unpredictable. In particular, due to the difficulties of storage and overseas transportation, the natural gas price is more volatile.

[0002] The separation of selected hydrocarbon components from a water-saturated hydrocarbon gas mixture (abbreviated as "raw gas" hereafter), in particular, the separation of the natural gas liquid (abbreviated as "NGL" hereafter) from natural gas, would consume a significant portion of the raw gas. The gas is consumed not only as the raw material, but also as the fuel to generate heat and power required for the energy-intensive separation process. The production cost of NGL, therefore, escalates with the gas price. However, the NGL is not the end-use product itself. As a liquid mixture, the NGL product must be further fractionated into its components and sold to different end users on the market. The market price of each NGL component would fluctuate according to their respective market demands. As a consequence, their respective net profit would be quite different. For instance, the market price of ethane rose much slower recently than other components when gas price went up. The net profit of ethane production may even become negative at peak gas price. On the contrary, the net profits of certain other NGL component, such as propane, would become more attractive due to the increasing LPG demand. [0003] In such a volatile NGL components market, the prevailing Cryogenic Process for NGL separation is facing a serious challenge. The energy-intensive Cryogenic process has been greatly favored in the past because of its high ethane recovery level $(\sim 90 \%)$. Facing the shrinking profit of ethane production, however, the overall net profits of existing Cryogenic separation plants have been declining. Many Cryogenic separation plants had to operate on so-called "ethane-rejection" cycle. In the ethane-rejection operations, the liquid ethane, separated after gas expansion, had to be re-evaporated from the NGL mixture and re-compressed to the original gas pressure, mixed with the residue gas, and sold at the same price of the raw gas. The energy consumed in the whole ethane-separation and ethane-rejection processes was completely wasted. The overall profit of the Cryogenic plants, therefore, would be decreasing faster due to its higher ethane recovery rate as compared with other separation processes. As a result, the future of the Cryogenic process becomes uncertain.

[0004] Numerous improvements have been proposed for lowering ethane-rejection costs. Among so-called "Next Generation" Cryogenic processes, a number of US patents have been granted, such as the U.S. Pat. Nos. 4,854,955; $4,889,545 ; 5,568,737 ; 5,711,712 ; 5,799,507$ and 5,881,569. Since all these patents are still based on cryogenic expansion cycle wherein the bulk raw gas must be expanded to sufficiently low pressure to create the required low cryogenic temperature, the residue gas has to be recompressed to pipeline pressure before sent out. The recompression power, therefore, could not be significantly reduced. On the other hand, the recovered NGL decreases substantially due to the rejection of ethane. As a result, the average energy cost per unit of the NGL products would rapidly increase, and the overall plant net profit drops accordingly. All these improved "Next Generation" cryogenic processes, therefore, could hardly make Cryogenic process more competitive in future NGL market.

[0005] A different approach based on the Absorption process has also been proposed to solve the problem. The traditional refrigerated oil absorption process (abbreviated as "ROA process" hereafter) failed to compete with Cryogenic process due to its lower ethane recovery rate and higher refrigeration energy consumption. Presently, the first weakness is no longer a drawback. To eliminate the second weakness, i.e., higher refrigeration energy consumption, a lot of efforts have been made to find alternative absorbents that could selectively absorb heavier hydrocarbons at ambient temperature. For example, Dr. Mehra has identified a number of such novel absorbents and received a series of U.S. Pat. Nos. 4,421,535; 4,511,381; 4,526,594; 4,578,094; $4,698,688 ; 5,561,988$; and 5,687,584, etc. He has also made notable progresses in commercializing his process during past two decades. However, due to the higher costs and the lack of industrial operational experience of those novel absorbents, the market share of the so-called "Mehra process" is still limited, and does not have significant impact on gas industry. It is, therefore, not expected to become a widely accepted alternative to the declining Cryogenic process in foreseeable future.

[0006] Another approach based on radically improvements on traditional ROA process has been proposed more recently by the present inventor to substantially reduce the refrigeration energy consumption while still using similar heavy oil absorbents under refrigeration temperature. The basic idea is to integrate the technology of refrigeration dehydration of natural gas with the improved heat-recuperated rich oil processing process. In April 2003, the U.S. Pat. No. 6,553,784 "Comprehensive Natural Gas Processor" based on an innovative "Improved Refrigeration Oil Absorption Process" (abbreviated as "IROA process" hereafter) was granted. The IROA process could substantially reduce the required refrigeration power and heat energy and effectively meet the challenge of the volatile NGL market, while still inheriting the matured experience of traditional ROA process. According to a preliminary computer simulation of the IROA process, as reported on AIChE 2005 Spring Meeting, April 11-14 2005, Atlanta, the IROA process could compete favorably with Cryogenic process over a wide range of ethane recovery rates, notably from $\sim 70 \%$ down to $\sim 2 \%$, for a wide variety of raw gas. The widely accepted expertise of the traditional ROA process currently in operation would give excellent market penetration prospect of the IROA process under development.

[0007] However, being emphasized on the improvements on the head and bottom ends of the ROA process, said patent has not fully explored the potential of the flexibility and energy-savings of the IROA process over the entire ethane recovery range between $>95 \%$ down to $<2 \%$, particularly at the highest and the lowest ends of the ethane recovery range, in comparison with the Mehra process.

[0008] Accordingly, it is an objective of the present invention to provide a fully flexible improved refrigerated absorption process (abbreviated as "FIRA process" hereafter) that could control the desirable ethane recovery rate from $>95 \%$ down to $<2 \%$ during operations to best meet the challenge of the volatile NGL market. 
[0009] Another objective of the present invention is to provide a fully energy-integrated FIRA apparatus that could substantially reduce the unit product energy consumption and maximize the profits of the FIRA gas separation plant during operations over the full range of ethane recovery rate from $>95 \%$ down to $<2 \%$.

[0010] A further objective is to provide high-performance components for the FIRA process and reduce the capital costs of the FIRA gas separation plant.

\section{BRIEF SUMMARY OF INVENTION}

[0011] With regard to the above and other objectives, the present invention provides a fully flexible improved refrigerated absorption (FIRA) process that could recover NGL from the raw gas and control the ethane recovery rate from $>95 \%$ to $<2 \%$ while maintaining high recovery rates ( $>90 \%$ ) of other heavier components. Besides the flexibility of the ethane recovery rates, the flexibility of the process also reflects in the following features: (1) flexibility of handling the raw gas with a wide range of water contents and gas composition; (2) flexibility of handling the raw gas under a wide range of pressure, e.g., from the deep-well gas $(>1000$ psia) to the refinery off-gas ( $<200$ psia); (3) flexibility of using various types of high gas solubility, highly selective, and low volatile absorbents (abbreviated as "lean oil" hereafter), including the heavy oils and other organic absorbents; and (4) flexibility of selecting and reorganizing various steps of the typical FIRA process to construct alternative processes to meet different production objectives and/or to retrofit exiting gas processing plants.

[0012] A typical FIRA process includes the following major steps: (1) pre-cooling and pre-dehydrating the raw gas with directly contact a counter-flowing liquid coolant comprising of an aqueous solution of a gas hydrate inhibitor to get the pre-cooled pre-dehydrated gas and the partial NGL condensate; (2) deep-cooling and deep-dehydrating the precooled pre-dehydrated gas with the refrigerant from an external refrigerator to get the deep-cooled deep-dehydrated gas and the deep-cooled NGL condensate; (3) flowing the NGL condensate into a condensate pre-demethanizer and a flexible condensate deethanizer in tandem to remove all the methane content therein and simultaneously reduce the ethane contend therein to any desirable level to get the deep-cooled purified NGL condensate; (4) flowing the deepcooled deep-dehydrated gas into a flexible absorber wherein contacting the gas with a counter-flowing liquid lean oil to get the cold residue gas and the cold rich absorbent (abbreviated as "rich oil" hereafter) with desirable level of ethane content; (5) flowing the cold rich oil into a rich oil predemethanizer and a flexible rich oil deethanizer in tandem to remove all the methane content therein and simultaneous reduce the ethane content therein to any desirable level to get the purified rich oil; (6) depressurizing the purified rich oil and distilling the purified rich oil in a rich oil fractionator to get the NGL vapor and the regenerated lean oil; (7) cooling the NGL vapor with a heat transport medium to transport the heat energy from the NGL vapor to the deep-cooled rich oil in the flexible rich oil deethanizer; (8) compressing the NGL vapor and mixing the compressed NGL vapor with the deep-cooled purified NGL condensate to get the low-temperature NGL vapor-liquid mixture; and (9) liquefying the low-temperature NGL vapor-liquid mixture to get final NGL product.
[0013] The present invention also provides a fully energyintegrated gas separation process that could substantially reduce the energy consumption over the entire range of ethane recovery rate from $>95 \%$ to $<2 \%$ and guarantee maximum profit of the separation plant in the volatile NGL market. The fully energy-integration process include the following features: (1) utilizing the cold residue gas and cold rich oil as coolants to directly or indirectly pre-cool the inlet raw gas and the pre-dehydrated gas in the pre-dehydrator; (2) utilizing the hot regenerated lean oil as heating medium to pre-heat the purified rich oil in the rich oil fractionator; (3) utilizing the hot regenerated lean oil as heating medium to heat the cold rich oil in the rich oil deethanizer; (4) utilizing the hot regenerated lean oil as heating medium to heat the deep-cooled NGL condensate in the condensate deethanizer; (4) utilizing the hot NGL vapor as heating medium to pre-heat the cold rich oil in the rich oil deethanizer; and (5) mixing the deep-cooled purified NGL condensate with the compressed NGL vapor to reduce the liquefaction power for NGL product.

[0014] The present invention also provides a fully energyintegrated FIRA apparatus with highly efficient components that could substantially reduce the energy consumption and maximize the profits of the FIRA gas separation plant. The high-performance components include, but not limited to, the cascade flexible deethanizers, and the dual-function exchanger-reactors.

\section{BRIEF DESCRIPTION OF SEVERAL VIEWS OF THE DRAWING}

[0015] The above and other features and advantages of the present invention will now be further described in the following detailed description section in conjunction with the attached drawings in which:

[0016] FIG. 1 illustrates the basic flow diagram of the typical flexible hydrocarbon gas separation process wherein an external refrigerator is used to provide the refrigerant for deep-cooling the raw gas and lean oil.

[0017] FIG. 2 illustrates examples of the high-performance dual-function exchanger-reactors used in FIG. 1 for carrying out heat- and mass-transfer simultaneously in critical components.

[0018] FIG. 3 illustrates the embodiments of the highperformance cascade flexible deethanizer that could replace the combination of a pre-demethanizer and a flexible deethanizer in tandem as illustrated in FIG. 1 for purifying NGL condensate and rich oil.

[0019] FIG. 4 illustrates another embodiment of the flexible hydrocarbon gas separation process as illustrated in FIG. 1 wherein the inlet gas has already been pre-dehydrated to pipeline gas spec.

[0020] FIG. 5 illustrates an alternative embodiment of the flexible hydrocarbon gas separation process as illustrated in FIG. 4 wherein the high-performance, integrated cascade flexible deethanizers are used.

[0021] FIG. 6 illustrates still another embodiment of the flexible hydrocarbon gas separation process as illustrated in FIG. 1 wherein the temperature of the pre-cooled gas is above the NGL dew point.

[0022] FIG. 7 illustrates still another embodiment of the flexible hydrocarbon gas separation process as illustrated in FIG. 1, wherein a solid adsorbent deep-dehydrator is installed before the pre-dehydrated gas is deep-cooled with a refrigerant. 
[0023] FIG. 8 illustrates an alternative embodiment of the flow diagram of flexible hydrocarbon gas separation process as illustrated in FIG. 7 wherein the raw gas pressure is so high that an internal gas expander could be used to replace the external refrigerator providing the refrigerant for deepcooling the gas and lean oil.

\section{DETAILED DESCRIPTION}

[0024] FIG. 1 illustrates the basic flow diagram of the typical flexible hydrocarbon gas separation process wherein an external refrigerator is used to provide the refrigerant for deep-cooling the raw gas and lean oil.

[0025] In FIG. 1, the raw hydrocarbon gas saturated with water vapor enters the primary heat exchanger 2 via gas inlet pipe 1. There are two coolant streams in the prime heat exchanger, i.e., (1) the deep-cooled residue gas stream, entering via the master residue gas pipe $\mathbf{1 6}$ and leaving via the residue gas discharge pipeline 19 to the external gas transport pipeline (not shown), and (2) the deep-cooled rich oil stream regulated with rich oil regulation valve $\mathbf{4 2}$, coming via rich oil pipe 17 , and leaving via rich oil pipe $17 a$. The raw gas is cooled to a temperature above the hydrate formation threshold, and then the cooled gas enters the moisture separator $\mathbf{3}$ wherein the entrained condensed water droplets are separated and discharged via the water drainage pipe 4. The pre-treated gas then enters the pre-cooler/ dehydrator 5 (abbreviated as "pre-dehydrator" hereafter) via the pre-treated gas inlet pipe $1 a$, flows upward, and is cooled by directly contacting a down-flowing liquid coolant. The liquid coolant is an aqueous solution of a gas hydrate inhibitor (e.g. ethylene glycol, calcium chloride, etc.), entering the pre-dehydrator at the top via coolant inlet pipe $\mathbf{6}$ and a booster pump 18. When the pre-treated gas is further cooled in the pre-dehydrator, the major portion of water vapor is condensed and dissolved into the coolant solution. A certain fraction of the heavier hydrocarbon vapor of the raw gas may also condense, according to the richness of the higher hydrocarbons in the raw gas. This portion of higher hydrocarbons condensate (abbreviated as "partial NGL condensate" hereafter), immiscible to the coolant, flows downward together with the coolant. The mixed flow is discharged via the liquid discharge pipe 12 into the two-phase separator 7 . The pre-dehydrated gas, flowing upward, leaves the pre-dehydrator via pre-dehydrator outlet pipe 8 . Since the moisture content therein is still exceeds the allowable level required by the subsequent refrigeration absorber, the pre-dehydrated gas is sent into the deep-cooler/dehydrator 9 (abbreviated as "deep-dehydrator" hereafter).

[0026] In the two-phase separator 7, the partial NGL condensate, if any, is separated from the liquid mixture. The separated partial NGL condensate is sent via condensate outlet pipe 10, though the condensate booster pump 11 and the shift valve $\mathbf{3 1}$ (normally open), into the deep-dehydrator. The boosted partial NGL condensate is mixed therein with the pre-dehydrated gas stream to increase the ethane content of the NGL condensate. In case the system needs to operate under ethane-rejection mode, the partial NGL condensate should directly flow into the condensate pre-demethanizer 35 by closing shift valve 31 (normally open) and opening the other shift valve $31 a$ (normally close).

[0027] The aqueous coolant, separated in the two-phase separator 7, is discharged via the coolant outlet pipe $12 a$ and split into two streams. The bulk coolant stream, sent via coolant transfer pipe $\mathbf{1 5}$ into the primary heat exchanger $\mathbf{2}$, is deeply cooled therein with the deep-cooled residue gas coming from the residue gas master pipe 16 and the deepcooled rich oil coming from rich oil inlet pipe 17 . The deep-cooled bulk coolant stream is then boosted with coolant booster pump 18 and re-enters the pre-dehydrator 5 via coolant inlet pipe 6.

[0028] The minor portion of the separated aqueous coolant, regulated with the regulating valve 13 , is sent via the regeneration coolant pipe $\mathbf{1 4}$ to the external glycol regenerator not shown in FIG. 1.

[0029] The make-up inhibitor solution is sent via inhibitor make-up pipe $\mathbf{2 0}$ to mix with the pre-dehydrated gas and the NGL condensate stream in the deep-dehydrator, 9. The gas-liquid mixture is deeply cooled therein to the low temperature required by the subsequent flexible absorber 28 . A considerable portion of the higher hydrocarbon gas is condensed therein as the NGL condensate, according to the richness of the higher hydrocarbon content of the raw gas. [0030] There are two streams of coolants flow in the deep-dehydrator 9: (1) the refrigerant, coming from an external refrigerator (not shown) via the refrigerant inlet pipe 24 and leaving via the refrigerant outlet pipe $24 a$, and (2) the deep-cooled rich oil regulated with rich oil regulation valve 21 , coming via the rich oil inlet pipe 22 and leaving via the rich oil outlet pipe $22 a$. The mixed flow of the deep-cooled gas, inhibitor solution and NGL condensate, is discharged at the bottom, via the mixture discharge pipe $8 a$, into the three-phase separator $\mathbf{2 6}$, wherein the three phases are separated. The separated deep-dehydrated gas (abbreviated as "cold dry gas" hereafter) is sent via cold dry gas outlet pipe $\mathbf{2 7}$ into the flexible absorber $\mathbf{2 8}$. The separated inhibitor solution is sent via the inhibitor solution outlet pipe $27 a$ into the coolant inlet pipe 6 . The separated NGL condensate is sent via the NGL condensate outlet pipe 29 into the NGL condensate pre-demethanizer 35.

[0031] The condensate pre-demethanizer 35 is heated with a stream of the hot regenerated lean oil regulated with the recycling lean oil regulating valve 33 , coming via the lean oil inlet pipe $32 c$ and leaving via the lean oil outlet pipe $32 d$. A significant portion of the methane, together with a small amount of ethane and trace of higher hydrocarbons, is evaporated as the residue gas from the condensate. The residue gas is then sent via residue gas outlet pipe $39 a$ to merge with the cold dry gas flowing in the cold dry gas pipe 27. The gas mixture is then sent into the flexible absorber 28 for further processing. The pre-demethanized NGL condensate is sent via the condensate de-pressurization valve $\mathbf{5 7 a}$ into the flexible condensate deethanizer $\mathbf{3 0}$ for complete demethanization and appropriate deethanization as required. [0032] The flexible condensate deethanizer 30 is made of an exchanger-reactor wherein the pre-demethanized NGL condensate flows down from the top as a liquid film covering the wall of the first group of narrow flow channels (shown as a thin-lined slender rectangular block). The liquid film is first cooled with a refrigerant flowing in the second group of channels, shown as a bold dotted line, coming via the refrigerant inlet pipe $\mathbf{2 3}$ and leaving via refrigerant outlet pipe $23 a$. The liquid film is then pre-heated with the recycling purified NGL condensate, flowing inside the third group of channels, shown as a bold dotted line, coming via recycling purified condensate pipe $\mathbf{3 4}$ and leaving via deepcooled NGL condensate pipe 36. The liquid film is eventually heated in the lower section of the flexible condensate deethanizer with the hot lean oil, flowing inside the fourth 
group of channels, shown as a bold dotted line, coming via lean oil inlet pipe $32 a$ and exits via lean oil outlet pipe $\mathbf{3 2} b$. All the methane and a desirable fraction of ethane in the NGL condensate liquid film are evaporated as the residue gas, together with trace of heavier hydrocarbon, when the liquid film arrives at the bottom of the flexible condensate deethanizer. The residue gas flows upward and directly contacts the down-flowing liquid film. The heaviest hydrocarbon components in the residue gas are essentially stripped back into the liquid film. By adjusting the working pressure and the bottom temperature of the flexible condensate deethanizer, the ethane content in the final purified condensate could be easily controlled. The residue gas, eventually leaving via residue gas outlet pipe 39 , is sent to the flexible rich oil deethanizer $\mathbf{5 5}$ for further processing.

[0033] In the flexible absorber 28, the deep-cooled, deepdehydrated gas mixture enters from the bottom, flows upward, and directly contacts the down-flowing lean oil coming from the top via lean oil inlet pipe 40. Although a variety of absorbents, either traditional or novel, could be used in this invention, the low-volatility heavy oil (e.g., average molecular weights higher than $\sim 140$ ) with high NGL solubility (e.g., $>20 \mathrm{scf} / \mathrm{gal}$ ) is generally preferred in view of their extensive operational practice in gas industry. By varying the molar flow ratio of the lean oil vs. gas, the ethane recovery rate may easily be controlled while keeping very high recovery rates of all other heavier $(\mathrm{C} 3+)$ components. However, since it is generally desirable to keep the $\mathrm{C} 3$ recovery higher than $95 \%$, the decrease of the molar flow ratio of the lean oil vs. gas would have a lower boundary. Subject to this constraint, the reasonable minimum value of $\mathrm{C} 2$ recovery rate with lean oil would be around $30 \%$. To further reduce the $\mathrm{C} 2$ recovery rate below $\sim 30 \%$, additional ethane-rejection in the subsequent flexible rich oil deethanizer is needed. The extra energy consumption of the ethanerejection operations in present invention, however, is much less than that in current Cryogenic process. It is because in present invention: (1) the maximum ethane content needs to be re-evaporated at the minimum $\mathrm{C} 2$ recovery level $(2 \%)$ is much less than current Cryogenic process, and (2) the pressure of the residue gas from ethane-rejection operations to be recompressed is significantly higher than current Cryogenic process. As a result, the extra energy consumption resulted from ethane-rejection operations in present invention would be much less than current Cryogenic process.

[0034] The deep-cooled residue gas is discharged from the top of the flexible absorber, via absorber residue gas discharge pipe 46, and mixed with the other stream of deepcooled residue gas stream flowing in the deethanizer residue gas discharge pipe 48. As described above, the mixed deep-cooled residue gas flows via residue gas master pipe 16 into the primary heat exchanger 2 to pre-cool the inlet raw gas. This is one important energy-integration measure of present invention.

[0035] The deep-cooled rich oil is discharged via rich oil discharge pipe $\mathbf{4 1}$ from the bottom of the flexible absorber. As another important energy-integration measure of present invention, the deep-cooled rich oil is used as a cooling medium in several heat-exchanging components, including (1) the primary heat exchanger 2 (regulated with the regulation valve 42); (2) the deep-dehydrator 9 (regulated with the regulation valve 21); and (3) the rich oil pre-demethanizer 45 (regulated with the regulation valve $\mathbf{4 3}$ ).
[0036] In the pre-demethanizer, the deep-cooled rich oil is pre-heated with the recycling lean oil, coming via cold recycling lean oil pipe 49 and leaving via deep-cooled recycling lean oil pipe $49 a$. A significant portion of the dissolved methane and a small portion of ethane are evaporated as the residue gas and return to the flexible absorber via residue gas transfer pipe 50. The pre-demethanized rich oil is then flashed via the rich oil de-pressurization valve $\mathbf{5 7}$ into a lower pressure vapor-liquid mixture. The vapor-liquid mixture enters the flexible rich oil deethanizer via rich oil mixture inlet pipe 58, at the junction between the upper and lower sections, $\mathbf{5 5}$ and $\mathbf{5 5} a$, wherein the liquid and the vapor are separated. The liquid rich oil flows downward as a liquid film covering the wall of the first group of narrow flow channels (shown as a thin-line slender rectangular block) of the lower section 55, while the residue gas flows upward along the other group of narrow flow channels (also shown as a thin-line slender rectangular block) of the upper deethanizer section $\mathbf{5 5} a$. In the lower deethanizer section, there is a second group of flow channels for the up-flowing hot lean oil, shown as bold dotted line, coming via recycling lean oil inlet pipe 59 and leaving via recycling lean oil outlet pipe $\mathbf{5 9} a$. The down-flowing rich oil is heated with the up-flowing hot lean oil. At the bottom of the lower deethanizer section, the dissolved methane in the rich oil is completely evaporated, together with a desired portion of the dissolved ethane, controlled with the bottom temperature of the liquid film. The evaporated residue gas, flowing upward, is stripped with the colder rich oil film. At the bottom of the lower deethanizer section, the purified rich oil, already completely demethanized and partially deethanized to desired level, is discharged via purified rich oil outlet pipe 63.

[0037] Inside the upper section of the flexible rich oil deethanizer, there is only one group of narrow flow channels (shown as a thin-line slender rectangular block). A stream of deep-cooled stripping lean oil, regulated with the stripping lean oil regulation valve $\mathbf{5 3}$, enters via the stripping lean oil inlet pipe 54. The trace hydrocarbons heavier than ethane is essentially stripped from the residue gas with the stripping lean oil. The portion of ethane stripped therein is controlled with changing the molar flow ratio of the stripping lean oil vs. the residue gas. The stripping lean oil eventually reaches the bottom of the upper deethanizer section, and merges with the rich oil liquid entering the lower deethanizer section.

[0038] The residue gas leaves the upper deethanizer section via residue gas outlet pipe $\mathbf{6 0}$. The residue gas is then split into two streams. The minor stream of the residue gas, regulated with the fuel gas de-pressurization valve 61 , is delivered as fuel gas, via fuel gas pipe 62 . The bulk stream of the residue gas is re-compressed with the residue gas compressor 56 to a pressure slightly higher than the raw gas. The high-pressure residue gas is then sent into the $\mathrm{T}$-joint $60 a$ to mix with the reflux pre-saturated lean oil, coming from reflux lean oil pipe 52, and the recycling lean oil, coming from deep-cooled recycling lean oil pipe $49 a$ and pressurized with the lean oil booster pump 51. The gasliquid mixture then enters the lean oil pre-saturator $\mathbf{4 7}$ under high turbulence, wherein the recycling lean oil is saturated with the methane of the residue gas, and deep-cooled in the lean oil pre-saturator with a refrigerant, entering via the refrigerant inlet pipe 25 and leaving via the refrigerant outlet pipe $25 a$. The deep-cooled residue gas and saturated lean oil are then separated in the pre-saturator. The separated residue 
gas is discharged via deethanizer residue discharge pipe $\mathbf{4 8}$ to merge with the other stream of residue gas, coming from the flexible absorber 28 via residue gas discharge pipe $\mathbf{4 6}$. The separated pre-saturated lean oil is divided into two streams. One stream, regulated by the first regulation valve $40 a$, is sent via the saturated lean oil pipe $\mathbf{4 0}$ into the flexible absorber $\mathbf{2 8}$ as the absorbent. The other stream is recycled via recycling lean oil pipe $\mathbf{5 2}$ into the pre-saturator to enhance the mixing process.

[0039] The next step is the separation of the desired NGL product from the purified rich oil. For this purpose, the purified rich oil is first flashed via the rich oil de-pressurization valve 64 into a vapor-liquid mixture. The vaporliquid mixture enters the rich oil fractionator via the flashed purified rich oil inlet pipe $\mathbf{6 5}$ at the junction between the lower and upper section, $\mathbf{6 6}$ and $\mathbf{6 6} a$, therein the liquid and the vapor are separated. The separated liquid purified rich oil flows downward as liquid film covering the wall of the lower first group of channels (shown as a thin-line slender rectangular block) of the lower fractionator section $\mathbf{6 6}$, while the separated NGL vapor flows upward along the upper first group of channels (shown as a thin-line slender rectangular block) of the upper fractionator section $66 a$.

[0040] In the lower fractionator section 66, the downflowing purified rich oil liquid film is heated by the upflowing recycling hot lean oil up-flowing inside the lower second group of channels, shown as bold dotted line, coming via the recycling hot lean oil discharge pipe $\mathbf{7 2}$ and leaving via the recycling lean oil outlet pipe $72 a$. The bottom temperature of the lower fractionator section is so controlled that the dissolved NGL components are essentially evaporated. The liquid is then purified as the regenerated lean oil. To further purify the regenerated lean oil to meet the reusable absorbent spec, the bottom liquid is discharged via lean oil discharge pipe $67 a$ into the re-boiler 67 . The regenerated lean oil is boiled therein with an external heat source (not shown, denoted with Q1), and the boiling liquid-vapor mixture is recycled into the fractionator via re-boiler recycling pipe $67 b$.

[0041] The purified regenerated lean oil re-enters the lower second group of channels via recycling hot lean oil discharge pipe $\mathbf{7 2}$ as described above. When leaving the fractionator via the recycling lean oil outlet pipe $72 a$, the recycling lean oil is still hot enough for heating the cold rich oil in the lower flexible deethanizer section $\mathbf{5 5}$. The recycling hot lean oil is first sent into an interim heater $\mathbf{7 3}$ to receive an extra amount of heat Q2 provided by an external heat source (not shown) to makeup the energy balance required in different operation conditions. Then the reheated hot lean oil, boosted with the lean oil transfer pump 74, is split into two streams. The bulk stream is sent via the lean oil inlet pipe 59 into the lower section of the flexible rich oil deethanizer $\mathbf{5 5}$ to heat the down-flowing cold rich oil. The cooled recycling lean oil leaves the lower deethanizer section via recycling lean oil outlet pipe $59 a$. The minor stream, regulated with the recycling lean oil regulation valve $\mathbf{3 3}$, is sent via the recycling lean oil pipe 32 into the flexible condensate deethanizer $\mathbf{3 0}$.

[0042] The cooled recycling lean oil flowing inside the recycling lean oil outlet pipe $\mathbf{5 9} a$ is split into two streams. The bulk stream is sent to the rich oil pre-demethanizer $\mathbf{4 5}$, via cold recycling lean oil inlet pipe 49 , to be further deep-cooled. The minor stream, regulated with the loop regulation valve $\mathbf{6 8}$, is sent via the loop inlet pipe $\mathbf{7 0}$ as the heat transport medium of the energy integration loop between the upper rich oil fractionator section $66 a$ and the lower rich oil deethanizer section $\mathbf{5 5}$.

[0043] In the upper fractionators section $66 a$, the temperature of the NGL vapor is still high, very close to the bottom temperature of the lower flexible deethanizer section $\mathbf{5 5}$. The NGL vapor needs to be cooled before compressed and liquefied as the final NGL product. Significant amount of heat energy in the hot NGL vapor could be utilized to heat the rich oil in the flexible deethanizer. For this purpose, an energy-integration loop is installed to transport the available heat energy from the hot NGL vapor to the flexible deethanizer. The energy-integrated loop comprises the following elements: the loop regulation valve 68, the loop booster pump 69, the loop inlet pipe 70, the upper second group of flow channels (shown as bold dotted line), and the loop outlet pipe 70a. A portion of the cold recycling lean oil is diverted from the recycling lean oil outlet pipe $\mathbf{5 9} a$ via the loop regulation valve $\mathbf{6 8}$, boosted with the loop booster pump 69, and flows via loop inlet pipe $\mathbf{7 0}$ into the upper second group of channels of the upper fractionator section $66 a$. The cold recycling lean oil cools the up-flowing hot NGL vapor in said upper fractionator section. The heated recycling lean oil then leaves via the loop outlet pipe $\mathbf{7 0} a$, merges with the other stream of hot recycling lean oil coming from recycling lean oil outlet pipe $\mathbf{7 2} a$, and enters the interim heater $\mathbf{7 3}$.

[0044] In summary, the recycling hot lean oil is used as the heating medium to provide heat to a number of energyintensive components, including: (1) the lower section of fractionators 66 ; (2) the lower section of flexible rich oil deethanizer 55 ; (3) the rich oil pre-demethanizer 45 ; (4) the condensate pre-demethanizer $\mathbf{3 5}$; (5) the flexible condensate deethanizer 30. This is another important measure of the full energy integration of the present invention.

[0045] The final step is to liquefy the NGL vapor into the liquid NGL product.

[0046] The cooled NGL vapor, left the upper fractionator section $66 a$ via the NGL vapor outlet pipe 75 , enters the NGL vapor pre-cooler $\mathbf{7 6}$ and is further cooled with cooling water (or ambient air), coming via water inlet pipe $\mathbf{7 7}$ and leaving via water outlet pipe $77 a$. The lean oil vapor condensate, if any, returns via reflux pipe $75 a$ into the upper fractionator section $66 \mathrm{a}$. The water-cooled NGL vapor, compressed with the NGL vapor compressor 78 to a higher pressure, is then sent to a T-joint mixer 79 to mix with the deep-cooled NGL condensate, coming from the deep-cooled condensate pipe 36 via the condensate de-pressurization valve 37. The mixing of the compressed NGL vapor with the deep-cooled condensate would greatly decrease the liquefaction pressure. The NGL vapor-condensate mixture is sent into the NGL liquefier 38 via NGL mixture pipe 80. The mixture is cooled therein with the cooling water (or ambient air), entering via water inlet pipe 81 $a$ and leaving via water outlet pipe 81. The liquefied final NGL product, boosted with the booster pump $\mathbf{8 2}$, is delivered as final NGL product via NGL product delivery pipe $\mathbf{8 3}$.

[0047] The mixing of the deep-cooled NGL condensate with the compressed NGL vapor to reduce the compressor power is another important measure of the full energy integration of the present invention.

[0048] To make the FIRA process more efficient and economic in the embodiment as illustrated in FIG. 1, high performance integrated exchanger-reactors are used in the 
following critical components, including: the flexible condensate deethanizer 30, the flexible rich oil deethanizer $\mathbf{5 5} / \mathbf{5 5} a$, and the rich oil fractionator $66 / 66 a$. These integrated exchanger-reactors could simultaneously perform the required mass- and heat-transfer functions in a single compact component with higher thermo-dynamic and masstransfer efficiency. As compared with the traditional discrete configuration comprising of a tower coupling with several side-reboilers, the integrated configuration has a number of additional merits, such as significant compactness, skidmounted capability, simplified field installation, and lower costs. It should also be recognized, however, that the discrete configuration comprising of a tower coupling with several side-reboilers is equally applicable in the FIRA process with excellent flexibility and significant energy savings as compared with both the traditional ROA process and the Cryogenic process.

[0049] Furthermore, the exceptional flexibility of the FIRA process of this invention could also give much freedom of selecting and combining a few steps of the typical FIRA process to meet particular production objectives and/ or to retrofit exiting gas processing plants for enhancing their performance. For example, the following combinations of selected steps of the process could provide an excellent simplified process for an independent gas processing facility:

[0050] (1) An independent refrigeration dehydration process comprising the following FIRA steps: (a) pre-dehydrating the inlet raw gas, and (b) separating the partial NGL condensate, if any. Such a dehydration facility could produce valuable NGL by-product in addition to the advantage of the elimination of BTEX emission from the traditional glycol dehydrators.

[0051] (2) An independent flexible straight refrigeration separation process with high efficiency and low operation costs comprising the following FIRA steps: (a) pre-cooling and pre-dehydrating the raw gas with the recycling deepcooled residue gas and the recycling deep-cooled NGL product; (b) deep-cooling the gas with an refrigerant to get the deep-dehydrated gas and deep-cooled NGL condensate; (c) removing all the methane content from the deep-cooled NGL condensate and simultaneously reduce the ethane contend therein to any desirable level to get the deep-cooled NGL product.

[0052] FIG. 2 illustrates examples of the high-performance dual-function exchanger-reactors used in FIG. 1 for carrying out heat- and mass-transfer simultaneously in critical components.

[0053] One of the outstanding merits of the present invention is the use of the high-efficiency, compact dual-function exchanger-reactor for simultaneous heat- and mass-transfer in critical components, such as the flexible NGL condensate deethanizer $\mathbf{3 0}$, the flexible rich oil deethanizer $\mathbf{5 5} / \mathbf{5 5} a$, and the fully energy-integrated purified rich oil fractionator 66/66a.

[0054] Although the exchanger-reactors have already been widely used in other chemical industries for years, the application to gas processing industry is still very limited. Specific requirements for gas processing present new challenge to current exchanger-reactor design.

[0055] The exchanger-reactors used in present invention could be classified roughly into two categories: the flexible deethanizer wherein the upper section is a stripper and the lower section an evaporator with intensive heat duty; and the fractionator wherein the upper section is a heat exchanger and the lower section a distiller with an external heat source. The key issue in such exchanger-reactor design is the generation and maintenance of a continuous, thin liquid film covering enormous surface of tens of thousands narrow flow channels within a compact exchanger-reactor. The innovative solution follows.

[0056] FIG. 2A illustrates one preferred embodiment of the plate-fin type exchanger-reactor applicable to the flexible rich oil deethanizer. Section A-A illustrates the general layout of various groups of flow channels therein. Section B-B illustrates the detailed internal configuration of these groups of flow channels.

[0057] The configuration of the flexible deethanizer is similar to an upright multi-stream plate-fin heat exchanger, comprising of an upper section $\mathbf{5 5} a$, and a lower section $\mathbf{5 5}$. As shown in Section B-B, there are three groups of narrow flowing channels, i.e. the main group of channels $\mathbf{5 5 2}$, extending from the top to the bottom of entire deethanizer, the upper group of channels 551, inside the upper section of the deethanizer, and the lower group of channels 553, inside the lower section of the deethanizer. The different groups of channels are separated with parting sheets and spacing bars as the conventional plate-fin heat exchanger.

[0058] The depressurized pre-demethanized rich oil, a mixture of NGL vapor and liquid rich oil, enters the bottom of the upper group of channels 551 of the rich oil deethanizer, via rich oil mixture inlet pipe 58, at the junction between the lower and upper deethanizer sections, $\mathbf{5 5}$ and 55 $a$. The vapor and liquid are separated therein with a special perforated fin separator $\mathbf{5 8 1}$. The separated rich oil liquid flows downward and passes through numerous small horizontal pinholes $\mathbf{5 8 2}$ on the parting sheets between the upper group of channels 551 and the main group of channels 552. The liquid then flows downward as thin liquid film covering the entire surface of the lower part of the main group of channels.

[0059] In the lower section of the deethanizer, the downflowing liquid film is heated with the hot recycling lean oil flowing up in the lower group of channels 553, coming via the recycling lean oil inlet pipe $\mathbf{5 9}$ and leaving via the recycling lean oil outlet pipe $\mathbf{5 9} a$. The residue gas, evaporated from the heated rich oil film, flows upward in the main group of channels $\mathbf{5 5 2}$ and, at the same time, is continuously stripped with the colder down-flowing liquid film. Trace of the heaviest hydrocarbon vapor is re-absorbed with the liquid film and carried down to the bottom.

[0060] In the upper section of the deethanizer, a stream of deepcooled stripping lean oil is introduced via stripping lean oil inlet pipe 54 and distributed with the distribution section 541 into both group of channels $\mathbf{5 5 1}$ and $\mathbf{5 5 2}$. The stripping lean oil flows downward as thin liquid film covering the entire surface of both group of channels $\mathbf{5 5 1}$ and $\mathbf{5 5 2}$. The residue gas, flowing up in both group of channels $\mathbf{5 5 1}$ and 552, is stripped with the down flowing lean oil film to remove the heaviest hydrocarbons components as well as the desired ethane content therein. Numerous horizontal pinholes 583 are provided on the parting sheets of the upper section of the deethanizer to equalize the vapor and liquid flowing inside both group of channels 551 and 552. At the bottom of the upper section of the deethanizer, the portion of the stripping liquid film flowing inside flow channel group 551 passes through the horizontal pinholes $\mathbf{5 8 2}$ and merges into the group channels $\mathbf{5 5 2}$. On the other hand, the residue 
gas, flowing to the top of the deethanizer, penetrates the falling liquid film via numerous pores of the special gasliquid separation section $\mathbf{6 0 1}$ and leaves the deethanizer via the residue gas outlet pipe $\mathbf{6 0}$.

[0061] By adjusting the molar ratio of the stripping lean oil vs. residue gas, as well as the bottom temperature of the deethanizer, the ethane recovery rate could be controlled to any desirable value between $>95 \%$ and $<2 \%$. The purified rich oil product with the desired ethane content is eventually discharged via the distribution section $\mathbf{6 3 1}$ and the purified rich oil outlet pipe 63 .

[0062] FIG. 2B illustrates one preferred embodiment of the plate-fin type exchanger-reactor applicable to the flexible rich oil fractionator. Section A-A illustrates the general layout of various groups of flow channels therein. Section $\mathrm{B}-\mathrm{B}$ illustrates the detailed internal configuration of these groups of flow channels.

[0063] The configuration of the flexible fractionator is similar to an upright multi-stream plate-fin heat exchanger, comprising of an upper section $\mathbf{6 6} a$ and a lower section $\mathbf{6 6}$. As shown in Section B-B, there are four groups of narrow flowing channels, i.e. the main group of channels $\mathbf{6 6 2}$, extending from the top to the bottom of entire fractionator; the upper group of channels 664, inside the upper section of the fractionator; the lower group of channels $\mathbf{6 6 3}$, inside the lower section of the fractionator, and the middle group of channels 661, between the upper and the lower group of channels. The different groups of channels are separated with parting sheets and spacing bars as the conventional plate-fin heat exchanger.

[0064] The depressurized purified rich oil, a mixture of NGL vapor and liquid rich oil, enters the middle group of channels 661 of the rich oil fractionator, via purified rich oil mixture inlet pipe $\mathbf{6 5}$, at the junction between the lower and upper sections, 66 and $\mathbf{6 6} a$. The vapor and liquid are separated therein with a special perforated fin separator $\mathbf{6 5 1}$. The separated liquid flows downward and passes through numerous small horizontal pinholes $\mathbf{6 5 2}$ on the parting sheets between the middle group of channels 661 and the main group of channels $\mathbf{6 6 2}$. The liquid then flows downward as thin liquid film covering the entire surface of the lower part of the main group of channels.

[0065] In the lower section of the fractionator, the downflowing liquid film is heated with the hot recycling lean oil flowing up in the lower group of channels $\mathbf{6 6 3}$, coming via the recycling lean oil inlet pipe $\mathbf{7 2}$ and leaving via the recycling lean oil outlet pipe $\mathbf{7 2} a$. The residue gas, evaporated from the heated rich oil film, mixed with the recycling NGL-lean oil vapor coming from the re-boiler recycling pipe $67 b$ through the lower special perforated fin separator 671 , flows upward in the main group of channels 662 and, at the same time, is continuously stripped with the colder down-flowing liquid film. Trace of the heaviest hydrocarbon vapor is re-absorbed with the liquid film and carried down to the bottom.

[0066] On the other hand, the NGL vapor of the inlet NGL mixture, separated in the special perforated fin separator 651, passes horizontally through numerous small pinholes 653 on the parting sheets into the main group of channels 662 and merges with the NGL vapor flowing up from the lower part of the same group of channels. Since the mixed NGL vapor is still rather hot, it is cooled with the downflowing cold lean oil in the upper group of channels $\mathbf{6 6 4}$, coming via loop inlet pipe $\mathbf{7 0}$ and leaving via loop outlet pipe 70 $\mathrm{a}$. The tiny amount of lean oil vapor condensed on the surfaces of the group channels $\mathbf{6 6 2}$ flows down to the junction between the upper and lower sections of the fractionator and merges with the input liquid purified rich oil. [0067] The cooled NGL vapor, on the other hand, flows to the top, passes through the upper special perforated fin separator 751, and leaves the fractionator via NGL vapor outlet pipe 75. The lean oil liquid condensed in external water cooler $\mathbf{7 6}$ (not shown here, ref. FIG. 1) returns as a reflux into the fractionator via the reflux pipe $75 a$. The reflux is distributed via the distribution section $\mathbf{7 5 2}$ into the main group of channels $\mathbf{6 6 2}$. The in-flow reflux is separated from the out-flow NGL vapor in the upper special perforated fin separator $\mathbf{7 5 1}$.

[0068] At the bottom of the fractionator, the very hot regenerated lean oil is eventually discharged via the lower special perforated fin separator 671 and the lean oil discharge pipe $67 a$ to the external re-boiler 67 (not shown here, ref. FIG. 1) for final purification.

[0069] It will be recognized that, based on similar principles as described in this section, other designs of exchanger-reactor could also perform simultaneous heatand mass-transfer functions in FIRA process as well.

[0070] FIG. 3 illustrates the embodiments of the highperformance cascade flexible deethanizer that could replace the combination of a pre-demethanizer and a flexible deethanizer in tandem as illustrated in FIG. 1 for purifying NGL condensate and rich oil.

[0071] The innovative application of exchanger-reactor in present invention makes possible to develop the more efficient and energy-saving cascade flexible deethanizers for purifying both NGL condensate and rich oil as illustrated in FIG. 3.

[0072] FIGS. 3-C through 3-E illustrate the evolution and the principle of the cascade flexible deethanizers for NGL condensate purification.

[0073] FIG. 3-C is the original flow diagram comprising of the combination of a condensate pre-demethanizer and a flexible condensate deethanizer in tandem as illustrated in FIG. 1.

[0074] The deep-cooled NGL condensate, under the high pressure of the flexible absorber $\mathbf{2 8}$ (not shown here, ref. FIG. 1), enters the pre-demethanizer 35 via the NGL condensate outlet pipe 29 . The high pressure deep-cooled condensate is heated with the recycling hot lean oil, coming via the lean oil inlet pipe $32 c$ and leaving via the lean oil outlet pipe $32 d$. A substantial portion of the condensed methane, together with a small portion of ethane and trace of higher hydrocarbons, is evaporated as the residue gas and sent back to the absorber via the residue gas outlet pipe $39 a$. The pre-methanized condensate is then de-pressurized with the condensate de-pressurization valve $\mathbf{5 7} a$ to a lower pressure and further purified in the flexible condensate deethanizer 30.

[0075] In the flexible condensate deethanizer 30, the depressurized condensate flows inside the first group of channels (shown as a thin-lined rectangular block). The condensate is first deep-cooled with the refrigerant flowing inside the second group of channels, shown as a bold dotted line, coming via the refrigerant inlet pipe 23 and leaving via the refrigerant outlet pipe $\mathbf{2 3} a$. Then the condensate is preheated with the recycling hot purified condensate flowing inside the third group of channels, shown as a bold dotted line, coming via the recycling condensate inlet pipe $\mathbf{3 4}$ and 
leaving via the deep-cooled NGL condensate pipe $\mathbf{3 6}$, and eventually heated with the recycling hot lean oil flowing inside the fourth group of channels, shown as a bold dotted line, coming via lean oil inlet pipe $\mathbf{3 2} a$ and leaving via lean oil outlet pipe $32 b$. The bottom temperature of the deethanizer is controlled according to the desired level of ethane content. The final purified condensate is discharged from the bottom via recycling condensate inlet pipe 34 and returned to the deethanizer to pre-heat the cold condensate as described above. The recycling condensate is deep-cooled and eventually discharged via deep-cooled NGL condensate pipe 36. The residue gas is sent via residue gas outlet pipe 39 to the rich oil deethanizer 55 (not shown here, ref. FIG. 1).

[0076] FIG. 3-D illustrates the new cascade flexible condensate deethanizing process comprising two similar deethanizer stages in tandem to replace the original embodiment illustrated in FIG. 3-C. The high-pressure deep-cooled NGL condensate is directly sent into the first stage of deethanizer 30A. The configuration of the first stage of deethanizer is similar to the original deethanizer 30 illustrated in FIG. 3-C, but operating under the same high pressure as the original pre-demethanizer $\mathbf{3 5}$.

[0077] In the first stage of new cascade flexible rich oil deethanizer $30 \mathrm{~A}$, the high pressure rich oil enters via the condensate inlet pipe 29 and flows inside the first group of channels (shown as a thin-lined slender rectangular block). The high-pressure condensate is first deep-cooled with the refrigerant flowing inside the second group of channels, shown as a bold dotted line, coming via the refrigerant inlet pipe 23 and leaving via the refrigerant outlet pipe $23 a$. Then the condensate is pre-heated with the recycling hot semipurified condensate flowing inside the third group of channels, shown as a bold dotted line, coming via the recycling semi-purified condensate inlet pipe $\mathbf{3 4}$ and leaving via the deep-cooled semi-purified condensate transfer pipe 129; and eventually the condensate is heated with the recycling hot lean oil flowing inside the fourth group of channels, shown as a bold dotted line, coming via lean oil inlet pipe $32 a$ and leaving via lean oil outlet pipe $\mathbf{3 2} b$. The bottom temperature of the first stage deethanizer is controlled according to the desired level of ethane content. The semi-purified condensate is discharged from the bottom via recycling condensate inlet pipe 34 and returned to the first stage deethanizer to pre-heat the cold condensate as described above. The recycling semi-purified condensate is deep-cooled and eventually flows via the de-pressurization valve 157 and the semi-purified condensate transfer pipe $\mathbf{1 2 9}$ into the second stage deethanizer $30 \mathrm{~B}$. The residue gas is sent out via the residue gas outlet pipe $39 a$ to the absorber 28 (not shown here, ref FIG. 1).

[0078] The working pressure and the bottom temperature of the second stage deethanizer $30 \mathrm{~B}$ depend on the partial deethanization level required. Should very high ethane recovery rate be required (i.e., no ethane-rejection), the high-pressure semi-purified condensate would be directly sent into the second stage deethanizer, without de-pressurization, for complete demethanization. Otherwise adequate depressurization is required: the pressure depends on the desirable level of the partial deethanization. The de-pressurized condensate, flashed into a vapor-liquid mixture, is sent into the second stage deethanizer and is separated therein. [0079] In the second stage deethanizer 30B, the liquid portion of the mixture flows inside the first group of channels (shown as a thin-lined slender rectangular block). The semi-purified condensate is first deep-cooled with the refrigerant flowing inside the second group of channels, shown as a bold dotted line, coming via the refrigerant inlet pipe 123 and leaving via the refrigerant outlet pipe $123 a$. Then the semi-purified condensate is pre-heated with the recycling hot purified condensate flowing inside the third group of channels, shown as a bold dotted line, coming via the recycling purified condensate inlet pipe 134 and leaving via the deep-cooled purified condensate pipe 136; and eventually the semi-purified condensate is heated with the recycling hot lean oil flowing inside the fourth group of channels, shown as a bold dotted line, coming via lean oil inlet pipe $132 a$ and leaving via lean oil outlet pipe 132b. The semi-purified condensate, flowing to the bottom, is processed into the purified condensate discharged via recycling purified condensate inlet pipe $\mathbf{1 3 4}$ and returns to the second stage deethanizer to pre-heat the cold condensate as described above. The recycling purified condensate is eventually deep-cooled and discharged via deep-cooled purified condensate pipe 136. The residue gas is sent via residue gas outlet pipe 39.

[0080] FIG. 3-E illustrates the integrated cascade flexible condensate deethanizer for NGL condensate purification.

[0081] To simplify the system and make the new embodiment more compact, the two stages of cascade condensate deethanizer could be constructed as a single integrated exchanger-reactor incorporating both $30 \mathrm{~A}$ and $30 \mathrm{~B}$ as illustrated in FIG. 3-E. Since all the numbers of relevant elements and the flow procedures in FIG. 3-E are identical to those described in FIG. 3-D, no redundant explanation is needed.

[0082] FIGS. 3-F through 3-H illustrate the evolution and the principle of the flexible cascade flexible deethanizer for rich oil purification.

[0083] FIG. 3-F is the original flow diagram comprising of the combination of a rich oil pre-demethanizer and a flexible rich oil deethanizer in tandem as illustrated in FIG. 1.

[0084] The deep-cooled rich oil, under the high pressure of the flexible absorber, 28 (not shown here, ref. FIG. 1), enters the pre-demethanizer 45 via the rich oil inlet pipe 44 . The high pressure deep-cooled rich oil is heated with the recycling lean oil coming via cold recycling lean oil pipe 49 and leaving via deep-cooled recycling lean oil pipe $49 a$. A substantial portion of the absorbed methane, together with a small portion of ethane and trace of higher hydrocarbons, is evaporated from the deep-cooled rich oil as the residue gas and sent back to the absorber via the residue gas transfer pipe 50. The pre-demethanized rich oil is then flashed with the condensate de-pressurization valve $\mathbf{5 7}$ into a rich oil liquid-vapor mixture that flows via rich oil mixture inlet pipe $\mathbf{5 8}$ into the flexible rich oil deethanizer at the junction of the upper and lower section of the deethanizer, $\mathbf{5 5} / \mathbf{5 5} a$.

[0085] The rich oil liquid-vapor is separated inside the flexible rich oil deethanizer. In the lower section of the deethanizer $\mathbf{5 5}$, the liquid rich oil flows downward as liquid film covering the channel wall of the first group of channels (shown as a thin-line slender rectangular block). The liquid rich oil is heated with the up-flowing recycling hot lean oil flowing inside the second group of channels, shown as a bold dotted line, coming via recycling lean oil inlet pipe 59 and leaving via recycling lean oil outlet pipe 59a. All the dissolved methane and a desirable portion of ethane are evaporated as the residue gas. The purified rich oil, containing adequate ethane content and all other heavier hydrocarbons, is discharged from the bottom via purified rich oil outlet pipe 63. The residue gas, on the other hand, flowing upward into the upper section, $\mathbf{5 5} a$, is stripped with the deep-cooled lean oil coming via the stripping lean oil inlet pipe 54 and sent out via residue gas outlet pipe 60 . 
[0086] FIG. 3-G illustrates the new cascade flexible rich oil deethanizing process comprising two similar deethanizer stages in tandem to replace the original embodiment as illustrated in FIG. 3-F. The high-pressure deep-cooled rich oil is directly sent into the first stage of rich oil deethanizer, $55 \mathrm{~A}$. The configuration of the first stage of rich oil deethanizer is similar to the lower section of the original rich oil deethanizer $\mathbf{5 5}$ illustrated in FIG. 3-F, but operating under the same high pressure as the original rich oil pre-demethanizer 45.

[0087] In the first stage of rich oil deethanizer 55A, the high-pressure rich oil enters via the rich oil inlet pipe 144, flowing as liquid film covering the channel wall of the first group of channels (shown as a thin-lined slender rectangular block). The rich oil is first pre-heated with the hot recycling semi-purified rich oil flowing inside the second group of channels, shown as a bold dotted line, coming via the recycling semi-purified rich oil inlet pipe 163 and leaving via the deep-cooled semi-purified condensate transfer pipe $\mathbf{5 8}$, and then heated with the recycling hot lean oil flowing inside the third group of channels, shown as a bold dotted line, coming via lean oil inlet pipe $132 a$ and leaving via lean oil outlet pipe $132 b$. The bottom temperature of the first stage rich oil deethanizer is controlled according to the desired level of ethane content. The final semi-purified rich oil is discharged from the bottom via recycling semi-purified rich oil pipe 163 , and returns to the first stage rich oil deethanizer to pre-heat the cold rich oil as described above. The recycling semi-purified rich oil is deep-cooled therein and then flows via the semi-purified rich oil de-pressurization valve $\mathbf{5 7}$ and the semi-purified rich oil transfer pipe $\mathbf{5 8}$ into the second stage rich oil deethanizer 55B. The residue gas is discharged via residue gas transfer pipe $\mathbf{5 0}$.

[0088] The working pressure and its bottom temperature of the second stage rich oil deethanizer $55 \mathrm{~B}$ depend on the desired deethanization level. Should very high ethane recov- ery rate be required (i.e., no ethane-rejection), the highpressure semi-purified condensate would be sent directly into the second stage rich oil deethanizer, without depressurization, for complete demethanization. Otherwise adequate depressurization is required: the pressure depends on the desired ethane level of the purified rich oil. The de-pressurized condensate, flashed into a vapor-liquid mixture, is sent into the second stage rich oil deethanizer and is separated therein.

[0089] The liquid portion of the mixture flows as liquid film covering the channel wall of the first group of channels (shown as a thin-lined slender rectangular block). Then the semi-purified rich oil is heated with the recycling hot lean oil flowing inside the second group of channels, shown as a bold dotted line, coming via lean oil inlet pipe $\mathbf{5 9}$ and leaving via lean oil outlet pipe $\mathbf{5 9} a$. The purified rich oil is discharged via purified rich oil outlet pipe 63 .

[0090] On the other hand, all the methane and a desirable portion of ethane, evaporated as the residue gas, flows into the upper section of the second stage deethanizer, and contacts with the stripping lean oil coming via the stripping lean oil inlet pipe 54. The stripped residue gas is discharged via the residue gas outlet pipe $\mathbf{6 0}$.

[0091] FIG. 3-H illustrates the integrated cascade flexible condensate deethanizer for rich oil purification.

[0092] To simplify the system and make the new embodiment more compact, the two stages of cascade condensate deethanizer could be constructed as a single integrated exchanger-reactor incorporating both $55 \mathrm{~A}$ and $55 \mathrm{~B}$ as illustrated in FIG. 3-H. Since all the numbers of relevant elements and the flow procedures in FIG. 3-H are identical to those described in FIG. 3-G, no redundant explanation is needed.

[0093] The advantages of the high performance cascade flexible deethanizer described above are best demonstrated with the comparison of the simulation results of the new configuration FIG. 3-G and the original one (FIG. 3-F).

TABLE 1

\begin{tabular}{|c|c|c|c|c|c|c|c|}
\hline \multirow[b]{2}{*}{ Stream No. } & \multicolumn{6}{|c|}{$\begin{array}{l}\text { Comparison of Flexible Rich Oil Deethanizer Simulation Results } \\
\text { (Unit: } \mathrm{lb}-\mathrm{mol} / \mathrm{hr} \text { for } 100 \mathrm{MMscfd} \text { input) } \\
\end{array}$} & \multirow[b]{2}{*}{$\mathrm{C} 10$} \\
\hline & P, psia & $\mathrm{T},{ }^{\circ} \mathrm{F}$ & $\mathrm{C} 1$ & $\mathrm{C} 2$ & $\mathrm{C} 3$ & $\mathrm{C} 4+$ & \\
\hline \multicolumn{8}{|c|}{ Original Case (FIG. 3-F): No Ethane-rejection, $95 \% \mathrm{C} 2$ Recovery } \\
\hline 44 (Inlet) & 890 & -40 & 3118.51 & 1336.47 & 550.21 & 437.72 & 1735.68 \\
\hline 50 & 890 & 40 & 1788.37 & 324.55 & 55.59 & 16.66 & 0.29 \\
\hline 58 & 450 & 32 & 1330.14 & 1011.56 & 494.56 & 421.05 & 1735.39 \\
\hline 54 & 450 & -40 & 0 & 0 & 0.06 & 2.09 & 1097.85 \\
\hline 60 & 45 & -29.6 & 1319.14 & 2.91 & 0 & 0 & 0 \\
\hline 63 (Outlet) & 450 & 261.7 & 11.00 & 1008.65 & 494.62 & 423.14 & 2833.24 \\
\hline \multicolumn{8}{|c|}{ Cascade Case (FIG. 3-G): No Ethane-rejection, 95\% C2 Recovery } \\
\hline 144 (Inlet) & 890 & -40 & 3118.51 & 1336.47 & 550.21 & 437.72 & 1735.68 \\
\hline 50 & 890 & -40 & 3021.50 & 224.97 & 25.73 & 6.73 & 0.04 \\
\hline 163 & 890 & 344.6 & 96.99 & 1111.50 & 524.48 & 430.99 & 1735.64 \\
\hline 58 & 890 & -40 & 96.99 & 1111.50 & 524.48 & 430.99 & 1735.64 \\
\hline 54 & 89 & -40 & 0 & 0 & 0 & 0 & 0 \\
\hline 60 & & -12 & 88.59 & 9.90 & 1.19 & 0.32 & 0.01 \\
\hline 63 (Outlet) & 890 & 393 & 8.04 & 1101.60 & 523.29 & 430.66 & 1735.63 \\
\hline \multicolumn{8}{|c|}{ Original Case (FIG. 3-F): Max. Ethane-rejection, 2\% C2 Recovery } \\
\hline 44 (Inlet) & & -40 & 2328.74 & 904.16 & 581.74 & 454.05 & 301.23 \\
\hline 50 & 890 & 40 & 1585.70 & 326.48 & 102.71 & 34.50 & 0.17 \\
\hline 58 & 450 & 32 & 743.04 & 577.68 & 479.03 & 419.55 & 307.06 \\
\hline 54 & 450 & -40 & 0 & 0 & 0.02 & 3.19 & 346.79 \\
\hline 60 & 450 & 38.4 & 743.52 & 555.96 & 9.49 & 0.55 & 0.08 \\
\hline 63 (Outlet) & 450 & 315.8 & Trace & 22.00 & 456.71 & 422.07 & 647.76 \\
\hline
\end{tabular}


TABLE 1-continued

\begin{tabular}{|c|c|c|c|c|c|c|c|}
\hline \multirow[b]{2}{*}{ Stream No. } & \multicolumn{6}{|c|}{$\begin{array}{l}\text { Comparison of Flexible Rich Oil Deethanizer Simulation Results } \\
\text { (Unit: } 1 \mathrm{~b}-\mathrm{mol} / \mathrm{hr} \text { for } 100 \mathrm{MMscfd} \text { input) } \\
\end{array}$} & \multirow[b]{2}{*}{$\mathrm{C} 10$} \\
\hline & P, psia & $\mathrm{T},{ }^{\circ} \mathrm{F}$ & $\mathrm{C} 1$ & $\mathrm{C} 2$ & $\mathrm{C} 3$ & $\mathrm{C} 4+$ & \\
\hline \multicolumn{7}{|c|}{ Cascade Case (FIG. 3-G): Max. Ethane-rejection, 2\% C2 Recovery } & \\
\hline 144(Inlet) & 890 & -40 & 2328.74 & 904.16 & 581.74 & 454.05 & 301.23 \\
\hline 50 & 890 & 40 & 2304.71 & 186.47 & 38.48 & 0.39 & 0.02 \\
\hline 163 & 890 & 32 & 24.03 & 717.69 & 543.26 & 443.67 & 301.21 \\
\hline 58 & 700 & -40 & 24.03 & 717.69 & 543.26 & 443.67 & 301.21 \\
\hline 54 & 700 & -40 & 0 & 0 & 0.02 & 3.15 & 250 \\
\hline 60 & 700 & 121.7 & 24.03 & 696.43 & 10.55 & 0.87 & 1.28 \\
\hline 63 (Outlet) & 700 & 400.1 & Trace & 21.26 & 532.73 & 445.95 & 549.93 \\
\hline
\end{tabular}

[0094] It is obvious in Table 1 that the advantages of the new cascade flexible deethanizer are: (1) for high ethane recovery, no ethane-rejection ( $95 \% \mathrm{C} 2$ recovery), complete demethanization could be carried out in new cascade process under higher pressures and the residue gas need no recompression; (2) for high ethane recovery, no ethane-rejection ( $95 \%$ C2 recovery), complete demethanization could be carried out in new cascade process without stripping lean oil; (3) maximum ethane-rejection process (2\% C2 recovery) could be carried out with lesser residue gas under higher pressure and as compared with the original process, and, hence, with much less residue gas recompression power; and (4) maximum ethane-rejection process ( $2 \% \mathrm{C} 2$ recovery) could be carried out with much less stripping lean oil. In short, the new process provides significant operational flexibility and energy-savings.

[0095] FIG. 4 illustrates another embodiment of the flexible hydrocarbon gas separation process as illustrated in FIG. 1 wherein the inlet gas has already been pre-dehydrated to pipeline gas spec.

[0096] In case the input gas had been already dehydrated elsewhere before entering the FIRA plant, a simpler embodiment of the present invention would be preferred. Since the water content of the input gas is much lower in the present case, the dehydration section in FIG. 4 could be much simpler than the basis flow diagram as illustrated in FIG. 1. The pre-treatment and the pre-dehydration section of FIG. 1 could be eliminated, including: the primary heat exchanger $\mathbf{2}$, the moisture deparator $\mathbf{3}$, the pre-dehydrator $\mathbf{5}$, and the three-phase separator 7 . The pipeline spec gas could be introduced directly to the deep-dehydrator 9 as shown in FIG. 4.

[0097] The internal structure of the deep dehydrator 9 of FIG. 4, however, would be a little complicated. The deepcooled residue gas would be sent directly into the deepdehydrator as the cooling medium, together with the deepcooled rich oil and the refrigerant from the external refrigerator. A multi-stream exchanger-reactor as illustrated in FIG. 2 should be used. The configuration given by the inventor in U.S. Pat. No. 6,694,786, "Non-frost Deepfreezing Gas Dehydrator", could serve as an example. The concentrate inhibitor solution introduced via the inhibitor make-up pipe $\mathbf{2 0}$ would generate an inhibitor liquid film to prevent the formation of gas hydrate.

[0098] The high concentrated inhibitor solution, diluted with the water condensed, flows into the three-phase separator 26, together with the immiscible NGL condensate and the deep-dehydrated hydrocarbon gas. The three immiscible fluids are separated therein. The separated diluted inhibitor solution is then discharged via inhibitor solution discharge pipe to the external inhibitor regeneration facility not shown in the FIG. 4.

[0099] The rest elements of the flow diagram and all the operation procedures in FIG. 4 are identical to those in FIG. 1. No redundant description is needed.

[0100] FIG. 5 illustrates an alternative embodiment of the flexible hydrocarbon gas separation process as illustrated in FIG. 4 wherein the high-performance, integrated cascade flexible deethanizers are used.

[0101] The major difference between FIG. 5 and FIG. 4 is the replacement of the combination of a pre-demethanizer and a flexible deethanizer in tandem with the integrated cascade flexible deethanizer. As already described in FIG. 3, the flexibility of the ethane-rejection operations in FIRA process could be more efficiently carried out with the high-performance, integrated cascade flexible deethanizer. Since the details of the internal configuration of the integrated cascade flexible deethanizer have already been described in FIGS. 3-E and 3-H, only the external connections of the deethanizers with other components need to be described in FIG. 5.

[0102] First, the external connections of the integrated cascade flexible NGL condensate deethanizer, $30 \mathrm{~A} / \mathbf{3 0 B}$.

[0103] There are 12 external connections as described below.

[0104] (1) The NGL discharge pipe 29 connects with the three-phase separator 26 providing deep-cooled NGL condensate stream to the first stage of the integrated flexible cascade deethanizer, $30 \mathrm{~A}$, via and the shift valve $31 \mathrm{a}$. The purpose of the pair of shift valves $31 a$ and $\mathbf{3 1} b$ is to bypass the NGL condensate directly into the absorber 28 in case the quantity of the NGL condensate is too small, not worthy to be purified specially in the cascade deethanizer.

[0105] (2) The residue gas outlet pipe 39 connects with the cold dry gas outlet pipe 27.

[0106] (3) The residue gas outlet pipe 139 connects with the second stage of the integrated cascade flexible deethanizer, $55 \mathrm{~A}$, via the residue gas de-pressurization valve 257 .

[0107] (4) The deep-cooled NGL condensate pipe 136 connects with the NGL liquefier $\mathbf{3 8}$, via the condensate depressurization valve 37 .

[0108] (5) The four refrigerant inlet and outlet pipes, 23, $23 a, 123$, and $123 a$, connect with the external refrigerator not shown in FIG. 5.

[0109] (6) The four lean oil inlet and outlet pipes, 32a, $32 b, \mathbf{1 3 2} a$, and $132 b$ connect with the hot recycling lean oil pipe 32 and the cold recycling pipe 49 , respectively. 
[0110] (7) The lean oil regulation valve 133 is installed to adjust the lean oil flow rates in the two stages of the deethanizer.

[0111] Secondly, the external connections of the integrated cascade flexible rich oil deethanizer, $\mathbf{5 5} \mathrm{A} / \mathbf{5 5} \mathrm{B}$.

[0112] There are 9 external connections as described below.

[0113] (1) The rich oil inlet pipe 144 connecting with the flexible absorber 28 providing deep-cooled rich oil stream to the first stage of the integrated flexible cascade deethanizer, $55 \mathrm{~A}$. The residue gas outlet pipe 39 connects with the cold dry gas outlet pipe 27.

[0114] (2) The residue gas transfer pipe 50 connects with the cold recycling lean oil pipe 49.

[0115] (3) The residue gas outlet pipe 60 connects with the residue gas discharge pipe 46 via the residue gas compressor 56 and the fuel gas pipe 62 via the fuel gas de-pressurization valve 61 .

[0116] (4) The purified rich oil outlet pipe 63 connects with the rich oil fractionator 66 , via the rich oil depressurization valve 64

[0117] (5) The stripping lean oil inlet pipe $\mathbf{5 4}$ connects with the lean oil pre-saturator 47 , via the stripping lean oil regulation valve $\mathbf{5 3}$.

[0118] (6) The lean oil inlet and outlet pipes 59 and $59 a$ connect with the lean oil transfer pump 74 and the cold recycling pipe 49 , respectively.

[0119] (7) The lean oil inlet and outlet pipes 232 $a$ and $232 b$ connect with the hot recycling lean oil pipe 32 and the cold recycling pipe 49 , respectively. The lean oil flow rate is adjusted with the lean oil regulation valve 233 .

[0120] The rest elements of the flow diagram and all the operation procedures in FIG. 5 are identical to those in FIG. 4. No redundant description is needed.

[0121] FIG. 6 illustrates still another embodiment of the flexible hydrocarbon gas separation process as illustrated in FIG. 1 wherein the temperature of the pre-cooled gas is above the NGL dew point.

[0122] The NGL dew point would be lower than the temperature of the pre-cooled gas under either of the following circumstances:

[0123] (1) The richness of the higher hydrocarbons of the raw gas is so low that the NGL dew point of the inlet raw gas would be lower than the temperature of the pre-dehydrated gas; or

[0124] (2) The inhibitor used in the coolant in the predehydrator has high foaming tendency so that the temperature of the pre-dehydrated gas has to be controlled below the NGL dew point.

[0125] Under either of the above circumstance, no partial NGL condensate would appear in the pre-dehydrator. As a result, the following components should be eliminated in FIG. 6, including: the two-phase separator 7, the condensate outlet pipe 10, the booster pump 11, and the pair of shifting valves 31 and $31 a$. No other modification to FIG. 1 is required.

[0126] The rest elements of the flow diagram and all the operation procedures in FIG. 6 are identical to those in FIG. 1. No redundant description is needed.

[0127] FIG. 7 illustrates still another embodiment of the flexible hydrocarbon gas separation process as illustrated in FIG. 1, wherein a solid adsorbent deep-dehydrator is installed before the pre-dehydrated gas is deep-cooled with a refrigerant.

[0128] As illustrated in FIG. 7, a solid desiccant deepdehydrator 84 is installed between the original pre-dehydrator $\mathbf{5}$ and the original deep-dehydrators $\mathbf{9}$ as illustrated in
FIG. 1. Since the moisture of the pre-dehydrated gas is very low, the required capacity of the solid desiccant dehydrator would be rather small. In most circumstance, a once-through cycle operation is preferred. The solid desiccant, loaded into the solid dehydrator via the desiccant inlet pipe $\mathbf{8 6}$, is discarded via the desiccant discharge pipe 87 without regeneration.

[0129] A special interesting case is the use of the deliquescent solid desiccant such as anhydrous $\mathrm{CaCl}_{2}$. The effluent from the deliquescent solid dehydrator could be used as the make-up inhibitor solution returned to the system via inhibitor make-up pipe 20.

[0130] The deep-dehydrated gas, leaving the solid desiccant dehydrator 84 via the solid-dehydrated gas pipeline 85 at the pre-cooling gas temperature, is sent to the deep-cooler $9 a$ (replacing prior deep-cooler/dehydrator 9 in FIG. 1). No water vapor would be condensed in the deep-cooler $9 a$; only NGL condensate would appear. The prior three-phase separator 26 in FIG. 1 is replaced with a two-phase gas-liquid separator $26 b$, and the prior recovered inhibitor pipe $27 a$ in FIG. 1 is eliminated.

[0131] For simplification, in FIG. 7, the partial NGL condensate, if any, is directly sent into the deep-cooler $9 a$. The prior condensate booster pump 11 and the shift valve 31 in FIG. 1 are eliminated.

[0132] The rest elements of the flow diagram and all the operation procedures in FIG. 7 are identical to those in FIG. 1. No redundant description is needed.

[0133] FIG. 8 illustrates an alternative embodiment of the flow diagram of flexible hydrocarbon gas separation process as illustrated in FIG. 7 wherein the raw gas pressure is so high that an internal gas expander could be used to replace the external refrigerator providing the refrigerant for deepcooling the gas and lean oil.

[0134] As illustrated in FIG. 8, wherein the inlet raw gas pressure is substantially higher than the residue gas transport pipeline, an integrated expander-compressor, 80/90, could be used to provide the refrigeration for the gas and the lean oil in the FIRA process. External refrigerator is no longer required. Significant energy savings are resulted.

[0135] The embodiment illustrated in FIG. 8 is based on that in FIG. 7 wherein a solid desiccant deep-dehydrator is used. There are three major differences between FIG. 8 and FIG. 7.

[0136] First, in FIG. 8, the expander 88 of an integrated expander-compressor unit (shown partly in solid line and partly in dotted line) is used to expand the deep-dehydrated gas to a sub-low temperature and get the sub-cooled residue gas and the NGL condensate. Both the prior deep-cooler 9 and the prior external refrigerator (not shown in FIG. 7) are eliminated.

[0137] The pre-cooled, deep-dehydrated gas leaving the solid desiccant dehydrator $\mathbf{8 4}$ enters the expander $\mathbf{8 8}$ of the integrated expander-compressor unit, via deep-dehydrated gas inlet pipe 85. The gas temperature after expansion drops substantially lower than the absorber temperature, i.e., $<-40^{\circ} \mathrm{F}$. A significant portion of the NGL vapor is condensed. The subcooled gas and NGL condensate mixture is separated in the two-phase separator $26 b$ into two streams. The sub-cooled gas is sent via sub-low temperature gas inlet pipe 25 into the refrigerant cooler $47 a$ and the lean oil pre-saturator 47 , cools the refrigerant and lean oil mixture, respectively. The sub-cooled gas temperature, when leaving the pre-saturator 47 , should be $-40 \mathrm{~F}$. Then the deep-cooled gas leaves the pre-saturator via the deep-cooled gas inlet pipe $25 a$ and enters the flexible absorber 28 . The sub-cooled NGL condensate is sent via NGL condensate outlet pipe 29 
through the shift valve $31 a$ into the first stage of NGL condensate deethanizer 30A. The partial NGL condensate, separated from the two-phase separator 7 , if any, is sent via the partial condensate pipe 11 $a$ through the depressurization valve 357 into the NGL condensate outlet pipe 29.

[0138] Secondly, in FIG. 8, the compressor 90 of the integrated expander-compressor unit (shown partly in solid line and partly in dotted line) is used for NGL vapor compression. The prior NGL vapor compressor 79 in FIG. 7 is eliminated.

[0139] The pre-cooled NGL vapor enters the compressor 90 via the NGL vapor transfer pipe 91. The NGL vapor is compressed therein, discharged via NGL vapor discharge pipe 92, mixed with the deep-cooled NGL condensate coming through the depressurization valve 37 , and then sent into the NGL liquefier 38.

[0140] The surplus gas expansion power, if any, could be utilized for other compressor/pumps in the FIRA process. (Not shown in FIG. 8).

[0141] Thirdly, in FIG. 8, the integrated cascade flexible deethanizer is used for both NGL condensate and rich oil deethanization. The prior combination of a pre-demethanizer and a flexible deethanizer for both NGL condensate and rich oil in FIG. 7 has been eliminated. The connections of these integrated cascade deethanizers are identical to those in FIG. 5.

[0142] The rest elements of the flow diagram and all the operation procedures in FIG. 8 are identical to those in FIG. 7. No redundant description is needed

[0143] In summary, present invention related to a flexible hydrocarbon gas separation process that could dehydrate the water-saturated hydrocarbon gas mixture and recover thereof the required higher hydrocarbons (NGL) therein with a controllable ethane recovery rate (ranging from $>95 \%$ to $<2 \%$ ) while keeping high recovery rate of all other heavier components. The flexible process comprises the following steps: deep-cooling and dehydrating the raw gas and get the NGL condensate; flowing the deep-dehydrated gas into the flexible absorber to get the rich oil with desirable ethane content; completely demethanizing and partially deethanizing as desired the rich oil and the NGL condensate to get purified rich oil and purified NGL condensate, respectively; separating the NGL vapor from the purified rich oil; cooling and compressing the NGL vapor; mixing the NGL vapor with the purified NGL condensate; and liquefying the mixture to get the final NGL product. The present invention also provides a flexible apparatus with highly efficient components for the flexible process.

[0144] Having described the present invention and preferable embodiments thereof, it will be recognized that numerous variations, substitutions and additions may be made to the present invention by those ordinary skills without departing from the spirit and scope of the appended claims.

What is claimed is:

1. A flexible hydrocarbon gas separation process that could dehydrate the water-saturated hydrocarbon gas ("raw gas" hereafter) and recover thereof the higher hydrocarbon liquid ("NGL" hereafter) with controllable ethane recovery rate ranging from $>95 \%$ to $<2 \%$ while keeping high recovery rate of all other heavier hydrocarbon components comprises the following steps:

(a) Pre-cooling and pre-dehydrating the raw gas with directly contact a counter-flowing liquid coolant comprising of an aqueous solution of a gas hydrate inhibitor to get the pre-cooled pre-dehydrated gas and the partial NGL condensate; (b) Deep-cooling and deep-dehydrating the pre-cooled pre-dehydrated gas with the refrigerant from an external refrigerator to get the deep-cooled deep-dehydrated gas and the deep-cooled NGL condensate;

(c) Flowing the NGL condensate into a condensate predemethanizer and a flexible condensate deethanizer in tandem to remove all the methane content therein and simultaneously reduce the ethane contend therein to any desirable level to get the deep-cooled purified NGL condensate;

(d) Flowing the deep-cooled deep-dehydrated gas into a flexible absorber wherein contacting the gas with a counter-flowing liquid absorbent ("lean oil" hereafter) to get the cold residue gas and the cold rich absorbent ("rich oil" hereafter) with desirable level of ethane content;

(e) Flowing the cold rich oil into a rich oil pre-demethanizer and a flexible rich oil deethanizer in tandem to remove all the methane content therein and simultaneous reduce the ethane content therein to any desirable level to get the purified rich oil;

(f) Depressurizing the purified rich oil and distilling the purified rich oil in a rich oil fractionator to get the NGL vapor and the regenerated lean oil;

(g) Cooling the NGL vapor with a heat transport medium to transport the heat energy from the NGL vapor to the deep-cooled rich oil in the flexible rich oil deethanizer;

(h) Compressing the NGL vapor and mixing the compressed NGL vapor with the deep-cooled purified NGL condensate to get the low-temperature NGL vaporliquid mixture; and

(i) Liquefying the low-temperature NGL vapor-liquid mixture to get final NGL product.

2. A flexible hydrocarbon gas separation process of claim 1 wherein in step (a) the liquid coolant comprising of an aqueous solution of a gas hydrate inhibitor is pre-cooled with the cold residue gas and the cold rich oil before being used as the coolant in step (a).

3. A flexible hydrocarbon gas separation process of claim 1 wherein in step (b) the pre-cooled pre-dehydrated gas is mixed with the partial NGL condensate before being deepcooled with a refrigerant to get the deep-cooled deepdehydrated gas and the NGL condensate.

4. A flexible hydrocarbon gas separation process of claim 1 wherein in step (b) the pre-cooled pre-dehydrated gas is mixed with a spray of concentrated gas hydrate inhibitor solution before being deep-cooled with a refrigerant to get the deep-cooled deep-dehydrated gas and the NGL condensate.

5. A flexible hydrocarbon gas separation process of claim 1 wherein in step (b) the pre-cooled pre-dehydrated gas is further deep-dehydrated with a solid desiccant to get the deep-dehydrated gas before further deep-cooled with a refrigerant.

6. A flexible hydrocarbon gas separation process of claim 5 wherein the solid desiccant is calcium chloride or other deliquesce solid desiccants that would be liquefied when absorbing sufficient water, and the deliquescent liquid is then used as the concentrated hydrate inhibitor solution.

7. A flexible hydrocarbon gas separation process of claim 1 wherein in step (c) and (e) the working pressure and bottom temperature of the deethanizer could be controlled to precisely reduce the ethane content of the purified condensate to desirable level. 
8. A flexible hydrocarbon gas separation process of claim 1 wherein in step (c) and (e) the tandem process with a pre-demethanizer and a deethanizer is replaced with the integrated process of the integrated cascade flexible deethanizer.

9. A flexible hydrocarbon gas separation process of claim 1 wherein in step (d) the absorbent used in the absorber could be either heavy oils (i.e., hydrocarbon mixture with molecular weight higher than 100) or other organic compounds with hydrocarbon gas solubility higher than 20 scf/gal.

10. A flexible hydrocarbon gas separation process of claim 1 wherein in step (d) the ethane recovery rate in the flexible absorber could be precisely controlled by changing the lean oil flow rate.

11. A flexible hydrocarbon gas separation process of claim 1 wherein in step (e) the regenerated lean oil is recycled as a heating medium.

12. A flexible hydrocarbon gas separation process of claim 1 wherein in step $(\mathrm{g})$ the heat transport medium is the cooled recycling lean oil.

13. A flexible hydrocarbon gas separation process that could dehydrate the water-saturated raw hydrocarbon gas under high pressure and recover thereof the NGL with controllable ethane recovery rate ranging from $>95 \%$ to $<2 \%$ while keeping high recovery rate (over $90 \%$ ) of all other heavier hydrocarbon components comprises the following steps:

(a) Pre-cooling and pre-dehydrating the raw gas with directly contact a counter-flowing liquid coolant comprising of an aqueous solution of a gas hydrate inhibitor to get the pre-cooled pre-dehydrated gas and the partial NGL condensate;

(b) Deep-dehydrating the pre-cooled pre-dehydrated gas with a solid desiccant to get the pre-cooled deepdehydrated gas;

(c) Expanding the pre-cooled deep-dehydrated gas to the lower pressure and a low temperature to get the deepcooled deep-dehydrated gas and the NGL condensate;

(d) Flowing the NGL condensate and the partial NGL condensate into a condensate pre-demethanizer and a flexible condensate deethanizer in tandem to remove all the methane content therein and simultaneous reduce the ethane content therein to any desirable level to get the deep-cooled purified NGL condensate;

(e) Flowing the deep-cooled deep-dehydrated gas into a flexible absorber wherein contacting the gas with a counter-flowing liquid absorbent ("lean oil" hereafter) to get the cold residue gas and the cold rich oil with desirable level of ethane content;

(f) Flowing the cold rich oil into a rich oil pre-demethanizer and a flexible rich oil deethanizer in tandem to remove all the methane content therein and simultaneous reduce the ethane content therein to any desirable level to get the purified rich oil;

(g) Depressurizing the purified rich oil and distilling the purified rich oil in a rich oil fractionator to get the NGL vapor and the regenerated lean oil;

(h) Cooling the NGL vapor with a heat transport medium to transport the heat energy from the NGL vapor to the deep-cooled rich oil in the flexible rich oil deethanizer; (i) Compressing the NGL vapor and mixing the compressed NGL vapor with the deep-cooled purified NGL condensate to get the low-temperature NGL vaporliquid mixture; and

(j) Liquefying the low-temperature NGL vapor-liquid mixture to get final NGL product.

14. A flexible hydrocarbon gas separation apparatus that could dehydrate the water-saturated raw hydrocarbon gas and recover thereof the NGL with controllable ethane recovery rate ranging from $>95 \%$ to $<2 \%$ while keeping high recovery rate (over $90 \%$ ) of all other heavier hydrocarbon components comprises the following components:

(a) A pre-cooler/dehydrator wherein the water-saturated raw hydrocarbon gas contact an cold aqueous solution of a gas hydrate inhibitor to get the pre-dehydrated gas and the partial NGL condensate (if any);

(b) A deep-cooler/dehydrator wherein the pre-dehydrated gas is mixed with a spray of gas hydrate inhibitor solution and cooled with cold rich oil and a refrigerant from an external refrigerator to get the deep-cooled, deep-dehydrated gas and the NGL condensate;

(c) A combination of a condensate pre-demethanizer and a flexible condensate deethanizer in tandem to completely demethanize the condensate and simultaneously reduce the ethane content herein to a desirable level to get the deep-cooled purified NGL condensate;

(d) A flexible absorber to recover NGL from the deepdehydrated gas to get the rich oil with desirable ethane content level;

(e) A rich oil pre-demethanizer and a flexible rich oil deethanizer in tandem to completely demethanize the rich oil and simultaneously reduce the ethane content therein to desirable level to get the purified rich oil;

(f) A rich oil fractionator to get the NGL vapor and the regenerated lean oil from the purified rich oil;

(g) An energy-integration loop between the rich oil fractionator and the flexible rich oil deethanizer to transport the heat energy of the NGL vapor inside the rich oil fractionator to heat the rich oil inside the flexible rich oil deethanizer;

(h) An NGL vapor compressor to compress the cooled NGL to the required liquefaction pressure; and

(i) An NGL liquefier to liquefy the mixture of the compressed vapor and the deep-cooled purified NGL condensate into final NGL product.

15. A comprehensive hydrocarbon gas separation apparatus of claim 14 wherein in item (b) the deep-cooler/ dehydrator is replaced with a solid desiccant deep-dehydrator and an integrated gas expander-compressor in tandem.

16. A comprehensive hydrocarbon gas separation apparatus of claim 14 wherein in item (c), (d) and (e) the flexible deethanizer is made of the integrated dual-functional exchanger-reactor.

17. A comprehensive hydrocarbon gas separation apparatus of claim 14 wherein in item (c) and (e) the combination of a pre-demethanizer and a flexible deethanizer in tandem is replaced with an integrated cascade flexible deethanizer.

18. A comprehensive hydrocarbon gas separation apparatus of claim 14 wherein in item (f) the rich oil fractionator is made of an integrated dual-functional exchanger-reactor. 